\title{
Molekulardynamische Untersuchungen zur Binnendynamik kollabierender Blasen
}

\author{
Dissertation \\ zur Erlangung des Doktorgrades \\ der Mathematisch-Naturwissenschaftlichen Fakultäten \\ der Georg-August-Universität zu Göttingen
}

vorgelegt von

Daniel Alexander Schanz

aus Mannheim

Göttingen 2008 
D7

Referent:

Prof. Dr. Werner Lauterborn

Korreferent:

Prof. Dr. Martin Rein

Tag der mündlichen Prüfung:

08.10.2008 


\section{Inhaltsverzeichnis}

1 Einleitung und Gliederung 1

2 Experimenteller Hintergrund 4

2.1 Kavitation . . . . . . . . . . . . . . . . 5

2.2 Lumineszenz . . . . . . . . . . . . . . . . . . . . . . . 10

2.2.1 Mehrblasensonolumineszenz (MBSL) . . . . . . . . . . . 10

2.2.2 Einzelblasensonolumineszenz (SBSL) . . . . . . . . . . . 10

2.2 .3 Ursachen des Leuchtens . . . . . . . . . . . . . . . . . . . . . . . . . . . .

2.3 Laser-induzierte Blasen . . . . . . . . . . . . . . . . . . . 17

3 Modellierung $\quad 20$

3.1 Modellierungsansätze . . . . . . . . . . . . . . . . . . . 21

3.2 Molekulardynamiksimulation . . . . . . . . . . . . 26

3.2 .1 Hartkugelmodell . . . . . . . . . . . . . . . . . 26

3.2.1.1 Ereignisse . . . . . . . . . . . . . . 26

3.2.1.2 Anfangsbedingung . . . . . . . . . . . . . 27

3.2.1.3 Teilchenkollisionen . . . . . . . . . . . . . . . . . 28

3.2.1.4 Wandkollisionen ............ . . . . 35

3.2.1.5 Zelleinteilung . . . . . . . . . . . . . 36

3.2 .2 Systemabhängige Einheiten . . . . . . . . . . . . . . . 37

3.2.3 Skalierung der Simulationsteilchen . . . . . . . . . . . . 37

3.2.4 Bestimmung der hydrodynamischen Größen . . . . . . . . 39

3.2.5 Bestimmung des Teilchendurchmessers . . . . . . . . . . 41

3.2.6 Glättung der Messwerte . . . . . . . . . . . . . . . . . . . 42

3.3 Blasendynamik . . . . . . . . . . . . . . . . . . . . 44

3.3.1 Blasenrandbewegung: Rayleigh-Plesset-Gleichung . . . . . 44

3.3.2 Festlegung der Simulationszeitspanne . . . . . . . . . . . . 45

3.4 Lichtemission . . . . . . . . . . . . . . . . . . . . . . 48 
4 Einfluß der Parameter des MD-Modells $\quad 50$

4.1 Randbedingung . . . . . . . . . . . . . . . . . . . 51

4.1 .1 Druck am Blasenrand . . . . . . . . . . . . . . . . 51

4.1.2 Wärmeaustausch mit dem Medium ... . . . . . . . . 51

4.1.2.1 Der thermische Akkomodationskoeffizient $\alpha_{t} \ldots$. 53

4.2 Kondensation und Verdampfung von Wasserdampf . . . . . . . . 63

4.2 .1 Vapor trapping . . . . . . . . . . . . . . . . . 65

4.2.2 Der $\mathrm{H}_{2} \mathrm{O}$-Akkomodationskoeffizient $\alpha_{v} \ldots \ldots \ldots$

4.2.2.1 Temperaturunabhängiges $\alpha_{v}$. . . . . . . . . . . 67

4.2.2.2 Temperaturabhängiges $\alpha_{v} \ldots \ldots$. . . . . . . 73

4.2.3 Einfluß auf die Dynamik des Blaseninneren . . . . . . . . . 75

4.2.4 Vergleich mit Kontinuumsmechanik . . . . . . . . . . . . 77

4.2.5 Rotationsfreiheitsgrade . . . . . . . . . . . . . 80

4.3 Chemisches Modell . . . . . . . . . . . . . . . . . . . . . . . 82

4.3 .1 Reaktionsarten ................ . . 83

4.3.2 Chemische Entwicklung im Hauptkollaps . . . . . . . . . . 87

4.3 .3 Langzeitentwicklung . . . . . . . . . . . . . . . . . 96

4.4 Genauigkeit der Approximation . . . . . . . . . . . . . . . . . 103

4.4.1 Einfluß der Simulationsteilchenanzahl . . . . . . . . . . 103

5 Ergebnisse für Sonolumineszenzblasen $\quad 110$

5.1 Variation der Wassertemperatur . . . . . . . . . . . . . . . 111

5.2 Variation des Anregungsdruckes . . . . . . . . . . . . . . . . . . 119

5.3 Verschiedene Edelgase . . . . . . . . . . . . . . . . . . 125

5.3.1 Segregation des Blaseninneren . . . . . . . . . . . . . . 131

5.3 .2 Edelgasgemische . . . . . . . . . . . . . . 137

6 Ergebnisse für Mikro-Blasen 142

6.1 1-MHz-Blasen . . . . . . . . . . . . . . . . . . . 143

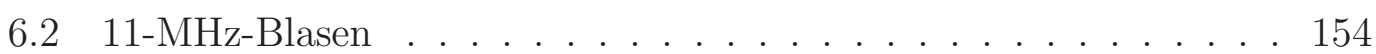

6.2.1 Skalierung mit der Teilchenzahl . . . . . . . . . . . . . . 164

7 Ergebnisse für laser-induzierte Blasen $\quad 169$

7.1 Anfangsbedingung . . . . . . . . . . . . . . . . . . . . 170

7.2 Blase in ruhendem Medium . . . . . . . . . . . . . . . . 174

7.3 Blase in angeregtem Medium . . . . . . . . . . . . . . . . . . . 178

8 Zusammenfassung und Ausblick 187 
$\begin{array}{ll}\text { A Symbolverzeichnis } & 195\end{array}$

A.1 Naturkonstanten und Abkürzungen . . . . . . . . . . . . . . . . 199

$\begin{array}{lr}\text { Literaturverzeichnis } & 200\end{array}$ 
Das Daumenkino in der unteren Ecke der Seiten mit ungerader Seitenzahl zeigt die Entwicklung der Temperatur in einer mit einer Frequenz $\nu_{a}=11 \mathrm{MHz}$ bei einem Druck $P_{a}=100$ bar angeregten Blase mit Ruheradius $R_{0}=130 \mathrm{~nm}$. Genauere Informationen zu dieser Rechnung finden sich in Kap. 6.2.

Die maximale Blasentemperatur (entspricht der Farbe 'weiß' in den Plots des Daumenkinos) beträgt ca. 450.000 K. Eine entsprechenden Farbskala ist in Abb. 6.11, a angegeben. Dargestellt ist ein Zeitraum von $\pm 2,7$ ps um den minimalen Radius, der Bildabstand beträgt 50 fs.

Das Daumenkino ist von der letzten Seite ausgehend nach vorne durchzublättern. 


\section{Kapitel 1}

\section{Einleitung und Gliederung}

Gegenstand dieser Arbeit ist die Simulation kollabierender Blasen in Wasser. Speziell werden Einzelblasensonolumineszenz in unterschiedlichen Regimen, Mehrblasensonolumineszenz bei hohen Anregungsfrequenzen und laser-induzierte Blasen mit und ohne akustische Anregung untersucht. Das Mittel der Modellierung ist die Molekulardynamiksimulation harter Kugeln.

In Kapitel 2 werden die Grundlagen der Kavitation und der Sonolumineszenz behandelt sowie die Entwicklung der Erforschung der genannten Erscheinungen dargelegt.

2.1 erläutert das Phänomen der Kavitation. Die Entstehung von Kavitationsblasen wird dargestellt sowie auftretende Effekte angesprochen.

2.2 behandelt das unter gewissen Umständen in Kavitationsblasen auftretende Leuchten gegen Ende des Kollaps - die sog. Kavitations- oder Sonolumineszenz. Die Fälle der Mehrblasensonolumineszenz (MBSL) und der Einzelblasensonolumineszenz (SBSL) sowie Ansätze zur Erklärung des Phänomens werden besprochen.

2.3 befaßt sich mit Blasen, die durch die Fokussierung eines kurzen Laserpulses in einer Flüssigkeit entstehen.

Kapitel 3 stellt Möglichkeiten der Modellierung einer Blase in einer Flüssigkeit dar und erläutert das hier gewählte Modell.

3.1 fasst zunächst verschiedene Möglichkeiten der Modellierung von Blasen in Flüssigkeiten und deren bisher erfolgte Anwendung zusammen. Es wird erläutert, warum hier die Molekulardynamiksimulation zum Einsatz kommt. 
Kap. 1 Einleitung und Gliederung

3.2 erklärt Einzelheiten der Molekulardynamiksimulation und ihre Anwendung auf das vorliegende Problem.

3.3 behandelt die Berechnung der Bewegung des Blasenrandes mit Hilfe der erweiterten Rayleigh-Plesset-Gleichung sowie die Ermittlung des Startpunktes der Simulation.

2.2 stellt das zur Simulation der Lichtemission benutzte Bremsstrahlungsmodell dar.

In Kapitel 4 werden Erweiterungen des grundsätzlichen Hartkugelmodells besprochen. In den einzelnen Unterkapiteln werden zunächst die dem implementierten Effekt zugrunde liegenden Überlegungen und Formeln dargestellt. Daran anschließend werden relevante Ergebnisse angegeben, die sich aus der Erweiterung des Modells ergeben. Die in diesem Kapitel dargestellten Simulationen beziehen sich auf typische Vertreter von Einzelblasensonolumineszenz.

4.1 behandelt den Einfluß unterschiedlicher Randbedingungen auf die Blasenoszillation und die Dynamik des Blaseninneren. Dabei wird vor allem auf den thermischen Akkomodationskoeffizienten $\alpha_{t}$ eingegangen, der den Energieübertrag der Teilchen an die Blasenwand steuert.

4.2 erläutert den Mechanismus der Verdampfung und Kondensation von Wasserdampf an der Blasenwand. Die Sensitivität der Blasenverhältnisse auf den Wasserdampfgehalt wird anhand einer Studie des Akkomodationskoeffizienten $\alpha_{v}$ erforscht.

4.3 befaßt sich mit dem Modell, das die in der Blase auftretenden chemischen Reaktionen darstellt. Der Einfluß dieser Reaktionen auf die Temperaturen der Blase sowie die Langzeitentwicklung der Spezieskonzentrationen werden dargestellt.

4.4 behandelt das Skalierungverhalten der Simulation bei Variation der Teilchenzahl.

Kapitel 5 stellt Ergebnisse dar, die durch verschiedene Parameterstudien unter typischen Einzelblasensonolumineszenz-Bedingungen gewonnen wurden.

5.1 zeigt die Auswirkungen von Änderungen der Wassertemperatur auf die äuBere und innere Blasendynamik. 
5.2 behandelt Simulationen von Blasen, die von verschiedenen Anregungsdrücken bei gleicher Frequenz angeregt werden. Möglichkeiten der Maximierung von Temperatur, Lichtemission und sonochemischer Aktivität werden erörtert.

5.3 beschreibt den Einfluß der Art des in der Blase vorkommenden Edelgases auf die Verhältnisse in der Blase. Eine räumliche Entmischung der Blasenbestandteile wird diskutiert.

Kapitel 6 beschreibt die Simulation sehr kleiner MBSL-Blasen, die mit hohen Frequenzen und Drücken angeregt werden. Die Anzahl an Teilchen in solchen Blasen nähert sich der Anzahl an simulierten Teilchen an. Vergleiche mit Experimenten werden durchgeführt.

6.1 behandelt Simulationen von mit $1 \mathrm{MHz}$ bei 4 bis 5 bar angeregten Blasen. Für verschiedene Ruheradien, Anregungsdrücke und Edelgase werden die Verhältnisse in der Blase dargelegt.

6.2 zeigt Ergebnisse von Rechnungen von Blasen, die mit $11 \mathrm{MHz}$ bei 100 bar angeregt werden. Für verschiedene Ruheradien werden die Blasenoszillation und die jeweiligen Verhältnisse im Kollaps diskutiert.

Kapitel 7 zeigt Ergebnisse von Simulationen von Blasen, die durch Fokussierung eines kurzen Laserpulses in das Blasenmedium erschaffen wurden.

7.1 beschreibt Überlegungen zu geeigneter Anfangsbedingungen zur Darstellung dieses Problems sowie die Ergebnisse der Simulation der Blase in den 10 ns nach dem Laserpuls.

7.2 zeigt die Simulationsergebnisse zur weiteren Entwicklung der erzeugten Kavität in einem ruhenden Medium.

7.3 zeigt für Blasen, die in akustisch angeregtem Wasser erzeugt werden, den Verlauf des Blasenrandes sowie die Binnendynamik im Hauptkollaps. Es werden verschiedene Phasen der Blasenentstehung relativ zum Schallfeld betrachtet.

Kapitel 8 fasst die erhaltenen Ergebnisse zusammen und gibt einen Ausblick auf weitere Simulationsszenarien sowie denkbare Erweiterungen des Modells. 


\section{Kapitel 2}

\section{Experimenteller Hintergrund}

In diesem Kapitel werden die den durchgeführten Simulationen zugrunde liegen-

den Effekte und Experimente dargestellt sowie auf deren bisherige Erforschung eingegangen. 


\subsection{Kavitation}

Als Kavitation bezeichnet man im Allgemeinen eine Hohlraumbildung ${ }^{\dagger}$ in Flüssigkeiten. Dieser Effekt kann durch einen Unterdruck erzeugt werden, der ein spontanes Ausdampfen der Flüssigkeit an sog. Kavitationskeimen bewirkt. Dies kann z.B. in schnell durchströmten Geräten hinter Ventilen oder ähnlichen Engstellen auftreten (hydrodynamische Kavitation). Ein gut untersuchtes Beispiel zeigt sich an den Kanten von schnell drehenden Propellern in Flüssigkeiten, wie z.B. Schiffschrauben (siehe Abb. 2.1). Durch den Unterdruck an der Rückseite (und vor allem an der Kante) des Propellers können sich Kavitationsblasen bilden oder, wie in Abb. 2.1, schlauchartige Kavitäten, die dem Verlauf des Propellers folgen. Auf Dauer kann der Kollaps von Kavitäten in der Nähe befindliche Flächen schädigen, ein bei Schiffsschrauben häufig beobachtetes Problem. Es kann sogar ein Absinken des Wirkungsgrades der Schraube bei Überschreiten einer gewissen Rotationsgeschwindigkeit beobachtet werden, da die auftretende Kavitation das Strömungsfeld stört [1].

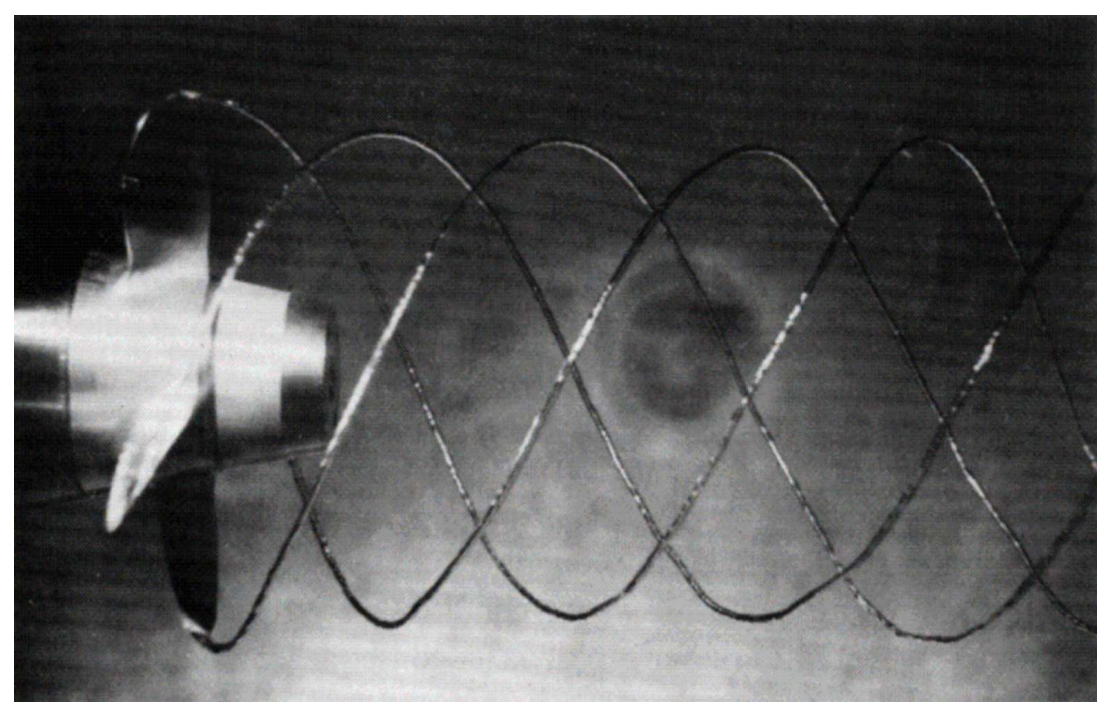

Abbildung 2.1: Aufnahme eines in einer Flüssigkeit rotierenden Propellers. Aus Luther [2]

In der Natur können Kavitationseffekte in Folge von Bodentrockenheit oder Frostereignissen in den Wurzeln, Stämmen und Blättern von Pflanzen auftreten. Wasser wird durch den Transpirationsstrom von den Wurzeln zu den Blättern transportiert. Da in Zeiten von Wassermangel oder bei Frost mehr Wasser transpiriert als nachgeleitet wird, baut sich im Leitgewebe, dem Xylem, eine Zugspannung

\footnotetext{
$\dagger$ lat. cavitas, cavum: Höhle, Hohlraum
} 
Kap. 2 Experimenteller Hintergrund

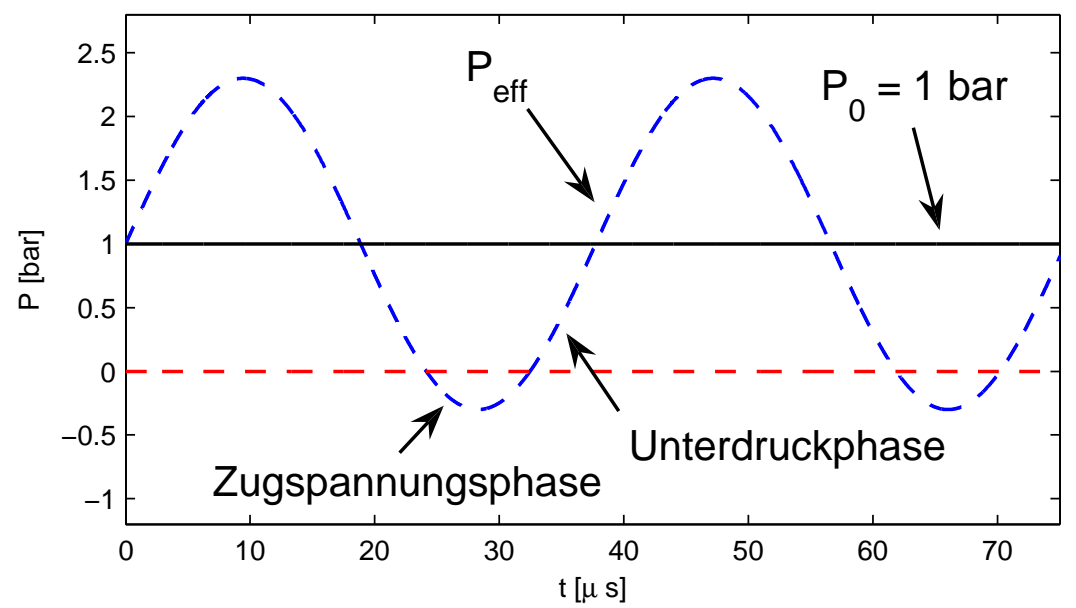

Abbildung 2.2: Verlauf des effektiven Druckes bei Anregung einer Flüssigkeit mit $P_{a}=1,3$ bar bei einer Frequenz $\nu_{a}=26,5 \mathrm{kHz}$. Der Umgebungsdruck $P_{0}$ beträgt 1 bar. Bildung von Kavitäten in den Unterdruck- und vor allem den Zugspannungsphasen.

auf. Überschreitet diese Spannung eine artspezifische Grenze, entstehen Embolien (Kavitäten). Diese führen zur teilweise irreversiblen Blockierung der Leitgefäße und behindern so zusätzlich die Wasseraufnahme. Die Entstehung von Embolien kann während Trockenperioden in situ an Baumstämmen mit Hilfe von Ultraschallmikrofonen verfolgt werden [3].

Kavitation kann im Labor besonders einfach durch ein oszillierendes Schallfeld hervorgerufen werden, welches mit der Periode der Oszillation Unterdruckphasen $\left(P_{\text {eff }}<P_{0}\right.$, der effektive Druck ist kleiner als der Umgebungsdruck) hervorruft, in denen sich Kavitäten durch ein Aufreißen der Flüssigkeit ausbilden können. Besonders ausgeprägt ist dieser Effekt, wenn Zugspannungsphasen auftreten $\left(P_{\text {eff }}<0\right.$, siehe Abb. 2.2). Dies bezeichnet man als akustische Kavitation. Diese Kavitäten können den in der Überdruckphase des Schallfeldes erfolgenden Kollaps überstehen und über viele Anregungsperioden existieren.

Eine weitere Methode der Erzeugung von Kavitationsblasen sind sog. laserinduzierte Kavitationsblasen (oder optische Kavitation), die durch einen lokalen Energieeintrag erzeugt werden. Hierbei wird durch die Fokussierung eines gepulsten ns- oder fs-Lasers in eine Flüssigkeit so viel Energie an diese abgegeben, dass eine Verdampfung und Ionisation (Plasma-Bildung) des Mediums stattfindet [4]. Daraufhin findet aufgrund des hohen Druckes der gebildeten Kavität eine schnelle Expansion mit nachfolgendem Kollaps statt. Wirkt auf das Blasenmedium zusätzlich noch ein Schallfeld, ist ein Übergang in eine stabile Oszillation möglich [5]. 

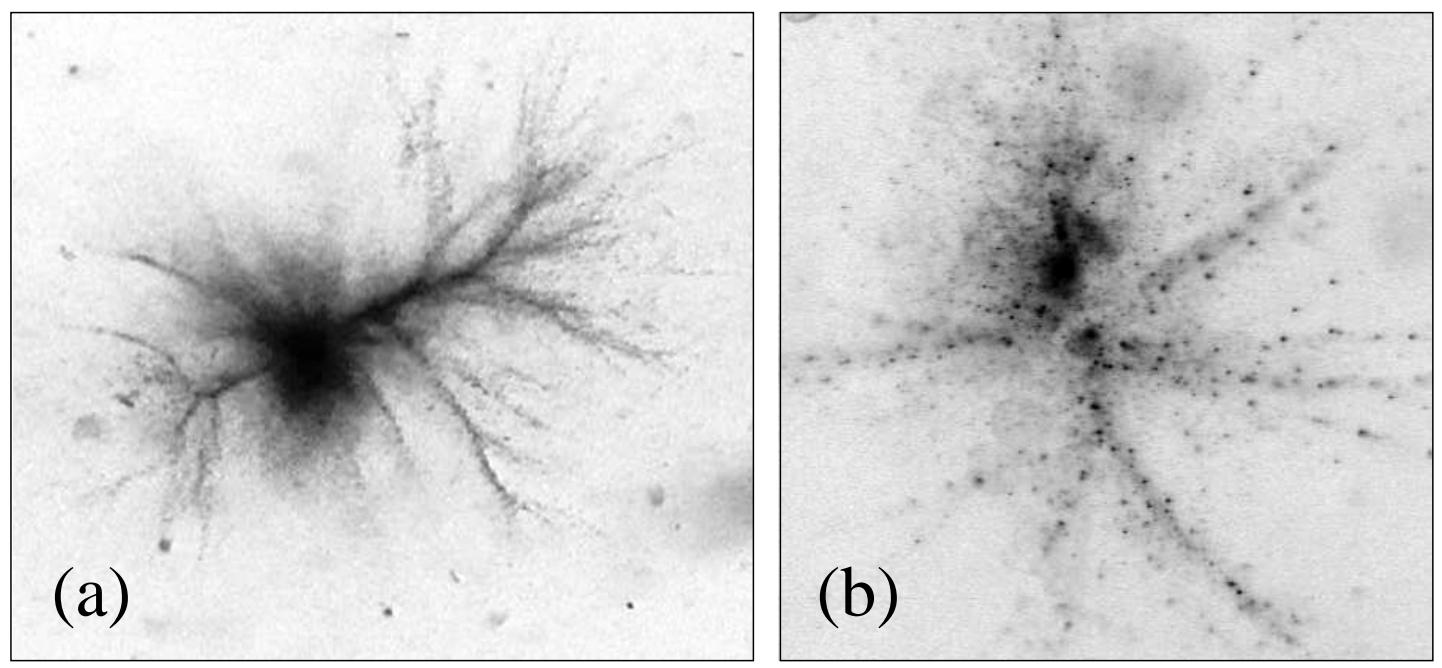

Abbildung 2.3: Streulichtaufnahmen einer sog. Streamer-Figur aus Kavitationsblasen bei $25 \mathrm{kHz}$ Anregung in invertierter Graustufendarstellung. (a) ist eine Langzeitaufnahme (20 ms Aufnahmezeit), die eine Bewegung der Blasen verdeutlicht, erstellt von MetTin [6]. (b) wurde mit kurzer Belichtungszeit ( $5 \mu \mathrm{s})$ aufgenommen, um einzelne Blasen identifizieren zu können. Aufgenommen von LUTHER [7].

Die Blasen sind vor allem in den Schwingungsbäuchen des Schallfeldes zu finden und bewegen sich in z. T. komplexen Figuren (siehe z.B. Abb. 2.3). Eine Abhandlung über die räumliche Organisation von Kavitationsblasen findet sich in [6]. Die Bewegung der Blasen in der Flüssigkeit wird durch die Interaktion der Blasenoszillation mit dem Schallfeld (Primäre und Sekundäre Bjerkneskraft) hervorgerufen [8][7][9].

Bei dem starken Kollaps von Kavitationsblasen kann kurz vor Ende des Hauptkollaps ein Leuchten ausgesandt werden, der Effekt der Kavitationslumineszenz. Diese ist vor allem unter Laborbedingungen bei Schallanregung zu beobachten. In diesem Fall spricht man von Sonolumineszenz. Je nach Anzahl der beteiligten

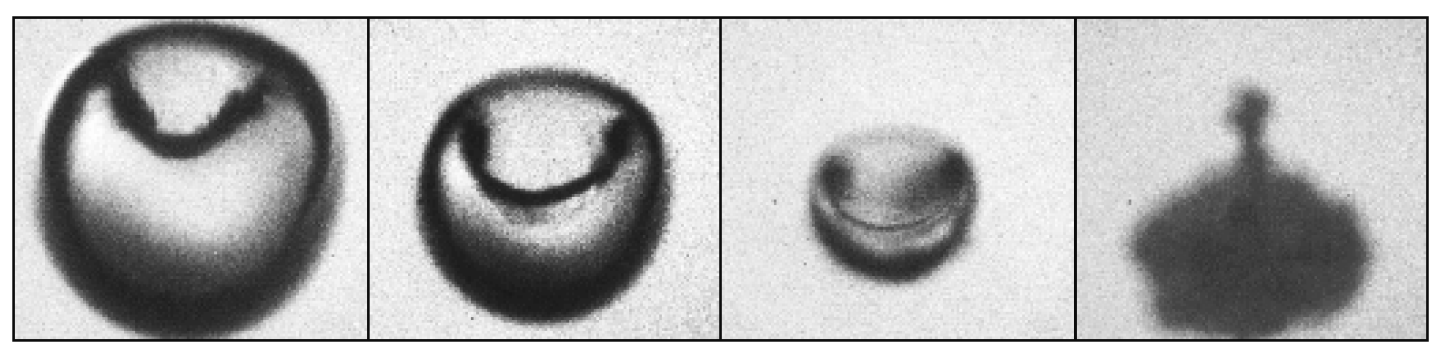

Abbildung 2.4: Aufnahme einer kollabierenden Kavitationsblase, die einen Jet in Richtung einer festen Grenzfläche ausbildet. Die Grenzfläche befindet sich unter der Blase, die Aufnahme wurde unter einem Blickwinkel von $45^{\circ}$ von oben erstellt. Aufnahme von LindAu [10] 
Kap. 2 Experimenteller Hintergrund

Kavitationsblasen werden die Mehrblasensonolumineszenz (MBSL, 'Multi Bubble SonoLuminescence') und die Einzelblasensonolumineszenz (SBSL, 'Single Bubble SonoLuminescence') unterschieden. Diese beiden Effekte werden in den nächsten Kapiteln dargestellt.

Der Kollaps einer Kavitationsblase muß nicht radialsymmetrisch verlaufen: Besonders bei Kollapsen in der Nähe von festen Grenzflächen beobachtet man eine Einstülpung der Blase auf der der Grenzfläche abgewandten Seite (siehe Abb. 2.4). Diese Einstülpung kann sich durch die gesamte Blase bewegen und gegen Ende des Kollaps die gegenüberliegende Blasenwand durchschlagen. Man spricht von einem Jet. Dieser ist auf die feste Grenzfläche gerichtet, weist sehr hohe Geschwindigkeiten auf und kann zu einer Schädigung der Grenzfläche führen. Ein in eine kavitierende Flüssigkeit gehaltenes Stück Aluminiumfolie wird innerhalb weniger Sekunden durch die auftreffenden Jets durchlöchert (siehe Abb. 2.5). In Ultraschallbädern wird derselbe Effekt, mit geringeren Anregungsdrücken, zur Reinigung eingesetzt. Die Energie der kollabierenden Blasen entfernt Verunreinigungen von Gegenständen - auch an schwer zu erreichenden Stellen. Diese Art der Reinigung wird z.B. gern bei Optikern zur Säuberung von Brillen genutzt.

In der Medizin wird die Kavitation zur Zertrümmerung von Blasen- und Nierensteinen ohne Eingriff in den Körper eingesetzt (Stoßwellenlithotripsie).

Die experimentelle Untersuchung der Sonolumineszenz und allgemein der Dynamik von Kavitationsblasen erweist sich als schwierig - die relevanten Zeitskalen sind äußerst kurz (einige ps), die räumliche Ausdehnung im Kollaps umfaßt maximal einige $\mu \mathrm{m}$. Die umgebende Flüssigkeit sowie die Interaktion der Kavitationsblasen erschwert experimentelle Messungen weiter. 


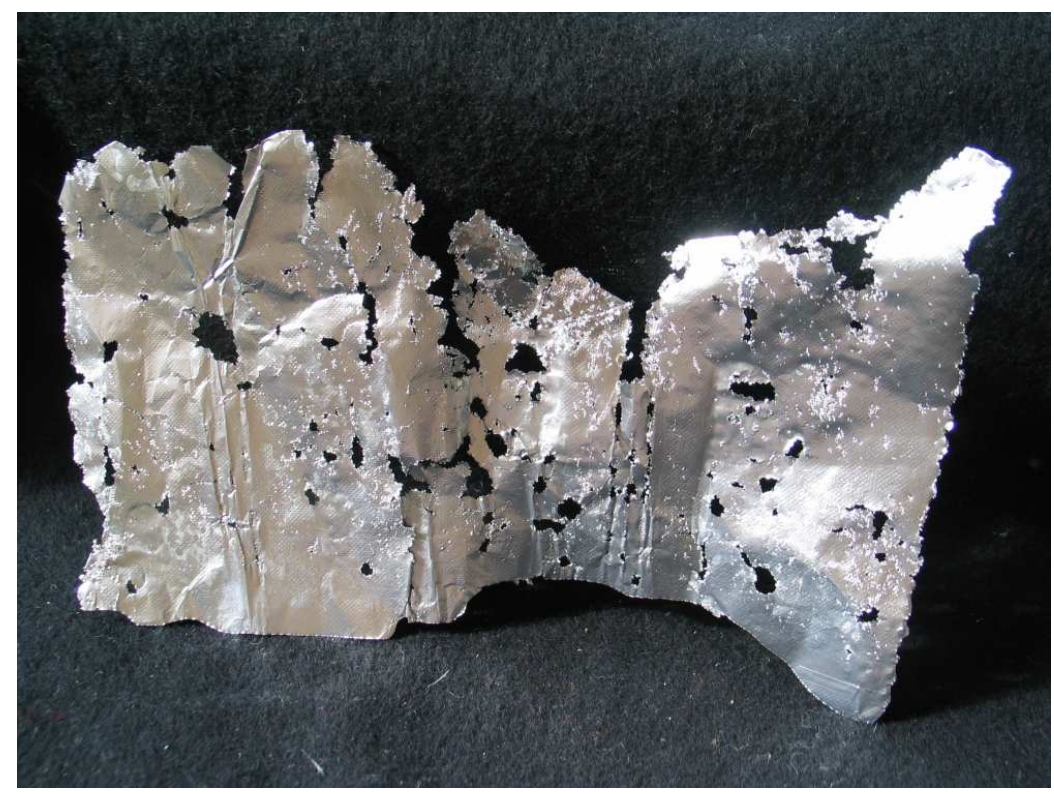

Abbildung 2.5: Eine durch Einwirkung von Kavitationsblasen durchlöcherte Aluminiumfolie. 
Kap. 2 Experimenteller Hintergrund

\subsection{Lumineszenz}

Im Folgenden werden die beiden Arten der im Labor auftretenden Lumineszenz von Blasen - Mehrblasensonolumineszenz und Einzelblasensonolumineszenz - vorgestellt, sowie auf Theorien der Lichtentstehung eingegangen.

\subsubsection{Mehrblasensonolumineszenz (MBSL)}

Bei Untersuchungen von Ultraschallbädern fanden MARINESCO und TrILlaT 1933, dass Ultraschallfelder in Flüssigkeiten in der Lage sind Fotoplatten zu schwärzen. FREnTzEL und SchulTES wiesen binnen Jahresfrist nach, dass von der beschallten, kavitierenden Flüssigkeit ein schwaches Leuchten ausgeht. HARVEY nannte den Effekt 'Sonolumineszenz' - die Entstehung von Licht aus Schall [11]. Der Begriff 'Mehrblasensonolumineszenz' (MBSL) wurde für diesen Effekt erst mit der Entdeckung der Einzelblasensonolumineszenz (siehe nächstes Kapitel) eingeführt.

MBSL läßt sich bei der Beschallung einer geeigneten Küvette durch ein Ultraschallfeld als schwaches Leuchten der entstehenden Kavitationsblasenwolken beobachten. Es läßt sich gut durch Langzeitbelichtungen von Fotographien sichtbar machen, durch Beigabe von Luminol verstärkt sich das Leuchten, so dass man es in einem abgedunkelten Raum nach einer kurzen Eingewöhnzeit mit dem bloßem Auge gut erkennen kann. Leuchtende Blasen dieser Art wurden von Domnitch und Gelfand in Kunstprojekten zur Visualisierung von Schall und Musik eingesetzt [12]. Abb. 2.6 zeigt ein Beispiel einer lumineszierenden Blasenwolke.

MBSL-Blasen überleben viele Perioden der Anregung, sind aber prinzipiell transient. Ihr Leben kann durch Fragmentation, langsame Diffusion der Blasenbestandteile in die Flüssigkeit oder Zusammenschluß mehrerer Blasen und anschließendem Aufsteigen beendet werden.

\subsubsection{Einzelblasensonolumineszenz (SBSL)}

Im Jahre 1989 gelang es GAITAN in einer schallangeregten Flüssigkeit eine einzelne Licht emittierende Blase zu erzeugen [13] [14]. Zu diesem Zweck entgaste er die benutzte Flüssigkeit auf ca. 20\% der Sättigungskonzentration von Luft, um die Kavitation bei Schallanregung zu minimieren. Bei einem Regime von $P_{a} \approx$ 


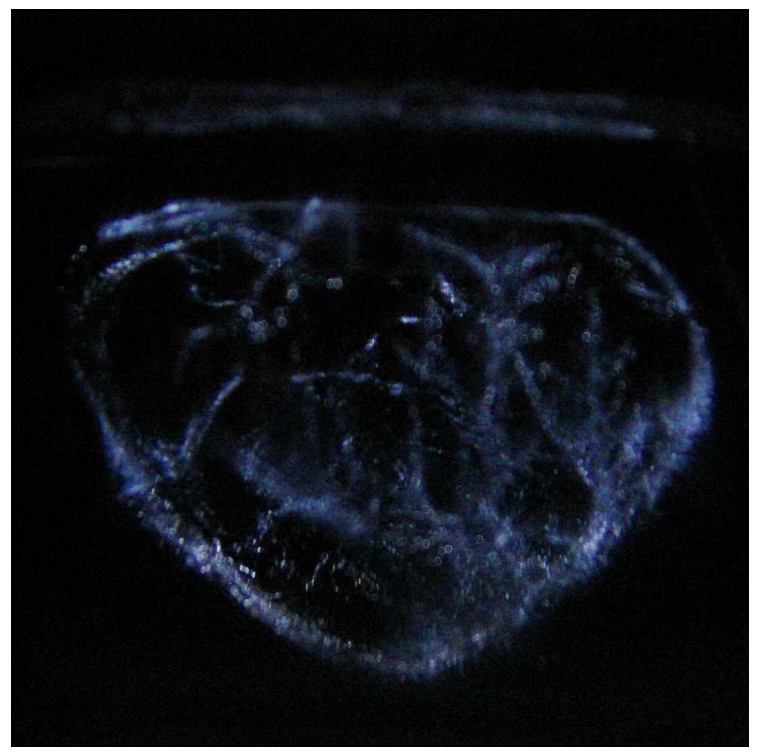

Abbildung 2.6: MBSL: Das Leuchten von Kavitationsblasen in einer ultraschallangeregten Flüssigkeit (Schwefelsäure). Belichtungszeit 1/9 s. Abbildung von Geisler und Lepoint [4].

1,2 - 1,4 bar (bei Umgebungsdruck $P_{0}=1$ bar) und resonanter Anregung war es möglich, eine einzelne, injizierte Blase in einem Druckbauch ortsstabil zu halten. Die so gefangene Blase vollführt, der Anregungsamplitude folgend, radiale Schwingungen und emittiert bei jedem Kollaps einen Lichtblitz. Die Lebensdauer einer solchen Blase ist nahezu unbegrenzt, sie kann im Labor über mehrere Tage auf ihrer Position gehalten werden. Abb. 2.7 zeigt das Leuchten einer solchen SBSL-Blase.

Die Lumineszenz einer SBSL-Blase ist deutlich größer als die von MBSL-Blasen: Das Licht einer SBSL-Blase läßt sich in einem abgedunkelten Raum gut erkennen, während das Leuchten von MBSL-Blasen diffus ist - einzelne Blasen lassen sich mit bloßem Auge nicht erkennen.

Die Entdeckung von SBSL führte zu einer deutlich besseren Untersuchbarkeit des Phänomens der Sonolumineszenz: Die verstärkte Lichtemission läßt mittels spektraler Untersuchungen genauere Rückschlüsse über das Blaseninnere zu. Desweiteren ist die Bewegung der Blase hier nicht durch die gleichzeitige Bewegung von Nachbarblasen überlagert, die eine experimentelle Untersuchung des Blasenkollaps verhindern, bzw. die Schwingung der Blase stören können. Die Bewegung des Blasenrandes wurde durch Streuung von Laserlicht sichtbar gemacht [15], später auch durch direkte Fotographie [16]. Es wurde klar, dass die Schwingung der Blase tatsächlich der über die Rayleigh-Plesset-Gleichung (siehe Abb. 3.3) 


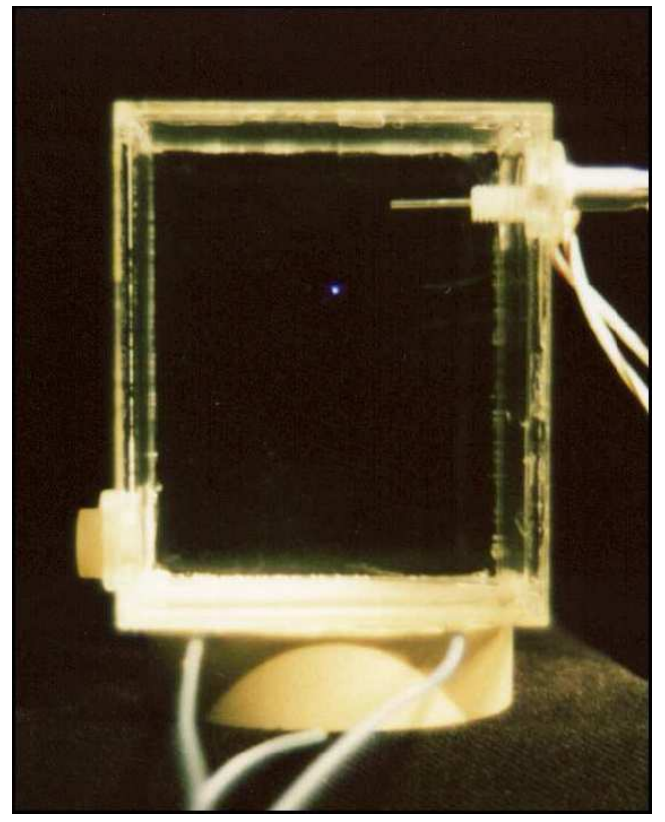

Abbildung 2.7: Fotographie einer in einer Blasenfalle schwingenden SBSL-Blase. Langzeitaufnahme mit 20 Minuten Belichtungszeit, aufgenommen von GEISLER[4].

vorhergesagten entspricht. Über die Entstehung der Lichtemission und den Zustand des Blaseninneren gab es viele Spekulationen und Ansätze. Mehrere Arbeitsgruppen [17][18][19][20] brachten die Idee ins Spiel, dass eine konvergierende sphärische Stoßwelle im Innern der Blase zu einem sehr kleinen, heißen Zentrum führt, was die sehr kurze Dauer des Lichtpulses erklärt (es waren Zeiten von weniger als 50 ps gemessen worden [21]). In Simulationen wurden für ein solches Energiefokussierungssystem Temperaturen im Bereich von $10^{8} \mathrm{~K}$ vorhergesagt. Die Aussicht auf solch hohe Temperaturen ließ sogar die Existenz von im Inneren der Blasen ablaufenden nuklearen Fusionsreaktionen möglich erscheinen [22].

Messungen des Spektrums des bei SBSL emittierten Lichts ergaben, dass es aus einem Kontinuum zusammengesetzt ist - spektrale Linien, wie sie bei MBSLBlasen zu sehen sind, treten nicht auf. Das Fehlen eines Linienspektrums bei SBSL-Blasen deutet auf höhere Temperaturen hin, da das von atomaren Übergängen ausgesandte Licht durch Kontinuumsemissionen (wie z.B. Bremsstrahlung) überlagert wird. HiLler konnte ein Schwarzkörperspektrum an des gemessene Spektrum anfitten, aus dem er schloß, dass die Temperatur mindestens $25.000 \mathrm{~K}$ beträgt [23].

Später wurde gezeigt, dass die Dauer des Lichtpulses deutlich länger sein kann als zunächst angenommen: GompF schätzte die Dauer zwischen 60 und 250 ps ab 
[24]. Ähnliche Ergebnisse wurden von Pecha [25] und Hiller erzielt [26]. Diese Werte ließen das Stoßwellenmodell als nicht geeignet erscheinen, um die Sonolumineszenz zu erklären. VuONG und SzERI fanden in numerischen Simulationen einer SBSL-Blase keine Stoßwellen in der Blase, wenn diffusive Transporteffekte berücksichtigt werden [27]. YASuis Modell ergab, dass sich SBSL-Blasen nahezu uniform durch quasi-adiabatische Kompression erhitzten, ca $10^{4} \mathrm{~K}$ erreichen und das Leuchten von der gesamten Blase ausgeht [28].

Die Intensität des Leuchtens zeigt eine starke Abhängigkeit von diversen Parametern: BARBER zeigte, dass die Lichtemission mit Erhöhung der Wassertemperatur stark abnimmt [29], Weninger fand eine Abhängigkeit von der Konzentration des in der Flüssigkeit gelösten Gases [30]. HiLler konnte nachweisen, dass die Art des gelösten Gases einen starken Einfluß auf die Leuchteigenschaften der Blase hat: Wenn nicht Luft in der Flüssigkeit gelöst wird, sondern eine Mischung aus Stickstoff und Sauerstoff, ist keinerlei Leuchten zu beobachten - erst die Zugabe eines Edelgases führt zu Lumineszenz [31]. Die Konzentration dieses Edelgases bestimmt die Stärke des Leuchtens, maximal wird sie bei ca. $1 \%$.

LÖFSTEDT wies darauf hin, dass eine Sättigung der Flüssigkeit mit Luft eines Partialdruckes von 200 Torr ein lumineszentes Verhalten hervorrief, obwohl ein Diffusionsgleichgewicht zwischen Innerem der Blase und dem in der Flüssigkeit gelösten Gas sehr viel niedrigere Partialdrücke fordert - ungefähr 3 Torr [32]. Er schloß daraus, dass es einen noch unbekannten Mechanismus des Massenstroms im System einer SBSL-Blase geben muß. Eine Theorie der Funktionsweise dieses Mechanismus wurde 1996 von LOHSE vorgeschlagen [33]. Er argumentierte, dass eine SBSL-Blase hauptsächlich aus Argon besteht und der Partialdruck dieses Edelgase für das Diffusionsgleichgewicht ausschlaggebend ist. Dies sei der Fall, da die Hauptbestandteile von Luft - Stickstoff und Sauerstoff - während des heißen Kollaps der Blase dissoziiert und so in wasserlösliche Substanzen umgewandelt werden, die die Blase verlassen können. Übrig bleibt das Edelgas Argon, welches in Luft einen Stoffmengenanteil von ca. $1 \%$ hat. Der Partialdruck von Argon in Luft eines Druckes von 200 Torr beträgt also 2 Torr, ein Wert der den von Löfstedt geforderten 3 Torr sehr nahe kommt. Lohses Hypothese wurde in den darauffolgenden Jahren experimentell (z.B. [34][35]) und theoretisch (z.B. [36]) mehrfach bestätigt.

Ein Faktor, der für den Zustand des Blaseninneren eine sehr große Rolle spielt, ist die Verdampfung und Kondensation von Wasser während der Blasenoszillation (wenn Wasser als Medium verwendet wird). КАматн entwickelte schon 1993 ein 
chemisches Modell für die Dissoziation von Wasser und die entstehenden Produkte. Yasui betonte die von Wasserdampf bewirkte Reduktion der Temperaturen durch endotherme Reaktionen sowie durch die Vergrößerung der Teilchenzahl [28][37]. Storey und Szeri zeigten, dass im schnellen Kollaps der Wasserdampf nicht genug Zeit hat an der Blasenwand zu kondensieren und signifikante Mengen in der Blase gefangen bleiben [38].

Eine umfassende Darstellung zur SBSL findet sich in dem exzellenten Review von BRENNER [39].

\subsubsection{Ursachen des Leuchtens}

Die Entdeckung von SBSL gab der Wissenschaft zwar eine Möglichkeit an die Hand, das Phänomen der Sonolumineszenz genauer zu untersuchen - definitive Aussagen über die Temperaturen in der Blase und den Lichtentstehungsmechanismus konnten bisher aber noch nicht getätigt werden.

In Abhängigkeit der in der Blase vermuteten Temperaturen gab es viele Ansätze der Erklärung des Leuchtens. LEPOINT machte eine dielektrische Entladung des Gases im Inneren der Blase für die Lichtentstehung verantwortlich [40]. PROsPERETTI vermutete, dass das harte Auftreffen eines Jets auf der gegenüberliegenden Blasenwand zu Fraktolumineszenz führt [41]. Schon 1952 wurde von Griffing die Chemilumineszenz ins Feld geführt, also ein Leuchten, das durch die chemischen Reaktionen im Blaseninneren hervorgerufen wird [42]. YAsui griff diesen Gedanken für MBSL-Blasen wieder auf [43]. FrommHold führte die Lumineszenz wiederum auf Dipole zurück, die bei Kollisionen der Teilchen induziert werden [44]. Später vermutete er, genauso wie Moss, die Bremsstrahlung von Elektronen aus dem entstehenden Plasma, die durch Kollisionen mit neutralen Edelgasatomen beschleunigt werden, als Ursache des Leuchtens [45][46]. Mit Bekanntwerden der Erkenntnisse, dass die Pulslänge des Leuchtens deutlich länger ist als anfangs angenommen (siehe oben), verloren diejenigen Modelle ihre Grundlage, die eine extrem kurze Leuchtdauer vorhersagten. Es rückten wieder 'einfache' Modelle in den Vordergrund - wie die adiabatische Erwärmung aufgrund des schnellen Kollaps, welcher keinen Wärmeausgleich der Blase mit der Umgebung gestattet. Zu diesen Modellen gehören die angesprochenen Bremsstrahlungsmodelle [47]. Ein von Wu und RoBerTs formuliertes Modell dieser Art wird in der vorliegenden Arbeit zur Simulation der Lumineszenz verwendet [17][48]. 
Das Spektrum von MBSL-Blasen unterscheidet sich von dem einer SBSL-Blase durch das Vorhandensein spektraler Linien, wie Matula zeigte [49]. Dieser Unterschied ließ zunächst den Gedanken aufkommen, den zwei Phänomenen lägen unterschiedliche Ursachen zu Grunde. Matula selber fand später, dass die Pulslängen von SBSL und MBSL vergleichbar sind und schloß, dass die Entstehungsmechanismen identisch sind. YASUI schlug vor, dass sich die beiden Blasenarten vor allem durch die erreichten Temperaturen unterscheiden [50]. Das Vorhandensein von sowohl Linien- als auch Kontinuumsspektrum bei MBSL erklärte er durch die Verschiedenheit der auftretenden Blasen, so dass einige von diesen sehr wohl die für das Auftreten eines kontinuierlichen Spektrums benötigten hohen Temperaturen aufweisen können.

2001 fand Young schließlich doch Linien in dem Leuchten von SBSL-Blasen: Indem er den Anregungsdruck immer weiter reduzierte (bis kurz vor die Schwelle, an der SL einsetzt), konnte er das Spektrum immer schwächer leuchtender SBSLBlasen aufzeichnen. Das Leuchten wurde bei den letzten Messungen so schwach, dass er über mehrere Tage Photonen zählen mußte, um auswertbare Ergebnisse zu erhalten. Ab einer bestimmten Schwelle der Anregung ist eine OH-Linie zu erkennen, die umso deutlicher hervortritt, je schwächer die Gesamtemission ist (siehe Abb. 2.8). Dieses Ergebnis zeigte endgültig die Identität der Lichtentstehung von

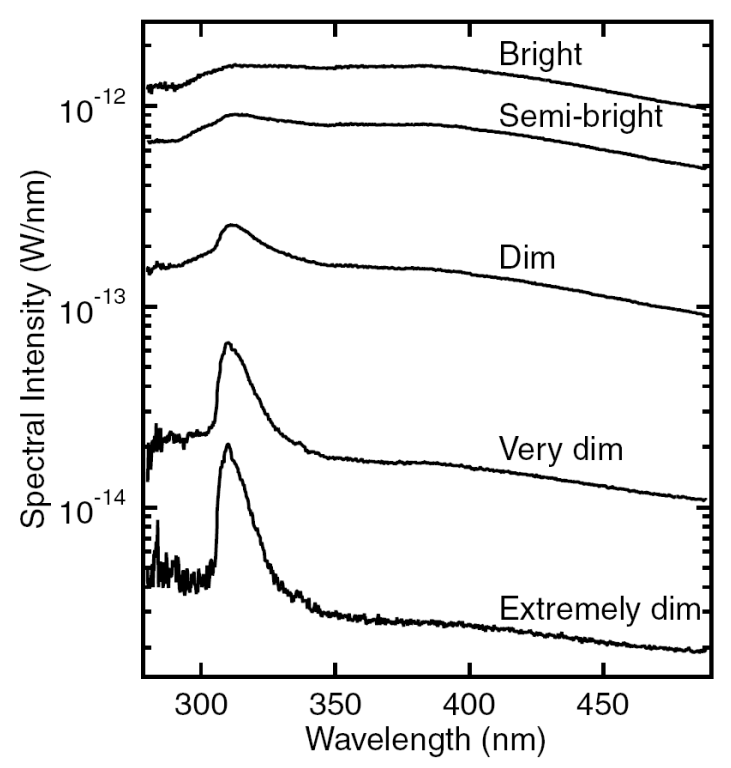

Abbildung 2.8: Spektrum einer SBSL-Blase in Abhängigkeit des Anregungsdruckes, für fünf Helligkeitsklassen. Klar tritt der OH-Peak bei niedrigen Intensitäten zutage. Abb. entnommen aus [51]. 
SBSL und MBSL.

Flannigan und Suslick fanden im Spektrum von SBSL-Blasen in Schwefelsäure erstmals Hinweise auf Atom-Emissionen von Argon. Diese Ergebnisse waren der erste experimentelle Nachweis eines schwach ionisierten Plasmas im Inneren der Blase [52]. 


\subsection{Laser-induzierte Blasen}

In der optischen Kavitation werden durch die Fokussierung eines Laserpulses in eine Flüssigkeit transiente Blasen erzeugt. Der die Blasenbildung auslösende Effekt ist der optische Durchbruch [53][54][55].

Bei der Fokussierung eines Laserstrahles in einem transparenten Medium ist es möglich, lokal sehr große Intensitäten zu erreichen. Hierdurch können im Fokusgebiet nichtlineare Ionisationsprozesse (Multiphotonen- und Kaskadenionisation) ausgelöst werden. Es wird ein Plasma gebildet und die Energie des Laserstrahls in kinetische Energie der Elektronen und Ionen umgewandelt. Wird eine gewisse Schwelle der Ionisation überschritten - bei der Durchbruchsenergie $E_{t h}$ - kommt es zu einem laser-induzierten Optischen Durchbruch. Durch die nichtlineare Art der Ionisationsprozesse nimmt der Ionisationsgrad oberhalb dieser Schwelle schlagartig zu. Der Wert von $E_{t h}$ ist abhängig von der Länge des Laserpulses $t_{p}$ :

$$
\frac{E_{t h}}{\sqrt{t_{p}}}=\text { const }
$$

Aus diesem Grund werden kurze Laserpulse im ns-, ps oder gar fs-Bereich bei der optischen Kavitation verwendet. Bei fs-Pulsen gilt 2.1 allerdings nicht mehr [4].

Nach dem Puls gibt das hochenergetische Plasma schnell seine Energie an die Teilchen des Fokusvolumens ab, wodurch sich dieses stark erhitzt und ausdehnt. Handelt es sich bei dem bestrahlten Medium um einen Festkörper, bleibt die Struktur rund um den Fokuspunkt thermisch zerstört (Aufschmelzung und Verdampfung). Dies wird z.B. bei der Vitrographie (Glasinnengravur) genutzt. Hier wird ein Laserstrahl auf viele verschiedene Punkte des Inneren eines Glasblocks fokussiert. In jedem Fokuspunkt entsteht ein wenige $\mu \mathrm{m}$ großes Gebiet, in dem das amorphe Glas auskristallisiert und als weißer Punkt sichtbar bleibt. Aus vielen dieser Punkte kann ein 3D-Bild im Inneren des Glases konstruiert werden (siehe Abb. 2.9).

Fokussiert man den Laserstrahl hingegen in eine Flüssigkeit, bildet sich eine mit Gas gefüllte Kavitationsblase, die aufgrund der großen Energie der Teilchen in ihrem Inneren schnell aufschwingt. In der Medizin wird dieser Effekt zum äußerst genauen Schneiden biologischen Gewebes genutzt: Das Aufschwingen einer Blase in einem Gewebe zerreißt dieses am Punkt der Blase und in der nächsten Umgebung. Da die Blasen bei niedrigen Energien nur einige Dutzend $\mu \mathrm{m}$ aufschwingen 


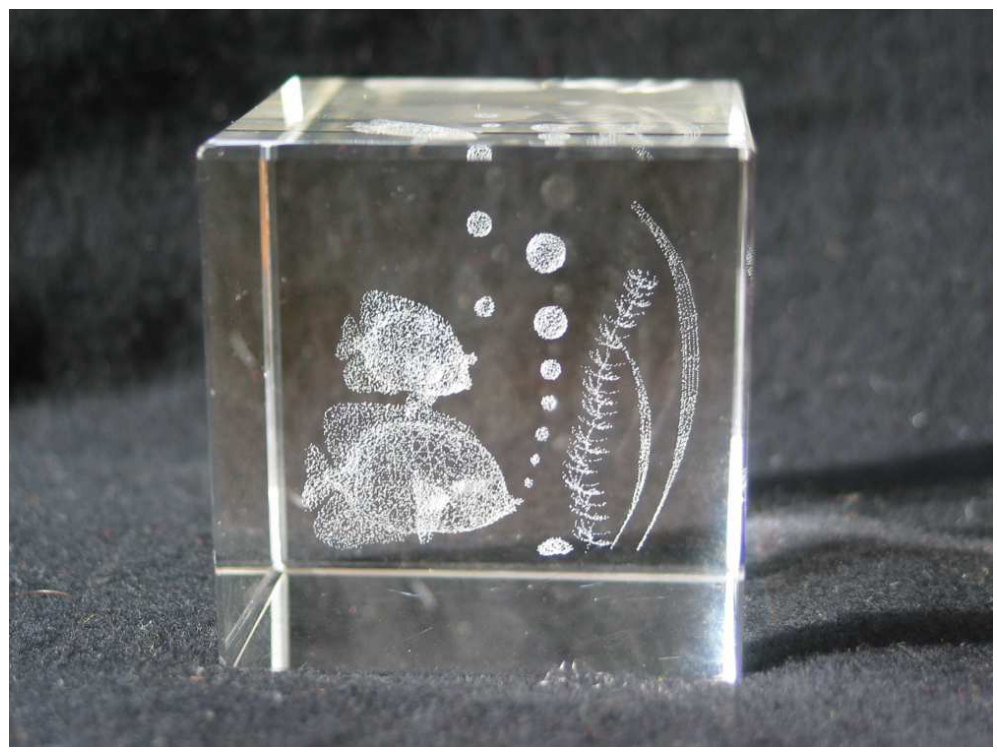

Abbildung 2.9: Vitrographie: Durch Fokussierung eines Lasers an vielen Orten innerhalb eines Glaskörpers kann durch die Effekte des optischen Durchbruchs ein dreidimensionales Bild geschaffen werden.

sind durch Aneinanderreihung von Laserpulsen sehr feine Schnitte möglich [56].

Mittels der laser-induzierten Kavitation ist es möglich, Kavitationsblasen an genau definierten Orten und Zeiten in einer Flüssigkeit entstehen zu lassen. Dies vereinfacht eine experimentelle Untersuchung der bei Kavitation auftretenden Phänomene sehr. Eine solche Blase sendet mit Beginn des Aufschwingens eine starke Stoßwelle in das umgebende Blasenmedium. Die Dynamik der Blasenbewegung und der Stoßwelle konnten fotographisch und holographisch untersucht werden [8]. Auf diese Weise konnte z.B. die Ausbildung eines Jets bei Kollaps der Blase nahe einer Grenzfläche aufgenommen werden. Im Kollaps von laser-induzierten Blasen wurde Kavitationslumineszenz nachgewiesen, deren Stärke klar von der Sphärizität der Blasen abhängt [57][58].

Abb. 2.10 zeigt eine Blase, die von einem fs-Puls erzeugt wurde. Durch nichtlineare Effekte, wie die Selbstfokussierung, sind mit solch kurzen Pulsen erzeugte Blasen anfänglich sehr schlauchförmig. Auch der Kollaps verläuft nicht sphärisch, wodurch bei fs-Blasen keine Lumineszenz beobachtet werden kann. Weitere Bilder sowie Simulationen von laser-induzierten Blasen finden sich in Kap. 7. 


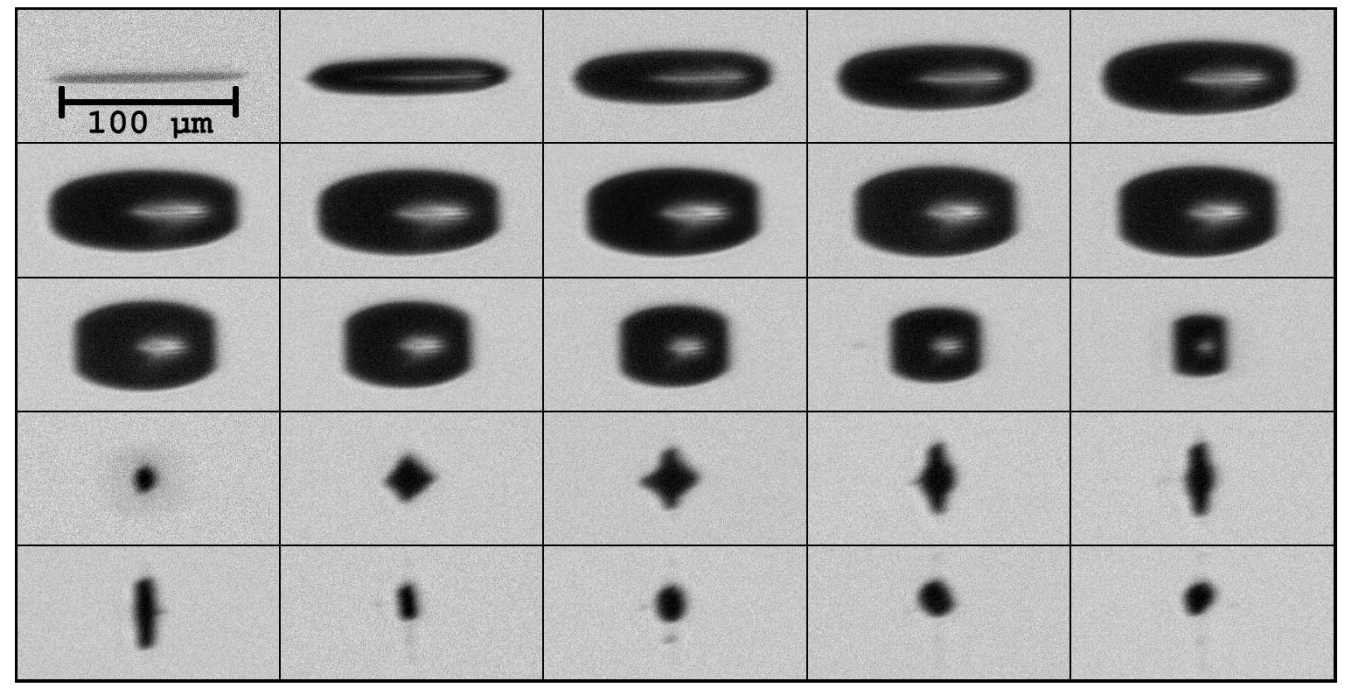

Abbildung 2.10: Fotographische Dokumentation der Entstehung und des Kollaps einer durch einen fs-Laser induzierten Kavitationsblase. Der Laserpuls liegt 100 ns vor dem ersten Bild und kommt mit $0.8 \mu \mathrm{J}$ von links. Der Bildabstand beträgt $400 \mathrm{~ns}$, die Dauer bis zum ersten Blasenkollaps rund $6 \mu \mathrm{s}$. Abbildung von GEISLER [4] 


\section{Kapitel 3}

\section{Modellierung}

In diesem Kapitel werden Möglichkeiten zur Modellierung von Kavitations- und Sonolumineszenz-Blasen dargelegt. Es wird auf die Gründe eingegangen, warum in diesem Fall molekulardynamische Methoden verwendet wurden sowie deren Anwendung auf das vorliegende Problem dargestellt. 


\subsection{Modellierungsansätze}

Die schwere Untersuchbarkeit der Kavitations- und Sonolumineszenz-Phänomene führte zu einer Vielzahl von Modellen, die die Verhältnisse im Blaseninneren darzustellen versuchten. Es erwies sich, dass die Oszillationen der Blase - für einen derart nichtlinearen Oszillator erstaunlich genau - von einer Differentialgleichung, die LORD RAYLEIGH im Jahre 1917 ableitete, beschrieben werden [59]. Erweiterungen dieser Gleichung, wie die Rayleigh-Plesset-Gleichung (3.50) [60], die Gilmore-Gleichung [61] oder die Keller-Miksis-Gleichung [62] wurden in fast allen Modellen verwendet. Somit war eine zufriedenstellende Beschreibung der Bewegung des Blasenrandes gegeben, man konnte sich also vor allem auf eine Simulation des Blaseninneren konzentrieren.

Die Modelle lassen sich grob in zwei Kategorien einteilen: solche, die das Blaseninnere als homogen annehmen und solche, die eine innere Blasendynamik berücksichtigen. Frühe Modelle der ersten Variante [63] nahmen meist eine adiabatische Zustandsgleichung an, was der Erkenntnis zuwider läuft, dass über den größten Teil der Blasenoszillation Energie sehr gut mit dem Blasenmedium ausgetauscht werden kann. Ein erweitertes Modell entwickelte YASUI, der eine Vielzahl an Effekten inkorporierte: Kondensation und Verdampfung von Wasser, Wärmeaustausch mit der Umgebung, eine Grenzschicht um die Blase, die Energie aufnehmen kann, sowie chemische Reaktionen. Yasuis Modell erwies sich als sehr flexibel und produzierte ein breites Spektrum an Ergebnissen (z.B. [28] [50] [64]).

Modelle dieses Typs eignen sich gut zur Vorhersage von Trends und über die Blase gemittelten Durchschnittswerten, wie STOREY und SzERI in einem Vergleich zu einem komplizierteren Modell zeigten [65].

Dennoch ist eine genauere Kenntnis des Zustandes des Blaseninneren erstrebenswert. Zu dessen Darstellung nehmen die meisten Modelle ein lokales Gleichgewicht an und beschreiben die Dynamik über die (nicht-dissipativen) Euler-Gleichungen oder die (dissipativen) Navier-Stokes-Gleichungen sowie die Energie- und Massenerhaltung [66]. Schließt man diese Gleichungen mit einer Zustandsgleichung sowie einer Randbedingung ab, können die interessierenden Größen wie Dichte, Druck und Temperatur orts- und zeitaufgelöst bestimmt werden. Die Modelle lassen sich z.B. durch Effekte wie Ionisation, Dissoziation oder Anregung von Vibrationsfreiheitsgraden erweitern.

In der Anfangszeit der Erforschung der Einzelblasensonolumineszenz waren die Modelle recht einfacher Natur: durch die Vernachlässigung von Viskosität sowie 
Massen- und Wärmeaustausch wurden sehr hohe Temperaturen (bis $10^{8} \mathrm{~K}$ ) und Pulslängen ermittelt, die gut mit dem damaligen Bild der sehr kurzen $(<50$ ps) Lichtblitze übereinstimmten [17]. Starke Stoßwellen wurden in den simulierten Blasen gefunden.

KONDIC erweiterte die Modelle um dissipative Anteile wie Wärmeleitung und Viskosität [67]. Diese Effekte setzten die Temperaturen auf maximal ca. 200.000 K herunter. VuONG und SzERI berücksichtigten den Wärmeübergang in die Flüssigkeit. Sie fanden, dass hierdurch die Ausbildung einer Stoßwelle im Blaseninneren verhindert wird [27].

Die Erfassung von Wasserdampf in den Modellen von Moss sowie Storey und SzERI senkte die berechneten Temperaturen dramatisch ab, da Energie nun in Rotationsfreiheitsgrade und Dissoziation der Wassermoleküle ausweichen kann [68] [38]. Zusätzlich wird der Adiabatenexponent $\gamma$ durch die große Zahl an gefangenen Wasserdampfmolekülen vermindert, was zu einer reduzierten Erwärmung führt. Mit der Implementierung all dieser Details wurden Temperaturen im Bereich $10.000 \mathrm{~K}$ bis $20.000 \mathrm{~K}$ erzielt, die viel besser mit den aktuellen experimentellen Ergebnissen des Spektrums und der Pulslänge des emittierten Lichts übereinstimmen. Neuere Simulationen stammen z.B. von HAUKE [69].

Eine Alternative zur Benutzung der Navier-Stokes-Gleichung zur Darstellung des Blaseninneren als ein Kontinuum ist eine direkte Simulation der Teilchen in der Blase mittels Molekulardynamiksimulationen (MDS). Die Benutzung von MDS bietet gegenüber kontinuumsbasierten Ansätzen einige Vorteile: durch den Teilchenansatz sind Effekte wie Wärmeleitung und Diffusion automatisch enthalten, es muß kein Modell dafür eingeführt werden. Es wird kein lokales thermisches Gleichgewicht vorausgesetzt und die Darstellung von Stößen ist realistischer [66]. Nachteile sind der hohe Rechenaufwand sowie die relativ geringe Anzahl an darstellbaren Teilchen (maximal ca. $10^{7}$ mit aktueller Computerhardware). Das Problem der Teilchenzahl kann durch den Einsatz von sog. kollektiven Teilchen, bei dem ein Simulationsteilchen für einen Verbund aus realen Teilchen steht, umgangen werden. Dieser Ansatz wurde durch die Einführung von Dissipativen Teilchen noch verfeinert [70][71].

Frühe molekulardynamische Simulationen eines Gases in einem kollabierenden kugelförmigen Container stammen von VLAdimiroff und zeigen eine Erhöhung der Temperatur des Zentrums gegenüber der Blasenwand gegen Ende des Kollaps [72]. Matsumoto [73] simulierte die Flüssigkeit als Lennard-Jones-Teilchen ${ }^{\dagger}$ und 
das Gas als Soft-Sphere-Partikel ${ }^{\ddagger}$.

RuUth führte die Simulation einer durch eine sphärische Randbedingung begrenzten Blase durch. Die Teilchen des Blaseninneren wurden durch ein Hartkugelmodell dargestellt (siehe Kap. 3.2.1). Die Bewegung der Blase war durch die einfache Rayleigh-Plesset-Gleichung gegeben, eine Kopplung des Blaseninneren an die Blasenrandbewegung wurde vernachlässigt. Das Modell enthielt eine Ionisierung der Teilchen, die Effekte von Wasserdampf und chemischen Reaktionen blieben hingegen unbeachtet. Es wird vor allem der Einfluß unterschiedlicher Randbedingungen herausgearbeitet [74]. OKUmuRA benutzte LennardJones-Teilchen, um die Entstehung und den nachfolgenden Kollaps einer Blase durch lokale Erhitzung einer Flüssigkeit zu simulieren [75].

Gaspard und Lutsko simulierten den Kollaps einer Kavität in einer Flüssigkeit mit 3 verschiedenen Modellen: Es kamen die einfachen Euler-Gleichungen, die dissipativen Navier-Stokes-Gleichungen sowie ein Modell harter Scheiben, das 2-D-Äquivalent der Hartkugelsimulation, zum Einsatz [76]. Sie fanden, dass die Euler-Gleichungen nicht in der Lage sind, den auftretenden Stoß adäquat aufzulösen: Die Stoßfront hat hier eine Breite von Null, im Moment der Fokussierung im Blasenzentrum werden unendliche Temperaturen und Geschwindigkeiten erreicht. Die Navier-Stokes-Gleichungen und die MD-Simulation können die Stoßfront hingegen deutlich besser darstellen. Die Breite der Front hat eine gewisse Ausdehnung, die erreichten Temperaturen und Geschwindigkeiten bleiben endlich. Es zeigt sich eine erstaunliche Übereinstimmung der beiden Modelle.

Das System einer Sonolumineszenzblase eignet sich aus mehreren Gründen gut für eine Anwendung molekulardynamischer Modelle. Zum einen ist die räumliche und zeitliche Ausdehnung gering: Durch die Größe von maximal ca. $50 \mu \mathrm{m}$ ist die Teilchenanzahl nicht zu groß, um sinnvoll durch kollektive Teilchen dargestellt zu werden; die typische Zeitskala von einigen $\mu$ s (im Kollaps sehr viel kürzer) läßt in einem vernünftigen Rahmen liegende Rechenzeiten zu. Zum anderen treten extreme Bedingungen in der Blase auf, unter denen die Gültigkeit vieler Annahmen, die für eine korrekte Modellierung mit Kontinuumsmodellen gefordert werden, unklar ist. Viele dieser Zusammenhänge (z.B. die Zustandsgleichung oder die Größe von Transportprozesse) müssen aus Bereichen, in denen die Eigenschaften bekannt sind, extrapoliert werden - mit Effekten, die je nach Art der Extrapolation

\footnotetext{
$\dagger$ Teilchen mit einem in der Nähe stark abstoßenden, aber in der Ferne leicht anziehenden Potential der Form $u\left(r_{i j}\right)=\left(d / r_{i j}\right)^{12}-\left(d / r_{i j}\right)^{6}, d$ : Teilchendurchmesser, $r_{i j}$ : Teilchenabstand

$\ddagger$ Teilchen mit einem in der Nähe stark abstoßenden Potential der Form $u\left(r_{i j}\right)=\left(d / r_{i j}\right)^{12}$
} 
variieren. Besonders fraglich ist die Annahme des lokalen thermischen Gleichgewichts, also der Eigenschaft, dass einzelne Teilchen des Systems immer mit ihren benachbarten Teilchen im Gleichgewicht sind - auch wenn es das Gesamtsystem vielleicht nicht ist. Kontinuumsmodelle sind von dieser Annahme abhängig. Bei den in Kavitationsblasen auftretenden Beschleunigungen ( $>10^{11} \mathrm{~g}$ ), Änderungen von Parametern innerhalb einiger ps sowie ggf. vorhandener Stöße ist ein lokales thermisches Gleichgewicht zumindest in den Endphasen des Kollaps nicht vorauszusetzen. Molekulardynamische Rechnungen sind hier deutlich im Vorteil, da der teilchenbasierte Ansatz keine Annahmen über lokale Verhältnisse benötigt. Es läßt sich festhalten, dass eine SBSL-Blase ein gutes 'Testgebiet' für die Möglichkeiten der MDS bietet.

In der vorliegenden Arbeit wurde ein Hartkugelmodell benutzt, um den Kollaps und nachfolgende Oszillationen einer Kavitationsblase zu modellieren. Die Verwendung harter Kugeln anstelle von Partikeln mit ausgedehnten Potentialen ist durch die hohen Temperaturen mit entsprechend hohen Teilchengeschwindigkeiten motiviert. Im Kollaps, dem interessantesten Teil der Simulation, sind die Geschwindigkeiten so hoch, dass schwache, langreichweitige Anteile eines Teilchenpotentials nur eine zu vernachlässigende Wirkung auf die Trajektorien der einzelnen Teilchen hätten. Durch die Verwendung des Hartkugelpotentials tauschen die Partikel nur bei Kollisionen Energie aus, die Trajektorien zwischen diesen verlaufen geradlinig gleichförmig. So wird eine rechenintensive Bestimmung der Teilchenbahnen mit konstantem Zeitschritt vermieden.

Durch Variation der Teilchenparameter lassen sich leicht verschiedene Spezies realisieren. Die Teilchen werden dabei immer als sphärisch angenommen. Molekülen werden Freiheitsgrade der Rotation in Form von Spin rauher Kugeln gegeben, in die Energie ausweichen kann. An der Blasenwand finden sowohl Kondensation und Verdampfung von Wasserdampf, als auch ein Wärmeübergang in die Umgebung der Blase statt. Die chemische Aktivität wird mittels eines Systems aus 25 Reaktionen modelliert.

Die Simulation baut in großen Teilen auf ein von Metten entwickeltes Modell auf, welches in seiner Promotionsarbeit "Molekulardynamik-Simulationen zur Sonolumineszenz" beschrieben wird [77][78]. Die dort dargestellten Ergebnisse werden hier erweitert und durch neue Betrachtungen ergänzt.

Die Programmierung geschah in der Programmiersprache 'C', die grundsätzlichen molekulardynamischen Algorithmen sind dem Standardwerk von RAPAPORT [79] entnommen und wurden dem hier betrachteten Problem entsprechend modifi- 
ziert und erweitert. Eine Übersicht der grundsätzlichen Herangehensweise findet sich in den restlichen Abschnitten dieses Kapitels. Genauere Informationen zur Implementation vieler Details lassen sich [80] entnehmen. 


\subsection{Molekulardynamiksimulation}

Das Innere der Blase wird durch ein teilchenbasiertes Modell dargestellt, dessen zeitliche Entwicklung abhängt von der Art der Interaktion der Partikel, den Randbedingungen und der Anfangsbedingung.

Die Wechselwirkung zwischen den Teilchen ist gekoppelt an die Art des gewählten Teilchenpotentials. In Systemen, die durch niedrige Teilchengeschwindigkeiten gekennzeichnet sind, empfiehlt sich die Verwendung eines kontinuierlichen Potentials (z.B. des Lennard-Jones-Potentials), da die Teilchen lange aufeinander einwirken können. Bei diesem Ansatz müssen die Newton'schen Bewegungsgleichungen kontinuierlich gelöst werden. Eine Alternative stellen Hartkugelpotentiale dar, bei denen die Teilchen nur durch direkte Kollisionen interagieren. Bei hohen Teilchengeschwindigkeiten und -temperaturen stellt diese Methode eine effiziente Alternative dar.

\subsubsection{Hartkugelmodell}

In einer Kavitationsblase kommt es, vor allem im physikalisch interessanten Kollaps, zu sehr hohen Temperaturen und Geschwindigkeiten im Bereich einiger $\mathrm{km} / \mathrm{s}$. Aus diesem Grund wurde für die vorliegende Arbeit ein Hartkugelmodell verwendet, dessen Eigenschaften und Parameter im Folgenden erklärt werden.

\subsubsection{Ereignisse}

Eine Hartkugelsimulation ist nicht auf einen systemweiten festen Zeitschritt angewiesen, da sich die Teilchen zwischen den durch Kollisionen hervorgerufenen Richtungsänderungen auf festen, linearen Trajektorien bewegen. Es reicht, für jedes Teilchen das zeitlich nächste Ereignis zu kennen, zu welchem sich der Geschwindigkeitsvektor des Teilchens ändern wird. Dieser Zeitpunkt wird für jedes Teilchen gespeichert, genauso wie eine persönliche Zeit, welche angibt, wann das Teilchen zuletzt betrachtet wurde.

Alle Ereignisse werden in einem effizienten Ereigniskalender abgelegt. Dies ist eine auf einem binären Baum basierende Datenstruktur, welche ein schnelles Einordnen der Ereignisse, sowie ein schnelles Auffinden des zeitlich nächsten Ereignisses ermöglicht [79]. Nicht nur Teilchen-Teilchen- und Teilchen-Wand-Kollisionen werden als Ereignis dargestellt - auch systemweite Operationen wie die Berechnung 
der hydrodynamischen Größen (siehe 3.2.4), eine Größenänderung der Zelleinteilung (siehe 3.2.1.5) oder die Neubestimmung der Rayleigh-Plesset-Parameter (siehe 3.3.1) sind als Ereignisse mit einer bestimmten Simulationszeit realisiert. So skaliert auch die zeitliche Ausführung dieser Operationen mit der Systemgröße. Eine genauere Darstellung der Implementation des Ereignismodells findet sich in $[80]$.

Das System extrahiert das zeitlich nächste Ereignis aus dem Ereignisbaum, setzt die Systemzeit $t_{S}$ auf die dort angegebene Zeit $t_{E}$ und aktualisiert Koordinaten sowie persönliche Zeit $t_{P}$ des/der betreffenden Teilchen:

$$
\vec{r}_{\text {neu }}=\vec{r}_{\text {alt }}+\vec{v}\left(t_{E}-t_{P, a l t}\right), \quad t_{P, \text { neu }}=t_{E} .
$$

Im Anschluß werden die Auswirkungen des Ereignisses auf das/die Teilchen oder auf das System berechnet sowie sämtliche Ereignisse, an denen die betrachteten Teilchen beteiligt sind, aus dem Baum entfernt. Zuletzt wird mit den neuen Geschwindigkeiten nach auftretenden Kollisionen gesucht und diese in den Baum eingetragen.

Ein Nachteil der ereignisgetriebenen Natur von Hartkugelmodellen sind die komplexen Zusammenhänge der Ereignisse innerhalb des Ereignisbaumes und der daraus resultierenden sehr schweren Parallelisierbarkeit des Algorithmusses [81].

\subsubsection{Anfangsbedingung}

Beim Start der Simulation werden die Teilchen auf einem kubischen Gitter innerhalb der Simulationsregion verteilt. Eine zufällige Verteilung der Teilchen erweist sich als aufwendig, da anfängliche Teilchenüberlappungen vermieden werden müssen. Da das Gas in den Phasen eines großen Blasenradius relativ gleichmäßig die Blase ausfüllt (die Diffusionszeitskala ist klein gegenüber der Zeitskala der Blasenrandbewegung) ist die gewählte Anfangsverteilung unproblematisch.

Die Geschwindigkeiten, die den Teilchen zu Anfang zugewiesen werden, ermitteln sich aus der Maxwell-Boltzmann-Verteilung:

$$
p(\vec{v})=\left(\frac{m}{2 \pi k_{B} T_{\text {init }}}\right)^{3 / 2} \exp \left(-\frac{m \vec{v}^{2}}{2 k_{B} T_{\text {init }}}\right) .
$$

Für die Wahrscheinlichkeitsdichte des Geschwindigkeitsbetrages $p(v)$ ergibt sich 
hieraus:

$$
p(v)=4 \pi\left(\frac{m}{2 \pi k_{B} T_{\text {init }}}\right)^{3 / 2} v^{2} \exp \left(-\frac{m v^{2}}{2 k_{B} T_{\text {init }}}\right) .
$$

Anschließend wird die resultierende Durchschnittsgeschwindigkeit $v_{k}^{\varnothing}$ für jede Koordinate $k$ berechnet und die Geschwindigkeit für alle Teilchen angepaßt, so daß der Massenschwerpunkt ruht:

$$
\Delta v_{k}^{i}=v_{k}^{i}-v_{k}^{\varnothing} / N_{\text {init }} \quad v_{k}^{\varnothing}=\sum_{i} v_{k}^{i} .
$$

Hierbei durchläuft $i$ alle Teilchen. Zur Erzeugung der zufallsverteilten MaxwellBoltzmann-Verteilung wird die Funktion gasdev aus [82] verwendet, welche das Box-Müller-Verfahren implementiert.

Falls die Rechnung zu einem späteren Zeitpunkt des Blasenkollaps gestartet wird, die Blasenwand also bereits eine gewisse Anfangsgeschwindigkeit $v_{W, \text { init }}$ besitzt, wird den Teilchen zusätzlich zu den zufallsverteilten Geschwindigkeiten eine radiale Geschwindigkeitskomponente $u(r)$ in Richtung des Blasenzentrums gegeben:

$$
u(r)=v_{W, i n i t} r / R
$$

Damit fällt der Betrag von $u(r)$ linear zum Zentrum ab, in welchem $u(0)=0$ gilt. Für die Rotationsgeschwindigkeiten zum Start der Simulation gelten analoge Überlegungen.

\subsubsection{Teilchenkollisionen}

\section{Kollisionszeit}

Betrachtet man zwei Teilchen 1 und 2, mit Ortsvektoren $\vec{r}_{1}, \vec{r}_{2}$ und Geschwindigkeitsvektoren $\vec{v}_{1}, \vec{v}_{2}$, so lautet die Kollisionsbedingung dieser Teilchen:

$$
\left(d_{1}+d_{2}\right) / 2=\left|\vec{r}_{12}+\vec{v}_{12} \cdot t_{K}\right|
$$

Dabei sind $\vec{r}_{12}=\vec{r}_{1}-\vec{r}_{2}$ der Abstandsvektor und $\vec{v}_{12}=\vec{v}_{1}-\vec{v}_{2}$ der Geschwindig- 
keitsdifferenzvektor der Teilchen. Man erhält für die Kollisionszeit $t_{K}$ :

$$
\begin{aligned}
\frac{\left(d_{1}+d_{2}\right)}{2} & =\sqrt{\left(\vec{r}_{12}+\vec{v}_{12} \cdot t_{K}\right)^{2}} \\
0 & =t_{K}^{2}+\frac{2\left(\vec{r}_{12} \cdot \vec{v}_{12}\right)}{\vec{v}_{12}^{2}} t_{K}+\frac{\vec{r}_{12}^{2}}{\vec{v}_{12}^{2}-\frac{\left(d_{1}+d_{2}\right)^{2}}{4 v_{12}^{2}}} \\
t_{K} & =-\frac{1}{\vec{v}_{12}^{2}} \cdot\left(\vec{r}_{12} \cdot \vec{v}_{12}+\sqrt{\left(\vec{r}_{12} \cdot \vec{v}_{12}\right)^{2}+\vec{r}_{12}^{2} \cdot\left(\vec{v}_{12}^{2}-\frac{\left(d_{1}+d_{2}\right)^{2}}{4}\right)}\right)
\end{aligned}
$$

Eine Kollision findet nur statt, wenn der Wurzelausdruck reell ist. (3.7) wird für alle potentiellen Kollisionpartner eines Teilchens ausgewertet und alle dabei gefundenen Kollisionszeiten in den Ereignisbaum eingetragen.

Im Folgenden wird die Berechnung der Geschwindigkeitsänderungen bei Kollision zweier Teilchen unter verschiedenen Bedingungen hergeleitet.

\section{Einfache Kollisionen}

Zunächst für eine Kollision ohne Rotationsfreiheitsgrade oder Massenveränderung:

Der Energieerhaltungssatz einer Kollision zweier Teilchen mit den Massen $m_{1}$ und $m_{2}$ sowie den Geschwindigkeiten $\vec{v}_{1}$ und $\vec{v}_{2}$ in einer Dimension läßt sich schreiben als:

$$
m_{1}\left(\vec{v}_{1}-\vec{v}_{1}^{\prime}\right)\left(\vec{v}_{1}+\vec{v}_{1}^{\prime}\right)=m_{2}\left(\vec{v}_{2}^{\prime}-\vec{v}_{2}\right)\left(\vec{v}_{2}^{\prime}+\vec{v}_{2}\right) .
$$

Sowie der Impulserhaltungssatz:

$$
m_{1}\left(\vec{v}_{1}-\vec{v}_{1}^{\prime}\right)=m_{2}\left(\vec{v}_{2}^{\prime}-\vec{v}_{2}\right)
$$

Teilt man (3.8) durch (3.9), erhält man: $\vec{v}_{1}-\vec{v}_{2}=\vec{v}_{2}^{\prime}-\vec{v}_{1}^{\prime}$. Die Relativgeschwindigkeit der beiden Teilchen ändert sich also nicht. Eingesetzt in die Impulserhaltung erhält man schließlich

$$
\vec{v}_{1}^{\prime}=\frac{\vec{v}_{1}\left(m_{1}-m_{2}\right)+2 m_{2} \vec{v}_{2}}{m_{1}+m_{2}}, \quad \text { bzw. } \quad \vec{v}_{2}^{\prime}=\frac{\vec{v}_{2}\left(m_{2}-m_{1}\right)+2 m_{1} \vec{v}_{1}}{m_{1}+m_{2}} .
$$

Bei der Kollision zweier Teilchen im freien Raum wird nur auf der Verbindungsachse der Teilchenzentren Energie ausgetauscht. Es gilt also: $\Delta \vec{v}_{i}=\vec{v}_{i}-\vec{v}_{i}^{\prime}=$ $\vec{v}_{i \perp}+\vec{v}_{i \|}-\vec{v}_{i \perp}-\vec{v}_{i \|}^{\prime}=\vec{v}_{i \|}-\vec{v}_{i \|}^{\prime}$. Dabei bezeichnet $\vec{v}_{i \|}=\left(\vec{v}_{i} \cdot \hat{\vec{r}}_{12}\right) \cdot \hat{\vec{r}}_{12}$ den Anteil der Geschwindigkeit von Teilchen i senkrecht zur Verbindungsachse, $\vec{v}_{i \perp}=\vec{v}_{i}-\vec{v}_{i \|}$ entsprechend den senkrechten Anteil. Hier ist $\hat{\vec{r}}_{12}=\left(\vec{r}_{1}-\vec{r}_{2}\right) /\left|\vec{r}_{1}-\vec{r}_{2}\right|$ ein Ein- 
heitsvektor in Richtung der Verbindungsachse. In diesem System gilt die eindimensionale Gleichung, daher folgt:

$$
\begin{aligned}
\Delta \vec{v}_{1} & =\left(\vec{v}_{1} \cdot \hat{\vec{r}}_{12}\right) \cdot \hat{\vec{r}}_{12}-\frac{\left(\vec{v}_{1} \cdot \hat{\vec{r}}_{12}\right) \cdot \hat{\vec{r}}_{12}\left(m_{1}-m_{2}\right)+2 m_{2}\left(\vec{v}_{2} \cdot \hat{\vec{r}}_{12}\right) \cdot \hat{\vec{r}}_{12}}{m_{1}+m_{2}} \\
& =\frac{2 m_{2}\left(\vec{v}_{1} \cdot \hat{\vec{r}}_{12}\right) \cdot \hat{\vec{r}}_{12}-2 m_{2}\left(\vec{v}_{2} \cdot \hat{\vec{r}}_{12}\right) \cdot \hat{\vec{r}}_{12}}{m_{1}+m_{2}} \\
& =\frac{-2 m_{2}\left(\vec{v}_{12} \cdot \hat{\vec{r}}_{12}\right) \hat{\vec{r}}_{12}}{m_{1}+m_{2}} .
\end{aligned}
$$

mit $\vec{v}_{12}=v_{1}-v_{2}$. Analog erhält man $\Delta \vec{v}_{2}=\frac{-2 m_{1}\left(\vec{v}_{12} \hat{\vec{r}}_{12}\right) \hat{\vec{r}}_{12}}{m_{1}+m_{2}}$.

\section{Kollision rotierender Teilchen}

Die Rechnung verkompliziert sich, wenn die Teilchen Freiheitsgrade der Rotation besitzen. Das Modell geht von rauhen Kugeln aus, so dass Energie zwischen den Freiheitsgraden ausgetauscht werden kann [83].

Bei der Kollision zweier rotierender Teilchen, mit den Winkelgeschwindigkeiten $\vec{\omega}_{i}$ und den Durchmessern $d_{i}$, gilt für die Geschwindigkeitsdifferenz am Kontaktpunkt, $\vec{g}_{12}$ :

$$
\vec{g}_{12}=\vec{v}_{12}-\frac{1}{2}\left(d_{1} \vec{\omega}_{1}+d_{2} \vec{\omega}_{2}\right) \times \hat{\vec{r}}_{12}
$$

Für den bei der Kollision übertragenen Impuls $\Delta \overrightarrow{p_{K}}$ gilt mit Drehimpulserhaltung und Impulserhaltung:

$$
\begin{aligned}
\frac{2 I_{1}}{d_{1}}\left(\vec{\omega}_{1}^{\prime}-\vec{\omega}_{1}\right) & =\frac{2 I_{2}}{d_{2}}\left(\vec{\omega}_{2}^{\prime}-\vec{\omega}_{2}\right)=-\hat{\vec{r}}_{12} \times \Delta \overrightarrow{p_{K}}, \\
-m_{1}\left(\vec{v}_{1}^{\prime}-\vec{v}_{1}\right) & =m_{2}\left(\vec{v}_{2}^{\prime}-\vec{v}_{2}\right)=\Delta \overrightarrow{p_{K}} .
\end{aligned}
$$

$I_{i}=m_{i} d_{i} / 10$ ist dabei das Trägheitsmoment einer Kugel. $\vec{g}_{12}^{\prime}$ bezeichne die Geschwindigkeitsdifferenz am Kontaktpunkt nach dem Stoß. So läßt sich mit der reduzierten Masse $M_{12}=\frac{m_{1} \cdot m_{2}}{m_{1}+m_{2}}$ schreiben:

$$
\begin{aligned}
\vec{g}_{12}-\vec{g}_{12}^{\prime} & =\vec{v}_{1}-\vec{v}_{1}^{\prime}-\vec{v}_{2}+\vec{v}_{2}^{\prime}-\frac{1}{2}\left(d_{1} \vec{\omega}_{1}-d_{1} \vec{\omega}_{1}^{\prime}\right) \times \hat{\vec{r}}_{12}-\frac{1}{2}\left(d_{2} \vec{\omega}_{2}-d_{2} \vec{\omega}_{2}^{\prime}\right) \times \hat{\vec{r}}_{12} \\
& =\frac{\Delta p_{K}}{m_{1}}+\frac{\Delta p_{K}}{m_{2}}+\left(\frac{5}{2} \cdot \frac{\hat{\vec{r}}_{12} \times \Delta \vec{k}_{K}}{m_{1} d_{1}}+\frac{5}{2} \cdot \frac{\hat{\vec{r}}_{12} \times \Delta \vec{p}_{K}}{m_{2} d_{2}}\right) \times \hat{\vec{r}}_{12} \\
& =\frac{\Delta p_{K}}{M_{12}}+\frac{5}{2 M_{12}} \cdot\left(\hat{\vec{r}}_{12} \times \Delta \overrightarrow{p_{K}}\right) \times \hat{\vec{r}}_{12} \\
& =\frac{\Delta p_{K}}{M_{12}}+\frac{5}{2 M_{12}} \cdot\left(\left(\hat{\vec{r}}_{12} \cdot \hat{\vec{r}}_{12}\right) \cdot \Delta \overrightarrow{p_{K}}-\hat{\vec{r}}_{12} \cdot\left(\hat{\vec{r}}_{12} \cdot \Delta \vec{p}_{K}\right)\right)^{\dagger} \\
& =\frac{7}{2 M_{12}} \Delta \vec{p}_{K}-\frac{5}{2 M_{12}} \cdot \hat{\vec{r}}_{12} \cdot\left(\Delta \vec{p}_{K} \cdot \hat{\vec{r}}_{12}\right) .
\end{aligned}
$$


Im allgemeinen Fall einer inelastischen Kollision gilt für die Geschwindigkeitsdifferenz am Kontaktpunkt nach der Kollision, $\vec{g}_{12}^{\prime}$ :

$$
\begin{aligned}
\hat{\vec{r}}_{12} \cdot \vec{g}_{12}^{\prime} & =-e \cdot \hat{\vec{r}}_{12} \cdot \vec{g}_{12} . \\
\hat{\vec{r}}_{12} \times \vec{g}_{12}^{\prime} & =-\beta_{0} \cdot \hat{\vec{r}}_{12} \times \vec{g}_{12} .
\end{aligned}
$$

Hierbei ist $e$ der Restitutionskoeffizient und $\beta_{0}$ der Restitutionskoeffizient der Rotation [84]. Mit (3.13) gilt dann:

$$
\begin{aligned}
& \vec{g}_{12}-\vec{g}_{12}^{\prime}=\frac{7}{2 M_{12}} \Delta \vec{p}_{K}-\frac{5}{2 M_{12}} \cdot \hat{\vec{r}}_{12} \cdot\left(\Delta \overrightarrow{p_{K}} \cdot \hat{\vec{r}}_{12}\right) \\
& \left(\vec{g}_{12}-\vec{g}_{12}^{\prime}\right) \cdot \hat{\vec{r}}_{12}=\frac{7}{2 M_{12}} \Delta \vec{p}_{K} \cdot \hat{\vec{r}}_{12}-\frac{5}{2 M_{12}} \cdot \hat{\vec{r}}_{12} \cdot\left(\Delta \vec{p}_{K} \cdot \hat{\vec{r}}_{12}\right) \cdot \hat{\vec{r}}_{12} \\
& 2 \cdot \vec{g}_{12} \cdot \hat{\vec{r}}_{12}=\Delta \vec{p}_{K} \cdot \hat{\vec{r}}_{12} / M_{12} \\
& \Delta \overrightarrow{p_{K}} \cdot \hat{\vec{r}}_{12}=M_{12}(e+1) \cdot \vec{g}_{12} \cdot \hat{\vec{r}}_{12} \text {. }
\end{aligned}
$$

Dies ist die Komponente von $\Delta \overrightarrow{p_{K}}$ in Richtung von $\hat{\vec{r}}_{12}$. Mit (3.13) und (3.16) erhält man weiter:

$$
\begin{aligned}
\vec{g}_{12} \times \hat{\vec{r}}_{12}-\vec{g}_{12}^{\prime} \times \hat{\vec{r}}_{12}= & \frac{7}{2 M_{12}} \Delta \vec{p}_{K} \times \hat{\vec{r}}_{12}-\frac{5}{2 M_{12}} \cdot \hat{\vec{r}}_{12} \cdot\left(\Delta \vec{p}_{K} \cdot \hat{\vec{r}}_{12}\right) \times \hat{\vec{r}}_{12} \\
\left(1+\beta_{0}\right) \vec{g}_{12} \times \hat{\vec{r}}_{12} \times \hat{\vec{r}}_{12}= & \frac{7}{2 M_{12}} \Delta \vec{p}_{K} \times \hat{\vec{r}}_{12} \times \hat{\vec{r}}_{12} \\
\left(1+\beta_{0}\right)\left(\left(\vec{g}_{12} \cdot \hat{\vec{r}}_{12}\right) \cdot \hat{\vec{r}}_{12}-\vec{g}_{12}\right)= & \frac{7}{2 M_{12}}\left(\left(\Delta \vec{p}_{K} \cdot \hat{\vec{r}}_{12}\right) \cdot \hat{\vec{r}}_{12}-\Delta \vec{p}_{K}\right) \\
-\frac{2 M_{12}}{7}\left(1+\beta_{0}\right)\left(\vec{g}_{12}-\left(\vec{g}_{12} \cdot \hat{\vec{r}}_{12}\right) \cdot \hat{\vec{r}}_{12}\right)= & \left(M_{12}(e+1) \cdot\left(\vec{g}_{12} \cdot \hat{\vec{r}}_{12}\right) \cdot \hat{\vec{r}}_{12}\right)-\Delta \vec{p}_{K} \\
\Rightarrow \Delta \vec{p}_{K}= & M_{12}(e+1) \cdot\left(\vec{g}_{12} \cdot \hat{\vec{r}}_{12}\right) \cdot \hat{\vec{r}}_{12} \\
& -\frac{2 M_{12}}{7}\left(1+\beta_{0}\right)\left(\vec{g}_{12}-\left(\vec{g}_{12} \cdot \hat{\vec{r}}_{12}\right) \cdot \hat{\vec{r}}_{12}\right) .
\end{aligned}
$$

Im betrachteten Fall einer elastischen Kollision rauher Kugeln gilt $e=1$ und $\beta_{0}=1$. Damit, sowie durch die Beziehungen $\vec{g}_{12 \|}=\left(\vec{g}_{12} \cdot \hat{\vec{r}}_{12}\right) \cdot \hat{\vec{r}}_{12}$ und $\vec{g}_{12 \perp}=$ $\vec{g}_{12}-\vec{g}_{12 \|}$, läßt sich vereinfachen:

$$
\Delta \overrightarrow{p_{K}}=-m_{1} \cdot \Delta \vec{v}_{1}=m_{2} \cdot \Delta \vec{v}_{2}=2 M_{12}\left(\vec{g}_{12 \|}-\frac{2}{7} \vec{g}_{12 \perp}\right) .
$$

Somit lassen sich die neuen Translationsgeschwindigkeiten berechnen. Für die

$\dagger$ Entwicklungssatz: $(\vec{a} \times \vec{b}) \times \vec{c}=(\vec{a} \cdot \vec{c}) \vec{b}-(\vec{b} \cdot \vec{c}) \vec{a}$ 
Rotationsfreiheitgrade liefert die Drehimpulserhaltung:

$$
\begin{aligned}
\frac{2 I_{1}}{d_{1}}\left(\vec{\omega}_{1}^{\prime}-\vec{\omega}_{1}\right) & =\frac{2 I_{2}}{d_{2}}\left(\vec{\omega}_{2}^{\prime}-\vec{\omega}_{2}\right)=-\hat{\vec{r}}_{12} \times \Delta \vec{p}_{K} \\
\frac{2 m_{1} d_{1}}{10} \Delta \vec{\omega}_{1} & =\frac{2 m_{2} d_{2}}{10} \Delta \vec{\omega}_{2}=-\hat{\vec{r}}_{12} \times m_{1} \Delta \vec{v}_{1} \\
m_{1} d_{1} \Delta \vec{\omega}_{1} & =m_{2} d_{2} \Delta \vec{\omega}_{2}=-10 m_{1} \overrightarrow{\vec{r}}_{12} \times \frac{\Delta \vec{v}_{1}}{\left(d_{1}+d_{2}\right)} .
\end{aligned}
$$

Wenn nur das Teilchen 1 Freiheitsgrade der Rotation besitzt, folgt in Analogie zur obigen Betrachtung:

$$
\begin{aligned}
\vec{g}_{12} & =\vec{v}_{12}-\frac{1}{2} \cdot d_{1} \vec{\omega}_{1} \times \hat{\vec{r}}_{12} \\
\vec{g}_{12}-\vec{g}_{12}^{\prime} & =\frac{\Delta p_{K}}{M_{12}}+\frac{5}{2 m_{1}} \cdot\left(\Delta \vec{p}_{K}-\hat{\vec{r}}_{12} \cdot\left(\hat{\vec{r}}_{12} \cdot \Delta \vec{p}_{K}\right)\right) \\
\Delta \vec{p}_{K} & =(1+e) M_{12} \cdot \vec{g}_{12} \\
\Delta \vec{p}_{K} & =\frac{2 M_{12} m_{1}}{2 m_{1}+5 M_{12}} \cdot\left(1+\beta_{0}\right)\left(\vec{g}_{12 \|}-\vec{g}_{12}\right)+(1+e) M_{12} \cdot \vec{g}_{12 \|} \cdot
\end{aligned}
$$

mit $e=1$ und $\beta_{0}=1$ :

$$
\Delta \overrightarrow{p_{K}}=-m_{1} \cdot \Delta \vec{v}_{1}=m_{2} \cdot \Delta \vec{v}_{2}=2 M_{12}\left(\vec{g}_{12 \|}-\frac{m_{1}}{m_{1}+5 / 2 M_{12}} \vec{g}_{12 \perp}\right),
$$

und damit:

$$
m_{1} d_{1} \Delta \vec{\omega}_{1}=-10 m_{1} \hat{\vec{r}}_{12} \times \frac{\Delta \vec{v}_{1}}{\left(d_{1}+d_{2}\right)}
$$

\section{Kollision chemisch reagierender Teilchen - ohne Rotation}

Geschieht beim Stoß zweier Teilchen eine chemische Reaktion, ändern sich Masse und Durchmesser der beteiligten Teilchen. Außerdem haben wir es mit einer inelastischen Kollision mit Energiegewinn/verlust $E_{d}$ zu tun. Um die neuen Translations- (nicht Rotations-) Geschwindigkeiten einer bimolekularen Reaktion zu bestimmen begibt man sich ins Schwerpunktsystem $S$ :

$$
\begin{array}{cc}
\vec{r}_{S}=\frac{m_{1} \cdot \vec{r}_{1}+m_{2} \cdot \vec{r}_{2}}{m_{1}+m_{2}} & \vec{v}_{S}=\frac{m_{1} \cdot \vec{v}_{1}+m_{2} \cdot \vec{v}_{2}}{m_{1}+m_{2}} \\
\vec{v}_{1_{S}}=\vec{v}_{1}-\vec{v}_{S} & \vec{v}_{2_{S}}=\vec{v}_{2}-\vec{v}_{S} .
\end{array}
$$

Hier sind $\vec{r}_{S}$ und $\vec{v}_{S}$ Ort bzw. Geschwindigkeit des Schwerpunktes; $\vec{v}_{1_{S}}$ und $\vec{v}_{2_{S}}$ sind die Geschwindigkeiten der Teilchen im Schwerpunktsystem. In $S$ gilt:

$$
m_{1} \cdot \vec{v}_{1_{S}}=-m_{2} \cdot \vec{v}_{2_{S}}
$$

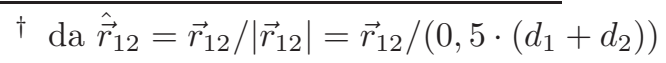


Für die kinetische Energie vor der Kollision gilt dann:

$$
\begin{aligned}
E_{k i n} & =\frac{1}{2} \cdot m_{1} \cdot \vec{v}_{1}^{2}+\frac{1}{2} \cdot m_{2} \cdot \vec{v}_{2}^{2}=\frac{1}{2} \cdot m_{1} \cdot\left(\vec{v}_{S}+\vec{v}_{1_{S}}\right)^{2}+\frac{1}{2} \cdot m_{2} \cdot\left(\vec{v}_{S}+\vec{v}_{2_{S}}\right)^{2} \\
& =\frac{1}{2} \cdot \vec{v}_{S}^{2}\left(m_{1}+m_{2}\right)+\frac{1}{2} \cdot\left(m_{1} \cdot \vec{v}_{1_{S}}^{2}+m_{2} \cdot \vec{v}_{2_{S}}^{2}\right)+\vec{v}_{S}\left(m_{1} \cdot \vec{v}_{1_{S}}+m_{2} \cdot \vec{v}_{2_{S}}\right) \\
& =\frac{1}{2} \cdot \vec{v}_{S}^{2} \cdot M_{12}+\frac{1}{2} \cdot\left(m_{1} \cdot \vec{v}_{1_{S}}^{2}+m_{2} \cdot \vec{v}_{2_{S}}^{2}\right) .
\end{aligned}
$$

Bei Kollision und Reaktion gilt Energie- und Massenerhaltung. Mit den neuen Massen $m_{3}, m_{4}$ und den neuen Geschwindigkeiten in $\mathrm{S} \vec{v}_{3_{S}}, \vec{v}_{4_{S}}$ :

$$
\begin{aligned}
& \frac{1}{2}\left(\vec{v}_{S}^{2} M_{12}+m_{1} \vec{v}_{1_{S}}^{2}+m_{2} \vec{v}_{2_{S}}^{2}\right)=\frac{1}{2}\left(\vec{v}_{S}^{2} \cdot M_{12}+m_{3} \cdot \vec{v}_{3_{S}}^{2}+m_{4} \cdot \vec{v}_{4_{S}}^{2}+2 \cdot E_{d}\right) \\
& m_{1} \cdot \vec{v}_{1_{S}}^{2}+m_{2} \cdot \vec{v}_{2_{S}}^{2}=m_{3} \cdot\left(-m_{4} / m_{3} \cdot \vec{v}_{4_{S}}\right)^{2}+m_{4} \cdot \vec{v}_{4_{S}}^{2}+2 E_{d}^{\dagger} \\
& m_{1} \cdot \vec{v}_{1_{S}}^{2}+m_{2} \cdot \vec{v}_{2_{S}}^{2}=\vec{v}_{4_{S}}^{2}\left(m_{4}^{2} / m_{3}+m_{4}^{2}\right)+2 E_{d} \\
& \vec{v}_{4_{S}}^{2}=\frac{1}{\left(m_{3}+m_{4}\right) / m_{3}}\left(m_{1}\left(\frac{\vec{v}_{12} \cdot m_{2}}{m_{1}+m_{2}}\right)^{2}+m_{2}\left(\frac{\vec{v}_{12} \cdot m_{1}}{m_{1}+m_{2}}\right)^{2}+2 E_{d}\right) \ddagger \\
& \vec{v}_{4_{S}}^{2}=\frac{M_{34}}{m_{4}^{2}} \cdot\left(\frac{\vec{v}_{12}^{2} \cdot M_{12}^{2}}{m_{1}}+\frac{\vec{v}_{12}^{2} \cdot M_{12}^{2}}{m_{2}}+2 E_{d}\right) \\
& v_{4_{S}}=\sqrt{\frac{M_{34}}{m_{4}^{2}} \cdot\left(\vec{v}_{12}^{2} \cdot M_{12}+2 E_{d}\right)} \text {. }
\end{aligned}
$$

Dies ist nun lediglich der Betrag der neuen Geschwindigkeiten (eindimensionale Rechnung). Der Richtungs-Einheitsvektor ergibt sich aus der Umkehr der Komponenten des Geschwindigkeitsdifferenzvektors $\vec{v}_{12}$ in Richtung des Verbindungsvektors $\vec{r}_{12}$ :

$$
\vec{v}_{34} / v_{34}=\vec{v}_{12} / v_{12}-2 \cdot\left(\vec{v}_{12} / v_{12}\right) \cdot \hat{\vec{r}}_{12} \cdot \hat{\vec{r}}_{12}=\hat{\vec{v}}_{12}-2 \cdot \hat{\vec{v}}_{12 \|} \cdot
$$

Damit ergibt sich insgesamt:

$$
\begin{aligned}
& \vec{v}_{4}=\frac{m_{1} \vec{v}_{1}+m_{2} \vec{v}_{1}}{m_{1}+m_{2}}+\sqrt{\frac{M_{34}}{m_{4}^{2}} \cdot\left(v_{12}^{2} \cdot M_{12}+2 E_{d}\right)} \cdot\left(\hat{\vec{v}}_{12}-2 \cdot \hat{\vec{v}}_{12 \|}\right), \\
& \vec{v}_{3}=\frac{m_{1} \vec{v}_{1}+m_{2} \vec{v}_{1}}{m_{1}+m_{2}}-\sqrt{\frac{M_{34}}{m_{3}^{2}} \cdot\left(v_{12}^{2} \cdot M_{12}+2 E_{d}\right)} \cdot\left(\hat{\vec{v}}_{12}-2 \cdot \hat{\vec{v}}_{12 \|}\right) .
\end{aligned}
$$

Die katalytische Reaktion besteht aus einem Zwei-Schritt-System, bei dessen ersten Schritt aus zwei Teilchen ein 'angeregter Komplex' $A^{*}$ gebildet wird. Im zweiten Schritt reagiert dieser unter Kollision mit einem Katalysatorteilchen zu einem Produkt weiter (siehe Kap. 4.3.1).

Für Geschwindigkeit und Masse des angeregten Komplexes liefern die Erhaltungs-

\footnotetext{
$\dagger$ Impulserhaltung: $\vec{v}_{3_{S}}=-m_{4} / m_{3} \cdot \vec{v}_{4_{S}}$

$\ddagger \vec{v}_{1_{S}}=\vec{v}_{1}-\vec{v}_{S}=\vec{v}_{1}-\left(m_{1} \cdot \vec{v}_{1}+m_{2} \cdot \vec{v}_{2}\right) /\left(m_{1}+m_{2}\right)=\vec{v}_{12} \cdot m_{2} /\left(m_{1}+m_{2}\right)$
} 
sätze:

$$
\vec{v}_{A^{*}}=\frac{m_{1} \vec{v}_{1}+m_{2} \vec{v}_{2}}{m_{1}+m_{2}} \quad m_{A^{*}}=m_{1}+m_{2}
$$

Stößt der angeregte Komplex während seiner Lebenszeit mit einem Katalysatorteilchen wird er in das Produkt umgewandelt. Dabei handelt es sich um eine bimolekulare Reaktion mit Energiegewinn/verlust, aber ohne Massenänderung. Die neuen Geschwindigkeiten werden nach Gl. (3.25) und (3.26) berechnet.

Bei der Rückreaktion mit drei zu beachtenden Teilchen gilt für die neuen Geschwindigkeiten $\vec{v}_{1}, \vec{v}_{2}$ und $\vec{K}^{\prime}$ unter Massen-, Impuls- und Energieerhaltung [77]:

$$
\begin{aligned}
\vec{v}_{1} & =\frac{m_{A^{*}} \vec{A}_{A^{*}}+m_{K} \vec{v}_{K}}{m_{1}+m_{2}+m_{K}}+\sqrt{\frac{M_{A^{*} K}}{m_{1}^{2}}\left(2 E_{d}+M_{A^{*}} v_{A^{*} K}^{2}\right)}\left(\frac{1}{2} \overrightarrow{n_{1}}+\frac{\sqrt{3}}{2} \overrightarrow{n_{2}}\right) \\
\vec{v}_{2} & =\frac{m_{A^{*}} \vec{v}_{A^{*}}+m_{K} \vec{v}_{K}}{m_{1}+m_{2}+m_{K}}+\sqrt{\frac{M_{A^{*} K}}{m_{2}^{2}}\left(2 E_{d}+M_{A^{*}} v_{A^{*} K}^{2}\right)}\left(\frac{1}{2} \vec{n}_{1}-\frac{\sqrt{3}}{2} \vec{n}_{2}\right) \\
{\overrightarrow{v_{K}}}^{\prime} & =\frac{m_{A^{*}} \vec{v}_{A^{*}}+m_{K} \vec{v}_{K}}{m_{1}+m_{2}+m_{K}}-\sqrt{\frac{M_{A^{*} K}}{m_{K}^{2}}\left(2 E_{d}+M_{A^{*}} v_{A^{*} K}^{2}\right)} \vec{n}_{1} .
\end{aligned}
$$

mit

$$
\begin{aligned}
\overrightarrow{n_{1}} & =\frac{\vec{r}_{A^{*}}-\vec{r}_{K}}{\left|\vec{r}_{A^{*}}-\vec{r}_{K}\right|} & \overrightarrow{n_{2}} & =\frac{\vec{r}_{A^{*}} \times \vec{r}_{K}}{\left|\vec{r}_{A^{*}} \times \vec{r}_{K}\right|} \\
M_{A^{*} K} & =\frac{m_{A^{*}} \cdot m_{K}}{m_{A^{*}}+m_{K}} & v_{A^{*} K} & =\left|\vec{v}_{A^{*}}-\overrightarrow{v_{K}}\right| .
\end{aligned}
$$

Findet während der Lebenszeit des angeregten Zustandes keine Kollision mit einem Katalysatorteilchen statt, zerfällt $A^{*}$ wieder in seine Ursprungsbestandteile 1 und 2. Für die Geschwindigkeiten gilt in diesem Fall:

$$
\begin{aligned}
& \vec{v}_{1}=\vec{v}_{A^{*}}+\frac{m_{A^{*}}}{m_{1}}\left(\vec{v}_{A^{*}}-2 \frac{\vec{v}_{A^{*}} \cdot \vec{r}_{A^{*}}}{r_{A^{*}}^{2}} \vec{r}_{A^{*}}\right), \\
& \vec{v}_{2}=\vec{v}_{A^{*}}-\frac{m_{A^{*}}}{m_{2}}\left(\vec{v}_{A^{*}}-2 \frac{\vec{v}_{A^{*}} \cdot \vec{r}_{A^{*}}}{r_{A^{*}}^{2}} \vec{r}_{A^{*}}\right) .
\end{aligned}
$$

\section{Kollision chemisch reagierender Teilchen - mit Rotation}

Die Kollisionsbedingungen rotierender Teilchen mit chemischen Reaktionen ließen sich analytisch leider nicht ermitteln. 


\subsubsection{Wandkollisionen}

Es muß berechnet werden, ob und wann ein Teilchen mit der bewegten Wand der Blase kollidiert. Sei $\left(r_{x}, r_{y}, r_{z}\right)$ der Ort eines Teilchens, $\left(v_{x}, v_{y}, v_{z}\right)$ seine Geschwindigkeit. Die Wand werde vom aktuellen Blasenradius $R_{W}$ aus konstant mit der Geschwindigkeit $v_{W}$ nach innen bewegt. Definiere:

$$
\begin{aligned}
& k_{1}:=v_{x}^{2}+v_{y}^{2}+v_{z}^{2}-v_{W}^{2}, \\
& k_{2}:=v_{x} r_{x}+v_{y} r_{y}+v_{z} r_{z}+v_{W} R_{W}, \\
& k_{3}:=r_{x}^{2}+r_{y}^{2}+r_{z}^{2}-R_{W}^{2} \leq 0 .
\end{aligned}
$$

Die Zeit, zu der das Teilchen mit der Wand kollidiert, berechnet sich dann durch:

$$
t_{W K}= \begin{cases}\left(-k_{2}+\sqrt{k_{2}^{2}-k_{1} k_{3}}\right) / k_{1} & \text { falls } \begin{array}{l}
k_{1} \neq 0 \wedge k_{2}^{2}-k_{1} k_{3} \geq 0 \wedge \\
\left(v_{W} \geq 0 \vee k_{1}>0 \vee k_{2}>0\right)
\end{array} \\
-k_{3} /\left(2 k_{2}\right) & \text { falls } k_{1}=0 \wedge k_{2}>0 \\
\infty & \text { sonst. }\end{cases}
$$

Wenn nun ein Teilchen auf die Wand trifft, wird davon ausgegangen, dass das Teilchen reflektiert wird. Dies entspricht grundsätzlich einer elastischen Kollision mit einer ruhenden Wand (Einfallswinkel = Ausfallswinkel, keine Änderung des Geschwindigkeitsbetrages). Allerdings wird dem Teilchen durch die Bewegung der Wand eine zusätzliche Geschwindigkeit in Richtung der Normalen der Wand mitgegeben. Da sich das Teilchen zum Zeitpunkt der Kollision direkt an der Wand befindet, stellt $\vec{n}_{W}:=\left(-r_{x},-r_{y},-r_{z}\right)$ einen Normalenvektor an die Wand dar. Wie in Abbildung 3.1 verdeutlicht, ist die resultierende Geschwindigkeitsänderung davon abhängig, ob sich Teilchen und Wand aufeinander zubewegen $\left(\vec{v}_{T} \cdot \vec{v}_{W}<0\right)$ oder die Wand das Teilchen 'von hinten' trifft $\left(\vec{v}_{T} \cdot \vec{v}_{W}>0\right)$. Im zweiten Fall gibt die Wand dem Teilchen lediglich einen Schubs in Richtung der Wandnormalen:

$$
\vec{v}_{T_{\text {neu }}}=\vec{v}_{T}+v_{W} \frac{\vec{n}_{W}}{n_{W}}
$$

Im ersten Fall findet eine Reflexion an der Wand statt. Trifft ein Teilchen mit dem Einfallsvektor $\vec{v}_{T}$ auf eine Wand, errechnet sich der Ausfallsvektor $\vec{v}_{T_{\text {neu }}}$ als Ergebnis verschiedener Projektionen auf den Normalenvektor sowie die Basisvektoren 


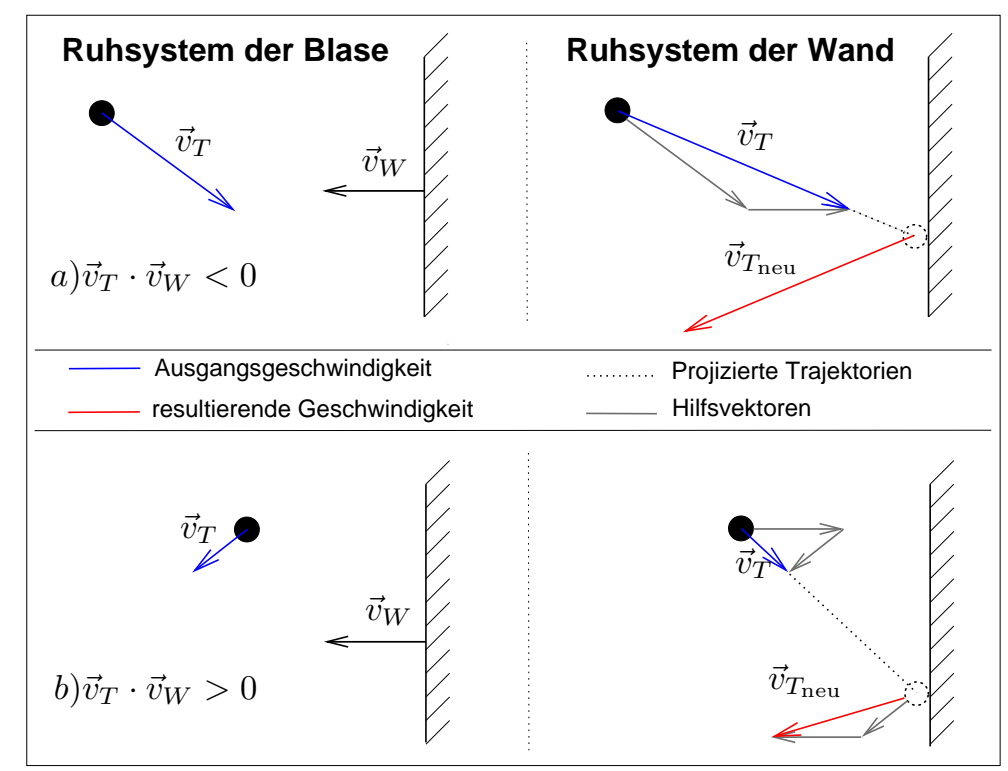

Abbildung 3.1: Wandkollision eines Teilchens: für die beiden relevanten Fälle jeweils links die Situation für einen Beobachter, rechts im System mit ruhender Wand. Die gekrümmte Wand wird lokal als linear betrachtet.

einer im Auftreffpunkt lokalisierten 'Spiegelebene':

$$
\vec{v}_{T_{\text {neu }}}=\vec{v}_{T_{\text {alt }}}-2\left(\vec{v}_{T_{\text {alt }}} \cdot \frac{\vec{n}_{W}}{n_{W}}\right) \frac{\vec{n}_{W}}{n_{W}}
$$

Nimmt man nun noch die von der Wandgeschwindigkeit übertragene Geschwindigkeitskomponente hinzu, ergibt sich:

$$
\vec{v}_{T_{\text {neu }}}=\vec{v}_{T_{\text {alt }}}+\left(v_{W}-2\left(\vec{v}_{T_{\text {alt }}} \cdot \frac{\vec{n}_{W}}{n_{W}}\right)\right) \frac{\vec{n}_{W}}{n_{W}}
$$

\subsubsection{Zelleinteilung}

Um die Effizienz des Algorithmus zu verbessern wird die Simulationsregion in Zellen unterteilt, die es ermöglichen, die Anzahl an potentiellen Kollisionspartnern pro Teilchen zu verringern. Ohne eine solche Struktur müßte nach jeder Richtungsänderung eines Teilchens die Kollisionsbedingung für alle anderen Teilchen im System geprüft werden, der Aufwand also mit $N_{\text {ges }}^{2}$ skalieren. Durch die praktizierte Einteilung müssen Kollisionspartner nur in der dem Teilchen eigenen Zelle sowie den 26 Nachbarzellen gesucht werden.

Es werden quaderförmige Zellen verwendet, deren Anzahl zu Beginn der Simu- 
lation ungefähr der Anzahl der simulierten Teilchen entspricht. Im Laufe des simulierten Blasenkollaps verkleinert sich die Simulationsregion drastisch. Um weiterhin eine effiziente Zelleinteilung zu gewährleisten wird die Größe der Zellen halbiert, sobald der Blasenradius um die Hälfte geschrumpft ist. Umgekehrt wird die Zellgröße vergrößert, wenn die Blase nach dem Kollaps wieder expandiert. Der Zeitpunkt des Wechsels eines Teilchen von der aktuellen Zelle in eine Nachbarzelle ist für jedes Teilchen als Ereignis im Ereignisbaum abgelegt, genauso die geschätzte Zeit für eine Größenänderung der Zellen.

\subsubsection{Systemabhängige Einheiten}

Da es sich bei der Simulation um eine Modellierung auf molekularer Ebene handelt empfiehlt es sich, in molekularen Einheiten zu rechnen. Eine Rechnung in SI-Einheiten würde aufgrund der resultierenden sehr großen und kleinen Zahlen das Auftreten von Over- oder Underflows sowie numerischer Fehler begünstigen. Daher werden folgende systemabhängige Größen eingeführt:

- Längeneinheit: der Teilchendurchmesser $d$.

- Masseneinheit: die Teilchenmasse $m$.

- Temperatureinheit: die Anfangstemperatur $T_{\text {init }}$.

- Ladungseinheit: die Elektronenladung $q_{0}=1.602 \cdot 10^{-19}$ As.

- Zeiteinheit: $t_{0}=\sqrt{\frac{m d^{2}}{k T_{\text {init }}}}$; da im Hartkugelmodell eine 'natürliche' Energieeinheit fehlt wird die thermische Energie $k T_{\text {init }}$ (k: Anzahl der Freiheitsgrade) als solche genutzt [85].

- Die Boltzmann-Konstante $k_{B}$ wird auf 1 gesetzt.

Aus diesen Umskalierungen ergeben sich die abgeleiteten Größen in Tabelle 3.1.

\subsubsection{Skalierung der Simulationsteilchen}

Die Anzahl an Teilchen, die simuliert werden können hängt von der verwendeten Computer-Hardware ab und bewegt sich in der Region einiger Millionen Teilchen. Eine Rechnung mit 10 Millionen Teilchen benötigt bereits mehrere Wochen, um 


\begin{tabular}{llr}
\hline Länge: & $r_{r}=$ & $d \cdot r$ \\
Zeit: & $t_{r}=$ & $\left(m d^{2} /\left(T_{\text {init }} k_{B}\right)\right)^{1 / 2} \cdot t$ \\
Dichte: & $\varrho_{r}=$ & $m / d^{3} \cdot \varrho$ \\
Temperatur: & $T_{r}=$ & $T_{\text {init }} \cdot T$ \\
Geschwindigkeit: & $u_{r}=$ & $\left(T_{\text {init }} k_{B} / m\right)^{1 / 2} \cdot u$ \\
Druck: & $p_{r}=$ & $T_{\text {init }} k_{B} / d^{3} \cdot p$ \\
\hline
\end{tabular}

Tabelle 3.1: Skalierungen der Messgrößen. Ganz rechts stehen jeweils die in der Simulation benutzten Größen

einen typischen Blasenkollaps zu simulieren (siehe Kap. 4.4). Aus diesem Grund ist es für die meisten interessierenden Fragestellungen nicht möglich, eine der realen Teilchenanzahl $\left(N_{r}\right)$ entsprechende Anzahl an Simulationsteilchen $\left(N_{\text {ges }}\right)$ bereitzustellen.

Um dennoch auch größere Systeme behandeln zu können läßt man ein einzelnes Simulationsteilchen für mehrere reale Teilchen stehen - man benutzt sog. kollektive Teilchen. Die Anzahl realer Teilchen, der ein simuliertes Teilchen entspricht, ist abhängig von der Systemgröße und der benutzten Teilchenzahl, sie wird durch den Skalierungsfaktor $S=N_{r} / N_{\text {ges }}$ beschrieben. Für die Größen Teilchenmasse $m$, Temperatur $T$ und Teilchendurchmesser $d$ in der Simulation werden nun die folgenden Transformationen eingeführt:

$$
\begin{aligned}
N_{r} m_{r} & =N_{g e s} m \\
N_{r} T_{r} & =N_{g e s} T \\
N_{r} d_{r}^{3} & =N_{g e s} d^{3} .
\end{aligned}
$$

Unter diesen Skalierungen bleiben die meisten physikalischen Größen invariant unter Teilchenzahländerungen. Dies sind im Einzelnen: Masse, Dichte, Druck, Geschwindigkeit, Volumen, Zeit, Energie und ausgeschlossenes Van-der-WaalsVolumen.

Allerdings werden durch diese Transformationen diffusive Prozesse überschätzt: Durch die Wahl von (3.39) wird das Volumen richtig transformiert, was für die korrekte Vorhersage der Kompression im Kollaps unerläßlich ist. Der von (zweidimensionalen) Stoßquerschnitten abhängige Wert der mittleren freien Weglänge $l$ würde zur korrekten Skalierung allerdings $N_{r} d_{r}^{2}=N_{g e s} d^{2}$ fordern. Aus diesem 
Grund wird $l$ um den Faktor $\left(N_{r} / N_{\text {ges }}\right)^{1 / 3}$ überschätzt. Die Effekte der Wärmeleitung, der Viskosität und der Teilchendiffusion, die alle linear von $l$ abhängen werden damit ebenfalls um diesen Faktor verstärkt.

Der Einfluß, den die Wahl des Skalierungsfaktors auf die Ergebnisse der Simulationen hat, wird in den Kapiteln 4.4 und 6.2.1 untersucht.

\subsubsection{Bestimmung der hydrodynamischen Größen}

Die Ermittlung der hydrodynamischen Größen stellt die 'Auswertung' der Simulation dar. Es werden die interessierenden physikalischen Parameter in der Blase bestimmt, anhand derer Einblick in die Dynamik und den Vorgang der Sonolumineszenz gewonnen werden kann. Diese Auswertung wird während eines Durchlaufs der Simulation typischerweise einige hundert Male durchgeführt, vor allem während des physikalisch interessanten Endes der Kollapsphase. Die Anweisung, die Auswertung durchzuführen, ist als Ereignis realisiert.

Zur Bestimmung der hydrodynamischen Größen wird die Blase in eine bestimmte, vom Benutzer vorgegebene Anzahl von Messzellen $\#_{M Z}$ eingeteilt. Diese werden so gewählt, dass das Volumen je Messzelle gleich ist. Durch die sphärische Randbedingung empfehlen sich Kugelschalen, da so die Symmetrien maximal ausgenutzt werden können. Da alle diese Kugelschalen das gleiche Volumen $V_{M Z}$ besitzen, ist die radiale Auflösung im Blasenzentrum geringer als am Blasenrand.

Die hydrodynamischen Größen werden durch Mittelung des Zustandes der in einer Messzelle enthaltenen Teilchen bestimmt. Im Einzelnen werden unter Verwendung von Kugelkoordinaten folgende Werte errechnet $\left(N_{j}\right.$ : Anzahl der Teilchen in Messzelle $j$ ):

- Die Dichte $\varrho$ als Masse der Teilchen je Volumen:

$$
\varrho(r, t)=\frac{1}{V_{M Z}} \sum_{i=1}^{N_{j}} m_{i} .
$$

- Die Schwerpunktsgeschwindigkeit als gewichtete Summe der Teilchengeschwindigkeiten:

$$
u(r, t)=\frac{\sum_{i=1}^{N_{j}} m_{i} \vec{v}_{i} \vec{r}_{i} / r_{i}}{\sum_{i=1}^{N_{j}} m_{i}} .
$$


- Die Temperatur der Translationsfreiheitsgrade ergibt sich aus dem Gleichverteilungssatz $E_{t l}=\frac{3}{2} k_{B} T_{t l}$. Die Energie der Translation $E_{t l}$ wird aus Summe der kinetischen Energien der Teilchen, abzüglich der kinetischen Schwerpunktsenergie berechnet:

$$
T_{t l}(r, t)=\frac{1}{3 k_{B} N_{j}}\left[\left(\sum_{i=1}^{N_{j}} m_{i} v_{i}^{2}\right)-u(r, t)^{2} \sum_{i=1}^{N_{j}} m_{i}\right] .
$$

- Die Temperatur der Rotationsfreiheitsgrade bestimmt sich genauso über die Summe der Rotationsenergien $E_{\text {rot }}=\frac{1}{2} J_{i} \vec{\omega}_{i}^{2}$, mit $J_{i}=\frac{1}{10} m_{i} d_{i}^{2}$ - dem Trägheitsmoment eines Teilchens:

$$
T_{r o t}(r, t)=\frac{1}{30 k_{B} N_{j}}\left(\sum_{i=1}^{N_{j}} m_{i} d_{i}^{2} \vec{\omega}_{i}^{2}\right)
$$

- Die Herleitung der Gleichung des Druckes ist ein wenig komplizierter. Es läßt sich zeigen, dass mittels des Virialsatzes folgende Relation gilt:

$$
p(r, t)=\frac{1}{V_{M Z}}\left[N_{j} k_{B} T+\frac{1}{3}\left(\sum_{k} \sum_{i<k}^{N_{j}} \vec{F}_{i k} \vec{r}_{i k}\right)\right] .
$$

Diese Beziehung gilt für kontinuierliche Potentiale $U_{i k}(r)$, wobei $F_{i k}=$ $-\frac{\partial U_{i k}}{\partial r} . F_{i k}$ ist also die von Teilchen $k$ auf Teilchen $i$ ausgeübte Kraft. Der zweite Term der rechten Seite stellt eine Erweiterung der idealen GasGleichung (1. Term) dar. Um auf die entsprechende Gleichung für Hartkugelpotentiale zu kommen, geht man von $F=m \cdot a$ aus. Da die Teilchen nur bei einer Kollision Kräfte austauschen, muß ein Zeitintervall $\Delta t$ definiert werden, in welchem die Kollisionen gezählt werden. Es ergibt sich:

$$
p(r, t)=\frac{1}{V_{M Z}}\left[N_{j} k_{B} T+\frac{1}{3 \Delta t}\left(\sum_{k} \sum_{i<k}^{N_{j}} m_{i k} \overrightarrow{\Delta v_{i}} \vec{r}_{i k}\right)\right] .
$$

In die Doppelsumme fließen nur diejenigen Teilchen ein, die während $\Delta t$ einer Kollision in der betrachteten Messzelle unterliegen, da für alle anderen die Geschwindigkeitsänderung $\Delta v$ Null ist.

Aufgrund der Symmetrien der Messzellen (Kugelschalen) kann man die erhaltenen 
Werte einfach gegen den Radius $r$ auftragen.

Die ermittelten hydrodynamischen Größen zeigen lokal eine Abhängigkeit von der Anfangsverteilung der Teilchen und sind - je nach initialer Teilchenanzahl $N_{\text {ges }, i}$ - unterschiedlich stark verrauscht. Aus diesem Grund werden die Messwerte im Laufe der Auswertung durch einen Gauß-Filter mit einstellbarer Breite räumlich geglättet. Eine zeitliche Glättung findet nicht statt.

\subsubsection{Bestimmung des Teilchendurchmessers}

Der Teilchendurchmesser wird durch das ausgeschlossene Van-der-WaalsVolumen der jeweiligen Teilchenspezies berechnet. Dabei wird davon ausgegangen, dass sich das System im thermodynamischen Gleichgewicht befindet, so dass der Zustand durch eine Van-der-Waals'sche Zustandsgleichung beschrieben werden kann:

$$
p(v, T)=\frac{1}{m} \cdot \frac{k_{B} T}{v-v_{a}} .
$$

Dabei bezeichnet $v=1 / \varrho$ das spezifische Volumen und $v_{a}$ das spezifische ausgeschlossene Volumen.

Das ausgeschlossene Volumen pro Teilchenpaar beträgt $\frac{4}{3} \pi d^{3}$ [86]. Für ein einzelnes Teilchen ergibt sich die Hälfte dieses Wertes: $\frac{2}{3} \pi d^{3}$. Für N Teilchen resultiert dies in einem ausgeschlossenen Van-der-Waals-Volumen von

$$
V_{a}=\frac{2 N \pi}{3} d^{3}
$$

woraus sich leicht der Teilchendurchmesser $d$ berechnen läßt:

$$
d=\left(\frac{3}{2 \pi} \cdot \frac{v_{a}}{N}\right)^{\frac{1}{3}}
$$

Liegt ein Gemisch verschiedener Gase vor, kann das ausgeschlossene Volumen berechnet werden über [87]:

$$
v_{a}=\sum_{i} v_{a, i}\left(\frac{N_{i}}{N_{g e s}}\right)^{2}+2 \sum_{i} \sum_{j=1}^{j<i}\left(\frac{1}{2}\left(v_{a, i}^{\frac{1}{3}}+v_{a, j}^{\frac{1}{3}}\right)\right)^{3}\left(\frac{N_{i}}{N_{g e s}}\right)\left(\frac{N_{j}}{N_{g e s}}\right) .
$$

Zur Ermittlung der Werte von $v_{a}$ für die vorkommenden Spezies wurden aus [88] die entsprechenden Werte für die verwendeten Edelgase sowie für $\mathrm{H}_{2} \mathrm{O}$ übernommen. Aus der Annahme, dass das Verhältnis der Volumina von $H$ und $O 3$ zu 2 


\begin{tabular}{ccc}
\hline Spezies & $v_{a}[\mathrm{ml} / \mathrm{mol}]$ & $d\left[10^{-10} \mathrm{~m}\right]$ \\
\hline $\mathrm{He}$ & 23,70 & 2,38 \\
$\mathrm{Ar}$ & 32,29 & 2,94 \\
$\mathrm{Xe}$ & 51,59 & 3.45 \\
$\mathrm{H}_{2} \mathrm{O}$ & 30,51 & 2,89 \\
$\mathrm{H}$ & 8,72 & 1,91 \\
$\mathrm{H}_{2}$ & 17,44 & 2,40 \\
$\mathrm{O}$ & 13,07 & 2,18 \\
$\mathrm{O}_{2}$ & 26,14 & 2,75 \\
$\mathrm{OH}$ & 21,79 & 2,59 \\
$\mathrm{HO}_{2}$ & 34,86 & 3,02 \\
$\mathrm{H}_{2} \mathrm{O}_{2}$ & 43,58 & 3,26 \\
\hline
\end{tabular}

Tabelle 3.2: Spezifisches Ausgeschlossenes Volumen aller verwendeter Spezies und der daraus bestimmte Teilchendurchmesser

beträgt wurde $v_{a}$ für alle weiteren Spezies berechnet. Durch diese Vorgehensweise wird gewährleistet, dass auch bei der Erhöhung der realen Gesamtteilchenzahl durch chemische Reaktionen immer ausreichend Raum für die entstehenden Teilchen ist. Die benutzten Werte von $v_{a}$ und der sich daraus ergebende Teilchendurchmesser sind in Tab. 3.2 angegebenen.

\subsubsection{Glättung der Messwerte}

Während eines Zeitintervalls $t+\mathrm{d} t_{\Delta K W}$ müssen viele der im Rahmen der Lösung der Rayleigh-Plesset-Gleichung (3.53) zum Zeitpunkt $t$ bestimmten Größen in der Zeit extrapoliert werden. Zu diesem Zweck wird zum Zeitpunkt $t$ mittels der Funktion svdfit aus [82] ein Polynom an die letzten 9 ermittelten Werte der jeweils behandelten Größe angefittet. Dabei wird eine Singulärwertzerlegung zur Fehlerquadratminimierung benutzt. Das erhaltene Polynom kann nun zu jedem Zeitpunkt des Intervalls $\left[t+\mathrm{d} t_{\Delta K W}\right]$ ausgewertet werden und man erhält eine Schätzung des Wertes der Größe zu diesem Zeitpunkt. Dieses Verfahren wird z.B. zur Ermittlung von $P_{W}$ und $\dot{P_{W}}$ an den Stützstellen des zur Lösung von (3.53) eingesetzten Runge-Kutta-Verfahrens eingesetzt.

Ein Polynom 1. Grades, also eine lineare Anpassung, hat sich als besonders stabil und ausreichend genau erwiesen. Ein Beispiel für den Fit von Polynomen 1. Grades an einen typischen Verlauf des Blasenwanddruckes ist in Abb. 3.2 angegeben.

Gerade bei Rechnungen, die nur eine relativ geringe Teilchenanzahl verwenden, ist ein hohes Rauschen der Messwerte nicht zu vermeiden. Um daraus resultierende 
Artefakte zu vermeiden, bzw. zu verringern, werden einige für den Fortlauf der Simulation kritischen Werte mittels des soeben beschriebenen Fits eines Polynoms geglättet. Dies betrifft z.B. die Temperatur an der Blasenwand $T_{W}$ (siehe Kap. 4.1.2) oder die Rate der Verdampfung $\dot{m}$ (siehe Kap. 4.2).

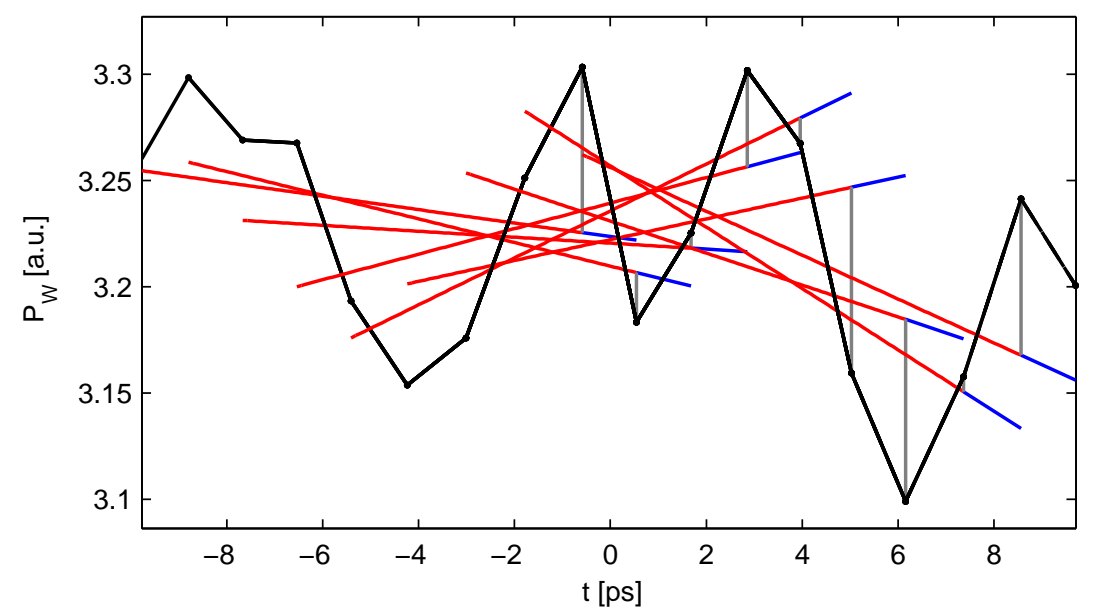

Abbildung 3.2: Beispiel für einen Polynomfit 1. Ordnung an den Wanddruck $P_{W}$. Die schwarze Kurve (-) gibt die aus der Simulation erhaltenen Druckwerte an. Die am Anfang jedes Intervalls ermittelten Polygone sind in ihrem Wertebereich - den neun jeweils vorigen Werten - dargestellt (-). Sie sind mit dem zugehörigen Zeitpunkt ihrer Berechnung zur besseren Darstellung verbunden $(-)$, sowie in den Bereich verlängert, in dem sie für die Schätzung der Ergebnisse verwendet werden (-). 


\subsection{Blasendynamik}

\subsubsection{Blasenrandbewegung: Rayleigh-Plesset-Gleichung}

Erste Arbeiten zur Beschreibung der radialen Dynamik einer Blase in einer Flüssigkeit wurden von LoRD RAYLEIGH durchgeführt [59]. Er beschrieb den Kollaps einer nicht-getriebenen Blase ohne Druck im Blaseninneren. Eine solche Blase schwingt nach dem Kollaps nicht wieder auf, sie verschwindet.

Später wurden seine Arbeiten durch MinnaERT um einen Blaseninnendruck erweitert [89]. Dadurch bekam die simulierte Blase einen Gleichgewichtsradius, den sie nach erfolgtem Kollaps unter abklingenden Nachschwingern annimmt.

Schließlich wurde von Plesset das Modell um einen die Oberflächenspannung $\sigma$ berücksichtigenden Term erweitert [60], Poritsky erfaßte die Viskosität $\nu$ der Flüssigkeit (im Folgenden als Wasser angenommen) [90]. Beide Effekte dämpfen die Bewegung des Blasenrandes.

Insgesamt ergab sich die Rayleigh-Plesset-Gleichung:

$$
R \ddot{R}+\frac{3}{2} \dot{R}^{2}=\frac{1}{\varrho_{W}}\left(P_{L}-P_{\infty}\right)
$$

Hierbei ist $P_{L}$ der Druck im Wasser direkt an der Blasenwand:

$$
P_{L}=P_{W}(t)-\frac{2 \sigma}{R}-4 \varrho_{W} \nu \frac{\dot{R}}{R}
$$

sowie $P_{\infty}$ der Druck in großer Entfernung zur Blase:

$$
P_{\infty}=P_{0}+P_{a}(t)=P_{0}-P_{a 0} \sin \left(\omega_{a} t\right) .
$$

$\varrho_{W}$ ist die Dichte von Wasser, $\sigma$ die Oberflächenspannung und $\nu$ die kinematische Viskosität des Wassers. $P_{W}(t)$ gibt den Gasdruck am Blasenrand, $P_{0}$ den konstanten Umgebungsdruck sowie $P_{a}(t)$ den akustischen Wechseldruck an.

Eine weitere wichtige Ergänzung des Modells war die Berücksichtigung des Energieverlustes, den die Blasendynamik durch die Abstrahlung einer Schallwelle in das schwach kompressible Blasenmedium erfährt. Dazu wird (3.50) ergänzt, so dass im hier vorliegenden Blasenmodell folgende erweiterte Form der Rayleigh- 
Plesset-Gleichung (RPG) genutzt wird:

$$
R \ddot{R}+\frac{3}{2} \dot{R}^{2}=\frac{1}{\varrho_{W}}\left(P_{L}-P_{\infty}\right)+\frac{R}{\varrho_{W} c_{W}}\left(\dot{P}_{W}(t)-\dot{P}_{\infty}\right)
$$

Der letzte Term beschreibt die Schallabstrahlung in die Flüssigkeit, $c_{W}$ ist die Schallgeschwindigkeit in Wasser.

Es existieren mehrere Varianten der Bewegungsgleichung einer Blase in einem schwach kompressiblen Medium, darunter die Keller-Miksis-Gleichung [62] und die Gilmore-Gleichung [61]. Diese Gleichungen weisen kleinere Unterschiede auf, sind aber alle, wie ProsperetTi zeigte, in 1. Ordnung richtig und äquivalent [91][92]. Lin zeigte, dass eine inhomogene Druckverteilung in der Blase, wie sie im vorliegenden Modell gegen Ende des Kollaps eintritt, die Gültigkeit der RPG nicht beeinflußt [93].

(3.53) ist eine gewöhnliche Differentialgleichung, die mit einem Runge-KuttaVerfahren 4. Ordnung gelöst wird [82]. Der Parameter $P_{W}(t)$, der Gasdruck am Blasenrand, muß nach Gl. (4.1) durch die Hartkugelsimulation bestimmt werden. Diese benötigt wiederum den Ort und die Geschwindigkeit des Blasenrandes aus der RPG, um den Zeitpunkt von Wandkollisionen korrekt vorhersagen zu können. Diese Teile der Simulation sind also voneinander abhängig und müssen abwechselnd bestimmt werden.

Die Rayleigh-Plesset-Gleichung wird gelöst und das Ergebnis ist für ein gewisses Zeitintervall gültig. Innerhalb dieses Intervalls wird die Geschwindigkeit des Blasenrandes als konstant angenommen. Das Zeitintervall wird so gewählt, dass die Anzahl der Wandkollisionen in einem Intervall, $\Delta K_{W}$, gleich bleibt. Damit erreicht man auch eine höhere zeitliche Auflösung gegen Ende der Simulation, da nun ungleich mehr Wandkollisionen auftreten. Der Wert von $\Delta K_{W}$ wird in Abhängigkeit der initialen Teilchenzahl festgelegt und bewegt sich zwischen 50 und 10.000 .

Die am Ende eines Intervalls ermittelten Werte für $P_{W}(t)$ und $\dot{P}_{W}(t)$ werden für die erneute Lösung der Rayleigh-Plesset-Gleichung benutzt.

\subsubsection{Festlegung der Simulationszeitspanne}

Durch den enormen Rechenaufwand, den Molekulardynamiksimulationen beanspruchen, ist es zumeist nur möglich, kleine (System-) Zeitspannen in akzepta- 
bler Rechenzeit zu simulieren. Aus diesem Grund ist es wichtig einen geeigneten Startpunkt für die Simulation zu finden, welcher zu korrekten Ergebnissen führt - und dabei Phasen vermeidet, in denen die MD-Simulation keine nennenswerten Vorteile zu einfacheren Modellen bietet.

Im Falle einer getriebenen Kavitationsblase ist hauptsächlich der schnelle Kollaps von Interesse, während das langsame Aufschwingen der Blase weniger aufschlußreich ist. Aus diesem Grund wird zunächst eine komplette Blasenschwingung in einem einfachen Modell berechnet, bei dem das Innere der Blase als homogen angenommen wird. Diese Betrachtung reicht aus, um die für den Start der Hartkugel-Simulation benötigten Anfangsbedingungen zu erhalten. Das vereinfachte Modell geht davon aus, dass die Zustandsänderung des betrachteten Vander-Waals-Gas polytrop erfolgen:

$$
p \cdot V^{\gamma}=\text { const }=p_{0} \cdot V_{0}^{\gamma} .
$$

$p$ ist der Blaseninnendruck, $V$ das Volumen, $p_{0}$ und $V_{0}$ die jeweiligen Werte im Gleichgewichtszustand. $\gamma$ bezeichnet den Polytropenexponenten, der je nach Prozeßart zwischen $\gamma=1.0$ (für den isothermen Fall) und $\gamma=\frac{c_{p}}{c_{v}}=\frac{f+2}{f}$ (für den adiabatischen Fall) variiert. Dabei ist $\frac{c_{p}}{c_{v}}$ das Verhältnis der spezifischen Wärmen und $f$ die Anzahl der angeregten Freiheitsgrade des Van-der-Waals-Gases. Da hier hauptsächlich der maximale Blasenradius von Interesse ist, bei dessen Erreichen die Blase als quasi-isotherm angesehen werden kann, wird im polytropischen Modell $\gamma=1.0$ genutzt.

Beachtet man, daß das Volumen durch das ausgeschlossene Volumen des Gases $V_{a}$, bzw. das spezifische ausgeschlossene Volumen des Gases $v_{a}=V_{a} / m$ nach unten begrenzt ist, läßt sich (3.54) schreiben als:

$$
\frac{p}{p_{0}}=\left(\frac{V_{0}-V_{a}}{V-V_{a}}\right)^{\gamma}=\left(\frac{1 / \varrho_{0}-v_{a}}{1 / \varrho-v_{a}}\right)^{\gamma} .
$$

Setzt man weiterhin die Massenerhaltung $\varrho R^{3}=\varrho_{0} R_{0}^{3}$ ein (keine Wasserdampfeffekte werden hier inkludiert) folgt weiter:

$$
p(t)=p_{0}\left(\frac{\varrho_{0} R_{0}^{3}\left(1 / \varrho_{0}-v_{a}\right)}{\varrho R^{3}\left(1 / \varrho-v_{a}\right)}\right)^{\gamma}=p_{0}\left(\frac{R_{0}^{3}-o^{3}}{R^{3}-o^{3}}\right)^{\gamma}, \text { mit } o^{3}=R_{0}^{3} \varrho_{0} v_{a} .
$$


Die Änderung des Druckes ergibt sich nach Zeitableitung:

$$
\dot{p}(t)=-\gamma p_{0} \frac{\left(R_{0}^{3}-o^{3}\right)^{\gamma-1}}{\left(R(t)^{3}-o^{3}\right)^{\gamma-1}} \cdot \frac{\left(R_{0}^{3}-o^{3}\right)}{\left(R^{3}-o^{3}\right)^{2}} \cdot 3 R^{2} \dot{R}=-\gamma p(t) \frac{3 R^{2} \dot{R}}{R(t)^{3}-o^{3}} .
$$

Mit den hier ermittelten Ausdrücken für $p$ und $\dot{p}$ wird nun die Rayleigh-PlessetGleichung (3.53) integriert und so der Blasenrandverlauf während einer kompletten Periode der Anregung berechnet. Abb. 3.3 zeigt den Verlauf des Blasenradius einer solche Rechnung für eine Blase unter typischen SBSL-Bedingungen.

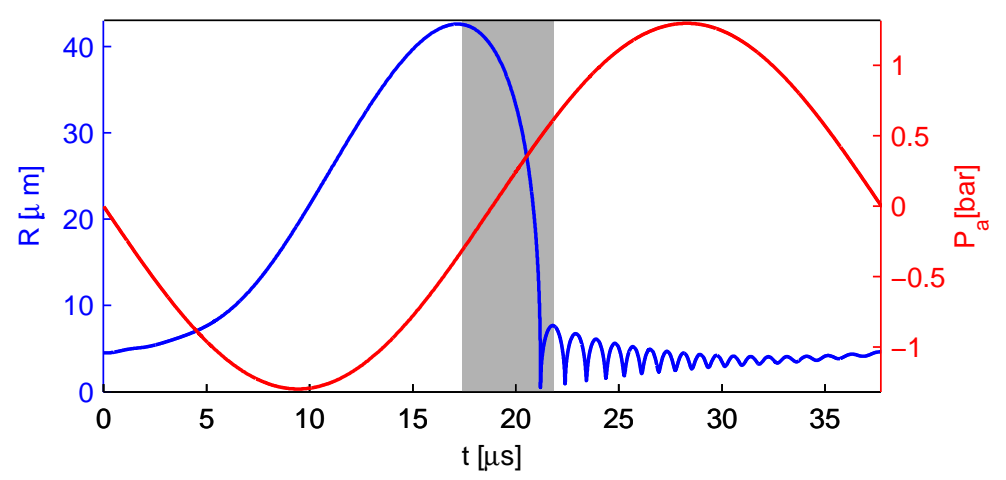

Abbildung 3.3: Verlauf von Blasenradius (-) und Anregungsdruck (-) für eine Periode der Anregung bei $\nu_{a}=\omega_{a} / 2 \pi=26,5 \mathrm{kHz}$ und $P_{a}=1,3$ bar. Berechnet in einem einfachen polytropischen Modell. Der graue Bereich zeigt eine typische Zeitspanne, während derer das Hartkugelmodell genutzt wird.

Die in diesem Modell bestimmte Radius-Zeit-Kurve wird nun benutzt, um den Startpunkt der Hartkugelsimulation zu bestimmen. Um den Kollaps komplett zu erfassen bietet sich ein Übergang zum Zeitpunkt des maximalen Blasenradius an (wie in Abb. 3.3 verdeutlicht). Aus Gründen der Rechenzeitersparnis kann auch ein späterer Zeitpunkt gewählt werden, zu dem sich die Blasenwand bereits mit einer gewissen Anfangsgeschwindigkeit $v_{W, \text { init }}$ bewegt.

Die Simulation wird typischerweise während der dem Kollaps folgenden Aufschwungsphase (der erste 'Rebound') beendet. Wird eine geringe Teilchenzahl gewählt können auch komplette Anregungsperioden mit mehreren Hauptkollapsen simuliert werden. 


\subsection{Lichtemission}

Wie in Kap. 2.2.3 dargelegt ist der momentan am weitesten akzeptierte Mechanismus der Lichtentstehung die thermische Strahlung. Das gemessene Spektrum kann gut über ein Bremsstrahlungsspektrum angenähert werden. Zur Abschätzung der Lumineszenz der simulierten Blasen wird ein von Wu und RoBERTs entwickeltes Emissionsmodell verwendet, welches auf der Bremsstrahlungsleistung des durch Kompressionserwärmung ionisierten Blaseninneren aufbaut [17][48].

Es wird davon ausgegangen, dass das Innere der Blase für Photonen durchsichtig ist, also keine Rückkopplung der Lichtentstehung auf das Teilchenmodell stattfindet. Dieser Ansatz scheint gerechtfertigt, da die mittlere freie Weglänge von Photonen mit $200 \mathrm{~nm}$ Wellenlänge in einem ionisierten Gas 8,6 $\mu \mathrm{m}$ beträgt, also deutlich mehr als die Größe der Emissionsregion [94].

Zur Abschätzung der emittierten Bremsstrahlungsleistung ist die Kenntnis des Ionisationsgrades $q=\frac{n_{i}}{n}$ nötig, also des Verhältnis von ionisierten Atomen $n_{i} \mathrm{zu}$ der Gesamtzahl der Atome $n$. Unter der Voraussetzung, dass in jeder Messzelle ein thermodynamisches Gleichgewicht zwischen Elektronen und Ionen herrscht, kann dieser mittels der Saha-Gleichung ermittelt werden [95]:

$$
\frac{q^{2}}{1-q} \approx 2.41 \cdot 10^{21} \cdot \frac{T^{3 / 2} \mathrm{e}^{\frac{-\chi_{1}}{k^{T}}}}{n}
$$

Hier ist $\chi_{1}$ die 1 . Ionisierungsenergie, $k_{B}$ die Boltzmannkonstante und $T$ die Temperatur. So kann für alle chemischen Spezies getrennt der Ionisationsgrad berechnet werden. Eine Übersicht der Ionisierungsenergien findet sich in Tab. 3.3. Nach Kenntnis des Ionisationsgrades kann die spektrale Bremsleistungsdichte pro Volumeneinheit berechnet werden [94]:

$$
\frac{\mathrm{d} P_{B r}}{\mathrm{~d} \lambda} \approx 1.57 \cdot 10^{-40} q^{2} n^{2} A_{l} T^{-1 / 2} \frac{\mathrm{e}^{\frac{-A_{l}}{\lambda T}}}{\lambda^{2}}, \quad \text { mit } \quad A_{l} \approx 1.44 \cdot 10^{-2} \mathrm{~K} \cdot \mathrm{m} .
$$

Es ist zu beachten, dass in dem vorliegenden Modell nur Einfach-Ionisation der Edelgasatome zum Tragen kommt. Würden $Z$ Elektronen ionisiert, müßte Gl. 3.59 mit $Z^{2}$ multipliziert werden.

Integriert man nun über den gesamten Wellenlängenbereich, erhält man die gesamte Bremsleistungsdichte jeder Messzelle:

$$
P_{B r} \approx 1.57 \cdot 10^{-40} q^{2} n^{2} T^{1 / 2} .
$$


Um die Daten besser mit den experimentellen Werten vergleichen zu können, sollte man diese Integration lediglich über den gemessenen Wellenlängenbereich durchführen:

$$
P_{B r} \approx 1.57 \cdot 10^{-40} q^{2} n^{2} T^{1 / 2}\left(\mathrm{e}^{\frac{-A_{l}}{\lambda_{\max T}}}-\mathrm{e}^{\frac{-A_{l}}{\lambda_{\min } T}}\right) .
$$

Eine Integration über das Volumen der Blase ergibt die Bremsstrahlungsleistung pro Zeiteinheit $P_{S L}$. Durch eine weitere Integration über die Zeit erhält man schließlich die gesamt emittierte Lichtenergie $E_{S L}$.

\begin{tabular}{cc}
\hline Spezies & $\chi_{1}[\mathrm{eV}]$ \\
\hline $\mathrm{He}$ & 24,587 \\
$\mathrm{Ar}$ & 15,769 \\
$\mathrm{Xe}$ & 12,130 \\
$\mathrm{H}_{2} \mathrm{O}$ & 13,605 \\
$\mathrm{H}$ & 13,598 \\
$\mathrm{H}_{2}$ & 13,598 \\
$\mathrm{O}$ & 13,618 \\
$\mathrm{O}_{2}$ & 13,618 \\
$\mathrm{OH}$ & 13,608 \\
$\mathrm{HO}_{2}$ & 13,611 \\
$\mathrm{H}_{2} \mathrm{O}_{2}$ & 13,608 \\
\hline
\end{tabular}

Tabelle 3.3: Die 1. Ionisierungsenergie $\chi_{1}$ für alle vorkommenden Spezies. Die Werte der gemischten Sauerstoff- und Wasserstoffverbindungen wurden aus den Werten der Elemente gemittelt. 


\section{Kapitel 4}

\section{Einfluß der Parameter des MD-Modells}

In diesem Kapitel sollen Erweiterungen des grundsätzlichen Hartkugelmodells besprochen werden. Dies sind z.B. die Berücksichtigung von Wasserdampf und chemischen Reaktionen oder die Einführung einer thermischen Grenzschicht am Blasenrand. In den einzelnen Unterkapiteln werden zunächst die dem implementierten Effekt zugrunde liegenden Überlegungen und Formeln dargestellt.

Daran anschließend werden relevante Ergebnisse angegeben, die sich aus der Erweiterung des Modells ergeben.

Desweiteren werden Auswirkungen angesprochen, die eine Änderung wichtiger Modellparameter - z.B. der Anzahl an simulierten Teilchen - auf die Ergebnisse der Simulationen haben. 


\subsection{Randbedingung}

\subsubsection{Druck am Blasenrand}

Um die Teilchensimulation des Blaseninneren an die durch die Rayleigh-PlessetGleichung (3.53) beschriebene Bewegung des Blasenrandes koppeln zu können, benötigt man den aktuellen an der Blasenwand herrschenden Gasdruck $P_{W}(t)$. $P_{W}(t)$ wird direkt über den Impuls, der innerhalb der letzten $\Delta K_{W}$ Wandkollisionen von den kollidierenden Teilchen an die Blasenwand übertragen wurde, berechnet.

$$
P_{W}(t)=\frac{1}{4 \pi R^{2} \Delta t_{\Delta K W}} \sum_{\Delta K_{W}} m_{i} \Delta \overrightarrow{v_{i}} \cdot \frac{\vec{r}}{r} .
$$

$\Delta \overrightarrow{v_{i}}$ ist die Geschwindigkeitsänderung des Teilchens nach (3.34) oder (3.36) und $\vec{r} / r$ stellt einen Normalenvektor an die Blasenwand dar (da sich das Teilchen an der Blasenwand befindet). $\Delta K_{W}$ ist die Anzahl an Wandkollisionen, die zwischen den Neuberechnungen von (3.53) stattfindet. Dieser Wert wird bei Start der Simulation je nach anfänglicher Teilchenanzahl $N_{\text {ges }, i}$ passend vorgegeben. $\Delta t_{\Delta K W}$ ist die Zeit, die zum Erreichen der $\Delta K_{W}$ Wandkollisionen verstreicht. Während dieser Zeit wird die Bewegung der Blase als konstant angesehen.

Durch die im Kollaps zunehmende Zahl an Wandkollisionen wird eine Erhöhung der zeitlichen Auflösung bei zunehmender Blasenwandgeschwindigkeit erreicht. $\Delta t_{\Delta K W}$ wird am Anfang eines Intervalls geschätzt und ein entsprechendes Ereignis in den Ereignisbaum eingetragen. Die Abweichung des erreichten $\Delta K_{W}$ zum vorgegebenen Wert fließt in die nächste Bestimmung von $\Delta t_{\Delta K W}$ ein.

Um zu starke Oszillationen des Wanddruckes zu vermeiden, wird $P_{W}$ über die Anpassung eines Polynoms erster Ordnung an die 9 letzten Werte berechnet (siehe Kap. 3.2.6). $\dot{P}_{W}(t)$ ergibt sich nach zeitlicher Ableitung des erhaltenen Polynoms.

\subsubsection{Wärmeaustausch mit dem Medium}

Man nimmt in der Flüssigkeit eine thermische Grenzschicht der Dicke $\delta$ mit einem quadratischen Profil an [96][97]:

$$
\frac{T_{L}(r, t)-T_{\text {water }}}{T_{W}-T_{\text {water }}}=\left(1-\frac{r-R}{\delta}\right)
$$


Damit gilt für die Flüssigkeitstemperatur $T_{L}: T_{L}(R)=T_{W}$ (die Temperatur der Blasenwand), $T_{L}(R+\delta)=T_{\text {water }}$ (die Flüssigkeitstemperatur in großer Entfernung zur Blase). Die Wärmeleistung $\dot{Q}$ der Grenzschicht kann nach dem Fourierschen Gesetz $\dot{Q}=-A \lambda_{W} \frac{\mathrm{d} T_{L}}{\mathrm{~d} r}$ bestimmt werden:

$$
\dot{Q}=\frac{8 \pi R^{2} \lambda_{W}}{\delta}\left(T_{W}-T_{w a t e r}\right)
$$

Hier bezeichnet $\lambda_{W}=0,609 \mathrm{~J} /(\mathrm{msK})$ die Wärmeleitzahl von Wasser. Die Änderung der Dicke der Grenzschicht läßt sich aus der Intergration der Energiegleichung der Flüssigkeit über die Grenzschicht berechnen [77]. Vereinfacht man dann mittels des Ansatzes $\delta<<R$ weiter, kommt man auf folgende Beziehung:

$$
\frac{\mathrm{d}}{\mathrm{d} t}\left(R^{2}\left(T_{W}-T_{\text {water }}\right) \delta\right)=6 D_{W} R^{2}\left(T_{W}-T_{\text {water }}\right) / \delta
$$

Dabei ist $D_{W}=1,462 \cdot 10^{-7} \mathrm{~m}^{2} / \mathrm{s}$ die thermische Diffusivität von Wasser. Mit Hilfe dieser Beziehung läßt sich (4.3) nach der Zeit integrieren. Man erhält für die gesamte abgegebene Wärmemenge Q:

$$
Q=\frac{4}{6} \frac{\pi R^{2} \lambda_{W}}{D_{W}}\left(T_{W}-T_{w a t e r}\right) \delta
$$

Ersetzt man hier wiederum $\delta$ wie in (4.3) angegeben, erhält man für die aktuelle Temperatur des Mediums an der Blasenwand $T_{W}$ :

$$
T_{W}=T_{\text {water }} \pm \frac{\sqrt{6 D_{W} Q \dot{Q}}}{8 \pi R^{2} \lambda_{W}} .
$$

Durch das quadratische Profil der Grenzschicht ist eine Berechnung von $\mathrm{T}_{W}$ nur möglich, wenn $Q$ und $\dot{Q}$ das selbe Vorzeichen besitzen. Sind $Q, \dot{Q}>0$, so gilt in (4.6) die Addition, für den negativen Fall die Subtraktion. Haben $Q$ und $\dot{Q}$ verschiedene Vorzeichen wird $T_{W}=T_{\text {water }}$ gesetzt. Diese Vorgehensweise läßt sich durch die Argumentation rechtfertigen, dass ein vorhandener Energieüberschuss im Wärmespeicher (den die thermische Grenzschicht darstellt) erst abgebaut werden muß, bevor $T_{W}$ einen Vorzeichenwechsel relativ zur Umgebungstemperatur vollziehen kann.

Mittels (4.6) kann die für das aktuelle Intervall $\Delta t_{\Delta K W}$ gültige Wandtemperatur bestimmt werden. Die Wärmemengen werden dabei direkt aus der Hartkugelsimulation bestimmt (mit $\dot{Q}=\dot{Q}_{G}$, die vom Gas an die Blasenwand abgegebene 
Wärmeleistung):

$$
\dot{Q}_{G}=\frac{3}{2} k_{B} \alpha_{t} \frac{\Delta K}{\mathrm{~d} t_{\Delta K W}}\left(T_{i}-T_{W}^{\prime}\right) .
$$

$T_{W}^{\prime}$ bezeichnet die für das letzte Intervall ermittelte Wandtemperatur, $k_{B}$ die Boltzmann-Konstante, $\alpha_{t}$ den thermischen Akkomodationskoeffizienten (siehe nächstes Kapitel), $\Delta K$ bedie Anzahl der in der Intervalllänge $\Delta t_{\Delta K W}$ erfolgten Kollisionen, $T_{i}$ ist die Temperatur der einfallenden Teilchen. Unter der Annahme, dass die Gruppengeschwindigkeit der Teilchen in der äußersten Schicht der Blase der Blasenwandgeschwindigkeit $v_{R}$ entspricht, gilt für diese:

$$
T_{i}=\frac{1}{3 k_{B} \Delta K} \sum_{j=1}^{\Delta K} m_{j}\left(\overrightarrow{v_{j}}-v_{R}\left(-\frac{\overrightarrow{r_{j}}}{r_{j}}\right)\right)^{2} .
$$

Da sich das Teilchen zum betrachteten Zeitpunkt direkt an der Blasenwand befindet, stellt $(-\vec{r} / r)$ einen Normalenvektor an die Wand dar.

Wird die Verdampfung und Kondensation von Wasserdampf berücksichtigt, muß die Wärmeleistung noch um die latente Wärme erweitert werden (siehe Kap. 4.2).

Die gesamte abgegebene Wärmemenge $Q$ bestimmt sich dann einfach aus der Summe der Wärmeleistungen aller j Kopplungsintervalle:

$$
Q=\sum_{j} \frac{3}{2} k_{B} \frac{\Delta K(j)}{\mathrm{d} t_{\Delta K W}(j)}\left(T_{i}(j)-T_{W}^{\prime}(j)\right) .
$$

Zum Ausgleich statistischer Schwankungen wird die Wandtemperatur aus einem linearen Fit an die letzten 5 Werte ermittelt (Siehe Kap. 3.2.6) .

\subsubsection{Der thermische Akkomodationskoeffizient $\alpha_{t}$}

Die Menge der vom Blaseninneren in das Medium gelangenden Energie ist abhängig von der Art der Wechselwirkung von Blasenwand und Teilchen bei einer Wandkollision. Wird von einer spiegelnden Reflektion ausgegangen, die lediglich die Richtung der Teilchengeschwindigkeit ändert sowie den Betrag um die Wandgeschwindigkeit in Normalenrichtung erhöht (siehe 3.2.1.4), findet keinerlei Wärmeaustausch statt und es gilt immer $T_{W}=T_{\text {water }}$ sowie $T_{r}=T_{i}$. Dies ist der adiabatische Grenzfall.

Geht man hingegen davon aus, dass die Teilchen alle ihre Energie an die Wand abgeben, bewegen sie sich nach einer Wandkollision mit Wandgeschwindigkeit 

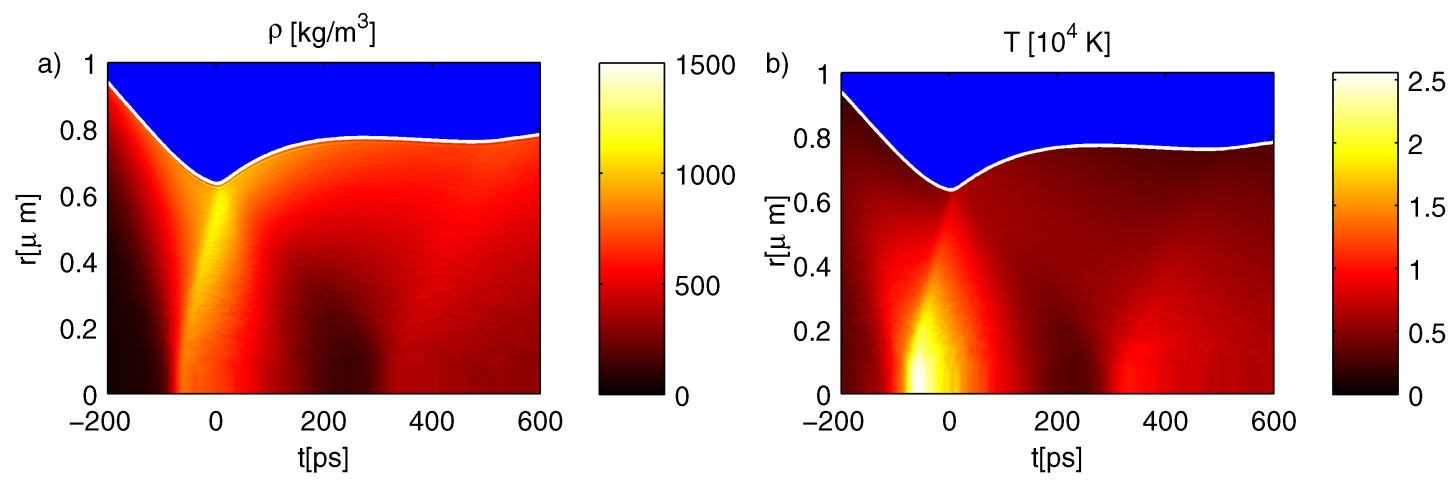

Abbildung 4.1: Entwicklung der Dichte und der Temperatur um die Zeit des minimalen Blasenradius ( $\mathrm{t}=0$ ) für den Fall $\alpha_{t}=1,0$ (isotherm). Sonstige Parameter siehe Tab. 4.1.

und weisen eine der Wandtemperatur entsprechende Temperatur auf $\left(T_{r}=T_{W}\right)$. Hiermit ist der isotherme Grenzfall realisiert. Die Wandtemperatur wird in diesem Fall nach (4.6) errechnet.

In der Realität muß davon ausgegangen werden, dass weder ein rein adiabatischer noch rein isothermer Wärmeübergang stattfindet. Aus diesem Grund wurde eine variable thermische Randbedingung eingeführt, die eine präzise Steuerung des Anteils der übertragenen Energie über einen thermischen Akkomodationskoeffizienten $\alpha_{t}$ erlaubt:

$$
T_{r}=\left(1-\alpha_{t}\right) \cdot T_{i}+\alpha_{t} \cdot T_{W}
$$

Für $\alpha_{t}=1$ bzw. $\alpha_{t}=0$ ergeben sich der isotherme bzw. der adiabatische Grenzfall. Im Folgenden soll der Einfluß des Wertes von $\alpha_{t}$ auf die Verhältnisse in der Blase und auf die Parameter der Blasenwand erläutert werden. Es wurden Vergleichrechnungen für verschiedene Werte von $\alpha_{t}$ durchgeführt. Die sonstigen verwendeten Parameter finden sich in Tab. 4.1.

\begin{tabular}{ccc|ccc|ccc}
\hline$P_{a}$ & - & $1,3 \mathrm{bar}$ & $N_{N G}$ & - & 216.000 & $v_{W, \text { init }}$ & - & $25 \mathrm{~m} / \mathrm{s}$ \\
$\nu_{a}$ & - & $26,5 \mathrm{kHz}$ & $S$ & - & $\approx 56.000$ & $\alpha_{t}$ & - & $0,0-1,0$ \\
$R_{0}$ & - & $4,5 \mu \mathrm{m}$ & Edelgas & - & Argon & $A_{v}$ & - & 0,3 \\
$T_{\text {water }}$ & - & $300 \mathrm{~K}$ & $\Delta K_{W}$ & - & 3.000 & & & \\
\hline
\end{tabular}

Tabelle 4.1: Parameter der Vergleichsrechnungen zum Einfluß von $\alpha_{t}$. $P_{a}$ bezeichnet den Anregungsdruck, $\nu_{a}$ die Anregungsfrequenz, $R_{0}$ den Ruheradius, $T_{w a t e r}$ die Wassertemperatur, $N_{N G}$ die simulierte Anzahl an Edelgasteilchen in der Blase, $S$ den Skalierungsfaktor (siehe Kap. 4.4), $\Delta K_{W}$ die Anzahl der Wandkollisionen zwischen zwei Lösungen der Randbedingung, $v_{W \text {, init }}$ die Geschwindigkeit der Blasenwandbei Start der Simulation, $\alpha_{t}$ den themischen Akkomodationskoeffizient und $A_{v}$ den Faktor des variablen Wasserdampf-Akkomodationskoeffizienten (siehe Kap. 4.2). 

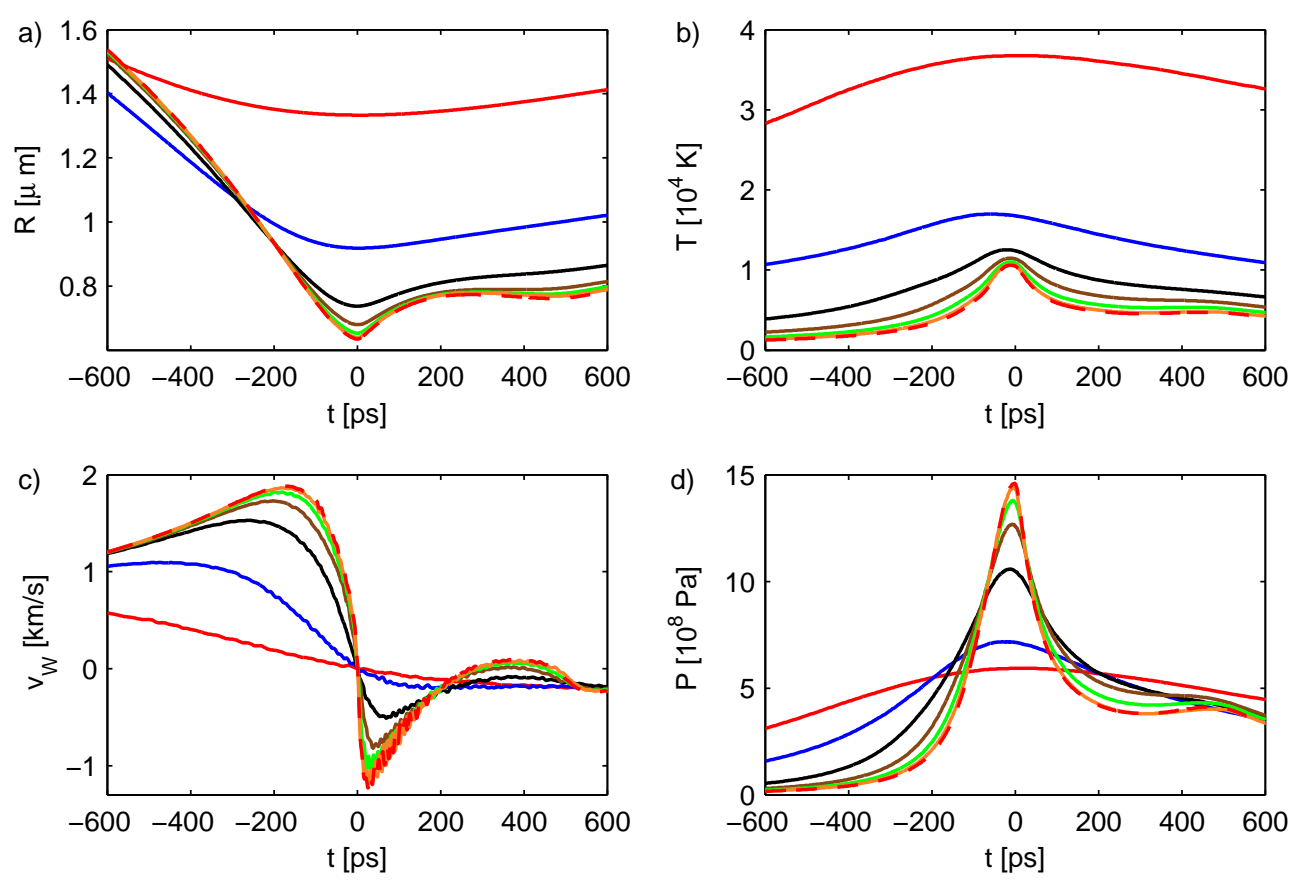

Abbildung 4.2: Verlauf des Blasenradius (a), der Durchschnittstemperatur (b), der Blasenwandgeschwindigkeit (c) und des durchschnittlichen Druckes in der Blase (d) um den Hauptkollaps $(\mathrm{t}=0)$. Parameter siehe Tab. 4.1.

Legende: $\alpha_{t}=0,0(-) ; 0,1(-) ; 0,3(-) ; 0,5(-) ; 0,7(-) ; 0,9(-) ; 1,0(-\mathbf{-})$.

Die nachfolgenden Abbildungen zeigen den Verlauf diverser Blasenkenngrößen für Variationen von $\alpha_{t}$ im Intervall von 0 bis 1. Dabei stellen Abb. 4.2 und 4.4 gemittelte Werte (über das gesamte Blasenvolumen, bzw. über die Blasenwand) dar, während Abb. 4.3 Werte für die innerste Messzelle (das Blasenzentrum) sowie einer äußeren Messzelle (in der Nähe des Blasenrandes) zeigt. Dies erlaubt eine Einsicht in die raumzeitliche Dynamik des Blaseninneren. Abb. 4.1 stellt diese Entwicklung speziell für $\alpha_{t}=1,0$ noch einmal raumzeitlich dar. Tab. 4.2 fasst die relevanten Ergebnisse nochmals zusammen.

Mit sinkendem $\alpha_{t}$ verbleibt mehr Energie in der Blase, was erwartungsgemäß zu höheren Durchschnittstemperaturen (Abb. 4.2,b) führt. Für $\alpha_{t}=1,0$ werden im Maximum fast viermal so hohe Werte erreicht als bei $\alpha_{t} \leq 0,5$. Die heiße Phase ist für kleine Werte des thermischen Akkomodationskoeffizienten deutlich ausgeprägter, die Dynamik langsamer.

Da die Teilchen in der Blase bei kleinem $\alpha_{t}$ eine höhere kinetische Energie besitzen, herrscht über den längsten Teil des Kollaps hinweg ein größerer Druck in der Blase (Abb. 4.2,d), der auch an die Blasenwand weitergegeben wird (Abb. 4.4, c). 

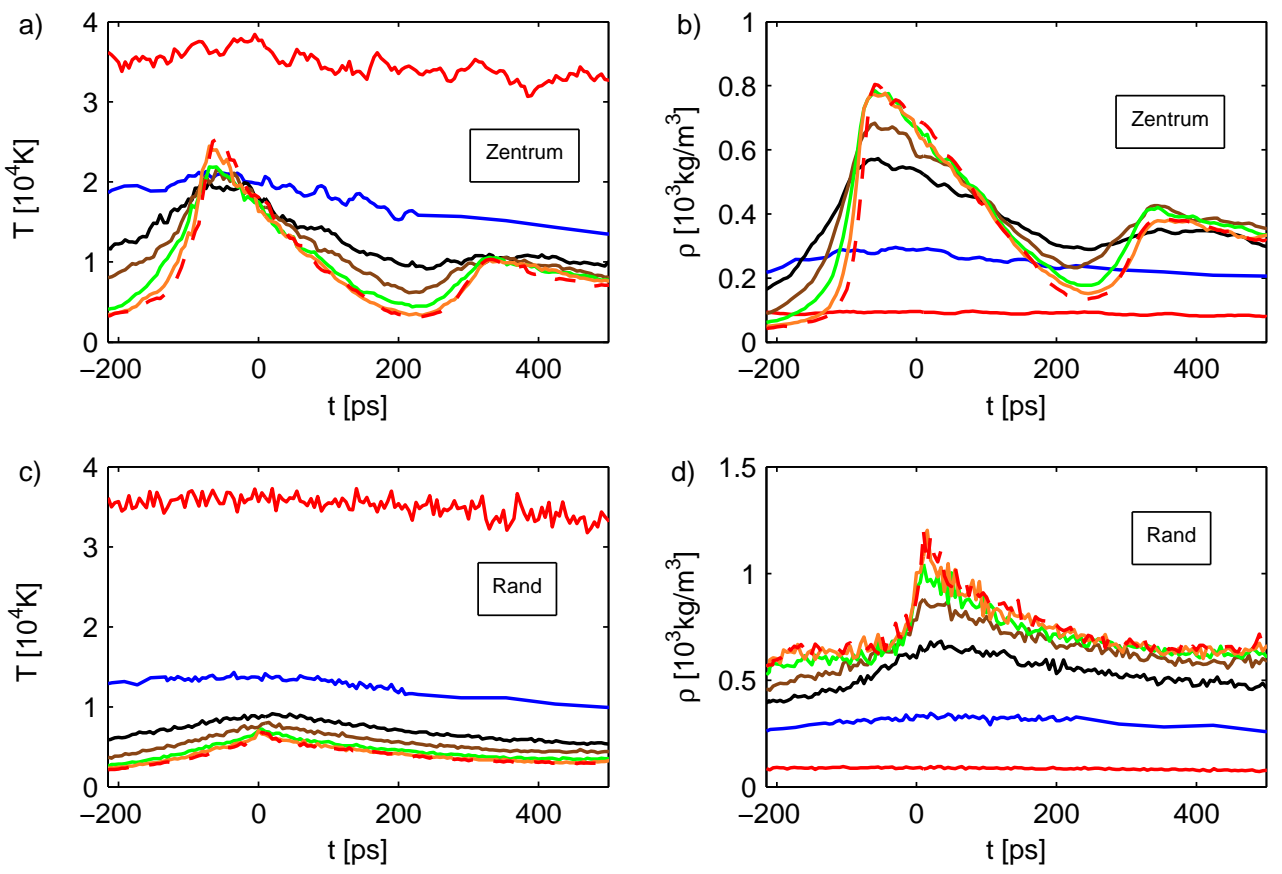

Abbildung 4.3: Verlauf von Temperatur und Dichte an Blasenrand und Blasenzentrum. Parameter siehe Tab. 4.1.

Legende: $\alpha_{t}=0,0(-) ; 0,1(-) ; 0,3(-) ; 0,5(-) ; 0,7(-) ; 0,9(-) ; 1,0(--)$.

Dieser Gegendruck bremst die Radiusdynamik und führt zu einer verminderten Blasenwandgeschwindigkeit (Abb. 4.2,c) und somit zu einem abgeschwächten Kollaps mit einem geringeren Minimalradius (Abb. 4.2,a). In der letzten Phase des Kollaps steigen Druck und Wanddruck dafür umso stärker, je größer $\alpha_{t}$ gewählt wird. Dies erklärt sich durch die größere Kompression im stärkeren Kollaps.

Bereits der Verlauf der Durchschnittstemperatur und des Durchschnittsdrucks zeigen, daß die Zeitskala der Dynamik in der Blase mit steigendem $\alpha_{t}$ kleiner wird. Noch deutlicher wird dies, wenn man die Dynamik im Blasenzentrum $(4.3, \mathrm{a} \& \mathrm{~b})$ mit der am Blasenrand $(4.3, \mathrm{c} \& d)$ vergleicht. Für $\alpha_{t}=0$ ist das Innere der Blase für den betrachteten Zeitraum örtlich und zeitlich homogen, am Rand und im Zentrum herrschen gleiche Drücke und Temperaturen. Je größer $\alpha_{t}$ gewählt wird, umso inhomogener werden die Blasenverhältnisse. Bei $\alpha_{t}=0,1$ sind bereits größere räumliche Unterschiede auszumachen (21.000 K im Zentrum gegenüber $14.000 \mathrm{~K}$ am Rand). Ab $\alpha_{t}=0,3$ läßt sich auch eine zeitliche Inhomogenität im Blasenzentrum erkennen, mit einem deutlichen Temperatur- und Dichtepeak 50 bis 70 ps vor der Zeit des minimalen Radius $\left(t_{R m i n}\right)$. Diese Peaks steilen sich für wachsendes $\alpha_{t}$ immer mehr auf, die Inhomogenität in der Blase nimmt weiter zu. 


\begin{tabular}{lllllllllll}
\hline$\alpha_{t}$ & $\begin{array}{l}R_{\min } \\
{[\mu \mathrm{m}]}\end{array}$ & $\begin{array}{l}v_{W} \\
{[\mathrm{~km} / \mathrm{s}]}\end{array}$ & $\begin{array}{l}T_{W} \\
{\left[10^{4} \mathrm{~K}\right]}\end{array}$ & $\begin{array}{l}P_{W} \\
{[\mathrm{GPa}]}\end{array}$ & $\begin{array}{l}T_{\varnothing} \\
{[\mathrm{K}]}\end{array}$ & $\begin{array}{l}T_{i} \\
{[\mathrm{~K}]}\end{array}$ & $\begin{array}{l}T_{\max } \\
{[\mathrm{K}]}\end{array}$ & $\begin{array}{l}F_{S} \\
{\left[\mathrm{~kg} / \mathrm{m}^{3} \mathrm{~s}\right]}\end{array}$ & $\begin{array}{l}E_{S L} \\
{[\mathrm{pJ}]}\end{array}$ & $\begin{array}{l}\Delta t_{S L} \\
{[\mathrm{ps}]}\end{array}$ \\
\hline 0,0 & 1,33 & 0,67 & 0,42 & 0,95 & 3,68 & 5,16 & 3,96 & - & 46,80 & 1.010 \\
0,1 & 0,92 & 1,09 & 8,09 & 1,92 & 1,70 & 2,01 & 2,15 & 0,49 & 0,319 & 320 \\
$\mathbf{0 , 3}$ & $\mathbf{0 , 7 3}$ & $\mathbf{1 , 5 3}$ & $\mathbf{9 , 9 6}$ & $\mathbf{3 , 4 1}$ & $\mathbf{1 , 2 5}$ & $\mathbf{1 , 2 6}$ & $\mathbf{2 , 0 1}$ & $\mathbf{2 , 8 7}$ & $\mathbf{0 , 0 3 9}$ & $\mathbf{1 2 5}$ \\
0,5 & 0,68 & 1,73 & 9,94 & 4,50 & 1,15 & 1,10 & 2,15 & 5,97 & 0,024 & 70 \\
0,7 & 0,65 & 1,82 & 9,30 & 5,25 & 1,10 & 0,96 & 2,19 & 9,92 & 0,022 & 50 \\
0,9 & 0,64 & 1,87 & 9,20 & 5,41 & 1,07 & 0,94 & 2,44 & 15,14 & 0,021 & 45 \\
1,0 & 0,63 & 1,88 & 9,26 & 5,50 & 1,06 & 0,95 & 2,55 & 18,04 & 0,024 & 45 \\
\hline
\end{tabular}

Tabelle 4.2: Parameterstudie $\alpha_{t}: R_{\text {min }}$ bezeichnet den minimalen Blasenradius, $v_{W}$ die maximale Blasenrandgeschwindigkeit, $T_{W}$ die maximale Wandtemperatur, $P_{W}$ den maximalen Wanddruck, $T_{\varnothing}$ die maximale Durchschnittstemperatur, $T_{i}$ die maximale Temperatur der einfallenden Teilchen, $T_{\max }$ die absolute Maximaltemperatur, $F_{S}$ die Stoßstärkekennzahl, $E_{S L}$ die gesamt emittierte Lichtenergie, $\Delta t_{S L}$ die Halbwertsdauer der Lichtemission. Der hervorgehobenen Wert von $\alpha_{t}=0,3$ findet im weiteren Verlauf der Arbeit Benutzung.

Es fällt auf, daß gerade diejenigen Rechnungen, welche die geringsten maximalen Durchschnittstemperaturen $\mathrm{T}_{\varnothing}$ aufweisen, kurzfristig hohe Zentrumstemperaturen erreichen. So beträgt $\mathrm{T}_{\varnothing} 17.000 \mathrm{~K}$ für $\alpha_{t}=0,1$, während für $\alpha_{t}=0,9$ lediglich $10.700 \mathrm{~K}$ erreicht werden. Dennoch ist die absolut maximale Temperatur im letzteren Fall mit 24.400 K deutlich größer als im Erstgenannten (21.500 K).

Diese Beobachtung lässt sich durch eine dichte, relativ kalte Randschicht erklären, welche sich für hohe Werte von $\alpha_{t}$ während des gesamten Kollapses ausbildet. Durch die langanhaltende Beschleunigung der Blasenwand bleibt diese Schicht bis kurz vor Ende des Kollaps eng am Rand der Blase. Erst wenn die Wandgeschwindigkeit $v_{W}$ schließlich aufgrund des hohen Gasdruckes in der Blase zu sinken beginnt, löst sich diese Schicht von der Blasenwand, läuft als Verdichtungswelle oder -stoss durch die Blase und konvergiert im Zentrum. Dort thermalisiert die Energie teilweise und führt zu dem beobachteten Temperatursprung. Das Volumen der heißen Region ist jedoch so klein, dass die Durchschnittstemperaturen relativ niedrig bleiben.

Die Welle wird im Zentrum reflektiert und läuft im Anschluss an das Temperaturmaximum unter weiter zunehmender Dichte zum Blasenrand zurück. Es werden Dichten von bis $1.200 \mathrm{~kg} / \mathrm{m}^{3}\left(=1,2 \varrho_{W}\right)$ erreicht. Die auslaufende Welle hinterläßt ein vergleichsweise kaltes, wenig popularisiertes Blasenzentrum.

Das Auftreffen der reflektierten Welle auf den Blasenrand führt zu einem relativ abrupten Ende des Kollaps und einem nachfolgenden Aufschwingen der Blase. Dies ist auch der Zeitpunkt der maximalen Blasenwandtemperatur, die für $\alpha_{t}>$ 0,1 zwischen 9.000 und $10.000 \mathrm{~K}$ liegt, also weit über der kritischen Temperatur. 

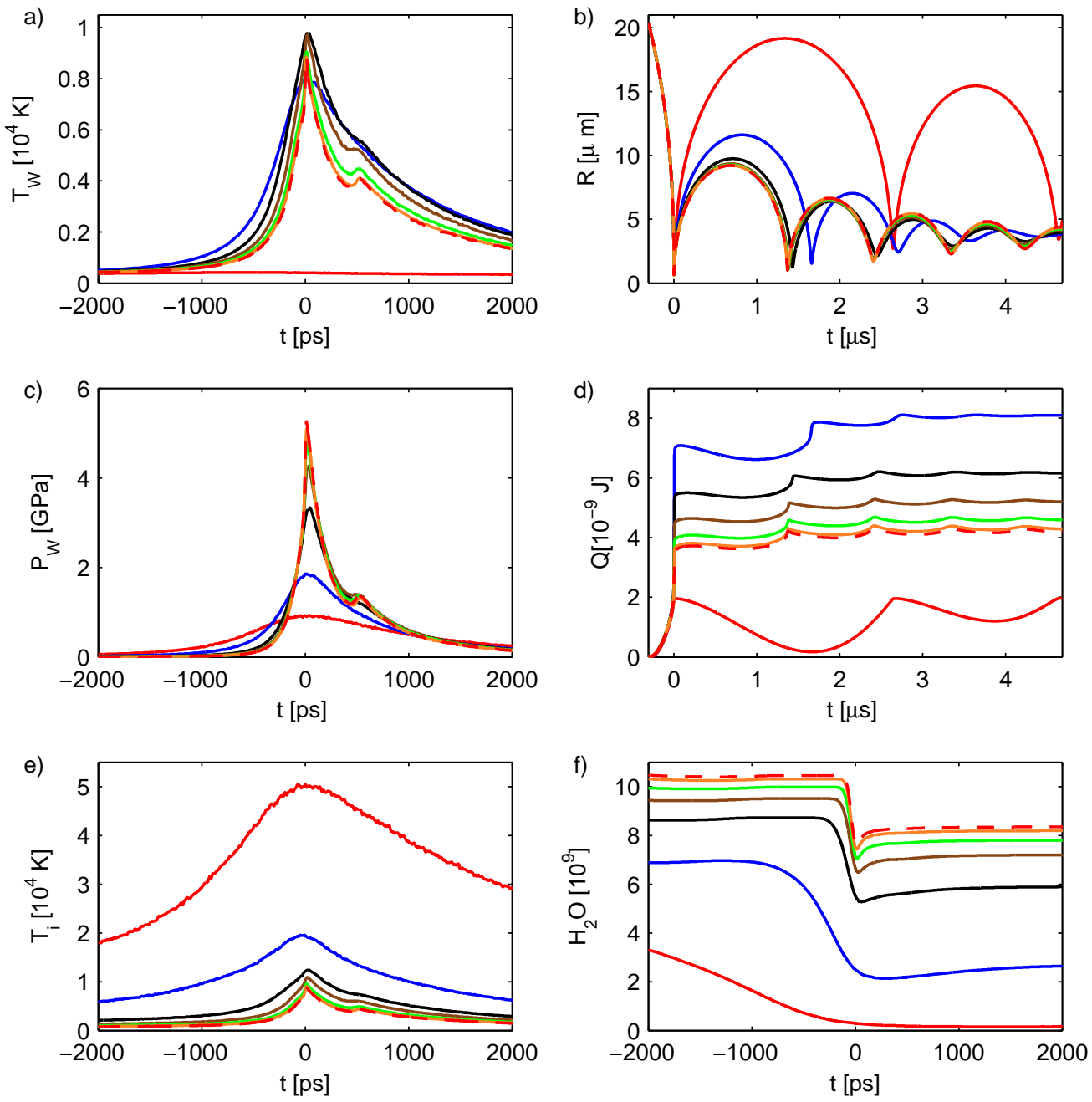

Abbildung 4.4: Parameterstudie $\alpha_{t}$ :Verlauf der Wandtemperatur(a), des Wanddruckes (c), der Temperatur der einfallenden Teilchen (e) und der Anzahl der $\mathrm{H}_{2} \mathrm{O}$ - Moleküle (f) jeweils während des Hauptkollapses $(t=0)$; sowie des Blasenradiusses (b) und der abgegebenen Wärmemenge (d) jeweils in den ersten $5 \mu \mathrm{s}$. a), c), e) wurden leicht entrauscht. Parameter siehe Tab. 4.1. Legende: $\alpha_{t}=0,0(-) ; 0,1(-) ; 0,3(-) ; 0,5(-) ; 0,7(-) ; 0,9(-) ; 1,0(-)$.

Nachfolgend läßt sich beobachten, dass die Welle an der Blasenwand reflektiert wird und zu einem zweiten lokalen Maximum der Zentrumstemperatur (bis $\approx$ $10.000 \mathrm{~K}$ ) -und -dichte (bis $\approx 400 \mathrm{~kg} / \mathrm{m}^{3}$ ) führt.

Nach der Reflektion der Verdichtungswelle an der Blasenwand kommt es zu einem abrupten Absinken des Wanddrucks $P_{W}$. In dessen Folge wird die zu diesem Zeitpunkt hohe Expansionsgeschwindigkeit des Blasenrandes stark vermindert. Gilt $\alpha_{t}>0,5$, kommt es sogar zu einem Vorzeichenwechsel der Blasenwandgeschwindigkeit $v_{W}$ - die Blase wird also kurzfristig wieder komprimiert. Diese Phase 
dauert je nach $\alpha_{t}$ ca. 100 - 150 ps. Erst wenn die Verdichtungswelle, nach einer erneuten Reflektion am Blasenzentrum, ein weiteres Mal die Blasenwand erreicht stabilisiert sich $P_{W}$ und $v_{W}$ dreht wieder ins Negative. Nun hat die Verdichtungswelle so viel Energie verloren, dass sie nicht mehr beobachtbar ist. In Abb. 4.1 lassen sich diese Ausführungen für den Fall $\alpha_{t}=1,0$ gut nachvollziehen. VuONG fand in einem Kontinuumsmodell vergleichbare Ergebnisse, obwohl Wasserdampfeffekte vernachlässigt und somit Temperaturen $>10^{5} \mathrm{~K}$ auftraten [20] [27].

Für kleine Werte von $\alpha_{t}$ wird die Ausbildung einer dichten Randschicht aufgrund der - auch nach einer Kollision mit der Blasenwand - hohen Teilchengeschwindigkeiten verhindert. Dies führt zu einer annähernd homogenen Verteilung der Teilchen in der Blase, wodurch die Entstehung von Verdichtungswellen, oder gar -stößen, unterbunden wird. Für $\alpha_{t}=0$ ist dieser Effekt sehr ausgeprägt. Aufgrund der in diesem Fall sehr trägen Blasenranddynamik ändert sich die Temperatur im in Abb. 4.3 dargestellten Zeitraum kaum. Das Maximum liegt bei sehr hohen 36.800 K. Die Dichte ändert sich ebenfalls nur geringfügig, das Maximum liegt bei lediglich ca. $90 \mathrm{~kg} / \mathrm{m}^{3}$. Ein Temperatur- oder Dichtegradient ist zu keinem Zeitpunkt vorhanden.

Der Verlauf der Wandtemperatur (4.4,a) zeigt, dass für alle Fälle, bis auf $\alpha_{t}=0$ relative ähnliche Maximalwerte erreicht werden (zwischen 8.000 und $10.000 \mathrm{~K}$ ). Die zeitliche Dynamik ist von der Dynamik der Verdichtungswelle im Blaseninneren geprägt. Deutlich ist deren zweites Auftreffen auf die Blasenwand ca. 600 ps nach $t_{R \text { min }}$, zu erkennen. Auffallend ist der abrupte Anstieg der Wandtemperatur beim Übergang von $\alpha_{t}=0,0 \mathrm{zu} \alpha_{t}=0,1$. Während im ersten Fall (erwartungsgemäß) keine Veränderung von $T_{W}$ eintritt, zeigt der zweite Fall bereits eine mit allen anderen Fällen vergleichbare Dynamik. Die hohe Temperatur gleicht den Effekt des geringeren Wärmeübergangs nahezu aus. Erst bei weiterer Reduktion von $\alpha_{t}$ sinkt $T_{W}$ merklich ab. Für $\alpha_{t}=0,05$ werden maximal $5.900 \mathrm{~K}$ erreicht, für $\alpha_{t}=0,01$ sind es $2.000 \mathrm{~K}$ (nicht graphisch gezeigt).

Durch die unterschiedliche Menge an in der Blase verbleibender Energie führt die Variation von $\alpha_{t}$ zu einer deutlichen Beeinflussung der Dynamik des Blasenrandes (Abb. 4.4,b): der hohe Druck im Inneren der Blase für kleine $\alpha_{t}$ lässt sie im ersten Rebound erheblich weiter aufschwingen und verzögert den Zeitpunkt des zweiten Kollaps. Die Blase schwingt für $\alpha_{t} \geq 0,5$ bis $\approx 9,3 \mu \mathrm{m}$ auf. Bei $\alpha_{t}=0,3$ sind es $9,7 \mu \mathrm{m}$, bei $\alpha_{t}=0,1$ bereits $11,6 \mu \mathrm{m}$ und bei $\alpha_{t}=1,0$ schließlich 19,1 $\mu \mathrm{m}$. Der Zeitpunkt des zweiten Kollapses verschiebt sich um 1,3 $\mu$ s.

Der Blick auf die zeitliche Entwicklung der abgegebenen Wärmemenge $Q(4.4, \mathrm{~d})$ 

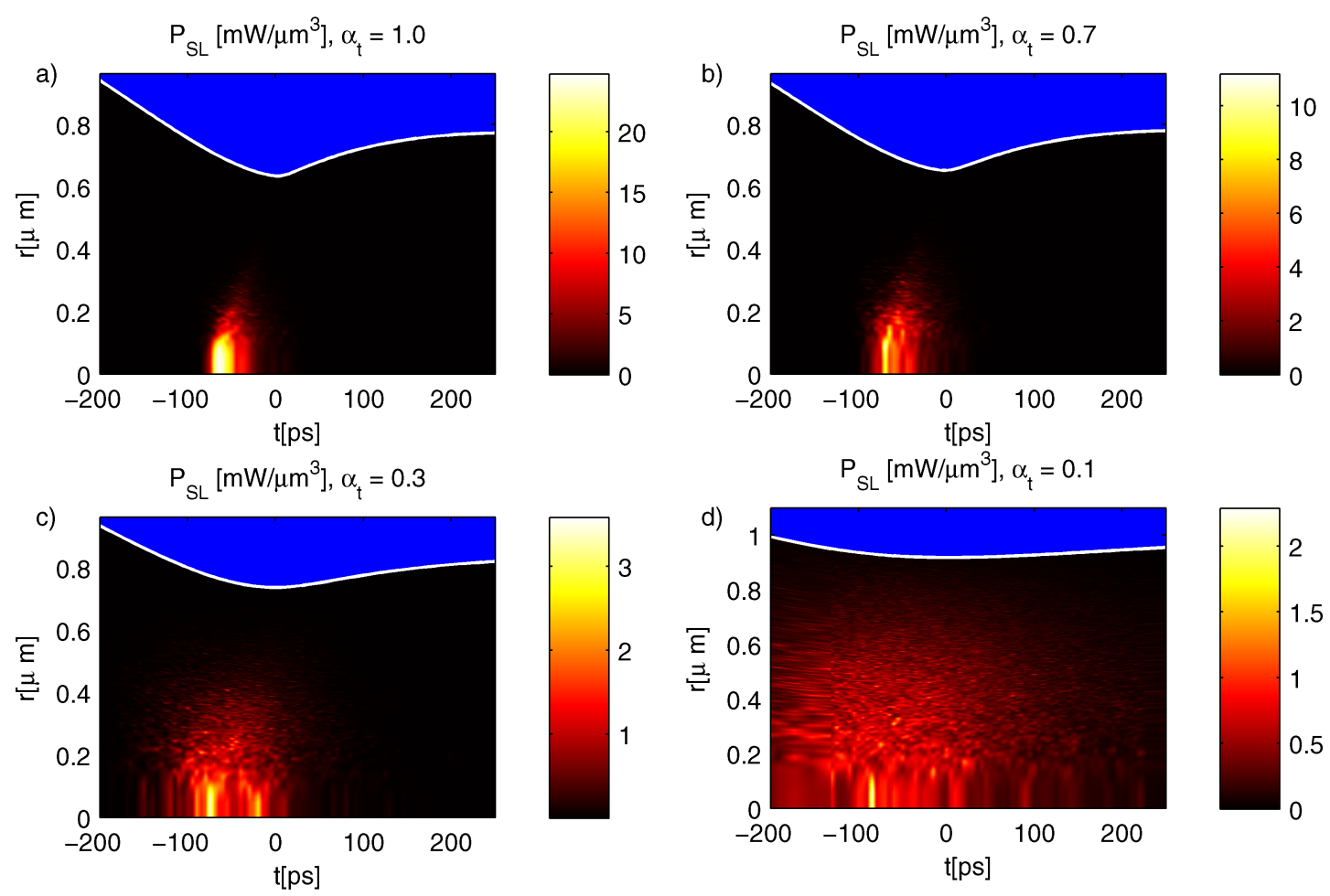

Abbildung 4.5: Lichtleistung in der Blase gegen Ende des Kollaps für $\alpha_{t}=1,0$ (a), $\alpha_{t}=0,7$ (b), $\alpha_{t}=0,3$ (c) und $\alpha_{t}=0,1$ (d). Sonstige Parameter siehe Tab. 4.1.

überrascht zunächst, da gerade für kleine $\alpha_{t}$ die größten $Q$-Werte erreicht werden. Es ist ersichtlich, daß der Hauptteil der Wärmeleistung in der letzten Phase des Hauptkollapses erbracht wird, in welchem Teilchen hoher Temperatur auch zum Blasenrand getragen werden. Bei kleinen $\alpha_{t}$-Werten weisen zum einen die auf die Blasenwand einfallenden Teilchen eine deutlich höhere Temperatur auf (4.4,e), zum anderen ist diese Phase deutlich ausgedehnter. Diese beiden Effekte kompensieren den geringeren Energieübertrag. Betrachtet man die Phase des langsamen Kollaps (hier nicht extra gezeigt), so wird erwartungsgemäß für großes $\alpha_{t}$ am meisten Wärme abgegeben. Erst ca. $0,1 \mu$ s vor Erreichen von $R_{\text {min }}$ dreht sich dieses Verhältnis um.

Die höheren Teilchengeschwindigkeiten bei kleinem $\alpha_{t}$ führen $\mathrm{zu}$ vermehrten Wandkollisionen und somit zu einer erhöhten Kondensation von Wasserdampf aus der Blase. Abb. 4.4, f zeigt die Menge des in der Blase verbliebenen Wasserdampfes (als Plateau zu erkennen). Der starke Abfall der Teilchenzahl um $t_{\text {Rmin }}$ kennzeichnet das Einsetzen der chemischen Reaktionen. Eine vermehrte Kondensation führt zu einer größeren Kondensationswärmeleistung $P_{L}$ (siehe Kap. 4.2). 
Dies ist eine weitere Erklärung für das unerwartet hohe $Q$ für kleine $\alpha_{t}$. Im Fall von $\alpha_{t}=0,0$ ist $P_{L}$ sogar der einzige für den Verlauf von $Q$ verantwortliche Faktor, da sonst keinerlei Wärmeübergang an der Blasenwand stattfindet.

Als Maß für die Stärke des Stoßes, bzw. der Verdichtungswelle, wird der Wert $F_{S}$ eingeführt, der sich aus $F_{S}=\delta \varrho / \delta t_{\varrho_{1 / 2}}$ ergibt. Dabei bezeichnet $\delta t_{\varrho_{1 / 2}}$ die Zeit, in der die Dichte des Blasenzentrums von dem halben Maximalwert zum vollen Maximalwert ansteigt; $\delta \varrho$ ist die dabei auftretende Dichtedifferenz. Tab. 4.2 gibt $F_{S}$ sowie eine Zusammenfassung der Ergebnisse für alle betrachteten Rechnungen an. Dieser Wert dient lediglich dem Vergleich der Dynamik der verschiedenen Rechnungen und ist nicht zu einer Klassifizierung in Stoß- oder Verdichtungswellen geeignet.

Liegt $\alpha_{t}$ unter 0,3 steigt die emittierte Lichtenergie $E_{S L}$ (siehe Tab. 4.2) stark an. Sowohl die Emissionsdauer als auch die Größe des Emissionsgebietes sind an diesem Anstieg beteiligt. Abb. 4.5 zeigt die Lichtemission für unterschiedliche $\alpha_{t}$ - Werte. Deutlich ist zu erkennen, dass mit abnehmendem $\alpha_{t}$ immer größere Anteile des Blaseninneren Licht emittieren und $\Delta t_{S L}$ zunimmt. Gleichzeitig sinkt die maximal erreichte Leuchtstärke ab (zu beachten sind die unterschiedlichen Skalen). Dies wird aber durch die erstgenannten Effekte mehr als kompensiert. Bei $\alpha_{t}=0,1$ liegt die gesamte Strahlungsemission mit $E_{S L}=0,31$ pJ bereits um eine Größenordnung über den Werten von $\alpha_{t}=0,3-1,0$. Bei $\alpha_{t}=0,0$ beträgt $E_{S L}=46,8$ pJ mit einer Emissionsdauer von ca. 1 ns.

Zur Wahl des in künftigen Rechnungen verwendeten Wertes von $\alpha_{t}$ soll auf die Ergebnisse bisheriger Untersuchungen zurückgegriffen werden und anhand des Vergleichs mit den verschiedenen hier vorgestellten Rechnungen ein passender Wert ausgewählt werden [46].

Frühe Messungen deuteten auf extrem kurze Lichtpulse hin, deren Entstehung mit in der Blase auftretenden Stoßwellen, die einen starken, kurzzeitigen 'Hot Spot' im Blasenzenrum erzeugen, erklärt wurde. Eine derartige Betrachtung des Blaseninneren würde im vorliegenden Modell für den Einsatz eines $\alpha_{t}$-Wertes nahe 1 sprechen, für die eine starke Konzentration der Energie, verbunden mit kurzen Lichtemissionsdauern (45 ps) gefunden wurde.

Später wurde jedoch anhand von Einzel-Photonen-Zählung [24] sowie mit 'streak camera' - Aufnahmen [25] gezeigt, dass die Pulsbreite deutlich größer ist als bis dahin angenommen (bestätigt in [98] [26]). Für typische SBSL-Regime wurden Werte von $\Delta t_{S L}$ von 100 - 250 ps gefunden. Diese Ergebnisse widerlegten einen 
starken Einfluß von Stoßwellen auf die Lichterzeugung und sprechen eher für eine aus einem größeren Volumen stammende Emission. Anhand der Simulationsergebnisse erscheint daher eine Wahl von $\alpha_{t}<0,5$ als sinnvoll. Für $\alpha_{t}=0,0$ und $\alpha_{t}=0,1$ ergeben sich Werte von $\Delta t_{S L}$, die weit (1010 ps), bzw. knapp (320 ps) außerhalb des experimentell gefunden Intervalls liegen. Die Rechnung mit $\alpha_{t}=$ 0,3 liegt mit $\Delta t_{S L}=125$ ps genau im zu erwartenden Bereich.

Messungen der Kollapsgeschwindigkeit [99] zeigten, dass der maximale Wert von $v_{W}$ zwischen 1.200 und $1.600 \mathrm{~m} / \mathrm{s}$ liegt. Auch dieses experimentelle Ergebnis wird bei Rechnungen mit $\alpha_{t}=0,3\left(v_{W}\right.$ maximal $\left.1.530 \mathrm{~m} / \mathrm{s}\right)$ am besten bestätigt. In nachfolgenden Rechnungen wird daher zumeist $\alpha_{t}=0,3$ verwendet. 


\subsection{Kondensation und Verdampfung von Wasser- dampf}

Neben dem sich in der Blase ansammelnden Edelgas (je nach dem in der Flüssigkeit gelösten Gas) besteht die Blase hauptsächlich aus Wasserdampfmolekülen, deren Anzahl im Laufe einer Blasenschwingung stark variiert. Es wird davon ausgegangen, dass sich der Wasserdampfgehalt im Inneren der Blase über einen Großteil der Zeit im Gleichgewicht mit dem Äußeren befindet - d.h., dass der Partialdruck des Wasserdampfes am Blasenrand $P_{v}(R)$ mit dem Sättigungsdampfdruck von Wasser bei Umgebungstemperatur $P_{v, \text { sat }}\left(T_{\infty}\right)$ übereinstimmt. Dies gilt aufgrund der langsamen Zeitskala während des gesamten Aufschwingvorganges sowie der ersten Phase des Kollaps. Lediglich gegen Ende des Kollaps, wenn die Zeitskala der Bewegung des Blasenrandes deutlich kleiner wird als diejenige des Phasenüberganges, gilt diese Annahme nicht mehr. Zur korrekten Erfassung dieser Überlegungen muß eine Bedingung gefunden werden, welche für ein mit der Blasenwand kollidierendes Teilchen eine der folgenden Interaktionen bestimmt:

- Das Teilchen bleibt stecken (es kondensiert)

- Das Teilchen wird reflektiert sowie ein weiteres Teilchen aus der Flüssigkeit wird dem Inneren der Blase hinzugefügt (eine Verdampfung findet statt)

- Das Teilchen wird reflektiert, es findet keinerlei Phasenübergang statt.

Bei der Bestimmung dieser Bedingung geht man zunächst von den Ausdrücken für die Rate der Verdampfung $\left(\dot{m}_{\text {eva }}\right)$ und der Rate der Kondensation $\left(\dot{m}_{\text {cond }}\right)$ [28][100] aus:

$$
\dot{m}_{\text {eva }}=\frac{\alpha_{v}}{\sqrt{2 \pi R_{V}}} \frac{P_{v, s a t}\left(T_{l}(R)\right)}{\sqrt{T_{l}(R)}} \quad \dot{m}_{\text {cond }}=\frac{\alpha_{v}}{\sqrt{2 \pi R_{V}}} \frac{\Gamma_{C} \cdot P_{v}(R)}{\sqrt{T_{g}(R)}} .
$$

Dabei bezeichnet $R_{V}$ die Gaskonstante für Wasserdampf, $T_{l}(R)$ die Temperatur der Flüssigkeit am Blasenrand, $T_{g}(R)$ die Temperatur des Gases am Blasenrand. $\alpha_{v}$ ist der Akkomodationskoeffizient von Wasser, dessen Bedeutung im Folgenden herausgearbeitet wird; $\Gamma_{C}$ ist ein Korrekturfaktor für die Massenbewegung zum Blasenrand:

$$
\Gamma_{C}=e^{-\Omega^{2}}-\Omega \sqrt{\pi}\left(1-\frac{2}{\sqrt{\pi}} \int_{0}^{\Omega} e^{-x^{2}} d x\right) \quad \Omega=\frac{\dot{m}}{P_{v}(R)}\left(\frac{R_{V} T}{2}\right)^{1 / 2} .
$$


Hier bezeichnet $\dot{m}=\left(\dot{m}_{\text {eva }}-\dot{m}_{\text {cond }}\right) /\left(4 \pi R^{2} \cdot \mathrm{d} t_{\Delta K W}\right)$ die Rate der Verdampfung je Zeit- und Flächeneinheit. Diese wird direkt aus der Simulation anhand der im letzten Intervall $\mathrm{d} t_{\triangle K W}$ erfolgten Phasenübergänge bestimmt. Die Phasenübergangswahrscheinlichkeit für ein einzelnes Teilchen ergibt sich dann aus dem Verhältnis der Raten sowie $\alpha_{v}$ :

$$
P_{p c}=\alpha_{v}\left(1-\frac{\dot{m}_{e v a}}{\dot{m}_{c o n d}}\right)=\alpha_{v}\left(1-\sqrt{\frac{T_{g}(R)}{T_{l}(R)}} \frac{P_{v, s a t}}{\Gamma_{C} \cdot P_{v}(R)}\right) .
$$

Gilt $P_{p c}>0$, gibt $P_{p c}$ die Wahrscheinlichkeit für eine Kondensation an, im negativen Fall steht $P_{p c}$ für die Wahrscheinlichkeit einer Verdampfung.

Bei jeder Wandkollision eines Teilchens $p_{1}$ wird die Summe der bisherigen Wahrscheinlichkeiten gebildet. Übersteigt diese Summe den Wert '1' wird eine Kondensation vollzogen, das aktuelle Teilchen also aus der Blase entfernt. Unterschreitet die Summe dagegen den Wert '-1' findet eine Verdampfung statt: ein zusätzliches Teilchen $p_{2}$ wird am gegenüberliegenden Punkt der Blasenwand im Inneren der Blase plaziert. Diesem Teilchen wird die Geschwindigkeit $\vec{v}\left(p_{2}\right)=-\vec{v}\left(p_{1}\right)$ zugewiesen. Eine etwaige anfängliche Teilchenüberlappung wird ignoriert, bzw. es wird bei der Erstellung der Ereignisliste für $p_{2}$ die entsprechende Kollision verworfen.

Der Sättigungsdampfdruck von Wasser berechnet sich wie folgt [101]:

$$
P_{v, s a t}=133,322 \cdot e^{\left(18,3036-\frac{3816,53}{T_{l}(R)-46,13}\right)}[\mathrm{Pa}] .
$$

Die Rayleigh-Plesset-Gleichung (3.53) wird um die Druckterme des Wasserdampfes ergänzt:

$R \ddot{R}+\frac{3}{2} \dot{R}^{2}=\frac{1}{\varrho_{W}}\left(P_{L}-P_{\infty}\right)+\frac{R}{\varrho_{W} c_{W}}\left(\dot{p}(R, t)-\dot{P}_{\infty}\right)+\frac{\ddot{m}_{v} R}{\varrho_{W}}+\frac{\dot{m}_{v}}{\varrho_{W}}\left(\dot{R}+\frac{\dot{m}_{v}}{2 \varrho_{W}}\right)$.

An der Grenzfläche gilt nunmehr

$$
P_{L}=p(R, t)-P_{v}(R)-\frac{2 \sigma}{R}-4 \varrho_{W} \nu \frac{\dot{R}}{R}+\frac{4 \varrho_{W} \nu \dot{m}_{v}}{R \cdot \varrho_{W}}
$$

$\dot{m}_{v}$ sowie dessen Ableitung $\ddot{m}_{v}$ an den Stützstellen der Integration werden durch einen Polynom - Fit an die letzten 5 Werte geschätzt (siehe 3.2.6). 
Beim Phasenübergang des Wassers wird eine gewisse Wärmemenge, die latente Wärme $L_{v}$, vom Blasenmedium aufgenommen (Kondensation) oder abgegeben (Verdampfung). Die mikroskopische Erklärung des Auftretens der latenten Wärme liegt in der Überwindung der intermolekularen Potentialkräfte - daher ist sie mit keiner Temperaturänderung verbunden. Sie kann für den relevanten Temperaturbereich näherungsweise bestimmt werden aus der Formel

$$
L_{v}=-730.000 \cdot \log \left(660,373-T_{W}\right)+1.876 .488 .
$$

Bei vorhandenem Phasenübergang gilt nun nicht mehr $\dot{Q}=\dot{Q}_{G}$, da sich die Wärmeleistung im Gas von der Wärmeleistung in der Flüssigkeit um die Phasenübergangswärmeleistung $\dot{Q}_{L}=4 \pi R^{2} \dot{m}_{v} L_{v}$ unterscheidet: $\dot{Q}=\dot{Q}_{G}-\dot{Q}_{L}$. Dies muß in (4.6), bzw. (4.7) beachtet werden.

Oberhalb der kritischen Temperatur von Wasser $\left(T_{k r i t}=647,4 \mathrm{~K}\right)$ gibt es keinen Unterschied zwischen gasförmiger und flüssiger Phase, die Gleichung (4.12) verliert ihre Gültigkeit. Übersteigt $T_{W}$ die kritische Temperatur wird daher angenommen, dass der Phasenübergang zum Erliegen kommt. Der Wasserdampfgehalt der Blase bleibt also in der Endphase des Kollaps bis auf chemisch induzierte Veränderungen konstant. Diese Vorgehensweise wird auch durch die im Vergleich zur Zeitskala der Diffusion extrem kurzen Zeitskala des Kollaps motiviert [38]. Um Oszillationen der Phasenübergangswahrscheinlichkeit zu vermeiden wird mittels der Abbildung $P_{p c}=P_{p c} \cdot\left(\frac{1}{2}-\frac{1}{2} \cdot \tanh \left(T_{W}-T_{k r i t}\right)\right)$ ein stetiger Abfall, bzw. Anstieg, von $P_{p c}$ um den kritischen Punkt erreicht.

\subsubsection{Vapor trapping}

Durch die hohe Geschwindigkeit des Blasenrandes gegen Ende des Kollaps kann die Kondensation der Wassermoleküle nicht mehr im Gleichgewicht geschehen. Es bleibt also eine gewisse Menge an $\mathrm{H}_{2} \mathrm{O}$ in der Blase, dessen Dampfdruck z.T. weit über dem Sättigungsdampfdruck liegt. Der Wasserdampf sammelt sich vor allem im Blasenzentrum, da zum Einen die sich dort befindenden Teilchen nicht genug Zeit haben, um zur Blasenwand zu diffundieren und zum Anderen die leichten $\mathrm{H}_{2} \mathrm{O}$-Moleküle ins Blasenzentrum getrieben werden (dies gilt nur bei Verwendung eines Edelgases, dessen Masse über der von $\mathrm{H}_{2} \mathrm{O}$ liegt, siehe Kap. 5.3). Dieser Effekt wird als 'vapor trapping' bezeichnet. Die Menge des gefangenen Wasserdampfes hat einen großen Einfluß auf die Blasenverhältnisse, da die Blase gegen 
Ende des Kollaps als nahezu adiabatisch angesehen werden kann und die Masse des Wasserdampfes der Kompressionserwärmung entgegenwirkt. Desweiteren nehmen die Rotationsfreiheitsgrade der Wassermoleküle zusätzliche Energie auf.

Abb. 4.6 illustriert die Verteilung von Argon und $\mathrm{H}_{2} \mathrm{O}$ gegen Ende des Kollaps. Es ist zu erkennen, dass sich während des Kollaps eine dichte Schicht an Argon -
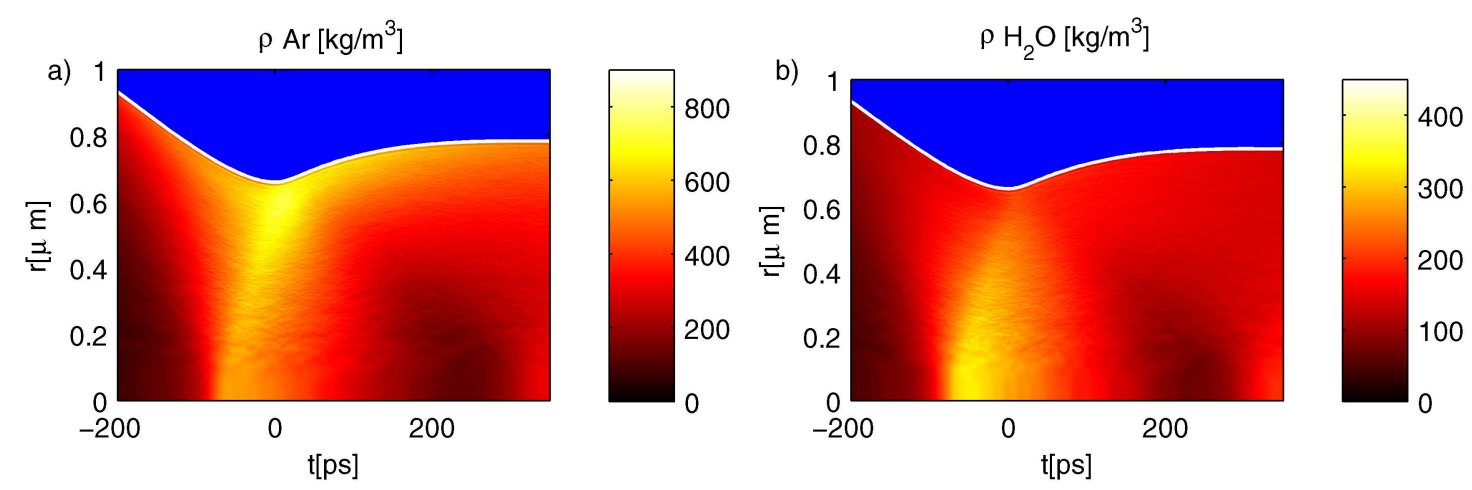

Abbildung 4.6: Dichte von Argon und $\mathrm{H}_{2} \mathrm{O}$ um den Zeitpunkt des minimalen Blasenradius $(\mathrm{t}=0) \cdot \alpha_{v}=0,1$. Sonstige Parameter siehe Tabelle 4.3 .

Atomen am Blasenrand ausgebildet hat, während die leichteren $\mathrm{H}_{2} \mathrm{O}$ - Moleküle diffuser in der Blase verteilt sind. Ungefähr 200 ps vor Erreichen des minimalen Radius lösen sich die Argon - Atome von der Blasenwand und treiben den Wasserdampf ins Blasenzentrum. Dort erreicht dieser sein Dichtemaximum, in der auslaufenden Verdichtungswelle sinkt die Wasserdampfdichte wieder ab. Das Blasenzentrum wird durch die Wasserdampfatome zu einem gewissen Grad abgeschirmt. Die Dichte der Argon-Atome nimmt in der auslaufenden Welle immer weiter zu, das Maximum wird beim Auftreffen auf die Blasenwand erreicht. Ein weiterer Effekt, der die Argon-Atome in der heißen Phase nach außen drückt ist die Thermodiffusion (Ludwig - Soret - Effekt): Schwere Teilchen (in diesem Fall Argon-Atome, verglichen mit den Wasserdampfmolekülen), die sich in einem Temperaturgradienten befinden, erfahren einen Nettoimpuls in Richtung der kalten Seite.

\subsubsection{Der $\mathbf{H}_{2} \mathbf{O}$-Akkomodationskoeffizient $\alpha_{v}$}

Die Menge des in der Blase verbleibenden Wasserdampfes hängt direkt mit der Phasenübergangswahrscheinlichkeit $P_{p c}$ zusammen, welche wiederum über den Parameter $\alpha_{v}$ beeinflußt werden kann. In der Literatur lassen sich Werte von $\alpha_{v}=$ 
0, 03 bis $\alpha_{v}=1,0$ finden [102][103]. Unlängst wurde bei einer simultanen Messung von $\alpha_{v}$ und $\alpha_{t}$ an einem Mikrotropfen Werte von $\alpha_{v}=0,12$ und $\alpha_{t}=0,65$ gefunden [104]. Storey und Szeri benutzen $\alpha_{v}=0,4$ [38]. Dieser Wert wurde zunächst auch von YASUI verwendet [105]. Später setzte er einen temperaturabhängigen Akkomodationskoeffizienten ein, dessen Wert von 0,35 (für $T_{l}<350 \mathrm{~K}$ ) bis 0,05 $\left(T_{l}>500 \mathrm{~K}\right)$ reicht [28]; siehe auch Kap. 4.2.2.2.

Die Verhältnisse in der Blase zeigen eine starke Abhängigkeit von der Menge des gefangenen Wasserdampfes, daher ist eine genaue Untersuchung mit dem Ziel einer möglichst korrekten Wahl von $\alpha_{v}$ unumgänglich.

\subsubsection{Temperaturunabhängiges $\alpha_{v}$}

Zunächst folgt eine allgemeine Betrachtung des Einflusses von $\alpha_{v}$ auf die Verhältnisse im Inneren der Blase. Zu diesem Zweck wurde zunächst eine Parameterstudie durchgeführt, bei der $\alpha_{v}$ im Intervall von 0,05 bis 0,8 variiert wurde; sonstige Kenngrößen blieben unverändert. Die Parameter der simulierten Blase finden sich in Tab. 4.3. Bei diesen Rechnungen wurde von einer Benutzung des chemischen Modells abgesehen, um die Abschätzung der Menge des in der Blase verbleibenden Wasserdampfes nicht zu verfälschen.

\begin{tabular}{ccc|ccc|ccc}
\hline$P_{a}$ & - & $1,3 \mathrm{bar}$ & $N_{N G}$ & - & 8.000 & $v_{W, \text { init }}$ & - & 0 bzw. $25 \mathrm{~m} / \mathrm{s}$ \\
$\nu_{a}$ & - & $26,5 \mathrm{kHz}$ & $S$ & - & $\approx 1.500 .000$ & $\alpha_{t}$ & - & 0,3 \\
$R_{0}$ & - & $4,5 \mu \mathrm{m}$ & Edelgas & - & Argon & $A_{v}$ & - & $0,25-2,0$ \\
$T_{\text {water }}$ & - & $300 \mathrm{~K}$ & $\Delta K_{W}$ & - & 200 & $\alpha_{v}$ & - & $0,05-0,8$ \\
\hline
\end{tabular}

Tabelle 4.3: Parameter der Vergleichsrechnungen zur Bestimmung von $\alpha_{v}$ und $A_{v}$.

Abb. 4.7, a zeigt den zeitlichen Verlauf der Anzahl an Wasserdampfteilchen $\left(\mathrm{N}_{\mathrm{H}_{2} \mathrm{O}}\right)$ in der Blase für verschiedene $\alpha_{v}$ während der ersten $9 \mu$ s der Blasenschwingung, Abb. 4.7,b eine Vergrößerung um $t_{R \min }$. In Abb. 4.7, c und 4.7,d sind die zugehörigen Radiusverläufe dargestellt. Tab. 4.4,a fasst die Ergebnisse dieser Simulationen zusammen.

Die starke Abhängigkeit des Wasserdampfgehaltes vom Akkomodationskoeffizienten wird deutlich: Für große Werte $(>0,15)$ zeigen sich scharfe Peaks in der Teilchenanzahl, die Zeitskala des Phasenübergangs ähnelt derjeniger der Blasendynamik. Je geringer $\alpha_{v}$ gewählt wird, umso träger folgt der Wasserdampfgehalt den Blasenrandoszillationen. Für $\alpha_{v}=0,05$ verschwindet sogar das dem Hauptkollaps folgende lokale Minimum. Dieses Minimum verschiebt sich mit steigendem 

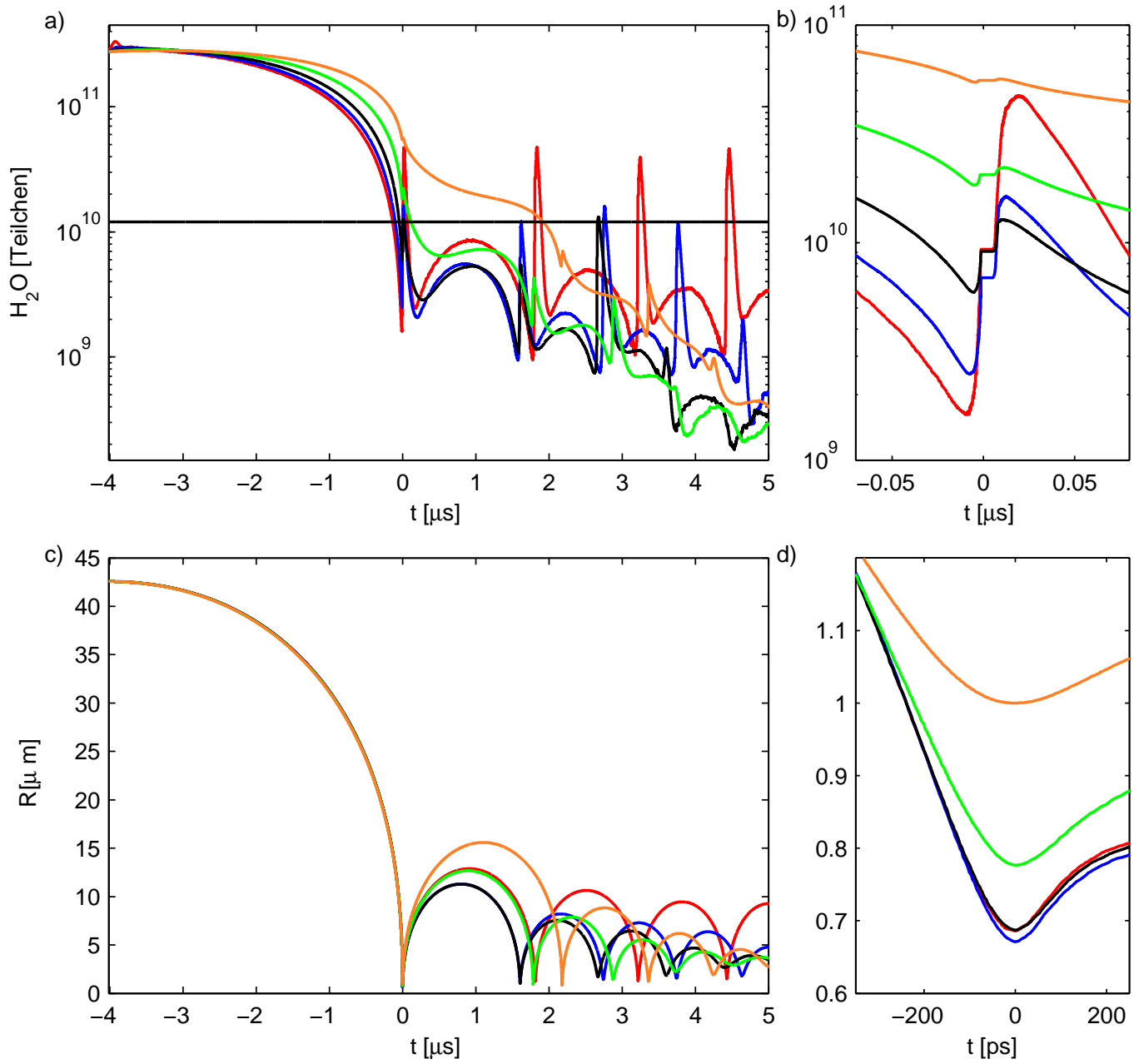

Abbildung 4.7: (a): Logarithmische Darstellung der realen Anzahl an $\mathrm{H}_{2} \mathrm{O}$ - Teilchen in der Blase für variiertes $\alpha_{v}$ mit $v_{W, \text { init }}=0 \mathrm{~m} / \mathrm{s}$. Die schwarze horizontale Linie gibt die Anzahl an Argon-Atomen an. (b): Ausschnittsvergrößerung von (a) im Hauptkollaps ( $=0)$. (c): Radiusverlauf für variiertes $\alpha_{v}$. (d): Ausschnittsvergrößerung von (c) im Hauptkollaps

Legende: $\alpha_{v}=0,8(-) ; 0,4(-) ; 0,2(-) ; 0,1(-) ; 0,05(-)$. Parameter siehe Tab. 4.3 .

Akkomodationskoeffizienten immer näher an $t_{R m i n}$ heran. Diese Verschiebung erklärt sich durch den höheren Partialdruck des Wasserdampfes für kleine $\alpha_{v}$, der zu einer fortgeführten Kondensation nach dem Hauptkollaps führt.

Im weiteren Verlauf sinkt der Wasserdampfgehalt unter mehreren Oszillationen weiter ab. Ca. $5 \mu$ s nach dem Hauptkollaps wird für $\alpha_{v} \leq 0,4$ ein von $\alpha_{v}$ unabhängiges Gleichgewicht zwischen Dampf- und Partialdruck erreicht. Die Simulation mit $\alpha_{v}=0,8$ zeigt auch zu diesem Zeitpunkt noch deutliche Schwankungen des Wasserdampfgehaltes, die den Nachschwingungen des Hauptkollaps folgen. Bei einer derart hohen Phasenübergangswahrscheinlichkeit treten starke Schwankun- 

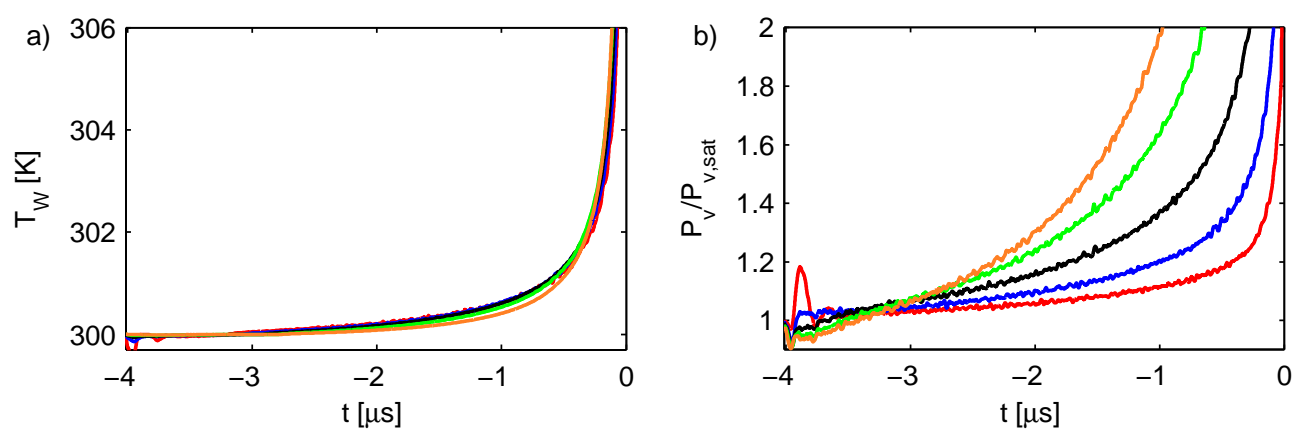

Abbildung 4.8: (a): Wandtemperatur in der langsamen Kollapsphase für variiertes $\alpha_{v}$ mit $v_{W, \text { init }}=0 \mathrm{~m} / \mathrm{s}$. (b): Verhältnis des aktuellen Dampfdrucks des Wassers in der Blase zum Sättigungsdampfdruck bei aktueller $T_{W}$.

Legende: $\alpha_{v}=0,8(\boldsymbol{(}) ; 0,4(\boldsymbol{-}) ; 0,2(\boldsymbol{-}) ; 0,1(-) ; 0,05(-)$. Parameter siehe Tab. 4.3.

gen der Blasenwandtemperatur auf, da der Einfluß der latenten Wärme wächst. Dies führt bei $\alpha_{v}=0,8$ zu einem Aufschauklungseffekt der Nachschwinger.

Abb. 4.7,b macht deutlich, daß die Menge an gefangenem $\mathrm{H}_{2} \mathrm{O}$ kurz vor dem Ende des Kollaps der erwarteten Tendenz entspricht: Je größer $\alpha_{v}$, desto weniger Wasserdampf befindet sich in der Blase. Direkt (ca. $5 \mathrm{~ns}$ ) vor $t_{R \min }$ nimmt $\mathrm{N}_{\mathrm{H}_{2} \mathrm{O}}$ jedoch, je nach $\alpha_{v}$, z.T. sehr deutlich zu. Dies erklärt sich durch die zu diesem Zeitpunkt einsetzende Erhitzung der Blasenwand, welche den Sättigungsdampfdruck erhöht und so eine Verdampfung bewirkt. Die Stärke dieses Effektes hängt direkt mit der Wahl von $\alpha_{v}$ zusammen, so daß sich bei $\alpha_{v}=0,8$ die Anzahl der Wasserdampfteilchen innerhalb dieser kurzen Zeit fast verfünffacht. Die Verdampfung wird erst mit Erreichen des kritischen Punktes gestoppt. Diese Phase ist an dem Plateau des $\mathrm{H}_{2} \mathrm{O}$ - Gehaltes um $\mathrm{t}=0$ zu erkennen. Die Höhe dieses Plateaus ist von entscheidender Bedeutung für die Bedingungen in der Blase gegen Ende des Kollaps.

$N_{\mathrm{H}_{2} \mathrm{O}}$ ist zum Zeitpunkt des minimalen Blasenradius für $\alpha_{v}=0,8$ höher als für $\alpha_{v}=0,4$ und $\alpha_{v}=0,25$. Sinkt die Wandtemperatur wieder unter den kritischen Punkt setzt zunächst eine erneute Verdampfung ein. Nach ca. 1 - 2 ns ist die Blasenwand so weit abgekühlt, dass Kondensation stattfindet. Es tritt also um den Hauptkollaps eine starke Oszillation des Wasserdampfgehaltes auf, welche auch bei den kleineren Kollapsen der Nachschwinger auszumachen ist.

Die Bewegung des Blasenrandes zeigt eine starke Abhängigkeit vom Wasserdampfgehalt: Für kleine $\alpha_{v}$ kollabiert die Blase deutlich weniger, schwingt dafür aber weiter auf. Dies erklärt sich durch die größere vorhandene Masse und das größere Gesamtvolumen der Teilchen in der Blase. Der stärkste Kollaps zeigt sich 
bei $\alpha_{v}=0,4$. Dies paßt zu der Beobachtung, dass bei diesem Wert der niedrigste Wasserdampfgehalt gegen Ende des Kollaps (Höhe des Plateaus) vorherrscht.

Abb. 4.8 zeigt die Wandtemperatur (a) sowie das Verhältnis des Druckes der momentan in der Blase vorhandenen Wasserdampfteilchen zum Sättigungsdampfdruck bei der aktuellen Wandtemperatur (b) für den 'langsamen' Teil des Hauptkollaps. Die Wandtemperatur steigt für alle Werte von $\alpha_{v}$ nur sehr langsam an und verläuft näherungsweise deckungsgleich. Der Dampfdruck des gefangenen Wasserdampfes zeigt hingegen schon in frühen Stadien des Kollaps eine deutliche Abhängigkeit vom Akkomodationskoeffizienten: Während für hohe Werte ( $\geq$ $0,4)$ auch noch $1 \mu$ s vor $t_{R m i n}$ nahezu Sättigungsdampfdruck in der Blase herrscht, steigt der Dampfdruck bei Verkleinerung von $\alpha_{v}$ immer schneller an. Bei $\alpha_{v}=$ 0,05 wird bereits $1 \mu \mathrm{s}$ vor $t_{R \min }$ der doppelte Wert von $P_{v, \text { sat }}\left(T_{W}\right)$ erreicht.

Die Annahme, dass über einen großen Teil des Blasenkollaps der Phasenübergang im Gleichgewicht geschieht, wird hier bei niedriger Wahl von $\alpha_{v}$ verletzt. Der Fall $\alpha_{v}=0,8$ zeigt hingegen bereits z.T. experimentell nicht beobachtetes Verhalten (lange, große Nachschwinger), so dass ein Wert von $\alpha_{v}=0,4$ als passendste Wahl erscheint. Dieses Ergebnis steht in Übereinstimmung mit dem bereits in anderen Publikationen verwendeten Wert [105][38].

\section{Verzögerter Start der Simulation}

Die gerade besprochenen Ergebnisse wurden mit Simulationen erzielt, die bei maximalem Blasenradius $\left(v_{W, \text { init }}=0 \mathrm{~m} / \mathrm{s}\right)$ gestartet wurden. Diese haben den Nachteil, dass sich am Anfang der Rechnung sehr viel Wasserdampf in der Blase befindet (in diesem Fall ca. 22 Teile $\mathrm{H}_{2} \mathrm{O}$ zu 1 Teil Argon). Dies erschwert eine hohe Wahl von $N_{N G}$, da die Rechnung durch die vielen $\mathrm{H}_{2} \mathrm{O}$ - Teilchen sehr aufwendig wird. Es ist also wünschenswert, die Molekulardynamiksimulation erst zu starten, wenn die Blase schon zu gewissem Maße kollabiert ist, da nun entsprechend weniger $\mathrm{H}_{2} \mathrm{O}$ - Teilchen dargestellt werden müssen. Um den Einfluß dieses späteren Startpunktes auf den Mechanismus des 'vapor trappings' zu untersuchen, wurden alle besprochenen Fälle von $\alpha_{v}$ noch einmal mittels bei $v_{W \text {,init }}$ $=25 \mathrm{~m} / \mathrm{s}$ gestarteten Simulationen berechnet. Dabei wurde davon ausgegangen, dass zu diesem Zeitpunkt noch $P_{v}(R)=P_{v, \text { sat }}\left(T_{\infty}\right)$ gilt. Die Blase erreicht die vorgegebene Wandgeschwindigkeit ca. $0,31 \mu \mathrm{s}$ vor $t_{R m i n}$. Der Zeitpunkt des maximalen Blasenradius lag ca. $4 \mu \mathrm{s}$ vor $t_{R \min }$.

Abb. 4.9 zeigt die Anzahl der Wasserdampfteilchen und den Blasenradius für die ersten 5,5 $\mu$ s des Blasenzyklus dieser Rechnungen. Tab. 4.4,b fasst die Ergebnisse 

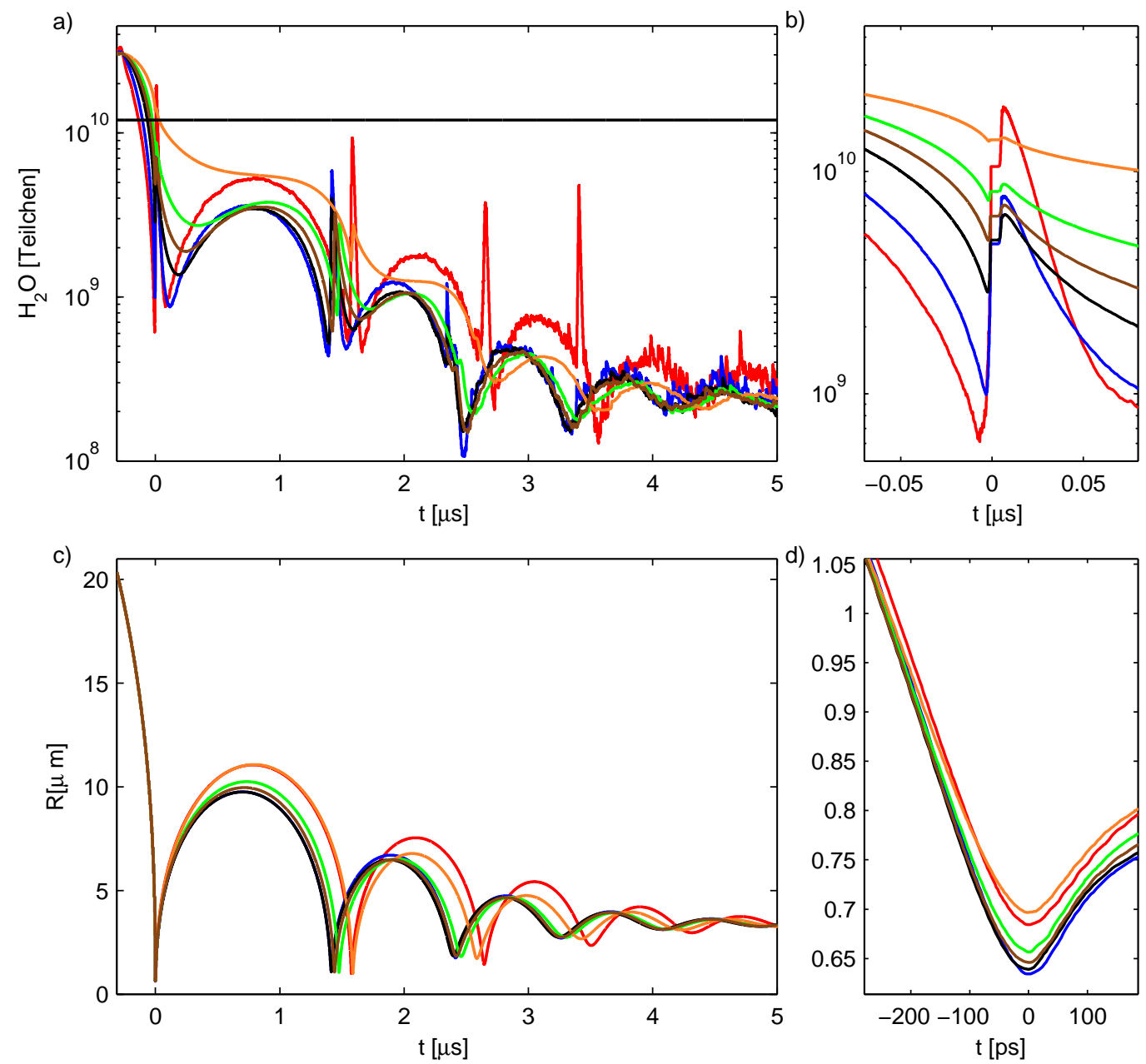

Abbildung 4.9: (a): Logarithmische Darstellung der realen Anzahl an $\mathrm{H}_{2} \mathrm{O}$ - Teilchen in der Blase für variiertes $\alpha_{v}$ mit $v_{W, \text { init }}=25 \mathrm{~m} / \mathrm{s}$. Die schwarze horizontale Linie gibt die Anzahl an Argon-Atomen an. (b): Ausschnittsvergrößerung von (a) im Hauptkollaps $(\mathrm{t}=0)$. (c): Radiusverlauf. (d): Ausschnittsvergrößerung von (c) im Hauptkollaps

Legende: $\alpha_{v}=0,8(-) ; 0,4(-) ; 0,2(-) ; 0,14(-) ; 0,1(-) ; 0,05$ (一). Parameter siehe Tab. 4.3.

zusammen. Die Anzahl an Edelgasatomen wurde gegenüber den vorigen Rechnungen nicht verändert $\left(N_{N G}=8000\right)$, das Verhältnis von $\mathrm{H}_{2} \mathrm{O}$ zu Argon beträgt zum Zeitpunkt des Starts der Simulation nun allerdings nur noch ca. 2,5 zu 1. Alle anderen Parameter entsprechen den in Tab. 4.3 angegebenen.

Im Vergleich zu der bei maximalem Blasenradius gestarteten Rechnung ist deutlich zu erkennen, dass die Menge des gefangenen Wasserdampfes stark abnimmt. Bei $\alpha_{v}=0,05$ befinden sich gegen Ende des Kollaps lediglich ungefähr ein Viertel so viele $\mathrm{H}_{2} \mathrm{O}$ - Atome in der Blase wie bei der Vergleichsrechnung. Nimmt $\alpha_{v} \mathrm{zu}$, schwächt sich dieser Effekt ab, ist aber in allen Fällen vorhanden. Die Erklärung 
hierfür ist, dass der Dampfdruck im Blaseninneren zum Zeitpunkt von $v_{\text {wall }}=25$ $\mathrm{m} / \mathrm{s}$ bei den Rechnungen mit $v_{W, \text { init }}=0 \mathrm{~m} / \mathrm{s}$ höher liegt als bei denjenigen mit $v_{W, \text { init }}=25 \mathrm{~m} / \mathrm{s}$ (siehe Abb. 4.8,b). Dieser Unterschied im Wasserdampfgehalt kann in der kurzen verbleibenden Zeit des Restkollaps nicht ausgeglichen werden. Während der Nachschwinger gleichen sich die Ergebnisse der unterschiedlichen Rechnungen durch die größere Zeitskala einander an.

Auch die Blasendynamik wird beeinflußt: Durch die geringere Anzahl an Teilchen können die Blasen stärker kollabieren. Vor allem bei $\alpha_{v}=0,05$ ist dies erwartungsgemäß sehr ausgeprägt. Es ist zu erkennen, dass bei den Rechnungen mit $\alpha_{v}=$ 0,05 und mit $\alpha_{v}=0,8$ in der entscheidenden Phase des Kollaps ähnlich viele $\mathrm{H}_{2} \mathrm{O}$ Teilchen in der Blase enthalten sind (durch die massive Eindampfung bei $\alpha_{v}=0,8$ sobald sich die Blasenwand erwärmt). Dies erklärt den zunächst sehr ähnlichen Radiusverlauf dieser beiden, eigentlich sehr unterschiedlichen, Rechnungen.

\begin{tabular}{llllllllll}
\hline & $\alpha_{v}$ & $A_{v}$ & $P_{A}$ & $v_{W, \text { init }}$ & $\begin{array}{l}R_{\min } \\
{[\mu \mathrm{m}]}\end{array}$ & $\begin{array}{l}v_{W} \\
{[\mathrm{~m} / \mathrm{s}]}\end{array}$ & $\begin{array}{l}T_{\varnothing} \\
{[\mathrm{K}]}\end{array}$ & $\begin{array}{l}\mathrm{H}_{2} \mathrm{O} \\
{\left[10^{9}\right]}\end{array}$ & $\begin{array}{l}\mathrm{H}_{2} \mathrm{O} \\
{[\%]}\end{array}$ \\
\hline a) & - & & & & & & & & \\
& 0,05 & - & 1,3 & 0,0 & 1,01 & 1000 & 4100 & 55,5 & 82,2 \\
& 0,1 & - & 1,3 & 0,0 & 0,78 & 1420 & 7700 & 20,5 & 63,1 \\
& 0,2 & - & 1,3 & 0,0 & 0,69 & 1680 & 12200 & 9,2 & 43,4 \\
& $\mathbf{0 , 4}$ & - & $\mathbf{1 , 3}$ & $\mathbf{0 , 0}$ & $\mathbf{0 , 6 7}$ & $\mathbf{1 7 8 0}$ & $\mathbf{1 4 1 0 0}$ & $\mathbf{6 , 9}$ & $\mathbf{3 6 , 5}$ \\
& 0,8 & - & 1,3 & 0,0 & 0,69 & 1720 & 12200 & 9,4 & 43,9 \\
\hline b) & & & & & & & & & \\
& 0,0 & - & 1,3 & 25,0 & 0,70 & 1600 & 9400 & 13,9 & 53,7 \\
& $\mathbf{0 , 1}$ & - & $\mathbf{1 , 3}$ & $\mathbf{2 5 , 0}$ & $\mathbf{0 , 6 6}$ & $\mathbf{1 7 6 0}$ & $\mathbf{1 2 5 0 0}$ & $\mathbf{8 , 1}$ & $\mathbf{4 0 , 3}$ \\
& $\mathbf{0 , 1 4}$ & - & $\mathbf{1 , 3}$ & $\mathbf{2 5 , 0}$ & $\mathbf{0 , 6 5}$ & $\mathbf{1 8 0 0}$ & $\mathbf{1 4 1 0 0}$ & $\mathbf{6 , 3}$ & $\mathbf{3 4 , 4}$ \\
& 0,2 & - & 1,3 & 25,0 & 0,64 & 1870 & 15700 & 4,9 & 29,0 \\
& 0,4 & - & 1,3 & 25,0 & 0,63 & 1900 & 16100 & 4,7 & 28,1 \\
& 0,8 & - & 1,3 & 25,0 & 0,68 & 1830 & 11600 & 10,5 & 46,7 \\
\hline c) & & & & & & & & & \\
& - & 0,25 & 1,3 & 0,0 & 0,80 & 1270 & 7100 & 23,8 & 66,5 \\
& - & 0,3 & 1,3 & 0,0 & 0,76 & 1390 & 8500 & 17,7 & 59,6 \\
& - & 0,4 & 1,3 & 0,0 & 0,71 & 1550 & 10900 & 11,5 & 48,9 \\
& - & 0,5 & 1,3 & 0,0 & 0,69 & 1650 & 12700 & 8,5 & 41,5 \\
& - & $\mathbf{1 , 0}$ & $\mathbf{1 , 3}$ & $\mathbf{0 , 0}$ & $\mathbf{0 , 6 6}$ & $\mathbf{1 7 6 0}$ & $\mathbf{1 5 6 0 0}$ & $\mathbf{5 , 4}$ & $\mathbf{3 1 , 0}$ \\
& - & 2,0 & 1,3 & 0,0 & 0,68 & 1720 & 13400 & 7,6 & 38,8 \\
\hline d) & & & & & & & & & \\
& - & 0,25 & 1,3 & 25,0 & 0,66 & 1760 & 12100 & 8,6 & 41,7 \\
& - & 0,3 & 1,3 & 25,0 & 0,65 & 1790 & 13100 & 7,2 & 37,5 \\
& - & $\mathbf{0 , 4}$ & $\mathbf{1 , 3}$ & $\mathbf{2 5 , 0}$ & $\mathbf{0 , 6 4}$ & $\mathbf{1 8 5 0}$ & $\mathbf{1 5 2 0 0}$ & $\mathbf{5 , 2}$ & $\mathbf{3 0 , 2}$ \\
& - & 0,5 & 1,3 & 25,0 & 0,63 & 1690 & 16900 & 3,9 & 24,5 \\
\hline
\end{tabular}

Tabelle 4.4: Übersicht der Rechnungen mit variiertem $\alpha_{v}$, bzw. $A_{v}$ für $N_{N G}=8.000$. $v_{W}$ ist die maximale Wandgeschwindigkeit, $T_{\varnothing}$ bezeichnet die maximale Durchschnittstemperatur. Der Wasserdampfgehalt ist sowohl in absoluter Anzahl, als auch im molaren Anteil gegeben. Die hervorgehobenen Zeilen entsprechen Parameterkombinationen, die im weiteren Verlauf der Arbeit Benutzung finden (siehe Text). 
Es zeigt sich also, dass eine unveränderte Wahl von $\alpha_{v}=0,4$ bei Verschiebung des Startpunktes der Simulation zu deutlichen Unterschieden im Wasserdampfgehalt und den erreichten Temperaturen (siehe Tab. 4.4) führt. Anhand der Simulationsergebnisse wurde ermittelt, dass Werte von $\alpha_{v}=0,1$ bis $\alpha_{v}=0,14$ bei $v_{W \text {,init }}=$ $25 \mathrm{~m} / \mathrm{s}$ die größte Übereinstimmung zu den Ergebnissen von $\alpha_{v}=0,4$ bei $v_{W, \text { init }}$ $=0 \mathrm{~m} / \mathrm{s}$ erziehlt. Versuche, den Dampfdruck bei Start der Simulation zu erhöhen und $\alpha_{v}$ unverändert zu lassen lieferten schlechtere Ergebnisse.

\subsubsection{Temperaturabhängiges $\alpha_{v}$}

Die in Kap. (4.2.2.1) dargelegten Ergebnisse beziehen sich auf einen über den gesamten Kollaps festen Wert von $\alpha_{v}$. Experimentelle Ergebnisse legen jedoch einen temperaturabhängigen Akkomodationskoeffizient nahe [106][107]. Es wurde eine Abnahme der Phasenübergangswahrscheinlichkeit mit steigender Temperatur gefunden. Um diesen Effekt zu simulieren wurde hier ein von der aktuellen Wandtemperatur abhängiger Akkomodationskoeffizient $\alpha_{v, v a r}$ benutzt [28]. Dieser wird mittels der Gregory-Newton-Formel [108] bestimmt:

$$
\begin{aligned}
\alpha_{v, \text { var }}\left(T_{W}\right) & = \begin{cases}0,35 & \text { falls } T_{W}<350 K \\
0,35-0,05 k^{(1)}-0,05 k^{(2)}+0,025 k^{(3)} & \text { sonst } \\
0,05 & \text { falls } T_{W}>500 K\end{cases} \\
k & =\left(T_{W} / 50\right)-7, \text { mit } k^{(m)}=k(k-1) \cdots[k-(m-1)] .
\end{aligned}
$$

Diese Berechnung führt zu dem in Abb. 4.10,a gezeigten Verlauf von $\alpha_{v, v a r}$. Der
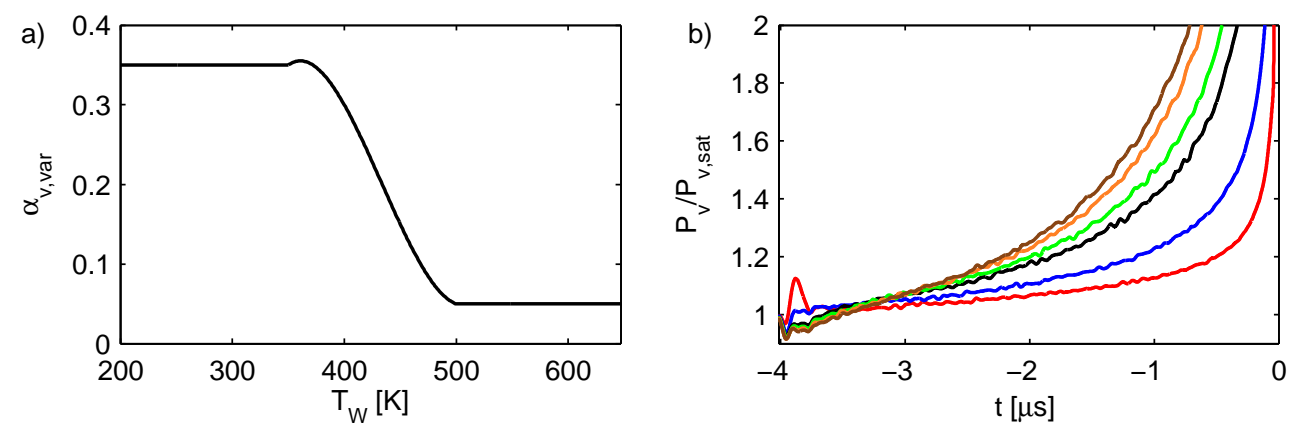

Abbildung 4.10: (a): Verlauf des Akkomodationskoeffizienten $\alpha_{v, v a r}$ mit der Wandtemperatur. (b): Verhältnis des Dampfdrucks zum Sättigungsdampfdruck bei aktueller Wandtemperatur für verschiedene Werte von $A_{v}$.

Legende: $A_{v}=2,0(-) ; 1,0(-) ; 0,5(-) ; 0.4(-) ; 0,3(-) ; 0,25$ (-). Parameter siehe Tab. 4.3 . 

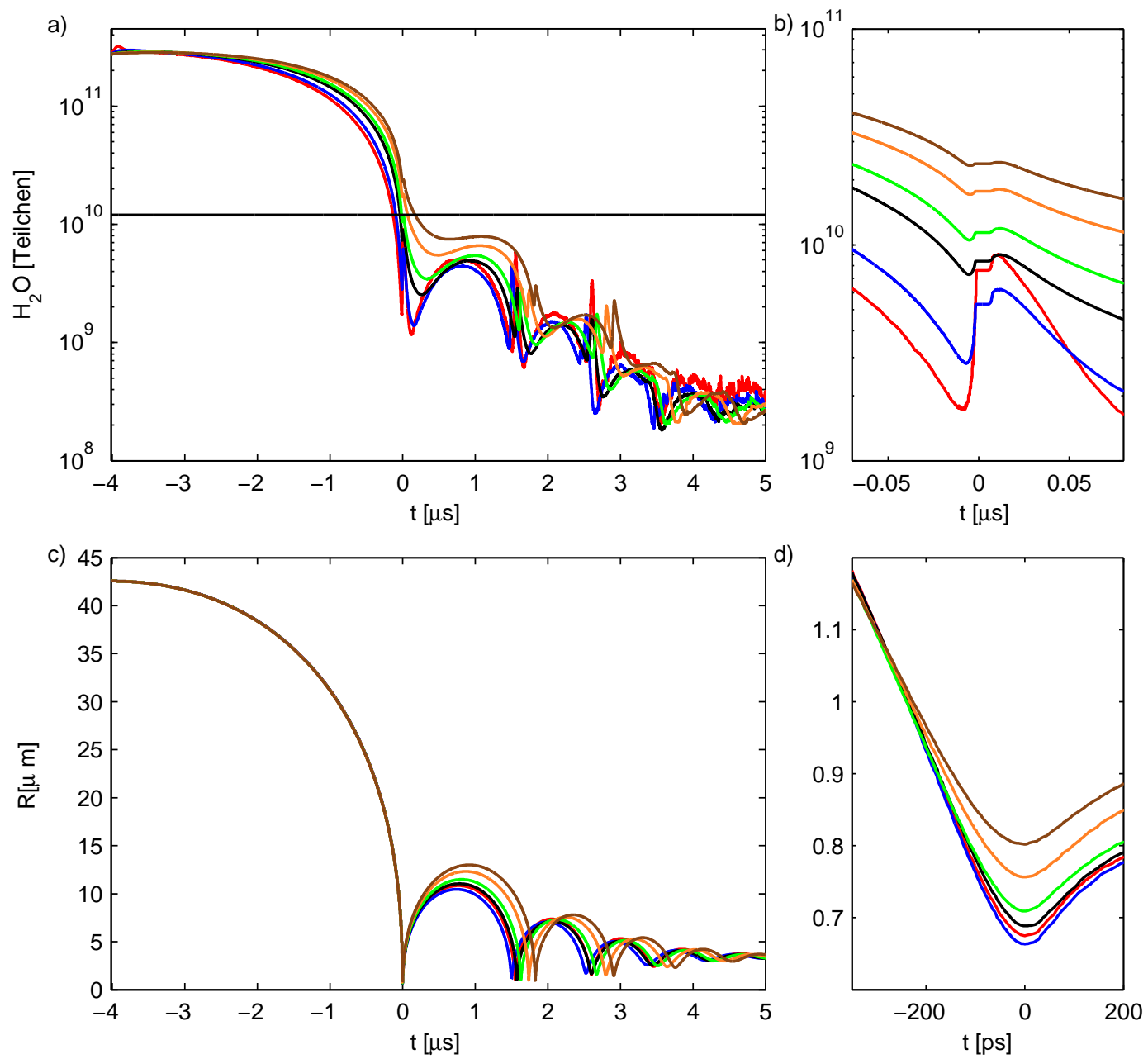

Abbildung 4.11: (a): Logarithmische Darstellung der Anzahl an $\mathrm{H}_{2} \mathrm{O}$ - Teilchen in der Blase für variiertes $A_{v}$ mit $v_{W, \text { init }}=0 \mathrm{~m} / \mathrm{s}$. Die schwarze horizontale Linie gibt die Anzahl an ArgonAtomen an. (b): Ausschnittsvergrößerung von (a) im Hauptkollaps $(\mathrm{t}=0)$. (c): Radiusverlauf. (d): Ausschnittsvergrößerung von (c) im Hauptkollaps. Parameter siehe Tab. 4.3.

Legende: $A_{v}=2,0(-) ; 1,0(-) ; 0,5(-) ; 0.4(-) ; 0,3(-) ; 0,25(-)$.

variable Akkomodationskoeffizient wird seinerseits mit dem Vorfaktor $A_{v}$ versehen, um den Vergleich verschiedener Werteintervalle von $\alpha_{v \text {,var }}$ zu ermöglichen: $A_{v, v a r}=A_{v} \cdot \alpha_{v, v a r}$.

Abb. 4.11 zeigt den Wasserdampfgehalt und den Radiusverlauf für Werte von $A_{v}$ $=2,0$ bis $A_{v}=0,25$, Tab. 4.4, c fasst die Ergebnisse zusammen. Im Vergleich zu Abb. 4.7 ist zu erkennen, dass der generelle Verlauf von $\mathrm{N}_{H_{2} O}$ auf einer $\mu$ s - Skala sehr ähnlich verläuft (vor allem für kleine Werte von $\alpha_{v}$, bzw. $A_{v}$ ). Auf der kurzen Zeitskala des Kollaps wird deutlich, dass das massive Eindampfen von Wassermolekülen in der heißen Phase bei einem variablen Akkomodationskoeffizienten 
deutlich abgemindert wird. Bei hohem $A_{v}$ sind die starken Oszillationen des Wasserdampfgehaltes kaum mehr vorhanden, die extrem hohen Nachschwinger sind auf ein zu erwartendes Niveau zurückgegangen. Für alle Werte von $A_{v}$ wird nach ca. $5 \mu$ s ein von $A_{v}$ unabhängiges Gleichgewicht erreicht. Abb. 4.10,b zeigt den für $A_{v} \leq 0,5$ schnellen Anstieg des Dampfdruckes in der Blase. Ein Wert von $A_{v}$ $=1,0$ erscheint als beste Wahl.

Es wurde eine zweite Betrachtung derselben Blasenparameter mit verzögertem Start der MD-Simulation bei $v_{W \text {,init }}=25 \mathrm{~m} / \mathrm{s}$ durchgeführt (die Gründe hierfür wurden im vorigen Abschnitt dargelegt). Die Ergebnisse sind in Tab. 4.4, d zusammengefaßt. Es wurde ein Wert von $A_{v}=0,4$ als derjenige identifiziert, der die mit $A_{v}=1,0$ bei $v_{W, \text { init }}=0 \mathrm{~m} / \mathrm{s}$ vergleichbarsten Ergebnisse liefert.

Allgemein liefert die temperaturabhängige Berechnung des Akkomodationskoeffizienten konsistentere Werte des Wasserdampfgehaltes und wird daher bei den meisten der folgenden Rechnungen benutzt.

\subsubsection{Einfluß auf die Dynamik des Blaseninneren}

Eine aussagekräftige Darstellung der räumlichen und zeitlichen Dynamik des Blaseninneren erfordert Rechnungen mit einer erhöhten Teilchenzahl. Daher wurden die Rechnungen aus Kap. 4.2.2.2 mit einer Anzahl von 216.000 Edelgasteilchen wiederholt. Die Simulationen wurden bei einer Wandgeschwindigkeit von $v_{W, \text { init }}=$ $25 \mathrm{~m} / \mathrm{s}$ gestartet und nach dem Hauptkollaps beendet. Die restlichen Parameter finden sich in 4.5 .

Abb. 4.12 zeigt die Temperatur im Blasenzentrum und am Blasenrand (a,c), die jeweilige Dichte von Wasserdampf $(b, d)$ sowie das Temperatur- und $\mathrm{H}_{2} \mathrm{O}$-Profil in der Blase für den Zeitpunkt der maximalen Temperatur (e,f). Es ist zu erkennen, dass das Temperaturmaximum im Blasenzentrum 40-70 ps vor Erreichen des minimalen Radius auftritt. Das Maximum verschiebt sich mit zunehmendem $\alpha_{v}$ in Richtung von $t_{R \min }$. Diese erklärt sich durch den abnehmenden Minimalradius,

\begin{tabular}{ccc|ccc|ccc}
\hline$P_{a}$ & - & $1,3 \mathrm{bar}$ & $N_{N G}$ & - & 216.000 & $v_{W, \text { init }}$ & - & $25 \mathrm{~m} / \mathrm{s}$ \\
$\nu_{a}$ & - & $26,5 \mathrm{kHz}$ & $S$ & - & $\approx 58.000$ & $\alpha_{t}$ & - & 0,3 \\
$R_{0}$ & - & $4,5 \mu \mathrm{m}$ & Edelgas & - & Argon & $A_{v}$ & - & $0,25-2,0$ \\
$T_{\text {water }}$ & - & $300 \mathrm{~K}$ & $\Delta K_{W}$ & - & 3.000 & & & \\
\hline
\end{tabular}

Tabelle 4.5: Parameter der Vergleichsrechnungen des Blaseninneren bei variiertem $A_{v}$. 
wodurch die reflektierte Verdichtungswelle den Blasenrand früher erreicht und den Kollaps stoppt (siehe Kap. 4.1).

Der starke Einfluß des Wasserdampfgehaltes auf die erreichten Temperaturen wird deutlich. Für $A_{v}=1,0$ wird eine maximale Wasserdampfdichte im Zentrum von $74 \mathrm{~kg} / \mathrm{m}^{3}$ erreicht, die Maximaltemperatur beträgt ca. $44.000 \mathrm{~K}$. Bei $A_{v}=0,25$ findet sich hingegen eine Wasserdampf-Dichte von $355 \mathrm{~kg} / \mathrm{m}^{3}$, wodurch nur noch maximal ca. $20.000 \mathrm{~K}$ erreicht werden. Am Blasenrand findet sich eine analoge Beziehung, hier sind die Temperaturen allerdings generell niedriger. Zwischen 7.300 K und $12.500 \mathrm{~K}$ werden für die verschiedenen Rechnungen erreicht. Die Temperatur- und $\mathrm{H}_{2} \mathrm{O}$-Dichte-Unterschiede für verschiedene Akkomodationskoeffizienten bleiben über den dargestellten Zeitraum der Endphase des Kollaps in der gesamten Blase erhalten.

Zum Zeitpunkt der maximalen Temperatur kann man für alle Rechnungen ein annähernd glockenförmiges Temperaturprofil erkennen. Die Dichte des Wasserdampfes fällt für $A_{v}<1,0$ linear vom Blasenzentrum zum Blasenrand ab. Der Vergleich von $A_{v}=1,0$ und $A_{v}=2,0$ zeigt, dass in der Nähe des Blasenrandes die vermehrte Eindampfung gegen Ende des Kollaps zu höheren $\mathrm{H}_{2} \mathrm{O}$-Dichte-Werten bei $A_{v}=2,0$ führt, während im Blasenzentrum die Rechnung mit $A_{v}=1,0$ leicht höhere Werte aufweist. Dies zeigt, dass die neu in die Blase verdampften Teilchen nicht genug Zeit haben, bis zum Blasenzentrum zu gelangen.

Die experimentellen ([109][110]) und numerischen ([111]) Hinweise, dass die Blasentemperatur nicht deutlich über $20.000 \mathrm{~K}$ liegt, deuten darauf hin, dass der im vorigen Kapitel ausgewählte Wert von $A_{v}=0.4$ in der richtigen Größenordnung liegt. 

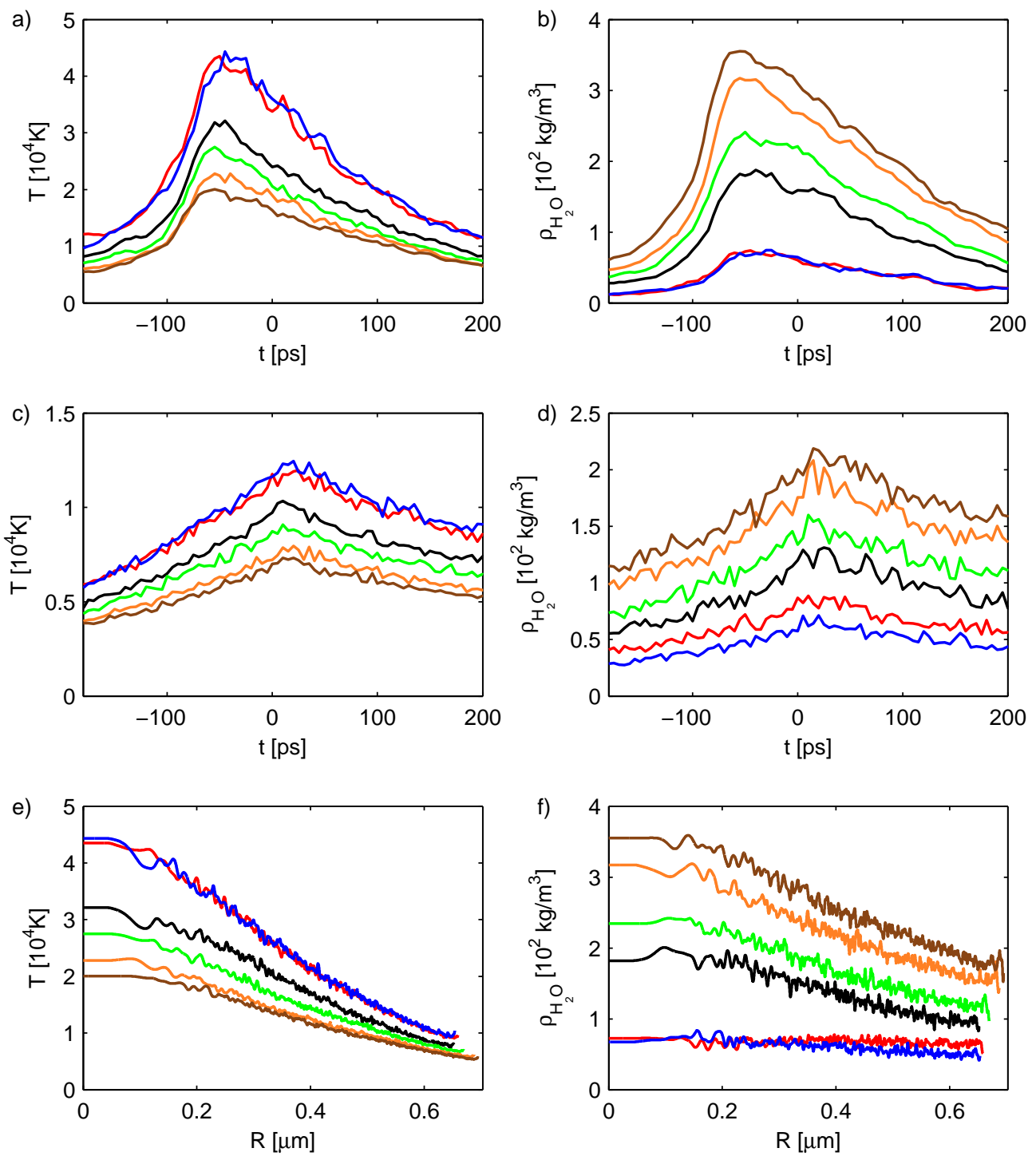

Abbildung 4.12:

(a): Temperatur im Blasenzentrum.

(b): Dichte von $\mathrm{H}_{2} \mathrm{O}$ im Blasenzentrum.

(c): Temperatur nahe am Blasenrand.

(d): Dichte von $\mathrm{H}_{2} \mathrm{O}$ nahe am Blasenrand.

(e): Temperaturprofil in der Blase zum Zeitpunkt der maximalen Temperatur.

(f): Wasserdampfverteilung in der Blase zum Zeitpunkt der maximalen Temperatur.

Legende: $A_{v}=2,0(-) ; 1,0(-) ; 0,5(-) ; 0.4(-) ; 0,3$ (-); 0,25 (-). Parameter siehe Tab. 4.5.

\subsubsection{Vergleich mit Kontinuumsmechanik}

An dieser Stelle sollen die Ergebnisse des vorliegenden molekulardynamischen Modells mit denjenigen aus einem kontinuumsmechanischem Navier-Stokes-Modell 
Kap. 4 Einfluß der Parameter des MD-Modells

\begin{tabular}{ccc|ccc|ccc}
\hline$P_{a}$ & - & $1,2 \mathrm{bar}$ & $N_{N G}$ & - & 216.000 & $v_{W, \text { init }}$ & - & $25 \mathrm{~m} / \mathrm{s}$ \\
$\nu_{a}$ & - & $26,5 \mathrm{kHz}$ & $S$ & - & $\approx 56.000$ & $\alpha_{t}$ & - & 0,3 \\
$R_{0}$ & - & $4,5 \mu \mathrm{m}$ & Edelgas & - & Argon & $A_{v}$ & - & 0,4 \\
$T_{\text {water }}$ & - & $300 \mathrm{~K}$ & $\Delta K_{W}$ & - & 3.000 & & & \\
\hline
\end{tabular}

Tabelle 4.6: Parameter der Vergleichsrechnungen zu Fall II aus [38].

(N-S-Modell) von Storey und Szeri [38] verglichen werden. Storey und Szeri berechnen zur Untersuchung der Rolle des Wasserdampfes eine typische SBSLBlase $\left(R_{0}=4,5 \mu \mathrm{m}, \nu_{a}=26,5 \mathrm{kHz}, \mathrm{P}_{a}=1,2\right.$ bar $)$. Sie benutzen einen statischen Akkomodationskoeffizienten mit einem Wert von 0,4 und lassen zunächst chemische Reaktionen außer acht (Fall II in [38]).

$\mathrm{Zu}$ Vergleichszwecken wird die Simulation einer Blase mit den vorgenannten Parametern durchgeführt. Dabei wurde ein variabler Akkomodationskoeffizient mit $A_{v}=0,4\left(v_{W, \text { init }}=25 \mathrm{~m} / \mathrm{s}\right)$ gewählt. Die restlichen Parameter lassen sich Tab. 4.6 entnehmen.

Abb. 4.13 zeigt die Entwicklung der durchschnittlichen Blasentemperatur und der Anzahl an Wassermolekülen für 10 ns um den Hauptkollaps. In Übereinstimmung mit den Ergebnissen von Storey und Szeri ist zu erkennen, dass die Temperatur während dieses Intervalls starke Schwankungen zeigt, während der Wasserdampfgehalt relativ konstant bleibt. Die erreichten Werte liegen in sehr guter Übereinstimmung zu [38] (siehe auch Tab. 4.7). Die Maximalwerte der Durchschnittstemperatur (8.400 K vs. $8.800 \mathrm{~K}$ ) sind sehr ähnlich, ebenso die Anzahl der in der Blase verbleibenden $\mathrm{H}_{2} \mathrm{O}$-Moleküle. Es fällt allerdings auf, dass im MD-Modell eine schnellere Dynamik vorherrscht: Die Temperatur steigt etwas schneller an und fällt vor allem deutlich rascher wieder ab. Der Kompressionsfaktor ist nahezu identisch.
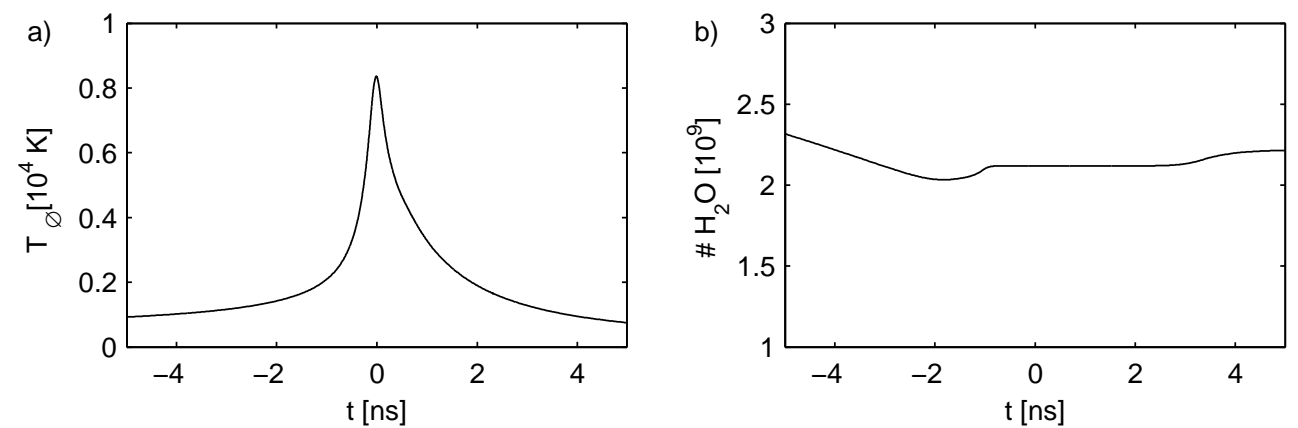

Abbildung 4.13: Durchschnittstemperatur und Anzahl der Wasserdampfmoleküle einer mit $P_{a}=1,2$ bar angeregten Blase. Parameter siehe Tab. 4.6. 

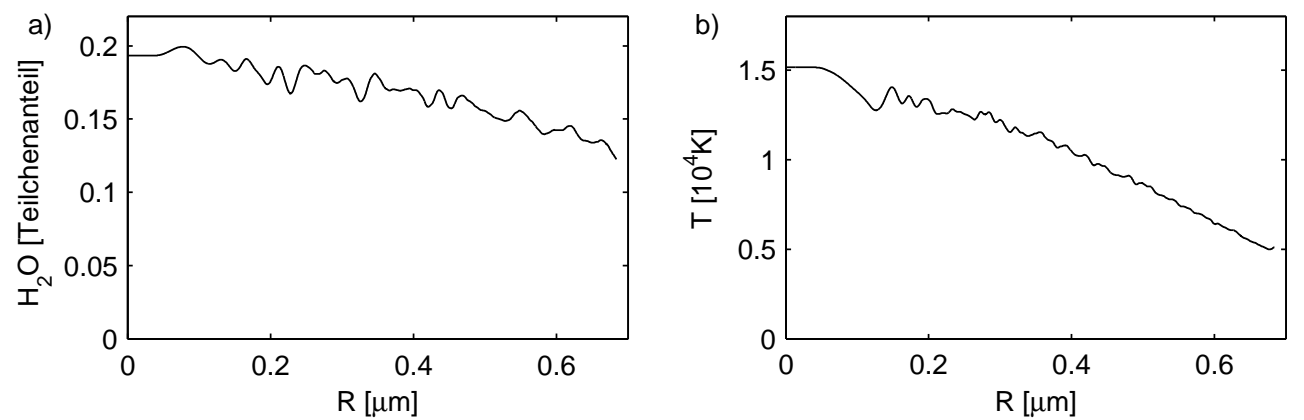

Abbildung 4.14: Teilchenanteil von Wasser und Temperatur in der Blase zum Zeitpunkt der maximalen Blasentemperatur einer mit $P_{a}=1,2$ bar angeregten Blase. Parameter siehe Tab. 4.6.

Der Blick auf die Verteilung des Wasserdampfes und die Temperatur im Inneren des Blase zum Zeitpunkt der maximalen Blasentemperatur (Abb. 4.14) zeigt, dass der Wasserdampfgehalt zum Blasenrand hin leicht abfällt. Die geringere Geschwindigkeit des Kollaps im Vergleich zu den Rechnungen mit $P_{a}=1,3$ bar (siehe Kap. 4.2.2.2) mindert das 'vapor trapping'. Dies steht in klarem Gegensatz zu den Ergebnissen aus [38], wo sich in den letzten 200 ns des Kollaps ein sehr deutliches Gefälle des Wasserdampfanteils zum Blasenrand hin ausbildete. In der dortigen Untersuchung lag der Wasserdampfanteil im Zentrum zum Zeitpunkt $t_{\text {Rmin }}$ bei 0,28, am Blasenrand hingegen bei ca. 0,02. In der hier vorliegenden Simulation ist zu keiner Zeit ein solches Gefälle zu erkennen.

Die Temperatur in der molekulardynamisch modellierten Blase sinkt von einem Wert von ca. 15.200 K im Zentrum linear zu einem Wert von ca. 5.000 K am Blasenrand ab. Die im Vergleich zum N-S-Modell (Zentrumstemperatur dort: 9700 K) größeren lokalen Unterschiede der Temperatur erklären sich durch den ausgeglicheneren Wasserdampfgehalt: Im (heißen) Blasenzentrum befinden sich vergleichsweise weniger $\mathrm{H}_{2} \mathrm{O}$-Teilchen, am (kälteren) Blasenrand dagegen mehr.

Insgesamt kann man sagen, dass die Übereinstimmung der beiden Modelle bei globalen (Durchschnitts-) Parametern sehr gut ist, während die innere Dynamik (vor allem aufgrund unterschiedlicher Diffusionszeitskalen) Unterschiede aufweist.

\begin{tabular}{llllllll}
\hline Modell & $R_{\max }[\mu \mathrm{m}]$ & $R_{\min }[\mu \mathrm{m}]$ & $K$ & $T_{\varnothing}[\mathrm{K}]$ & $T_{\max }[\mathrm{K}]$ & $\mathrm{H}_{2} \mathrm{O}\left[10^{9}\right]$ & $\mathrm{H}_{2} \mathrm{O}[\%]$ \\
\hline $\mathrm{N}-\mathrm{S}$ & 31,3 & 0,70 & 44,7 & 8800 & 9700 & 2,0 & 14.2 \\
MDS & 31,1 & 0,69 & 45,0 & 8400 & 15200 & 2,1 & 15.0 \\
\hline
\end{tabular}

Tabelle 4.7: Vergleich der Navier-Stokes (N-S) und Molekulardynamiksimulation (MDS) für Fall II aus [38]. $K$ ist der Kompressionsfaktor, $\mathrm{T}_{\varnothing}$ gibt die maximale Durchschnittstemperatur an, $T_{\max }$ die maximale Zentrumstemperatur. Die $\mathrm{H}_{2} \mathrm{O}$-Angaben beziehen sich auf $t_{R \min }$. 


\subsubsection{Rotationsfreiheitsgrade}

\begin{tabular}{ccc|ccc|ccc}
\hline$P_{a}$ & - & $1,3 \mathrm{bar}$ & $N_{N G}$ & - & 216.000 & $v_{W, \text { init }}$ & - & $25 \mathrm{~m} / \mathrm{s}$ \\
$\nu_{a}$ & - & $26,5 \mathrm{kHz}$ & $S$ & - & $\approx 56.000$ & $\alpha_{t}$ & - & 0.3 \\
$R_{0}$ & - & $4,5 \mu \mathrm{m}$ & Edelgas & - & Argon & $\alpha_{v}$ & - & 0.1 \\
$T_{\text {water }}$ & - & $300 \mathrm{~K}$ & $\Delta K_{W}$ & - & 5.000 & & & \\
\hline
\end{tabular}

Tabelle 4.8: Parameter der Vergleichsrechnungen zum Einfluß der Rotationsfreiheitsgrade.

Durch ihren nicht-sphärischen Aufbau besitzen Wasserdampfmoleküle Freiheitsgrade der Rotation, in welche Energie ausweichen und gespeichert werden kann (siehe Kap. 3.2.1.3). Hier soll der Einfluß dieses Effektes auf die äußere und innere Dynamik einer Kavitationsblase behandelt werden. Dazu wurden Vergleichsrechnungen mit und ohne aktivierter Rotation durchgeführt. Die entsprechenden Parameter sind Tab. $4.8 \mathrm{zu}$ entnehmen. Abb. 4.15 zeigt Ergebnisse beider Rechnungen in der letzten Phase des Kollaps. Die Blase ohne Rotationsfreiheitsgrade der $\mathrm{H}_{2} \mathrm{O}$-Moleküle kollabiert deutlich weniger stark, da die auftreffenden Teilchen eine größere kinetische Energie besitzen (siehe Abb. 4.15,a). Dies führt für den größten Teil des Kollaps zu einem erhöhten Wanddruck gegenüber der Vergleichs-
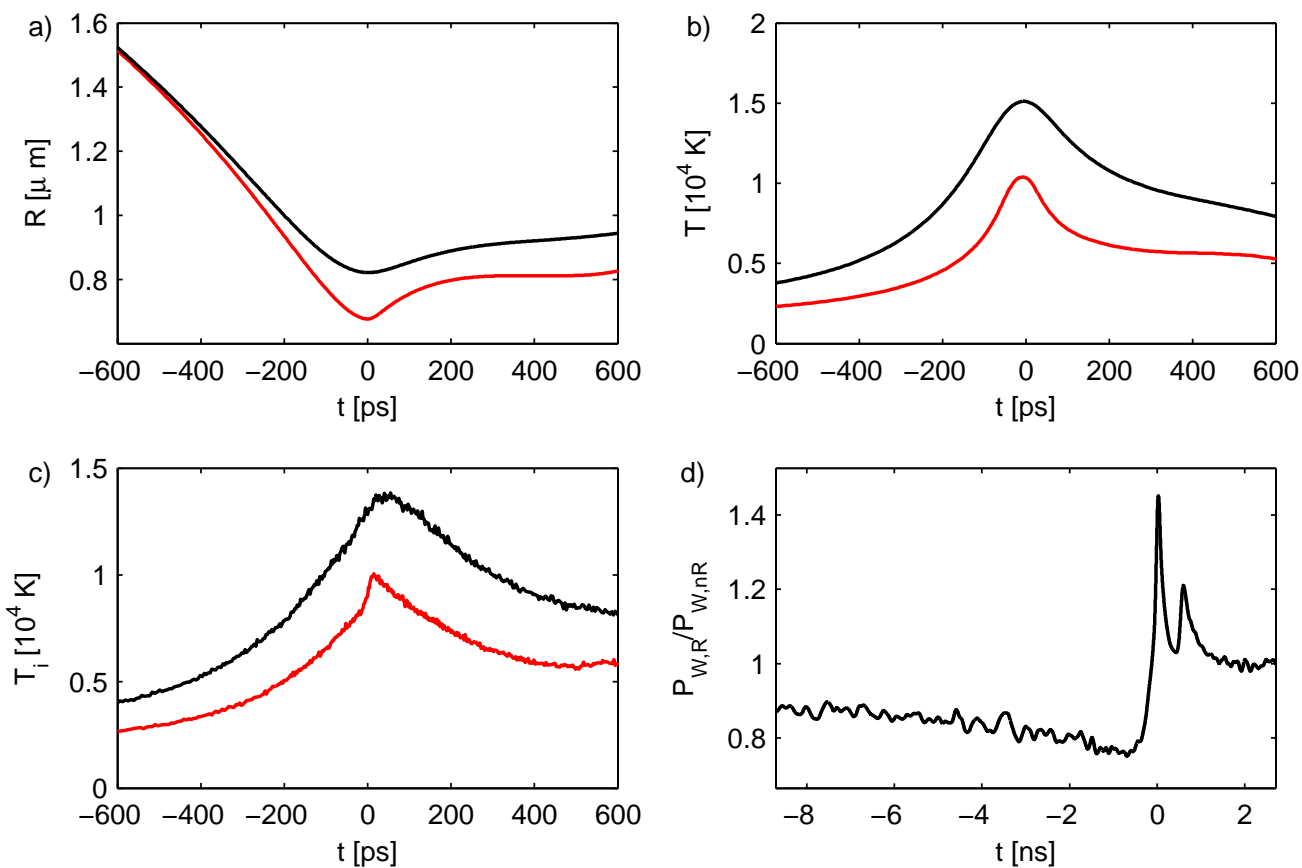

Abbildung 4.15: Verlauf von Radius (a), Durchschnittstemperatur (b), Temperatur der einfallenden Teilchen $\mathrm{T}_{i}$ (c) für Rechnungen mit Rotation (-) und ohne Rotation (-) der $\mathrm{H}_{2} \mathrm{O}$ Moleküle. (d) zeigt den Quotienten des Wanddrucks der Rechnung ohne Rotation und desjenigen der Rechnung mit Rotation. Parameter siehe Tab. 4.8. 

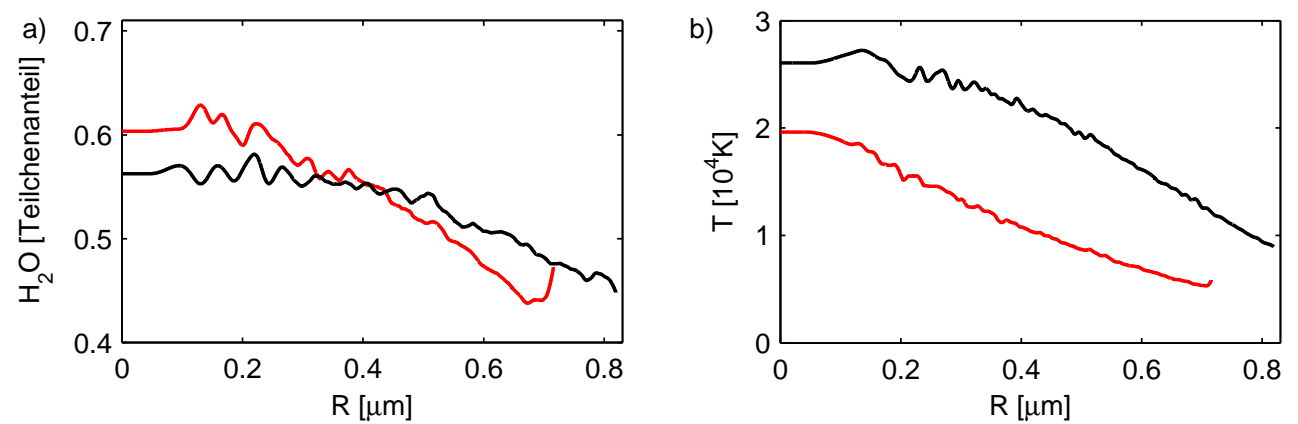

Abbildung 4.16: (a): Teilchenanteil von Wasser und (b): Temperatur in der Blase zum Zeitpunkt der maximalen Blasentemperatur für Rechnungen mit Rotation (-) und ohne Rotation (一) der $\mathrm{H}_{2} \mathrm{O}$ - Moleküle. Parameter siehe Tab. 4.8

rechnung mit Rotation. Lediglich in der letzten Phase, ca. 300 ns vor erreichen des minimalen Radius, dreht sich das Verhältnis der Wanddrücke durch den stärkeren Kollaps der Vergleichsrechnung. Die Durchschnittstemperatur liegt für die Rechnung ohne Rotation stets deutlich höher. Im Maximum werden ca. $15.100 \mathrm{~K}$ gegenüber ca. $10.050 \mathrm{~K}$ erreicht.

Der Wasserdampfgehalt der Blase wird kaum beeinflußt. Abb. 4.16, a zeigt, dass bei vorhandener Rotation die Wasserdampfteilchen inhomogener verteilt sind, das Gefälle zum Blasenrand ist stärker. Dies erklärt sich durch die geringer Diffusion aufgrund der langsameren Teilchengeschwindigkeiten. Die Temperaturen werden in der gesamten Blase herabgesetzt, das Temperaturprofil (Abb. 4.16,b) wird insgesamt nach unten verschoben. In Abb. 4.17 kann man erkennen, dass die heiße Phase bei der Rechnung ohne Rotation länger anhält, die Dynamik verlangsamt ist und die heiße Region vergrößert wird.
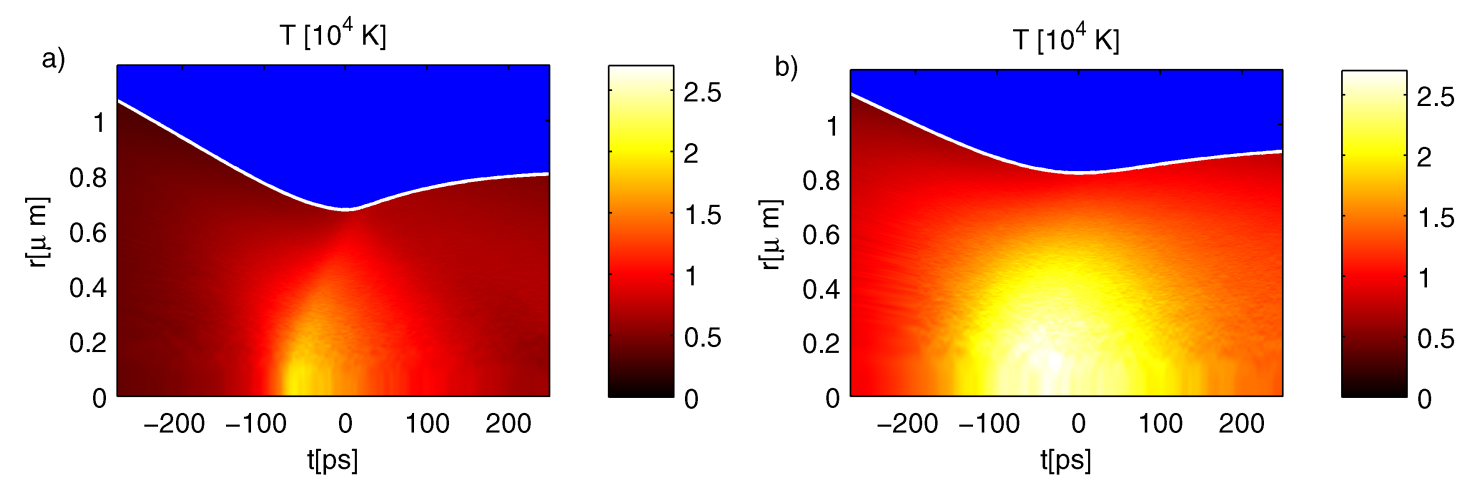

Abbildung 4.17: Temperaturverteilung für Rechnungen mit Rotation (a) und ohne Rotation (b). Parameter siehe Tab. 4.8 


\subsection{Chemisches Modell}

Die in Kavitations- und Sonolumineszenzblasen ablaufenden chemischen Reaktionen sind ein eigenes weites Forschungsfeld: die Sonochemie. SusLICK wies auf die Kombination aus extrem kurzer Zeitskala, hohem Druck und großer pro Molekül zur Verfügung stehender Energie hin, wie sie nur durch Ultraschallanregung von Flüssigkeiten zu erreichen sind [112][113]. Schon lange werden diese Eigenschaften bei der Auslösung und Beschleunigung von chemischen Reaktion durch MBSL benutzt [114][115]. Unterschiedliche Effekte kommen dabei zum tragen: Zum einen gelangen Stoffe, die im Blasenmedium gelöst werden, in der Unterdruckphase in das Innere einer Blase, bleiben dort während des Kollaps gefangen, reagieren durch die auftretenden hohen Temperaturen und werden schließlich z.B. durch einen Jet oder durch Kondensation wieder an das die Blase umgebende Medium abgegeben. Zum anderen werden aus der Blase austretende Radikale, vor allem $\mathrm{OH}^{-}$, für Reaktionen verantwortlich gemacht, die außerhalb der Blase stattfinden.

Die Art des Austritts der Radikale ist umstritten. Entweder könnte zu einem Zeitpunkt nahe des minimalen Blasenradius der momentane Blaseninhalt durch Jets oder andere Deformationen in der umgebenden Flüssigkeit verteilt werden [116]. Vor allem bei den instabileren MBSL-Blasen könnte dieser Mechanismus auftreten. Ebenso ist denkbar, dass in der heißen Phase des Kollaps, wenn auch an der Blasenwand überkritische Temperaturen erreicht werden, viele Radikale in die Flüssigkeit diffundieren könnten. Eine weitere Möglichkeit ist das langsamere Kondensieren der entstandenen Spezies an der Blasenwand [38]. Dieser Mechanismus ist um mehrere Größenordnungen weniger effektiv als die zuerst erwähnten.

Von LEPOINT et al. wurde gezeigt, dass SBSL-Blasen ebenso chemisch aktiv sind - auch in Regimes, in denen noch kein Leuchten beobachtbar ist [117].

Die hohen Temperaturen führen in jedem Fall zu einer signifikanten Dissoziation des gefangenen Wasserdampfes. Durch den endothermen Charakter diese Vorgangs ist mit einer starken Beeinflussung der Verhältnisse in der Blase zu rechnen, wie YASUi sowie STOREy und Szeri anhand von kontinuumsmechanischen Modellen gezeigt haben [28][38].

Im vorliegenden Modell sind 27 chemische Reaktionen implementiert, die die Bildung folgender Spezies - neben den Edelgasen und $\mathrm{H}_{2} \mathrm{O}$ - bewirken: $\mathrm{O}, \mathrm{O}_{2}, \mathrm{O}_{3}, \mathrm{H}$, $\mathrm{H}_{2}, \mathrm{OH}, \mathrm{HO}_{2}$ und $\mathrm{H}_{2} \mathrm{O}_{2}$. Da in der Simulation eine direkte Ionisation der Teilchen 
nicht realisiert ist wird hier, und im weiteren Verlauf, auf die Kennzeichnung der Ionen (durch Index ${ }^{+}$oder $^{-}$) verzichtet.

Die vorkommenden Reaktionen sind in Tab. 4.9 angegeben. Sie teilen sich in zwei grundsätzliche Klassen, die im Folgenden beschrieben werden.

\subsubsection{Reaktionsarten}

Eine Reaktion kann bei einem direkten Stoß zweier Teilchen, von denen mindestens eines reaktionsfähig ist, stattfinden. Die Energie, die für eine Reaktion benötigt wird, kann auf zwei verschiedene Weisen bereitgestellt werden:

- zum Einen kann die Energie aus der Stoßenergie $E_{S}$ der beiden beteiligten Teilchen gezogen werden. In diesem Fall spricht man von einer bimolekularen Reaktion.

- zum Anderen kann ein Katalysator die Energie bereitstellen. Dieser Katalysator tritt in Form eines dritten Teilchens, das an dem Stoß beteiligt ist, auf. In diesem Fall spricht man von einer katalytischen Reaktion.

Auf die beiden vorkommenden Reaktionsarten wird im Folgenden eingegangen.

\section{Bimolekulare Reaktionen}

Bei einer bimolekularen Reaktion werden zwei Teilchen 1 und 2 unter Ausnutzung ihrer kinetischen Energie in andere Teilchen 3 und 4 umgesetzt:

$$
1+2 \rightleftharpoons 3+4
$$

Die kinetische Energie, die dem Stoß der Teilchen zur Verfügung steht, berechnet sich im Schwerpunktsystem mittels

$$
E_{S}=\frac{1}{2} M_{12} v_{12}^{2}
$$

Dabei bezeichnet $M_{12}$ die reduzierte Masse und $v_{12}$ die Geschwindigkeitsdifferenz $\left|\vec{v}_{1}-\vec{v}_{2}\right|$ der Teilchen.

Eine Reaktion kann dann stattfinden, falls die Stoßenergie $E_{S}$ die Aktivierungsenergie $E_{a}$ der Reaktion übersteigt. Ist dies gegeben, so läßt sich die Wahrscheinlichkeit $p_{R}$, dass die beiden Teilchen tatsächlich reagieren, mittels des Quotienten 
aus totalem Reaktionsquerschnitt $\sigma_{R}$ und totalem Stoßquerschnitt $\sigma_{T}$ berechnen: $p_{R}=\sigma_{R} / \sigma_{T}$ [118]. Unter $\sigma_{T}$ stellt man sich am anschaulichsten die geometrische Querschnittsfläche vor, die ein Objekt einem anfliegenden Teilchen bietet, so dass beim Auftreffen des Teilchens in diese Fläche irgendeine Wirkung eintritt. Im Falle zweier unterschiedlicher sphärischer Teilchen mit Durchmessern $d_{1}$ und $d_{2}$ ergibt dies:

$$
\sigma_{T}=\pi\left(\frac{d_{1}+d_{2}}{2}\right)^{2} .
$$

Zur Berechnung von $\sigma_{R}$ betrachtet man zunächst die von der Temperatur abhängige Ratengleichung der Reaktion:

$$
-\dot{n}_{1}=k_{H}(T) n_{1} n_{2}-k_{R}(T) n_{3} n_{4} .
$$

$n_{x}$ bezeichnet hier die Teilchenanzahldichte der Spezies, $k_{H}(T)$ und $k_{R}(T)$ sind die Ratenkoeffizienten für Hin- und Rückreaktion:

$$
k(T)=\Lambda T^{\eta} \mathrm{e}^{-\frac{E_{a}}{k_{B} T}}
$$

mit den reaktionsabhängigen Konstanten $\Lambda$ und $\eta$.

Unter Verwendung der Reaktionswahrscheinlichkeit und der Kollisionsdichte $\frac{n_{1} n_{2} \sigma_{T}<v_{12}>}{1+\delta_{12}}$ (Produkt der Teilchenanzahldichten, des Stoßquerschnittes und der gemittelten Geschwindigkeit; $\delta_{12}$ : Kroneckersymbol) erhält man einen weiteren Ausdruck für die Änderung der Teilchenanzahl:

$$
-\dot{n}_{1}=\frac{\sigma_{R}}{1+\delta_{12}} n_{1} n_{2} \sqrt{\frac{8 k_{B} T}{\pi M_{12}}} \mathrm{e}^{-\frac{E_{a}}{k_{B} T}} .
$$

Hier wurde $\frac{\sigma_{R}}{\sigma_{T}}$ als Reaktionswahrscheinlichkeit, $\mathrm{e}^{-\frac{E_{a}}{k_{B} T}}$ als Anteil der Kollisionen, deren Energie für eine Reaktion ausreicht, sowie $\sqrt{\frac{8 k_{B} T}{\pi M_{12}}}$ als gemittelte Teilchengeschwindigkeit benutzt. Der Reaktionsquerschnitt ist abhängig von der Stoßenergie $E_{S}=m_{12} v_{12}^{2}[118]$ :

$$
\sigma_{R}=\sigma_{0}\left(1-\frac{E_{a}}{E_{S}}\right)\left(\frac{E_{S}}{E_{a}}-1\right)^{\eta-1 / 2}
$$

mit noch unbekanntem Faktor $\sigma_{0}$. Man erhält mit der Gammafunktion $\Gamma$ [77]:

$$
-\dot{n}_{1}=\frac{\sigma_{0}}{1+\delta_{12}} n_{1} n_{2} \sqrt{\frac{8 k_{B} T}{\pi M_{12}}} \Gamma(\eta+3 / 2)\left(\frac{k_{B} T}{E_{a}}\right)^{\eta-1 / 2} \mathrm{e}^{-\frac{E_{a}}{k_{B} T}} .
$$


Der Vergleich dieses Ausdrucks mit (4.20) bzw. (4.21) liefert eine Gleichung für $\Lambda$, die sich leicht nach $\sigma_{0}$ auflösen läßt:

$$
\sigma_{0}=\frac{\Lambda \cdot\left(1+\delta_{12}\right)}{\Gamma(\eta+3 / 2)} \sqrt{\frac{\pi M_{12}}{8 k_{B} T}}\left(\frac{E_{a}}{k_{B}}\right)^{\eta-1 / 2} .
$$

Mit den nun bekannten Querschnitten kann $p_{R}$ berechnet werden. Diese wird bei jeder Kollision ausgewertet. Sollte sich eine Reaktion ergeben, werden die Geschwindigkeiten der Produkte nach Gl. 3.25 und 3.26 berechnet.

\section{Katalytische Reaktionen}

Das Vorhandensein eines Katalysators setzt die Aktivierungsenergie einer Reaktion herab. In der Simulation wird davon ausgegangen, dass eine katalytische Reaktion immer stattfindet, falls ein Katalysatoratom zur Verfügung steht. Daher wird die Aktivierungsenergie dieser Reaktionen mit Null festgesetzt, die Wahrscheinlichkeit $p_{R}=\sigma_{R} / \sigma_{T}$ mit Eins. Als Reaktionsgleichung ergibt sich:

$$
1+2+K \rightleftharpoons 3+K
$$

Da in der Simulation keine Dreier-Stöße implementiert sind wird für die Hinreaktion ein zweischrittiger Lindemann-Mechanismus angenommen [119], bei dem die Edukte vorübergehend einen angeregten Komplex $A^{*}$ bilden:

$$
1+2 \rightleftharpoons A^{*}
$$

Falls $A^{*}$ nun mit einem Katalysatoratom stößt wird die Reaktion abgeschlossen:

$$
A^{*}+K \rightarrow 3+K
$$

Die Geschwindigkeit des angeregten Komplexes wird nach Gl. (3.27) berechnet, die Lebenszeit bestimmt sich durch

$$
t_{A^{*}}=\frac{a_{T, A^{*}} \sqrt{\sigma_{A^{*}}}}{v_{12}}
$$

Dabei bezeichnet $a_{T, A^{*}}$ einen von der Reaktion abhängigen konstanten Parameter, $\sigma_{A^{*}}$ den Reaktionsquerschnitt des angeregten Paares sowie $v_{12}$ die Geschwindigkeitsdifferenz der beiden ursprünglichen Teilchen. 
Die ternäre Reaktion wird also durch zwei bimolekulare Reaktionen dargestellt. Der zweite Reaktionsschritt besitzt dabei eine Reaktionsenergie von

$$
E_{d}^{\prime}=E_{d}+\frac{1}{2} M_{12} v_{12}^{2}
$$

Stößt das angeregte Teilchen während der Lebenszeit des angeregten Zustandes mit keinem anderen Teilchen, zerfällt es wieder in seine Ursprungsbausteine. Für die beiden Teilchen gelten nach dem Zerfall die in Gl. (3.31) und (3.32) angegebenen Geschwindigkeiten.

Bei der Rückreaktion einer katalytischen Reaktion sind drei Teilchen zu beachten: die wieder getrennten Reaktanden sowie der Katalysator. Für deren Teilchengeschwindigkeiten gelten die in Gl. (3.28) - (3.30) beschriebenen Beziehungen.

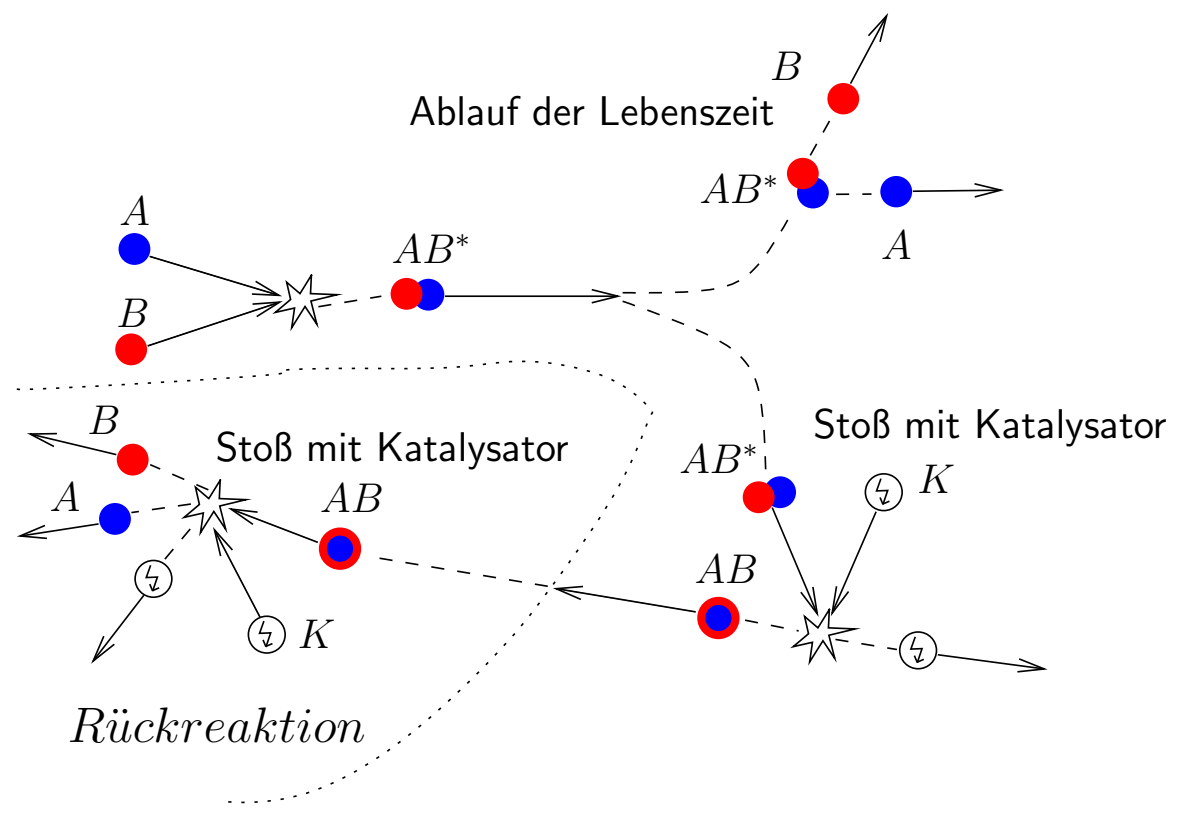

Abbildung 4.18: Schema der ternären katalytischen Reaktion.

In Abb. 4.18 ist der Ablauf einer ternären katalytischen Reaktion schematisch dargestellt.

Für ein und dieselben Edukte können auch mehrere unterschiedliche Reaktionen ablaufen. So kann z.B. der Stoß von einem H-Atom mit einem $\mathrm{HO}_{2}$-Molekül zur Bildung von entweder $\left(\mathrm{O}+\mathrm{H}_{2} \mathrm{O}\right)$ oder $\left(\mathrm{O}_{2}+\mathrm{H}_{2}\right)$ oder aber $(\mathrm{OH}+\mathrm{OH})$ führen. Wenn zwei Teilchen stoßen, die unterschiedliche Reaktionen eingehen können, wird für jede Reaktion anhand der benötigten Energie und der Reaktionswahr- 


\begin{tabular}{|c|c|c|c|c|c|c|c|}
\hline Nr. & & eak & & $\Lambda\left[\mathrm{m}^{3} / \mathrm{mol}\right]$ & $\eta$ & $E_{a}[\mathrm{~K}]$ & $E_{D}[\mathrm{~kJ} / \mathrm{mol}$ \\
\hline 1 & $\mathrm{O}+\mathrm{O}+\mathrm{M}$ & $\rightarrow$ & $\mathrm{O}_{2}+\mathrm{M}$ & $1,2 \cdot 10^{5}$ & $-1,0$ & 0 & $-505,40$ \\
\hline 2 & $\mathrm{O}+\mathrm{H}+\mathrm{M}$ & $\rightarrow$ & $\mathrm{OH}+\mathrm{M}$ & $5,0 \cdot 10^{5}$ & $-1,0$ & 0 & $-436,23$ \\
\hline 3 & $\mathrm{O}+\mathrm{H}_{2}$ & $\rightarrow$ & $\mathrm{H}+\mathrm{OH}$ & $3,8 \cdot 10^{-2}$ & 2,7 & 3152 & 8,23 \\
\hline 4 & $\mathrm{O}+\mathrm{HO}_{2}$ & $\rightarrow$ & $\mathrm{OH}+\mathrm{O}_{2}$ & $2,0 \cdot 10^{8}$ & 0,0 & 0 & $-231,85$ \\
\hline 5 & $\mathrm{O}+\mathrm{H}_{2} \mathrm{O}_{2}$ & $\rightarrow$ & $\mathrm{OH}+\mathrm{HO}_{2}$ & 9,6 & 2,0 & 2014 & $-56,08$ \\
\hline 6 & $\mathrm{H}+\mathrm{O}_{2}+\mathrm{M}$ & $\rightarrow$ & $\mathrm{HO}_{2}+\mathrm{M}$ & $2,8 \cdot 10^{6}$ &,- 86 & 0 & $-204,80$ \\
\hline 7 & $\mathrm{H}+\mathrm{O}_{2}+\mathrm{O}_{2}$ & $\rightarrow$ & $\mathrm{HO}_{2}+\mathrm{O}_{2}$ & $2,0 \cdot 10^{7}$ & $-1,24$ & 0 & $-204,80$ \\
\hline 8 & $\mathrm{H}+\mathrm{O}_{2}+\mathrm{H}_{2} \mathrm{O}$ & $\rightarrow$ & $\mathrm{HO}_{2}+\mathrm{H}_{2} \mathrm{O}$ & $11,0 \cdot 10^{6}$ &,- 76 & 0 & $-204,80$ \\
\hline 9 & $\mathrm{H}+\mathrm{O}_{2}+\mathrm{Ar}$ & $\rightarrow$ & $\mathrm{HO}_{2}+\mathrm{Ar}$ & $7,0 \cdot 10^{5}$ &,- 80 & 0 & $-204,80$ \\
\hline 10 & $\mathrm{H}+\mathrm{O}_{2}$ & $\rightarrow$ & $\mathrm{O}+\mathrm{OH}$ & $2,6 \cdot 10^{10}$ &,- 67 & 8581 & 69,17 \\
\hline 11 & $\mathrm{H}+\mathrm{H}+\mathrm{M}$ & $\rightarrow$ & $\mathrm{H}_{2}+\mathrm{M}$ & $1,0 \cdot 10^{6}$ & $-1,0$ & 0 & $-444,47$ \\
\hline 12 & $\mathrm{H}+\mathrm{H}+\mathrm{H}_{2}$ & $\rightarrow$ & $\mathrm{H}_{2}+\mathrm{H}_{2}$ & $9,0 \cdot 10^{4}$ &,- 60 & 0 & $-444,47$ \\
\hline 13 & $\mathrm{H}+\mathrm{H}+\mathrm{H}_{2} \mathrm{O}$ & $\rightarrow$ & $\mathrm{H}_{2}+\mathrm{H}_{2} \mathrm{O}$ & $6,0 \cdot 10^{7}$ & $-1,25$ & 0 & $-444,47$ \\
\hline 14 & $\mathrm{H}+\mathrm{OH}+\mathrm{M}$ & $\rightarrow$ & $\mathrm{H}_{2} \mathrm{O}+\mathrm{M}$ & $2,2 \cdot 10^{10}$ & $-2,0$ & 0 & $-508,82$ \\
\hline 15 & $\mathrm{H}+\mathrm{HO}_{2}$ & $\rightarrow$ & $\mathrm{O}+\mathrm{H}_{2} \mathrm{O}$ & $3,9 \cdot 10^{6}$ & 0,0 & 337 & $-236,09$ \\
\hline 16 & $\mathrm{H}+\mathrm{HO}_{2}$ & $\rightarrow$ & $\mathrm{O}_{2}+\mathrm{H}_{2}$ & $4,4 \cdot 10^{7}$ & 0,0 & 537 & $-239,67$ \\
\hline 17 & $\mathrm{H}+\mathrm{HO}_{2}$ & $\rightarrow$ & $\mathrm{OH}+\mathrm{OH}$ & $0,8 \cdot 10^{8}$ & 0,0 & 319 & $-162,26$ \\
\hline 18 & $\mathrm{H}+\mathrm{H}_{2} \mathrm{O}_{2}$ & $\rightarrow$ & $\mathrm{HO}_{2}+\mathrm{H}_{2}$ & 12,0 & 2,0 & 2618 & $-64,32$ \\
\hline 19 & $\mathrm{H}+\mathrm{H}_{2} \mathrm{O}_{2}$ & $\rightarrow$ & $\mathrm{OH}+\mathrm{H}_{2} \mathrm{O}$ & $1,0 \cdot 10^{7}$ & 0,0 & 1812 & $-290,93$ \\
\hline 20 & $\mathrm{OH}+\mathrm{H}_{2}$ & $\rightarrow$ & $\mathrm{H}+\mathrm{H}_{2} \mathrm{O}$ & $2,1 \cdot 10^{-6}$ & 1,51 & 1727 & $-64,35$ \\
\hline 21 & $\mathrm{OH}+\mathrm{OH}+\mathrm{M}$ & $\rightarrow$ & $\mathrm{H}_{2} \mathrm{O}_{2}+\mathrm{M}$ & $2,3 \cdot 10^{6}$ &,- 90 & -856 & $-217,89$ \\
\hline 22 & $\mathrm{OH}+\mathrm{OH}$ & $\rightarrow$ & $\mathrm{H}_{2} \mathrm{O}+\mathrm{O}$ & $3,5 \cdot 10^{-2}$ & 2,40 & -1062 & $-66,79$ \\
\hline 23 & $\mathrm{OH}+\mathrm{HO}_{2}$ & $\rightarrow$ & $\mathrm{O}_{2}+\mathrm{H}_{2} \mathrm{O}$ & $1,4 \cdot 10^{7}$ & 0,0 & -252 & $-304,44$ \\
\hline 24 & $\mathrm{OH}+\mathrm{H}_{2} \mathrm{O}_{2}$ & $\rightarrow$ & $\mathrm{HO}_{2}+\mathrm{H}_{2} \mathrm{O}$ & $2,0 \cdot 10^{6}$ & 0,0 & 215 & $-128,67$ \\
\hline 25 & $\mathrm{HO}_{2}+\mathrm{HO}_{2}$ & $\rightarrow$ & $\mathrm{O}_{2}+\mathrm{H}_{2} \mathrm{O}_{2}$ & $1,3 \cdot 10^{5}$ & 0,0 & -821 & $-175,35$ \\
\hline 26 & $\mathrm{H}_{2} \mathrm{O}+\mathrm{Ar}$ & $\rightarrow$ & $\mathrm{OH}+\mathrm{H}+\mathrm{Ar}$ & $1,96 \cdot 10^{16}$ & $-1,62$ & 59700 & 508,82 \\
\hline 27 & $\mathrm{H}_{2} \mathrm{O}+\mathrm{H}_{2} \mathrm{O}$ & $\rightarrow$ & $\mathrm{OH}+\mathrm{H}+\mathrm{H}_{2} \mathrm{O}$ & $3,13 \cdot 10^{17}$ & $-1,62$ & 59700 & 508,82 \\
\hline
\end{tabular}

Tabelle 4.9: Die im Modell verwendeten Reaktionen, nach der Gri-Mech 3.0 - Datenbank [120] sowie [28], bzw. [121] . $\Lambda$ und $\eta$ bezeichnen die Konstanten der Ratengleichung der Reaktion, $E_{a}$ die Aktivierungsenergie, $E_{D}$ die Reaktionsenthalpie. $M$ steht für ein beliebiges Katalysatorteilchen.

scheinlichkeit entschieden, ob sie stattfinden kann. Unter diesen wird nun die Wahrscheinlichste ermittelt und schließlich ausgeführt.

\subsubsection{Chemische Entwicklung im Hauptkollaps}

Abb. 4.19 zeigt die raumzeitliche Entwicklung der Temperatur, der Dichte des Wasserdampfes und der Dichte ausgewählter Spezies während der heißen Kollaps- 

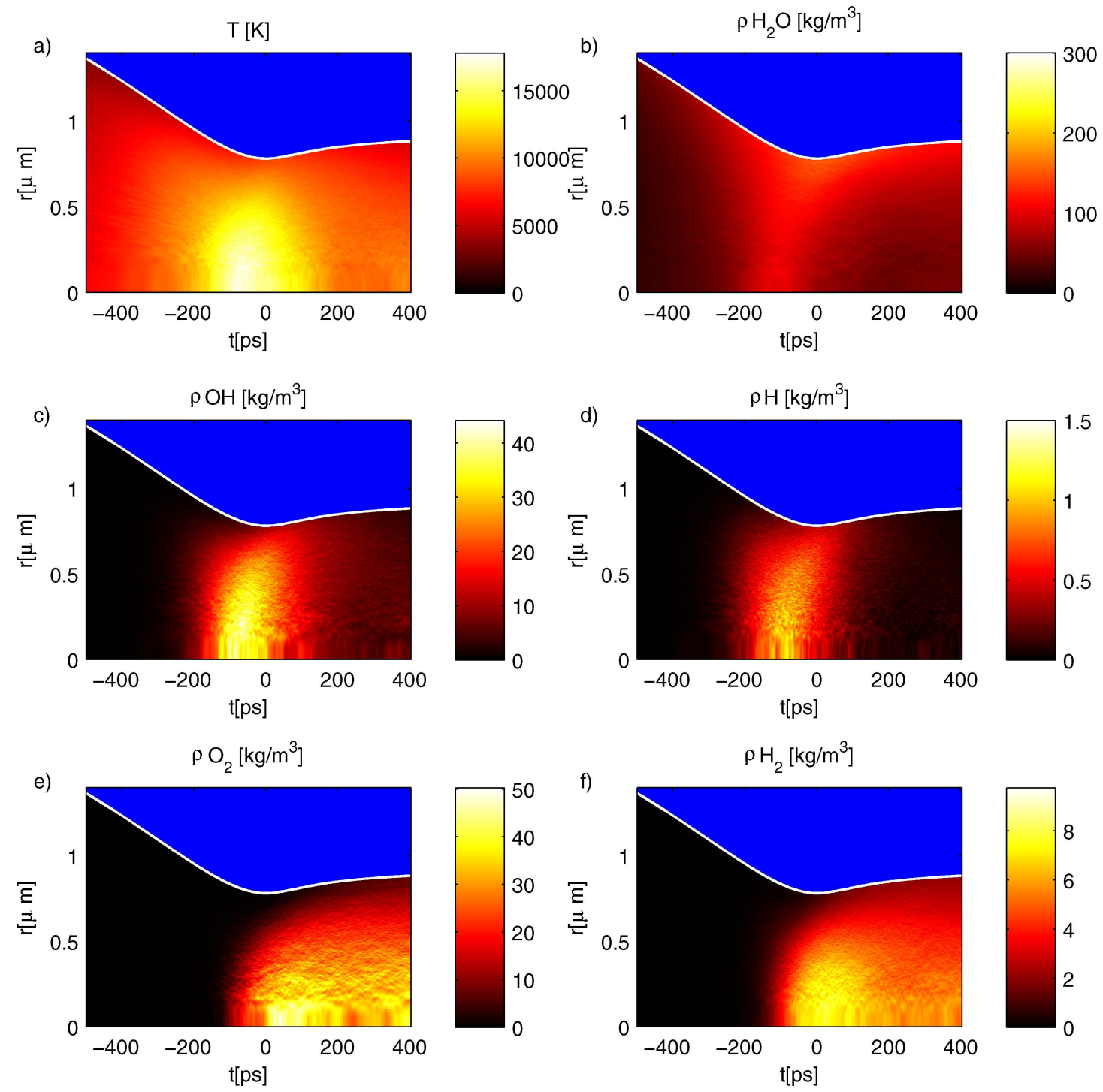

Abbildung 4.19: Raumzeitliche Entwicklung der Temperatur (a) sowie der Dichte von $\mathrm{H}_{2} \mathrm{O}$ (b), $\mathrm{OH}$ (c), $\mathrm{H}(\mathrm{d}), \mathrm{O}_{2}$ (e) und $\mathrm{H}_{2}$ um den Zeitpunkt des minimalen Blasenradius. Parameter siehe Tab. 4.10 .

phase. Es ist gut zu erkennen, dass durch die hohen Temperaturen, die infolge der Fokussierung der einlaufenden Verdichtungswelle im Blasenzentrum auftreten, die dort befindlichen $\mathrm{H}_{2} \mathrm{O}$-Teilchen teilweise dissoziiert werden. Die chemischen Reaktionen setzen ein, wenn die Temperaturen des Blasenzentrums ca. $8.500 \mathrm{~K}$ erreichen. Zunächst treten die Dissoziationsprodukte $\mathrm{OH}$ und $\mathrm{O}$ (nicht dargestellt) in nennenswerten Mengen auf. Diese Spezies sind hochreaktiv und gehen sehr schnell weitere Verbindungen ein. Die chemische Aktivität findet hauptsächlich in den Zentrumsbereichen der Blase statt. Die niedrigeren Temperaturen am 


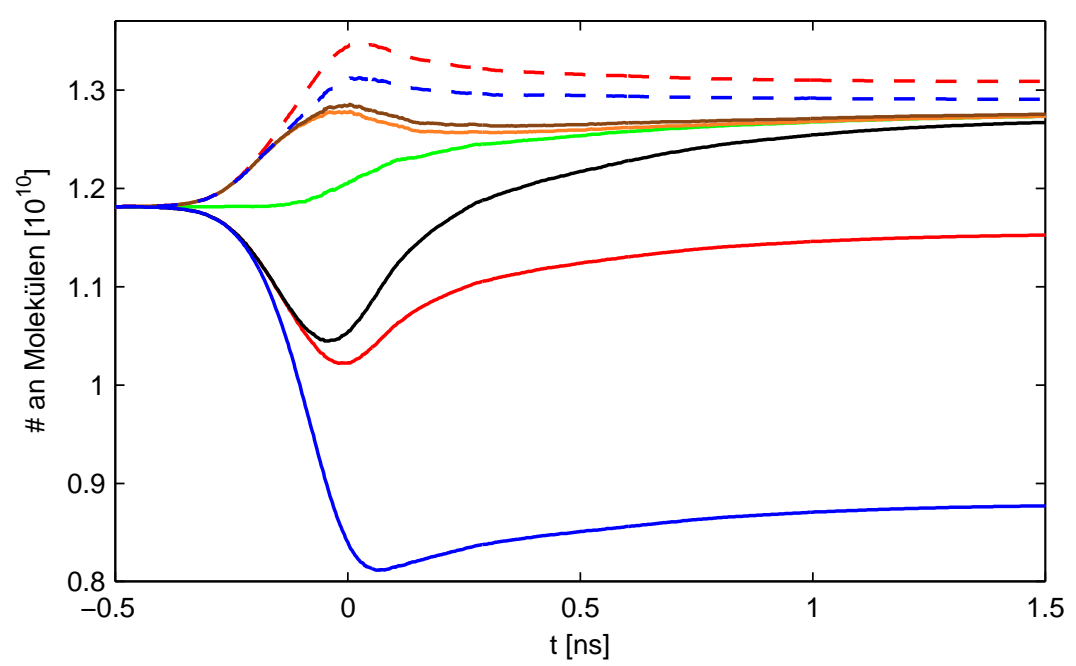

Abbildung 4.20: Entwicklung der realen Anzahl aller betrachteter Spezies um den Hauptkollaps. Kumulative Darstellung - jede Kurve besteht aus der Summe der darunter liegenden sowie der Teilchenanzahl der jeweiligen Spezies. Parameter siehe Tab. 4.10.

Legende: $\mathrm{H}_{2} \mathrm{O}(-) ; \mathrm{H}_{2}(-) ; \mathrm{O}_{2}(-) ; \mathrm{OH}(-) ; \mathrm{H}(-) ; \mathrm{O}(-) ; \mathrm{HO}_{2}(--) ; \mathrm{H}_{2} \mathrm{O}_{2}$ (- -) .

Blasenrand reichen nicht aus, um relevant viele Wassermoleküle zu dissoziieren. Dementsprechend finden sich auch Dissoziationsprodukte sowie kurz darauf deren Reaktionsprodukte zum größten Teil in der Nähe des Blasenzentrums. Erst im Laufe der Zeit verteilen sie sich in der Blase.

Abb. 4.20 zeigt die Anzahl aller Spezies für 2 ns um den minimalen Blasenradius. Der Zeitpunkt der Bildung, die Menge an gebildeten Teilchen sowie die Stabilität der Spezies lassen sich hier gut ablesen. Viele der bei der Dissoziation gebildeten Produkte reagieren schnell zu $\mathrm{H}_{2} \mathrm{O}$ zurück. Ein weiterer großer Teil der H-Atome bildet das stabile $\mathrm{H}_{2}$, während $\mathrm{OH}$ zunächst in großen Mengen $\mathrm{zu}_{2} \mathrm{O}_{2}$ umgesetzt

\begin{tabular}{ccc|ccc|ccc}
\hline$P_{a}$ & - & $1,3 \mathrm{bar}$ & $N_{N G}$ & - & 216.000 & $v_{W, \text { init }}$ & - & $20 \mathrm{~m} / \mathrm{s}$ \\
$\nu_{a}$ & - & $26,5 \mathrm{kHz}$ & $S$ & - & $\approx 59.000$ & $\alpha_{t}$ & - & 0,3 \\
$R_{0}$ & - & $4,5 \mu \mathrm{m}$ & Edelgas & - & Argon & $\alpha_{v}$ & - & 0,1 \\
$T_{\text {water }}$ & - & $300 \mathrm{~K}$ & $\Delta K_{W}$ & - & 5000 & & & \\
\hline
\end{tabular}

Tabelle 4.10: Parameter der Rechnungen zum Einfluß chemischer Reaktionen

wird. Dieses reagiert wiederum entweder mit $\mathrm{OH}$ weiter $\mathrm{zu} \mathrm{HO}_{2}$ und $\mathrm{H}_{2} \mathrm{O}$ oder mit $\mathrm{H}$ zu $\mathrm{OH}$ und $\mathrm{H}_{2} \mathrm{O}$. Die Bildung von $\mathrm{O}_{2}$ setzt vergleichsweise spät ein, hält dann aber über einen relativ langen Zeitraum (ca. 2 ns) an. Dies rührt vermutlich daher, dass zu dessen Produktion hauptsächlich $\mathrm{HO}_{2}$ benötigt wird, welches erst in ausreichender Menge gebildet werden muß. O tritt nur in sehr geringer Menge auf, da auch zu dessen Bildung $\mathrm{HO}_{2}$ benötigt wird und atomarer Sauerstoff schnell 
$\mathrm{zu} \mathrm{O}_{2}$ reagiert.

Insgesamt kann man sagen, dass die reaktiven Spezies nur einige hundert ps in nennenswerter Anzahl in der Blase vorkommen. Sobald die Temperaturen absinken und somit keine weitere Dissoziation für einen Nachschub an $\mathrm{OH}$ - und

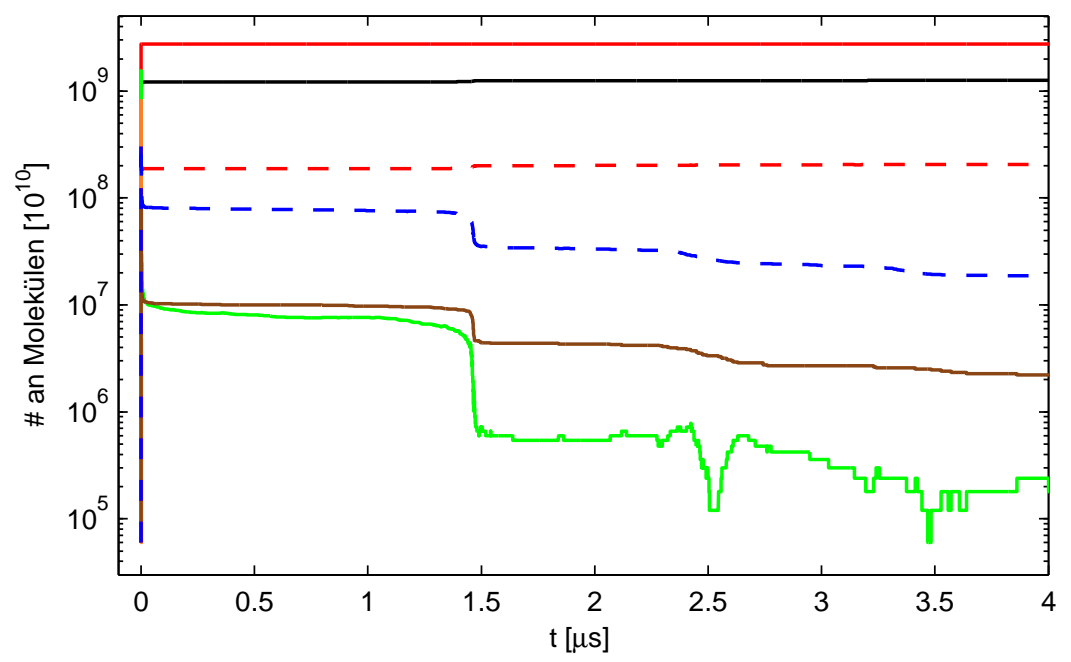

Abbildung 4.21: Entwicklung der realen Teilchenanzahl der entstehenden chemischen Spezies für $4 \mu s$ nach dem minimalen Blasenradius $(\mathrm{t}=0)$ in logarithmische Darstellung. Rebounds der Blasenschwingung treten bei ca. 1,5 ps, 2,5 ps und 3,5 ps auf. Parameter siehe Tab. 4.10. Legende: $\mathrm{H}_{2}(-) ; \mathrm{O}_{2}(-) ; \mathrm{OH}(-) ; \mathrm{H}(-) ; \mathrm{O}(-) ; \mathrm{HO}_{2}(--) ; \mathrm{H}_{2} \mathrm{O}_{2}(--)$.

H-Teilchen sorgt, sinkt deren Anzahl stark ab.

Abb. 4.21 zeigt die weitere Entwicklung der Teilchenanzahl der gebildeten Spezies für $4 \mu$ s nach dem Zeitpunkt des minimalen Blasenradius. Es ist zu erkennen, dass in Zeiten, in denen das Blaseninnere kühl ist, die Anzahl aller Spezies relativ konstant bleibt. Lediglich $\mathrm{OH}$ zeigt eine leichte Abnahme. $\mathrm{H}$ ist hier schon gar nicht mehr vorhanden.

Heizt sich die Blase wieder auf (was bei den Rebounds geschieht), kommen wieder chemische Prozesse in Gang - allerdings in deutlich verringertem Maße, verglichen mit dem Hauptkollaps. $\mathrm{OH}$ wird gut erkennbar umgesetzt, auch $\mathrm{O}$ und $\mathrm{HO}_{2}$ reagieren in relevanter Menge. Gebildet werden $\mathrm{O}_{2}$ und $\mathrm{H}_{2} \mathrm{O}_{2}$ (aufgrund der logarithmischen Auftragung schwer zu erkennen). Für die Bildung von $\mathrm{H}_{2}$ ist nicht genug Energie vorhanden.

Abb. 4.22 zeigt die durchschnittliche Temperatur alles Spezies in der heißen Phase des Hauptkollaps. Am Anfang der Kurven sind bei den neu entstehenden Spezies größere Schwankungen der Durchschnittstemperatur zu beobachten, die auf 


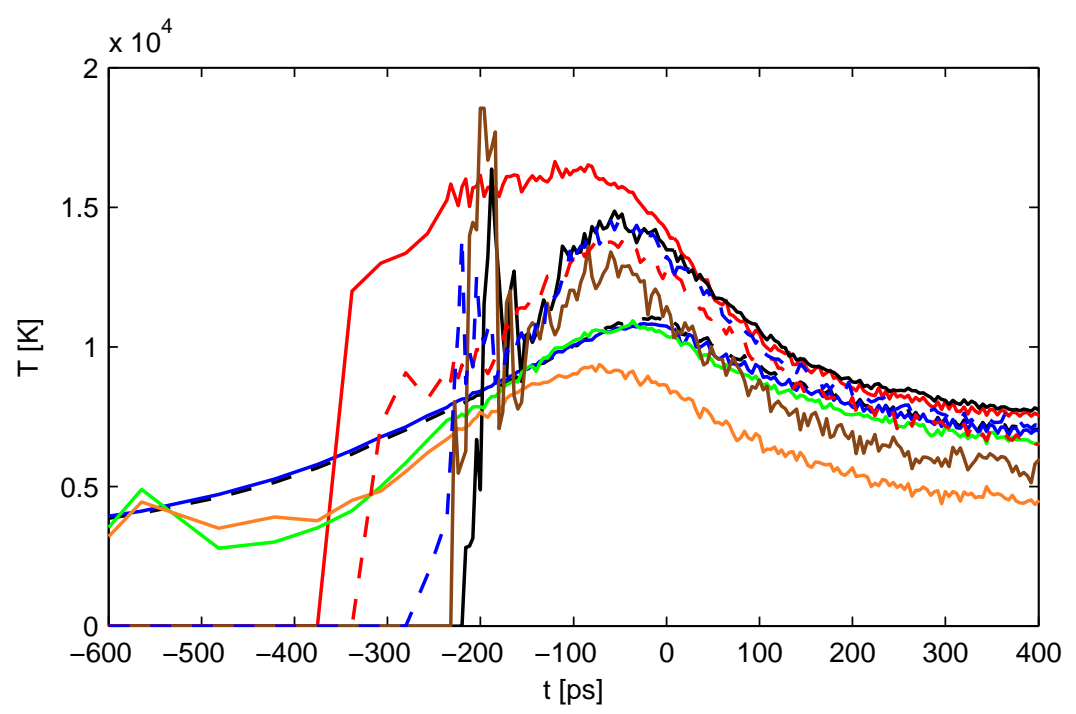

Abbildung 4.22: Entwicklung der Durchschnittstemperatur für alle betrachteten Spezies um den Hauptkollaps. Parameter siehe Tab. 4.10.

Legende: $\mathrm{Ar}(-\mathbf{-}) ; \mathrm{H}_{2} \mathrm{O}(-) ; \mathrm{H}_{2}(-) ; \mathrm{O}_{2}(-)$; $\mathrm{OH}(-) ; \mathrm{H}(-) ; \mathrm{O}(-) ; \mathrm{HO}_{2}(--) ; \mathrm{H}_{2} \mathrm{O}_{2}$ (- -).

der zu diesem Zeitpunkt sehr geringen Teilchenzahl der entsprechenden Spezies beruhen.

Die Temperaturen von Argon und $\mathrm{H}_{2} \mathrm{O}$ unterscheiden sich im gesamten beobachteten Zeitraum nur marginal. Beide erreichen ca. 50 ps vor $t_{R m i n}$ ein Maximum von ca. $11.000 \mathrm{~K}$.

Die Dissoziationsprodukte $\mathrm{OH}$ und $\mathrm{H}$ zeigen vor allem anfänglich, wenn die Dissoziation die mit Abstand häufigste Reaktion ist, niedrigere Temperaturen: 400 ps vor $t_{R \min }$ werden ca. $3.500 \mathrm{~K}$, bzw. $3.900 \mathrm{~K}$ erreicht, während $\mathrm{H}_{2} \mathrm{O}$ zu diesem Zeitpunkt im Durchschnitt bereits ca. $5.000 \mathrm{~K}$ heiß ist. Hier ist der endotherme Charakter der Dissoziation gut zu erkennen: Ein Großteil der Energie einer Teilchenkollison wird in der chemischen Reaktion gebunden, die Produkte sind entsprechend kalt. Mit der Zeit erwärmen sich $\mathrm{OH}$ und $\mathrm{H}$ - die entstehenden Teilchen nehmen durch Kollisionen wieder Energie auf. Gerade bei $\mathrm{OH}$ ist zu beobachten, dass in späteren Phasen des Kollaps auch andere - exotherme - Reaktionen an dessen Bildung beteiligt sind und so die Durchschnittstemperatur bis auf die von $\mathrm{H}_{2} \mathrm{O}$ und Argon ansteigt. $\mathrm{H}$ bleibt auch in diesen Phasen kälter als alle anderen Spezies.

Das andere Extrem ist bei $\mathrm{H}_{2}$-Teilchen zu beobachten. Da die für die Bildung von $\mathrm{H}_{2}$-Molekülen verantwortlichen Reaktionen ausnahmslos exotherm sind, erhalten die entstehenden Teilchen eine große thermische Energie, die zuvor bei 


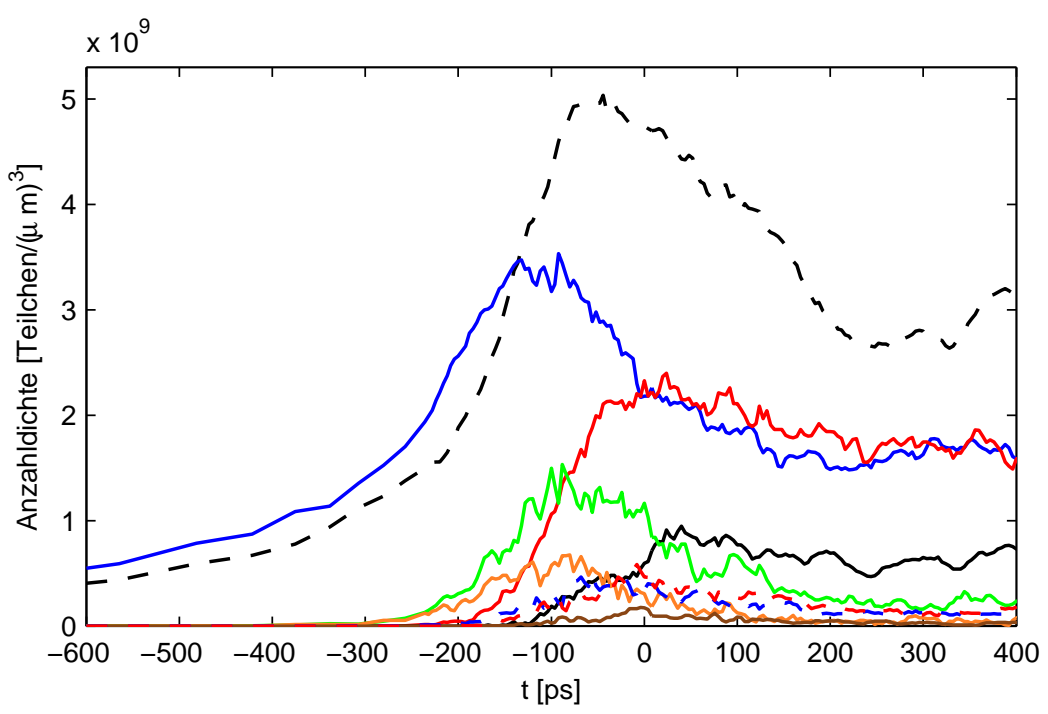

Abbildung 4.23: Entwicklung der Anzahldichte im Blasenzentrum für alle betrachteter Spezies um den Hauptkollaps. Parameter siehe Tab. 4.10.

Legende: $\mathrm{Ar}(-\boldsymbol{-}) ; \mathrm{H}_{2} \mathrm{O}(-) ; \mathrm{H}_{2}(-) ; \mathrm{O}_{2}(-) ; \mathrm{OH}(-) ; \mathrm{H}(-) ; \mathrm{O}(-) ; \mathrm{HO}_{2}(-\boldsymbol{-}) ; \mathrm{H}_{2} \mathrm{O}_{2}$ (- -).

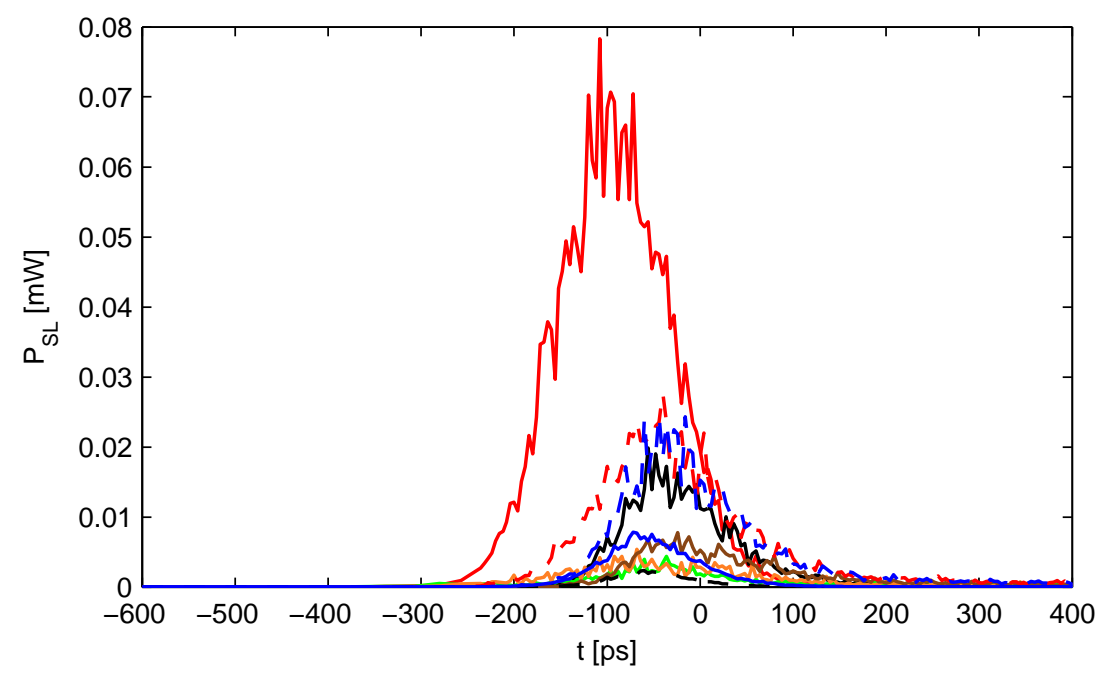

Abbildung 4.24: Lichtemission für alle betrachteten Spezies um den Hauptkollaps. Parameter siehe Tab. 4.10,

Legende: $\mathrm{Ar}(--) ; \mathrm{H}_{2} \mathrm{O}(-) ; \mathrm{H}_{2}(-) ; \mathrm{O}_{2}(-) ; \mathrm{OH}(-) ; \mathrm{H}(-) ; \mathrm{O}(-) ; \mathrm{HO}_{2}(--) ; \mathrm{H}_{2} \mathrm{O}_{2}(--)$.

der Dissoziation gebunden wurde. Dementsprechend liegt die Temperatur der $\mathrm{H}_{2}$-Moleküle weit über derjeniger von Argon und Wasserdampf. Im Maximum beträgt die durchschnittliche $\mathrm{H}_{2}$-Temperatur ca. $16.600 \mathrm{~K}$, während $\mathrm{H}_{2} \mathrm{O}$ lediglich knapp über $10.800 \mathrm{~K}$ erreicht. Auch bei der Bildung von $\mathrm{O}, \mathrm{O}_{2}, \mathrm{HO}_{2}$ und $\mathrm{H}_{2} \mathrm{O}_{2}$ sind hauptsächlich exotherme Reaktionen beteiligt. Deren maximale Durchschnittstemperaturen liegen zwischen $13.600 \mathrm{~K}$ und $14.900 \mathrm{~K}$. Tab. 4.11 fasst die- 
se Ergebnisse, sowie weitere Werte - sowohl über die Blase gemittelte, als auch einzeln für die verschiedenen Spezies - zusammen.

In den Abb. 4.23 und 4.24 sind schließlich die Anzahldichte im Zentrum und die gesamt emittierte Lichtenergie, jeweils aufgeschlüsselt nach Spezies, für die heiße Kollapsphase dargestellt. Die Anzahldichte im Zentrum entspricht der nach den Werten für die gesamte Blase zu erwartenden. Gut ist zu erkennen, wie $\mathrm{H}_{2} \mathrm{O}$ abgebaut wird und dafür andere Spezies entstehen. Nach einem anfänglichen $\mathrm{OH}$ Peak entstehen hauptsächlich stabile Spezies wie $\mathrm{O}_{2}$ und $\mathrm{H}_{2}$. Zum Zeitpunkt des minimalen Blasenradius befinden sich ungefähr dieselbe Anzahl an $\mathrm{H}_{2}$ - wie $\mathrm{H}_{2} \mathrm{O}$ Moleküle im Blasenzentrum.

Beim Blick auf die Lichtenergie fällt auf, dass nicht etwa das Edelgas oder der Wasserdampf eine entscheidene Rolle bei der Lichtemission spielen. Trotz der vergleichsweise hohen Anzahl an Teilchen dieser Spezies im Zentrum - vor allem von Argon - ist die pro Zeiteinheit emittierte Lichtmenge ungleich geringer als die von anderen Spezies, vor allem von $\mathrm{H}_{2}$. Die hohen Temperaturen der Wasserstoffmoleküle und ihre vergleichsweise hohe Anzahl führen dazu, dass $\mathrm{H}_{2}$ mit Abstand am meisten Licht emittiert $\left(E_{S L}=9,8\right.$ pJ, fast die Hälfte der gesamten Lichtleistung von 22,7 pJ - siehe Tab. 4.11). Auch die anderen entstehenden Spezies, deren Temperaturen im Durchschnitt über denen von Argon und Wasserdampf liegen, tragen mehr zu der gesamten Lichtleistung bei als die beiden

\begin{tabular}{lllll}
\hline & $\begin{array}{l}T_{\max }[\mathrm{K}]: \\
E_{S L}[\mathrm{fJ}]:\end{array}$ & $\begin{array}{l}19.700 \\
22,70\end{array}$ & $\begin{array}{l}T_{\varnothing}[\mathrm{K}]: \\
\mathrm{R}_{\text {min }}[\mu \mathrm{m}]:\end{array}$ & $\begin{array}{l}11.440 \\
0,78\end{array}$ \\
\hline \multirow{2}{*}{ Spezies } & $N_{\max }\left[10^{9}\right]$ & $\# W C\left[10^{10}\right]$ & $T_{\varnothing}[\mathrm{K}]$ & $E_{S L}[\mathrm{fJ}]$ \\
\hline $\mathrm{Ar}$ & - & - & 11,0 & 0,23 \\
$\mathrm{H}_{2} \mathrm{O}$ & - & - & 10,8 & 0,86 \\
$\mathrm{H}$ & 0,81 & 0,25 & 9,3 & 0,85 \\
$\mathrm{H}_{2}$ & 2,75 & 80,62 & 16,6 & 9,80 \\
$\mathrm{O}_{2}$ & 0,09 & 0,11 & 13,6 & 1,13 \\
$\mathrm{O}_{2}$ & 1,25 & 8,73 & 14,9 & 2,15 \\
$\mathrm{OH}$ & 1,53 & 0,25 & 10,9 & 0,57 \\
$\mathrm{HO}_{2}$ & 0,35 & 0,59 & 14,5 & 3,16 \\
$\mathrm{H}_{2} \mathrm{O}_{2}$ & 0,36 & 1,33 & 13,8 & 3,94 \\
\hline
\end{tabular}

Tabelle 4.11: Ergebnisse für eine Blase mit Wasserdampf und chemischen Reaktionen (Parameter siehe Tab. 4.10) für die gesamte Blase (obere zwei Zeilen) und für die einzelnen Spezies. $T_{\max }$ ist die absolut größte Temperatur, $T_{\varnothing}$ das Maximum der Durchschnittstemperatur, $E_{S L}$ die gesamte emittierte Lichtleistung, $N_{\max }$ die maximale Anzahl der entsprechenden Spezies zu irgendeinem Zeitpunkt und $\#_{W C}$ die Anzahl der Wandkollisionen von Teilchen der entsprechenden Spezies bis zum ersten Rebound-Kollaps (ca. 1,6 $\mu$ s nach $t_{R \min }$ ). 

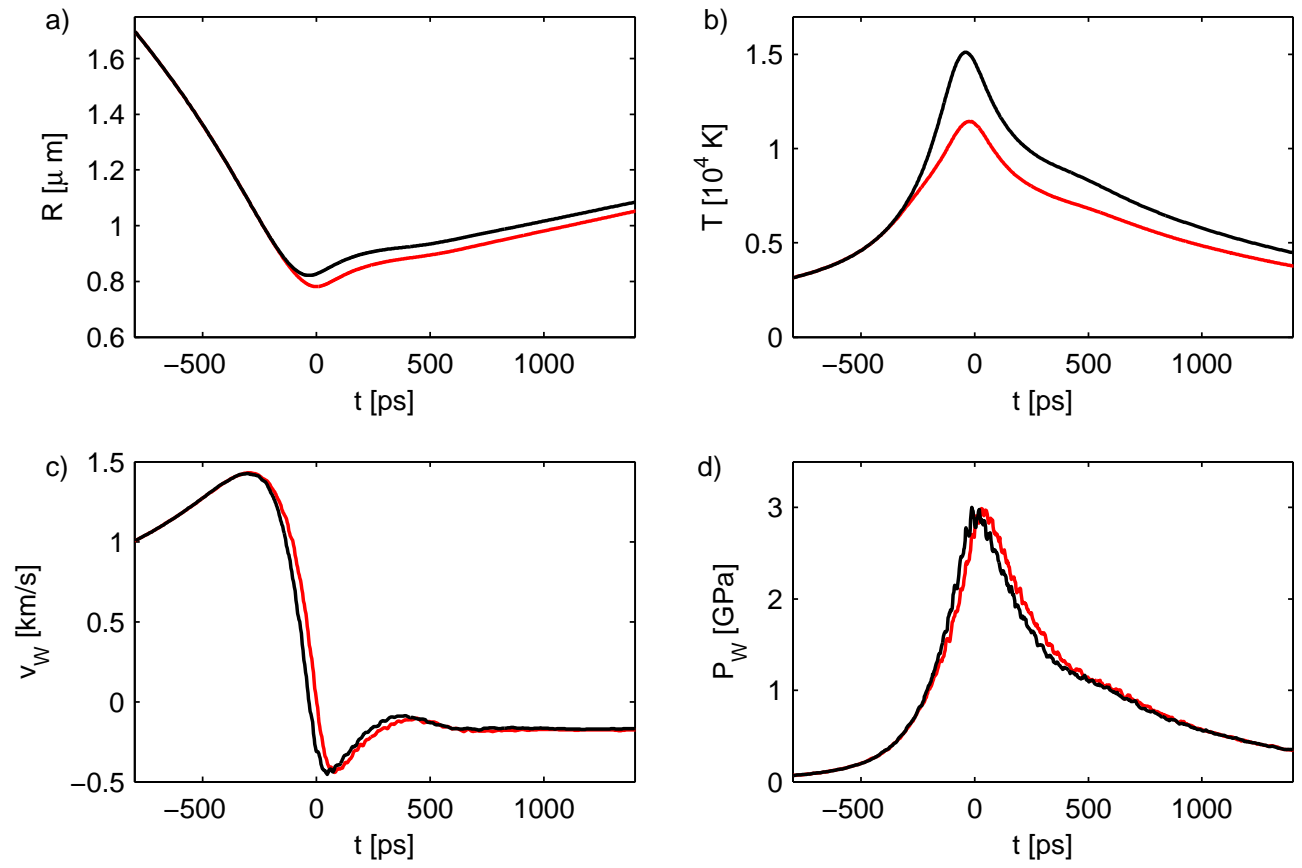

Abbildung 4.25: Verlauf von Radius (a), Durchschnittstemperatur (b), Wandgeschwindigkeit (c) sowie Wanddruck (d) für Rechnungen mit chemischen Reaktionen ( - ) und ohne chemischen Reaktionen (-). Parameter siehe Tab. 4.10.

häufigsten Spezies. Die Emission von Argon ist durch die relativ hohe Ionisierungsenergie nochmals deutlich niedriger als die von $\mathrm{H}_{2} \mathrm{O}$. Es ist ersichtlich, dass die Temperatur entscheidend die Lichtleistung beeinflußt, die Anzahldichte hat nur sekundären Einfluß (siehe Kap. 2.2).

Abb. 4.25 zeigt den Vergleich einiger Blasenparameter einer Argon-Blase mit Wasserdampf und chemischen Reaktionen (Fall 1) und einer Argon-Blase mit Wasserdampf, bei der keine chemischen Reaktionen simuliert werden (Fall 2). $\mathrm{Zu}$ beachten ist, dass für diesen Vergleich in beiden Fällen keine Freiheitsgrade der Rotation für die Wasserdampfteilchen erfaßt werden. Dies geschieht, da für inelastische Teilchenkollisionen mit Masseänderung keine Lösung der Kollisionsbedingungen mit Rotation der Teilchen gefunden werden konnte. Um eine bessere Vergleichbarkeit zu gewährleisten wurde also auch bei Fall 2 auf die Simulation der Rotation verzichtet (diese Blase entspricht der in Kap. 4.2.5 betrachteten).

Die Bewegung des Blasenrandes wird erwartungsgemäß erst gegen Ende des Kollaps beeinträchtigt, da vorher keine chemischen Reaktionen auftreten. Die Blase mit chemischen Reaktionen kollabiert etwas stärker, da die Kollapsenergie zum Teil in chemischen Reaktionen gebunden wird. Der minimale Radius be- 
trägt $0,78 \mu \mathrm{m}$, ohne Reaktionen werden $0,82 \mu \mathrm{m}$ erreicht. Die maximale Blasenwandgeschwindigkeit ist nahezu identisch, der Kollaps dauert nur etwas länger. Große Unterschiede zeigt die erreichte Durchschnittstemperatur: Bis ca. 350 ps vor $t_{R m i n}$ verläuft die entsprechende Kurve deckungsgleich, dann, mit Einsetzen der chemischen Reaktion im ersten Fall, steigen die Temperaturen deutlich weniger stark an, verglichen mit Fall 2. Das Maximum beträgt ohne chemische Reaktionen ca. $15.100 \mathrm{~K}$, mit chemischen Reaktionen sind es lediglich ca. $11.450 \mathrm{~K}$. Dabei erreicht die Blase ohne chemische Reaktionen das Temperaturmaximum etwas weiter vor $t_{\text {Rmin }}$ (ca. 20 ps) als die Vergleichsblase. Dies dürfte durch den größeren Minimalradius zu erklären sein.

Der Druck an der Blasenwand $P_{W}$ ist bei beiden Rechnungen vergleichbar groß. Dies liegt vermutlich daran, daß die Teilchen nahe der Blasenwand, die $P_{W}$ maßgeblich bestimmen, nur in sehr geringem Maße chemisch reagieren.
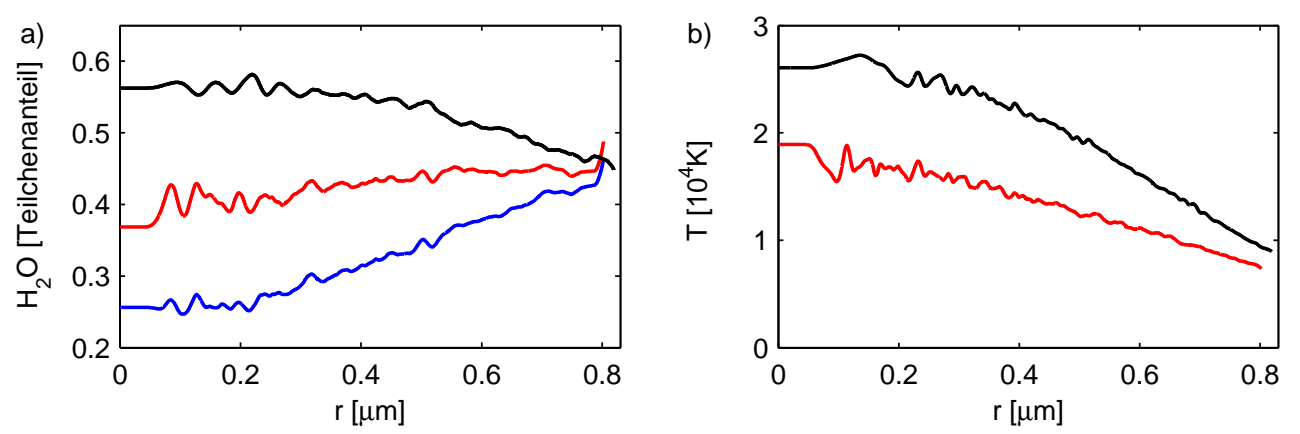

Abbildung 4.26: (a): Teilchenanteil von Wasser zum Zeitpunkt der maximalen Blasentemperatur für Rechnungen ohne chemische Reaktionen (-) und mit chemischen Reaktionen ( - : $\left.N_{\mathrm{H}_{2} \mathrm{O}} /\left(N_{\mathrm{H}_{2} \mathrm{O}}+N_{\mathrm{Ar}}\right),-: N_{\mathrm{H}_{2} \mathrm{O}} / N_{\text {ges }}\right)$

(b): Temperatur in der Blase zum Zeitpunkt der maximalen Blasentemperatur für Rechnungen ohne chemische Reaktionen (-) und mit chemische Reaktionen (-). Parameter siehe Tab. 4.10.

In Abb. 4.26 ist der Teilchenanteil von $\mathrm{H}_{2} \mathrm{O}$ sowie die Temperatur als radiale Funktion zum Zeitpunkt der maximalen Blasentemperatur für beide betrachteten Fälle dargestellt. Für die Rechnung mit chemischen Reaktionen ist das Teilchenanzahlverhältnis sowohl in Bezug auf lediglich das Edelgas, als auch in Bezug auf die Gesamtteilchenzahl, also inklusive aller entstehenden Spezies, dargestellt. Während in der Blase ohne chemische Reaktionen Wasserdampf die häufigste Spezies im Teilchenzentrum zum betrachteten Zeitpunkt darstellt, ist dies bei zusätzlich simulierten chemischen Reaktionen nicht mehr der Fall: Die $\mathrm{H}_{2} \mathrm{O}$ Anzahl wird umso stärker verringert, je dichter man dem Blasenzentrum kommt. Argon kommt nun häufiger vor als Wasserdampf. Wenn man auch die anderen 
Spezies betrachtet, ist nur noch ca. jedes vierte Teilchen im Zentrum ein Wasserdampfteilchen. Am Blasenrand sind praktische keine Unterschiede zwischen den Rechnungen zu erkennen.

Die Temperatur in der Blase fällt für beide Fälle nahezu linear zum Blasenrand hin ab. Dort sind die Unterschiede gering, aber vorhanden (ca. $7.500 \mathrm{~K}$ vs. ca. 9.000 K). Im Fall ohne chemische Reaktionen steigt die Temperatur stärker an. Im Maximum werden 27.200 K erreicht, in der Vergleichsrechnung nur ca. $18.900 \mathrm{~K}$.

\subsubsection{Langzeitentwicklung}

Es ist von Interesse, was mit entstehenden Spezies passiert, wenn die Blase über mehrere Zyklen oszilliert und so mehrere Phasen der chemischen Produktion durchläuft. Akkumulieren gewisse Spezies über die Zeit oder werden sie im Laufe der Zeit abgebaut? Gibt es ein Gleichgewicht, ab dem bei einem Kollaps keine Netto-Zunahme an chemischen Produkten mehr zu beobachten ist? Welcher Anteil der Spezies gelangt in die Flüssigkeit?

Die Antworten auf diese Fragen hängen stark mit der Stabilität der Blase und der Kondensationswahrscheinlichkeit der einzelnen Spezies zusammen. Bildet die Blase gegen Ende des Kollaps Jets aus, oder wird sie sogar teilweise zerstört, so werden bedeutend mehr reaktive Spezies von der Flüssigkeit aufgenommen, als wenn die Blase eine sphärische Geometrie beibehält. Geht man davon aus, dass kein Material gegen Ende des Kollaps aus der Blase 'gespült' wird, ist die Entwicklung der Anzahl von Spezies $X$ abhängig von der Möglichkeit der Kondensation an der Blasenwand - in der Simulation also von dem Akkomodationskoeffizienten $\alpha_{X}$.

Die Bildung von Jets oder eine etwaige Zerstörung der Blase kann in diesem Modell nicht erfaßt werden. Hier soll untersucht werden, welche Auswirkung verschiedene Werte von $\alpha_{X}$ auf den Inhalt des Blaseninneren haben. Eine genaue Bestimmung von $\alpha_{X}$ ist schwierig und konnte bisher nur für einzelne Spezies -

\begin{tabular}{ccc|ccc|ccc}
\hline$P_{a}$ & - & $1,3 \mathrm{bar}$ & $N_{N G}$ & - & 3.908 & $v_{W, \text { init }}$ & - & $25 \mathrm{~m} / \mathrm{s}$ \\
$\nu_{a}$ & - & $26,5 \mathrm{kHz}$ & $S$ & - & $\approx 3.000 .000$ & $\alpha_{t}$ & - & 0,3 \\
$R_{0}$ & - & $4,5 \mu \mathrm{m}$ & Edelgas & - & Argon & $\alpha_{v}$ & - & 0,1 \\
$T_{\text {water }}$ & - & $300 \mathrm{~K}$ & $\Delta K_{W}$ & - & 70 & & & \\
\hline
\end{tabular}

Tabelle 4.12: Parameter der Langzeit-Rechnungen von Blasen mit Wasserdampf und chemischen Reaktionen. 

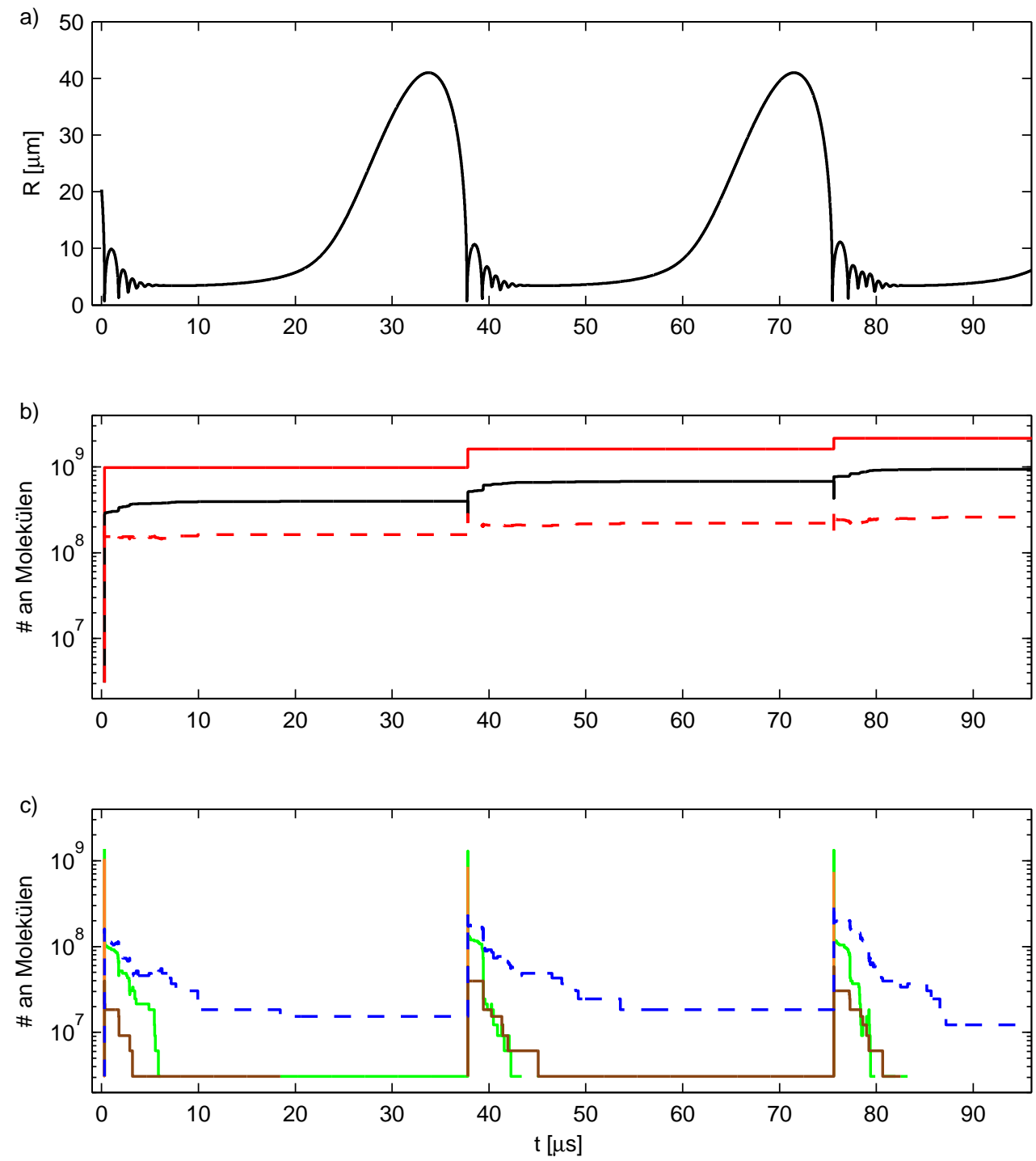

Abbildung 4.27: Langzeitentwicklung des Blasenradius (a) und der Anzahl der gebildeten Spezies für $\alpha_{X}=0(\mathrm{~b}, \mathrm{c})$. Parameter siehe Tab. 4.12.

Legende - (b): $\mathrm{H}_{2}(-), \mathrm{O}_{2}(-), \mathrm{H}_{2} \mathrm{O}_{2}$ (- -); (c): $\mathrm{OH}(-), \mathrm{H}(-), \mathrm{O}(-), \mathrm{HO}_{2}(--)$.

und auch dort nur mit einem großen Fehler behaftet - durchgeführt werden. Aus Experimenten und Simulationen kann für das OH-Radikal ein Wert von $\alpha_{O H}=$ 0,001 bis $\alpha_{O H}=0,01$ geschätzt werden [122][123].

Um ein Bild von der Langzeitentwicklung des Blaseninneren zu erhalten, wurde eine Blase unter SBSL-Bedingungen für ca. $100 \mu$ s simuliert. Bei einer Anregungsfrequenz von $\nu_{a}=26,5 \mathrm{kHz}$ treten in diesem Zeitraum drei Hauptkollapse auf. Die Rechnung wurde für verschiedene Werte des Akkomodationskoeffizienten $\alpha_{X}$ 
Kap. 4 Einfluß der Parameter des MD-Modells

\begin{tabular}{lllllll} 
Spezies & $N_{X} \mathrm{~K} 1\left[10^{8}\right]$ & $N_{X} \mathrm{~K} 2\left[10^{8}\right]$ & $N_{X} \mathrm{~K} 3\left[10^{8}\right]$ & $N_{K ; 0,01}\left[10^{7}\right]$ & $N_{K ; 0,001}\left[10^{7}\right]$ & $N_{r ; 0,001}\left[10^{7}\right]$ \\
\hline $\mathrm{H}$ & 10,11 & 8,52 & 7,43 & 4,3 & 0,3 & 0,0 \\
$\mathrm{H}_{2}$ & 9,85 & 6,20 & 5,50 & 93,2 & 101,0 & 0,0 \\
$\mathrm{O}$ & 0,40 & 0,58 & 0,58 & 2,8 & 0,3 & 0,0 \\
$\mathrm{O}_{2}$ & 3,95 & 2,80 & 2,60 & 26,9 & 32,1 & 5,8 \\
$\mathrm{OH}$ & 13,5 & 12,9 & 13,2 & 24,2 & 1,5 & 0,0 \\
$\mathrm{HO}_{2}$ & 1,62 & 0,58 & 0,39 & 9,2 & 4,3 & 0,6 \\
$\mathrm{H}_{2} \mathrm{O}_{2}$ & 1,52 & 2,32 & 3,37 & 19,0 & 14,1 & 2,8 \\
\hline
\end{tabular}

Tabelle 4.13: Ergebnisse der Langzeitbetrachtung der Blasen aus Tab. 4.12, $N_{X}$ K1/K2/K3 geben die im ersten/zweiten/dritten Kollaps gebildete Anzahl der jeweiligen Spezies an, $N_{K ; 0,01}$ und $N_{K ; 0,001}$ geben die Anzahl der im Anschluß an den ersten Kollaps (bis ungefähr t=30 $\mu \mathrm{s}$ ) aus der Blase kondensierten Teilchen für $\alpha_{X}=0,01$, bzw. $\alpha_{X}=0,001$ an. $N_{r ; 0,001}$ steht für die Anzahl der in der Blase verbleibenden Teilchen bei $\alpha_{X}=0,001$ (bis ungefähr $\mathrm{t}=30 \mu \mathrm{s}$ ).

durchgeführt $\left(\alpha_{X}=0 ; \alpha_{X}=0,01 ; \alpha_{X}=0,001\right)$. Die Transferwahrscheinlichkeit des Wasserdampfes wird nach wie vor über den $\mathrm{H}_{2} \mathrm{O}$ - Akkomodationskoeffizienten $\alpha_{v}$ und (4.11) berechnet, für Argon ist sie Null. Bei einer derartigen Rechnung über mehrere Anregungsperioden können nur relativ wenige Teilchen simuliert werden, ansonsten würde die Rechnung zu lange dauern. Der Skalierungsfaktor $S$ ist somit sehr hoch, was zum Teil auftretende Unterschiede zu den Werten im letzten Kapitel erklärt. Die Parameter der Rechnung sind in Tab. 4.12 angegeben.

Abb. 4.27 zeigt die Entwicklung des Blasenradius sowie der Teilchenanzahl aller entstehenden Spezies für den Fall $\alpha_{X}=0$. Die Spezies teilen sich in zwei Klassen, von denen die eine $\left(\mathrm{H}_{2}, \mathrm{O}_{2}\right.$ und $\left.\mathrm{H}_{2} \mathrm{O}_{2}\right)$ in der heißen Kollapsphase entsteht und in den langen Phasen der Nachschwinger und des Aufschwingens der Blase in konstanter Anzahl in der Blase erhalten bleiben. Die andere Klasse (OH, H, O und $\mathrm{HO}_{2}$ ) wird noch während der heißen Phase zu einem Großteil wieder umgesetzt, ist in der Phase der Nachschwinger nur noch in geringer Anzahl vorhanden und verschwindet während des Aufschwingens nahezu vollständig aus der Blase (siehe auch voriges Kapitel, Abb. 4.21).

Die Bildung der langlebigen Spezies verringert sich von Hauptkollaps zu Hauptkollaps. Beim ersten Kollaps werden noch 9,8·10 $\mathrm{H}_{2}$ - Moleküle gebildet, beim zweiten sind es nur noch $6,2 \cdot 10^{8}$, beim dritten schließlich $5,5 \cdot 10^{8}$ (siehe auch Tab. 4.13). Dies läßt auf einen gewissen Sättigungseffekt schließen. Bei $\mathrm{O}_{2}$ und $\mathrm{HO}_{2}$ sind ähnliche Entwicklungen zu beobachten. Die kurzlebigen Spezies verhalten sich hingegen uneinheitlich: Während sich die Produktion von $\mathrm{OH}$ zwischen den Kollapsen nicht sonderlich ändert, wird $\mathrm{H}$ weniger produziert - $\mathrm{O}$ und $\mathrm{H}_{2} \mathrm{O}_{2}$ zeigen hingegen eine aufsteigende Tendenz. Es ist zu vermuten, dass diese Ergebnisse 
von der geringen Teilchenzahl beeinflußt sind und größeren Schwankungen je nach genauer Anfangsbedingung unterliegen. Die geringe Teilchenzahl ist gut an dem bei niedrigen Werten treppenförmigen Verlauf der Kurven in Abb. 4.27- $4.29 \mathrm{zu}$ erkennen, der deutlich macht, dass die Werte hier von einzelnen Simulationsteilchen abhängen.
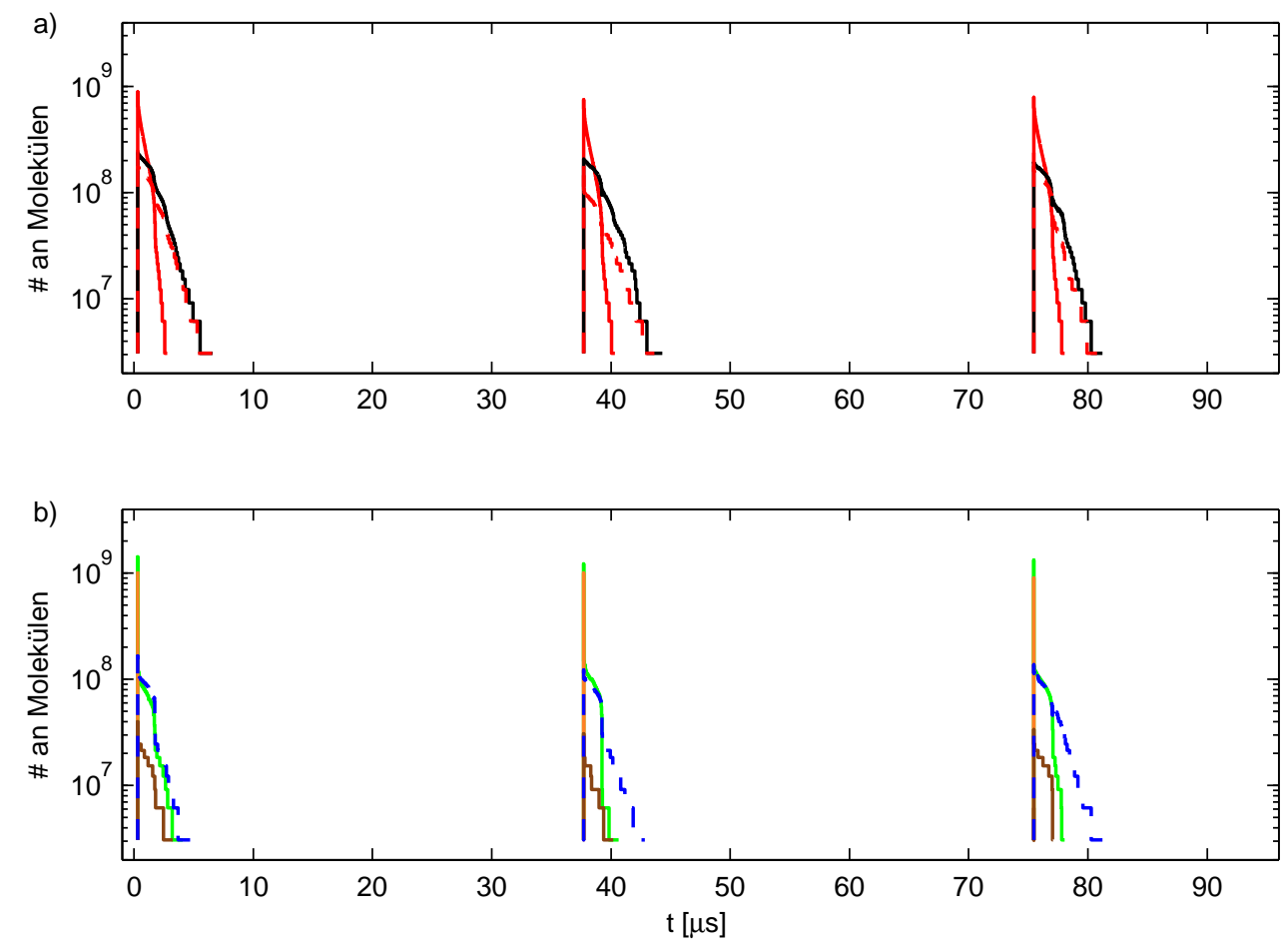

Abbildung 4.28: Langzeitentwicklung der Anzahl der gebildeten Spezies für $\alpha_{X}=0,01$. Parameter siehe Tab. 4.12.

Legende - (a): $\mathrm{H}_{2}(-), \mathrm{O}_{2}(-), \mathrm{H}_{2} \mathrm{O}_{2}(--)$; (b): $\mathrm{OH}(-), \mathrm{H}(-), \mathrm{O}(-), \mathrm{HO}_{2}(--)$.

Abb. 4.28 zeigt die Teilchenanzahl der gebildeten Spezies bei einer Rechnung mit $\alpha_{X}=0,01$ für alle Spezies. Die anderen Parameter und das dargestellte Zeitintervall sind identisch zu Abb. 4.27, auf eine Darstellung des Radiusverlauf wird daher verzichtet.

Es wird deutlich, dass für einen solchen Wert von $\alpha_{X}$ sämtliche im Kollaps gebildeten Spezies die Blase verlassen. Dies geschieht relativ schnell, schon mit Abklingen der Nachschwinger ist der Prozeß abgeschlossen. Die Geschwindigkeit der Kondensation an der Blasenwand richtet sich stark nach dem Gewicht - und damit der Geschwindigkeit - der Teilchen. H ist unmittelbar nach dem Kollaps komplett abgebaut, $\mathrm{H}_{2}$ nach ca. $2 \mu$ s. Obwohl $\mathrm{O}_{2}$ direkt nach Ende des Kollaps im Vergleich zu $\mathrm{H}_{2}$ bedeutend weniger oft vorkommt, sind mehr als $5 \mu$ s für den 
kompletten Abbau notwendig. Dasselbe gilt für $\mathrm{H}_{2} \mathrm{O}_{2}$.

Abb. 4.29 zeigt eine Rechnung derselben Blase, nun mit $\alpha_{X}=0,001$ für alle Spezies. Die auf ein Zehntel verkleinerte Kondensationswahrscheinlichkeit bewirkt deutliche Unterschiede in der Zusammensetzung der Blase über längere Zeiträume. Der Abbau der stabilen Spezies erfolgt deutlich langsamer, $\mathrm{H}_{2}$ ist nun erst nach ca. $15 \mu$ s vollständig verschwunden. Die schwereren Spezies wie $\mathrm{O}_{2}$ und $\mathrm{H}_{2} \mathrm{O}_{2}$ kondensieren zu langsam, um während einer Anregungsperiode komplett abgebaut zu werden, es verbleibt ein Rest bis zum darauffolgenden Kollaps (siehe auch Tab. 4.13). Die kurzlebigen Spezies H, O und $\mathrm{OH}$ werden wie im letzten Fall schnell abgebaut. $\mathrm{HO}_{2}$ verbleibt aufgrund seines hohen Gewichtes in geringem Maße in der Blase.
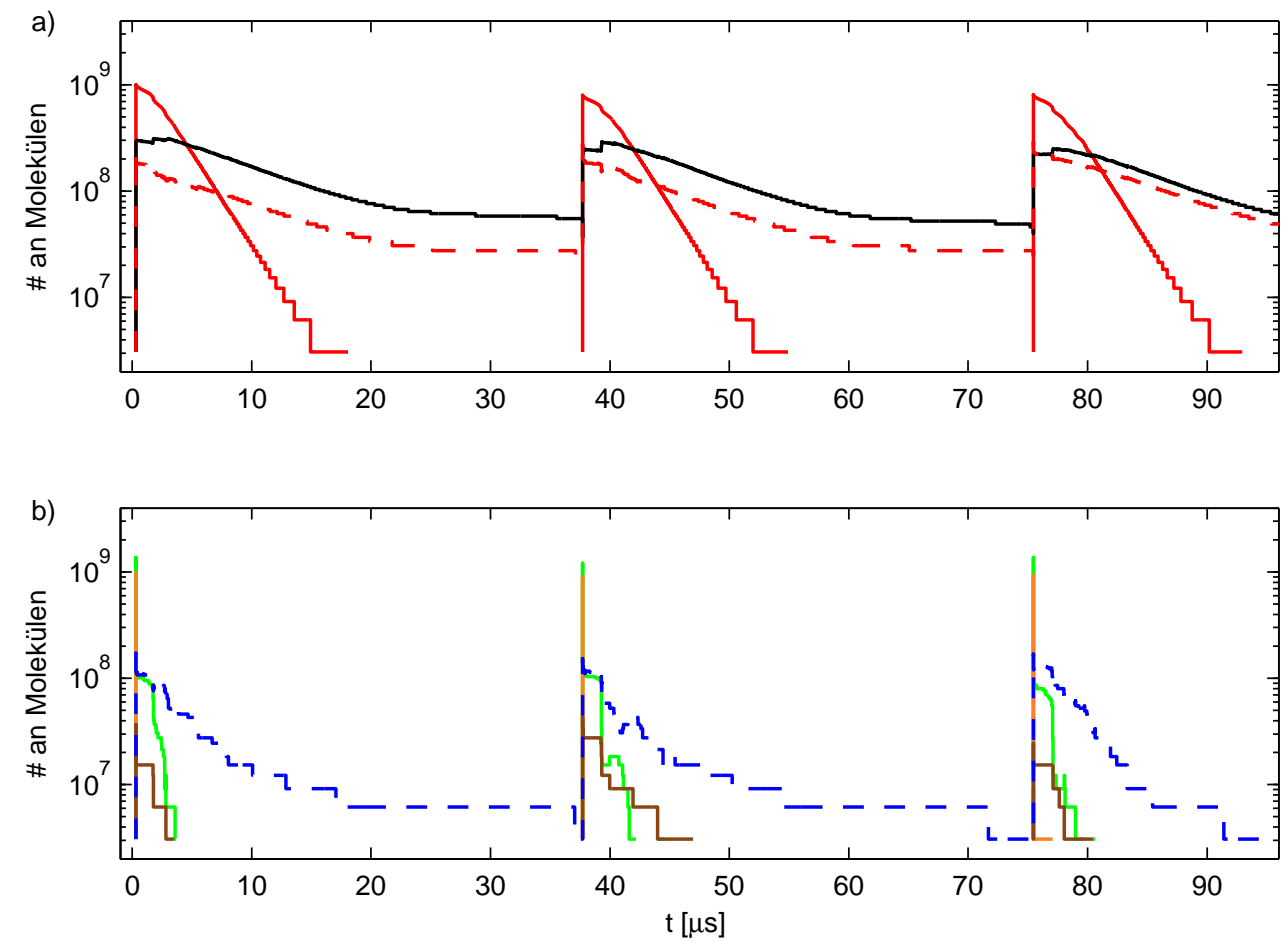

Abbildung 4.29: Langzeitentwicklung der Anzahl der gebildeten Spezies für $\alpha_{X}=0,001$. Parameter siehe Tab. 4.12,

Legende: - (a): $\mathrm{H}_{2}(-), \mathrm{O}_{2}(-), \mathrm{H}_{2} \mathrm{O}_{2}(--)$; (b): $\mathrm{OH}(-), \mathrm{H}(-), \mathrm{O}(-), \mathrm{HO}_{2}(--)$.

Abb. 4.30 zeigt die aus den beiden Werten von $\alpha_{X}$ resultierende Kondensationsanzahl für alle Spezies. Für die stabilen Spezies $\mathrm{O}_{2}$ und $\mathrm{H}_{2}$ ergibt sich mit sinkendem $\alpha_{X}$ interessanterweise eine leicht ansteigende Tendenz der kondensierten Teilchen (besser zu erkennen in Tab. 4.13). Bei $\alpha_{X}=0,01$ kondensieren $93,2 \cdot 10^{7} \mathrm{H}_{2}$-Teilchen, bei $\alpha_{X}=0,001$ sind es $101,0 \cdot 10^{7}$. Für $\mathrm{O}_{2}$-Teilchen ergeben 
sich $26,9 \cdot 10^{7}$ gegenüber $32,1 \cdot 10^{7}$, obwohl weitere $5,8 \cdot 10^{7}$ Teilchen in der Blase verbleiben. Diese Trends erklären sich mit Blick auf die reaktiveren Spezies (siehe unten). Insgesamt ist bei den relativ stabilen Spezies für beide $\alpha_{X}$-Werte eine ähnliche Entwicklung zu beobachten, lediglich die Geschwindigkeit, mit der das jeweilige Niveau erreicht wird, verlangsamt sich mit sinkendem $\alpha_{X}$.
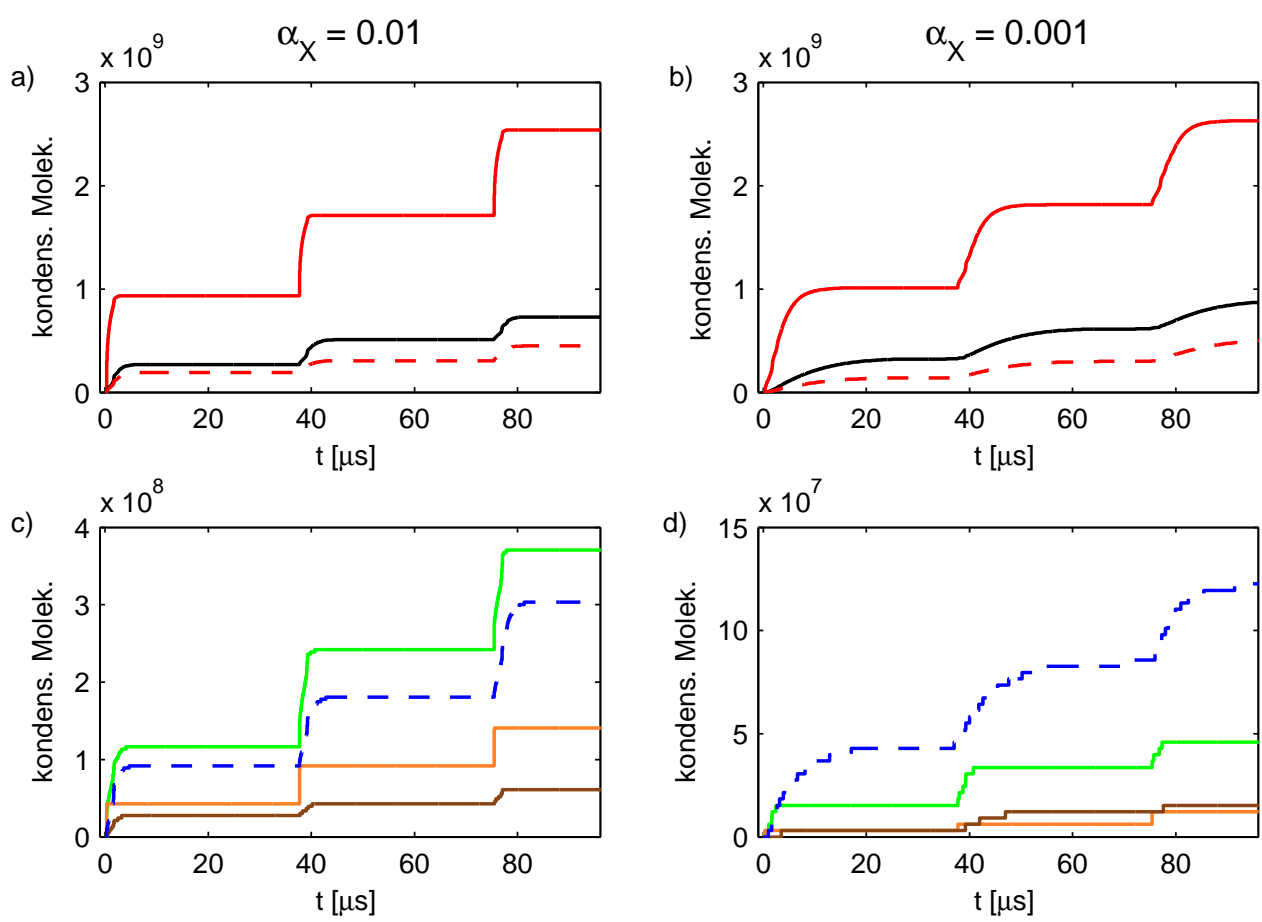

\begin{abstract}
Abbildung 4.30: Entwicklung der Anzahl der an der Blasenwand kondensierten Teilchen aller Spezies für $\alpha_{X}=0,01$ (linke Spalte, (a) und (c)) sowie $\alpha_{X}=0.001$ (rechte Spalte, (b) und (d)). Parameter siehe Tab. 4.12,

Legende: $\mathrm{H}_{2}(-), \mathrm{O}_{2}(-), \mathrm{H}_{2} \mathrm{O}_{2}(--) ; \mathrm{OH}(-), \mathrm{H}(-), \mathrm{O}(-), \mathrm{HO}_{2}(--)$.
\end{abstract}

Bei den reaktiven Verbindungen in der unteren Spalte zeigt sich ein anderes Bild (man beachte die unterschiedlichen Skalierungen): je größer $\alpha_{X}$, desto mehr dieser Teilchen schaffen es, die Blase zu verlassen. Bei $\alpha_{X}=0,01$ kondensieren nach dem ersten Kollaps ca. 9,2 $10^{7} \mathrm{HO}_{2}$-Teilchen an der Blasenwand, bei $\alpha_{X}=0,001$ sind es nur 4,3 $10^{7}$. Ausgeprägter ist dieser Effekt bei $\mathrm{OH}: 11,6 \cdot 10^{7}$ kondensierte Teilchen bei $\alpha_{X}=0,01$ stehen hier $1,5 \cdot 10^{7}$ bei $\alpha_{X}=0,001$ gegenüber. Für $H$ und $\mathrm{O}$ sind noch größere Verhältnisse zu beobachten.

Die größere Reaktivität von $\mathrm{OH}$ im Vergleich zu $\mathrm{HO}_{2}$ erklärt das sich umkehrende Verhältnis der Anzahl der kondensierten Moleküle dieser Spezies beim Übergang $\mathrm{zu} \alpha_{X}=0,001$ (grüne und blau gestrichelte Linie in Abb. 4.30, c und 4.30, d): 
$\mathrm{OH}$ ist von der länger dauerenden Kondensation mehr betroffen, da es schneller umgesetzt wird und somit im Verhältnis weniger kondensieren kann.

Insgesamt läßt sich sagen, dass der Effekt von $\alpha_{X}$ umso größer wird, je reaktiver die betrachtete Spezies ist: je höher die Reaktionswahrscheinlichkeit, desto niedriger die Wahrscheinlichkeit eines Teilchens, mehrere Wandkollisionen zu erfahren. Daher ist die Wahrscheinlichkeit, bei einer der wenigen vorkommenden Wandkollisionen zu kondensieren, entscheidend für die Anzahl der kondensierten Teilchen. Bei niederigem $\alpha_{X}$ werden mehr Teilchen durch Reaktionen abgebaut. Dies erklärt auch die zunehmenden Kondensationswerte für $\mathrm{O}_{2}$ und $\mathrm{H}_{2}$ : Da weniger reaktive Spezies die Blase verlassen, stehen diese für chemische Rekationen zur Verfügung, bei denen die genannten Spezies gebildet werden. Diese können wiederum vermehrt die Blase verlassen.

Eine Abschätzung, wieviele OH-Radikale in die Flüssigkeit gelangen, ist vom Mechanismus des Austritts abhängig. Bei einer angenommenen Zerstörung der Blase läge das theoretische Maximum bei ca. 1,5·10 ${ }^{9}$ Teilchen, dem Maximum der OH-Teilchenzahl. Findet keine Zerstörung statt und der Austritt der Teilchen geschieht über Kondensation, liegt die Zahl - je nach Akkomodationskoeffizient in der Größenordnung $10^{7}$ bis $10^{8}$. Diese Zahlen liegen im groben Bereich anderer Veröffentlichungen, die allerdings unterschiedliche Blasenparameter verwenden [28][38][124]. 


\subsection{Genauigkeit der Approximation}

Die hier zumeist untersuchten Sonolumineszenzblasen mit einem Ruheradius von 4,5 $\mu \mathrm{m}$ enthalten in der Realität eine Anzahl an Edelgasatomen in der Größenordnung von $10^{11}$ und zum Zeitpunkt des maximalen Blasenradius noch eine erheblich größere Menge an Wasserdampfteilchen. Mit aktuellen Computern ist die Simulation einer der dem Experiment entsprechenden Menge an Teilchen nicht möglich. Daher werden in der hier vorliegenden Simulation Teilchen verwendet, die für einen kompletten Teilchenverbund in der Realität stehen. Die Anzahl der realen Teilchen, die ein Simulationsteilchen darstellt ist der Skalierungsfaktor $S$ der Simulation (siehe Kap. 3.2.3). Da z.B. diffusive Effekte von $S$ abhängen (Faktor: $S^{1 / 3}$ ) ist eine Untersuchung des Skalierungsverhalten wichtiger Blasenparameter mit der verwendeten Teilchenanzahl unerläßlich. So kann zum einen der Fehler abgeschätzt werden, der durch die Skalierung unweigerlich eingeführt wird. Zum anderen läßt sich - je nach Ziel der Rechnung - eine untere Grenze der Teilchenzahl finden, unter der die Ergebnisse zu ungenau werden, um aussagekräftig zu sein.

\subsubsection{Einfluß der Simulationsteilchenanzahl}

\begin{tabular}{ccc|ccc|ccc}
\hline$P_{a}$ & - & $1,3 \mathrm{bar}$ & $N_{N G}$ & - & variiert & $v_{W, \text { init }}$ & - & $20 \mathrm{~m} / \mathrm{s}$ \\
$\nu_{a}$ & - & $26,5 \mathrm{kHz}$ & $S$ & - & variiert & $\alpha_{t}$ & - & 0,3 \\
$R_{0}$ & - & $4,5 \mu \mathrm{m}$ & Edelgas & - & Argon & $\alpha_{v}$ & - & 0,1 \\
$T_{\text {water }}$ & - & $300 \mathrm{~K}$ & $\Delta K_{W}$ & - & variiert & $\#_{M Z}$ & - & 2.000 \\
\hline
\end{tabular}

Tabelle 4.14: Parameter der Vergleichsrechnungen mit unterschiedlicher Teilchenanzahl. Weitere Angaben in Tab. 4.15.

Es wurden Rechnungen durchgeführt, bei denen die anfängliche Teilchenzahl $N_{\text {ges }, i}$ von ca. $10^{4}$ bis ca. $10^{7}$ variiert wurde. Eine exakt den Zehnerpotenzen entsprechende Anzahl ließ sich aufgrund der gleichmäßigen Anfangsverteilung nicht realisieren. Der Übersicht halber wird im Folgenden die tatsächliche Teilchenzahl (siehe Tab. 4.15) auf die nächste Zehnerpotenz gerundet.

Die Anzahl der Wandkollisionen zwischen zwei Lösungen der Rayleigh-PlessetGleichung $\Delta K_{W}$ wurde $N_{\text {ges }, i}$ entsprechend skaliert. Sämtliche andere Parameter der Simulationen (angegeben in Tab. 4.14), inklusive der Anzahl der Messzellen $\#_{M Z}$, wurden zunächst unverändert gelassen. 

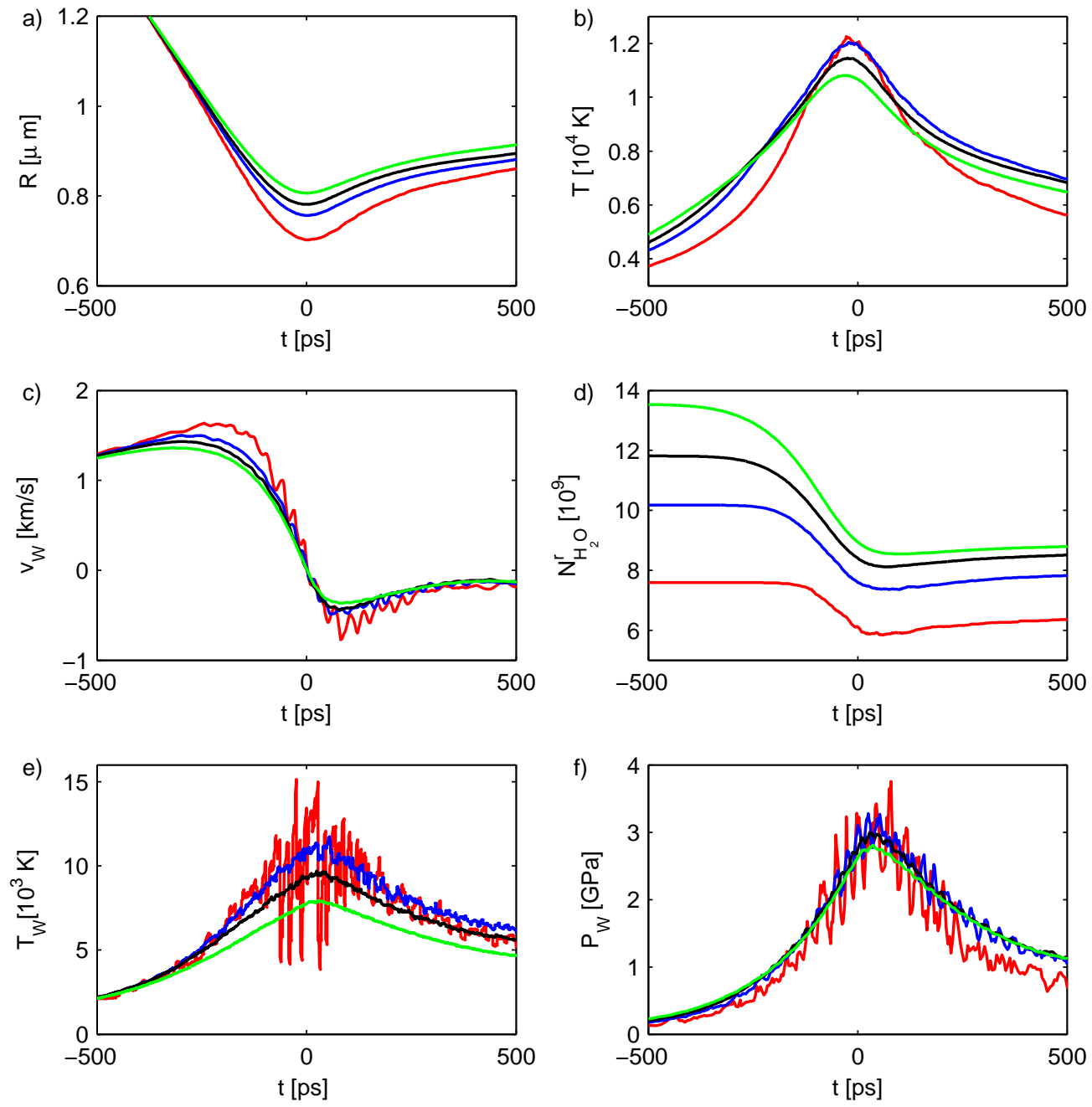

Abbildung 4.31: Entwicklung des Blasenradius (a), der Durchschnittstemperatur (b), der Blasenwandgeschwindigkeit (c), der realen Anzahl der in der Blase befindlichen $\mathrm{H}_{2} \mathrm{O}$-Moleküle (d), der Temperatur an der Blasenwand (e) sowie des Druckes am Blasenrand (f) für die in Tab. 4.14 beschriebenen Rechnungen.

Legende: $N_{g e s, i}=10^{4}(-) ; N_{g e s, i}=10^{5}(-) ; N_{g e s, i}=10^{6}(-) ; N_{g e s, i}=10^{7}(-)$.

Tab. 4.15 gibt Kennzahlen dieser Rechnungen an, in Abb. 4.31 ist die Entwicklung des Blasenradius, der Wandgeschwindigkeit, der durchschnittlichen Temperatur, der Anzahl an Wasserdampfteilchen, der Temperatur an der Blasenwand sowie des Druckes an der Blasenwand gegen Ende des Kollaps dargestellt. Es ist zu erkennen, dass die Blasen mit wachsender Teilchenzahl geringere Wandgeschwindigkeiten $v_{W}$ aufweisen und weniger stark kollabieren. Bei sehr wenigen Teilchen zeigt $v_{W}$ stärkere Oszillationen, die bei Erhöhung von $N_{g e s, i}$ schnell verschwinden.

Von Metten wurde eine Analyse der Teilchenzahlabhängigkeit von Sonolumi- 
neszenzblasen ohne die Simulation von Wasserdampf und chemischen Reaktionen durchgeführt [77]. Dabei zeigte sich, dass mit der Teilchenzahl die Stärke der einlaufenden Verdichtungswelle zunimmt und höhere Temperaturen erreicht werden. Bei den vorliegenden Untersuchungen ist hingegen eine leichte Abnahme der Temperaturen bei Erhöhung der Teilchenzahl zu beobachten. Dies läßt sich mit Blick auf die Anzahl der in der Blase gefangenen Wasserdampfteilchen erklären: Durch die stärkeren Diffusionseffekte gelangen bei geringen Teilchenzahlen im Verhältnis mehr $\mathrm{H}_{2} \mathrm{O}$-Teilchen an die Blasenwand und können dort kondensieren. Während bei $N_{\text {ges }, i}=10^{7}$ gegen Ende des Kollaps noch 13,6 $10^{9}$ Wasserdampfteilchen in der Blase befinden, sind es bei $N_{\text {ges }, i}=10^{4}$ nur noch $7,6 \cdot 10^{9}$. Die Blase mit kleinem Wert von $N_{g e s, i}$ kann also zum einen aufgrund der geringeren Teilchenzahl weiter und stärker kollabieren. Zum anderen wird durch den niedrigeren Wasserdampfanteil die Stoßdynamik weniger herabgesetzt und die Temperaturen in geringerem Maße durch chemische Reaktion vermindert. Diese Effekte kompensieren die stoßfördernde Wirkung einer erhöhten Teilchenzahl und erklären den beobachteten stärkeren Kollaps mit kleinerem Minimalradius und erhöhten Temperaturen bei Blasen mit kleiner Teilchenzahl.

Der Skalierungseffekt der Diffusion läßt sich gut an der Anzahl der Wand- und Teilchenkollisionen beobachten (siehe Tab. 4.15). Die (mit $S$ skalierte) Anzahl der Teilchenkollisionen steigt mit zunehmender Simulationsteilchenzahl stark an. Die Zunahme entspricht in groben Zügen dem Skalierungsfaktor der Diffusion $\left(\left(N_{i} / N_{j}\right)^{1 / 3}\right)$. Durch diesen Effekt wird der Blasenrand zunehmend vor Teilchen aus dem Blaseninneren abgeschirmt, es kommt (skaliert) zu weniger Wandkollisionen, je größer $N_{g e s, i}$.

Die Werte für Druck und Temperatur am Blasenrand (berechnet nach (4.1) und (4.6)) zeigen bei niedriger Teilchenzahl gegen Ende des Kollaps starke Oszillationen. Die (unskaliert) niedrige Anzahl an Wandkollisionen führt zu einer nicht ausreichenden Statistik, welche starke Schwankungen der Messwerte bewirkt. Bei Erhöhung von $N_{\text {ges }, i}$ verschwinden vor allem bei $T_{W}$ die Oszillationen schnell, die maximalen Wandtemperaturwerte sinken mit wachsender Teilchenzahl - vermutlich ein Effekt der abnehmenden Diffusion und Teilchentemperatur.

Insgesamt kann man sagen, dass für globale Parameter - wie die Bewegung des Blasenrandes, den Wanddruck oder gemittelte Werte wie Durchschnittstemperatur und -dichte - ab Teilchenzahlen von $N_{\text {ges }, i}=10^{5}$ akzeptable Ergebnisse erzielt werden. Die Resultate liegen zumeist in einem Bereich von ca. $20 \%$ ober- oder unterhalb derjeniger, die mit $N_{g e s, i}=10^{7}$ - dem momentanen Maximum an in 
Kap. 4 Einfluß der Parameter des MD-Modells

\begin{tabular}{lllll}
\hline Fall & 1 & 2 & 3 & 4 \\
\hline Parameter & & & & \\
$N_{\text {ges }, i}$ & 9.216 & 97.336 & 941.192 & 9.800 .344 \\
$S$ & 6.095 .681 & 579.973 & 59.979 & 5.760 \\
$\Delta K_{W}$ & 50 & 500 & 5.000 & 50.000 \\
$N_{\text {ges }, c}$ & 3.211 & 38.196 & 396.586 & 4.429 .389 \\
\hline Ergebnisse & & & & \\
$\#$ PC $\left[10^{11}\right]$ & 3,32 & 8,64 & 20,63 & 50,52 \\
$\#_{W C}\left[10^{11}\right]$ & 11,09 & 10,09 & 9,35 & 8,99 \\
$T_{\max }[\mathrm{K}]$ & 28.335 & 18.109 & 18.098 & 16.450 \\
$T_{\varnothing}[\mathrm{K}]$ & 12.257 & 12.030 & 11.447 & 10.809 \\
$R_{\min }[\mu \mathrm{m}]$ & 0,702 & 0,756 & 0,781 & 0,807 \\
$v_{W}[\mathrm{~km} / \mathrm{s}]$ & 1,63 & 1,50 & 1,43 & 1,36 \\
$\mathrm{OH}\left[10^{9}\right]$ & 1,30 & 1,60 & 1,54 & 1,41 \\
$\mathrm{H}_{2} \mathrm{O}\left[10^{9}\right]$ & 7,6 & 10,2 & 11,8 & 13,6 \\
\hline
\end{tabular}

Tabelle 4.15: Ergebnisse der Rechnungen unter Verwendung verschiedener Teilchenzahlen bei Start der Simulation. $N_{g e s, c}$ ist die Anzahl an Simulationspartikeln gegen Ende des Kollaps, $\#_{P C}$ die skalierte Gesamtzahl der realen Teilchenkollisionen bis $t_{R m i n}$, \#WC die skalierte Gesamtzahl der realen Wandkollisionen bis $t_{R \min }, T_{\max }$ bezeichnet die absolut größte Temperatur in der Blase, $T_{\varnothing}$ die maximale Durchschnittstemperatur, $R_{\text {min }}$ den minimalen Blasenradius, $v_{W}$ die maximale Blasenwandgeschwindigkeit, $\mathrm{OH}$ die maximale reale $\mathrm{OH}-$ Teilchen-Anzahl in der Blase und $\mathrm{H}_{2} \mathrm{O}$ die Anzahl der in der Blase gefangenen Wasserdampfatome (gemessen kurz vor Einsetzen der chemischen Reaktionen)

angemessener Zeit simulierbarer Teilchen - erzielt werden. Auch für $N_{g e s, i}=10^{4}$ werden Ergebnisse erhalten, die beim Ermitteln von Trends zu Testzwecken gute Dienste leisten. Die dabei auftretenden Oszillation wichtiger Parameter zeigen jedoch, dass Ergebnisse aus Rechnungen mit derart niedriger Teilchenzahl mit Vorsicht zu behandeln und mit einer größeren Teilchenzahl nachzuprüfen sind.

Abb. 4.32 und 4.33 geben einen Einblick in das Innere der Blase. Abb. 4.32 zeigt dabei die Entwicklung der Temperatur, der Dichte, des Druckes sowie der $\mathrm{H}_{2} \mathrm{O}-$ Dichte, jeweils für das Blasenzentrum. Es wird deutlich, dass eine Erhöhung der Simulationsteilchenzahl einen kontinuierlicheren Verlauf der betrachteten Werte bewirkt. Bei $N_{g e s, i}=10^{4}$ springt die Zentrumstemperatur sehr stark hin und her - ein statistischer Effekt des zu kleinen Messvolumens für eine derart geringe Teilchenzahl. Der Zustand einzelner Simulationsteilchen bewirkt hier große

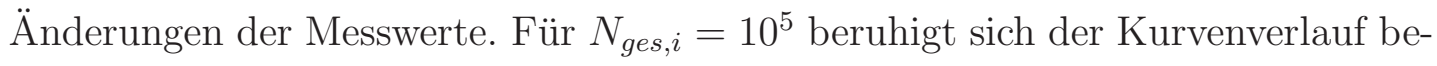
reits deutlich, die beiden anderen Kurven verlaufen glatt und fast deckungsgleich. Das durchschnittliche Niveau der Zentrumstemperatur steigt mit Erhöhung der Teilchenzahl, was auf eine stärkere Fokussierung der Energie hinweist. Die heiße Region schrumpft bei steigendem $N_{g e s, i}$.

Die Dichte im Blasenzentrum verläuft für Teilchenzahlen ab $N_{g e s, i}=10^{5}$ sehr ähnlich, nur in einem Bereich von ca. 200 ps um $T_{R \min }$ zeigt sich, dass die Blase 

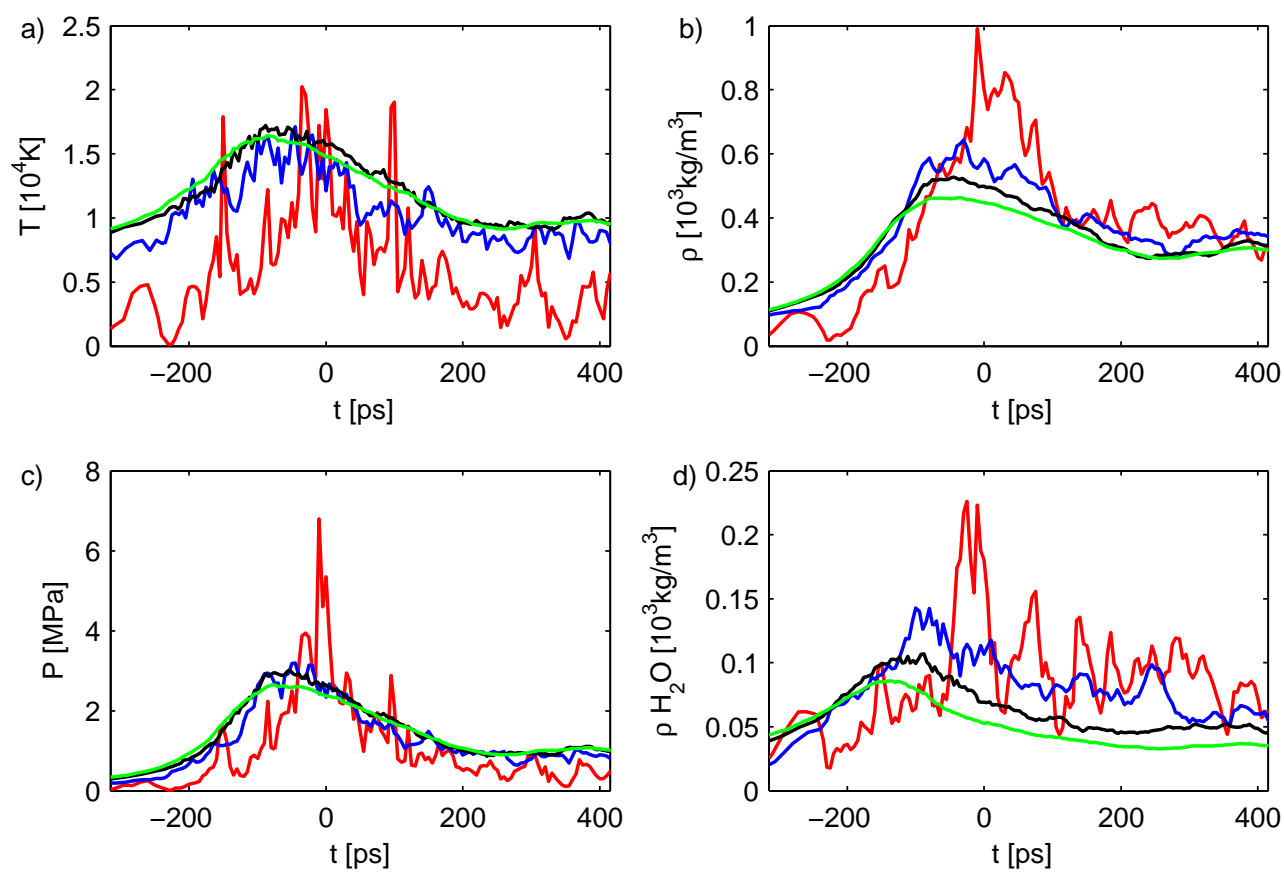

Abbildung 4.32: Entwicklung der Temperatur (a), der Dichte (b) und des Druckes (c) und der Dichte von Wasserdampf (d), jeweils im Blasenzentrum, für die in Tab. 4.14 beschriebenen Rechnungen. Parameter siehe Tab. 4.14.

Legende: $N_{\text {ges }, i}=10^{4}(-) ; N_{\text {ges }, i}=10^{5}(-) ; N_{\text {ges }, i}=10^{6}(-) ; N_{\text {ges }, i}=10^{7}(-)$.

für kleine Teilchenzahlen stärker komprimiert wird und höhere Dichten erreicht werden. In der Dichte des Wasserdampfes zeigt sich dies noch deutlicher, eine Separierung der Kurven stellt sich hier früher ein und dauert auch länger an. Der Druck im Blasenzentrum verläuft, bis auf die starken Schwankungen bei der Rechnung mit $10^{4}$ Teilchen, für alle Rechnungen vergleichbar.

Abb. 4.33 zeigt die raumzeitliche Entwicklung der Temperatur für die vier Rechnungen. Das sehr fleckige Erscheinungsbild von Abb. 4.33, a zeigt erneut die zu große Anzahl an Messzellen für kleine Werte von $N_{\text {ges }, i}$ (mehr dazu am Ende dieses Abschnitts). Die Temperaturverteilung der restlichen Rechnungen zeigt bereits große Ähnlichkeiten. Mit wachsender Teilchenzahl werden die Ergebnisse homogener und die heiße Region schrumpft in gewissem Maße. Das Temperaturverhältnis von Blasenzentrum zu Blasenrand nimmt zu.

Es bleibt festzustellen, dass sich ab $N_{\text {ges }, i}=10^{5}$ Ergebnisse für das Blaseninnere erzielen lassen, die zumindest im Trend richtig liegen. Für eine korrekte Auflösung der Hydrodynamik ist jedoch eine höhere Teilchenzahl empfehlenswert.

Bei noch kleineren Teilchenzahlen läßt sich die Wiedergabe der inneren Dynamik 

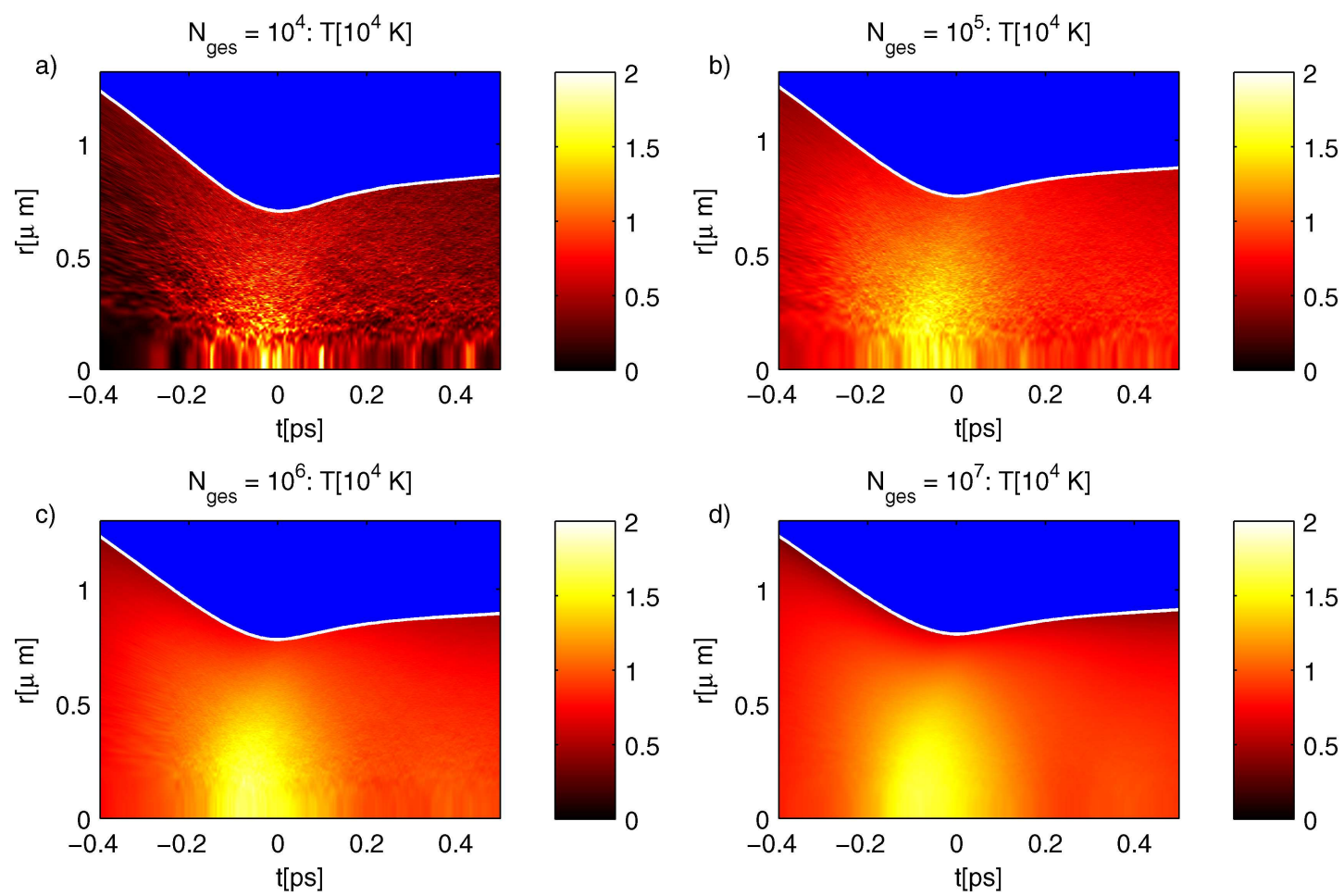

Abbildung 4.33: Raumzeitliche Entwicklung der Temperatur für die in Tab. 4.14 beschriebenen Rechnungen mit verschiedenen Teilchenanzahlen. In gleicher Skalierung dargestellt. (a) weist auch Temperaturen größer als $20.000 \mathrm{~K}$ auf. $\#_{M Z}=2000$ für alle Rechnungen.

verbessern, indem eine Reduzierung der örtlichen Aufösung vorgenommen wird. Für $N_{\text {ges }, i}=10^{4}$ sind im interessierenden Teil des Kollaps nur noch ca. 3.200 Teilchen in der Blase. Die in den soeben vorgestellten Ergebnissen benutzte Anzahl an Messzellen von $\#_{M Z}=2.000$ ist in diesem Fall viel zu hoch, da keine ausreichende statistische Verteilung der Teilchen vorliegt.

Abb. 4.34 zeigt die Zentrumsdichte sowie die raumzeitliche Temperaturverteilung für die Rechnung mit $N_{g e s, i}=10^{4}$, allerdings mit $\#_{M Z}=100$. Die Unterschiede zu den Ergebnissen bei Verwendung von mehr Messzellen (siehe Abb. 4.32,b und 4.33 ,a) sind offensichtlich: Die starken Sprünge in den Messwerten sind verschwunden, die Kurven reihen sich in die Trends der anderen Rechnungen ein. Die Temperaturverteilung ist viel kontinuierlicher und ähnelt bis auf die reduzierte Auflösung derjeniger der anderen Rechnungen. Bei Rechnungen mit $N_{\text {ges }, i}<10^{5}$ erscheint eine Reduktion der räumlichen Auflösung also dringend angebracht.

Beim Blick auf die für unterschiedliche Teilchenzahlen benötigten Rechenzeiten wird offenbar, warum eine Minimierung von $N_{\text {ges }, i}$ für bestimmte Zwecke wün- 

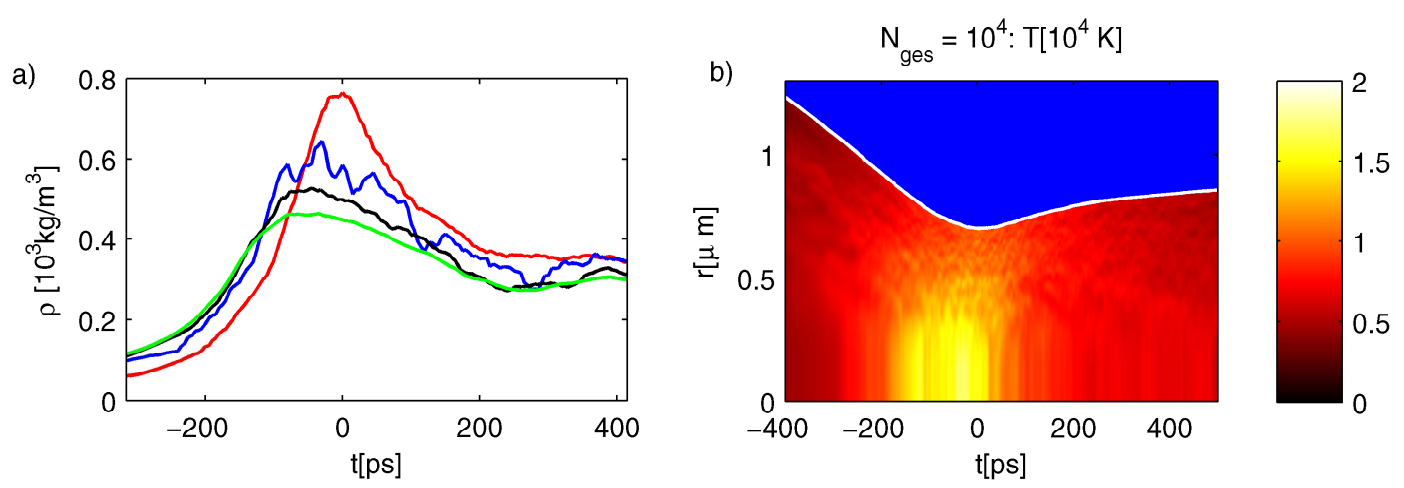

Abbildung 4.34: (a): Entwicklung der Dichte im Blasenzentrum für $N_{g e s, i}=10^{4}(-) ; N_{\text {ges }, i}$ $=10^{5}(-) ; N_{\text {ges }, i}=10^{6}(-) ; N_{\text {ges }, i}=10^{7}(-)$. Dabei gilt $\#_{M Z}=100$ für $N_{g e s, i}=10^{4}$ und $\#_{M Z}=2000$ für alle anderen Rechnungen. (b): Raumzeitliche Entwicklung der Temperatur für $N_{\text {ges }, i}=10^{4}$ mit $\#_{M Z}=100$.

schenswert ist. Die Simulation mit $N_{\text {ges }, i}=10^{4}$ benötigt für die Berechnung des Blasenkollaps - ausgehend von $v_{W, \text { init }}=20 \mathrm{~m} / \mathrm{s}$ bis ca. 1 ns nach $t_{R \min }$ (insgesamt $\approx 0,44 \mu \mathrm{s}$ ) - eine Zeit von ungefähr 30 Minuten. Für $N_{\text {ges }, i}=10^{5}$ dauert dieselbe Rechnung ca. 2 Stunden, bei $N_{\text {ges }, i}=10^{6}$ sind es bereits ca. 36 Stunden und für $N_{g e s, i}=10^{7}$ schließlich mehrere Wochen. Für eine möglichst korrekte Darstellung vor allem der inneren Dynamik ist eine Maximierung der Teilchenzahl natürlich wünschenswert. Liegt der Sinn einer Rechnung eher in der Ermittlung makroskopischer Parameter, kann auch eine geringere Teilchenzahl sinnvoll sein. Sollen längere Phasen der Blasenschwingung berechnet werden (z.B. ein kompletter Zyklus) kommt man kaum über $N_{\text {ges }, i}=10^{5}$ hinaus, ohne inakzeptabel hohe Rechenzeiten in Kauf nehmen zu müssen.

Die Daten zeigen, dass bei der Erhöhung der Teilchenzahl von $N_{g e s, i}=10^{4}$ auf $N_{\text {ges }, i}=10^{7}$ bei vielen Parametern Ergebnisse erzielt werden, deren Abweichungen im Bereich unter 20 Prozent liegen. Beim Übergang zu Blasen, deren Teilchenzahl mit der Realität zu vergleichen ist (in diesem Fall ca. $5 \cdot 10^{10}$ ), wären, dem Trend folgend, Ergebnisse zu erwarten die mit den hier erhaltenen Werten in guter Übereinstimmung liegen.

In Kap. 6.2.1 werden sehr kleine, im MHz-Bereich getriebene Blasen untersucht, die geringe Werte des Skalierungsfaktors erlauben. Es werden Ergebnisse von Blasen mit $S=1, S=10$ und $S=100$ verglichen. 


\section{Kapitel 5}

\section{Ergebnisse für Sonolumineszenzblasen}

In diesem Teil der Arbeit werden Ergebnisse präsentiert, die durch Simulationen von Blasen unter typischen SBSL-Bedingungen gewonnen wurden.

Dabei wird die Abhängigkeit der äußeren und inneren Dynamik sowie der chemischen Entwicklung von mehreren Parametern (Wassertemperatur $T_{\text {water }}$, Anregungsdruck $P_{a}$ und Art des vorkommenden Edelgases) untersucht. 


\subsection{Variation der Wassertemperatur}

Schon früh wurde bei Mehrblasensonolumineszenz (MBSL) beobachtet, dass Änderungen der Flüssigkeitstemperatur zu starken Variationen in der emittierten Photonenzahl führen [125][126]. Später wurde dies auch bei Einzelblasensonolumineszenz (SBSL) nachgewiesen [23][29]. Für nahezu alle Flüssigkeiten zeigt sich bei niedrigen Temperaturen die stärkste Lumineszenz [30].

Als Erklärung dieses Phänomens werden mehrere Effekte herangezogen. VuONG et al. machten die Änderung der Löslichkeit des Edelgases in Wasser (und damit des Gleichgewichtsradius der Blase) bei Temperaturänderung für die beobachteten Schwankungen der Lumineszenz verantwortlich [127]. Wasserdampfeffekte wurden in dem verwendeten Modell allerdings nicht betrachtet . Experimentell fanden VAZQUez und PUtTERMAN eine bis zu 100-fache Verstärkung der Lichtintensität von SBSL-Blasen bei Verminderung der Wassertemperatur $\left(T_{\text {water }}\right)$ [128]. Gleichzeitig ändert sich der Ruheradius kaum, abweichend von den Überlegungen in [127]. Sie schlossen daher auf einen überwiegenden Einfluß von Wasserdampf auf das Lichtentstehungssystem. HiLGenfeld et al. fanden eine Möglichkeit zur weiteren Erhöhung der erzeugten Lichtmenge durch die Anwendbarkeit höherer Anregungsdrücke bei niedrigerer Wassertemperatur [111]. Solche Blasen zeigten eine geringere Neigung zur Fragmentation, was Hilgenfeldt durch ein Anwachsen der Viskosität und der Oberflächenspannung mit dem Sinken von $T_{\text {water }}$ erklärte. YASUI untersuchte den Einfluß von $T_{\text {water }}$ auf MBSL- und SBSL-Blasen anhand eines Kontinuum-Modells mit Phasenübergang und chemischen Reaktionen [64]. Er fand er eine Zunahme von Maximaltemperatur und Lumineszenz mit sinkendem Wasserdampfgehalt bei niedrigen Wassertemperaturen. Yasuis Modell beinhaltet neben einem Plasma-Bremsstrahlungs-Modell ein ChemolumineszenzModell für angeregte OH-Radikale (siehe auch [43]). Bei hoher Anregung sah Yasui eine Zunahme der Chemolumineszenz aufgrund verstärkter OH-Bildung. Plasma-Emission stellte sich allerdings als der dominierende Lichtentstehungsmechanismus heraus.

\section{Parameter}

Es wurde eine Blase unter typischen SBSL-Bedingungen für verschiedene Werte der Wassertemperatur im Intervall von $T_{\text {water }}=277 \mathrm{~K}$ bis $T_{\text {water }}=310 \mathrm{~K}$ simuliert. Eine mögliche Änderung des Gleichgewichtsradius durch veränderte 

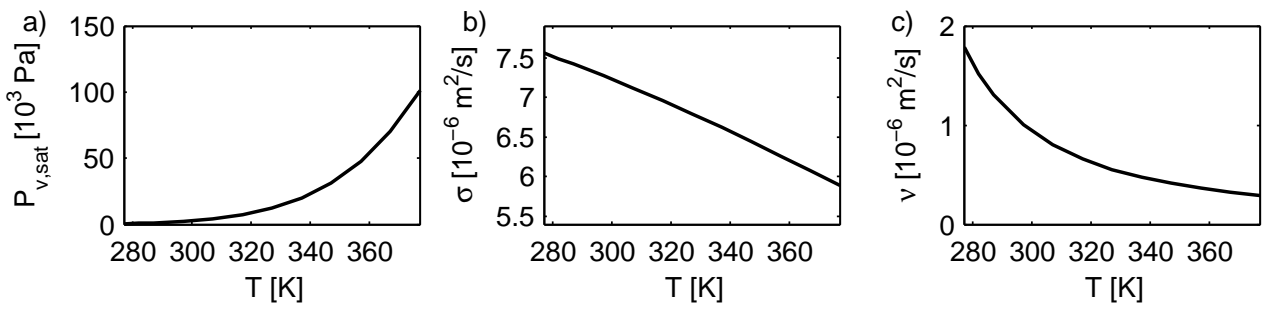

Abbildung 5.1: Verlauf von Sättigungsdampfdruck (a), Oberflächenspannung (b) und Viskosität (c) in Abhängigkeit der Wassertemperatur.

Löslichkeit des Edelgase in Wasser wurde vernachlässigt.

Die Abhängigkeit der simulationsrelevanten Größen Sättigungsdampfdruck $\left(P_{v, s a t}\right)$, Oberflächenspannung $(\sigma)$ und Viskosität $(\nu)$ von $T_{\text {water }}$ ist in Abb. 5.1 dargestellt. $P_{v, \text { sat }}$ steigt exponentiell mit $T_{\text {water }}$ (siehe Gl. (4.12)). Es befinden sich bei Start der Simulation also stark unterschiedliche Mengen an $\mathrm{H}_{2} \mathrm{O}$ in der Blase. Die Abhängigkeit der Kondensationswahrscheinlichkeit (Gl. 4.11) von $P_{v, \text { sat }}$ bewirkt vor allem in den Phasen langsamer Blasenrandgeschwindigkeiten einen Einfluß von $T_{\text {water }}$ auf die Menge des in der Blase verbleibenden Wasserdampfes. Das Absinken von $\sigma$ und $\nu$ bei Erhöhung von $T_{\text {water }}$ führt zu einer geringeren Dämpfung der Blasenoszillation.

Tabelle 5.1 gibt die verwendeten Parameter an. Aufgrund des sehr hohen Wasserdampfanteils für hohe Werte von $T_{\text {water }}$ wurde ein relativ großer Skalierungsfaktor S verwendet. Die experimentelle Beobachtung, dass für niedrigere Werte von $T_{\text {water }}$ die steigende Oberflächenspannung eine stärkere Anregung der Blase erlaubt, ohne diese zu zerstören [111], wurde bewußt nicht beachtet, da hier die reine Auswirkung der Änderung physikalischer Parameter mit $T_{\text {water }}$ dokumentiert werden soll.

\begin{tabular}{|c|c|c|c|c|c|c|c|}
\hline$P_{a}$ & 1,3 bar & $N_{A r, i n i t}$ & - & $\begin{array}{r}216.000 \\
\sim 58000\end{array}$ & $v_{\text {init }}$ & - & $25 \mathrm{~m} / \mathrm{s}$ \\
\hline $\begin{array}{l}\nu a \\
R_{0}\end{array}$ & $\begin{array}{c}26,5 \mathrm{kHz} \\
4,5 \mu \mathrm{m}\end{array}$ & $\begin{array}{c}\mathrm{S} \\
\text { Edelgas }\end{array}$ & - & $\begin{array}{c}\approx 58.000 \\
\text { Argon }\end{array}$ & $\begin{array}{l}\alpha_{t} \\
A_{v}\end{array}$ & - & $\begin{array}{l}0.3 \\
0.3\end{array}$ \\
\hline$T_{\text {water }}$ & $277-333 \mathrm{~K}$ & & & & & & \\
\hline
\end{tabular}

Tabelle 5.1: Parameter der Vergleichsrechnungen von $T_{\text {water }}$.

\section{Ergebnisse}

Abb. 5.2 zeigt den zeitlichen Verlauf des Blasenradius und der Anzahl an Wasserdampfteilchen in der Blase. Tabelle 5.2 fasst verschiedene Ergebnisse zusammen. 
Eine Erhöhung von $T_{\text {water }}$ führt zu einem stärkeren Aufschwingen der Blase, verursacht durch den größeren Dampfdruck des Wassers sowie die geringere Dämpfung der Blasenrandbewegung durch Oberflächenspannung und Viskosität. Bei $T_{\text {water }}=310 \mathrm{~K}$ wird ein Maximalradius $R_{\max }=46,4 \mu \mathrm{m}$ erreicht, während dieser Wert für $T_{\text {water }}=277 \mathrm{~K}$ bei 38,4 $\mu \mathrm{m}$ liegt. Der exponentielle Anstieg des Wasserdampfgehaltes mit $T_{\text {water }}$ zu Anfang der Simulation bleibt bis zum Erreichen von $R_{\text {min }}$ erhalten. Der große Wert von $R_{\max }$ führt bei hoher $T_{\text {water }}$ zunächst zu einer erhöhten Blasenrandgeschwindigkeit. Eine Vergrößerung der Kompression wird aber durch den zunehmend dämpfenden Einfluß des Wasserdampfes verhindert. Die erreichten Minimalradien bleiben bis zu $T_{W} \approx 290 \mathrm{~K}$ näherungsweise konstant. Durch den größeren Dampfdruck bei hohen $T_{\text {water }}$-Werten schwingt die Blase bei den Rebounds deutlich weiter auf.
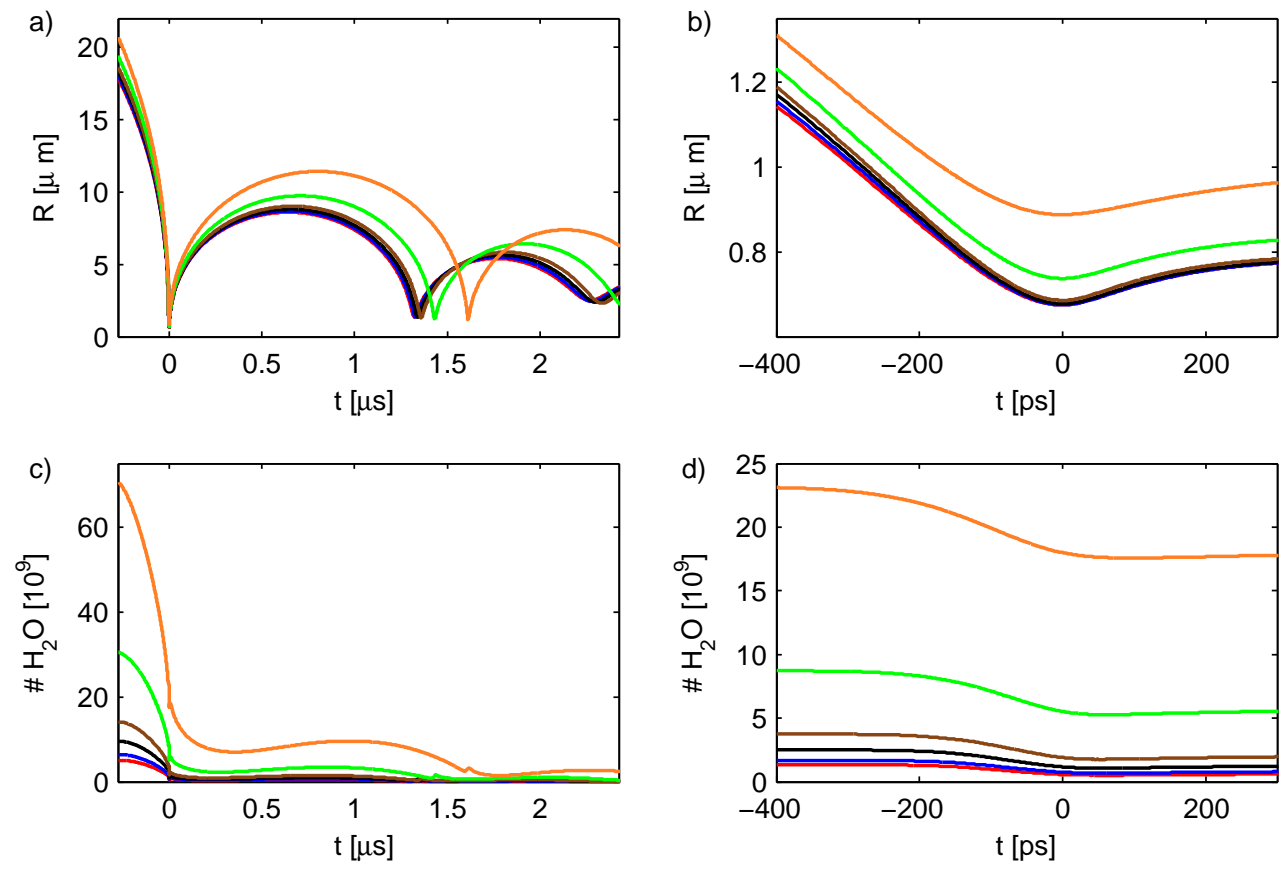

Abbildung 5.2: Radiusverlauf (a,b) und Anzahl an Wasserdampfteilchen (c,d) für die ersten $3 \mu s$ und in der Vergrößerung um den Zeitpunkt des min. Blasenradius $(\mathrm{t}=0)$ für verschiedene Werte von $T_{\text {water }}$. Parameter siehe Tab. 5.1.

Legende: $T_{\text {water }}=277 \mathrm{~K}(-), 280 \mathrm{~K}(-), 285 \mathrm{~K}(-), 290 \mathrm{~K}(-), 300 \mathrm{~K}(-), 310 \mathrm{~K}(-)$.

Abb. 5.3 zeigt die Entwicklung der Temperatur im Blasenzentrum für die Endphase des Kollaps, sowie die Bremsstrahlleistung der gesamten Blase in diesem Zeitraum. Die Rechnungen mit $T_{\text {water }}=277 \mathrm{~K}, 280 \mathrm{~K}$ und $285 \mathrm{~K}$ erreichen vergleichbare Maximaltemperaturen $T_{\max }$ um $27.000 \mathrm{~K}$. Für $T_{\text {water }}=290 \mathrm{~K}$ sinkt 
Kap. 5 Ergebnisse für Sonolumineszenzblasen

\begin{tabular}{lllllll}
\hline$T_{\text {water }}[\mathrm{K}]$ & 277 & 280 & 285 & 290 & 300 & 310 \\
\hline Parameter & & & & & & \\
$\sigma\left[10^{-2} \mathrm{~N} / \mathrm{m}\right]$ & 7,50 & 7,46 & 7,39 & 7,32 & 7,20 & 7,01 \\
$\nu\left[10^{-6} \mathrm{~m}^{2} / \mathrm{s}\right]$ & 1,57 & 1,43 & 1,25 & 1,09 & 0,86 & 0,69 \\
$P_{v, \text { sat }}\left[10^{3} \mathrm{~Pa}\right]$ & 0,78 & 0,97 & 1,37 & 1,89 & 3,51 & 6,20 \\
\hline Dynamik & & & & & & \\
$R_{\text {max }}[\mu \mathrm{m}]$ & 38,4 & 38,8 & 39,5 & 40,5 & 42,5 & 46,4 \\
$R_{\text {min }}[\mu \mathrm{m}]$ & 0,675 & 0,675 & 0,677 & 0,686 & 0,737 & 0,888 \\
$K$ & 56.8 & 57.5 & 58.3 & 59.03 & 57.7 & 52.3 \\
$T_{\text {max }}$ & 27.150 & 27.050 & 26.510 & 24.870 & 20.060 & 15.440 \\
$T_{\varnothing}$ & 14.670 & 14.750 & 14.530 & 14.100 & 12.550 & 10.060 \\
$\mathrm{H}_{2} \mathrm{O}_{\text {init }}\left[10^{9}\right]$ & 5,0 & 6,4 & 9,5 & 14,1 & 30,5 & 72,0 \\
$\mathrm{H}_{2} \mathrm{O}_{\text {coll }}\left[10^{9}\right]$ & 1,34 & 1,69 & 2,51 & 3,75 & 8,72 & 16,0 \\
$\mathrm{H}_{2}\left[10^{9}\right]$ & 0,50 & 0,64 & 0,97 & 1,40 & 2,60 & 4,36 \\
$\mathrm{OH}\left[10^{9}\right]$ & 0,46 & 0,53 & 0,71 & 0,91 & 1,43 & 2,20 \\
$\Delta_{S L}[\mathrm{ps}]$ & 100 & 95 & 95 & 100 & 125 & 185 \\
$d_{S L}[\mu \mathrm{m}]$ & 0,5 & 0,6 & 0,7 & 0,6 & 0,7 & 0,8 \\
$E_{S L}\left[10^{-2} \mathrm{pJ}\right]$ & 7,39 & 7,98 & 8,19 & 7,44 & 3,93 & 1,19 \\
\hline
\end{tabular}

Tabelle 5.2: Auswirkungen der Änderung der Wassertemperatur $T_{W}$ auf die physikalischen Parameter $\sigma, \nu$ und $P_{v, \text { sat }}$ sowie auf Simulationsergebnisse:

$R_{\max }$ ist der maximale Blasenradius, $R_{\min }$ der minimale Blasenradius, $K$ der Kompressionsfaktor, $T_{\max }$ die absolut maximale Temperatur, $T_{\varnothing}$ die maximale über die Blase gemittelte Durchschnittstemperatur, $\mathrm{H}_{2} \mathrm{O}_{\text {init }}$ die Anzahl der Wasserteilchen bei Start der Simulation, $\mathrm{H}_{2} \mathrm{O}_{\text {coll }}$ die Anzahl der Wasserteilchen bei Kollapsende, $\mathrm{H}_{2}$ die Anzahl der gebildeten $\mathrm{H}_{2}$-Moleküle, $\mathrm{OH}$ die maximale Anzahl an OH-Molekülen, $\Delta t_{S L}$ die Halbwertsbreite der Lichtemission, $d_{S L}$ der Durchmesser der Licht emittierenden Region und $E_{S L}$ die gesamt emittierte Lichtenergie.

$T_{\max }$ auf ca. $25.000 \mathrm{~K}$, obwohl dieser Fall die höchste Kompression aufweist. Wird $T_{\text {water }}$ weiter erhöht fällt $T_{\text {max }}$ stark ab, bei $T_{\text {water }}=310 \mathrm{~K}$ werden ca. $15.000 \mathrm{~K}$ erreicht. Ein vermehrter Wasserdampfgehalt führt zu verminderten Temperaturen, da die $\mathrm{H}_{2} \mathrm{O}$ - Dissoziation viel Energie binden kann (siehe Kap. 4.2)
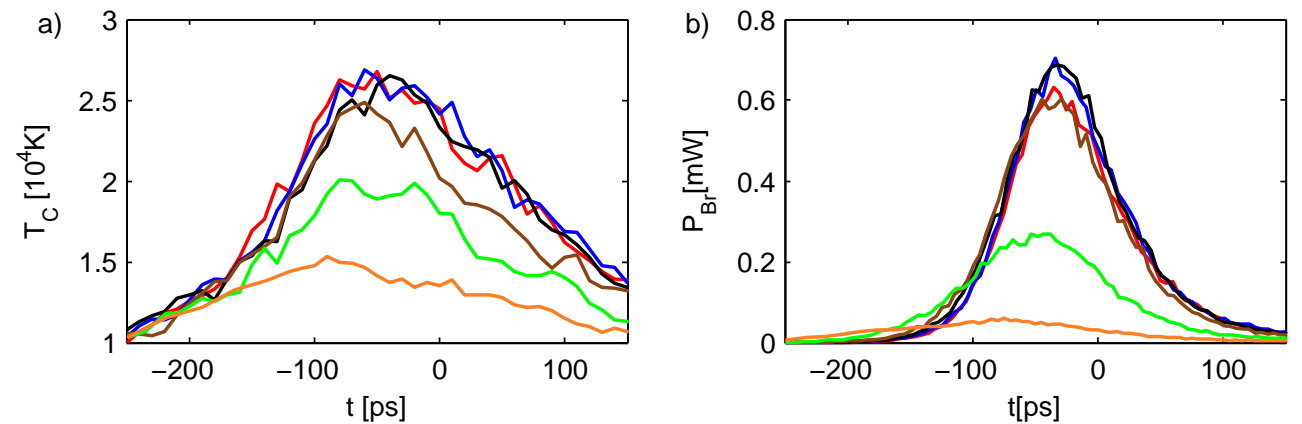

Abbildung 5.3: Temperatur im Blasenzentrum (a) sowie Bremsstrahlleistung der gesamten Blase (b) um den Zeitpunkt des min. Blasenradius $(\mathrm{t}=0)$ für verschiedene Werte von $T_{\text {water }}$. Parameter siehe Tab. 5.1.

Legende: $T_{\text {water }}=277 \mathrm{~K}(-), 280 \mathrm{~K}(-), 285 \mathrm{~K}(-), 290 \mathrm{~K}(-), 300 \mathrm{~K}(-), 310 \mathrm{~K}(-)$. 
Besonders am Fall $T_{\text {water }}=300 \mathrm{~K}$ wird dies deutlich, da hier dieselbe Kompression wie bei $T_{\text {water }}=285 \mathrm{~K}$ erreicht wird, die Temperaturen aber stark herabgesetzt sind. Bis $T_{\text {water }}=285 \mathrm{~K}$ werden die Effekte des Wasserdampfs von der steigenden Kompression kompensiert. Der Zeitpunkt der maximalen Temperatur verschiebt sich mit sinkender $T_{\text {water }}$ in Richtung des Zeitpunkts des minimalen Blasenradius, da $R_{\min }$ abnimmt.

Die Lichtleistung der Blase zeigt im Modell die experimentell beobachtete Abhängigkeit von der Wassertemperatur. Das Maximum der emittierten Lichtenergie $E_{S L}$ liegt zwischen $T_{\text {water }}=280 \mathrm{~K}$ und $T_{\text {water }} 285 \mathrm{~K}$. Für $T_{\text {water }}=277 \mathrm{~K}$ zeigt sich trotz vergleichbarer Temperaturen ein leichter Abfall von $E_{S L}$, da die Größe der emittierenden Region $d_{S L}$ abnimmt. Für $T_{\text {water }}>290 \mathrm{~K}$ verringert sich $E_{S L}$ drastisch. Gleichzeitig nehmen sowohl $d_{S L}$, als auch insbesondere die Dauer der Emission $\Delta t_{S L}$ zu. Die Peak - Leistung sinkt also noch drastischer als die Gesamtenergie. Bei $T_{\text {water }}=310 \mathrm{~K}$ sind die Temperaturen nicht ausreichend, um einen nennenswerten Ionisationsgrad zu erreichen. Exemplarisch zeigt Abb. 5.4 Temperatur und Lichtleistung raumzeilich aufgelöst für den Fall $T_{\text {water }}=285 \mathrm{~K}$.
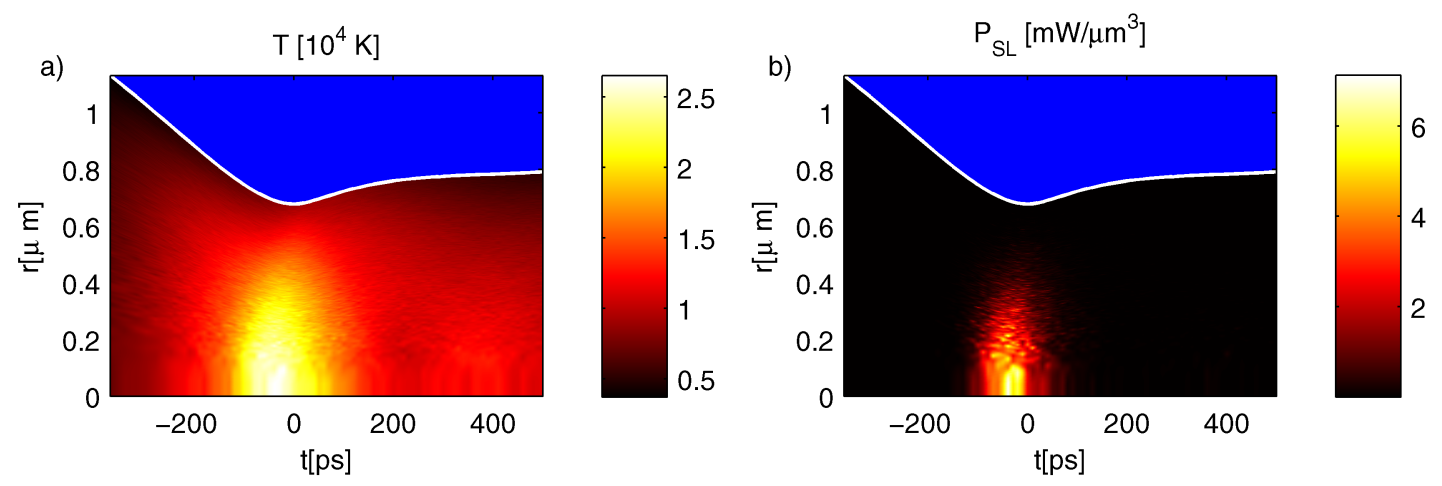

Abbildung 5.4: Temperatur (a) und Lichtleistung (b) für $T_{\text {water }}=285 \mathrm{~K}$.

Die chemische Aktivität der Blase erscheint hauptsächlich an den Wasserdampfgehalt gekoppelt, weniger an die erreichten Maximaltemperaturen. Abb. 5.5, a zeigt die maximale Anzahl an Teilchen der Spezies $\mathrm{H}, \mathrm{H}_{2}, \mathrm{O}_{2}$ und $\mathrm{OH}$, die sich zu irgendeinem Zeitpunkt in der Blase befinden. $\mathrm{H}_{2}$ und $\mathrm{O}_{2}$ sind dabei 'stabile' Spezies, die während der heißen Kollapsphase gebildet werden und danach über längere Zeit in der Blase verbleiben, während $\mathrm{O}$ und $\mathrm{OH}$ schnell weiter reagieren und somit nur für kurze Zeit in nennenswerter Menge vorkommen. Für diese zeigt Abb. 5.5, a die Peak-Werte im Kollaps.

Bei allen betrachteten Spezies zeigt sich eine starke Zunahme der Bildung bei 

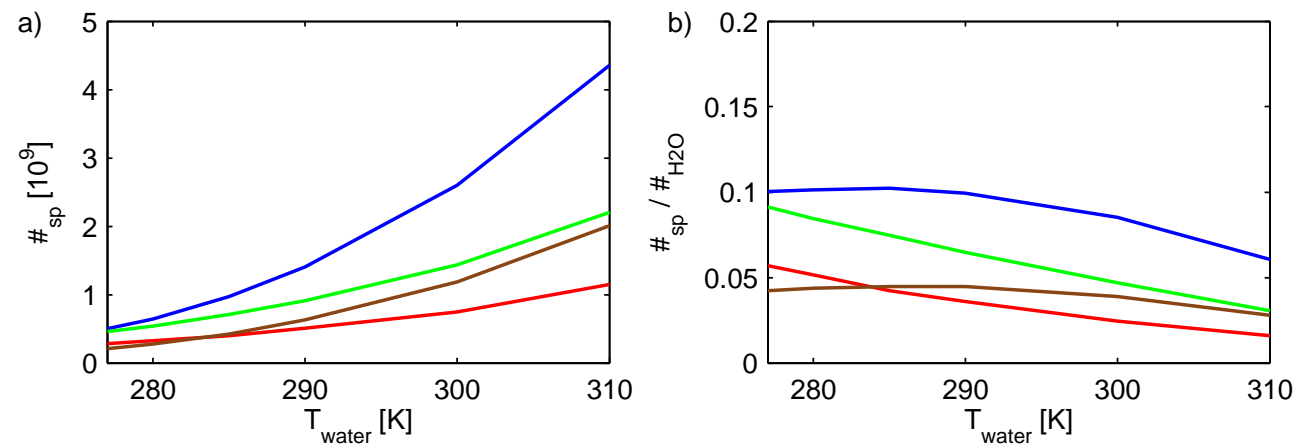

Abbildung 5.5: (a): Maximale Anzahl an Teilchen für verschiedene Spezies in Abhängigkeit von der Wassertemperatur. (b): wie (a), normiert auf die Anzahl an $\mathrm{H}_{2} \mathrm{O}$ - Molekülen gegen Ende des Kollaps.

Spezies: $\mathrm{H}(-), \mathrm{H}_{2}(-), \mathrm{O}_{2}(-), \mathrm{OH}(-)$.

Erhöhung der Wassertemperatur. Trotz deutlich niedriger Blasentemperaturen wird bei $T_{\text {water }}=310 \mathrm{~K}$ nahezu die zehnfache Menge an $\mathrm{H}_{2}$ gebildet verglichen $\mathrm{zu} T_{\text {water }}=277 \mathrm{~K}$. Dies läßt sich durch einen Mangel an dissoziationsfähigem $\mathrm{H}_{2} \mathrm{O}$ bei niedrigen Wassertemperaturen erklären. Gut zu erkennen ist dies in Abb. 5.6, welche die raumzeitliche Entwicklung der Dichten von $\mathrm{H}_{2} \mathrm{O}, \mathrm{OH}$ und $\mathrm{H}_{2}$ für $T_{\text {water }}$ $=285 \mathrm{~K}$ und $T_{\text {water }}=310 \mathrm{~K}$ vergleicht. Bei $T_{\text {water }}=285 \mathrm{~K}$ werden nahezu alle sich im (heißen) Blasenzentrum befindlichen $\mathrm{H}_{2} \mathrm{O}$ - Moleküle dissoziiert (5.6,a). Es folgt eine Phase, in der hochreaktive Produkte wie $\mathrm{OH}$ (5.6, c), $\mathrm{O}$ und $\mathrm{H}$ vorhanden sind, die schnell zu stabilen Spezies wie $\mathrm{O}_{2}$ (5.6,e), $\mathrm{H}_{2}, \mathrm{HO}_{2}$ oder $\mathrm{H}_{2} \mathrm{O}_{2}$ weiterreagieren. Im Vergleich dazu ist bei $T_{\text {water }}=310 \mathrm{~K}$ eine deutlich größere Menge an Wasserdampf verfügbar (man beachte die unterschiedlichen Skalierungen von Abb. 5.6, a und 5.6,b. Die thermische Energie reicht nicht für eine vollständige Dissoziation des sich im Blasenzentrum befindlichen $\mathrm{H}_{2} \mathrm{O}$ aus, es werden aber deutlich höhere Dichten der Produkte erreicht. In diesem Fall ist die vorhandene Energie der limitierende Faktor, bei $T_{\text {water }}=285 \mathrm{~K}$ ist es die vorhandene Menge an Wasserdampf.

Abb. 5.5,b zeigt, dass bei niedriger Wassertemperatur verhältnismäßig mehr reaktive Produkte gebildet werden. Für $T_{\text {water }}=277 \mathrm{~K}$ entstehen annähernd genauso viele $\mathrm{OH}$ - wie $\mathrm{H}_{2}$-Moleküle. Diese Verhältnis sinkt bei $T_{\text {water }}=310 \mathrm{~K}$ auf ca. 1 zu 2. Dies erklärt sich durch die bei niedriger $T_{\text {water }}$ vorhandene Energie, die eine erneute Bildung der reaktiven Spezies ermöglicht. So findet z.B. die Reaktion 'O $+\mathrm{H}_{2} \rightarrow \mathrm{H}+\mathrm{OH}^{\prime}$ (mit hoher Aktivierungsenergie, siehe Kap. 4.3) bei $T_{\text {water }}=$ $285 \mathrm{~K}$ deutlich häufiger statt als bei $T_{\text {water }}=310 \mathrm{~K}$. Allgemein sinkt das Verhältnis von gebildeten Spezies zu in der Blase verbleibenden Wasserdampfteilchen. 

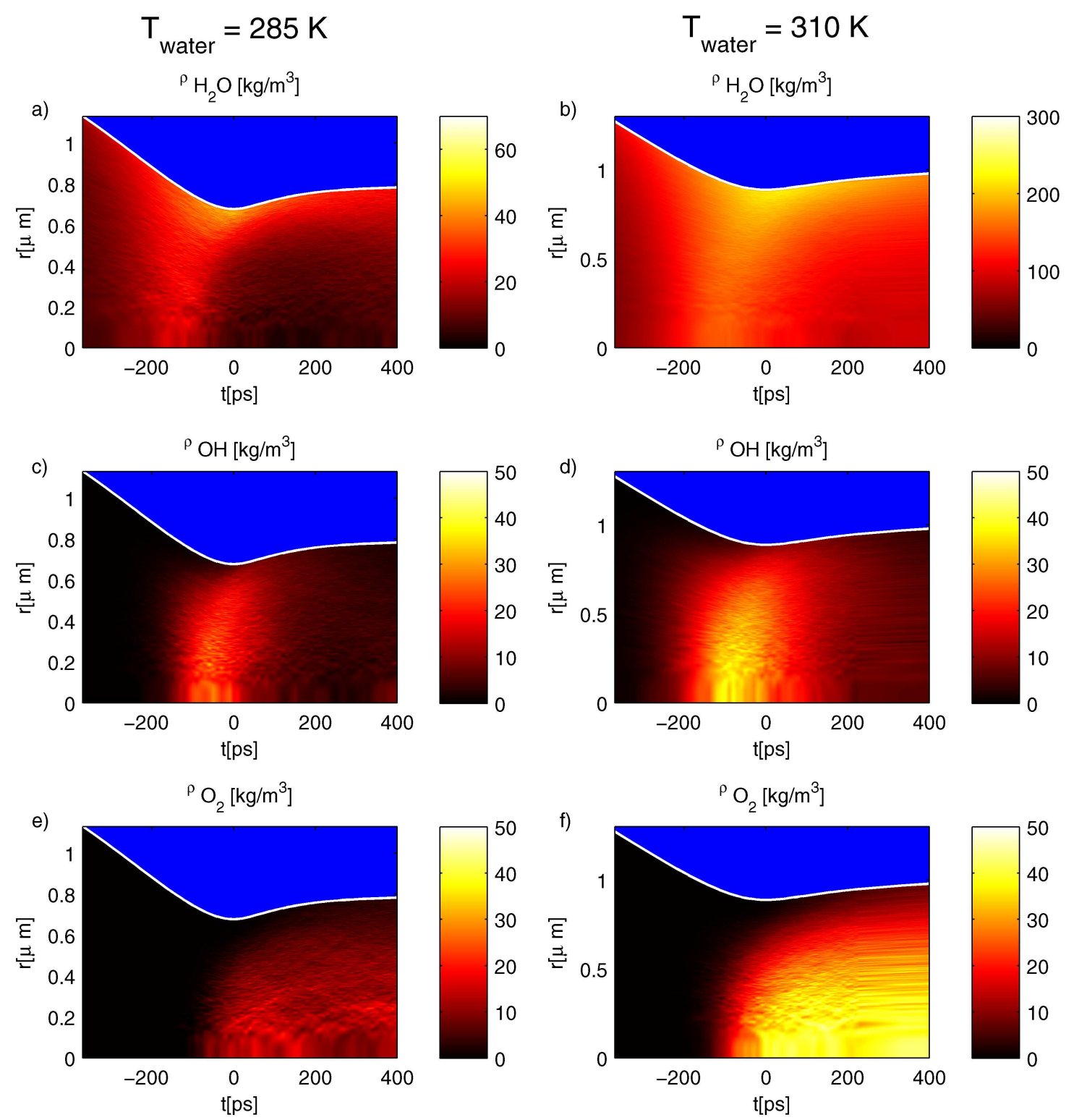

Abbildung 5.6: Entwicklung der Dichte von $\mathrm{H}_{2} \mathrm{O}, \mathrm{OH}$ und $\mathrm{H}_{2}$ für $T_{\text {water }}=285 \mathrm{~K}$ (a,c,e) und $T_{\text {water }}=310 \mathrm{~K}(\mathrm{~b}, \mathrm{~d}, \mathrm{f})$ um den Zeitpunkt des minimalen Blasenradius $(\mathrm{t}=0)$.

Die gefundenen Trends und Zusammenhänge setzen sich fort, bzw. finden Bestätigung in einer erweiterten Vergleichsrechnung von Blasen mit einer Wassertemperaturen von $T_{\text {water }}=285 \mathrm{~K}$ bis $T_{\text {water }}=333 \mathrm{~K}$. Diese mußte aufgrund der extremen Menge an $\mathrm{H}_{2} \mathrm{O}$ - Teilchen bei $T_{\text {water }}=333 \mathrm{~K}$ mit einer geringen Edelgasteilchenzahl $(S \approx 200.000)$ und einer höheren Anfangswandgeschwindigkeit $\left(\mathrm{v}_{W, \text { init }}=45 \mathrm{~m} / \mathrm{s}\right)$ durchgeführt werden. Daher wird auf eine detaillierte Darstellung der Ergebnisse für diese hohen Wassertemperaturen verzichtet. Auch hier 
zeigte sich bei jeder Erhöhung der Wassertemperatur eine deutliche Abnahme der Lichtemission bei gleichzeitiger Zunahme der chemischen Aktivität. 


\subsection{Variation des Anregungsdruckes}

Die in Sonolumineszenz- und Kavitationsblasen auftretenden hohen Temperaturen führen zu interessanten Anwendungen in der Sonochemie und anderen Gebieten. Es wurden sogar Möglichkeiten erörtert, ob die thermale Energie einer solchen Blase ausreichend sein könnte, um nukleare Fusionsprozesse zu erlauben [22]. Positive Messungen zu diesen Überlegungen erwiesen sich allerdings als nicht reproduzierbar. Ein experimenteller Nachweis steht bisher also aus.

Wenn eine Maximierung der Blasentemperatur gewünscht wird, liegt eine Erhöhung des Anregungsdruckes $P_{a}$ nahe, um die Blasen heftiger kollabieren zu lassen. Welche Auswirkungen eine Änderung von $P_{a}$ auf die Blasenparameter und das Blaseninnere hat, soll im Folgenden erörtert werden.

\begin{tabular}{|c|c|c|c|c|c|c|c|c|}
\hline$P_{a}$ & - & 1,33-1,45 bar & $\begin{array}{c}N_{N G} \\
S\end{array}$ & - & $\begin{array}{l}216.000 \\
\sim 27000\end{array}$ & $v_{W, \text { init }}$ & - & $30 \mathrm{~m} / \mathrm{s}$ \\
\hline $\begin{array}{l}\nu a \\
R_{0}\end{array}$ & - & $\begin{array}{c}26,5 \mathrm{kHz} \\
3,85-7,72 \mu \mathrm{m}\end{array}$ & Edelgas & - & $\begin{array}{c}\text { Argon } \\
\text { Argon }\end{array}$ & $\begin{array}{l}\alpha_{t} \\
\alpha_{v}\end{array}$ & - & $\begin{array}{l}0,3 \\
0,1\end{array}$ \\
\hline$T_{\text {water }}$ & - & $300 \mathrm{~K}$ & $\Delta K_{W}$ & - & 200 & & & \\
\hline
\end{tabular}

Tabelle 5.3: Parameter der Rechnungen zum Einfluß des Anregungsdruckes $P_{a}$.

Für einen gegebenen Wert von $P_{a}$ ergibt sich nur bei bestimmten Werten des Gleichgewichtsradius $R_{0}$ ein diffusives Gleichgewicht, welches eine stabile Anregung der Blase erlaubt. Die $R_{0}$-Werte sind darüber hinaus abhängig vom Gasdruck des in der Flüssigkeit gelösten Gases, $P_{G}[33]$. Wird ein Edelgas gelöst, muß die Flüssigkeit bis auf einen Wert von ungefähr $P_{G} / P_{0}=0,004$ entgast werden. $P_{O}$ entspricht hierbei 1 bar. Wird stattdessen Luft verwendet, ist eine Entgasung nur bis zu Werten von $P_{G} / P_{0}=0,1-0,4$ nötig [129]. in der vorliegenden Untersuchung wird ein Wert von $P_{G} / P_{0}=0,2$ benutzt. Eine Implementation des Diffusiven-Gleichgewichts-Modells, das FyrILlas in [130] beschrieben hat wurde genutzt, um für verschiedene Werte von $P_{a}$ zugehörige Werte von $R_{0}$ zu ermitteln, die stabil in einer Blasenfalle bei $26,5 \mathrm{kHz}$ (siehe Tab. 5.3) schwingen. Die gefundenen Ruheradien sind in Tab. 5.4 angegeben.

Der Radiusverlauf für sechs Werte von $P_{a}=1,33$ bar bis $P_{a}=1,45$ bar, berechnet im polytropischen Modell (siehe Kap. 3.3.2), ist in Abb. 5.7 gezeigt. Ein stärkerer Anregungsdruck führt zusammen mit dem steigenden Ruheradius zu deutlich größeren Maximalradien. Beim Übergang von $P_{a}=1,33$ bar zu $P_{a}=1,45$ bar nimmt der Maximalradius von 43,5 $\mu \mathrm{m}$ auf 67,6 $\mu \mathrm{m}$ zu. Die Nachschwinger sind umso größer, je höher $P_{a}$, was sich durch die steigende Anzahl an Edelgasatomen 


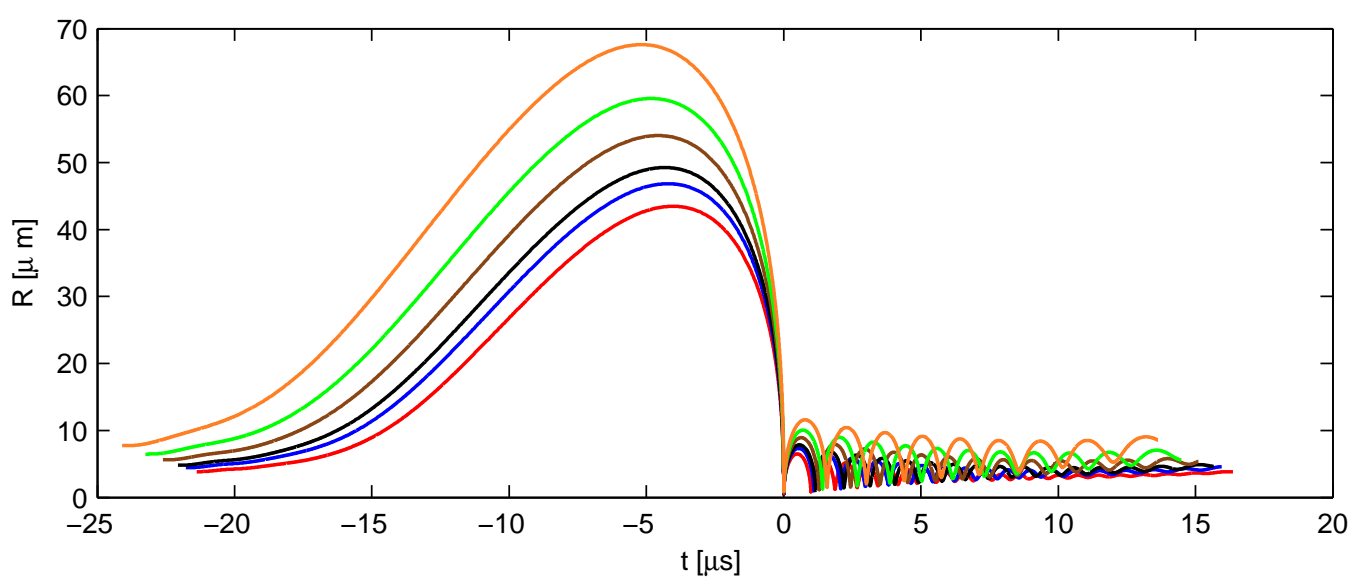

Abbildung 5.7: Verlauf der Blasenschwingung im polytropischen Modell für verschiedene Werte von $P_{a}$. Parameter siehe Tab. 5.3.

Legende: $P_{a}=1,33(-) ; 1,34(-) ; 1,35(-) ; 1,37(-) ; 1,40(-) ; 1,45(-)$.

in der Blase erklärt. Die Kollapsphase wird nun ab einer Blasenrandgeschwindigkeit von $30 \mathrm{~m} / \mathrm{s}$ im Molekulardynamikmodell berechnet. Um in allen Fällen eine genügende räumliche Auflösung zu erhalten, wird mit einer konstanten Anzahl an simulierten Edelgasatomen gerechnet. Dadurch wächst der Skalierungsfaktor der Simulation mit steigendem $P_{a}$. Die restlichen Parameter sind in Tab. 5.3 angegeben.

Abb. 5.8 zeigt Ergebnisse dieser Rechnungen für die Zeit um den minimalen Blasenradius $(t=0)$. Weitere Ergebnisse werden in Tab. 5.4 zusammengefaßt, ebenso werden die Rechnungen dort in sechs Fälle gegliedert. Die steigende Anzahl an Argon-Atomen führt bei hohem $P_{a}$ zu deutlich größeren Minimalradien. Bei Fall 1 beträgt $R_{\min } 0,63 \mu \mathrm{m}$, bei Fall 6 sind es 1,24 $\mu \mathrm{m}$. Der ebenfalls wachsende Maximalradius kann das Wachstum von $R_{\text {min }}$ nicht kompensieren, so dass für größeres $P_{a}$ kleinere Kompressionsverhältnisse $K$ erzielt werden. Der Mechanismus des 'vapor trappings' skaliert linear mit dem Anregungsdruck: Der Prozentsatz von Wasserdampfteilchen in der Blase kurz vor Einsetzen der chemischen Reaktionen ändert sich nur geringfügig zwischen den verschiedenen Rechnungen. Der Wert bewegt sich zwischen 45,75\% und 47,04\%. Je größer $P_{a}$, desto schneller verläuft der Kollaps - bis kurz vor Erreichen $t_{R m i n}$. Der vermehrte Blaseninhalt bei hohem $P_{a}$ macht sich schneller durch einen hohen Innendruck bemerkbar, so dass diese Blasen zu einem früheren Zeitpunkt abgebremst werden. In Fall 6 erreicht die Blase ca. 400 ps vor $t_{R m i n}$ das Wandgeschwindigkeitsmaximum, im Fall 1 geschieht die ca. 180 ps vor $t_{R m i n}$. Damit erreichen die Fälle mit kleinem $P_{a}$ eine größere maximale Wandgeschwindigkeit. 

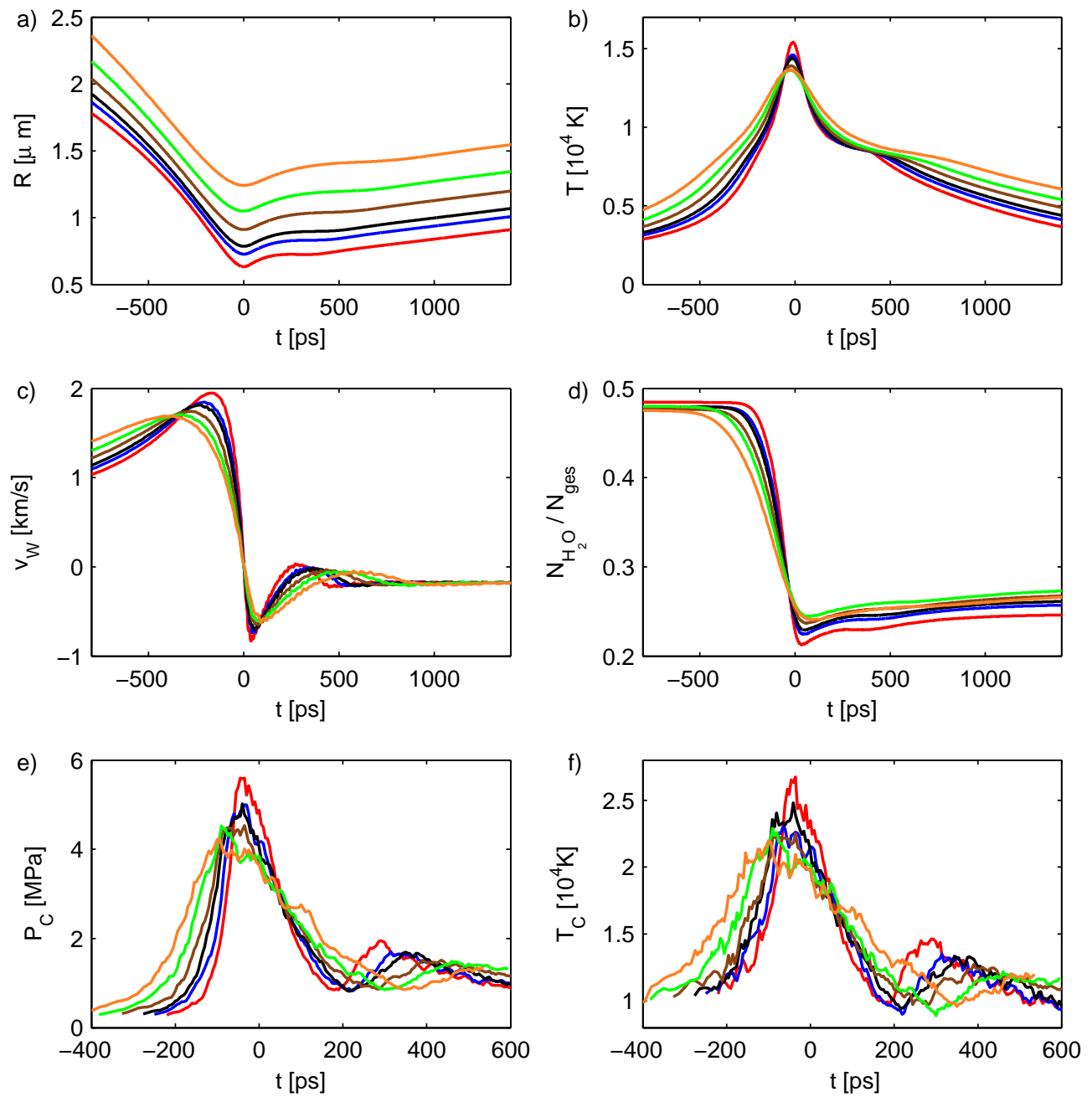

Abbildung 5.8: Verlauf von Radius (a), Durchschnittstemperatur (b), Blasenwandgeschwindigkeit (c), dem Verhältnis von gefangenem Wasserdampf zur gesamten Teilchenzahl (d), dem Druck im Blasenzentrum (e) sowie der Temperatur im Blasenzentrum (f) für verschiedene Werte von $P_{a}$. Parameter siehe Tab. 5.3.

Legende: $P_{a}=1,33(-) ; 1,34(-) ; 1,35(-) ; 1,37(-) ; 1,40(-) ; 1,45(-)$.

Es wird also deutlich, dass nicht etwa die am höchsten angeregten Blasen am heftigsten kollabieren - das Gegenteil ist der Fall! Dies wird auch in den erreichten maximalen Durchschnittstemperaturen deutlich, die von $15.400 \mathrm{~K}$ im Fall 1 auf 13.600 K im Fall 6 zurückgehen. Die Maximaltemperaturen, die im Blasenzentrum erreicht werden, gehen noch etwas stärker zurück (von $26.470 \mathrm{~K}$ auf ca. 21.990 K). Dieser Rückgang wird sowohl durch das verminderte Kompressionsverhältnis verursacht, als auch durch den größeren minimalen Blasenradius, der zu einer längeren Zeitskala der Binnendynamik führt. Dies ist gut an den Plots von Zentrumsdruck und -temperatur zu erkennen, die für niedrige Werte von $P_{a}$ 

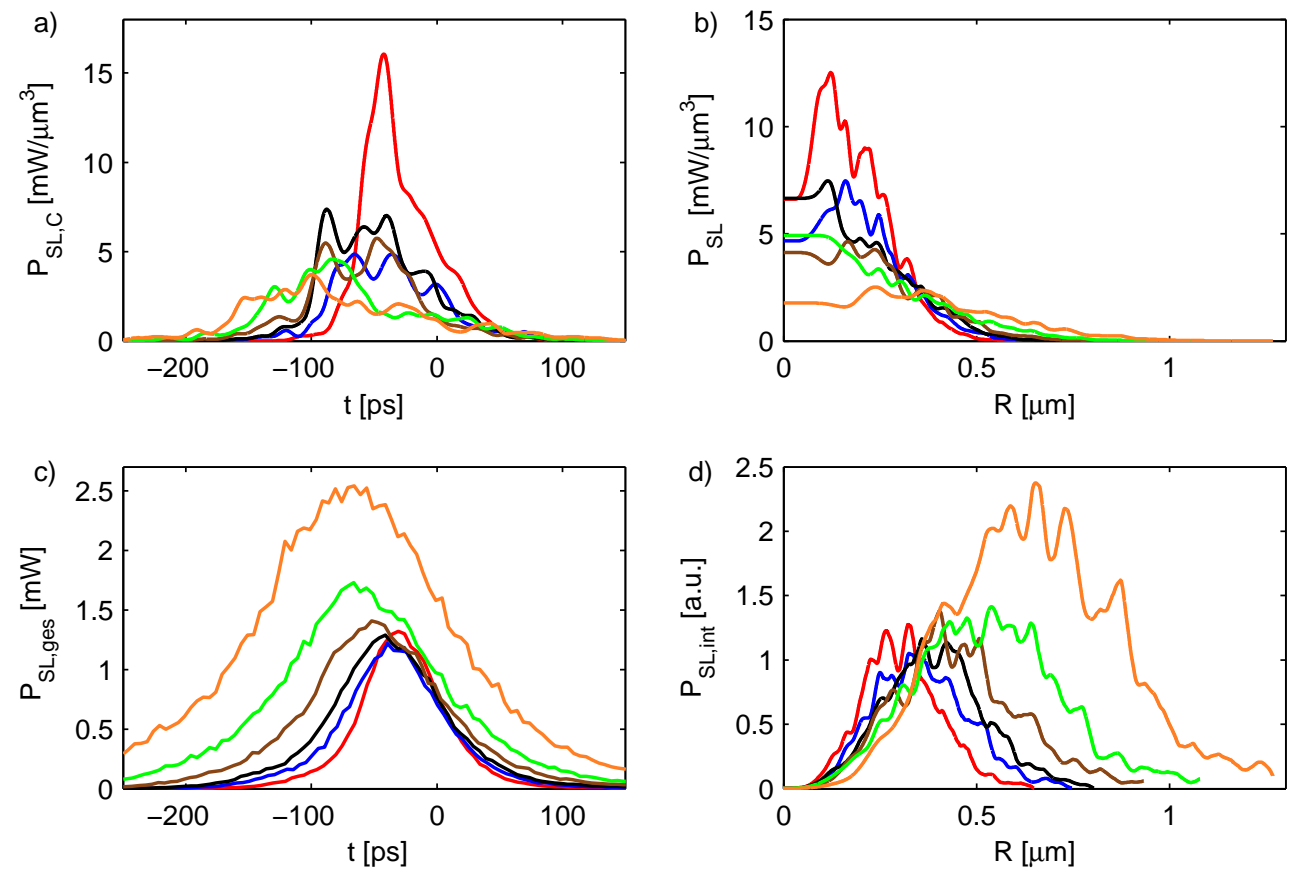

Abbildung 5.9: Lichtemission der Blasen mit variiertem $P_{a}$. (a): Emission des Blasenzentrums während der heißen Phase des Kollaps. (b): Radiale Verteilung der Lichtleistungsdichte zum Zeitpunkt der maximalen Gesamtemission. (c): Gesamte Lichtleistung der Blase während der heißen Phase des Kollaps. (d): Wie (b), allerdings skaliert mit dem Radius der jeweiligen Kugelschale zur Verdeutlichung des Volumeneffektes. In beliebigen Einheiten. Parameter siehe Tab. 5.3.

Legende: $P_{a}=1,33(-) ; 1,34(-) ; 1,35(-) ; 1,37(-) ; 1,40(-) ; 1,45(-)$.

eine deutlich kürzere Periode extremer Bedingungen im Blaseninneren zeigen.

Abb. 5.9 zeigt Aspekte der Lichtemission der betrachteten Blasen. Es wird deutlich, dass die verminderten Temperaturen in den Fällen mit größerem $P_{a}$ nicht zu verminderter Lumineszenz führen. Die Emission des Blasenzentrums ist zwar erwartungsgemäß für Fall 1 am größten, dieses trägt aufgrund seines geringen Volumens aber nur einen sehr kleinen Teil zur gesamten Emission bei. Der Schnitt durch die Blase zeigt, dass die Blasen mit wachsendem $P_{a}$ zwar lokal weniger Licht emittieren, durch ihre Größe insgesamt aber deutlich größere Lichtleistungen aufweisen. An dem mit dem Kugelschalenvolumen skalierten Plot (Abb. 5.9, d) läßt sich dies deutlich erkennen. Nicht nur die pro Zeiteinheit emittierte Lichtleistung steigt mit wachsendem $P_{a}$, durch die langsamere Blasendynamik wächst auch die Dauer der Lichtemission. Die Halbwertsbreite $\Delta t_{S L}$ steigt von 70 ps auf 170 ps. Insgesamt ergibt sich eine Steigerung der Lichtleistung von 0,096 fJ im Fall 1 auf 0,478 fJ im Fall 6, also ungefähr eine Verfünffachung. 

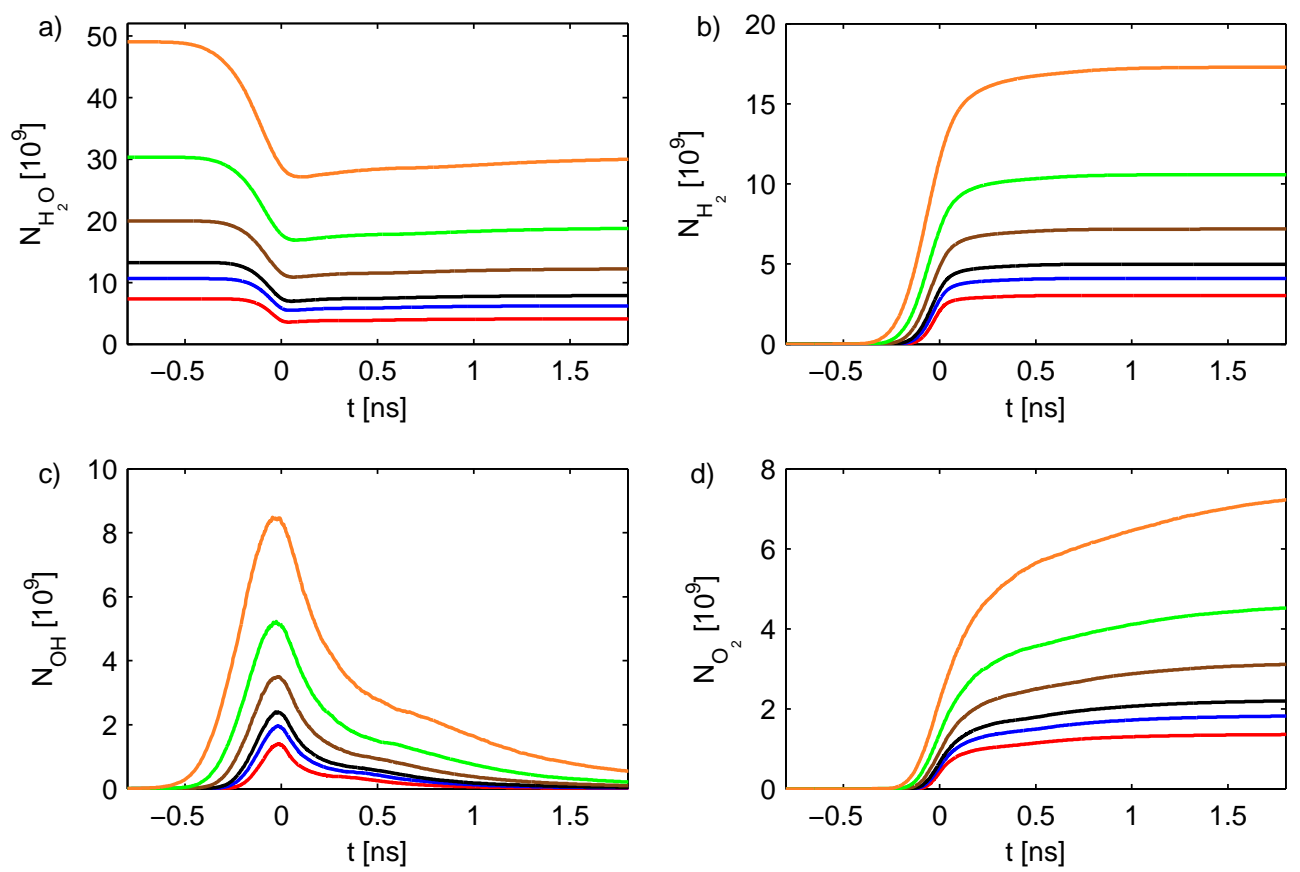

Abbildung 5.10: Chemische Aktivität der Blasen mit variiertem $P_{a}$. Gesamtzahl an vorhandenem $\mathrm{H}_{2} \mathrm{O}(\mathrm{a}), \mathrm{H}_{2}$ (b), $\mathrm{OH}$ (c) und $\mathrm{O}_{2}$ (d). Parameter siehe Tab. 5.3.

Legende: $P_{a}=1,33(-) ; 1,34(-) ; 1,35(-) ; 1,37(-) ; 1,40(-) ; 1,45(-)$.

Abb. 5.10 stellt die Entwicklung der Teilchenanzahl einiger gebildeter Spezies dar. Es läßt sich ein ähnlicher Trend wie bei der Lichtemission ausmachen: Die Temperaturen sind für hohes $P_{a}$ zwar deutlich reduziert, die zugenommene Systemgröße und der vermehrte $\mathrm{H}_{2} \mathrm{O}$-Vorrat $\left(49,0 \cdot 10^{9}\right.$ im Fall 6 zu 7,3·10 im Fall 1) bewirken jedoch eine starke Steigerung der chemischen Aktivität der Blase. So werden im Fall 6 ca. 17,3·10 $\mathrm{H}_{2}$-Moleküle gebildet, im Fall 1 sind es lediglich $3,1 \cdot 10^{9}$. Ähnliche Verhältnisse ergeben sich für $\mathrm{OH}\left(8,5 \cdot 10^{9} \mathrm{zu} 1,4 \cdot 10^{9}\right)$ und $\mathrm{O}_{2}\left(7,8 \cdot 10^{9} \mathrm{zu} 1,4 \cdot 10^{9}\right)$. Betrachtet man alle Spezies ergibt sich eine Spanne des Verhältnisses der Teilchenanzahlen im Fall 6 zum Fall 1 von 5,4 bis 6,3. Der Zuwachs der chemischen Aktivität ist also gut zu vergleichen mit dem Zuwachs an Lichtemission.

Es bleibt festzustellen, dass eine Erhöhung der Blasentemperaturen durch eine Erhöhung des Anregungsdruckes nicht zu erreichen scheint. Das Eintreten des diffusiven Gleichgewichtes bei einem größeren Ruheradius und die damit einhergehende Vermehrung der Blaseninhaltes führen im Gegenteil zu kleineren Temperaturen. Eine Erhöhung der Temperaturen scheint eher durch eine Verminderung der Blasengröße bei gleichzeitiger Erhöhung der Anregungsfrequenz möglich zu 
Kap. 5 Ergebnisse für Sonolumineszenzblasen

\begin{tabular}{lllllll}
\hline Fall & 1 & 2 & 3 & 4 & 5 & 6 \\
\hline Parameter & & & & & & \\
$P_{a}[\mathrm{bar}]$ & 1,33 & 1,34 & 1,35 & 1,37 & 1,40 & 1,45 \\
$R_{0}[\mu \mathrm{m}]$ & 3,85 & 4,44 & 4,81 & 5,60 & 6,47 & 7,72 \\
$N_{A r}\left[10^{9}\right]$ & 0,781 & 1.153 & 1.440 & 2.196 & 3.290 & 5.420 \\
$\mathrm{~S}$ & 36.695 & 54.342 & 67.557 & 102.899 & 154.761 & 254.682 \\
\hline Dynamik & & & & & & \\
$R_{\max }[\mu \mathrm{m}]$ & 43,5 & 46,9 & 49,3 & 54,0 & 59,5 & 67,6 \\
$R_{\min }[\mu \mathrm{m}]$ & 0,63 & 0,73 & 0,79 & 0,91 & 1,05 & 1,24 \\
$K$ & 69,04 & 64,24 & 62,40 & 59,34 & 56,66 & 54,51 \\
$T_{\varnothing}[\mathrm{K}]$ & 15.410 & 14.600 & 14.390 & 13.920 & 13.640 & 13.650 \\
$T_{\max }[\mathrm{K}]$ & 26.740 & 24.700 & 24.790 & 23.170 & 22.900 & 21.990 \\
$\mathrm{H}_{2} \mathrm{O}\left[10^{9}\right]$ & 7,3 & 10,6 & 13,3 & 20,0 & 30,3 & 49,0 \\
$\mathrm{H}_{2} \mathrm{O}[\%]$ & 47,05 & 46,40 & 46,20 & 45,75 & 45,80 & 45,90 \\
$\mathrm{OH}\left[10^{9}\right]$ & 1,4 & 2,0 & 2,4 & 3,5 & 5,2 & 8,5 \\
$\Delta t_{S L}[\mathrm{ps}]$ & 70 & 85 & 90 & 110 & 130 & 170 \\
$P_{S L}[\mathrm{pJ}]$ & 0,096 & 0,108 & 0,129 & 0,175 & 0,258 & 0,478 \\
\hline
\end{tabular}

Tabelle 5.4: Parameter und Ergebnisse der Rechnungen mit variiertem Anregungsdruck $P_{a}$. $R_{0}$ ist der Ruheradius, $N_{A r}$ die Anzahl an Argon-Atomen, $R_{\max }$ der Maximalradius, $R_{\min }$ der Minimalradius, $K$ der Kompressionsfaktor, $T_{\varnothing}$ das Maximum der Durchschnittstemperatur, $T_{\text {max }}$ das absolute Temperaturmaximum, $\mathrm{H}_{2} \mathrm{O}$ die Anzahl an $\mathrm{H}_{2} \mathrm{O}$-Teilchen kurz vor Einsetzen der chemischen Reaktionen, $\mathrm{H}_{2} \mathrm{O}[\%]$ der prozentuale Anteil von $\mathrm{H}_{2} \mathrm{O}$ an der Gesamtteilchenzahl zu diesem Zeitpunkt, $\mathrm{OH}$ die maximale Anzahl an $\mathrm{OH}, \Delta t_{S L}$ die Halbwertsbreite der Lichtemission, $P_{S L}$ die gesamt emittierte Lichtleistung. Weitere Parameter in Tab. 5.3.

sein (mehr dazu in Kap. 6).

Ist hingegen eine Maximierung der chemischen Aktivität oder der Lichtemission gewünscht, kommt eine Erhöhung des Anregungsdruckes durchaus in Frage, da das vergrößerte System zum vermehrten Auftreten beider Effekte führt.

Die gefundenen Pulsbreiten und Trends der Lichtemission entsprechen sehr gut Ergebnissen, die GompF durch Einzel-Photonen-Zählung[24] und PEcha mittels einer Streak-Kamera [25] fanden. Die beobachteten Trends der Sonochemie werden durch Ergebnisse von MARK unterstützt, der eine Zunahme der $\mathrm{OH}$ Produktion bei Erhöhung der akustischen Anregung bei MBSL-Experimenten fand [131]. ToEgEL versuchte, das Sonolumineszenzleuchten zu maximieren, indem er Blasen mit geringer Frequenz $(7,1 \mathrm{kHz})$ anregte, um den Maximalradius zu vergrößern. Er sah bedeutend geringere Effekte als die theoretische vorhergesagten (diese prognostizierten die 100- bis 1000-fache Lichtleistung). Mittels Modellrechnungen sah er, dass der zusätzlich in die Blase verdampfende Wasserdampf extremeren Temperaturen entgegenwirkte. 


\subsection{Verschiedene Edelgase}

Als das in Sonolumineszenz- und Kavitationsblasen enthaltene Gas wird in den meisten Fällen Argon beschrieben. Dies ist der Fall, da eine in einer Flüssigkeit oszillierende Luftblase im Laufe vieler Oszillationen die nicht-edlen Bestandteile durch chemische Reaktionen in lösliche Spezies umwandelt und sich so das bei weitem häufigste Edelgas - also Argon - in der Blase ansammelt. Dieser Mechanismus wurde zunächst von LoHSE et al. vermutet [33] und später von MATULA et al. [35] und Ketterling et al. [34] experimentell nachgewiesen. Wird die beschallte Flüssigkeit mit Argon gesättigt, ist es möglich, die Stabilität der Sonolumineszenz zu verbessern und die Leuchtintensität stark zu vergrößern [31].

Durch eine Sättigung mit einem anderen Edelgas kann die Art des in der Blase vorhandenen Gases gezielt gesteuert werden [132]. Je nach verwendetem Gas sind dabei unterschiedliche Effekt beobachtbar. DiDEnKo et al. sahen bei MBSLVersuchen eine extreme Zunahme der emittierten Lichtenergie bei Verwendung von Edelgasen mit höherer relativer Atommasse. Beim Vergleich von Xenon- und Heliumblasen wurde eine knapp 100-fache Zunahme von $E_{S L}$ durch den Übergang zu Xenon gemessen. Die chemische Aktivität, ausgedrückt in der Anzahl der gebildeten $\mathrm{OH}-$ Moleküle, stieg um ca. das vierfache. HILlER zeigte, dass vergleichbare Trends für SBSL-Blasen existieren [31].

\begin{tabular}{|c|c|c|c|c|c|c|c|}
\hline$P_{a}$ & 1,3 bar & $N_{N G}$ & - & 216.000 & $v_{W, \text { init }}$ & - & $25 \mathrm{~m} / \mathrm{s}$ \\
\hline$\nu_{a}$ & $26,5 \mathrm{kHz}$ & $S$ & - & $\approx 58.000$ & $\alpha_{t}$ & - & 0,3 \\
\hline$R_{0}$ & $4,5 \mu \mathrm{m}$ & Edelgas & - & Argon, Xenon, Helium & $\alpha_{v}$ & - & 0,1 \\
\hline$T_{\text {water }}$ & $300 \mathrm{~K}$ & $\Delta K_{W}$ & - & 3.000 & & & \\
\hline
\end{tabular}

Tabelle 5.5: Parameter der Rechnungen mit unterschiedlichen Edelgasen.

Im Folgenden soll der Einfluß des in der Blase vorhandenen Edelgases auf die Dynamik des Blaseninneren untersucht werden. Dazu wurden Vergleichsrechnungen mit den Edelgasen Helium, Argon und Xenon, sowie Mischungen dieser Gase im Verhältnis 1:1 durchgeführt. Die weiteren Blasenparameter dieser Rechnungen sind in Tab. 5.5 angegeben. Einige relevante Eigenschaften der betrachteten Edelgase sind in Tab. 5.6 dargestellt.

\section{Hydrodynamik}

Abb. 5.11 zeigt die Entwicklung des Blasenradius, der Durchschnittstemperatur, der Wandgeschwindigkeit sowie des durchschnittlichen Druckes in der Blase um $t_{R m i n}(t=0)$ für eine Helium-, eine Argon- und eine Xenon-Blase. Tab. 5.7 fasst 

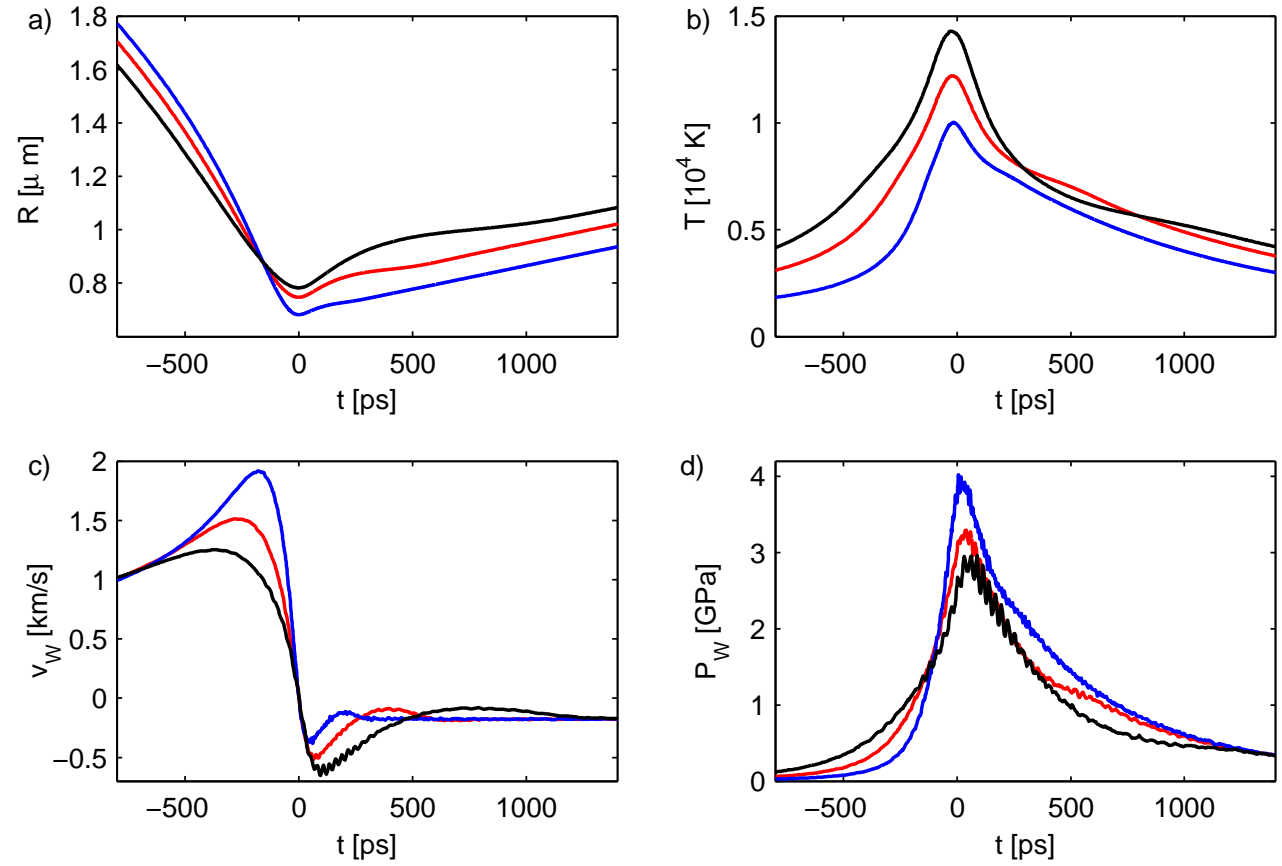

Abbildung 5.11: Rechnungen von Blasen mit unterschiedlichem Edelgasinhalt. (a): Radiusentwicklung. (b): Durchschnittstemperatur der Blase. (c): Blasenwandgeschwindigkeit. (d): Druck an der Blasenwand. Sonstige Parameter siehe Tab. 5.5.

Legende: $\mathrm{He}(-)$; $\operatorname{Ar}(-)$; Xe (-).

wichtige Ergebnisse zusammen. Es ist zu erkennen, dass die Blase umso weiter kollabiert, je leichter (und kleiner) das vorhandene Edelgas ist. Für die Helium (He) - Blase wird $R_{\text {min }}=0,685 \mu \mathrm{m}$ erreicht, bei der Xenon (Xe) - Blase sind es $0,785 \mu \mathrm{m}$. Die Blasenwandgeschwindigkeit ist bis ca. $700 \mathrm{ps}$ vor $t_{R m i n}$ annähernd gleich, danach wird der Rand der Helium-Blase weiter beschleunigt, während die Bewegung der Argon- und Xenon-Blase zunehmend abgebremst wird. Der Innendruck an der Blasenwand $P_{W}$ ist für die meiste Zeit des Kollaps umso größer, je schwerer die Teilchen in der Blase sind. Erst ca. 150 ps vor $t_{R m i n}$ steigt $P_{W}$ für die Helium-Blase stark an und weist in dieser Phase die höchsten Werte auf.

Bei der Xenon-Blase herrscht für eine vergleichsweise lange Zeit ein hoher Druck an der Blasenwand, so dass diese Blase die größte Rebound-Geschwindigkeit erreicht. Die Temperaturen zeigen eine starke Abhängigkeit von der Teilchenmasse: die schweren Xenon-Teilchen können die Energie des Kollaps am besten absorbieren und schließlich in thermische Energie umsetzen. Im Maximum ergibt sich mit $14300 \mathrm{~K}$ eine fast 50-prozentige Temperaturerhöhung, verglichen mit Helium $(10068 \mathrm{~K})$. 


\begin{tabular}{lllllll}
\hline Edelgas & $A[\mathrm{u}]$ & $R_{v d W}[\mathrm{pm}]$ & $\chi_{1}[\mathrm{eV}]$ & $v_{N G, \text { init }}[\mathrm{m} / \mathrm{s}]$ & $v_{N G, \max }[\mathrm{m} / \mathrm{s}]$ & $D_{\text {Ekin }}$ \\
\hline $\mathrm{He}$ & 4,0026 & 140 & 24,58 & 1260 & 7270 & 33,3 \\
$\mathrm{Ar}$ & 39,948 & 188 & 15,76 & 400 & 2570 & 41,3 \\
$\mathrm{Xe}$ & 131,29 & 216 & 12,13 & 220 & 1690 & 59,0 \\
\hline
\end{tabular}

Tabelle 5.6: Eigenschaften und Energieaufnahme der verwendeten Edelgase. $A$ ist die Atommasse, $R_{v d W}$ der van-der-Waals Radius, $\chi_{1}$ die 1 . Ionisierungsenergie, $v_{N G \text {, init }}$ die anfängliche durchschnittliche Teilchengeschwindigkeit des Edelgases, $v_{N G, \max }$ die maximale durchschnittliche Teilchengeschwindigkeit des Edelgases. $D_{E k i n}=E k i n_{N G, \max } / E k i n_{N G, \text { init }}$, der Quotient aus maximaler und initialer durchschnittlicher kinetischer Energie der Edelgasteilchen, ist ein Maß für die im Kollaps von den Teilchen aufgenommene Energie.

Die innere Dynamik der Blase wird sichtbar durch Lage und Ausprägung des 'Huckels' der Blasenrandbewegung, welcher dem minimalen Radius direkt folgt. Diese Phase der hohen negativen Wandgeschwindigkeit wird durch das Auftreffen der im Blasenzentrum reflektierten Verdichtungswelle auf den Blasenrand erzeugt (siehe Kap. 4.1.2.1). Im Falle der Helium-Blase ist sie sehr kurz, die Blase geht innerhalb von ca. 200 ps in ein gleichmäßiges Wachstum über. Bei Argon ist die Phase bereits ausgeprägter (Dauer ca. 600 ps), bei Xenon schließlich dauert sie ca. 1,5 ns, die Ausbeulung des $R(t)$ - Verlaufes ist gut zu erkennen. Diese zeitliche Ausdehnung ist durch die deutlich sinkenden Teilchengeschwindigkeiten zu erklären - die im Zentrum reflektierte Welle wirkt über eine längere Zeit auf den Blasenrand ein und transportiert eine größere Energie. Begleitet wird diese Phase durch zunächst schnell sinkende Durchschnittstemperaturen (aufgrund der abnehmenden Kompression). Verlangsamt sich das Wachstum der Blase, sinken die Temperaturen wieder langsamer.

Ein Grund für die deutlich herabgesetzten Temperaturen mit sinkender Atommasse liegt in dem geringeren Vermögen der Teilchen, die Energie des Kollaps der Blasenwand aufzunehmen. Tab. 5.5 vergleicht die durchschnittliche kineti-

\begin{tabular}{|c|c|c|c|c|c|c|c|c|c|c|c|c|}
\hline $\begin{array}{l}\text { Nr. } \\
-\end{array}$ & $\begin{array}{l}\text { NG } \\
-\end{array}$ & $\begin{array}{l}T_{\max } \\
{[\mathrm{K}]}\end{array}$ & $\begin{array}{l}T_{\varnothing} \\
{[\mathrm{K}]}\end{array}$ & $\begin{array}{l}R_{\min } \\
{[\mu \mathrm{m}]}\end{array}$ & $\begin{array}{l}K \\
-\end{array}$ & $\begin{array}{l}v_{W} \\
{[\mathrm{~km} / \mathrm{s}]}\end{array}$ & $\begin{array}{l}p_{W} \\
{[\mathrm{GPa}]}\end{array}$ & $\begin{array}{l}\mathrm{OH} \\
{\left[10^{9}\right]}\end{array}$ & $\begin{array}{l}\mathrm{O}_{2} \\
{\left[10^{9}\right]}\end{array}$ & $\begin{array}{l}\mathrm{H}_{2} \mathrm{O} \\
{\left[10^{9}\right]}\end{array}$ & $\begin{array}{l}E_{S L} \\
{[\mathrm{pJ}]}\end{array}$ & $\begin{array}{l}\Delta t_{S L} \\
{[\mathrm{ps}]}\end{array}$ \\
\hline 1 & $\mathrm{He}$ & 13154 & 10068 & 0,685 & 62,4 & 1,92 & 4,02 & 1,20 & 0,71 & 8,81 & 0.004 & 110 \\
\hline 2 & $\mathrm{Ar}$ & 18870 & 12269 & 0,750 & 57,1 & 1,51 & 3,30 & 1,49 & 1,05 & 8,59 & 0.034 & 120 \\
\hline 3 & $\mathrm{Xe}$ & 23337 & 14334 & 0,785 & 54,5 & 1,26 & 2,95 & 1,74 & 1,47 & 8,77 & 0.211 & 130 \\
\hline
\end{tabular}

Tabelle 5.7: Ergebnisse der Rechnungen unter Verwendung verschiedener Edelgase (NG). $T_{\max }$ bezeichnet die absolut größte Temperatur in der Blase, $T_{\varnothing}$ die maximale Durchschnittstemperatur, $R_{\text {min }}$ den minimalen Blasenradius, $K$ den Kompressionsfaktor, $v_{W}$ die maximale Blasenwandgeschwindigkeit, $p_{W}$ den maximalen Blasenwanddruck, $\mathrm{OH}$ und $\mathrm{O}_{2}$ die jeweils maximale Anzahl der Spezies in der Blase, $\mathrm{H}_{2} \mathrm{O}$ die Anzahl der in der Blase gefangenen Wasserdampfatome (gemessen kurz vor Einsetzen der chemischen Reaktionen), $E_{S L}$ die gesamte Lichtemission der Blase (siehe auch Tab. 5.8) und $\Delta t_{S L}$ die Halbwertsdauer des Leuchtens. 
sche Energie der Edelgasteilchen am Anfang der Simulation mit dem im Kollaps erreichten Maximalwert. Dieses Maximum wird bei Xenon ca. 200 ps vor Erreichen von $t_{\text {Rmin }}$ angenommen, bei Argon ca. 100 ps, bei Helium ca. 20 ps. Das Maximum der durchschnittlichen Teilchengeschwindigkeit wird deutlich später erreicht als das Maximum der Blasenwandgeschwindigkeit. Zu diesem Zeitpunkt läuft eine Verdichtungswelle durch die Blase, hat das Zentrum aber noch nicht erreicht.

Es ist zu erkennen, dass Teilchen mit großer Masse einen größeren Zuwachs an kinetischer Energie im Blasenkollaps verzeichnen. Durch die relativ größere Geschwindigkeitszunahme bei Wandkollisionen haben Xenon-Atome am Ende des Kollaps im Durchschnitt die 59,0-fache kinetische Energie, verglichen mit dem Start der Simulation; Helium-Atome weisen lediglich die 33,3-fache Energie auf. Bei Start der Simulationen haben die Atome aller Edelgase die selbe mittlere kinetische Energie, da die Anfangstemperatur gleich ist. Die bei Wandkollisionen stattfindende Beschleunigung der Teilchen in Wandnormalenrichtung (siehe Kap. 4.1) bewirkt bei schwereren Spezies einen größeren Zuwachs an kinetischer Energie. Die kinetische Energie der Teilchen thermalisiert schließlich im Blasenzentrum, was die hohen Temperaturen für die Xenon-Rechnung erklärt.

\section{Lichtemission}

Die unterschiedlichen Temperaturen und Eigenschaften der Gase führen zu erheblichen Änderungen im Lumineszenzverhalten der Blasen. Abb. 5.12 zeigt verschiedene Aspekte der Lichtemission der eben besprochenen Blasen, Tab. 5.7 gibt die gesamt emittierte Energie und die Halbwertsdauer der Emission an. Abb. 5.13 stellt die Temperatur- und Lichtemissionsentwicklung für die verschiedenen Edelgase raumzeitlich dar.

Es ist zu erkennen, dass bei Erhöhung der Atommasse des Edelgases die maximale Lichtleistung drastisch ansteigt, während die Leuchtdauer nur leicht zunimmt. Die Zunahme des Leuchtens ist in der gesamten Blase zu beobachten. Den größten Anteil an der Lichtemission haben die mittleren Regionen der Blase (0,3 $0,5 \mu \mathrm{m}$ vom Blasenzentrum entfernt), da dort noch ausreichend hohe Temperaturen herrschen und ein großes Volumen zur Verfügung steht. Die Zentrumsregionen strahlen zwar mit der höchsten Lichtleistung, haben aber aufgrund ihres geringen Volumens kaum Einfluß auf die Gesamtemission.

Die Xenon-Blase emittiert ca. 50 mal so viel Energie wie die Helium-Blase (0,211 pJ vs. $0,004 \mathrm{pJ})$ und ca. $6 \mathrm{mal}$ so viel wie die Argon-Blase (0,034 pJ). Diese 

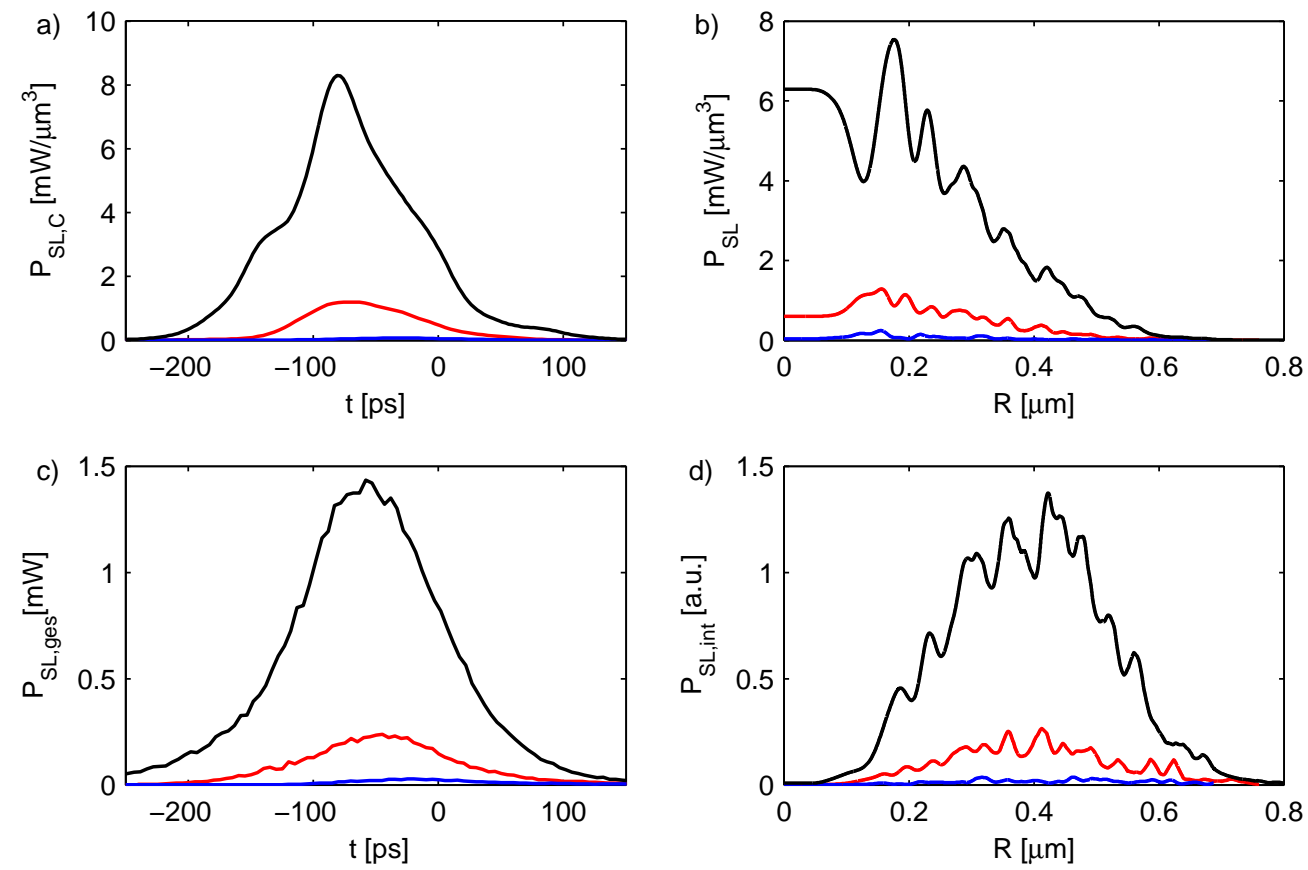

Abbildung 5.12: Lichtemission der Blasen mit unterschiedlichem Edelgasinhalt. (a): Emission des Blasenzentrums während der heißen Phase des Kollaps. (b): Radiale Verteilung der Lichtleistung zum Zeitpunkt der maximalen Gesamtemission (c): Gesamte Lichtleistung der Blase während der heißen Phase des Kollaps. (d): Wie (b), allerdings skaliert mit dem Radius der jeweiligen Kugelschale zur Verdeutlichung des Volumeneffektes. In beliebigen Einheiten. Sonstige Parameter siehe Tab. 5.5.

Verwendetes Edelgas: He (-); Ar (-); Xe (-).

großen Differenzen resultieren zum einen aus den erhöhten Temperaturen in der Xenon-Blase, zum anderen aus der niedrigeren Ionisierungsenergie von Xenon. Wie in Tab. 5.8 zu erkennen, trägt im Helium-Fall das Edelgas nahezu nichts zur Lichtleistung bei. Die Temperaturen sind nicht ausreichend, um Helium nennenswert $\mathrm{zu}$ ionisieren, die erhaltene Lichtleistung wird vom Wasserdampf und vor allem den entstehenden Spezies erbracht. Dass deren Leistung deutlich niedriger ausfällt als bei der Argon- und vor allem der Xenon-Rechnung ist ein alleiniger Temperatureffekt.

Auch bei Argon ist zu beobachten, dass das Edelgas eine zu vernachlässigende Rolle bei der Lichtemission spielt (weniger als $2 \%$ von $E_{S L}$, siehe auch Kap. 4.3.2). Erst bei Xenon ist ein größerer Anteil des Edelgases an der Gesamtemission zu beobachten (ca. $24 \%$ ). Dies resultiert aus der niedrigen 1. Ionisierungsenergie von Xenon, welche auch unter derjeniger der anderen chemischen Spezies liegt, und so zu einem höheren Ionisationsgrad führt. 

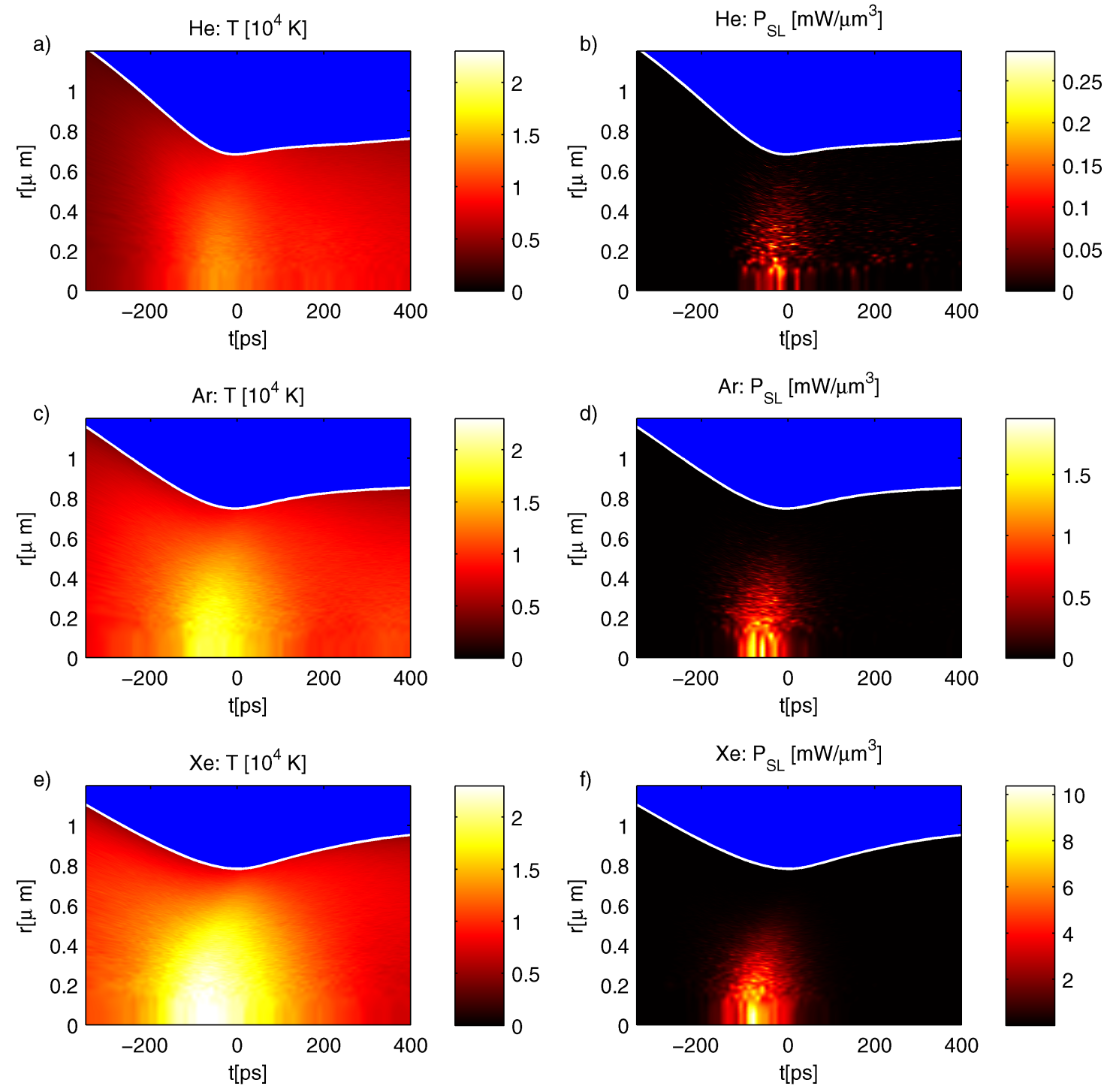

Abbildung 5.13: Temperatur und Lichtleistung für Rechnungen mit Helium (a,b), Argon $(\mathrm{c}, \mathrm{d})$ und Xenon $(\mathrm{e}, \mathrm{f})$ als in der Blase vorhandenem Edelgas. Die Temperaturen wurden zu Vergleichszwecken in der selben Skala geplottet. Bei der Lichtleistung war dies aufgrund der extremen Unterschiede nicht möglich. Sonstige Parameter siehe Tab. 5.5.

Der starke Anteil, den die entstehenden Spezies an $E_{S L}$ haben ist eine Folge des exothermen Charakters der an der Bildung dieser Verbindungen beteiligten Reaktionen. Aus diesem Grund weisen die aus den Dissoziationsprodukten gebildeten Spezies $\mathrm{H}_{2}, \mathrm{O}, \mathrm{O}_{2}, \mathrm{HO}_{2}$ und $\mathrm{H}_{2} \mathrm{O}_{2}$ eine deutliche höhere Temperatur als das Edelgas oder der Wasserdampf auf. Lediglich die durch die endotherme Dissoziationsreaktion entstehenden $\mathrm{H}$ und $\mathrm{OH}$ haben mit Wasserdampf vergleichbare Temperaturen. Dieser Wärmeüberschuss erklärt die hohe Strahlungsleistung der 
entstehenden Spezies. Das häufig gebildete $\mathrm{H}_{2}$ strahlt im Falle von Argon und Xenon klar am meisten Licht aus. Wird Helium als Edelgas verwendet, wird die $\mathrm{H}_{2}$-Bildung aufgrund der niedrigeren Temperaturen verzögert. In diesem Fall emittieren die stabilen Spezies $\mathrm{O}_{2}, \mathrm{HO}_{2}$ und $\mathrm{H}_{2} \mathrm{O}_{2}$ mit $\mathrm{H}_{2}$ vergleichbar viel Energie.

\begin{tabular}{lllllllllll}
\hline \multicolumn{1}{c}{ Spezies } & $\mathrm{NG}$ & $\mathrm{H}_{2} \mathrm{O}$ & $\mathrm{H}$ & $\mathrm{H}_{2}$ & $\mathrm{O}$ & $\mathrm{O}_{2}$ & $\mathrm{OH}$ & $\mathrm{HO}_{2}$ & $\mathrm{H}_{2} \mathrm{O}_{2}$ & gesamt \\
\hline a) & & & & & & & & & & \\
$E_{S L}[\mathrm{NG}=\mathrm{He}]$ & $5^{*} 10^{-6}$ & 0,09 & 0,28 & 0,84 & 0,38 & 0,63 & 0,26 & 0,82 & 0,96 & 4,29 \\
$E_{S L}[\mathrm{NG}=\mathrm{Ar}]$ & 0,66 & 1,76 & 1,32 & 14,80 & 1,51 & 3,13 & 1,05 & 4,53 & 5,66 & 34,45 \\
$E_{S L}[\mathrm{NG}=\mathrm{Xe}]$ & 51,00 & 9,08 & 3,71 & 82,57 & 5,02 & 17,73 & 2,86 & 19,32 & 21,09 & 212,39 \\
\hline $\mathrm{b})$ & & & & & & & & & & \\
$T[\mathrm{NG}=\mathrm{He}]$ & 0,99 & 0,94 & 0,82 & 1,16 & 0,98 & 1,18 & 1,01 & 1,11 & 1,06 & 1,00 \\
$T[\mathrm{NG}=\mathrm{Ar}]$ & 1,16 & 1,12 & 0,98 & 1,72 & 0,89 & 1,49 & 1,13 & 1,36 & 1,32 & 1,22 \\
$T[\mathrm{NG}=\mathrm{Xe}]$ & 1,30 & 1,24 & 1,12 & 2,09 & 1,46 & 1,99 & 1,25 & 1,91 & 1,81 & 1,43 \\
\hline
\end{tabular}

Tabelle 5.8: (a): Gesamt emittierte Lichtenergie, aufgeschlüsselt nach chemischen Spezies für die Blasen aus Tab. 5.5. Alle Angaben in fJ. (b): Maximum der Durchschnittstemperatur, aufgeschlüsselt nach chemischen Spezies für die Blasen aus Tab. 5.5. Alle Angaben in $10^{4} \mathrm{~K}$. NG bezeichnet das jeweilige Edelgas.

\subsubsection{Segregation des Blaseninneren}

Tritt eine Entmischung des Blaseninhalts auf, ist durch die räumliche Änderung der Schallgeschwindigkeit eine Beeinflussung der Hydrodynamik des Blaseninneren zu erwarten. Abb. 5.14 stellt die Dichteverteilung im Kollaps von Wasserdampf und Edelgas für eine Helium- und eine Xenon-Blase gegenüber. Deutlich ist zu erkennen, dass die im Verhältnis leichteren Teilchen im Blasenzentrum gesammelt werden, während schwerere Teilchen tendenziell am Blasenrand verbleiben.

Abb. 5.15 verdeutlicht die Entwicklung der Segregation von Edelgas und Wasserdampf über einen größeren Zeitraum. Dargestellt sind für unterschiedliche Größen das Verhältnis zwischen den Werten im Blasenzentrum und am Blasenrand. Dabei ist jeweils links ein Zeitraum vom Start der Simulation bis 300 ns nach $t_{R m i n}$ aufgetragen, rechts eine Vergrößerung auf $1 \mathrm{~ns}$ um $t_{R m i n}$. Die dargestellten Werte sind die Dichte des Wasserdampfes (a,b), die Dichte des Edelgases (c,d) sowie das Teilchenanzahlverhältnis von $\mathrm{H}_{2} \mathrm{O}$ zur Gesamtteilchenzahl als ein Maß der Entmischung.

Die Verteilung des Wasserdampfes in der Blase unterscheidet sich lediglich für eine sehr kurze Zeit (ca. 2 ns) um $t_{R m i n}$ wesentlich zwischen den verschiedenen 

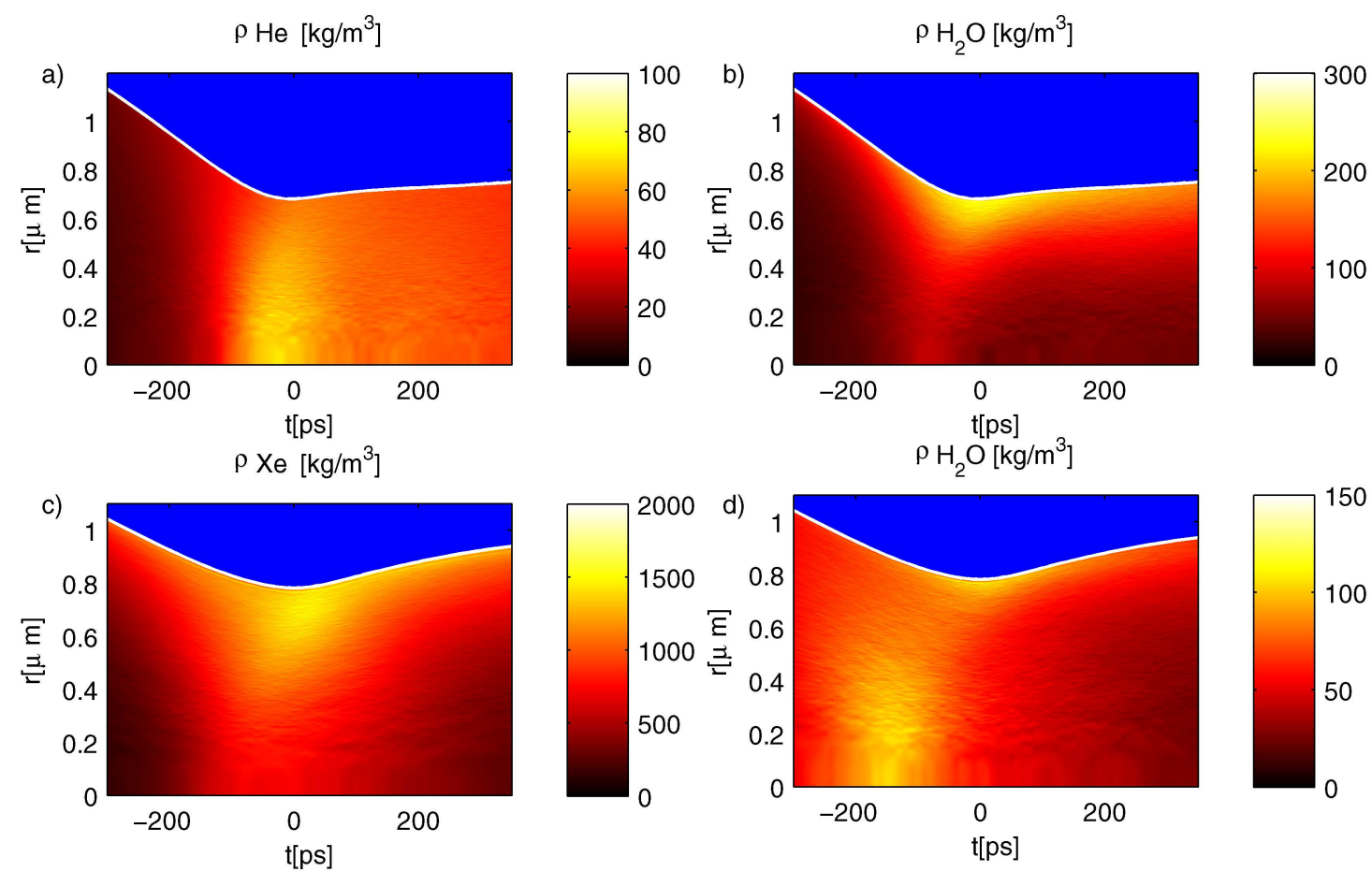

$\rho \mathrm{H}_{2} \mathrm{O}\left[\mathrm{kg} / \mathrm{m}^{3}\right]$

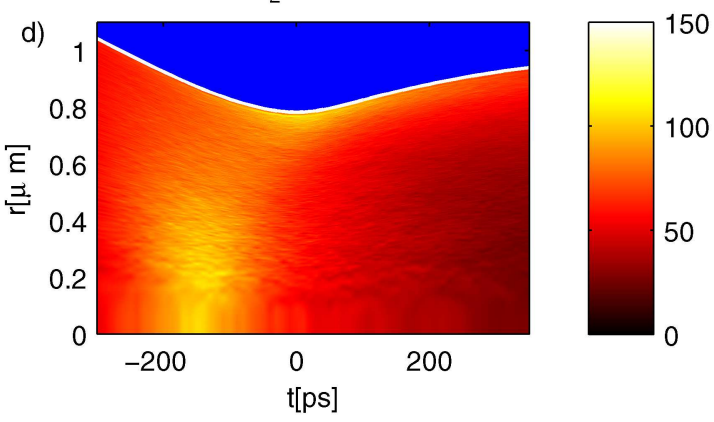

Abbildung 5.14: Dichteverteilung von Edelgas und Wasserdampf im Falle von Helium (a,b) und Xenon (c,d). Sonstige Parameter siehe Tab. 5.5.

Rechnungen (Abb. 5.15,b). Während des Kollaps ergibt sich in allen Fällen ein langsam zunehmendes Gefälle der Wasserdampfdichte vom Blasenrand zum Blasenzentrum, da nicht alle $\mathrm{H}_{2} \mathrm{O}$-Teilchen verdampfen und sich an der Blasenwand sammeln (Abb. 5.15,a). Erst mit dem Abbremsen der Blasenwand löst sich diese Schicht von der Wand und der Quotient $\varrho \mathrm{H}_{2} \mathrm{O}, \mathrm{C} / \varrho \mathrm{H}_{2} \mathrm{O}, \mathrm{W}$ beginnt zu steigen. Da das Abbremsen bei der Xenon-Blase am ehesten einsetzt ist auch dieser Effekt zuerst bei der Xenon-Blase zu beobachten. Je schwerer das eingesetzte Edelgas ist, umso mehr Wasserdampfteilchen treibt die einlaufende Verdichtungswelle vor sich her. Bei Xenon beträgt die $\mathrm{H}_{2} \mathrm{O}$-Dichte im Zentrum maximal das 1,5fache der Dichte am Blasenrand. Dieser Wert ist durch einsetzende chemische Reaktionen, welche die Wasserdampfdichte im Zentrum herabsetzen, gedämpft. Zählt man die entstehenden Produkte hinzu ergibt sich für Xenon ein maximaler $\varrho_{\mathrm{H}_{2} \mathrm{O}, \mathrm{C}} / \varrho_{\mathrm{H}_{2} \mathrm{O}, \mathrm{W}}$-Wert von 3,5 (nicht graphisch gezeigt). Bei Argon übersteigt die $\mathrm{H}_{2} \mathrm{O}$-Dichte im Zentrum niemals die entsprechende Dichte am Blasenrand. Betrachtet man auch die chemischen Produkte ergibt sich allerdings ein Faktor von ca. 2,0. Bei Helium zeigt sich das das hier umgekehrte Gewichtsverhältnis von Edelgas und Wasserdampf. Es ist zwar eindeutig ein Peak der Zentrumsdichte 
von $\mathrm{H}_{2} \mathrm{O}$ erkennbar, die Dichte am Blasenrand ist aber zu allen Zeiten bedeutend höher. Dies gilt auch unter Berücksichtigung der chemischen Produkte.

Im Anschluß an den heißen Kollaps steigt für alle Rechnungen der DichteQuotient auf einen Wert um 2.0. Von dort fällt er langsam wieder ab. Dies läßt sich erklären, wenn man sich verdeutlicht, dass die sich entfernende Blasenwand Bereiche geringer Dichte hinterläßt und zusätzlich aufgrund des immer noch hohen Druckes eine Kondensation der $\mathrm{H}_{2} \mathrm{O}$-Atome an der Blasenwand stattfindet.

Betrachtet man hingegen das Verhältnis der Dichte des Edelgases im Blasenzentrum $\varrho_{N G, C}$ und am Blasenrand $\varrho_{N G, W}$ (Abb. 5.15,c\&d) fällt auf, dass sich schon in der langsamen Phase des Kollaps deutliche Unterschiede zwischen den Rechnungen ergeben. Diese starken Unterschiede lassen sich durch die verschiedenen Teilchengeschwindigkeiten erklären. Während bei Helium die Verteilung der Edelgasatome bis in die letzte Phase des Kollaps relativ homogen bleibt (minimaler Wert von $\left.\varrho_{N G, C} / \varrho_{N G, W} \approx 0,5\right)$, da die schnellen Atome von der Blasenwand wieder in das Zentrum gelangen können, bildet sich für Argon und Xenon ein immer ausgeprägterer Dichte-Gradient aus. Der Dichte-Quotient erreicht minimal 0,18 für Argon und 0,07 für Xenon. D.h., dass kurz vor Ende des Kollaps am Blasenrand die ca. 13-fache Xenon-Dichte, verglichen mit der im Blasenzentrum, herrscht.

Mit dem Abbremsen der Blasenwand vergrößert sich der Dichte-Quotient und steigt für Xenon auf ca. 0,7, für Argon auf ca. 1,0 und für Helium auf ca. 1,6. Durch die Reflektion der einlaufenden Dichtewelle sinkt er dann schnell wieder ab. In der Phase des Blasenwachstums im ersten Rebound ( $\mathrm{t}=50$ - 300 ps in 5.15, c) pendelt sich der Dichtequotient je nach Edelgasmasse auf ein unterschiedliches Niveau ein. Hier sieht man den umgekehrten Effekt des Blasenkollaps: durch die sich entfernende Blasenwand wird ein Bereich geringer Dichte erzeugt, der umso ausgeprägter ist, je langsamer die Teilchen sind.

Die Segregation des Blaseninneren wird in Abb. 5.15 ,e\&f dargestellt. Das Verhältnis des Teilchenzahlanteil des Wasserdampfes $M F_{H_{2} \mathrm{O}}$ ('molar fraction', $M F_{\mathrm{H}_{2} \mathrm{O}}=$ $\mathrm{N}_{\mathrm{H}_{2} \mathrm{O}} / N_{\text {ges }}$ ) im Zentrum und am Blasenrand gibt an, in welchem Maße sich Edelgas und Wasserdampf entmischt haben. Am Anfang des Kollaps, bei niedrigen Wandgeschwindigkeiten, sind beide Spezies noch annähernd gleich verteilt. Eine Entmischung setzt zuerst bei der Xenon-Rechnung, ca. 130 ns vor $t_{\text {Rmin }}$, ein. Die langsamen Xenon-Atome sammeln sich deutlich früher an der Blasenwand als die schnelleren $\mathrm{H}_{2} \mathrm{O}$-Moleküle. Dieser Effekt verstärkt sich bis kurz vor dem Erreichen des minimalen Blasenradius. Zu diesem Zeitpunkt beträgt $M F_{\mathrm{H}_{2} \mathrm{O}} \mathrm{im}$ 

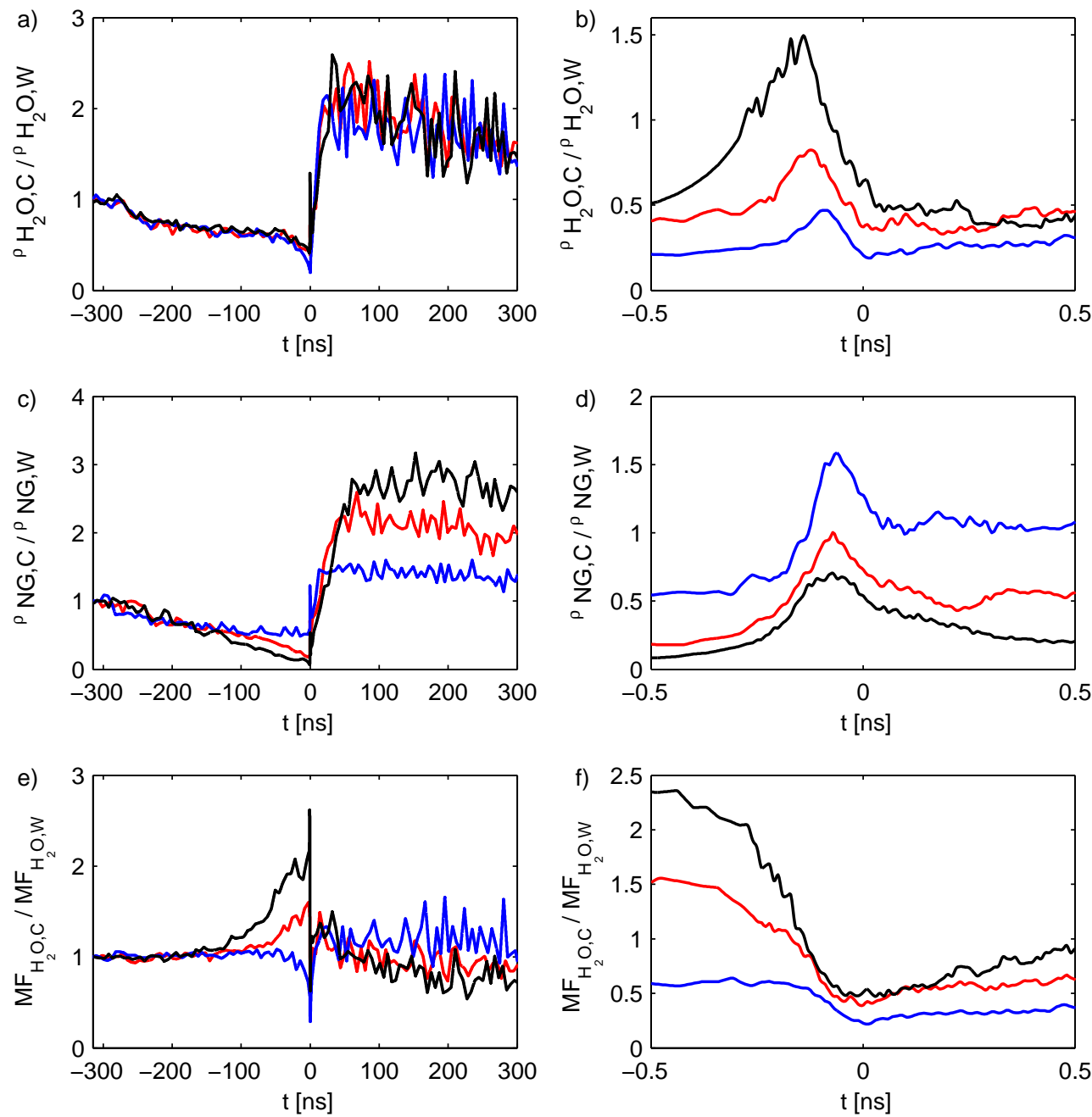

Abbildung 5.15: (a,b): Verhältnis der Dichte des Wasserdampfes in Blasenzentrum und Blasenrand. (c,d): Verhältnis der Dichte des Edelgases in Blasenzentrum und Blasenrand. (e,f): Verhältnis des Teilchenanzahlverhältnisses von $\mathrm{H}_{2} \mathrm{O}$ in Blasenzentrum und Blasenrand. Parameter siehe Tab. 5.5.

Verwendetes Edelgas: He (-); Ar (-); Xe (-).

Zentrum das knapp 2,5-fache des Wertes am Blasenrand. Ca. 500 ps vor $t_{R m i n}$ fällt der Quotient stark ab und erreicht in seinem Minimum bei $t_{R m i n}$ nur noch den Wert 0,5. Dies resultiert sowohl aus der Diffusion aufgrund der auslaufenden Verdichtungswelle, als auch aus den einsetzenden chemischen Reaktionen, die die Anzahl der Wasserdampfteilchen stark herabsetzen. Ungefähr die Hälfte aller $\mathrm{H}_{2} \mathrm{O}$-Moleküle wird dissoziiert. Im Anschluß an die heiße Phase pendelt sich der Quotient auf einen Wert um 1 ein, die Segregation ist also während der 
Aufschwungphase niedrig.

Für Argon ergibt sich ein ähnlicher Verlauf. Die Segregation setzt später ein (ca. $90 \mathrm{~ns}$ vor $\left.t_{R \min }\right)$ und ist weniger stark ausgeprägt. Der Abfall des Quotienten fällt weniger stark aus, da durch die insgesamt niedrigeren Temperaturen weniger $\mathrm{H}_{2} \mathrm{O}$-Moleküle dissoziiert werden (ca. $37 \%$ ).

Im Helium-Fall setzt die Segregation nochmals später ein (ca. 30 ns vor $t_{R m i n}$ ). Der Quotient ist in dieser Phase kleiner 1, in diesem Fall findet also eine umgekehrte Entmischung statt, da das Edelgas die leichtere Komponente darstellt. Der Quotient erreicht kurz vor $t_{R m i n}$ einen Wert von ca. 0,6 und wird durch chemische Reaktionen sowie die auslaufenden Verdichtungswelle auf ca. 0,22 gedrückt. Ungefähr $25 \%$ des Wasserdampfes werden dissoziiert.
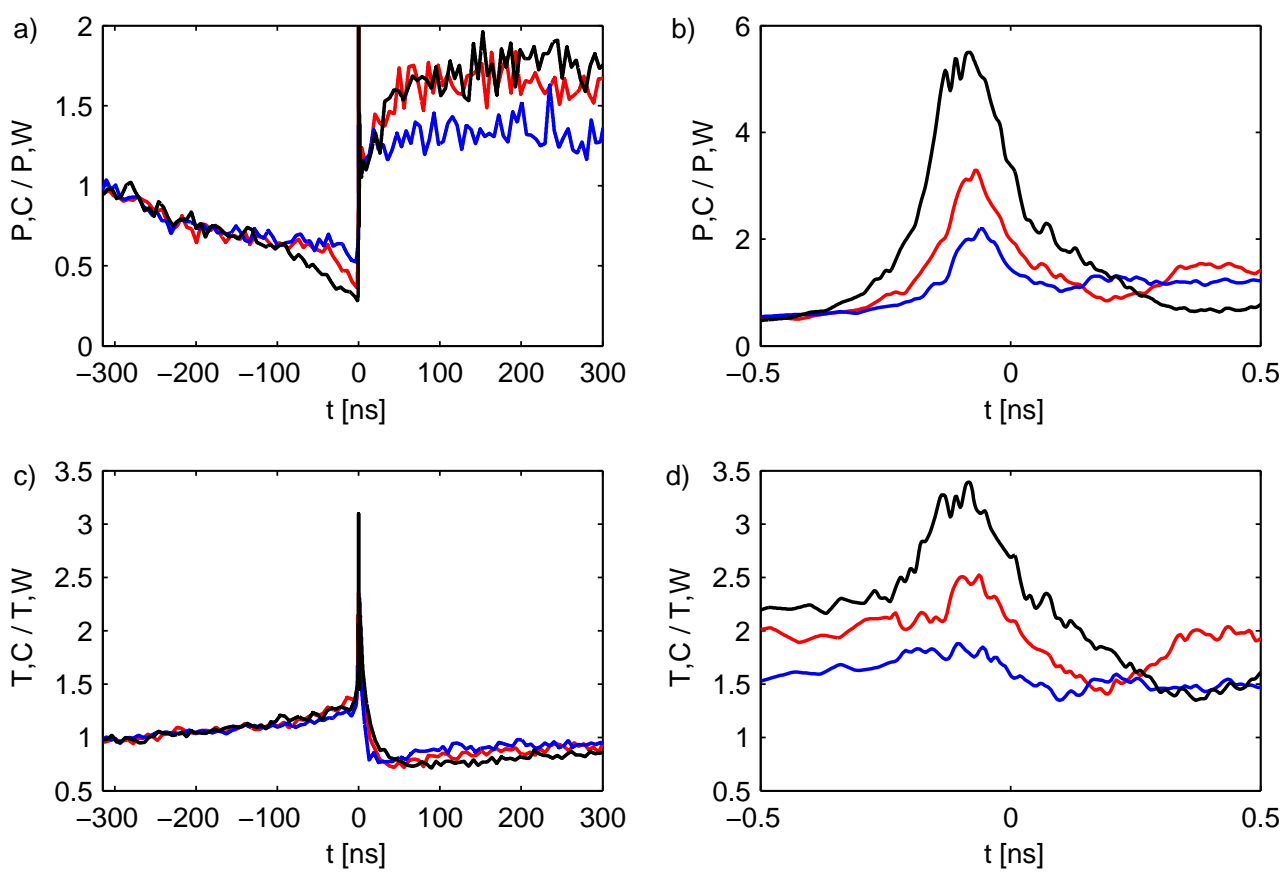

Abbildung 5.16: (a,b): Verhältnis des Druckes im Blasenzentrum zum Druck am Blasenrand (c,d): Verhältnis von Temperatur im Blasenzentrum zu Temperatur am Blasenrand. Parameter siehe Tab. 5.5.

Verwendetes Edelgas: Ar (-); He (-); Xe (-).

Zusammenfassend läßt sich sagen, dass eine Segregation der Blasenbestandteile schon während des langsamen Teils des Kollaps geschieht. Die Entmischung wird durch die kurz vor Erreichen des minimalen Blasenradius ins Blasenzentrum laufende Verdichtungswelle noch verstärkt. Nach diesem Maximum der Segregation 
wird sie durch Reflektion der Verdichtungswelle sowie die einsetzenden chemischen Reaktionen schnell wieder abgebaut und ist in der expansiven Phase nicht erkennbar.

Der die Segregation bewirkende Effekt ist vermutlich die Druck-Diffusion. Dies bezeichnet die Tendenz, dass in einem Teilchengemisch welches einem Druckgefälle ausgesetzt ist, leichte Teilchen in Bereiche niedrigen Druckes getrieben werden, schwere Teilchen entsprechend in Bereiche hohen Druckes. Ein weiterer Effekt, der die Segregation beeinflussen könnte, ist die Thermo-Diffusion. Dies bezeichnet das Phänomen, dass in einem Teilchengemisch, welches einem Temperaturgradienten ausgesetzt ist, leichte Teilchen in Bereiche hoher Temperatur getrieben werden.

Abb. 5.16 stellt die Entwicklung des Druck- und Temperaturgradienten in der Blase dar. Es ist zu erkennen, dass sich relativ schnell ein vom Blasenrand zum Blasenzentrum abfallender Druckgradient einstellt, welcher ab ca. 100 ns vor $t_{R m i n}$ deutliche Unterschiede zwischen den Rechnungen aufweist. Bei Xenon ist er am stärksten ausgeprägt, bei Helium am schwächsten. Mit dem Einlaufen der Verdichtungswelle dreht sich der Gradient um, nun ist der Druck im Zentrum am größten (Abb. 5.16,c). Danach fällt der Druckgradient schnell wieder ab, um während der Expansionsphase auf einen relativ stabilen Wert zu wachsen.

Der Temperaturgradient bildet sich bedeutend im Vergleich langsamer aus und läßt bis kurz vor Erreichen von $t_{R m i n}$ keine Unterschiede zwischen den Rechnungen erkennen. Im Maximum ist der Gradient sehr ausgeprägt (aber schwächer als der Dichtegradient). Die unterschiedliche Dynamik der Verdichtungswelle, je nach Teilchengewicht, läßt sich gut erkennen. Der Temperaturgradient wird nach dem Kollaps schnell wieder abgebaut.

Die Zeitskala der Spezies-Segregation ähnelt derjeniger der Entwicklung des Druckgradienten. Die Verteilung des Edelgases und des Wasserdampfes in der Blase (Abb. 5.15,a\&c) paßt sich in Phasen langsamer Blasenrandbewegung dem Druckgradienten (Abb. 5.16,a) an. Die Änderungen des Temperaturgradienten spielen sich auf einer deutlich kleineren Zeitskala ab. Der Effekt der DruckDiffusion ist also vermutlich für den langsamen Aufbau der Segregation verantwortlich. In der heißen Phase des Kollaps, mit einer schnell laufenden Verdichtungswelle wirken Druck- und Thermo-Diffusion zunächst in eine Richtung (einlaufende Verdichtungswelle), später entgegengesetzt (auslaufende Verdichtungswelle). Auch auf dieser kurzen Zeitskala scheint die Druck-Diffusion zu überwiegen, da mit dem Auslaufen der Welle eine deutliche Abnahme der Segregation zu 
beobachten ist.

Es ist erwähnenswert, dass das unterschiedliche Segregationsverhalten praktisch ausschließlich auf einer unterschiedlichen Verteilung der Edelgase beruht. In den langsamen Phasen des Kollaps ist die Verteilung des Wasserdampf für alle Rechnungen nahezu gleich. Lediglich in dem von durch die Blase laufenden Verdichtungswellen gekennzeichneten Ende des Kollaps ergeben sich nennenswerte Unterschiede zwischen den verschiedenen Fällen. Es scheint, dass die Dichte des Edelgases nur in den Phasen extremer Kompression groß genug ist, die Bewegung des Wasserdampfes nennenswert zu beeinflussen.

\subsubsection{Edelgasgemische}

In diesem Abschnitt sollen Segregationseffekte beim Vorhandensein mehrerer Edelgase untersucht werden. Dazu wurden Rechnungen mit einem 1:1Teilchenzahlverhältnis von Argon und Helium, Argon und Xenon sowie Xenon und Helium durchgeführt. Die restlichen Blasenparameter entsprechen denjenigen aus Tab. 5.5.

Tab. 5.9 fasst die Ergebnisse dieser Rechnungen für Hydrodynamik, Blasendynamik, chemische Entwicklung sowie Lichtemission zusammen. Die im vorigen Kapitel gefundenen Ergebnisse finden sich hier zumeist wieder: es ist mit wachsender Edelgasmasse eine Zunahme der Maximaltemperatur, der Durchschnittstemperatur, des Minimalradius, der Lichtemission und der chemischen Aktivität sowie einer Abnahme der Blasenwandgeschwindigkeit und des maximalen Blaseninnendruckes zu verzeichnen. Die erreichten Werte reihen sich zwischen den Werten der Rechnungen mit nur einem Edelgas ein. Bei fast allen beobachteten Messwerten ergibt sich eine klare ab- oder aufsteigende Tendenz nach der durchschnittlichen Masse des verwendeten Edelgases, unabhängig von einer eventuellen Mischung verschiedener Gase (siehe auch Tab. 5.7).

\begin{tabular}{|c|c|c|c|c|c|c|c|c|c|c|c|c|c|}
\hline $\begin{array}{l}\text { Nr. } \\
-\end{array}$ & $\begin{array}{l}\text { NG1 } \\
-\end{array}$ & $\begin{array}{l}\mathrm{NG} 2 \\
{[\mathrm{~K}]}\end{array}$ & $\begin{array}{l}T_{\max } \\
{[\mathrm{K}]}\end{array}$ & $\begin{array}{l}T_{\varnothing} \\
{[\mu \mathrm{m}]}\end{array}$ & $\begin{array}{l}R_{\text {min }} \\
-\end{array}$ & $\begin{array}{l}K \\
{[\mathrm{~km} / \mathrm{s}]}\end{array}$ & $\begin{array}{l}v_{W} \\
{[\mathrm{GPa}]}\end{array}$ & $\begin{array}{l}p_{W} \\
{\left[10^{9}\right]}\end{array}$ & $\begin{array}{l}\mathrm{OH} \\
{\left[10^{9}\right]}\end{array}$ & $\begin{array}{l}\mathrm{O}_{2} \\
{\left[10^{9}\right]}\end{array}$ & $\begin{array}{l}\mathrm{H}_{2} \mathrm{O} \\
{[\mathrm{pJ}]}\end{array}$ & $\begin{array}{l}E_{S L} \\
{[\mathrm{ps}]}\end{array}$ & $\Delta t_{S L}$ \\
\hline 4 & $\mathrm{Ar}$ & $\mathrm{He}$ & 15199 & 11113 & 0.720 & 59,4 & 1,70 & 3.73 & 1,37 & 0.91 & 8.76 & 0,016 & 95 \\
\hline 5 & $\mathrm{Xe}$ & $\mathrm{He}$ & 17689 & 12409 & 0.730 & 58,6 & 1,49 & 3.40 & 1,62 & 1.30 & 8.79 & 0,051 & 110 \\
\hline 6 & $\mathrm{Xe}$ & $\mathrm{Ar}$ & 20498 & 13419 & 0.770 & 55,6 & 1,36 & 3.05 & 1,66 & 1.32 & 8.67 & 0,128 & 125 \\
\hline
\end{tabular}

Tabelle 5.9: Ergebnisse der Rechnungen mit zwei Edelgasen (NG1 und NG2), Teilchenanzahlverhältnis 1:1. Zum Vergleich mit Rechnungen unter Verwendung eines einzelnen Edelgases sowie Parametererklärungen: siehe Tab. 5.7. 

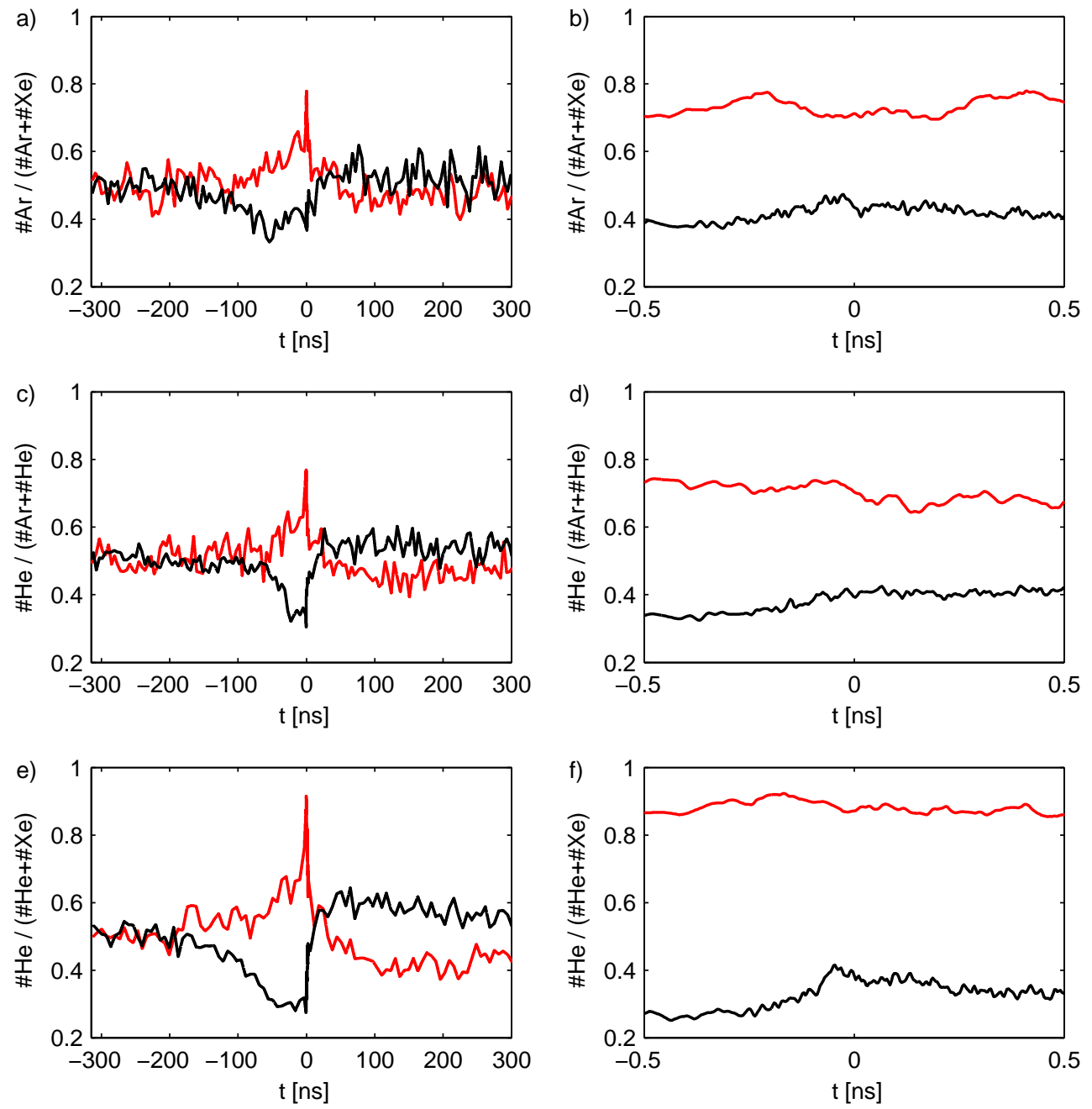

Abbildung 5.17: Segregation der Edelgase. Dargestellt ist jeweils das Verhältnis der Atome des leichteren Edelgases zur Gesamtzahl der Edelgasatome. Vergleich zwischen dem Blasenrand (-) und dem Blasenzentrum (-) für die Edelgasgemische Argon-Xenon (a,b), Argon-Helium (c,d), Xenon-Helium (e,f). Parameter siehe Tab. 5.5.

Ein gewisser Effekt der Entmischung läßt sich am Vergleich der Rechnung einer Xenon-Helium-Mischung zur Rechnung mit reinem Argon feststellen. Die Mischung weist ein höheres durchschnittliches Atomgewicht (67,6 u) als Argon $(39,995 \mathrm{u})$ auf. Der allgemeinen Tendenz entsprechend werden im erstgenannten Fall höhere Durchschnittstemperaturen (12409 K vs. 12269 K) erzielt. Die absolut maximale Temperatur (im Blasenzentrum) liegt allerdings niedriger als bei der reinen Argon-Rechnung (17869 K vs. 18870 K) - vermutlich ein Effekt der hohen Konzentration an Helium-Teilchen im Blasenzentrum (siehe unten, Abb. 5.17,f), welche durch ihre hohe Teilchengeschwindigkeit extremen Temperaturen 
entgegenwirken. Die höheren Teilchengeschwindigkeiten können auch die leicht gesunkene Leuchtdauer (110 ps vs. 120 ps) erklären.

Abb. 5.17 zeigt die Entmischung der beteiligten Edelgase vom Start der Simulation bis 300 ns nach dem Kollaps (linke Spalte) sowie in der Vergrößerung von 1 ns um $t_{R \min }$ (rechte Spalte). Wasserdampf und dessen chemische Produkte werden nicht erfaßt, dargestellt ist das Anzahlverhältnis des jeweils leichteren Edelgases zur Gesamtedelgasanzahl - jeweils für das Blasenzentrum und den Blasenrand. Deutlich ist zu erkennen, dass bereits im langsamen Teil des Kollaps ein Übergewicht leichter Spezies im Blasenzentrum entsteht, während am Blasenrand schwere Spezies angesammelt werden. Diese Tendenz setzt umso früher ein, je größer der Massenunterschied $(\Delta m)$ der betrachteten Edelgase ist: Bei Xenon und Helium $(\Delta m=127,19 \mathrm{u})$ ist ab ca. $200 \mathrm{~ns}$ vor $t_{R \min }$ ein Gradient im Teilchenanzahlverhältnis sichtbar, bei Argon und Xenon $(\Delta m=91,34 \mathrm{u})$ tritt dieser ca. $150 \mathrm{~ns}$ vor $t_{R m i n}$ auf, bei Helium und Argon $(\Delta m=35,95 \mathrm{u})$ ab ca. 110 ns vor $t_{R m i n}$. Die Stärke des Gradienten nimmt bis zum Erreichen des minimalen Blasenradius zu und kehrt sich danach schnell um. In der Zeit $t_{\text {Rmin }}+/-$ $0,5 \mathrm{~ns}$ - ein Zeitraum, in dem sich die Zentrumstemperatur fast verfünffacht und danach wieder abfällt - ändert sich die Segregation der Spezies kaum. Bei der Helium-Xenon-Mischung sind in diesem Zeitraum neun von zehn Edelgasatomen im Blasenzentrum Helium-Atome.

In Abb. 5.18 ist für zwei Mischungen (Helium-Xenon und Helium-Argon) das Verhältnis der Dichte im Blasenzentrum zur Dichte am Blasenrand dargestellt, aufgeschlüsselt nach Wasserdampf, leichtem Edelgas und schwerem Edelgas. Der Verlauf der Kurven für Wasserdampf und das leichte Edelgas (Helium) ist für beide Rechnungen nahezu identisch, ausgenommen eines kurzen Zeitraums um $t_{\text {Rmin }}$ (rechte Spalte). Die Graphen des schweren Edelgases zeigen erwartungsgemäß Unterschiede zwischen den Rechnungen auf, da es sich um verschiedene Gase handelt (Argon bzw. Xenon). Für alle betrachteten Gase bildet sich im Kollaps zunehmend ein Dichtegradient von der Blasenwand zum Blasenzentrum aus. Je schwerer das betrachtete Gas, umso ausgeprägter ist der Dichteunterschied.

Im Falle von Wasserdampf wird die Ausbildung des Gradienten zunächst durch eine Kondensation der Wasserdampfteilchen an der Blasenwand verlangsamt. In der letzten Phase des Kollaps, wenn sich die Temperatur der Blasenwand erhöht und eine Verdampfung einsetzt, verstärkt sich der Gradient stark. Die leichten Helium-Atome sind schnell genug, um sich von der kollabierenden Blasenwand zu entfernen, während Argon und vor allem Xenon zu langsam sind, um von der Bla- 

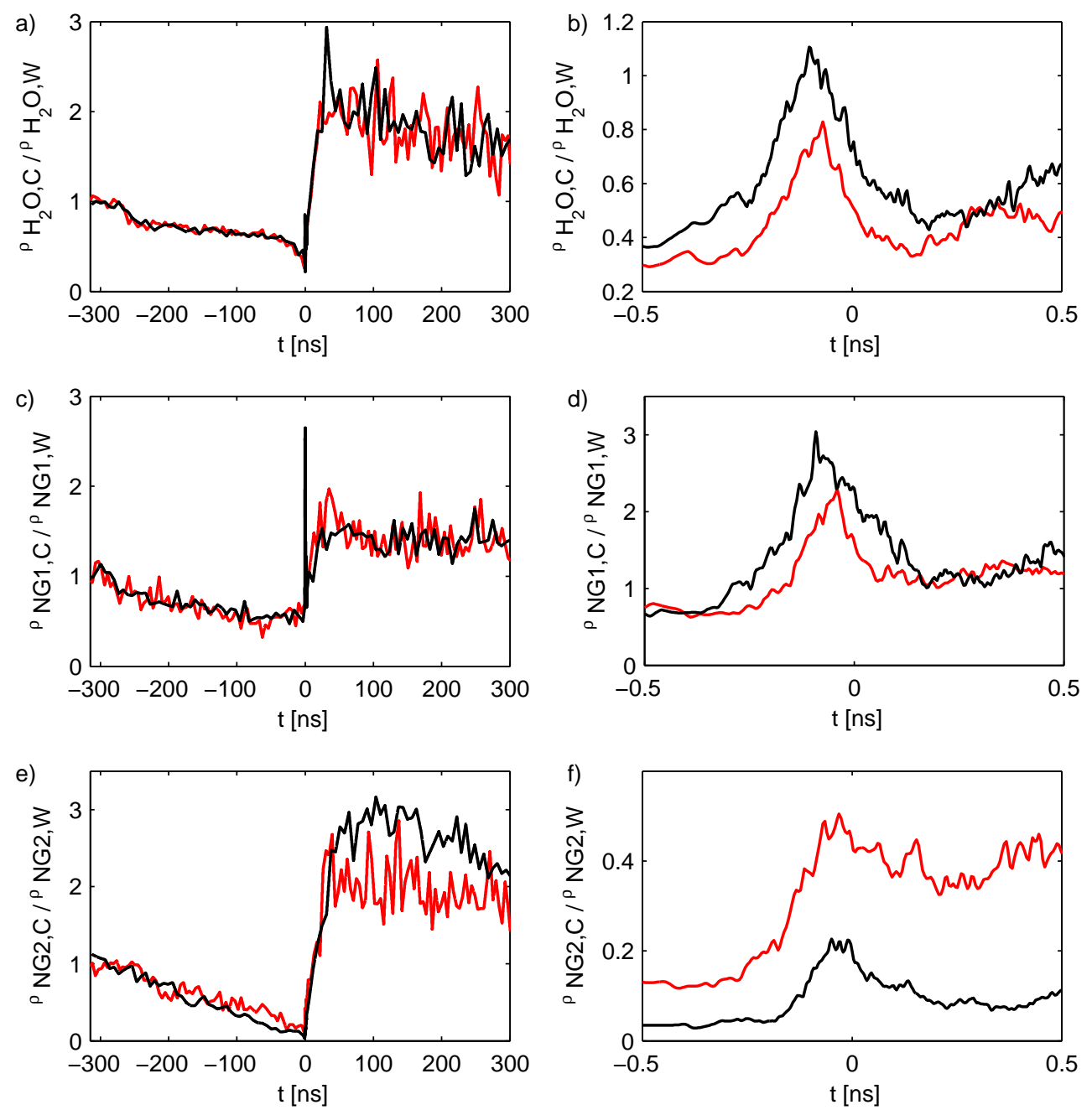

Abbildung 5.18: Dichteverhältnis zwischen Blasenzentrum und Blasenwand für die Rechnungen mit 1:1-Mischungen von Helium-Xenon (-) sowie Helium-Argon (-). (a,b): Wasserdampf; (c,d): das leichte Edelgas (jeweils Helium); (e,f): das schwere Edelgas (— : Xenon, — : Argon). Parameter siehe Tab. 5.5 .

senwand ins Blasenzentrum zu gelangen und den Gradienten auszugleichen. Bei der Xenon-Helium-Mischung herrscht gegen Ende des Kollaps an der Blasenwand ungefähr die 30-fache Xenon-Dichte, verglichen mit dem Blasenzentrum.

Der in beiden Mischungen nahezu identische Kurvenverlauf für Wasserdampf und Helium zeigt, dass in Phasen geringer Dichte in der Blase die Zusammensetzung des Gasgemisches einen geringen Einfluß auf die räumliche Verteilung der einzelnen Komponenten hat. Auftretende Inhomogenitäten in der Dichte einer Spezies 
sind in deren Eigenschaften (hauptsächlich der Masse) begründet, nicht in der Interaktion mit Teilchen einer anderen Spezies.

In den Phasen großer Dichte in der Blase, gegen Ende des Hauptkollaps, ändert sich dies. Durch die Herabsetzung der mittleren freien Weglänge kollidieren die Teilchen bedeutend häufiger, wodurch Effekte der Druck-Diffusion (siehe Kap. 5.3.1) sichtbar werden. Je größer das Massenverhältnis zwischen den vorhandenen Spezies, umso mehr leichte Teilchen sammeln sich während der einlaufenden Verdichtungswelle im Blasenzentrum. Dies erklärt die größeren Dichten von Wasserdampf und Helium im Blasenzentrum bei der Helium-Xenon-Mischung, verglichen mit der Mischung aus Helium und Argon (Abb. 5.18,b\&d). Das schwere Edelgas wird in dieser Phase ebenfalls tendenziell zum Blasenzentrum getrieben, erreicht dort aber nie die Dichten vom Blasenrand (Abb. 5.18,f). Auffallend ist, dass sowohl für Argon als auch für Xenon deutlich niedrigere Werte erreicht werden, verglichen zu den Rechnungen, in denen diese das jeweils einzige Edelgas darstellen (vgl. Abb. 5.15,d). Das Vorhandensein des sehr leichten Helium schirmt anscheinend das Blasenzentrum besser gegen das schwere Edelgas ab, als Wasserdampf alleine dies tut.

In der folgenden Phase des Blasenwachstums drehen sich die Dichtegradienten um. Die Unterschiede im Wasserdampf- und Helium-Dichtegradienten zwischen den verschiedenen Rechnungen gleichen sich schnell aus. Je schwerer und langsamer das Gas, umso ausgeprägter ist der Gradient auch in dieser Phase. Durch die weitestgehend unbeeinflußte Verteilung des Wasserdampfes in der Blase ist eine Beeinflussung des Wasserdampfgehaltes gegen Ende des Kollaps nicht zu beobachten. Alle Rechnungen weisen eine vergleichbare Anzahl an $\mathrm{H}_{2} \mathrm{O}$-Partikeln zu diesem Zeitpunkt aus (nicht gezeigt).

Der Vergleich mit den Ergebnissen eines kontinuumsmechanischen Modells in [133] zeigt, dass beide Modelle ähnliche Ergebnisse liefern. Für eine HeliumXenon-Mischung sagt das kontinuumsmechanische Modell ebenfalls eine sich langsam aufbauende Segregation vorher, welche sich in der heißen Phase kaum ändert, danach abgebaut wird und sich in der Aufschwungphase umkehrt. Die Entmischung setzt allerdings im Vergleich zum hier besprochenen Modell später ein (ca. 60 ns vor Erreichen des minimalen Radius). Die maximale Segregation ist geringer als hier beobachtet, baut sich dafür aber deutlich langsamer wieder ab. Die Nichtberücksichtigung von Wasserdampf und chemischen Reaktionen in [133] kann für einige dieser Unterschiede verantwortlich sein. 


\section{Kapitel 6}

\section{Ergebnisse für Mikro-Blasen}

In diesem Teil der Arbeit werden Ergebnisse von Simulationen sehr kleiner Blasen dargestellt, die mit hohen Drücken und Frequenzen angeregt werden.

Wie schon in Kap. 4.4 besprochen ist das Verhältnis $S=N_{\text {real }} / N_{\text {sim }}$ zwischen

realer und simulierter Teilchenzahl ein Maß für die Über- oder Unterschätzung diffusiver Prozesse, da die mittlere freie Weglänge $l$ der Teilchen mit $S^{1 / 3}$ skaliert. Im Sinne einer möglichst exakt abbildenden Simulation ist es erstrebenswert, $S$ zu minimieren. Dies kann zum Einen, wie in Kap. 4.4 geschehen, über eine Erhöhung von $N_{\text {sim }}$ realisiert werden. Eine andere, weniger rechenintensive und zugleich interessante Möglichkeit ist die Simulation kleinerer Blasen, also eine Verkleinerung von $N_{\text {real }}$.

Typische SBSL-Blasen, die mit Frequenzen im Bereich 20 - $40 \mathrm{kHz}$ angeregt werden, haben einen Ruheradius von $R_{0} \approx 3-6 \mu \mathrm{m}$ [9]. Dies entspricht einer Anzahl an Edelgasatomen im Bereich von $10^{10}$ bis $10^{11}$ und führt auf Skalierungen, je nach $N_{\text {sim }}$, von $S=40.000$ bis $\mathrm{S}=500.000$.

Beim Übergang zu deutlich kleineren Blasen lassen sich hingegen Skalierungsfaktoren hinunter bis zu $S=1$ realisieren. Um solch kleine Blasen ( $R_{0}$ minimal $70 \mathrm{~nm}$ ) zu nichtlinearen Oszillationen anzuregen, bei denen Lichtemission stattfindet, sind hohe Anregungsfrequenzen und -drücke notwendig. Die für die Blasenoszillation kritische Blake-Schwelle [134] verschiebt sich mit sinkendem $R_{0} \mathrm{zu}$ höheren Drücken, der Einfluß der Oberflächenspannung steigt an.

Für die Simulation dieser Mikro-Blasen wurden Experimente von WENINGER, VAzQuez und CAmara zum Vorbild genommen, die Blasen bei Anregungsfrequenzen von $\nu_{a}=1 \mathrm{MHz}$ und $\nu_{a}=11 \mathrm{MHz}$ erzeugt haben [135][136][137]. Die entstehenden Blasen bildeten dichte Wolken transienter MBSL-Blasen, die stark lumineszierten. 


\subsection{1-MHz-Blasen}

Eine Untersuchung von MBSL - Blasen, die mit einer Frequenz von $\nu_{a}=$ $1 \mathrm{MHz}$ angeregt werden, wurde von Weninger und CAmara et. al durchgeführt [135][137]. Der Ruheradius der Blasen wurde von Camara als zwischen 300 und $600 \mathrm{~nm}$ liegend abgeschätzt. Der Anregungsdruck betrug zwischen 4 und 5 bar. In [137] vermuten die Autoren aufgrund des auftretenden Breitbandspektrum sehr hohe Temperaturen (ca. 500.000 K) im Inneren der Blase.

In der vorliegenden Arbeit wurden diverse Parameter-Kombinationen der in [137] untersuchten 1- MHz-Blasen simuliert und die Ergebnisse teilweise mit den experimentell gefundenen verglichen. Die variierten Parameter waren: der Ruheradius $R_{0}$ (Rechnungen mit $R_{0}=300 \mathrm{~nm}, R_{0}=450 \mathrm{~nm}$ und $R_{0}=600 \mathrm{~nm}$ ), der Anregungsdruck $P_{a}$ (variiert zwischen $P_{a}=4$ bar, $P_{a}=4,5$ bar und $P_{a}=5$ bar) sowie das verwendete Edelgas (Xenon, Argon oder Helium). Desweiteren wurde für zwei Fälle mit größeren Werten der Akkomodationskoeffizienten $\left(A_{v}=1,0\right.$ statt 0,3 und $\alpha_{t}=1,0$ statt 0,3$)$ gearbeitet, um den Einfluß dieser Parameter auch auf derart kleine Blasen zu erfassen (Überlegungen zu $A_{v}$ und $\alpha_{t}$ finden sich in Kap. 4.2.2, bzw. Kap. 4.1.2.1). Die Anfangsgeschwindigkeit $v_{\text {init }}$ wurde so gewählt, dass die anfängliche Gesamtteilchenzahl für alle Rechnungen ca. $N_{\text {ges }, i}$ $=700.000$ betrug. Die sonstigen Parameter der Rechnungen sind in Tab. 6.1 angegeben, weitere Angaben sowie ausgewählte Ergebnisse enthält Tab. 6.2. Die im Folgenden beschriebenen Abbildungen vergleichen den Einfluß der verschiedenen Parameter und beleuchten einige Aspekte genauer.
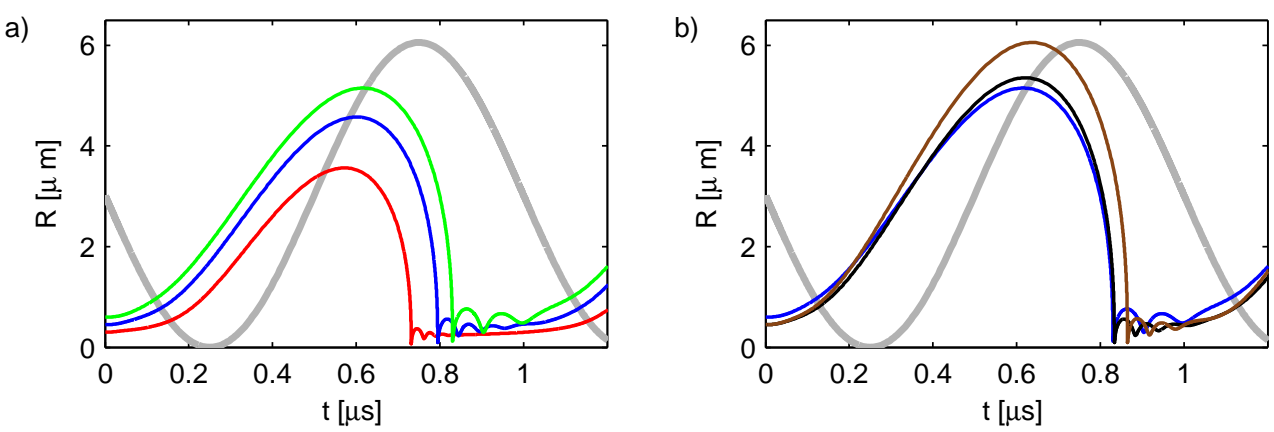

Abbildung 6.1: (a): Radiusverlauf für die Fälle 1, 2 und 3 aus Tab. 6.2; (b): Radiusverlauf für die Fälle 2, 4 und 5 aus Tab. 6.2. Berechnet mittels eines Kontinuumsmodells ohne Dynamik des Blaseninneren.

Fall 1(-): $R_{0}=300 \mathrm{~nm}, P_{a}=4$ bar ; Fall 2(一): $\mathrm{R}_{0}=450 \mathrm{~nm}, P_{a}=4$ bar; Fall $3(-): \mathrm{R}_{0}=$ $600 \mathrm{~nm}, P_{a}=4$ bar ; Fall 4(-): $R_{0}=450 \mathrm{~nm}, P_{a}=4,5$ bar ; Fall $5(-): R_{0}=450 \mathrm{~nm}, P_{a}=$ 5 bar ; $(-)$ : $\mathrm{P}_{a}(\mathrm{t})$ in a.u.. 
Abb. 6.1 zeigt zunächst den groben Radiusverlauf der Fälle 1-5 aus Tab. 6.2 für ca. eine Periode der Anregung. Dieser Radiusverlauf wurde in dem einfachen polytropischen Modell berechnet, das auch für die Bestimmung der Startbedingungen der MD-Simulation verantwortlich ist (siehe Kap. 3.3.2). Es ist zu erkennen, dass

\begin{tabular}{|c|c|c|c|c|c|c|c|}
\hline$P_{a}$ & $4,0-5,0$ bar & $N_{N G}$ & - & 343.000 & $v_{W, \text { init }}$ & - & $28-60 \mathrm{~m} / \mathrm{s}$ \\
\hline$\nu_{a}$ & $1,0 \mathrm{MHz}$ & $S$ & - & $49-224$ & $\alpha_{t}$ & - & $0,3,1,0$ \\
\hline$R_{0}$ & $300-600 \mathrm{~nm}$ & Edelgas & - & Argon, Xenon, Helium & $A_{v}$ & - & 0,$3 ; 1,0$ \\
\hline$T_{\text {water }}$ & $290 \mathrm{~K}$ & $\Delta K_{W}$ & - & 1.800 & & & \\
\hline
\end{tabular}

Tabelle 6.1: Parameter der Rechnungen von Blasen mit $1 \mathrm{MHz}$ Anregung. Weitere Angaben finden sich in Tab. 6.2.

ein größerer Ruheradius auch zu einem größeren maximalen Radius führt, genauso wie eine stärke Anregungsamplitude. Die Anzahl der Nachschwinger des Hauptkollaps ist deutlich geringer als bei einer im selben Modell gerechneten 26,5 kHz-Blase (siehe z.B. Abb 3.3). Insgesamt erscheint dieser Teil deutlich gedämpfter als bei größeren Blasen. Dies dürfte an der großen Oberflächenspannung liegen, die bei kleinen Blasen wirkt.
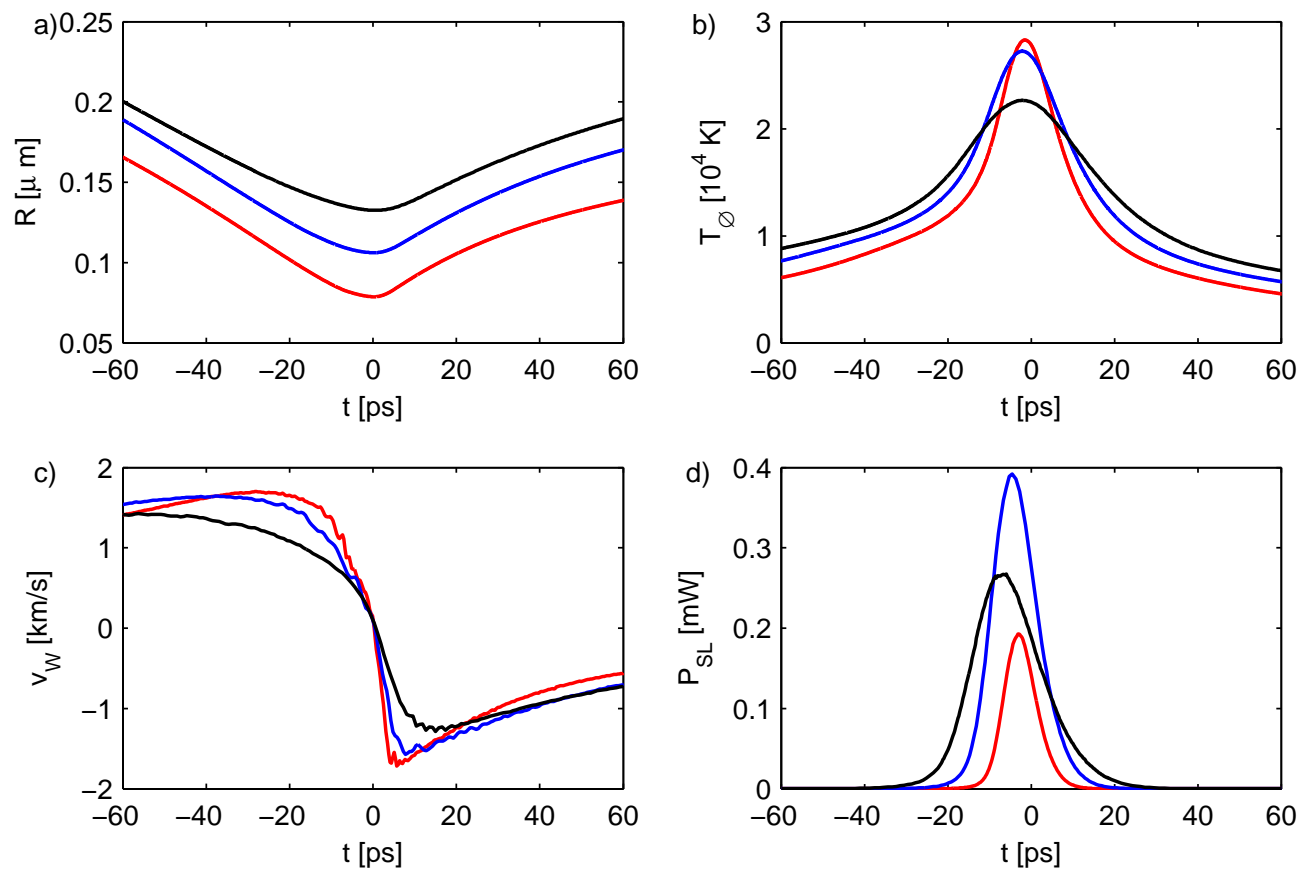

Abbildung 6.2: Verlauf des Blasenradius (a), der Durchschnittstemperatur (b), der Blasenwandgeschwindigkeit (c) und der Lichtemission der gesamten Blase (d) um den Hauptkollaps $(\mathrm{t}=0)$ für die Fälle 1, 2 und 3 aus Tab. 6.2. Sonstige Parameter siehe Tab. 6.1.

Fall $1(-): R_{0}=300 \mathrm{~nm}$; Fall 2(-): $\mathrm{R}_{0}=450 \mathrm{~nm}$; Fall $3(-): \mathrm{R}_{0}=600 \mathrm{~nm}$.

Abb. 6.2 zeigt den Vergleich der molekulardynamisch simulierten Blasen mit va- 
riiertem Ruheradius (Fälle 1 bis 3 aus Tab. 6.2). Es wird deutlich, dass der aus einem größeren Ruheradius folgende größere Maximalradius nicht zu einem stärkeren Kollaps führt. Die Blase mit $\mathrm{R}_{0}=300 \mathrm{~nm}$ (Fall 1) kollabiert auf den kleinsten Minimalradius und weist das größte Kompressionsverhältnis auf ( $K=$ 45,2 - verglichen mit $K=39,3$ für Fall 3). Der Grund hierfür liegt in dem deutlich mit dem Ruheradius ansteigenden Wasserdampfgehalt. In Fall 3 sind gegen Ende des Kollaps noch 24,2·10 $\mathrm{H}_{2} \mathrm{O}$-Teilchen in der Blase, im Fall 1 sind es lediglich $5,9 \cdot 10^{6}$. Dementsprechend sinken auch die erreichten Maximaltemperaturen mit dem Ruheradius: im Blasendurchschnitt von 28.290 K für Fall 1 auf $22.650 \mathrm{~K}$ im Fall 3. Die lokal höchsten Temperaturen sinken noch stärker: von $68.300 \mathrm{~K}$ auf $49.300 \mathrm{~K}$.

Sinkende Temperaturen bedeuten in diesem Fall nicht eine sinkende Lichtleistung. Mit $P_{a}$ steigt auch der Durchmesser der Licht emittierenden Region $d_{S L}$ sowie die Dauer des Leuchtens $\Delta t_{S L}$ an: $d_{S L}$ beträgt im ersten Fall 111 nm, im zweiten $147 \mathrm{~nm}$, im dritten sind es $176 \mathrm{~nm}$. $\Delta t_{S L}$ nimmt von 9 ps auf $19 \mathrm{ps}$ zu. Diese Effekte kompensieren die deutlich stärkere Leuchtdichte im Blasenzentrum bei niedrigem $R_{0}$. Die gesamt emittierte Lichtleistung $E_{S L}$ steigt von 1,9 fJ für $\mathrm{R}_{0}$ $=300 \mathrm{~nm}$ über 5,3 fJ für $\mathrm{R}_{0}=450 \mathrm{~nm}$ auf 5,6 fJ für $\mathrm{R}_{0}=600 \mathrm{~nm}$. Abb. 6.3 verdeutlicht den Effekt der Größe der Licht emittierenden Region für den Zeitpunkt der maximalen Emission.
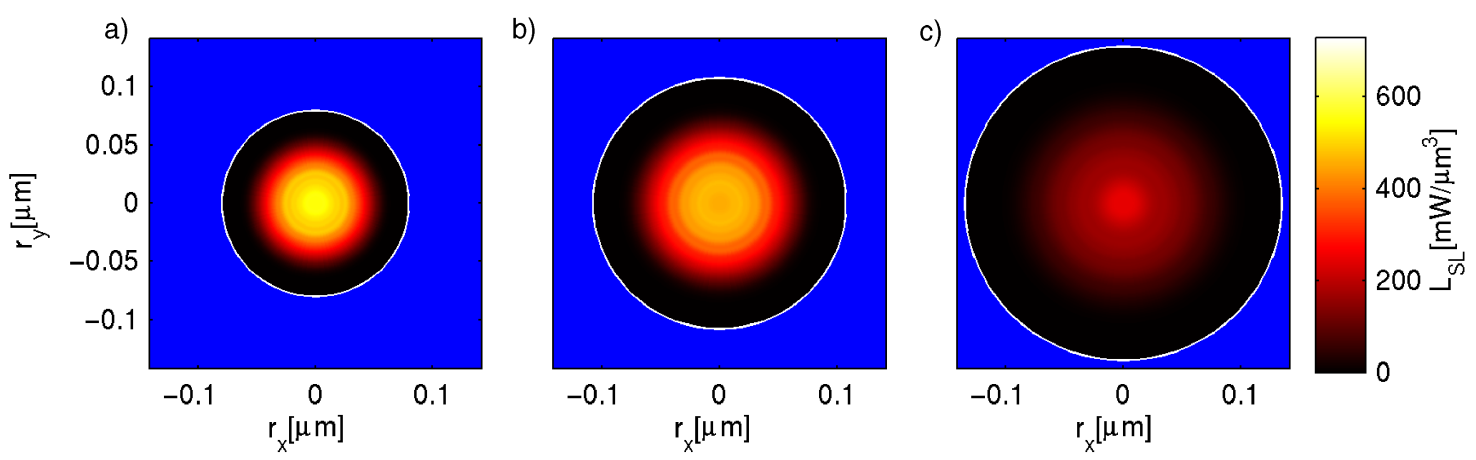

Abbildung 6.3: Lichtemission zum Zeitpunkt der maximalen Leuchtstärke für Fall 1, 2 und 3 aus Tab. 6.2. $\mathrm{R}_{0}=300 \mathrm{~nm}(\mathrm{a}), \mathrm{R}_{0}=450 \mathrm{~nm}$ (b) und $\mathrm{R}_{0}=600 \mathrm{~nm}$ (c).

Abb. 6.4 vergleicht Ergebnisse der Fälle 2, 4 und 5 aus Tab. 6.2, also Rechnungen einer Blase mit $\mathrm{R}_{0}=450 \mathrm{~nm}$ und in 3 Stufen variiertem Anregungsdruck $\left(P_{a}\right.$ $=4 / 4,5 / 5$ bar). Aus Abb. 6.1 ist zu erkennen, dass die Blasen umso weiter aufschwingen, je höher sie angeregt werden $\left(R_{\max }=6,01 \mu \mathrm{m}\right.$ für Fall 5 , verglichen mit $R_{\max }=4,57 \mu \mathrm{m}$ für Fall 2). In Abb. 6.4,a sieht man, dass in allen drei Fällen 
die Blasen auf einen vergleichbaren Minimalradius komprimiert werden, die Fälle mit höherem $P_{a}$ ein wenig mehr $\left(R_{\min }=101 \mathrm{~nm}\right.$ für Fall $5, R_{\min }=106 \mathrm{~nm}$ für Fall 2). Der Kollaps der Blasen mit großer Anregung verläuft also deutlich stärker (der Kompressionsfaktor $K$ steigt von 43,1 über 52,0 auf 59,5 für die betrachteten Fälle). Dieses Ergebnis zeichnet ein anderes Bild, als das in Kap. 5.2
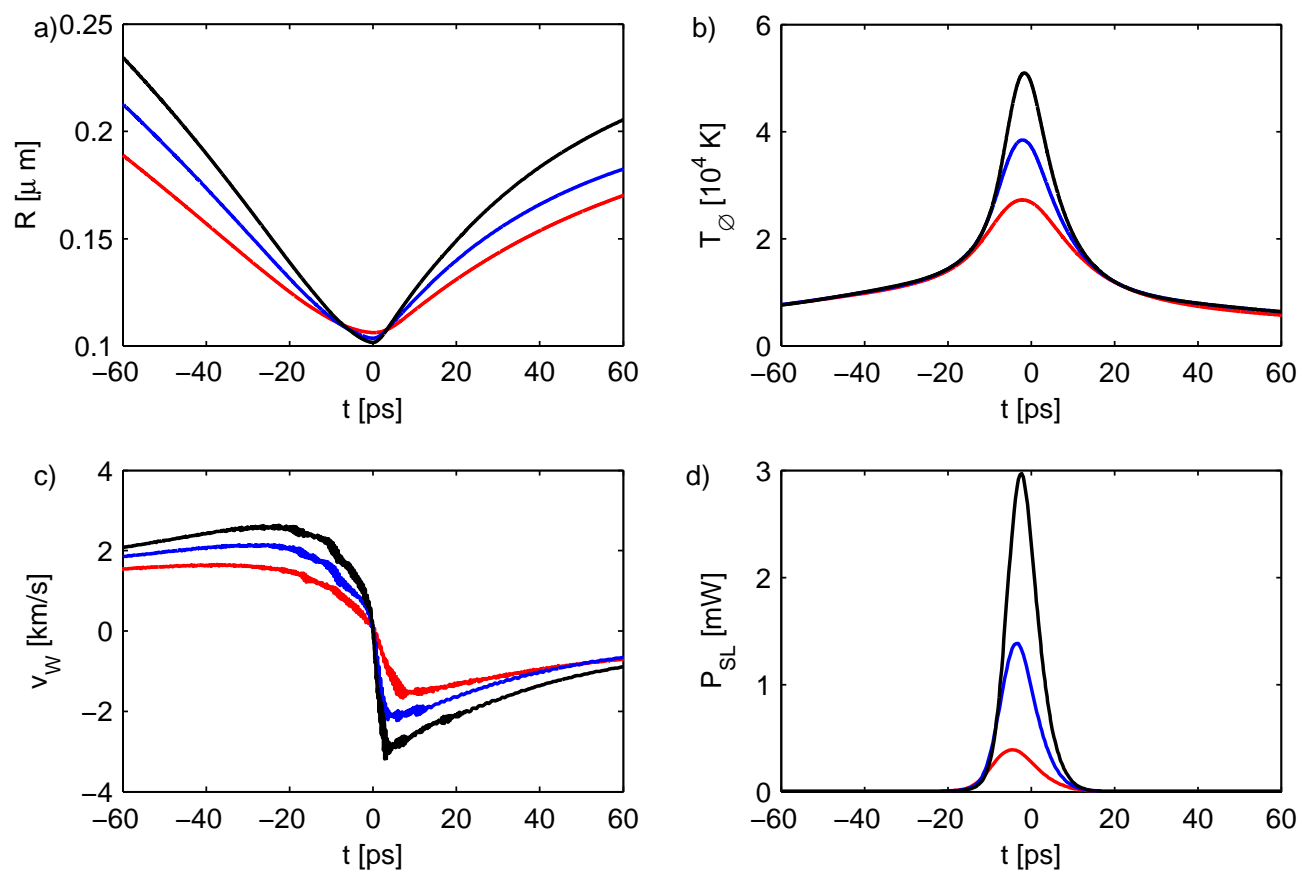

Abbildung 6.4: Verlauf des Blasenradius (a), der Durchschnittstemperatur (b), der Blasenwandgeschwindigkeit (c) und der Lichtemission der gesamten Blase (d) um den Hauptkollaps $(\mathrm{t}=0)$ für die Fälle 2, 4 und 5 aus Tab. 6.2. Sonstige Parameter siehe Tab. 6.1.

Fall 2(-): $\mathrm{P}_{a}=4,0$ bar ; Fall 4(-): $\mathrm{P}_{a}=4,5$ bar; Fall 5(-): $\mathrm{P}_{a}=5,0$ bar.

gefundene, wo die Anregungsdruckabhängigkeit typischer SBSL-Blasen (26,5 kHz Anregung) untersucht wurde. Dort wurde ein abnehmendes Kompressionsverhältnis mit zunehmendem $P_{a}$ festgestellt. Bei den hier betrachteten Blasen handelt es sich allerdings um transiente MBSL-Blasen, so dass nicht von einem diffusiven Gleichgewicht wie in Kap. 5.2 ausgegangen wurde und somit die Größe von $R_{0}$ für die $P_{a}$-Vergleichsrechnungen unverändert blieb. Die maximale Durchschnittsdichte steigt, auch durch einen leicht steigenden Wasserdampfgehalt, mit wachsendem $P_{a}$ an (siehe Tab. 6.2). Der stärkere Kollaps der höher angeregten Blasen führt zu deutlich heraufgesetzten Temperaturen: Die maximale Durchschnittstemperatur steigt von 27.240 K in Fall 2 über $38.440 \mathrm{~K}$ in Fall 4 auf 50.950 K in Fall 5. Die absoluten Maximaltemperaturen steigen sogar auf bis zu 129.300 K (Fall 5). Die kompensierende Wirkung des zusätzlichen Wasserdampfes kann hier also nicht 
den Energiezuwachs durch den Kollaps aufwiegen.

Den Temperaturen entsprechend steigt auch die emittierte Lichtenergie: Mit 27,8 fJ wird in Fall 5 mehr als fünf mal soviel Licht emittiert wie in Fall 2 (5,3 fJ). Ebenso steigt die chemische Aktivität. Die hohen Temperaturen führen in allen Fällen zu einem sehr hohen Dissoziationgrad: in Fall 2 werden 86,8 \% der gegen Ende des Kollaps vorhandenen $\mathrm{H}_{2} \mathrm{O}$-Teilchen in andere Spezies umgesetzt, in Fall 4 sind es $91,7 \%$, in Fall 5 sogar 94,1 \% (siehe Tab. 6.2, Werte für $\mathrm{H}_{2} \mathrm{O}_{r}$ und $\mathrm{H}_{2} \mathrm{O}_{\text {min }}$ ). Bei $P_{a}=5$ bar sind gegen Ende des Kollaps fast zehnmal mehr $\mathrm{H}_{2}{ }^{-}$ als $\mathrm{H}_{2} \mathrm{O}$-Teilchen in der Blase. Abb. 6.5 verdeutlicht die Temperaturverteilung,
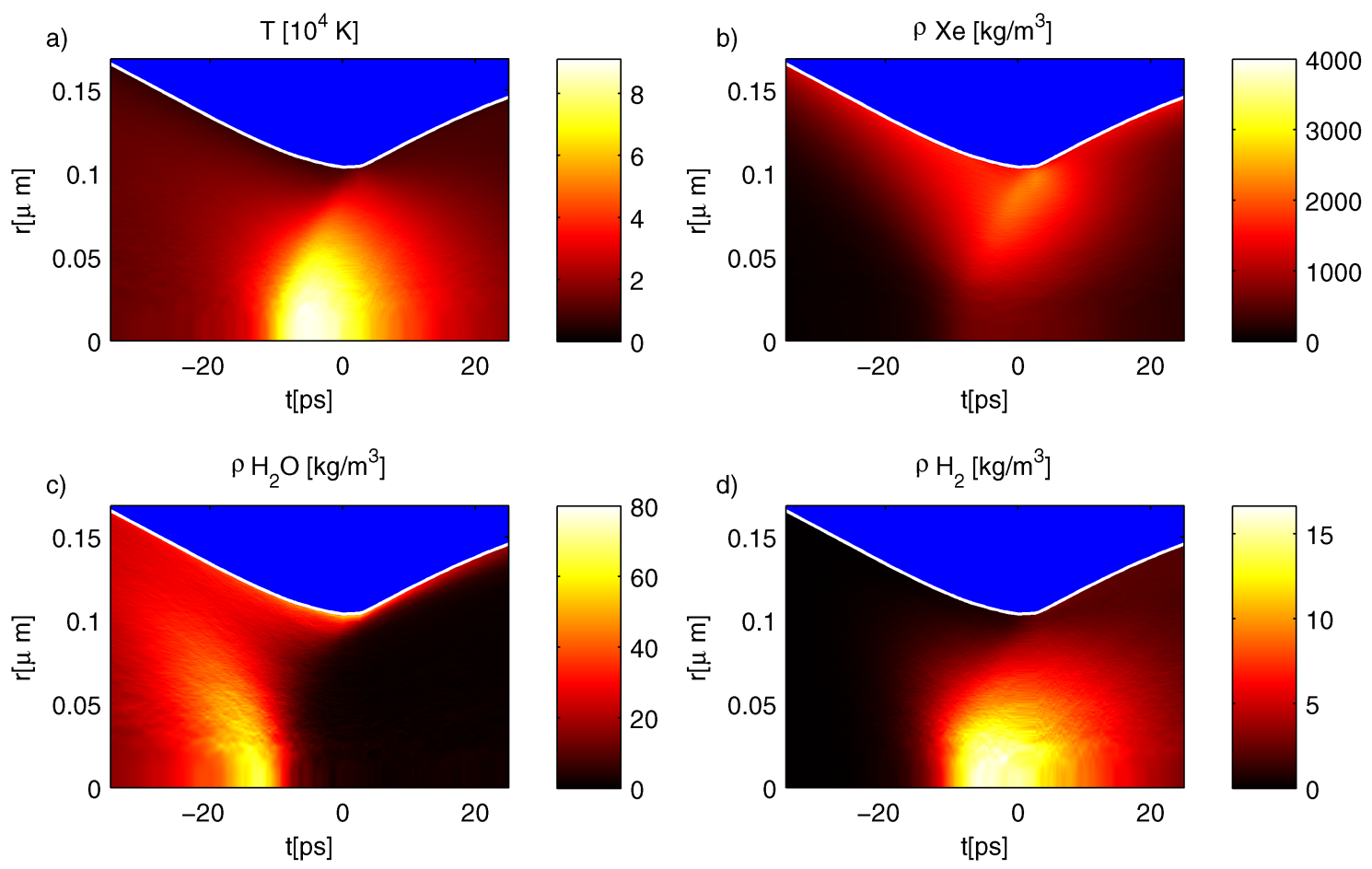

Abbildung 6.5: Temperatur (a), Dichte von Xenon (b), von Wasserdampf (c) und von $\mathrm{H}_{2}$ (d) um den Hauptkollaps $(\mathrm{t}=0)$ für Fall 4 aus Tab. 6.2. Sonstige Parameter siehe Tab. 6.1.

die Dissoziation des Wasserdampfes und die Entstehung chemischer Spezies, am Beispiel von $\mathrm{H}_{2}$, für den Fall $P_{a}=4,5$ bar. Es ist gut zu erkennen, dass im heißen Blasenzentrum fast der gesamte Wasserdampf umgesetzt wird, es entstehen chemische Produkte in großer Menge. Am Blasenrand sind die Temperaturen klein genug, um eine komplette Umsetzung des Wasserdampfes zu verhindern.

Der Vergleich des Einflusses verschiedener Edelgase auf die Blasenverhältnisse ist in Abb. 6.6 dargestellt. Gezeigt sind die Fälle 2, 6 und 7 aus Tab. 6.2, die Xenon, 

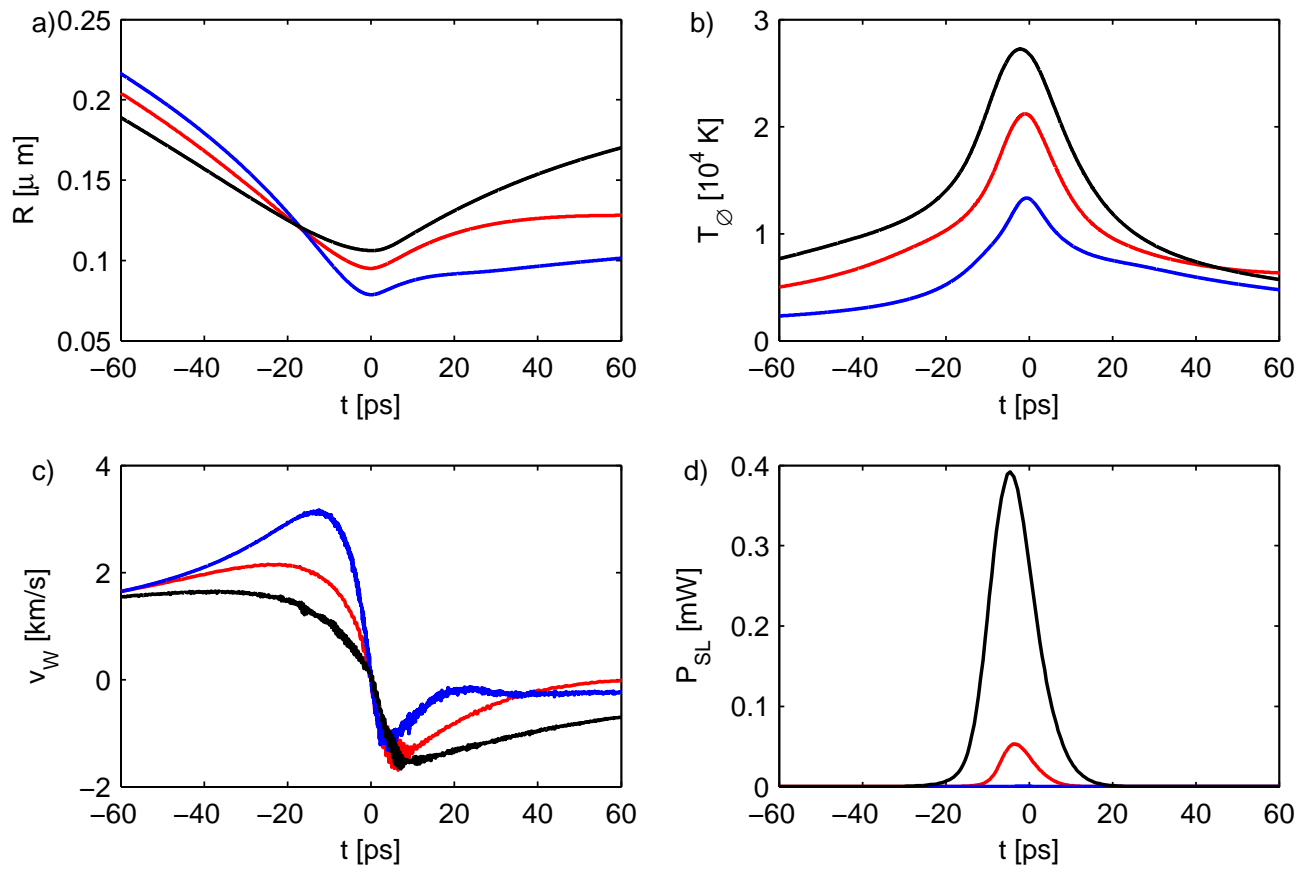

Abbildung 6.6: Verlauf des Blasenradius (a), der Durchschnittstemperatur (b), der Blasenwandgeschwindigkeit (c) und der Lichtemission der gesamten Blase (d) um den Hauptkollaps $(\mathrm{t}=0)$ für die Fälle 2, 6 und 7 aus Tab. 6.2. Sonstige Parameter siehe Tab. 6.1.

Fall 7(-): Argon; Fall 6(-): Helium; Fall 2(-): Xenon.

Argon und Helium als jeweils einziges vorkommendes Edelgas verwenden, einen Ruheradius von $R_{0}=450 \mathrm{~nm}$ haben und mit $P_{a}=4$ bar angeregt werden. $\mathrm{Im}$ polytropischen Modell ist der Blasenrandverlauf für die drei Fälle gleich, er entspricht der blauen Kurve in Abb. 6.1. Im molekulardynamischen Modell wirkt die innere Dynamik hingegen deutlich auf die Blasenrandbewegung ein: Aufgrund des kleineren Impulsübertrags der leichten Helium-Atome weist die Blase aus Fall 6 in den entscheidenden Phasen gegen Ende des Kollaps einen geringeren Wandinnendruck $P_{W}$ auf (nicht gezeigt), wodurch die Blase ab ca. 50 ps vor $t_{R \min }$ deutlich stärker beschleunigt als in den anderen Fällen (maximale Wandgeschwindigkeit: $3,10 \mathrm{~km} / \mathrm{s}$ bei Fall 6, 2,15 km/s bei Fall 7 und 1,65 km/s bei Fall 2). Entsprechend kollabiert die Blase mit den leichtesten Edelgasteilchen am weitesten $\left(R_{\min }=\right.$ $78 \mathrm{~nm}$ für die Helium-Blase). Insgesamt ähneln die Radiusverläufe - sieht man von den Skalen ab - sehr denjenigen aus Kap. 5.3, in welchem typische, bei $26,5 \mathrm{kHz}$ angeregte, SBSL-Blasen mit unterschiedlichen Edelgasen untersucht wurden. Die 1- MHz-Blasen zeigen höhere Kollaps-Geschwindigkeiten, die Unterschiede zwischen den einzelnen Edelgasen sind hier etwas stärker ausgeprägt. Ebenso wie in Kap. 5.3 erreicht die Helium-Blase, trotz ihres stärkeren Kollaps, sehr viel gerin- 
gere Durchschnittstemperaturen (max. $13.340 \mathrm{~K}$ ) als die Argon- (21.160 K) oder Xenon-Blase (27.240 K). Damit steigt die Temperatur beim Übergang von Helium zu Xenon um mehr als das Doppelte an. In Kap. 5.3 betrug das Verhältnis nur ca. 1:1,4 (siehe Abb. 5.11). Die Lichtleistung nimmt beim Übergang zu schwereren Edelgasen extrem zu, was anhand der starken Temperatur-Abhängigkeit der Emission zu erwarten ist. Die Helium-Blase emittiert lediglich 0,005 fJ, die Argon-Blase bereit 0.5 fJ und die Xenon-Blase schließlich 5,3 fJ. Die Emission der verschiedenen Blasen liegt also in einem drei Größenordnungen breiten Intervall. Bei SBSL-Blasen fiel dieser Unterschied beträchtlich niedriger aus (ca. 1:50, siehe Tab. 5.7). Durch den deutlich höheren Anregungsdruck bei 1-MHzAnregung (4 bar vs. 1,3 bar) scheinen die Edelgasatome deutlich mehr Energie aufnehmen zu können und so die großen Unterschiede zwischen den Blasenklassen hervorzurufen.
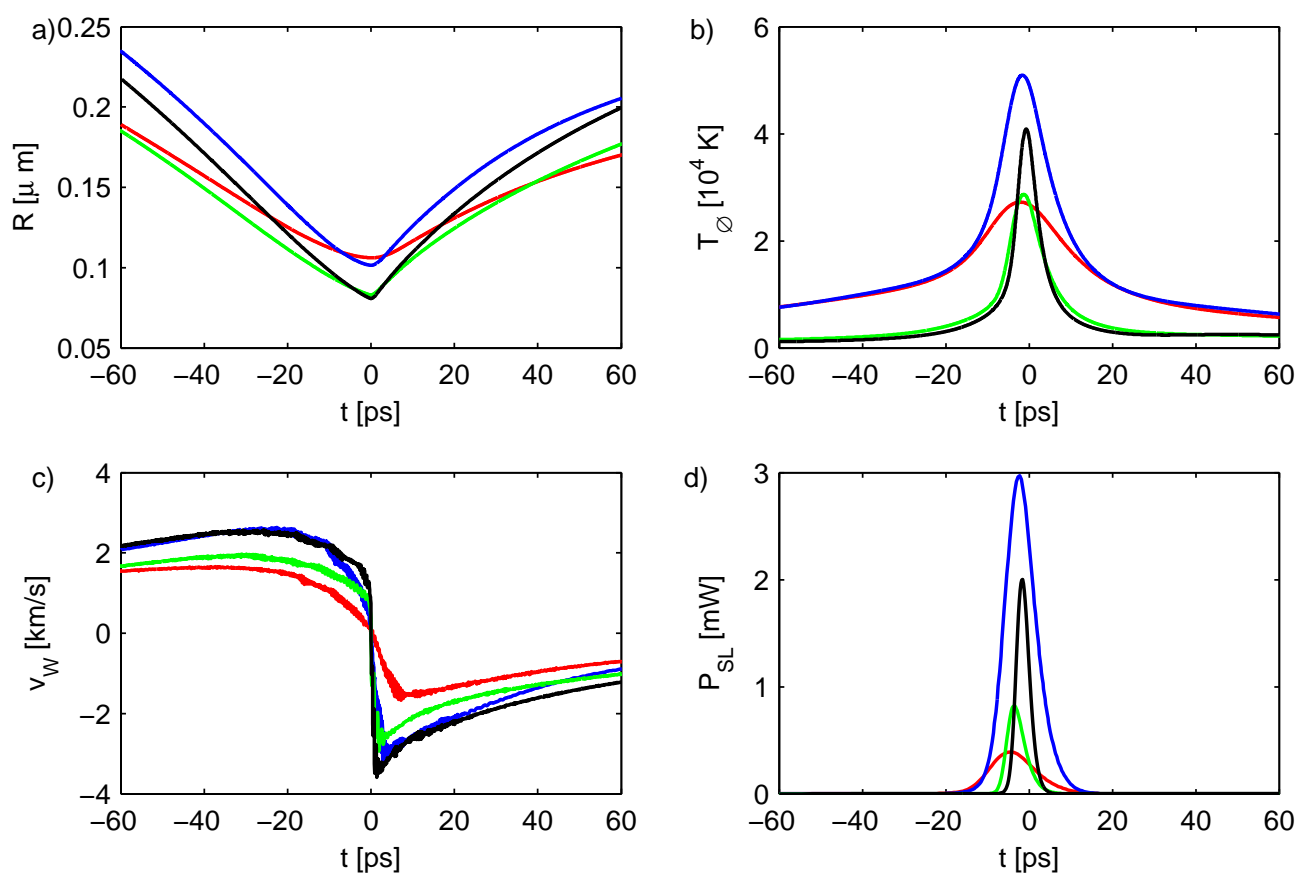

Abbildung 6.7: Verlauf des Blasenradius (a), der Durchschnittstemperatur (b), der Blasenwandgeschwindigkeit (c) und der Lichtemission der gesamten Blase (d) um den Hauptkollaps $(\mathrm{t}=0)$ für die Fälle 2, 5, 8 und 9 aus Tab. 6.2. Sonstige Parameter siehe Tab. 6.1.

Fall 2(-): $P_{a}=4$ bar, $A_{v}=0,3, \alpha_{v}=0,3$; Fall $5(-): P_{a}=5$ bar, $A_{v}=0,3, \alpha_{v}=0,3$; Fall $8(-): P_{a}=4$ bar, $A_{v}=1,0, \alpha_{v}=1,0$; Fall $9(-): P_{a}=5$ bar, $A_{v}=1,0, \alpha_{v}=1,0$.

Abb. 6.7 zeigt Ergebnisse der Fälle 2, 5, 8 und 9 aus Tab. 6.2. Fall 8 und 9 entsprechen dabei Fall 2 und 5, bis auf veränderte Akkomodationskoeffizienten: $A_{v}$ wurde von 0,3 auf 1,0 erhöht, so dass mehr Wasserdampf aus der Blase konden- 
sieren kann, $\alpha_{t}$ wurde ebenfalls von 0,3 auf 1,0 erhöht, so dass die Teilchen ihre gesamte Überschussenergie an die Blasenwand abgeben können. Entsprechende Untersuchungen an SBSL-Blasen in den Kapiteln 4.2.2 und Kap. 4.1.2.1 zeigen, dass eine erhöhte Wasserdampfkondensation zu extremeren Bedingungen in der Blase führt, sowie dass eine Erhöhung von $\alpha_{t}$ zu größeren Zentrumstemperaturen (aber sinkenden Durchschnittstemperaturen) führt. Diese Parameterstudie wurde hier durchgeführt, um abschätzen zu können, mit welchen Temperaturen in den betrachteten Blasen bei extremen Parameterkombinationen maximal zu rechnen ist.
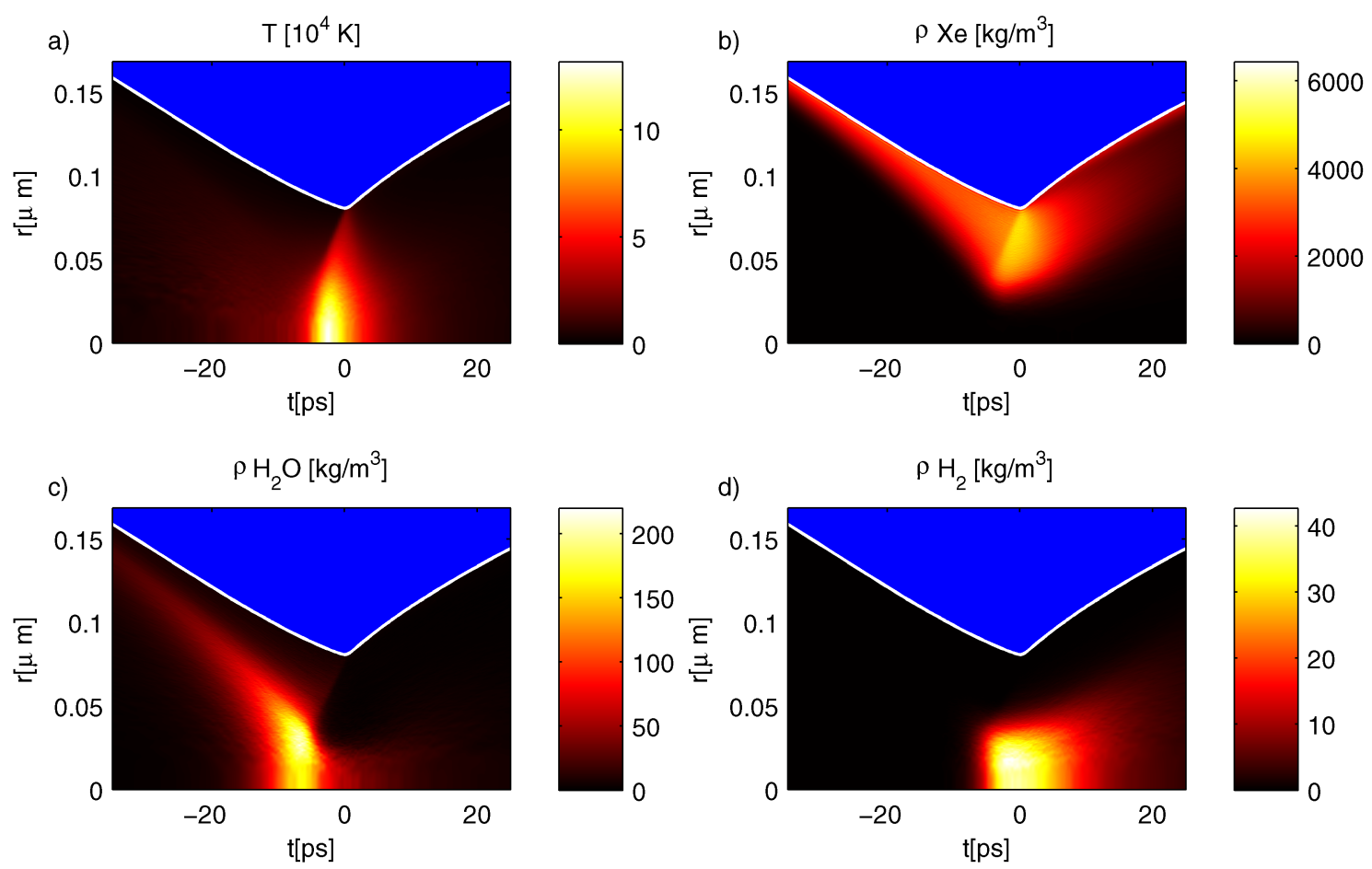

Abbildung 6.8: Temperatur (a), Dichte von Argon (b), Wasserdampf (c) und $\mathrm{H}_{2}$ (d) um den Hauptkollaps ( $\mathrm{t}=0$ ) für Fall 9 aus Tab. 6.2. Sonstige Parameter siehe Tab. 6.1.

Es läßt sich erkennen, dass die Blasen aus den Fällen 8 und 9 (mit 'extremen' Parametern) deutlich weiter kollabieren $\left(R_{\min }=83\right.$, bzw. $\left.80 \mathrm{~nm}\right)$ als ihre Gegenstücke mit moderateren Parametern $\left(R_{\min }=106\right.$, bzw. $\left.101 \mathrm{~nm}\right)$. Dies erklärt sich sowohl aus der geringeren Anzahl an $\mathrm{H}_{2} \mathrm{O}$-Molekülen (6,4·10 für Fall 9; 18,7·10 für Fall 5) in Folge des größeren Wertes von $A_{v}$, als auch aus der kleineren kinetischen Energie der Teilchen in der Blase (und damit kleinerem Wanddruck des Gases) aufgrund der gesteigerten Energieabgabe an das Blasenumgebungsmedium. Der Kollaps endet bei den Rechnungen mit $\alpha_{t}=1,0$ extrem plötzlich, 
gerade in Fall 9 zeigt sich ein jäher Vorzeichenwechsel der Wandgeschwindigkeit. Der Blick auf die in Abb. 6.8 für diesen Fall dargestellte innere Dynamik der Blase zeigt das Auftreffen einer am Blasenzentrum reflektierten Dichtewelle aus Xenon-Teilchen als Ursache für dieses jähe Ende des Kollaps.

Die Durchschnittstemperaturen steigen gegen Ende des Kollaps für die Fälle 8 und 9 rasant an, nachdem sie bis ca. 20 ps vor $t_{R m i n}$ auf deutlich niedrigerem Niveau verlaufen, verglichen mit den Fällen 2 und 5. Im Fall 8 herrschen in einer sehr kurzen Zeit (wenige ps) höhere Durchschnittstemperaturen als im Fall 2. Nach den Ergebnissen aus Kap. 4.1.2.1 war dies nicht zu erwarten und ist hier vermutlich auf den ebenfalls modifizierten Koeffizienten $A_{v}$ zurückzuführen. Die Rechnung aus Fall 9 erreicht hingegen nicht die Temperaturen ihres Gegenstückes (Fall 5). Die in Fall 5 von den Teilchen aus dem heftigen Kollaps gezogene Energie überwiegt hier den Effekt des vermehrten Wasserdampfes. Die absolut größten Temperaturen sind in Fall 8 deutlich höher als in Fall 2 (105.900 K vs. $64.300 \mathrm{~K})$, im Vergleich dazu ist der Unterschied von Fall 9 (131.700 K) zu Fall 5 (129.300 K) deutlich geringer. Die hohen absoluten Maximaltemperaturen erklären sich durch die sehr scharfe Fokussierung der Teilchen im Blasenzentrum. Während des Kollaps sammeln sich bei einem Wert von $\alpha_{t}=1,0$ die meisten Teilchen an der Blasenwand. Verlangsamt sich die Bewegung des Blasenrandes lösen sich die Teilchen von der Wand und bewegen sich als starke Dichtewelle in Richtung Blasenzentrum (in Abb. 6.8 gut zu erkennen). Die Segregation der Spezies ist dabei sehr ausgeprägt, da die Wasserdampfteilchen aufgrund ihres geringeren Gewichtes bedeutend schneller sind als die Xenon-Teilchen. Die $\mathrm{H}_{2} \mathrm{O}$-Moleküle erreichen vor den Xenon-Atomen das Blasenzentrum und schirmen dieses nahezu komplett von den Xenon-Atomen ab. Diese werden an dem 'Kern' aus $\mathrm{H}_{2} \mathrm{O}$-Teilchen (und den Dissoziationsprodukten) reflektiert und laufen zum Blasenrand zurück, wo das Auftreffen der Dichtewelle den Kollaps beendet.

Die $\mathrm{H}_{2} \mathrm{O}-$ Moleküle werden nahezu vollständig dissoziiert, nur im Blasenzentrum sind über $t_{R \min }$ hinaus eine geringe Anzahl vorhanden. Vermutlich werden diese aus Rückreaktionen der Dissoziationsprodukte gebildet. Im Gegensatz zu den Rechnungen mit moderateren Parametern gelangen nach dem Kollaps keine $\mathrm{H}_{2} \mathrm{O}$ Teilchen zum Blasenrand (siehe Abb. 6.5).

Zum Zeitpunkt des Auftreffens der reflektierten Dichtewelle treten hohe Temperaturen an der Blasenwand auf. Abb 6.9 zeigt die Wandtemperatur $T_{W}$ um den Hauptkollaps sowie die Temperaturverteilung in der Blase zum Zeitpunkt der maximalen Blasentemperatur. Bei den Fällen 8 und 9 steigt $T_{W}$ innerhalb 

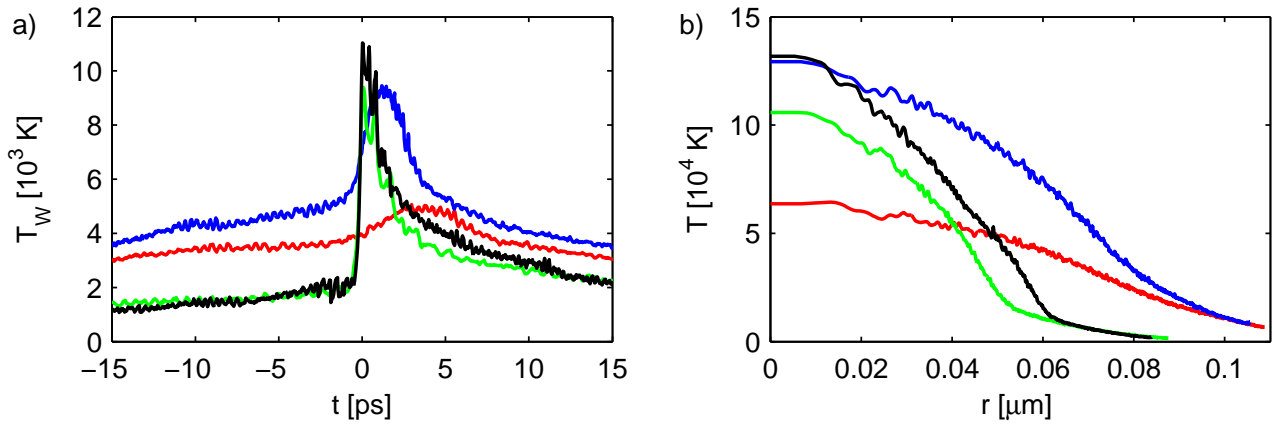

Abbildung 6.9: (a): Verlauf der Wandtemperatur für die Fälle 2(一), 5(一), $8(-)$ und $9(-)$ aus Tab. 6.2. (b) Verlauf der Temperatur in der Blase zum Zeitpunkt der maximalen Temperatur für die selben Fälle.

weniger ps von ca. $2.000 \mathrm{~K}$ auf über $10.000 \mathrm{~K}$ an. In den Fällen 2 und 5 verläuft der Anstieg deutlich langsamer, gerade für Fall 2 bleibt die Temperatur deutlich geringer (ca. $5.000 \mathrm{~K}$ ). Die Temperaturverteilung in der Blase ist für Rechnungen mit $\alpha_{t}=1,0$ deutlich inhomogener, es bildet sich eine relativ ausgedehnte kalte Schicht an der Blasenwand aus.

Allgemein läßt sich sagen, daß Blasen, die mit einer Frequenz von $\nu_{a}=1 \mathrm{MHz}$ und einem Druck von $P_{a}=4-5$ bar angeregt werden, deutlich extremere Bedingungen aufweisen als typische SBSL-Blasen. Die Kollapsgeschwindigkeit steigt, genauso wie die erreichten Temperaturen. Selbst in dem moderaten Fall 7, mit Argon als Edelgas, werden Temperaturen von bis zu $48.700 \mathrm{~K}$ erreicht, weit über den ca. $18.000 \mathrm{~K}$ einer SBSL-Blase mit vergleichbaren Werten für $A_{v}$ und $\alpha_{t}$. Verwendet man Xenon als Edelgas und steigert die Anregung auf $P_{a}=5$ bar steigen die Blasentemperaturen auf erstaunliche 130.000 K. Dieser Wert liegt allerdings immer noch deutlich unter der in [137] vorhergesagten Temperaturen von $500.000 \mathrm{~K}$.

In derselben Veröffentlichung wurde die Vermutung getätigt, dass in SBSLBlasen, die mit ca. $30 \mathrm{kHz}$ angeregt werden, ähnliche Temperaturen auftreten. Die Autoren argumentierten, dass diese Blasen eine deutlich größere Emissionsregion aufweisen, die - im Gegensatz derjeniger der hier beobachteten Blasen opak ist. Daher könne man lediglich die Temperatur der Oberfläche sehen, obwohl im Blasenzentrum deutlich höhere Temperaturen herrschten. Diese Theorie kann durch die hier vorliegenden Ergebnisse nicht bestätigt werden. Die in den vorigen Kapiteln besprochenen SBSL-Blasen weisen zwar ein deutlich größeres Emissionsgebiet auf (ca. 0,7 $\mu \mathrm{m}$, verglichen mit 0,1-0,2 $\mu \mathrm{m}$ ), die Temperaturen sind hingegen deutlich unter den hier gesehenen anzusiedeln.

Die Steigerung der Lichtleistung um drei Größenordnungen beim Übergang von 


\begin{tabular}{llllllllll}
\hline Fall & 1 & 2 & 3 & 4 & 5 & 6 & 7 & 8 & 9 \\
\hline Parameter & & & & & & & & & \\
$P_{A}[\mathrm{bar}]$ & 4,0 & 4,0 & 4,0 & 4,5 & 5,0 & 4,0 & 4,0 & 4,0 & 5,0 \\
Edelgas & $\mathrm{Xe}$ & $\mathrm{Xe}$ & $\mathrm{Xe}$ & $\mathrm{Xe}$ & $\mathrm{Xe}$ & $\mathrm{He}$ & $\mathrm{Ar}$ & $\mathrm{Xe}$ & $\mathrm{Xe}$ \\
$R_{0}[\mathrm{~nm}]$ & 300 & 450 & 600 & 450 & 450 & 450 & 450 & 450 & 450 \\
$A_{v}$ & 0,3 & 0,3 & 0,3 & 0,3 & 0,3 & 0,3 & 0,3 & 1,0 & 1,0 \\
$\alpha_{t}$ & 0,3 & 0,3 & 0,3 & 0,3 & 0,3 & 0,3 & 0,3 & 1,0 & 1,0 \\
$v_{W, \text { init }}[\mathrm{m} / \mathrm{s}]$ & 35 & 35 & 28 & 47 & 60 & 35 & 35 & 35 & 60 \\
$S$ & 49 & 117 & 224 & 117 & 117 & 117 & 117 & 117 & 117 \\
\hline Dynamik & & & & & & & & & \\
$R_{\text {min }}[\mathrm{nm}]$ & 78 & 106 & 132 & 103 & 101 & 79 & 95 & 83 & 80 \\
$R_{\text {max }}[\mathrm{nm}]$ & 3.560 & 4.570 & 5.200 & 5.360 & 6.010 & 4.580 & 4.570 & 4.580 & 6.010 \\
$K$ & 45,2 & 43,1 & 39,3 & 52,0 & 59,5 & 57,9 & 48,1 & 55,1 & 75,1 \\
$T_{\varnothing}[\mathrm{K}]$ & 28.290 & 27.240 & 22.650 & 38.440 & 50.950 & 13.340 & 21.160 & 28.690 & 40.870 \\
$T_{\text {max }}[\mathrm{K}]$ & 68.300 & 64.300 & 49.300 & 91.000 & 129.300 & 24.900 & 48.700 & 105.900 & 131.700 \\
$\varrho_{\varnothing}\left[\mathrm{kg} / \mathrm{m}^{3}\right]$ & 2.350 & 2.411 & 2.448 & 2.772 & 3.034 & 181 & 1.025 & 4.082 & 4.784 \\
\hline Lichtemission & & & & & & & & & \\
$E_{S L}[\mathrm{fJ}]$ & 1,9 & 5,3 & 5,6 & 14,9 & 27,8 & 0,005 & 0,5 & 4,4 & 8,0 \\
$d_{S L}[\mathrm{~nm}]$ & 111 & 147 & 176 & 155 & 163 & 95 & 117 & 102 & 109 \\
$\Delta t_{S L}[\mathrm{ps}]$ & 9,0 & 10,9 & 19,0 & 9,7 & 8,4 & 9,0 & 5,6 & 5,0 & 3,6 \\
\hline Chemie & & & & & & & & & \\
$\mathrm{H}_{2} \mathrm{O}_{r}\left[10^{6}\right]$ & 5,9 & 13,6 & 24,2 & 16,8 & 18,7 & 13,4 & 13,3 & 3,9 & 6,4 \\
$\mathrm{H}_{2} \mathrm{O}_{\min }\left[10^{6}\right]$ & 0,7 & 1,8 & 4,1 & 1,4 & 1,1 & 2,6 & 6,3 & 0,3 & 0,3 \\
$\mathrm{OH}_{1}\left[10^{6}\right]$ & 1,3 & 3,1 & 5,4 & 4,1 & 4,6 & 2,4 & 2,9 & 1,0 & 1,4 \\
$\mathrm{H}_{2}\left[10^{6}\right]$ & 2,6 & 6,1 & 10,9 & 8,1 & 9,3 & 3,7 & 5,5 & 1,8 & 3,2 \\
\hline
\end{tabular}

Tabelle 6.2: Kenngrößen verschiedener Rechnungen von Blasen mit $\nu_{a}=1 \mathrm{MHz}$.

$K$ ist der Kompressionsfaktor, $T_{\varnothing}$ die maximale Durchschnittstemperatur, $\mathrm{T}_{\max }$ die absolut maximale Temperatur in der Blase, $\varrho_{\varnothing}$ die maximale Durchschnittsdichte, $E_{S L}$ die gesamt abgegebene Lichtenergie, $d_{S L}$ der Durchmesser der Emissionregion, $\Delta t_{S L}$ die Halbwertsbreite der Lichtemission, $\mathrm{H}_{2} \mathrm{O}_{r}$ ist die Anzahl der in der Blase gefangenen $\mathrm{H}_{2} \mathrm{O}$-Moleküle kurz vor Einsetzen der chemischen Reaktionen, $\mathrm{H}_{2} \mathrm{O}_{\text {min }}$ die minimale Anzahl an $\mathrm{H}_{2} \mathrm{O}$-Molekülen kurz nach $t_{R \min }, \mathrm{OH}$ die maximale Anzahl an $\mathrm{OH}-$ Molekülen in der Blase und $\mathrm{H}_{2}$ die Anzahl an gebildeten $\mathrm{H}_{2}$-Molekülen.

Helium auf Xenon als Edelgas wird in [135] sehr gut bestätigt, auch wenn die hier gefundenen Halbwertsbreiten der Lichtemission (3,6 bis 19,0 ps) deutlich unter den dort gemessenen (98 ps) liegen.

Bei den hier vorliegenden extremen Bedingungen in der Blase ist die Gültigkeit vieler verwendeter Formeln natürlich eingeschränkt. Der im Vergleich zu typischen SBSL-Blasen geringere Durchmesser der betrachteten Blasen vergrößert den Einfluß der Oberflächenspannung. Dies sollte zu einer besseren Sphärizität führen, welche letztlich die Stabilität der Blase und die Energiefokussierung stärkt. Natürlich ist bei den auftretenden Drücken und Geschwindigkeiten dennoch eine teilweise Zerstörung oder Deformation der Blase möglich, welche hier nicht erfaßt wird. Daher sind die Ergebnisse sicherlich nicht absolut zu sehen. Der experimentell ermittelte Trend zu einer extremeren Radius- und Binnendynamik läßt sich jedoch gut erkennen. 


\subsection{1-MHz-Blasen}

Zusätzlich zu den Untersuchungen von Blasen, die mit $\nu_{a}=1 \mathrm{MHz}$ angeregt werden, zeigen Weninger und CAMARA in [135] auch einige Ergebnisse von Experimenten, die eine Anregung von $\nu_{a}=11 \mathrm{MHz}$ verwenden. Um lumineszierende MBSL-Blasen bei einer derart hohen Frequenz zu erzeugen, mußte ein Anregungsdruck von ca. 100 bar eingesetzt werden. Das Experiment konnte leider nur für kurze Zeit betrieben werden, da der verwendete Transducer den auftretenden Belastungen auf Dauer nicht gewachsen war. Dennoch konnten einige Messungen durchgeführt werden. Der gewählte Anregungsdruck liegt weit über der Kavitationsschwelle [138], daher wurde ein sehr dichtes Blasenfeld beobachtet, das ein mit dem bloßen Auge gut sichtbares Leuchten produzierte. Die Autoren schätzten den Ruheradius der entstandenen Blasen als zwischen $25 \mathrm{~nm}$ und $750 \mathrm{~nm}$ liegend ab. Dieser sehr große Bereich an Blasenvarianten läßt eine große Variabilität an Leuchtintensität und Blasentemperatur der einzelnen Blasen in der Blasenwolke vermuten. Diese Annahme wird durch ein gemessenes breitbandiges Spektrum des emittierten Lichts gestützt.

In dieser Arbeit sollen die von Weninger und Camara getätigten Experimente durch die Simulation des Blaseninneren vergleichbarer Blasen nachvollzogen werden und ungefähre Aussagen über Temperaturen, Lumineszenz und chemische Aktivität getätigt werden. Dafür wurden Blasen mit unterschiedlichen Ruheradien, und somit unterschiedlicher Teilchenzahl, auf Unterschiede sowohl der Radiusdynamik, als auch der inneren Hydrodynamik untersucht. Besonders interessant werden hochfrequent angeregte Blasen dadurch, dass ihre geringe Größe es erlaubt, teilchenbasierte Simulationen durchzuführen, deren Teilchenzahl näherungsweise der Realität entspricht. Die hier simulierten Fälle haben einen Skalierungsfaktor von $S=8,2-378,9$.

\begin{tabular}{ccc|ccc|ccc}
\hline$P_{a}$ & - & $100 \mathrm{bar}$ & $N_{N G}$ & - & 343.000 & $v_{W, \text { init }}$ & - & $50-80 \mathrm{~m} / \mathrm{s}$ \\
$\nu_{a}$ & - & $11,0 \mathrm{MHz}$ & $S$ & - & $4,8-19,8$ & $\alpha_{t}$ & - & 0,3 \\
$R_{0}$ & - & $130-750 \mathrm{~nm}$ & Edelgas & - & Argon & $A_{v}$ & - & 0,3 \\
$T_{\text {water }}$ & - & $290 \mathrm{~K}$ & $\Delta K_{W}$ & - & 4.000 & & & \\
\hline
\end{tabular}

Tabelle 6.3: Parameter der Rechnungen von Blasen mit $11 \mathrm{MHz}$ Anregung. Weitere Angaben finden sich in Tab. 6.4.

Wie LAUTERBORN zeigte, hängen die Resonanzkurven getriebener Blasen bei der Hauptresonanz in Richtung langsamer Frequenzen über und weisen super- und subharmonische Maxima auf [139][140]. Dies ist ein für nichtlineare Oszillatoren 


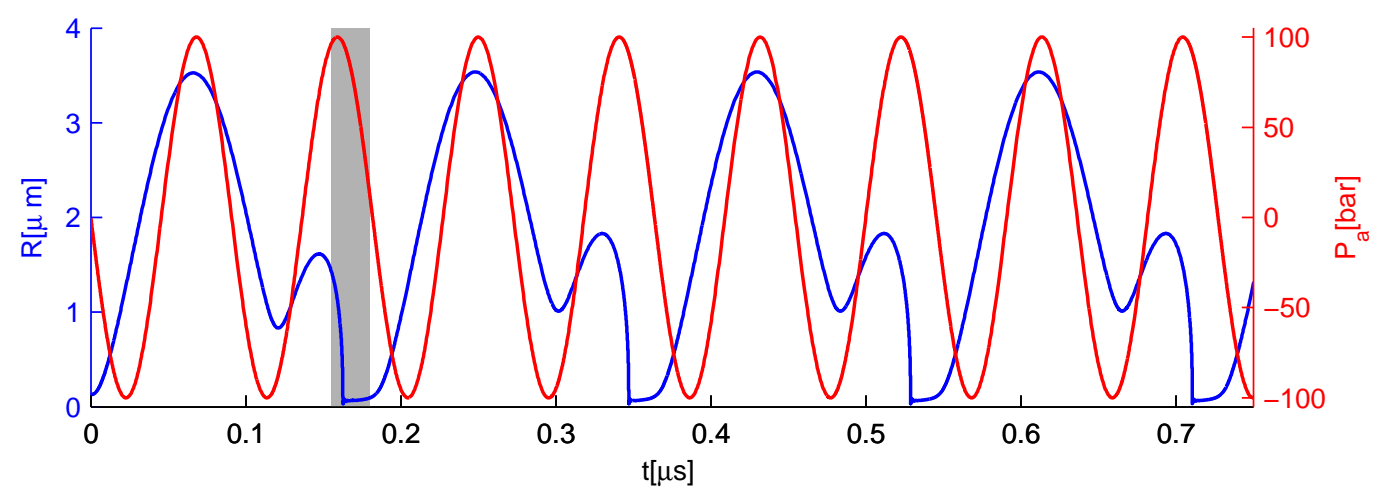

Abbildung 6.10: Radiusverlauf (-) und Anregungsdruck (-) für Fall 1 aus Tab. 6.4 $\left(R_{0}\right.$ $=130 \mathrm{~nm}$ ). Berechnet in einem einfachen polytropischen Modell. Der graue Bereich gibt die ungefähre Zeitspanne der MD-Simulation an.

typisches Verhalten. Auch Blasen, die unter der Resonanzfrequenz getrieben werden können starke subharmonische Komponenten aufweisen[141]. An den hier betrachteten Blasen läßt sich ein solches Verhalten beobachten: Der hohe Anregungsdruck führt bei allen simulierten Blasen zu subharmonischem Antwortverhalten. Die Blasen haben nicht mehr genug Zeit, um jeder Kompressionsphase mit einem Kollaps zu folgen und weisen daher nur jede zweite oder dritte Periode der Anregung einen schnellen Hauptkollaps auf. Da die Radiuskurven eine Abhängigkeit von Anregungsfrequenz und Ruheradius aufweisen, die in bestimmten Parameterbereichen chaotisch werden kann [142][143], treten für alle betrachteten Blasen unterschiedliche Radius-Zeit-Kurven auf.

Unter einem Ruheradius von $R_{0} \approx 100 \mathrm{~nm}$ wurde die Lösung der RayleighPlesset-Gleichung im polytropischen Modell instabil, so dass in der vorliegenden Untersuchung Werte zwischen $R_{0}=130 \mathrm{~nm}$ und $R_{0}=750 \mathrm{~nm}$ verwendet wurden. Eine kleinere Blase $\left(R_{0}=70 \mathrm{~nm}\right)$, für die einige Anregungsperioden stabil gerechnet werden konnten, wird im nächsten Unterkapitel diskutiert.

Im Folgenden sind für die Fälle $R_{0}=130 \mathrm{~nm}, 250 \mathrm{~nm}, 400 \mathrm{~nm}, 550 \mathrm{~nm}$ und 700 nm zunächst die Radius-Zeit-Kurven für acht Perioden der Anregung angegeben. In diesen Abbildungen ist jeweils der stärkste Kollaps markiert, welcher im molekulardynamischen Modell simuliert wurde. Ergebnisse der MD-Rechnung werden jeweils in einem weiteren Plot sowie in Tab. 6.4 dargestellt. Tab. 6.3 enthält generelle Parameter.

Abb. 6.10 zeigt den Radiusverlauf einer Blase mit $R_{0}=130 \mathrm{~nm}$ (Fall 1 aus Tab. 6.4). Es ist zu erkennen, dass die Blase bei jeder zweiten Periode der Anregung einen starken Kollaps erfährt. Die Radiusbewegung folgt diesem Muster relativ 
stabil. Der Kollaps findet sein Ende zu einem Zeitpunkt, zu dem der maximale Kompressionsdruck herrscht, wird also maximal beschleunigt. Während die Höhe des großen Maximums konstant bleibt schwankt die Höhe des zweiten, lokalen Maximums vor dem Hauptkollaps. Da die Schwankungen relativ klein ausfallen wurde willkürlich eine dieser Oszillationen als Start der MD-Simulation gewählt (grau markiert). Der Radius, von welchem der Hauptkollaps beginnt, ist klein $(1,62 \mu \mathrm{m})$. Dennoch wird aufgrund des hohen Druckes eine vergleichsweise große Kompression erreicht $(K=42,6)$.

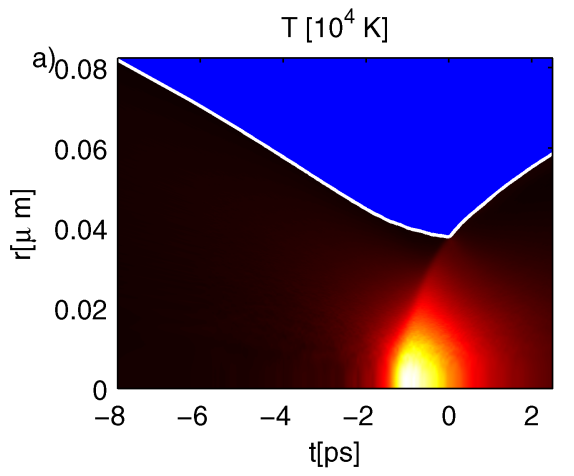

$\rho \mathrm{H}_{2} \mathrm{O}\left[\mathrm{kg} / \mathrm{m}^{3}\right]$

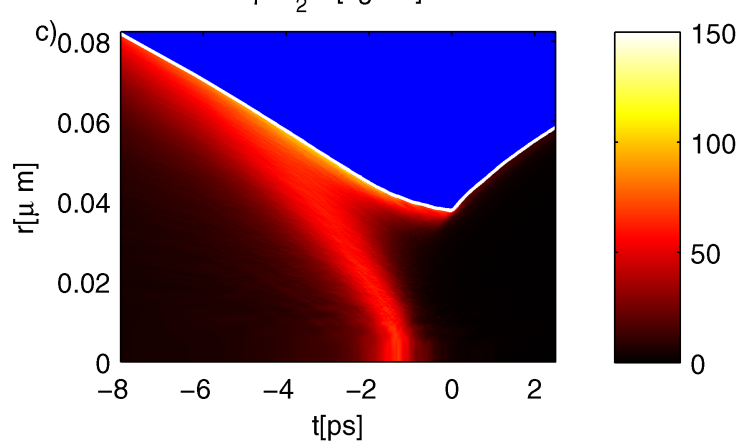

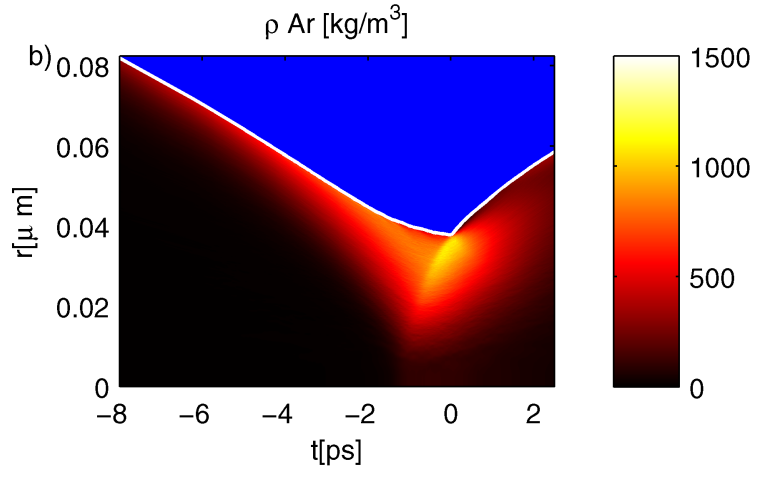

$P_{S L}\left[W / \mu m^{3}\right]$

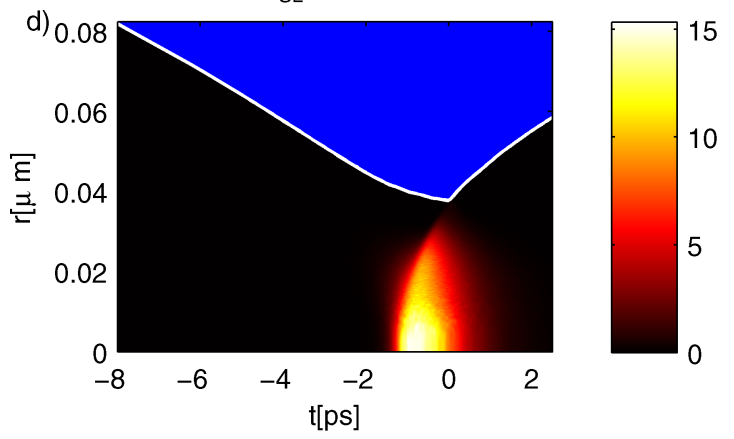

Abbildung 6.11: Verlauf von Temperatur (a), Dichte von Argon (b), Dichte von $\mathrm{H}_{2} \mathrm{O}$ (c) sowie der Lichtleistung für Fall 1 aus Tab. $6.4\left(R_{0}=130 \mathrm{~nm}\right)$.

Abb. 6.11 zeigt ausgewählte Ergebnisse der MD-Rechnung dieser Blase. Sie kollabiert bis auf $38 \mathrm{~nm}$ und weist ein kleines, heißes Zentrum auf. Der Durchmesser der Emissionsregion beträgt lediglich ca. $50 \mathrm{~nm}$. Eine Segregation der Spezies ist deutlich zu erkennen, das Edelgas dringt nur in sehr geringen Mengen in das Blasenzentrum vor, da dieses vom leichteren Wasserdampf und dessen chemischen Produkten (diese sind nicht gezeigt) bevölkert ist. Eine starke Argon-Dichtewelle beendet den Kollaps schlagartig. Die Temperaturen im Zentrum erreichen extrem hohe 459.000 K. Dies erklärt sich durch den sehr heftigen Kollaps sowie der geringen Menge an vorhandenem Wasserdampf (verursacht durch den in diesem Fall 


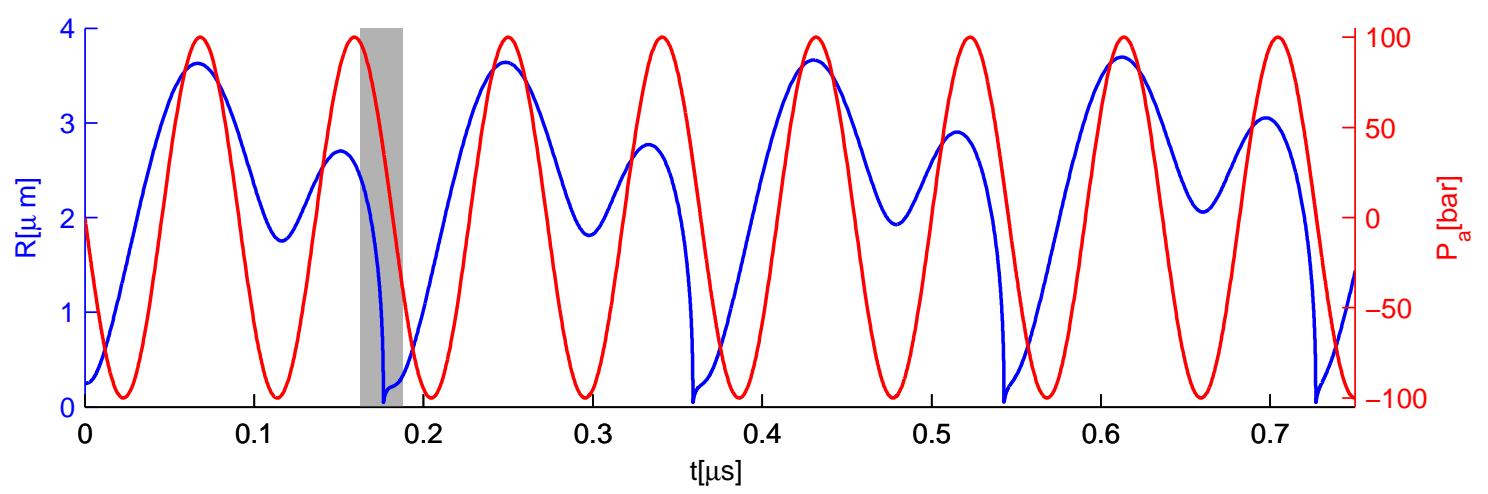

Abbildung 6.12: Radiusverlauf (-) und Anregungsdruck (-) für Fall 2 aus Tab. 6.4 $\left(R_{0}\right.$ $=250 \mathrm{~nm}$ ). Berechnet in einem einfachen polytropischen Modell. Der graue Bereich gibt die ungefähre Zeitspanne der MD-Simulation an.

sehr kleinen maximalen Radius. Die Lichtleistung erreicht bis zu $15 \mathrm{~W} / \mu \mathrm{m}^{3}$, ein Wert, der um drei Größenordnungen über den 2-10 $\mathrm{mW} / \mu \mathrm{m}^{3}$ einer typischen SBSL-Blase liegt. Dennoch beträgt die gesamt emittierte Lichtenergie mit 1,1 fJ weniger als die einer SBSL-Blase, da die Orts- und Zeitskalen $\left(\Delta t_{S L}=1,1 \mathrm{ps}\right)$

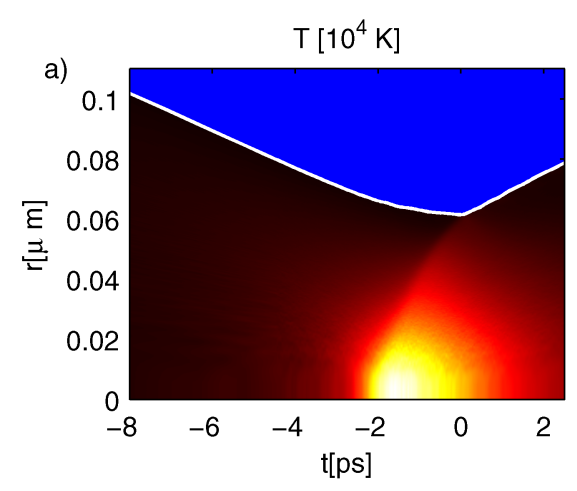

$\rho \mathrm{H}_{2} \mathrm{O}\left[\mathrm{kg} / \mathrm{m}^{3}\right]$

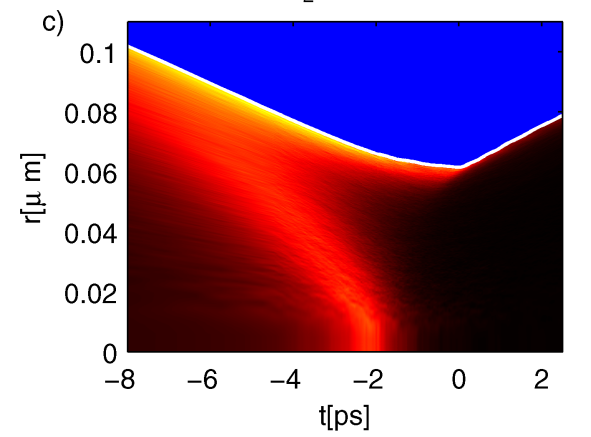

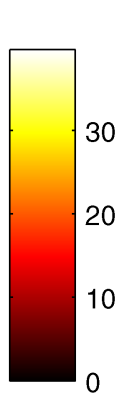

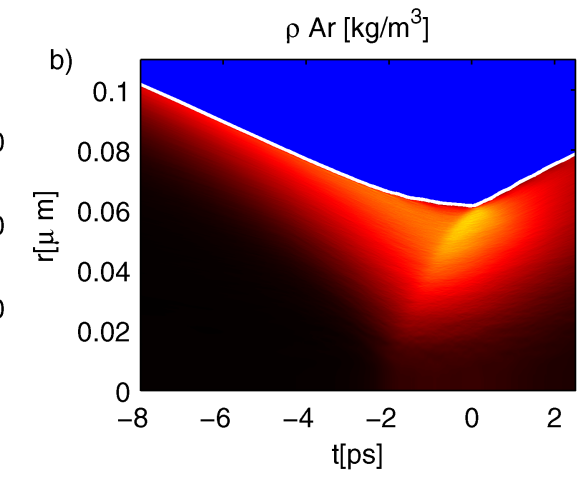

$P_{S L}\left[W / \mu m^{3}\right]$

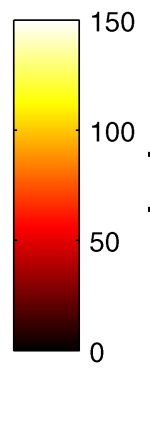

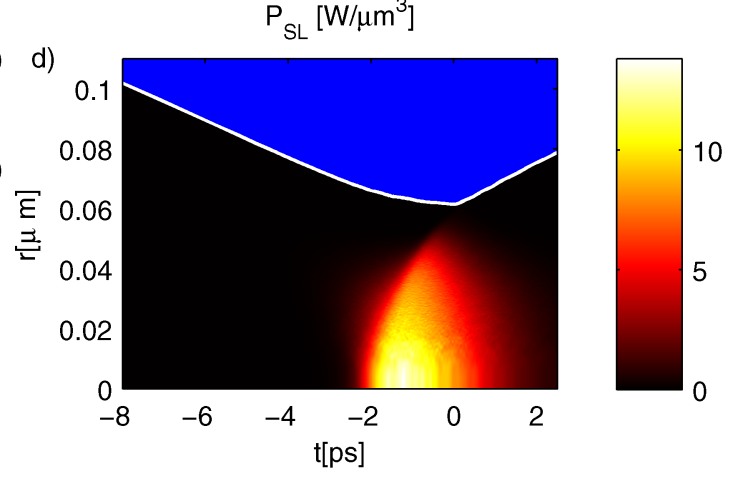

Abbildung 6.13: Verlauf von Temperatur (a), Dichte von Argon (b), Dichte von $\mathrm{H}_{2} \mathrm{O}$ (c) sowie der Lichtleistung für Fall 2 aus Tab. 6.4 $\left(R_{0}=250 \mathrm{~nm}\right)$. 
deutlich kleiner sind.

Abb. 6.12 zeigt den Radiusverlauf einer Blase mit $R_{0}=250 \mathrm{~nm}$ (Fall 2 aus Tab. 6.4). Auch diese Blase zeigt ein Periode-2-Verhalten, der Hauptkollaps findet gegenüber Fall $1 \mathrm{zu}$ einem etwas späteren Zeitpunkt statt. Daher ist zwar der Anregungsdruck zum Zeitpunkt des minimalen Blasenradius geringer, aber da die Blase vor dem Hauptkollaps weiter aufschwingt $\left(R_{\max }=2,70 \mu \mathrm{m}\right)$ als die $130 \mathrm{~nm}$ Blase wird eine etwas größere Kompression erreicht $\left(R_{\min }=62 \mathrm{~nm}, K=43,5\right)$. Wie in Abb. 6.13 zu erkennen sind die erreichten Temperaturen dennoch niedriger als in Fall 1, was auf einen gestiegenen Wasserdampfanteil gegen Ende des Kollaps (47,7\% vs. 44,3\%) sowie auf die generell gestiegene Teilchenzahl zurückzuführen ist. Im Maximum werden $396.000 \mathrm{~K}$ erreicht.

Die Lichtleistung pro Volumen liegt daher etwas unter derjeniger von Fall 1, die Leuchtdauer $\left(\Delta t_{S L}=1,9 \mathrm{ps}\right)$ und die Größe der emittierenden Region $\left(d_{S L}=\right.$ $83 \mathrm{~nm}$ ) steigen jedoch an, so dass mit $E_{S L}=7,2 \mathrm{fJ}$ deutlich mehr Licht emittiert wird.

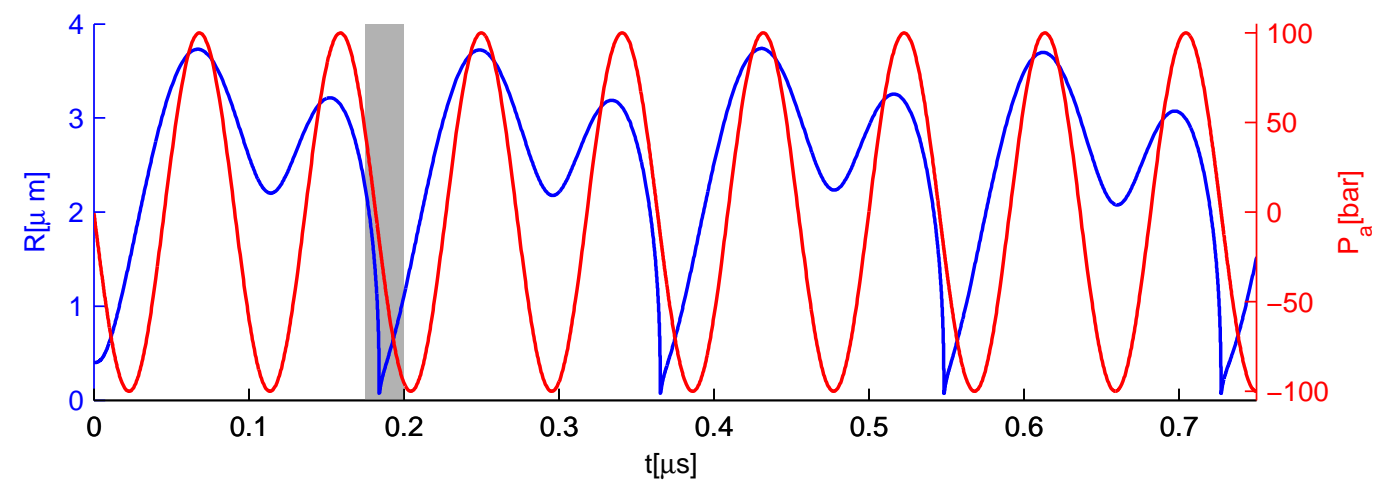

Abbildung 6.14: Radiusverlauf (-) und Anregungsdruck (-) für Fall 3 aus Tab. 6.4 $\left(R_{0}\right.$ $=400 \mathrm{~nm}$ ). Berechnet in einem einfachen polytropischen Modell. Der graue Bereich gibt die ungefähre Zeitspanne der MD-Simulation an.

In Abb. 6.14 ist der Radiusverlauf einer Blase mit dem Ruheradius $R_{0}=400 \mathrm{~nm}$ (Fall 3 aus Tab. 6.4) dargestellt. Weiterhin kollabiert die Blase stabil zu jeder zweiten Periode der Anregung. Das Minimum ist im Vergleich zum vorigen Fall noch weiter verschoben, so dass das Ende des Kollaps nun zu einer Phase erreicht wird, zu der der Anregungsdruck bereits wieder negativ wird. Das dem Kollaps vorausgehende lokale Maximum ist weiter angewachsen, der für den Hauptkollaps maßgebliche maximale Radius beträgt ca. 3,22 $\mu \mathrm{m}$.

Durch die fehlende unterstützende Wirkung des Anregungsdruckes in den letzten Phase des Kollaps fällt die Kompression deutlich geringer aus als in den Fällen 

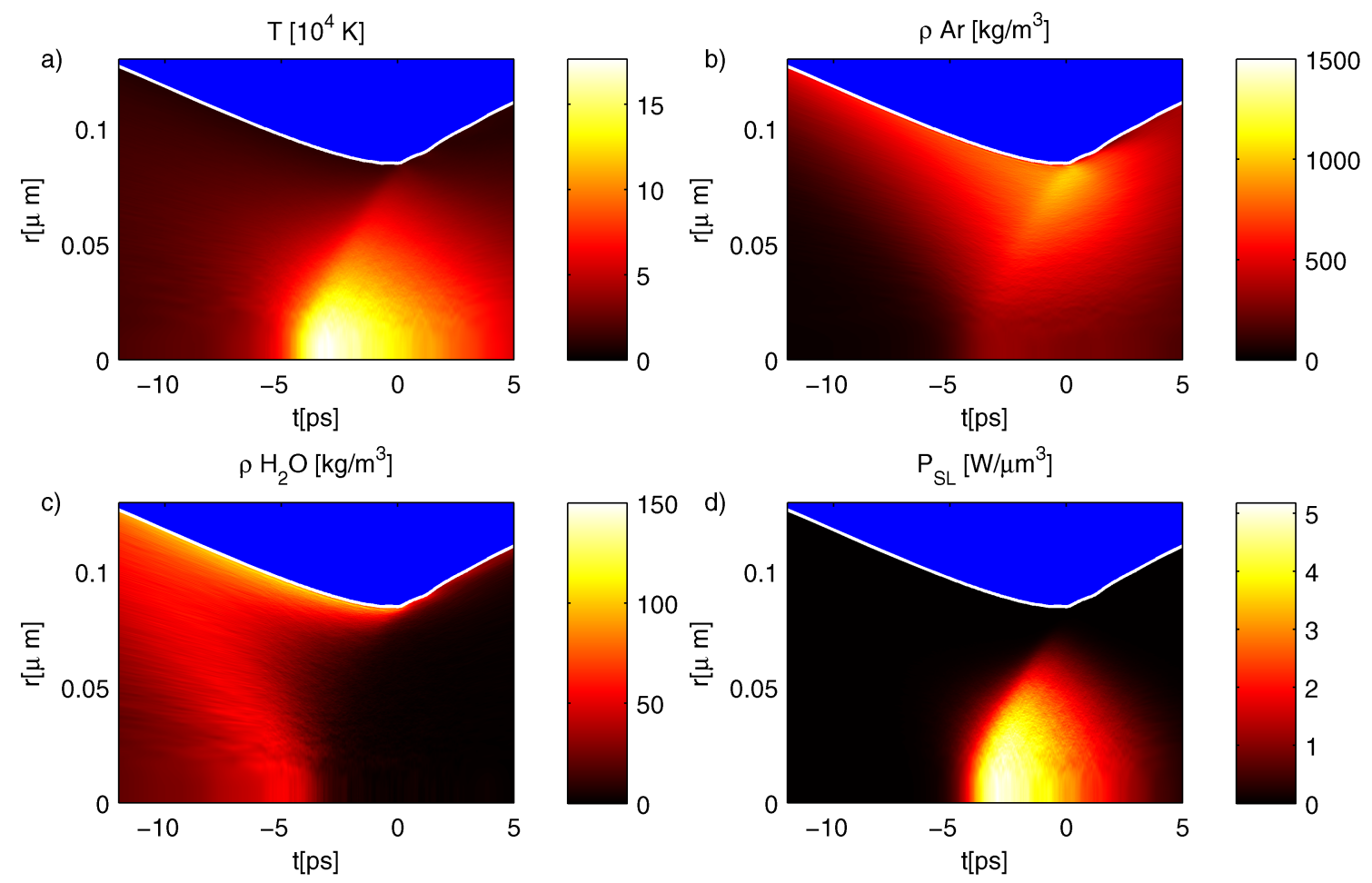

Abbildung 6.15: Verlauf von Temperatur (a), Dichte von Argon (b), Dichte von $\mathrm{H}_{2} \mathrm{O}$ (c) sowie der Lichtleistung für Fall 3 aus Tab. 6.4 $\left(R_{0}=400 \mathrm{~nm}\right)$.

1 und 2: der erreichte minimale Radius von $85 \mathrm{~nm}$ ergibt ein Kompressionsverhältnis $K=37,9$. Da gleichzeitig die Teilchenzahl steigt, verringern sich die Temperaturen deutlich: Es werden nunmehr maximal 176.000 K erreicht. Dies gilt, obwohl der Anteil der $\mathrm{H}_{2} \mathrm{O}$-Teilchen an der Gesamtteilchenzahl gegen Ende des Kollaps deutlich sinkt: bei Fall 2 beträgt der Anteil an Wasserdampf kurz vor Einsetzten der chemischen Reaktionen 47,7\%, in diesem Fall sind es 40,5\%. Da die Geschwindigkeit des Kollaps abnimmt, haben die Wasserdampfteilchen mehr Zeit an der Wand zu kondensieren.

Die emittierte Lichtenergie nimmt hingegen nochmals zu. Die doppelt so lange Leuchtdauer $\left(\Delta t_{S L}=3,98 \mathrm{ps}\right)$ und die gewachsene Emissionsregion $\left(d_{S L}\right.$ $=110 \mathrm{~nm}$ ) kompensieren die gesunkene Lichtleistung pro Volumen (maximal $\left.5 \mathrm{~W} / \mu \mathrm{m}^{3}\right)$. So wird insgesamt eine Energie von 12,5 fJ als Licht emittiert.

Abb. 6.16 zeigt den Radiusverlauf einer Blase mit $R_{0}=550 \mathrm{~nm}$ (Fall 4 aus Tab. 6.4). Es ist zu erkennen, dass die Blase nun nicht mehr einem Periode-2-Muster folgt, sondern sich die Blasenrandoszillationen ungefähr nach jeder vierten Oszillation wiederholen. Im weiteren Verlauf der Blasenschwingung sieht man auch 


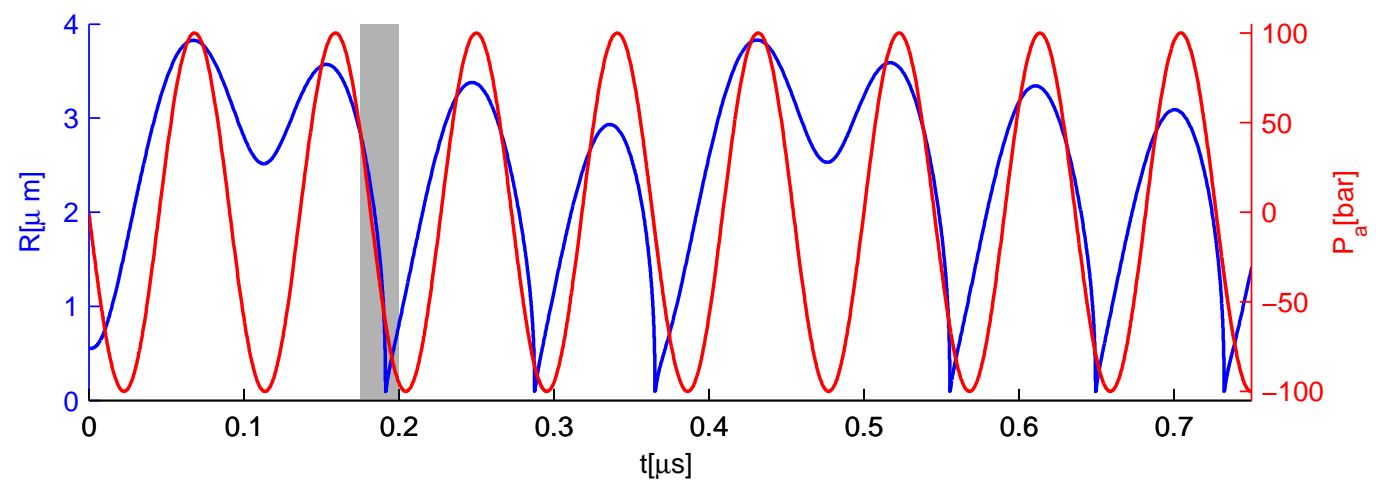

Abbildung 6.16: Radiusverlauf (-) und Anregungsdruck (-) für Fall 4 aus Tab. 6.4 $\left(R_{0}\right.$ $=550 \mathrm{~nm}$ ). Berechnet in einem einfachen polytropischen Modell. Der graue Bereich gibt die ungefähre Zeitspanne der MD-Simulation an.

Phasen, in denen das System in einer Periode-3 schwingt. Insgesamt ist die Blasenschwingung komplizierter geworden. Als Start der Hartkugelsimulation wurde ein Maximum vor einem vergleichsweise starken Kollaps ausgewählt, dessen Maximalradius $3,57 \mu \mathrm{m}$ beträgt.
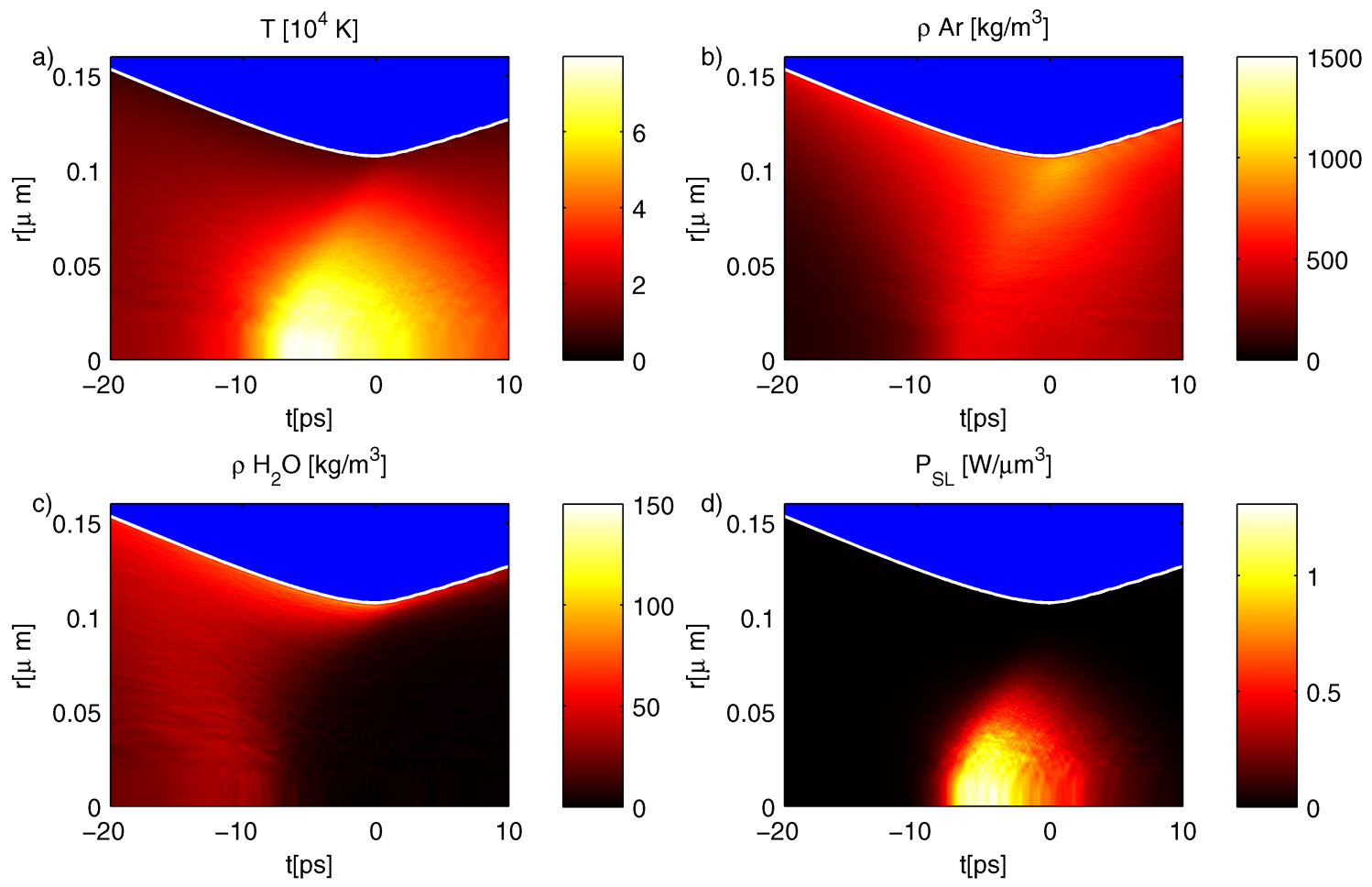

Abbildung 6.17: Verlauf von Temperatur (a), Dichte von Argon (b), Dichte von $\mathrm{H}_{2} \mathrm{O}$ (c) sowie der Lichtleistung für Fall 4 aus Tab. $6.4\left(R_{0}=550 \mathrm{~nm}\right)$. 
Abb. 6.17 zeigt Ergebnisse dieser Rechnung. Der minimale Blasenradius wird erreicht, wenn das Schallfeld bereits stark negativ ist, also ein hoher Unterdruck den Kollaps bremst. Daher wird ein vergleichsweise geringer Kompressionsfaktor von $K=31,1$ erreicht (mit $R_{\text {min }}=108 \mathrm{~nm}$ ). Aus diesem Grund - und aufgrund der weiter steigenden Teilchenzahl - sind die Temperaturen auf maximal $85.000 \mathrm{~K}$ reduziert. Die Kollapsgeschwindigkeit nimmt weiter ab, daher kann auch vergleichsweise mehr $\mathrm{H}_{2} \mathrm{O}$ der Blase entweichen. Die Dauer der Lichtemission steigt zwar weiter an $\left(\Delta t_{S L}=7,68 \mathrm{ps}\right)$, die aufgrund der niedrigeren Temperaturen stark verminderte Lichtleistung führt aber zu einer im Vergleich zu Fall 2 und 3 geringeren gesamt emittierten Lichtenergie von 6,5 fJ. Abb. 6.18 stellt zum

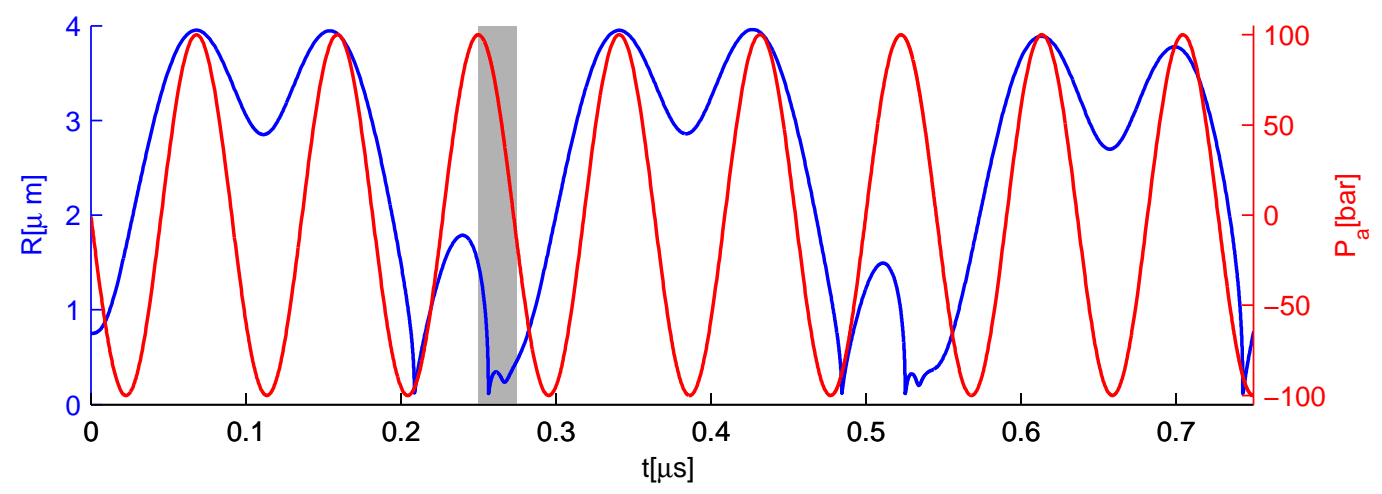

Abbildung 6.18: Radiusverlauf (-) und Anregungsdruck (-) für Fall 5 aus Tab. 6.4 $\left(R_{0}\right.$ $=750 \mathrm{~nm}$ ). Berechnet in einem einfachen polytropischen Modell. Der graue Bereich gibt die ungefähre Zeitspanne der MD-Simulation an.

Abschluß den Radiusverlauf einer Blase mit $R_{0}=750 \mathrm{~nm}$ (Fall 5 aus Tab. 6.4) dar. Es ist zu erkennen, dass die Blase zunächst zwei gleich große Maxima ohne dazwischenliegendem Kollaps aufweist. Im Anschluß kollabiert die Blase, allerdings verlaufen große Teile dieses Kollaps zu einer Zeit mit hohem negativem Druck, so dass er sehr schwach ausfällt. Danach schwingt die Blase relativ kurz auf, um wieder zu kollabieren. Dieser Kollaps endet in einer Überdruckphase, wodurch er trotz des kleinen Startradius heftiger abläuft als der vorige. Daher wird die MD-Simulation an diesem Kollaps gestartet. Am (nicht gezeigten) weiteren Verlauf der Blasenschwingung ist ersichtlich, dass die Größe des 'kleinen' Maximums recht stark schwankt, die vorliegende Hartkugelsimulation stellt also nur ein Beispiel unter vielen Möglichkeiten dar.

Die Ergebnisse dieser Rechnung, die in Abb. 6.19 dargestellt sind, zeigen, dass es sich bei diesem Fall um denjenigen mit den niedrigsten Temperaturen und der geringsten Lichtleistung handelt. Die Blase kollabiert bis auf 134 nm, was 

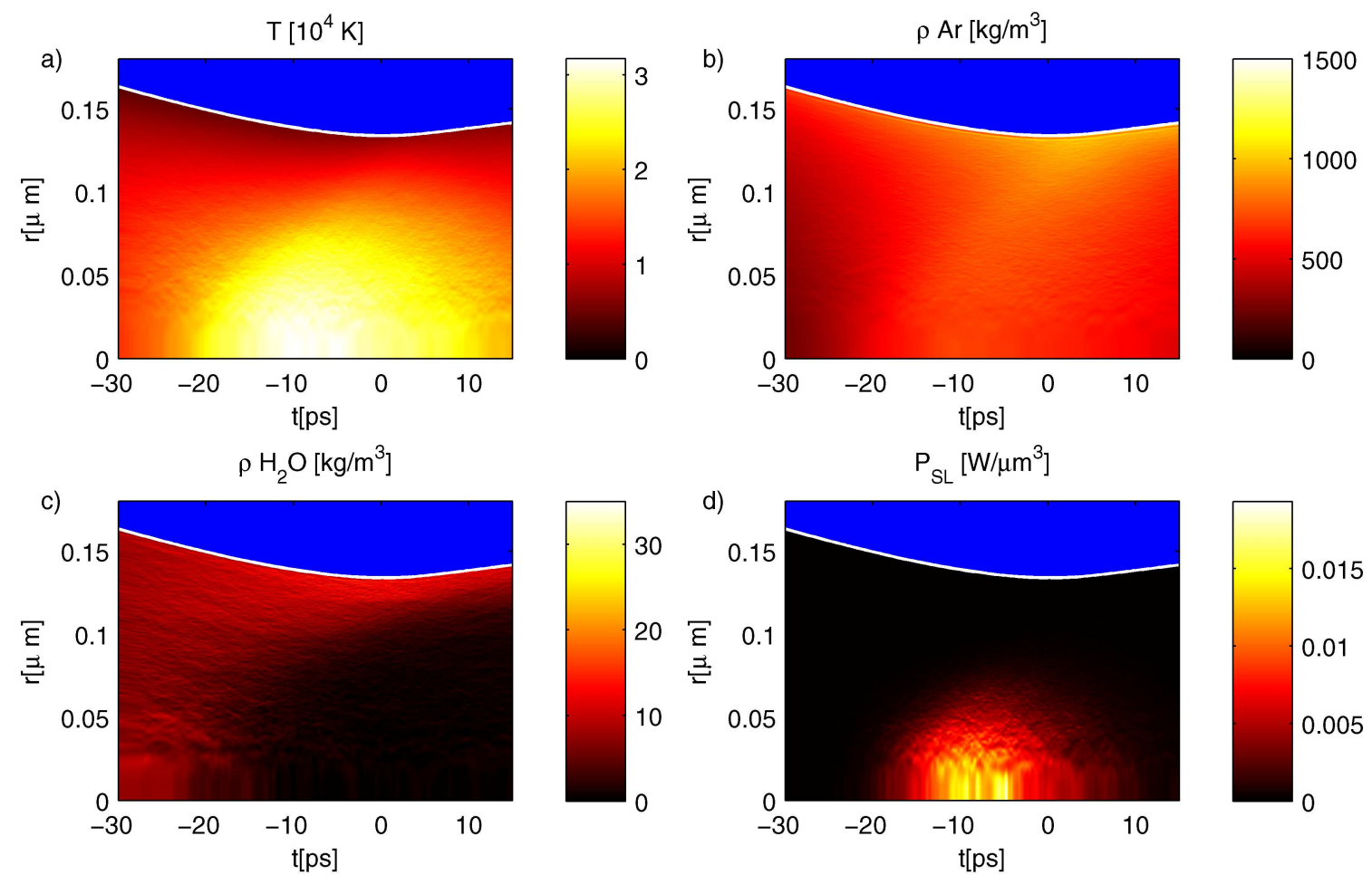

Abbildung 6.19: Verlauf von Temperatur (a), Dichte von Argon (b), Dichte von $\mathrm{H}_{2} \mathrm{O}$ (c) sowie der Lichtleistung für Fall 5 aus Tab. 6.4 $\left(R_{0}=750 \mathrm{~nm}\right)$.

zusammen mit dem geringen Maximalradius $\left(R_{\max }=1,79 \mathrm{~nm}\right)$ ein sehr niedriges Kompressionsverhältnis von $K=13,4$ ergibt. Daraus resultierend erreichen die Temperaturen maximal $31.700 \mathrm{~K}$. Die langsame Kollapsgeschwindigkeit führt $\mathrm{zu}$ einem geringen $\mathrm{H}_{2} \mathrm{O}$-Anteil von 25,6 \%. Die Lichtleistung ist mit maximal $0,02 \mathrm{~W} / \mu \mathrm{m}^{3}$ sehr gering, insgesamt werden lediglich $0,045 \mathrm{fJ}$ emittiert.

Aus den Ergebnissen der unterschiedlichen Fälle wird klar, dass man es in Blasenwolken, die mit hohen Frequenzen und Drücken angeregt werden, potentiell mit sehr unterschiedlichen Blasen zu tun hat, die vollkommen verschiedene Temperatur- und Leuchtcharakteristika aufweisen. Da die entstehenden Blasen transient sind, also nicht über lange Zeit, sondern ggf. nur einige hundert Perioden der Anregung (oder noch weniger) überleben, sind Blasen von sehr unterschiedlicher Anfangsgröße denkbar. Da die innere Dynamik, wie in den hier vorgestellten Untersuchungen erkennbar, stark von der Größe der Blasen abhängt, hat man es mit einem Bereich von Temperaturen zu tun, welcher sich über fast zwei Größenordnungen erstrecken kann. Die hieraus resultierenden Verschiebungen im Spektrum der einzelnen Blasen könnten durchaus das bei MBSL beobachtete 


\begin{tabular}{llllll}
\hline Fall & 1 & 2 & 3 & 4 & 5 \\
\hline Parameter & & & & & \\
$P_{A}[\mathrm{bar}]$ & 100,0 & 100,0 & 100,0 & 100,0 & 100,0 \\
Edelgas & $\mathrm{Ar}$ & $\mathrm{Ar}$ & $\mathrm{Ar}$ & $\mathrm{Ar}$ & $\mathrm{Ar}$ \\
$R_{0}[\mathrm{~nm}]$ & 130 & 250 & 400 & 550 & 750 \\
$N_{N G}^{r}\left[10^{6}\right]$ & 2,76 & 11,05 & 30,82 & 63,06 & 129,00 \\
$A_{v}$ & 0,3 & 0,3 & 0,3 & 0,3 & 0,3 \\
$\alpha_{t}$ & 0,3 & 0,3 & 0,3 & 0,3 & 0,3 \\
$v_{W, \text { init }}[\mathrm{m} / \mathrm{s}]$ & 80 & 80 & 50 & 50 & 50 \\
$S$ & 8,2 & 32,5 & 90,4 & 190,0 & 378,9 \\
\hline Dynamik & & & & & \\
$R_{\text {min }}[\mathrm{nm}]$ & 38 & 62 & 85 & 108 & 134 \\
$R_{\text {max }}[\mathrm{nm}]$ & 1.620 & 2.700 & 3.220 & 3.570 & 1.790 \\
$K$ & 42,6 & 43,5 & 37,9 & 33,1 & 13,4 \\
$T_{\varnothing}[\mathrm{K}]$ & 96.500 & 88.500 & 54.000 & 31.800 & 14.600 \\
$T_{\text {max }}[\mathrm{K}]$ & 459.000 & 396.000 & 176.000 & 85.000 & 31.700 \\
$\varrho_{\varnothing}\left[\mathrm{kg} / \mathrm{m}^{3}\right]$ & 1.500 & 1.510 & 1.410 & 1.190 & 890 \\
\hline Lichtemission & & & & & \\
$E_{S L}[\mathrm{fJ}]$ & 1,16 & 7,26 & 12,52 & 6,55 & 0,046 \\
$d_{S L}[\mathrm{~nm}]$ & 50 & 83 & 110 & 120 & 130 \\
$\Delta t_{S L}[\mathrm{ps}]$ & 1,13 & 1,90 & 3,98 & 7,68 & 15,43 \\
\hline Chemie & & & & & \\
$\mathrm{H}_{2} \mathrm{O}_{r}\left[10^{6}\right]$ & 2,2 & 10,1 & 21,0 & 26,0 & 5,2 \\
$\mathrm{H}_{2} \mathrm{O}_{r}[\%]$ & 44,3 & 47,7 & 40,5 & 29,2 & 25,9 \\
$\mathrm{H}_{2} \mathrm{O}_{\min }\left[10^{6}\right]$ & 0,1 & 0,3 & 1,2 & 2,8 & 1,7 \\
$\mathrm{OH}_{\mathrm{H}}\left[10^{6}\right]$ & 0,5 & 2,3 & 5,1 & 6,5 & 1,9 \\
$\mathrm{H}_{2}\left[10^{6}\right]$ & 1,1 & 5,0 & 10,3 & 12,4 & 1,6 \\
\hline & & & & &
\end{tabular}

Tabelle 6.4: Kenngrößen verschiedener Rechnungen von Blasen mit $\nu_{a}=11 \mathrm{MHz}$.

$R_{\max }$ bezeichnet hier die Höhe des (lokalen) Maximums vor dem Hauptkollaps, nicht unbedingt den absolut größten Radius. $K$ ist der Kompressionsfaktor, $T_{\varnothing}$ die maximale Durchschnittstemperatur, $\mathrm{T}_{\max }$ die absolut maximale Temperatur in der Blase, $\varrho_{\varnothing}$ die maximale Durchschnittsdichte, $E_{S L}$ die gesamte abgegebene Lichtenergie, $d_{S L}$ der Durchmesser der Emissionregion, $\Delta t_{S L}$ die Halbwertsbreite der Lichtemission, $\mathrm{H}_{2} \mathrm{O}_{r}$ ist die Anzahl der in der Blase gefangenen $\mathrm{H}_{2} \mathrm{O}-$ Moleküle kurz vor Einsetzen der chemischen Reaktionen (einmal in absoluter Menge, einmal als Anteil an der Gesamtteilchenzahl), $\mathrm{H}_{2} \mathrm{O}_{\text {min }}$ die minimale Anzahl an $\mathrm{H}_{2} \mathrm{O}$-Molekülen kurz nach $t_{R m i n}$, $\mathrm{OH}$ die maximale Anzahl an $\mathrm{OH}-$ Molekülen in der Blase und $\mathrm{H}_{2}$ die Anzahl an gebildeten $\mathrm{H}_{2}$-Molekülen.

kontinuierliche Spektrum des emittierten Lichts erklären. Da der Radiusverlauf der einzelnen Blasen zusätzlich noch in gewissen Grenzen fluktuiert, ist eine weiter Quelle der Verschmierung der Emissionen zu einem breitbandigen Spektrum existent.

Natürlich stößt das eingesetzte Modell bei derart extremen Bedingungen an seine Grenzen. Die Gültigkeit vieler Annahmen ist bei den auftretenden Temperaturen fragwürdig, die Eigenschaften des im Inneren auftretenden Plasmas sind nicht erfaßt. Auch ist der Anteil an Edelgas bei transienten Blasen nicht bekannt und im Modell vermutlich überschätzt. Dennoch vermitteln die erhaltenen Ergebnisse einen Eindruck von den auftretenden Trends, der sich zumindest teilweise auf die 
Realität übertragen läßt.

\subsubsection{Skalierung mit der Teilchenzahl}

An dieser Stelle sollen die Charakteristika identischer Blasen, die mit unterschiedlicher Teilchenanzahl molekulardynamisch simuliert wurden, verglichen werden. Wie schon in Kap. 4.4 angedeutet soll hier die Untersuchung der Skalierungsfaktoren nach unten abgeschlossen werden.

Mit dem Ziel, eine Blase mit dem Skalierungsfaktor $S=1$ zu simulieren, also eine mit der realen Teilchenzahl übereinstimmende Anzahl an Simulationsteilchen zu benutzen, wurde eine sehr kleine Blase mit einem Ruheradius von $R_{0}=70 \mathrm{~nm}$ ausgesucht. Diese enthält ca. $7,6 \cdot 10^{5}$ Edelgasteilchen. Damit zusätzlich relativ wenige Wasserdampfteilchen zu simulieren sind, wurde eine hohe Startgeschwindigkeit von $v_{\text {init }}=400 \mathrm{~m} / \mathrm{s}$ gewählt. Insgesamt sind bei $S=1$ damit ca. $1,5 \cdot 10^{6}$ Teilchen in der Blase. Die generellen Parameter entsprechen den im letzten Kapitel besprochenen. Von der selben Blase wurden auch Vergleichsrechnungen mit $S=10$ und $S=100$ durchgeführt. Die Parameter und einige Ergebnisse der drei Rechnungen sind in Tab. 6.5 zusammengefaßt. Abb. 6.20 zeigt zunächst den im polytropischen Modell ermittelten groben Radiusverlauf der Blase. Man erkennt, dass die Blase in einem Periode-2-Rhythmus schwingt mit einem schwachen und einem starken Kollaps je Schwingungsperiode. Der starke Kollaps findet in der Überdruckphase statt und startet von einem vergleichweise großen Maximalradius $\left(R_{\max }=2,3 \mu \mathrm{m}\right)$. Die Hartkugelsimulation wird in diesem Kollaps gestartet.

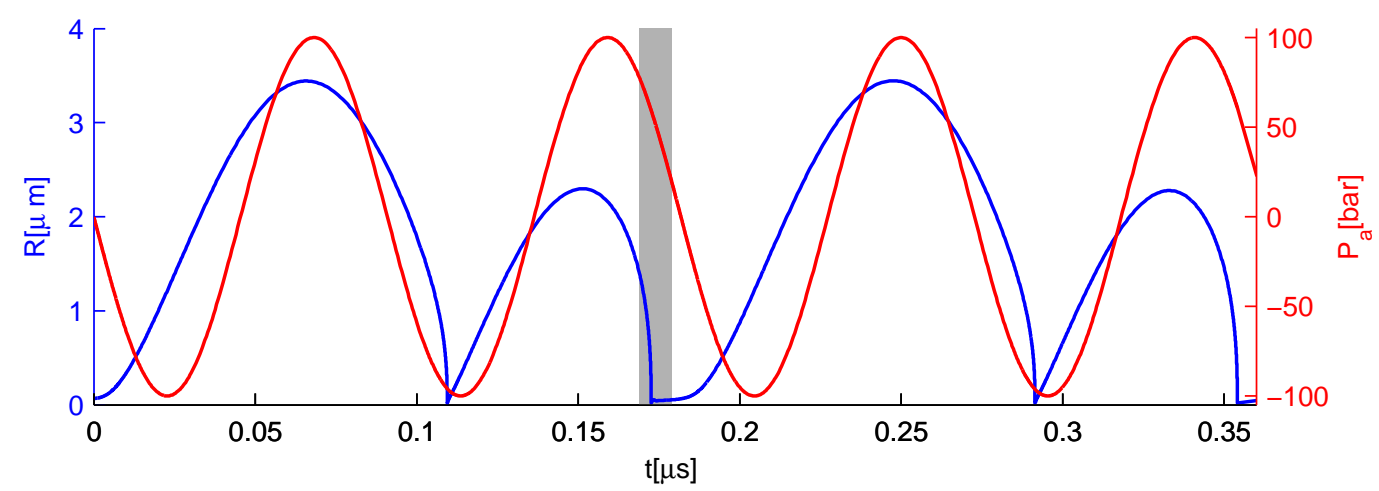

Abbildung 6.20: Radiusverlauf (-) und Anregungsdruck (-) für eine Blase mit $R_{0}=70 \mathrm{~nm}$ unter den im vorigen Kapitel besprochenen Bedingungen. Berechnet in einem einfachen polytropischen Modell. Der graue Bereich gibt die ungefähre Zeitspanne der MD-Simulation an. 

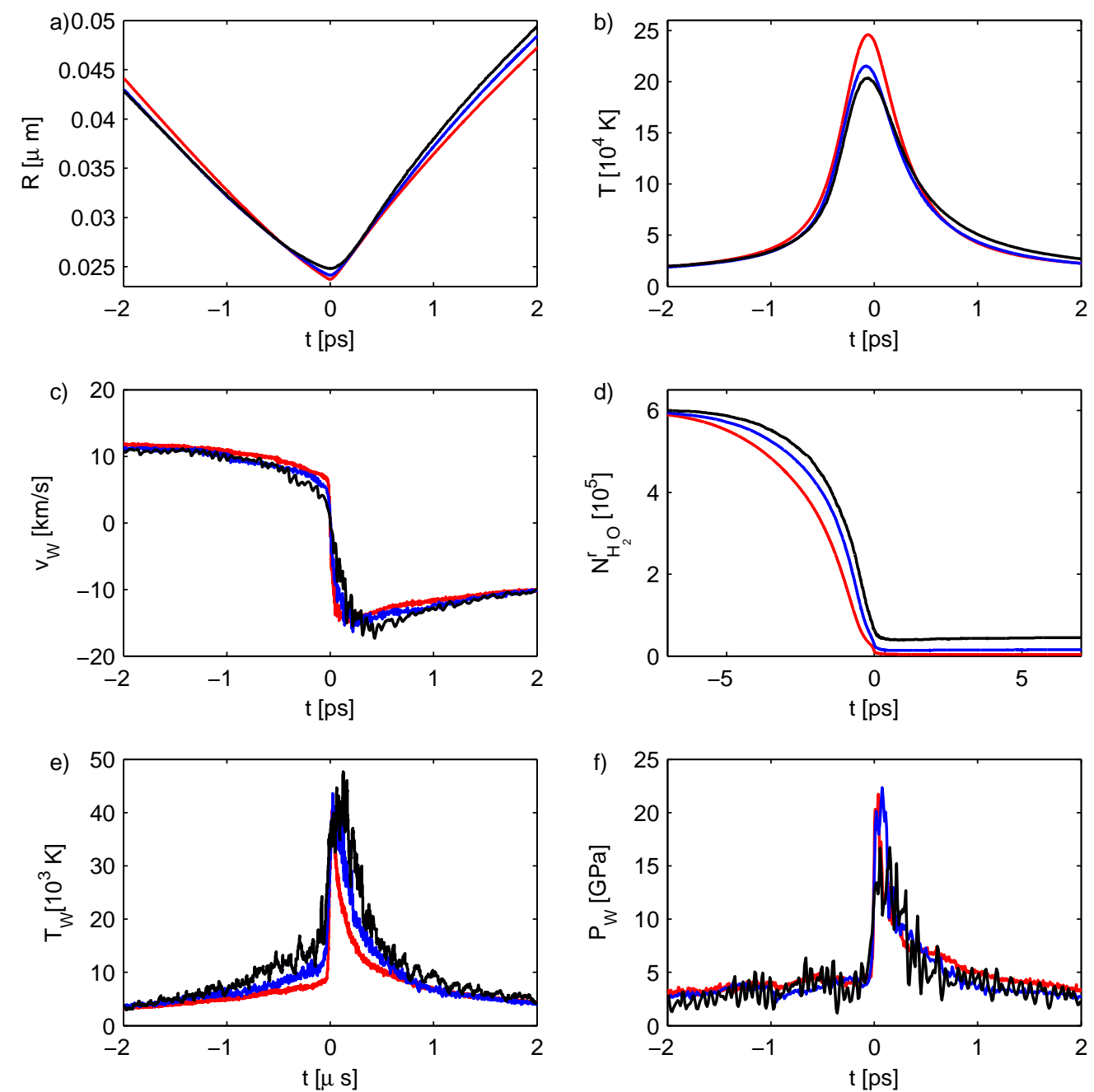

Abbildung 6.21: Entwicklung des Blasenradius (a), der Durchschnittstemperatur (b), der Blasenwandgeschwindigkeit (c), der realen Anzahl der in der Blase befindlichen $\mathrm{H}_{2} \mathrm{O}$-Moleküle (d), der Temperatur an der Blasenwand (e) sowie des Druckes am Blasenrand (f) für die in Tab. 6.5 beschriebenen Rechnungen.

Legende: $S=1($ ( ); $S=10($ ()); $S=100($ ()).

Abb. 6.21 zeigt den Vergleich einiger Blasenparameter für die unterschiedlichen Rechnungen. Für alle Fälle ergeben sich vergleichbare Kurvenverläufe, die Unterschiede betragen zumeist nur wenige Prozent. Je mehr Teilchen simuliert werden, desto glatter verlaufen die Graphen. Die Blase mit $S=1$ (Fall 1 aus Tab. 6.5) kollabiert am stärksten $\left(R_{\min }=23,8 \mathrm{~nm}\right)$ und weist die höchsten Durchschnittstemperaturen auf. Die Anzahl an gefangenem $\mathrm{H}_{2} \mathrm{O}$ ist für alle Fälle vergleichbar, die chemische Aktivität (Abbau der Wasserdampfmoleküle) ist durch die erhöhten Temperaturen in Fall 1 am ausgeprägtesten. Eine Diversifizierung 
des Wasserdampfanteils für verschiedenen $S$, wie in Kap. 4.4 beobachtet, wird hier vermutlich durch den späten Start der Simulation verhindert, da der Effekt der unterschiedlichen Teilchendiffusion nur kurz wirken kann. Wie schon in Kap. 4.4 festgestellt, gibt es einen starken Zusammenhang der skalierten Anzahl an Teilchenkollisionen mit dem Skalierungsfaktor, während die skalierte Anzahl an Wandkollisionen nahezu unverändert bleibt (siehe Tab. 6.5).
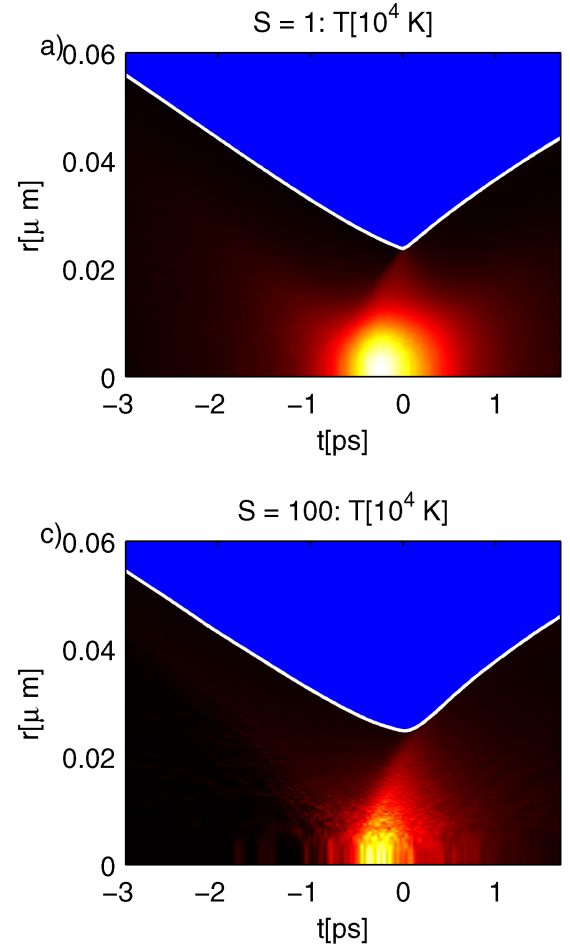
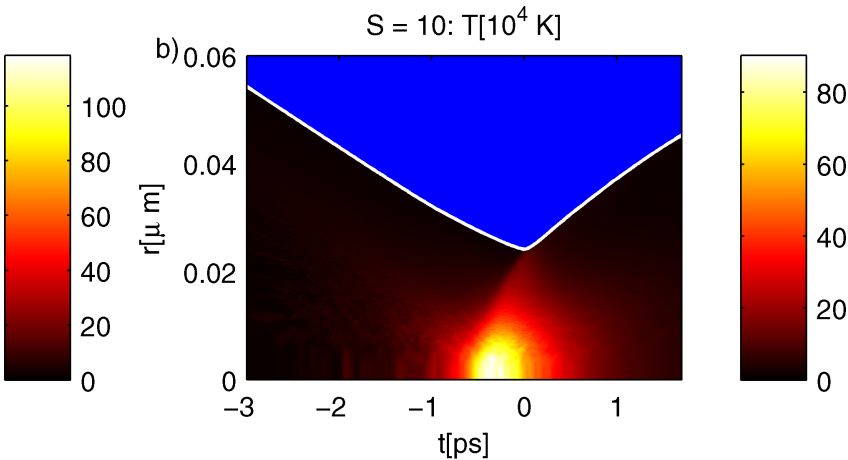

$$
S=1: P_{S L}\left[W / \mu m^{3}\right]
$$
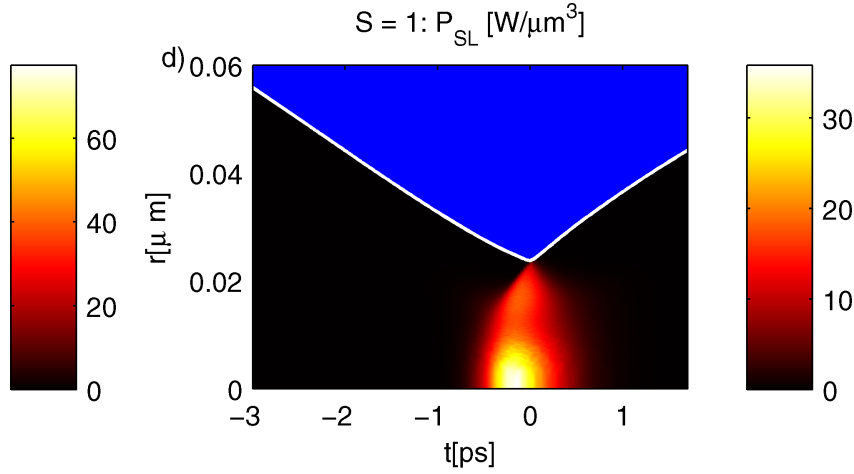

Abbildung 6.22: Entwicklung der Temperatur für die in Tab. 6.5 beschriebenen Rechnungen: (a): $S=1,(\mathrm{~b}): S=10,(\mathrm{c}): S=100$, sowie die Entwicklung der Lichtleistung für $S=1$ (d).

Abb. 6.22 zeigt die Entwicklung der Temperatur in den betrachteten Blasen um den Zeitpunkt des minimalen Radius. Die äußerst starke Kompression $(K \approx$ 120), die aus dem stark positiven Anregungsdruck, dem hohen Maximalradius und der geringen Teilchenzahl resultiert, führt für alle Fälle von $S$ zu extremen Temperaturen. Fall 1 weist mit maximal $11,8 \cdot 10^{5} \mathrm{~K}$ die höchsten Werte auf. Mit steigendem $S$ fallen diese auf $9,0 \cdot 10^{5} \mathrm{~K}$ (Fall 2) bzw. $7,7 \cdot 10^{5} \mathrm{~K}$ (Fall 1) ab. Die maximalen Durchschnittstemperaturen fallen genauso von $2,45 \cdot 10^{5}$ (Fall 3) auf $2,05 \cdot 10^{5}$ (Fall 3) ab. Die Maximal- und Durchschnittstemperaturen zeigen in diesem Fall also eine umgekehrte Abhängigkeit von der Skalierung, als dies in Kap. 4.4 der Fall war. Dies zeigt nochmals die Abhängigkeit der inneren Dynamik vom Wasserdampfgehalt: da bei den hier betrachteten Blasen die Anzahl 
an gefangenen $\mathrm{H}_{2} \mathrm{O}$-Molekülen für alle Fälle gleich ist, werden die Temperaturen für Rechnungen mit höherer Teilchenzahl nicht herabgesetzt, wie dies bei den Untersuchungen in Kap. 4.4 beobachtet wurde. Die durch eine höhere Simulationsteilchenzahl verstärkte Stoßdynamik kann hier voll in höhrere Temperaturen umgesetzt werden.

Trotz der verschiedenen Temperaturen ist die Lichtemission in allen drei Fällen vergleichbar groß, da die Dauer des Leuchtens mit Zunahme der Temperatur abnimmt. In Abb. 6.22, d ist die Lichtemission beispielhaft für Fall 1 dargestellt. Es läßt sich sagen, dass die in Kap. 4.4 gefundenen Trends bei Variation des Skalierungsfaktors $S$ hier auch für kleine Blasen bestätigt werden: Die Stoßdynamik nimmt mit wachsender Teilchenzahl zu, die erreichten Ergebnisse sind aber bei Skalierungen, die um 2 Größenordnungen auseinander liegen, durchaus miteinander zu vergleichen. Je nachdem, ob dem Wasserdampf genug Zeit bleibt, in relevantem Maße aus der Blase zu verdampfen, machen sich die Diffusionsunterschiede der Rechnungen bemerkbar. Eine kompensation der verstärkten Stoßdynamik durch eine abgeschwächte Kondensation von Wasserdampf am der Blasenwand wird durch die geringe Blasengröße und den schnellen Kollaps verhindert. 


\begin{tabular}{|c|c|c|c|}
\hline Fall & 1 & 2 & 3 \\
\hline \multicolumn{4}{|l|}{ Parameter } \\
\hline$P_{A}[\mathrm{bar}]$ & 100,0 & 100,0 & 100,0 \\
\hline Edelgas & $\mathrm{Ar}$ & $\mathrm{Ar}$ & $\mathrm{Ar}$ \\
\hline$R_{0}[\mathrm{~nm}]$ & 70 & 70 & 70 \\
\hline$N_{N G}^{r}\left[10^{5}\right]$ & 7,59 & 7,59 & 7,59 \\
\hline$A_{v}$ & 0,3 & 0,3 & 0,3 \\
\hline$\alpha_{t}$ & 0,3 & 0,3 & 0,3 \\
\hline$v_{W, \text { init }}[\mathrm{m} / \mathrm{s}]$ & 400 & 400 & 400 \\
\hline$N_{g e s, i}$ & 1.520 .875 & 148.877 & 15.625 \\
\hline$S$ & 1,04 & 10,69 & 101,88 \\
\hline$\Delta K_{W}$ & 618 & 500 & 150 \\
\hline \multicolumn{4}{|l|}{ Dynamik } \\
\hline$R_{\max }[\mathrm{nm}]$ & 2.295 & 2.295 & 2.295 \\
\hline$R_{\min }[\mathrm{nm}]$ & 23,8 & 24,1 & 24,8 \\
\hline$K$ & 123,9 & 122,4 & 118,9 \\
\hline$v_{W}[\mathrm{~km} / \mathrm{s}]$ & 11,8 & 11,3 & 11,0 \\
\hline$T_{\max }\left[10^{5} \mathrm{~K}\right]$ & 11,8 & 9,0 & 7,7 \\
\hline$T_{\varnothing}[\mathrm{K}]$ & 2,45 & 2,15 & 2,05 \\
\hline$N_{g e s, c}$ & 1.373 .198 & 131.945 & 13.735 \\
\hline$\#_{P C}\left[10^{7}\right]$ & 17,23 & 8,09 & 3,74 \\
\hline$\#_{W C}\left[10^{6}\right]$ & 7,99 & 7,62 & 7,48 \\
\hline \multicolumn{4}{|l|}{ Lichtemission } \\
\hline$E_{S L}[\mathrm{fJ}]$ & 0,46 & 0,42 & 0,46 \\
\hline$d_{S L}[\mathrm{~nm}]$ & 28 & 28 & 28 \\
\hline$\Delta t_{S L}[\mathrm{ps}]$ & 0,46 & 0,48 & 0,53 \\
\hline \multicolumn{4}{|l|}{ Chemie } \\
\hline $\mathrm{H}_{2} \mathrm{O}_{r}\left[10^{5}\right]$ & 6,1 & 6,1 & 6,1 \\
\hline $\mathrm{H}_{2} \mathrm{O}_{r}[\%]$ & 44,5 & 44,5 & 44,5 \\
\hline $\mathrm{H}_{2} \mathrm{O}_{\min }\left[10^{4}\right]$ & 0,1 & 1,5 & 4,0 \\
\hline $\mathrm{OH}\left[10^{5}\right]$ & 2,2 & 1,8 & 1,4 \\
\hline $\mathrm{H}_{2}\left[10^{5}\right]$ & 3,0 & 2,9 & 2,7 \\
\hline
\end{tabular}

Tabelle 6.5: Kenngrößen verschiedener Rechnungen von Blasen mit $\nu_{a}=11 \mathrm{MHz}$ und $R_{0}=$ $70 \mathrm{~nm}$ unter variierter Teilchenzahl.

$R_{\max }$ bezeichnet hier die Höhe des (lokalen) Maximums vor dem Hauptkollaps, nicht unbedingt den absolut größten Radius. $K$ ist der Kompressionsfaktor, $v_{W}$ die maximale Wandgeschwindigkeit, $T_{\varnothing}$ die maximale Durchschnittstemperatur, $T_{\max }$ die absolut maximale Temperatur in der Blase, $N_{g e s, c}$ die Anzahl an Simulationspartikeln gegen Ende des Kollaps, \# ${ }_{P C}$ die Gesamtzahl der realen Teilchenkollisionen bis $t_{R m i n}, \#_{W C}$ die Gesamtzahl der realen Wandkollisionen bis $t_{R m i n}, E_{S L}$ die gesamte abgegebene Lichtenergie, $d_{S L}$ der Durchmesser der Emissionregion, $\Delta t_{S L}$ die Halbwertsbreite der Lichtemission, $\mathrm{H}_{2} \mathrm{O}_{r}$ die Anzahl der in der Blase gefangenen $\mathrm{H}_{2} \mathrm{O}$ Moleküle kurz vor Einsetzen der chemischen Reaktionen (einmal in absoluter Menge, einmal als Anteil an der Gesamtteilchenzahl), $\mathrm{H}_{2} \mathrm{O}_{\min }$ die minimale Anzahl an $\mathrm{H}_{2} \mathrm{O}$-Molekülen kurz nach $t_{R \min }, \mathrm{OH}$ die maximale Anzahl an $\mathrm{OH}-$ Molekülen in der Blase und $\mathrm{H}_{2}$ die Anzahl an gebildeten $\mathrm{H}_{2}$-Molekülen. 


\section{Kapitel 7}

\section{Ergebnisse für laser-induzierte Blasen}

In diesem Teil der Arbeit werden Ergebnisse präsentiert, die von Simulationen einer laser-induzierten Blase gewonnen wurden. Das Prinzip der Kavitation durch optischen Durchbruch ist in Kap. 2.3 dargestellt.

Experimentelle Grundlage bilden die von KuRz et al. durchgeführten Versuche an akustisch angeregten Laserblasen [144]. Der bei diesen Experimenten verwendete Laser hatte eine Energie von $0.22 \mu \mathrm{J}$ bei einer Pulslänge von $130 \mathrm{fs}$. Ziel war es, den an sich relativ schwachen Kollaps einer laser-induzierten Blase durch akustische Anregung zu verstärken. In solchen Blasen läßt sich unter geeigneten Umständen Kavitationslumineszenz beobachten [145]. Hier sollen die in [144] durchgeführten Experimente in dem bereits bekannten Molekulardynamikmodell nachvollzogen und Aussagen über den Zustand des Blaseinneren gewonnen werden.

In diesem Kapitel wird zunächst die Simulation der Anfangsphase der Blasenbewegung - kurz nach dem optischen Durchbruch - beschrieben. Danach wird auf eine Blase in einem nicht-angeregten Medium eingegangen, zum Schluß dann auf getriebene laser-induzierte Blasen.

Als Blaseninhalt wird lediglich Wasserdampf sowie die in der heißen Phase des optischen Durchbruchs gebildeten chemischen Spezies angenommen. Eine Diffusion von Edelgas in die Blase ist aufgrund der kleinen Zeitskala vernachlässigbar [146]. 


\subsection{Anfangsbedingung}

Die Entstehung des Plasmas kann durch das Modell nicht erfaßt werden, da eine molekulardynamische Simulation der Elektronen zu aufwendig wäre. Die Rechnung startet zu dem Moment, an dem das Elektronengas seine Energie an das Medium abgegeben hat.

Es wurden folgende Annahmen zum Zustand der Blase zu diesem Zeitpunkt gemacht:

- Die Blase besteht aus Wasserdampf mit eine Dichte von $350 \mathrm{~kg} / \mathrm{m}^{3}$ und einer Temperatur von 3000 K. Da die Blase zu diesem Zeitpunkt bereits zu einem gewissen Teil expandiert sein muß, wird nicht von der Wasserdichte ausgegangen.

- Ein Drittel des Wasserdampfes wurde von der Laserenergie dissoziiert und liegt als freie $O$ - und $H$-Atome vor.

- Die Anfangsgröße der Blase wurde auf 1,4 $\mu \mathrm{m}$ festgesetzt. Dieser Wert wurde empirisch aus experimentellen Daten gewonnen.

- Die Blase wird als sphärisch angenommen. Eine eventuelle Elliptizität wird vernachlässigt.

Durch die explosionsartige Ausdehnung des Mediums wird eine Stoßwelle ausgesandt, welche das Wachstum der Blase hemmt. Da diese nicht durch das RayleighPlesset-Modell erfaßt wird, wird der Stoßdruck durch folgenden Maßnahmen simuliert:

- Zunächst wird die Bewegung des Blasenrandes am Start der Simulation festgehalten. Es findet ein langsamer Übergang statt, durch den die Bewegung des Blasenrandes bei ca. drei ns wieder voll durch die Rayleigh-PlessetGleichung beschrieben wird.

- Außerdem wird in der frühen Phase ein Gegendruck $P_{S W}$ von 1 kbar der Expansion der Blase entgegen gestellt. Auch dieser wird langsam relaxiert und erreicht bei $t=10 \mathrm{~ns}$ den Wert Null. 

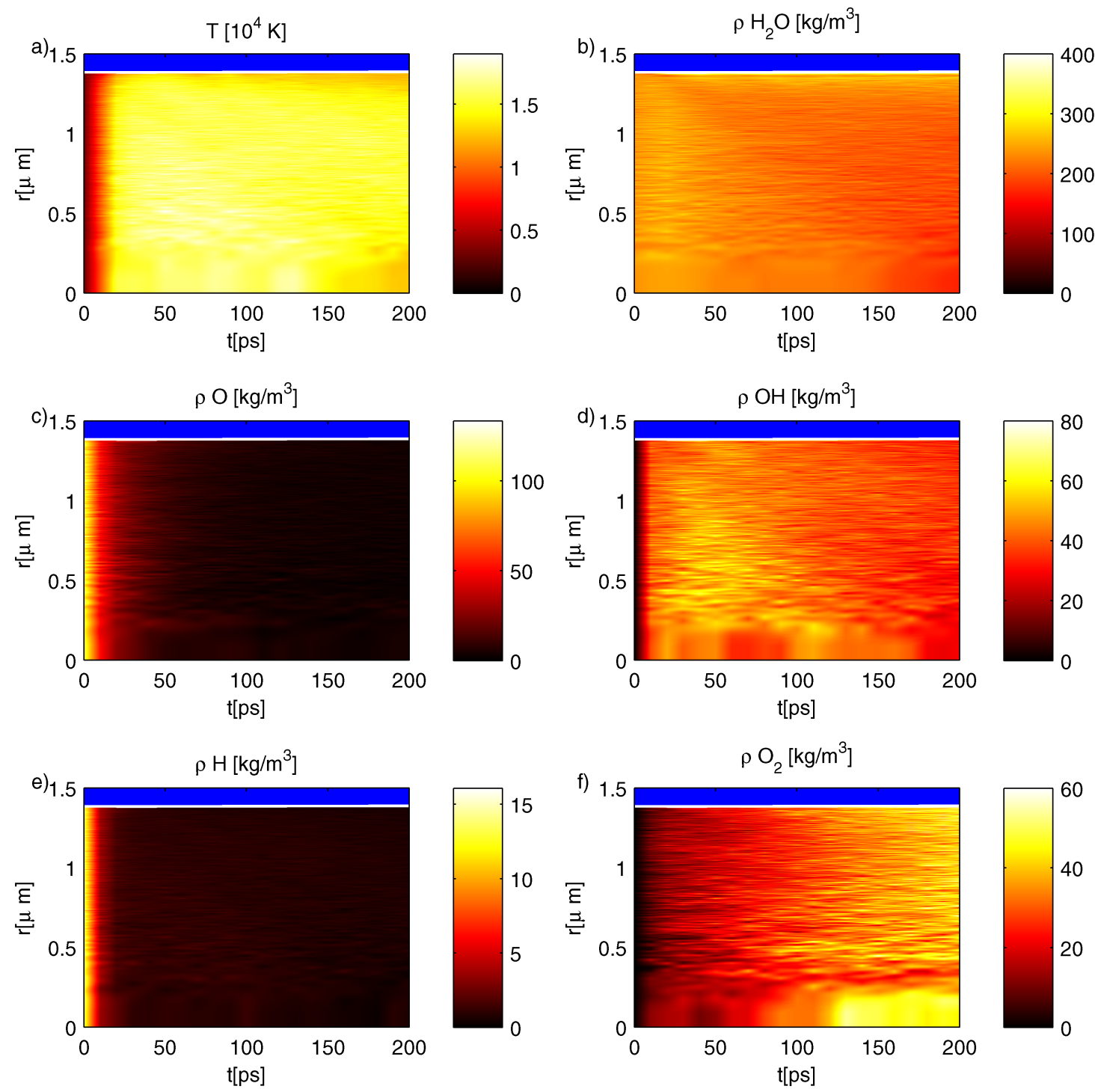

Abbildung 7.1: Entwicklung der Temperatur (a), sowie der Dichte von $\mathrm{H}_{2} \mathrm{O}$ (b), O (c), $\mathrm{OH}$ (d), $\mathrm{H}$ (e) und $\mathrm{O}_{2}$ (f) in den ersten 200 ps der Simulation. Parameter siehe Tab. 7.1.

Nach 10 ns wird keine Beeinflussung der Blasenrandbewegung mehr vorgenommen. Ohne diese Ausbremsung in frühen Stadien würde die Blase sofort rapide wachsen und sehr große Radien erreichen, da die Energie, die die Blase durch die Stoßwellenaussendung verliert, unterschätzt würde - entsprechend würde die zur Blasenexpansion zur Verfügung stehende Energie überschätzt. In diesem Abschnitt soll zunächst dargestellt werden, welche Bedingungen in der Blase während der Entstehungsphase der Blase herrschen. Für die Simulation werden 216.000 Teilchen verwendet. Darunter sind $86.400 \mathrm{H}_{2} \mathrm{O}$-Teilchen, 86.400 


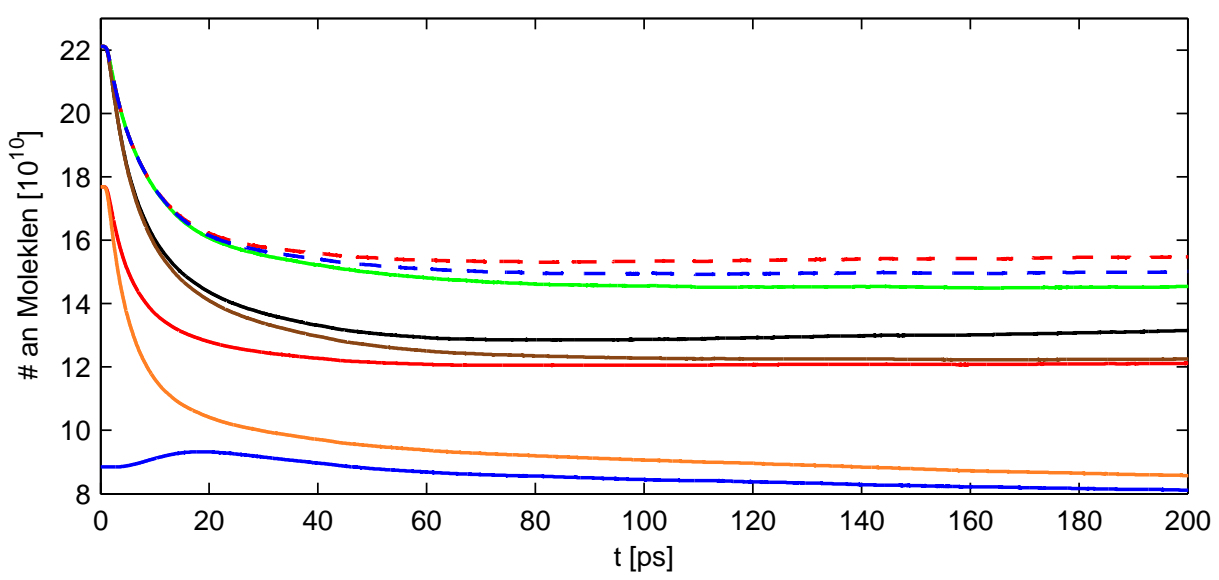

Abbildung 7.2: Entwicklung der realen Anzahl aller betrachteter Spezies für die ersten 200 ps der Simulation. Kumulierte Darstellung - jede Kurve besteht aus der Summe der darunter liegenden sowie der Teilchenanzahl der jeweiligen Spezies. Parameter siehe Tab. 7.1. Legende: $\mathrm{H}_{2} \mathrm{O}(-) ; \mathrm{H}(-) ; \mathrm{H}_{2}(-) ; \mathrm{O}(-) ; \mathrm{O}_{2}(-) ; \mathrm{OH}(-) ; \mathrm{HO}_{2}$ (- -); $\mathrm{H}_{2} \mathrm{O}_{2}$ (- -).

H-Teilchen und 43.200 O-Teilchen. Diese Verteilung basiert auf der Annahme, dass ein Drittel aller Wassermoleküle durch die Laserenergie dissoziiert wurde. Den Teilchen wird eine Temperatur von $3000 \mathrm{~K}$ gegeben, die Wassertemperatur liegt bei $300 \mathrm{~K}$. Weitere Parameter sind in Tab. 7.1 aufgeführt.

Abb. 7.1 zeigt die Entwicklung der Temperatur sowie der Dichte einiger Spezies während der ersten 200 ps der Simulation in Raum und Zeit. Abb. 7.2 zeigt für denselben Zeitraum die Entwicklung der Anzahl aller Spezies in kumulativer Darstellung. Es ist zu erkennen, dass die anfänglichen Temperaturen von $3.000 \mathrm{~K}$ sehr schnell durch exotherme Reaktionen auf ca. 18.000 K ansteigen. Die in der Dissoziation der Wassermoleküle gespeicherte Energie wird gleichmäßig in der gesamten Blase freigesetzt. Bereits nach ca. 20 ps ist dieser Prozeß abgeschlossen. Gleichzeitig ist eine starke Abnahme des atomaren Sauerstoffs und Wasserstoffs zu beobachten. - diese reagieren zunächst hauptsächlich zu $\mathrm{OH}$ und $\mathrm{H}_{2}$. Auch eine

\begin{tabular}{ccc|ccc|ccc}
\hline$P_{S W}$ & - & $1.000 \mathrm{bar}$ & $N_{\text {ges }, i}$ & - & 216.000 & $v_{W, \text { init }}$ & - & $0 \mathrm{~m} / \mathrm{s}$ \\
$t_{P_{S W}}$ & - & $10 \mathrm{~ns}$ & $S$ & - & $\approx 1.000 .000$ & $\alpha_{t}$ & - & 0.3 \\
$R_{\text {init }}$ & - & $1,4 \mu \mathrm{m}$ & Edelgas & - & - & $\alpha_{v}$ & - & 0.1 \\
$T_{\text {water }}$ & - & $300 \mathrm{~K}$ & $\Delta K_{W}$ & - & 3000 & $T_{G a s, i}$ & - & $3.000 \mathrm{~K}$ \\
$M F_{H_{2} O}$ & - & 0,4 & $M F_{H}$ & - & 0,4 & $M_{O}$ & - & 0,2 \\
\hline
\end{tabular}

Tabelle 7.1: Parameter der Rechnungen der Dynamik einer laser-induzierten Blase unmittelbar nach dem Laserpuls. Hier bezeichnet $P_{S W}$ den die Stoßwellenabstrahlung simulierenden Gegendruck, $t_{P_{S W}}$ den Zeitraum zu Anfang der Simulation, zu dem $P_{S W}$ wirkt, $R_{\text {init }}$ den initialen Blasenradius, $M F_{X}$ den anfänglichen Teilchenzahlanteil der verschiedenen Spezies und $T_{G a s, i}$ die anfängliche Temperatur des Blaseninneren. 
leichte Zunahme an $\mathrm{H}_{2} \mathrm{O}$-Molekülen ist zu beobachten. Der schnelle molekulare Wasserstoff wird als erstes abgebaut, molekularer Sauerstoff ist etwas länger in der Blase vorhanden. Nach 30 ps sind von anfänglich $8,85 \cdot 10^{10}$ realen H-Atomen nur noch ca. $0,82 \cdot 10^{10}$ übrig, bei O-Atomen sind es $0,92 \cdot 10^{10}$ von $4,43 \cdot 10^{10} \mathrm{zu}$ Beginn. Die maximale Durchschnittstemperatur der Blase wird nach ca. 50 ps erreicht, sie beträgt $16.300 \mathrm{~K}$. Die Blasentemperatur nimmt nun langsam ab, währenddessen werden die $\mathrm{OH}$-Verbindungen und zu einem gewissen Teil auch $\mathrm{H}_{2} \mathrm{O}$ zu stabilen Spezies wie $\mathrm{O}_{2}$ und $\mathrm{H}_{2}$ umgesetzt. Die Anzahl an H-Atomen sinkt nur noch langsam ab, da diese durch die immer noch hohen Temperaturen laufend nachproduziert werden. Abb. 7.3 zeigt die weitere Entwicklung der
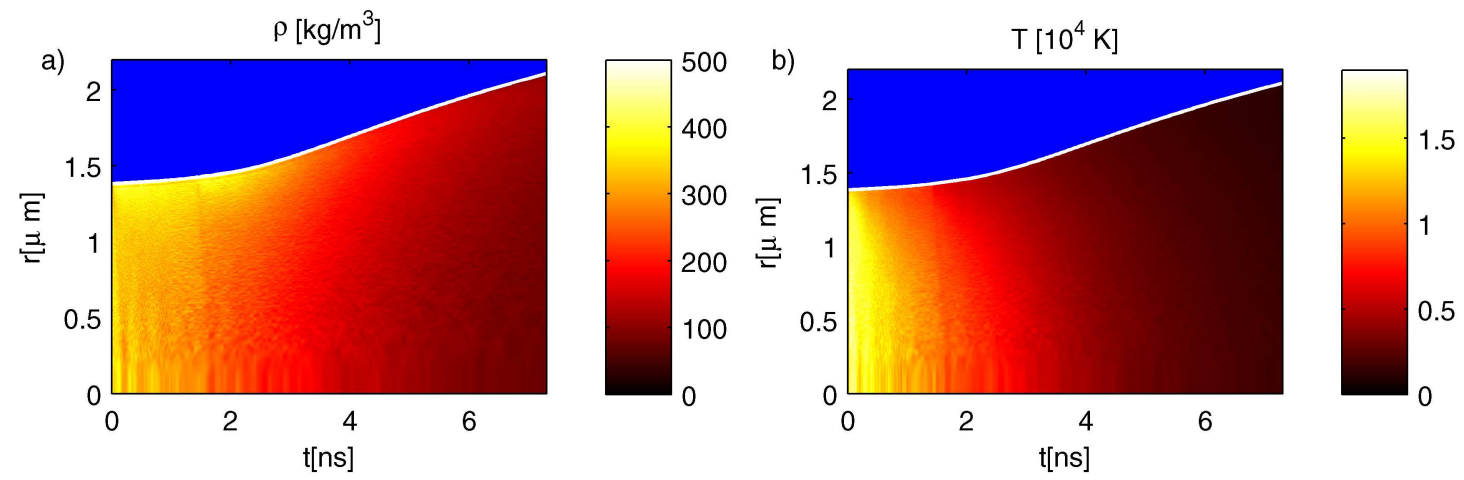

Abbildung 7.3: Entwicklung der Temperatur (a), und der Dichte (b) in den ersten 7 ns der Simulation einer laser-induzierten Blase mit den in Tab. 7.1. beschriebenen Parametern

Dichte und der Temperatur während der ersten 7 ns. Die Blase beginnt trotz des hohen Gegendruckes und der gehemmten Bewegungsfreiheit praktisch sofort zu expandieren. Diese Expansion ist zunächst langsam, nimmt aber ab ca. 2 ns deutlich zu und erreicht ein Maximum (ca. $150 \mathrm{~m} / \mathrm{s}$ bei $4 \mathrm{~ns}$ ). Danach nimmt die Blasenwandgeschwindigkeit wieder ab und erreicht nach 10 ns einen Wert von $60 \mathrm{~m} / \mathrm{s}$, der dann, mit dem kompletten Wegfall des Gegendruckes, nur langsam weiter sinkt.

Die Temperaturen sinken durch Expansion der Blase und Wärmeabgabe am Blasenrand schnell wieder ab. Die anfänglichen $3.000 \mathrm{~K}$ unterschreitet die Blasentemperatur 4,2 ns nach dem Laserpuls, bei $10 \mathrm{~ns}$ ist sie auf $900 \mathrm{~K}$ abgefallen. 


\subsection{Blase in ruhendem Medium}

In diesem Abschnitt wird die Dynamik des Blasenrandes und des Blaseninneren für eine Blase besprochen, die in stillem Wasser, ohne zusätzliche Schallanregung, von einem Laserpuls erzeugt wurde. Eine fotographische Aufnahme der Expansion und nachfolgendem Kollaps einer solchen Blase ist in Abb. 7.4 gegeben. Der

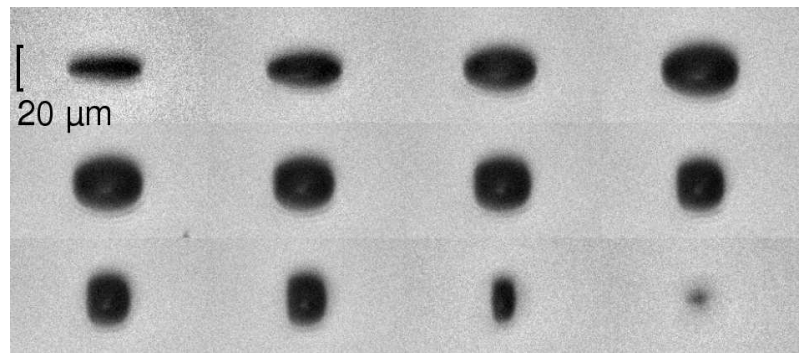

Abbildung 7.4: Fotographische Wiedergabe der Dynamik einer von einem 130 fs-Puls mit einer Energie von $0.22 \mu \mathrm{J}$ produzierten Blase. Der Bildabstand beträgt $200 \mathrm{~ns}$. Aufgenommen von KRÖNINGER [147].

Radiusverlauf einer mit dem in dieser Arbeit genutzten Molekulardynamikmodell simulierten Blase ist in Abb. 7.5 dargestellt. Aus Abb. 7.4 ist ersichtlich, dass die von fs-Pulsen erzeugten Blase nicht sphärisch sind: der Bereich, in dem der optische Durchbruch auftritt ist elongiert, woraus eine elliptische Blase resultiert. Diese hat eine maximale Größe von ca. $30 \mu \mathrm{m} \cdot 55 \mu \mathrm{m}$. Auch der Kollaps einer solchen Blase verläuft nicht sphärisch. An den Enden der längeren Achse bilden sich im Kollaps Einbuchtungen, so dass sich die Elliptizität der Blase ändert.

Die komplizierte Dynamik der Blasenform kann in dem vorliegenden Modell nicht erfaßt werden. Stattdessen wird hier von einer sphärischen Blase ausgegangen. Die sich ebenfalls bietende Möglichkeit der Nutzung einer elliptischen Randbedingung [77] erscheint in diesem Fall keine bessere Wahl, da diese eine feste Elliptizität im Kollaps zur Folge hätte. Bei dem betrachteten relativ langsamen Kollaps ist ein vergleichsweise geringer Fehler durch die Annahme von Sphärizität zu erwarten.

Für die Simulation einer solchen Blase über einen größeren Zeitraum (20 $\mu \mathrm{s})$

\begin{tabular}{ccc|ccc|ccc}
\hline$P_{S W}$ & - & $1.000 \mathrm{bar}$ & $N_{\text {ges }, i}$ & - & 64.000 & $v_{W, \text { init }}$ & - & $0 \mathrm{~m} / \mathrm{s}$ \\
$t_{P_{S W}}$ & - & $10 \mathrm{~ns}$ & $S$ & - & $\approx 3.450 .000$ & $\alpha_{t}$ & - & 0.3 \\
$R_{\text {init }}$ & - & $1,4 \mu \mathrm{m}$ & Edelgas & - & - & $\alpha_{v}$ & - & 0.1 \\
$T_{\text {water }}$ & - & $300 \mathrm{~K}$ & $\Delta K_{W}$ & - & 800 & $T_{\text {Gas }, i}$ & - & $3.000 \mathrm{~K}$ \\
$M F_{H_{2} O}$ & - & 0,4 & $M F_{H}$ & - & 0,4 & & $M F_{O}$ & - \\
\hline
\end{tabular}

Tabelle 7.2: Parameter der Rechnungen laser-induzierter Blasen. 


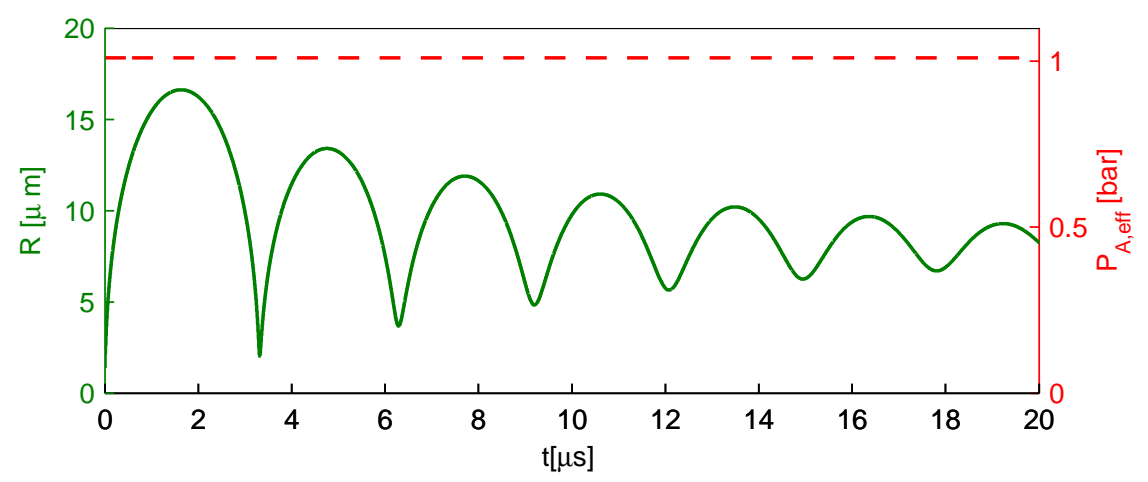

Abbildung 7.5: Im MD-Modell simulierter Radiusverlauf und konstanter Umgebungsdruck für eine Blase mit den in Tab. 7.2 angegebenen Parametern. Ohne Kondensation der chemischen Spezies an der Blasenwand.

wurde die Anzahl der Simulationsteilchen im Vergleich zu Kap. 7.1 verringert. Insgesamt befinden sich bei Start der Simulation 64.000 Partikel in der Blase, unter den in Kap. 7.1 besprochenen Anfangsbedingungen. Die weiteren verwendeten Parameter lassen sich Tab. 7.2 entnehmen, die simulierte Blase entspricht Fall 1 aus Tab. 7.3.

Die Entwicklung des Blasenradius für $20 \mu$ s findet sich in Abb. 7.5. Nach der im letzten Kapitel besprochenen Anfangsphase expandiert die Blase aufgrund der hohen Energie der Teilchen in ihrem Inneren auf einen maximalen Radius von $R_{\max }=16,6 \mu \mathrm{m}$. Diesem folgt ein relativ schwacher Kollaps, der die Blase auf ca. 2,02 $\mu \mathrm{m}$ komprimiert. Der minimale Radius des Hauptkollaps wird 3,31 $\mu$ s nach dem Laserpuls erreicht.

Die Entwicklung der Dichte und der Temperatur rund um den Zeitpunkt des minimalen Radius sind in Abb. 7.6 dargestellt. Es ist offensichtlich, dass keine extremen Bedingungen in dieser Blase erreicht werden. Die Temperaturen steigen maximal auf ca. $540 \mathrm{~K}$, im Blasendurchschnitt sogar nur auf ca. $347 \mathrm{~K}$. Die Dichte übersteigt nie $100 \mathrm{~kg} / \mathrm{m}^{3}$. Durch den langsamen Kollaps erhitzt sich die Blase gleichmäßig, ein heißeres Blasenzentrum ist nicht zu erkennen. Bei diesen Bedingungen tritt natürlich keinerlei Kavitationslumineszenz auf. Nach Erreichen des minimalen Blasenradius nimmt die Temperatur durch Wärmeübergang am Blasenrand und nachlassende Kompression schnell ab. Nach dem Hauptkollaps oszilliert die Blase mit sich abschwächender Amplitude um einen Ruheradius von ca. 8,5 $\mu \mathrm{m}$. Unter Laborbedingungen löst sich eine laser-induzierte Blase ohne Anregung schnell auf.

Daher wurde die eben besprochene Rechnung noch einmal durchgeführt, diesmal 

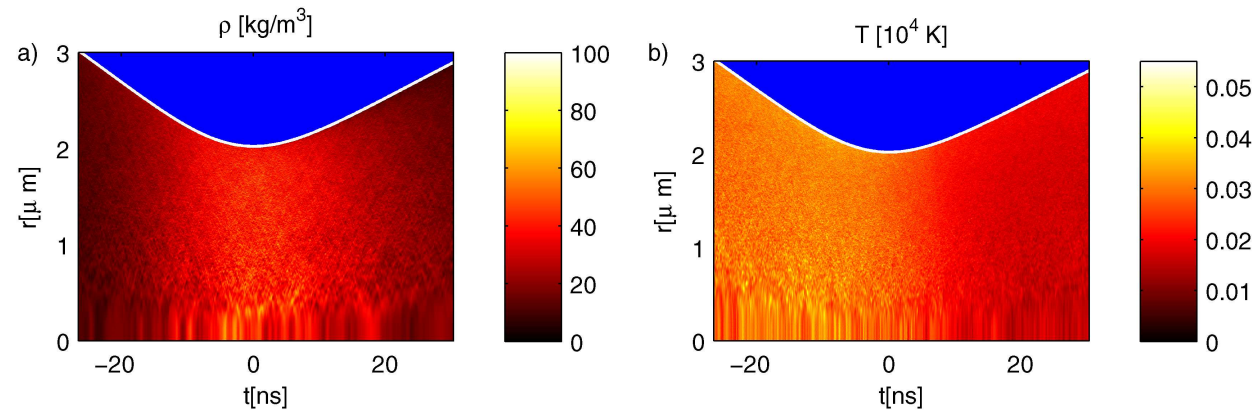

Abbildung 7.6: Dichte und Temperatur im ersten Kollaps einer in Tab. 7.2 beschriebenen laser-induzierten Blase ohne akustische Anregung. Parameter siehe Tab. 7.2.

mit einer gewissen Kondensation der entstandenen chemischen Spezies an der Blasenwand. Für alle Spezies (außer $\mathrm{H}_{2} \mathrm{O}$ ) wurde pauschal ein Akkomodationskoeffizient von $\alpha_{X}=0,001$ angenommen. Die resultierende Radius-Zeit-Kurve ist in Abb. 7.7 angegeben, weitere Ergebnisse finden sich unter 'Fall 2' in Tab. 7.3. Es läßt sich erkennen, dass die Blase nun unter Oszillationen, die schnell kleiner werden als in Fall 1, langsam schrumpft - nach $20 \mu$ s hat diese Blase einen Radius von $4 \mu \mathrm{m}$, eine Auflösung ist nach ca. $50 \mu \mathrm{s} \mathrm{zu}$ erwarten. Der Hauptkollaps tritt durch den laufenden Massenverlust etwas früher (nach 3,18 $\mu$ s) ein.

Abb. 7.8 vergleicht die Radiusentwicklung, die Durchschnittstemperatur, die Blasenwandgeschwindigkeit und die Anzahl der $\mathrm{H}_{2}$-Teilchen um den Hauptkollaps für die Fälle mit und ohne Kondensation der chemischen Spezies. Der geringere Innendruck im Fall 2 führt zu gesteigerten Kollapsgeschwindigkeiten und einem kleineren minimalen Radius $(1,52 \mu \mathrm{m})$. Der Kompressionsfaktor steigt von 8,2 auf 10,6. Dementsprechend sind die Temperaturen angehoben und die innere

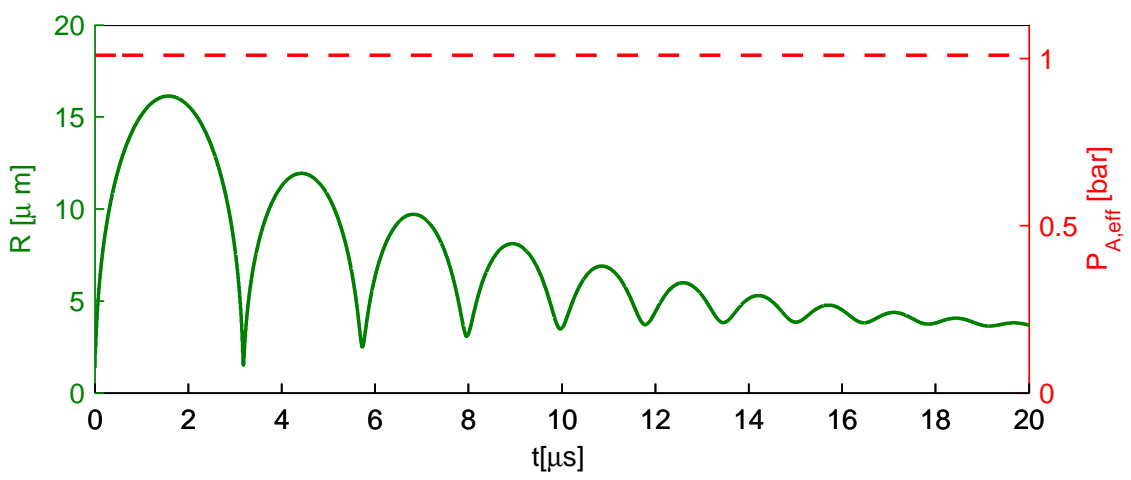

Abbildung 7.7: Im MD-Modell simulierter Radiusverlauf und konstanter Umgebungsdruck für eine Blase mit den in Tab. 7.2 angegebenen Parametern. Mit langsamer Kondensation der chemischen Spezies an der Blasenwand 
Dynamik verstärkt (gut zu sehen an dem Temperaturpeak kurz vor Ende des Kollaps). Maximal werden $405 \mathrm{~K}$ im Blasendurchschnitt erreicht. Die Temperaturen nehmen verglichen mit Fall 1 nach dem Kollaps deutlich schneller ab und erreichen kleinere Werte. Vermutlich können die Teilchen durch die kleinere Gesamtteilchenzahl ihre thermische Energie schneller an die Blasenwand abgeben. Mit unter $200 \mathrm{~K}$ wird es in der Expansionsphase sehr kalt.

Eine chemische Aktivität im Hauptkollaps ist in beiden Fällen nicht vorhanden. Die Anzahl an $\mathrm{H}_{2}$-Teilchen bleibt im Fall 1 konstant, im Fall 2 nimmt sie durch Kondensation langsam ab. Eine Produktion von $\mathrm{OH}$ ist nicht zu beobachten (siehe Tab. 7.3). Die berechneten Maximalradien (16,7 bzw. 16,1 $\mu \mathrm{m})$ liegen im Bereich
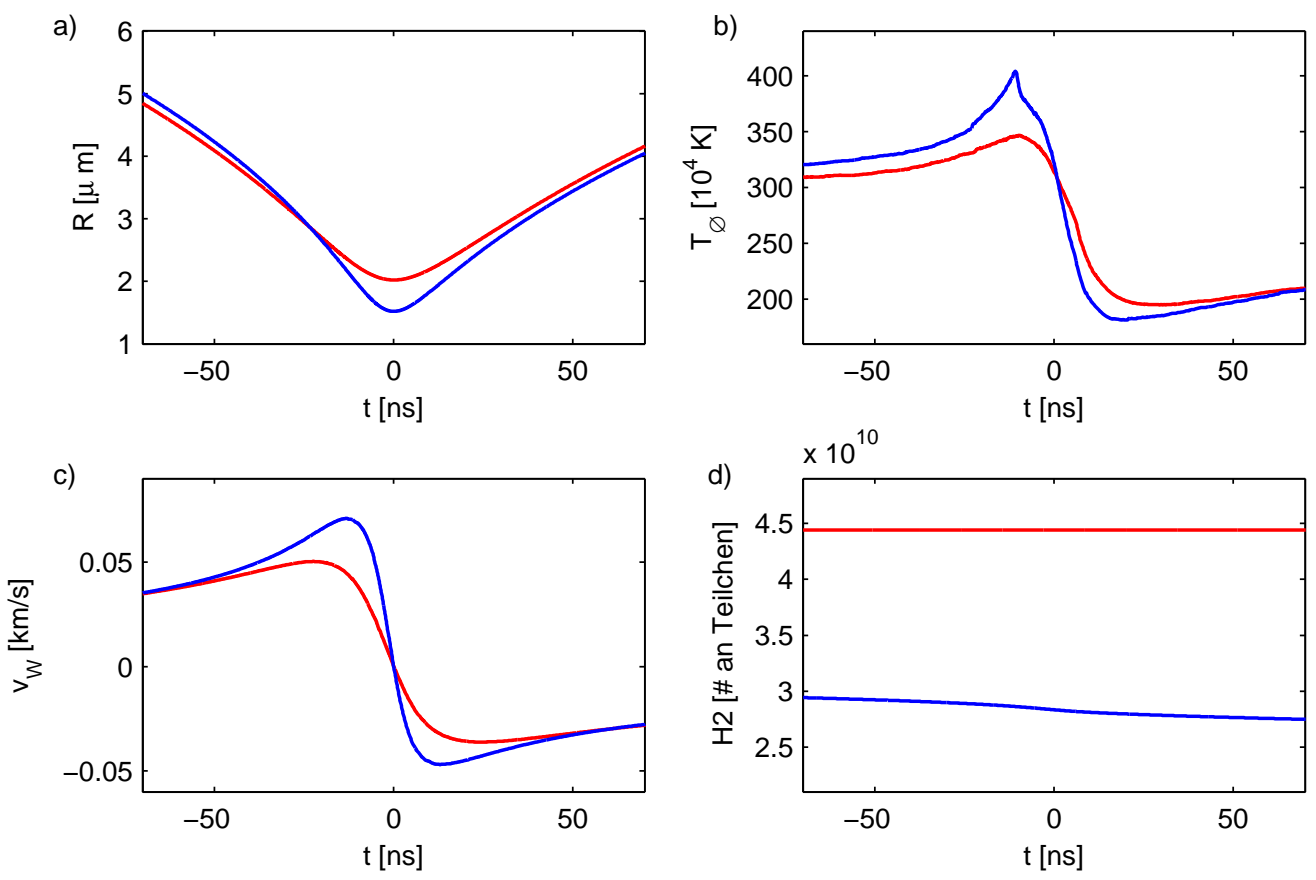

Abbildung 7.8: Verlauf von Radius (a), Durchschnittstemperatur (b), Blasenwandgeschwindigkeit (c) sowie der Anzahl an $\mathrm{H}_{2}$ - Teilchen (d) im ersten Kollaps der laser-induzierten Blasen ohne akustische Anregung (Fall 1 und 2 aus Tab. 7.3). Parameter siehe Tab. 7.2. Legende: Fall 1 (-): ohne Kondensation der chemischen Spezies; Fall 2 (-): mit Kondensation der chemischen Spezies

der in [144] experimentell ermittelten Radien der kurzen Halbachse (ca 15,0 $\mu \mathrm{m}$ ), die Zeit bis zum ersten Kollaps der Blase (ca. 3,2 $\mu$ s) ist etwas verlängert (experimentell ca. 2,6 $\mu \mathrm{s}$ ). Eine Lumineszenz konnte, genau wie im Experiment, nicht beobachtet werden. 


\subsection{Blase in angeregtem Medium}

Eine Möglichkeit zur Erzeugung lumineszenter laser-induzierter Blasen besteht in der akustischen Anregung des Blasenmediums. Die Beeinflussung der Blasendynamik fällt unterschiedlich aus, je nachdem zu welchem Zeitpunkt die Blase relativ zum Verlauf der Schallanregung erzeugt wird. Herrscht zum Zeitpunkt des Laserpulses ein Überdruck in der Flüssigkeit, wird die Expansion der Blase gebremst und der Kollaps, verglichen zu einem Fall ohne Schallfeld, früher eintreten. Nach diesem ersten Kollaps kann das Schallfeld in der Unterdruckphase die Blase wieder aufziehen und ggf. einen stärkeren zweiten Kollaps bewirken. Wird die Blase hingegen während einer Unterdruckphase produziert, so kann das Schallfeld die Expansion der Blase unterstützen und ein großer Maximalradius erreicht werden, dem ein starker Kollaps folgt.

Der Zeitpunkt der Blasenentstehung relativ zum Schallfeld wird durch den Parameter der Phase der Entstehung $\varphi_{S}$ (engl: seeding phase, gemessen in Grad) beschrieben. Die Entwicklung der Blasenranddynamik in Abhängigkeit von $\varphi_{S}$ ist in Abb. 7.9 dargestellt.

Die größeren Maximalradien, verglichen mit einem Fall ohne Anregung, und die Energie der akustischen Anregung führen zu deutlich extremeren Bedingungen in der Blase und damit ggf. zu Kavitationslumineszenz. Ein weiterer Effekt, der das Auftreten höherer Temperaturen unterstützt, ist eine stärkere Sphärizität der

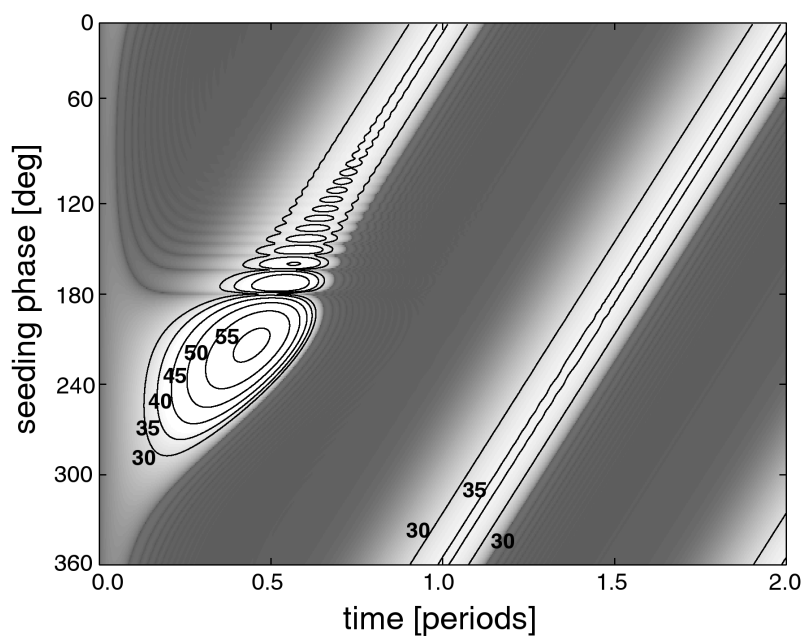

Abbildung 7.9: Der numerisch berechnete Blasenradius in Graustufendarstellung als Funktion der Phase der Entstehung $\varphi_{S}$ und der Zeit $t$. Angaben der Konturlinien in $\mu$ m. Entnommen aus [144]. Dort finden sich genauere Angaben über die verwendete Simulation. 
Blasen. Da der Hauptkollaps durch die Anregung der Blase verzögert wird, kann die Blase eine anfängliche Elliptizität durch eine lange Einwirkung der Oberflächenspannung ausgleichen und nahezu sphärisch kollabieren.

Abb. 7.10 zeigt eine fotographische Bildfolge einer laser-induzierten Blase, die bei $\varphi_{S}=192^{\circ}$ in Wasser produziert wurde, welches von einem Schallfeld mit Schalldruck $P_{a}=1,59$ bar bei einer Frequenz von $\nu_{a}=44 \mathrm{kHz}$ angeregt wurde. Die restlichen Parameter entsprechen denen der in Abb. 7.4 gezeigten Blase ohne akustische Anregung. Es ist gut zu erkennen, dass die Blase zunächst derjeniger ohne Anregung entspricht, dann aber von dem zunehmend negativen Schallfeld auf einen großen Maximalradius von ca. $100 \mu \mathrm{m}$ aufgezogen wird (man beachte die unterschiedlichen Raum- und Zeitskalen von Abb. 7.4 und 7.10). Im Zuge der langsamen Expansion der Blase wird diese zunehmend sphärisch, beim Kollaps bleibt dieser Zustand erhalten.

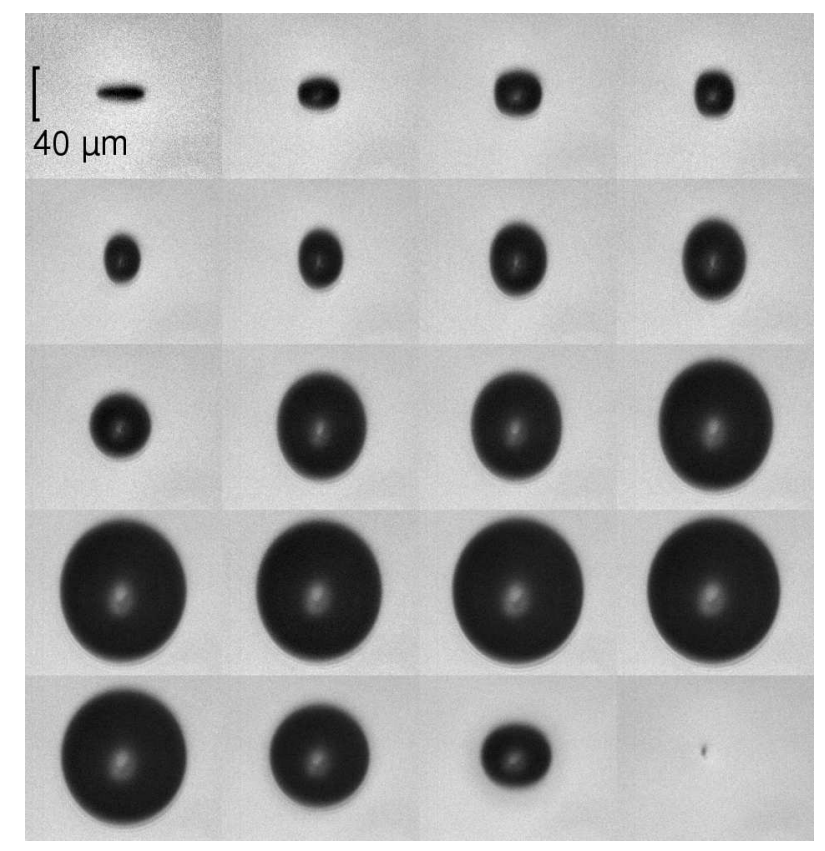

Abbildung 7.10: Fotographische Wiedergabe der Dynamik einer von einem 130 fs-Puls mit einer Energie von $0.22 \mu \mathrm{J}$ produzierten Blase, die von einem Schallfeld mit $P_{a}=1,59$ bar und $\nu_{a}=44 \mathrm{kHz}$ angeregt wird. Phase der Entstehung: $\varphi_{S}=192^{\circ}$. Der Bildabstand beträgt $800 \mathrm{~ns}$. Aufgenommen von KRÖNINGER [147].

Im Folgenden wird der Radiusverlauf, die innere Dynamik und die Lichtemission für Blasen mit drei verschiedenen Werten von $\varphi_{S}$ dargestellt und mit den entsprechenden Fällen aus [144] verglichen.

Abb. 7.11 zeigt den im MD-Modell berechneten Radiusverlauf für eine Blase, die 
bei $\varphi_{S}=184^{\circ}$ in Wasser geschossen wird, welches von einem Schallfeld mit $P_{a}=$ 1,59 bar und $\nu_{a}=44 \mathrm{kHz}$ angeregt wird. Die Anfangsbedingungen entsprechen den in Kap. 7.1 dargelegten, die Blase stellt Fall 3 aus Tab. 7.3 dar. Im unteren Teil der Abbildung finden sich Pseudo-Streak-Aufnahmen einer entsprechenden Blase aus [144]. Diese entstehen durch Ausschnitt eines Streifens aus der Mitte jedes Bildes aus einer Bildfolge wie in Abb. 7.10 und anschließender Aneinanderreihung. So ergibt sich eine Radius-Zeit-Aufnahme. Der Begriff Pseudo erklärt sich aus der Tatsache, dass nicht Bilder ein- und derselben Blase benutzt werden können, da hiefür die Zeitauflösung der Kamera nicht ausreicht. Stattdessen werden die aufeinanderfolgenden Bilder jeweils von einer neu produzierten Blase gemacht, wobei die Verzögerung der Aufnahme entsprechend dem Bildabstand erhöht wird. Dies erklärt auch den nicht völlig kontinuierlichen Verlauf des Blasenradius, da kleine Unterschiede der erzeugten Blasen nicht verhindert werden können.

Der Vergleich von Simulation und Experiment ergibt Ähnlichkeiten, die sich aber in einigen Punkten unterscheiden. So ist im Experiment zu beobachten, dass die
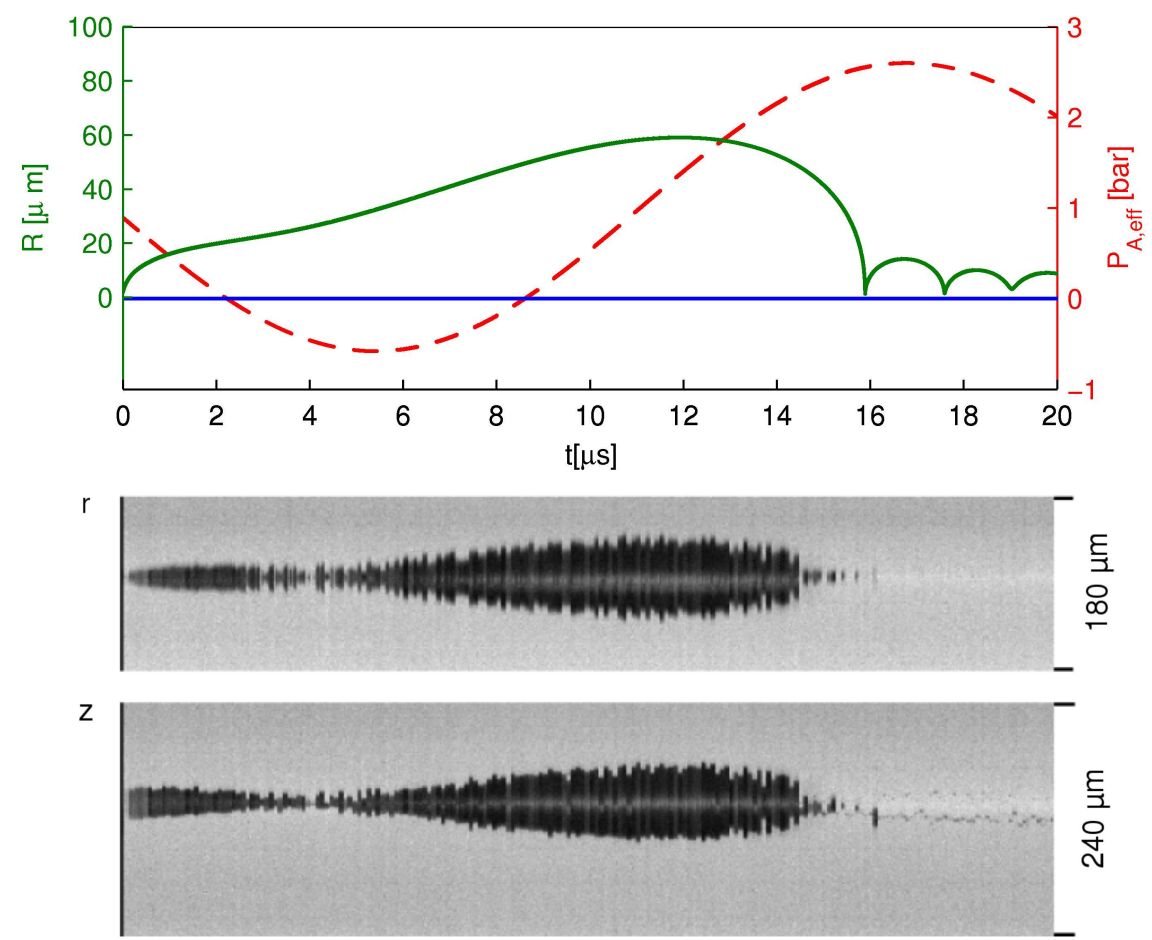

Abbildung 7.11: (a): Im MD-Modell simulierter Radiusverlauf und Anregung für eine bei $\varphi_{S}=184^{\circ}$ in das Schallfeld geschossene Blase. (b): Fotographische Streak-Aufnahme einer vergleichbaren Blase mit gleicher Zeitachse. (r): laterale Richtung (senkrecht zum Laserpuls); (z): longitudinale Richtung (parallel zum Laserpuls). Aufnahme aus [144]. 
Blase nach Erreichen eines lokalen Maximums bei ca. $2 \mu$ s zunächst schrumpft, um danach vom Schallfeld auf einen maximalen Radius von ca. $47 \mu \mathrm{m}$ aufgezogen zu werden. Es folgt ein Kollaps, der ca. 14,5 $\mu$ s nach dem Laserpuls abgeschlossen ist. In der Simulation wird hingegen kein lokales Maximum bei $2 \mu$ s angenommen, die Geschwindigkeit der Expansion verringert sich lediglich. Entsprechend wird die Blase auf einen größeren Maximalradius von $R_{\max }=59 \mu \mathrm{m}$ aufgezogen. Der Kollaps erfolgt hierdurch etwas verzögert bei ca. 15,9 $\mu \mathrm{s}$.

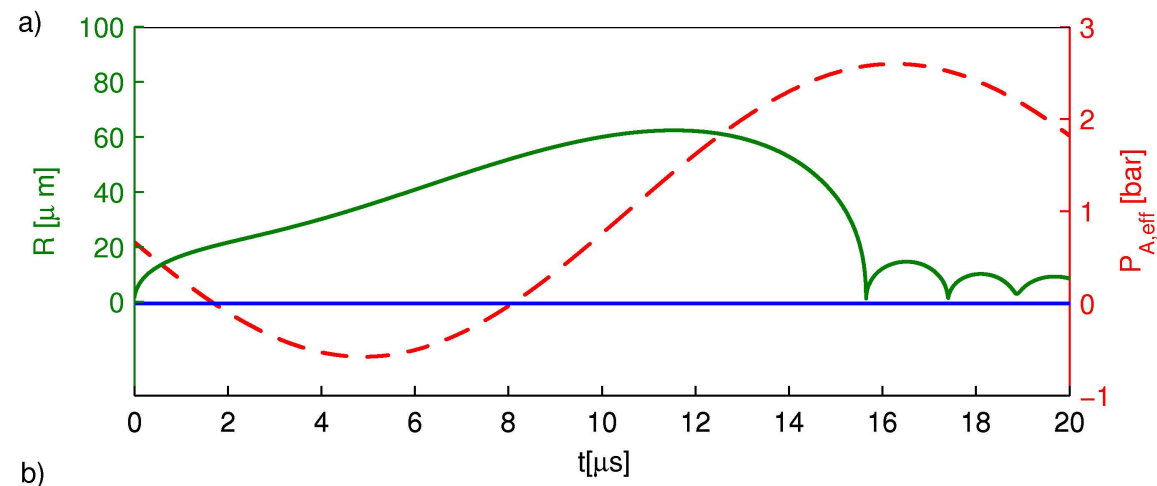

b)

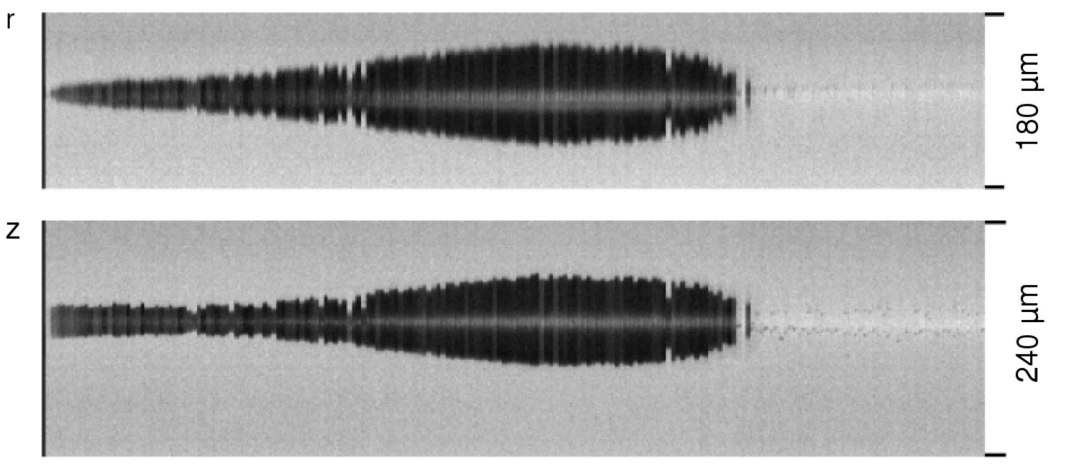

Abbildung 7.12: (a): Im MD-Modell simulierter Radiusverlauf und Anregung für eine bei $\varphi_{S}$ $=192^{\circ}$ in das Schallfeld geschossenen Blase. (b): Fotographische Pseudo-Streak-Aufnahme einer vergleichbaren Blase mit gleicher Zeitachse. (r): laterale Richtung (senkrecht zum Laserpuls); (z): longitudinale Richtung (parallel zum Laserpuls). Aufnahme aus [144].

Abb. 7.12 zeigt die Radiusentwicklung einer vergleichbaren Blase, diesmal mit $\varphi_{S}$ $=192^{\circ}$. Dies entspricht Fall 4 aus Tab. 7.3. In der fotographischen Darstellung des Experimentes ist zu erkennen, dass die Blase aufgrund des etwas späteren Entstehungszeitpunkts (daraus resultierend herrscht ein negativerer Druck) kein deutliches lokales Maximum vor Erreichen des maximalen Blasenradius mehr aufweist. Die Blase expandiert bis auf ca. 51,5 $\mu \mathrm{m}$ und kollabiert zu einer Zeit ca. 14,8 $\mu$ s nach dem Laserpuls.

Die Molekulardynamiksimulation zeigt ähnliche Ergebnisse. Der Maximalradius 
wird mit 62,6 $\mu \mathrm{m}$ auch hier überschätzt, genauso wie die Kollapszeit $(15,64 \mu \mathrm{m})$. Die Nachschwinger des Hauptkollaps erscheinen größer als auf der StreakAufnahme. Der generelle Verlauf der Blasenschwingung wird aber, wie schon im letzten Fall, gut reproduziert.
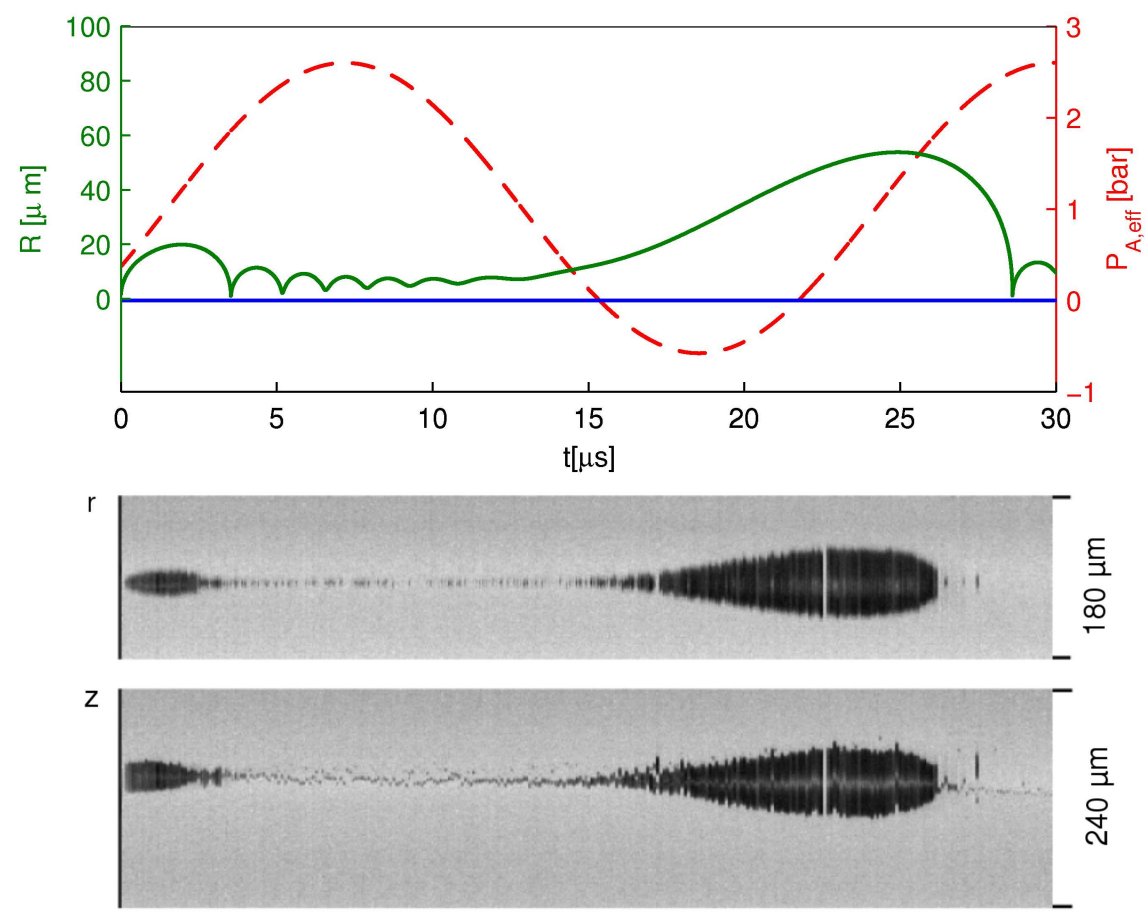

Abbildung 7.13: (a): Im MD-Modell simulierter Radiusverlauf und Anregung für eine bei $\varphi_{S}=331^{\circ}$ in das Schallfeld geschossenen Blase. (b): Fotographische Streak-Aufnahme einer vergleichbaren Blase mit selber Zeitachse. Aufnahme aus [144].

Abb. 7.13 zeigt die Radius- und Schallfeldamplitudenentwicklung für Fall 5 aus Tab. 7.3, eine Blase die bei $\varphi_{S}=331^{\circ}$ produziert wurde. Die Expansion dieser Blase wird in den ersten zwei $\mu$ s noch durch einen leichten Unterdruck des Schallfeldes unterstützt, danach wirkt ein immer stärkerer Überdruck auf die Blase. Daher expandiert die Blase zunächst auf einen Radius, der etwas größer ist als derjenige einer Blase ohne Anregung (siehe Abb. 7.5). Es folgt ein aufgrund des geringen Radius schwacher Kollaps und eine Phase, in der die Blase aufgrund des hohen Überdrucks kleine Oszillationen durchführt. Ungefähr $13 \mu$ s nach dem Laserpuls tritt das Schallfeld wieder in die Unterdruckphase ein und die Blase expandiert. Sie erreicht einen maximalen Radius von 53,8 $\mu \mathrm{m}$ - weniger als in den Fällen 3 und 4, da hier die Energie des Laserpulses zur Expansion fehlt. Der Hauptkollaps erfolgt ca. 28,6 $\mu$ s nach dem Laserpuls in einer Phase relativ hohen, 
aber nicht maximalen, Druckes.

Die Pseudo-Streak-Aufnahme des entsprechenden Experimentes zeigt ein ähnliches Bild, auch wenn in der Simulation wiederum der maximale Radius und der Zeitpunkt des Hauptkollaps überschätzt werden. Im Experiment werden $R_{\max }=$ $40,8 \mu \mathrm{m}$ sowie $t_{K o l l}=26,3 \mu$ s gefunden. Abb. 7.14 zeigt nun die durch das MD-
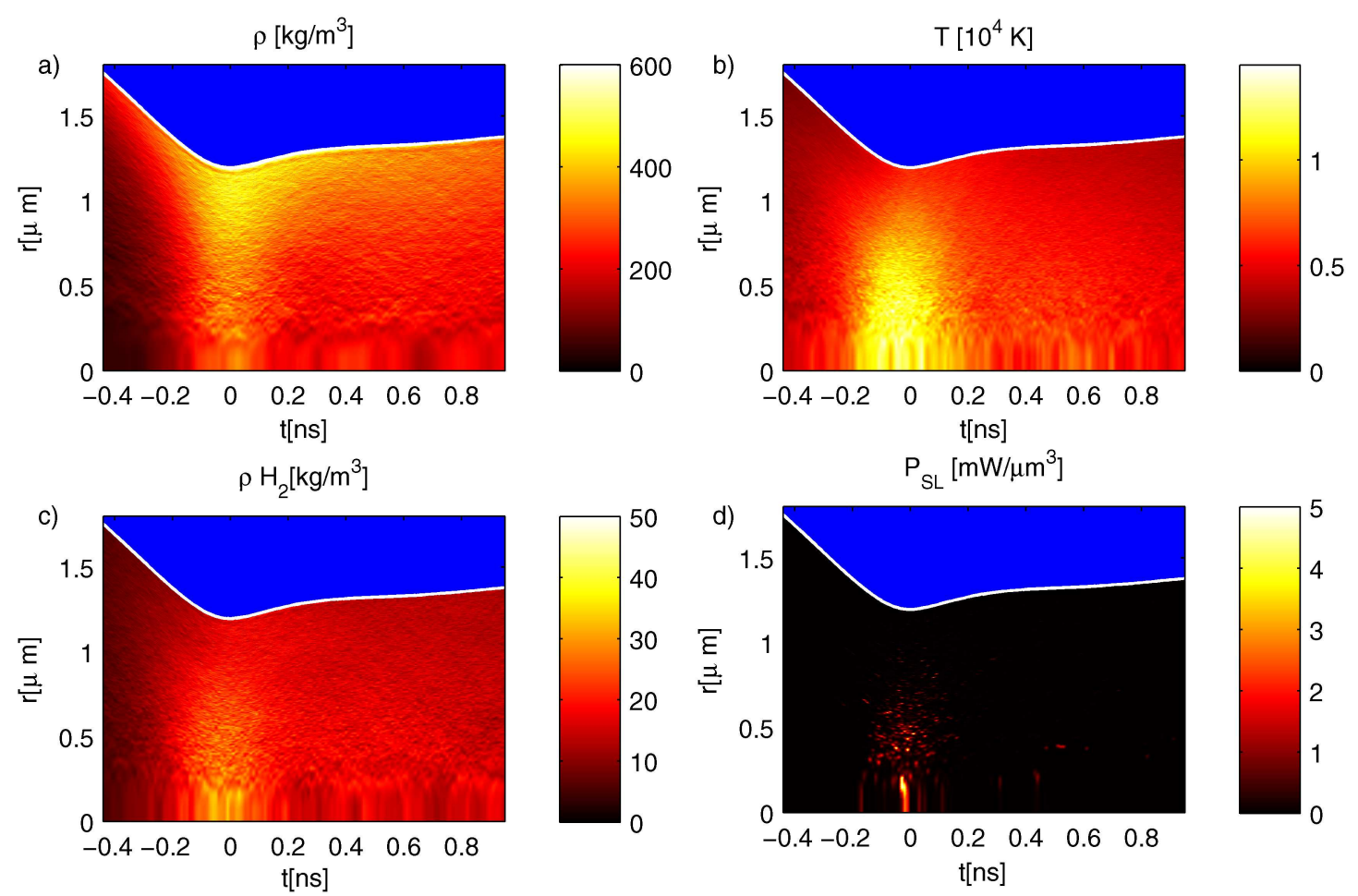

Abbildung 7.14: Verlauf von Dichte (a), Temperatur (b), Dichte von $\mathrm{H}_{2}$ (c) sowie der Lichtleistung für die Blase aus Abb. 7.12 (Fall 4 in Tab. 7.3) mit $\varphi_{S}=192^{\circ}$.

Modell ermittelte innere Dynamik der Blase mit $\varphi_{S}=192^{\circ}$. Dargestellt sind der raumzeitliche Verlauf der Dichte, der Temperatur, der Dichte von $\mathrm{H}_{2}$ sowie der Lichtleistung um den Zeitpunkt des minimalen Radius im Hauptkollaps.

Durch das Fehlen eines (schweren) Edelgases werden im Vergleich zu SBSL-Blasen niedrige Dichten bis ca. $600 \mathrm{~kg} / \mathrm{m}^{3}$ erreicht. Die größten Dichten werden hier nicht im Blasenzentrum erreicht, was sich durch das Vorhandensein leichter Spezies schon vor der heißen Kollapsphase erklärt: In der Entstehungsphase der Blase wurden durch die Energie des Laserpulses viele leichte $\mathrm{H}_{2}$ - Teilchen gebildet, die bis zum Hauptkollaps in der Blase verblieben sind. Diese sammeln sich nun aufgrund ihrer hohen Geschwindigkeit im Blasenzentrum und schirmen es vor den schweren Teilchen ab. Trotz des hohen Anregungsdrucks (1,59 bar, verglichen 
mit 1,3 bar bei typischen SBSL-Blasen) und der höheren Frequenz (44 kHz statt $26,5 \mathrm{kHz}$ ) werden vergleichsweise geringe Temperaturen erreicht. Die absolute Maximaltemperatur beträgt ca. $17.760 \mathrm{~K}$, die maximale über die Blase gemittelte Durchschnittstemperatur ca. 10.200 K. Die im Vergleich relativ niedrigen Temperaturen resultieren vor allem aus der Tatsache, dass in diesen Teilchen keine chemisch inerten Edelgase vorkommen: Alle vorhandenen Teilchen können potentiell Energie durch chemische Reaktionen aufnehmen.

Aufgrund der niedrigen Teilchenzahl der Simulation sind die Temperaturwerte vermutlich etwas überschätzt. Dies ist auch der Grund für den ungleichmäßigen Charakter der Lichtemission. Einzelne Meßzellen können aufgrund der wenigen vorhandenen Teilchen deutlich heißer werden als ihre Umgebung und so für helle 'Lichtpunkte' sorgen. Das Emissionsgebiet ist aufgrund des großen Minimalradius der Blasen recht ausgedehnt (ca. 1,8 $\mu \mathrm{m}$ im Durchmesser). Insgesamt emittiert die Blase eine Lichtenergie von $E_{S L}=0,44 \mathrm{pJ}$.

Abb. 7.15 vergleicht einige Kenngrößen der Fälle 3, 4 und 5 aus Tab. 7.3. Die Blase mit $\varphi_{S}=331^{\circ}$ (Fall 5) weist den kleinsten maximalen Radius auf und kollabiert aufgrund der geringeren Masse in der Blase auf den kleinsten minimalen Radius. Die Kompression und die maximale Blasenwandgeschwindigkeit ist in diesem Fall am geringsten. In Übereinstimmung mit den experimentellen Daten treten in der Blase mit $\varphi_{S}=192^{\circ}$ (Fall 4) die extremsten Bedingungen auf. Der Zeitpunkt der Blasenentstehung nutzt in diesem Fall optimal die Unterdruckphase für das Erreichen eines großen Maximalradius, der Kollaps findet zu einer Zeit maximalen Überdrucks statt. die kleine zeitliche Verschiebung gegenüber Fall 3 $\left(\varphi_{S}=184^{\circ}\right)$ reicht aus, um die emittierte Lichtenergie fast zu verdoppeln $(0,44 \mathrm{pJ}$ vs. $0,27 \mathrm{pJ})$. In Fall 5 wird nochmals deutlich weniger Licht emittiert (0,12 pJ). Ähnlich verhält es sich mit der chemischen Aktivität im Hauptkollaps. Es existiert bereits eine große Menge an stabilen chemischen Spezies (wie $\mathrm{H}_{2}$ ) aus der Entstehungsphase der Blase. Die Anzahl dieser Moleküle ändert sich im Hauptkollaps nur geringfügig. Die Temperaturen sind zwar vor allem bei Fall 4 nicht viel geringer als direkt nach dem Laserpuls, die Ausdehnung des heißen Gebietes und die zeitliche Dauer der heißen Phase sind aber deutlich geringer.

In [144] wurde eine sehr starke Abhängigkeit der Lichtemission von der Phase der Entstehung $\varphi_{S}$ gefunden. Es wurde ein schmaler Bereich von $7^{\circ}$ um $\varphi_{S}=192^{\circ}$ gesehen, in dem die Blasen nennenswert lumineszierten. Bei $\varphi_{S}=192^{\circ}$ wurde mehr als zehn mal mehr Licht detektiert als bei $\varphi_{S}=184^{\circ}$. Der Trend einer vermehrten Emission ist im MD-Modell ebenfalls zu sehen, er ist allerdings deutlich 
weniger ausgeprägt. KURZ vermutete einen Zusammenhang der Lichtemission mit der verbleibenden Exzentrizität der Blasen. Dieser Zusammenhang kann im vorliegenden sphärischen Modell nicht erfaßt werden.

Die sonochemische Entwicklung folgt der bei der Lichtemission beobachteten: Die Blase mit den größten erreichten Temperaturen $\left(\varphi_{S}=192^{\circ}\right)$ zeigt die stärkste chemische Aktivität.

Die generellen Trends werden von dem Modell wiedergegeben, einige Ergebnisse lassen allerdings darauf schließen, dass die gewählten Anfangsbedingungen nicht exakt den realen Gegebenheiten entsprechen. Die konstant überschätzten Maximalradien lassen eine zu hohe initiale Teilchendichte und/oder -temperatur vermuten. $\mathrm{Zu}$ einer genaueren Untersuchung dieser Parameter und einer besseren Anpassung an experimentelle Parameter fehlte leider die Zeit.

\begin{tabular}{|c|c|c|c|c|c|}
\hline Fall & 1 & 2 & 3 & 4 & 5 \\
\hline \multicolumn{6}{|l|}{ Parameter } \\
\hline$P_{A}[\mathrm{bar}]$ & 0 & 0 & 1,59 & 1,59 & 1,59 \\
\hline$\varphi_{S}^{\circ}$ & 0 & 0 & 184 & 192 & 331 \\
\hline$\alpha_{s p}$ & 0 & 0.01 & 0 & 0 & 0 \\
\hline \multicolumn{6}{|l|}{ Dynamik } \\
\hline$R_{\min }[\mu \mathrm{m}]$ & 2,02 & 1,52 & 1,26 & 1,30 & 1,20 \\
\hline$R_{\max }[\mu \mathrm{m}]$ & 16,6 & 16,1 & 59,0 & 62,5 & 53,8 \\
\hline$t_{K o l l}[\mu \mathrm{s}]$ & 3,31 & 3,18 & 15,88 & 15,64 & 28,61 \\
\hline$K$ & 8,2 & 10,6 & 46,8 & 48,1 & 44,8 \\
\hline$T_{\varnothing}[\mathrm{K}]$ & 347 & 405 & 9.650 & 10.200 & 8.870 \\
\hline$T_{\max }[\mathrm{K}]$ & 540 & 650 & 16.170 & 17.660 & 14.510 \\
\hline$\varrho_{\varnothing}\left[\mathrm{kg} / \mathrm{m}^{3}\right]$ & 36 & 61 & 304 & 305 & 307 \\
\hline \multicolumn{6}{|l|}{ Lichtemission } \\
\hline$E_{S L}[\mathrm{pJ}]$ & 0 & 0 & 0,27 & 0,44 & 0,12 \\
\hline$d_{S L}[\mu \mathrm{m}]$ & - & - & 1,6 & 1,8 & 1,3 \\
\hline$\Delta t_{S L}[\mathrm{ps}]$ & - & - & 90 & 160 & 60 \\
\hline \multicolumn{6}{|l|}{ Chemie } \\
\hline $\mathrm{H}_{2} \mathrm{O}_{r}\left[10^{9}\right]$ & 1,40 & 1,60 & 8,3 & 10,0 & 6,1 \\
\hline $\mathrm{OH}_{r}\left[10^{9}\right]$ & 0 & 0 & 0 & 0 & 0 \\
\hline $\mathrm{OH}_{\text {coll }, \max }\left[10^{9}\right]$ & 0 & 0 & 3,2 & 4,8 & 1,6 \\
\hline $\mathrm{H}_{2 r}\left[10^{10}\right]$ & 4,42 & 2,92 & 4,47 & 4,39 & 4,38 \\
\hline $\mathrm{H}_{2 \text { coll, } \max }\left[10^{10}\right]$ & 4,42 & 2,82 & 4,53 & 4,50 & 4,36 \\
\hline
\end{tabular}

Tabelle 7.3: Kenngrößen verschiedener Rechnungen von Blasen mit $\nu_{a}=11 \mathrm{MHz}$.

$R_{\max }$ bezeichnet hier die Höhe des (lokalen) Maximums vor dem Hauptkollaps, nicht unbedingt den absolut größten Radius. $K$ ist der Kompressionsfaktor, $T_{\varnothing}$ die maximale Durchschnittstemperatur, $\mathrm{T}_{\max }$ die absolut maximale Temperatur in der Blase, $\varrho_{\varnothing}$ die maximale Durchschnittsdichte, $E_{S L}$ die gesamte abgegebene Lichtenergie, $d_{S L}$ der Durchmesser der Emissionregion, $\Delta t_{S L}$ die Halbwertsbreite der Lichtemission, $\mathrm{H}_{2} \mathrm{O}_{r}$ ist die Anzahl der in der Blase gefangenen $\mathrm{H}_{2} \mathrm{O}$-Moleküle kurz vor Einsetzen der chemischen Reaktionen (einmal in absoluter Menge, einmal als Anteil an der Gesamtteilchenzahl), $\mathrm{H}_{2} \mathrm{O}_{\text {min }}$ die minimale Anzahl an $\mathrm{H}_{2} \mathrm{O}-$ Molekülen kurz nach $t_{R m i n}, \mathrm{OH}$ die maximale Anzahl an $\mathrm{OH}-$ Molekülen in der Blase und $\mathrm{H}_{2}$ die Anzahl an gebildeten $\mathrm{H}_{2}$-Molekülen. 
a)
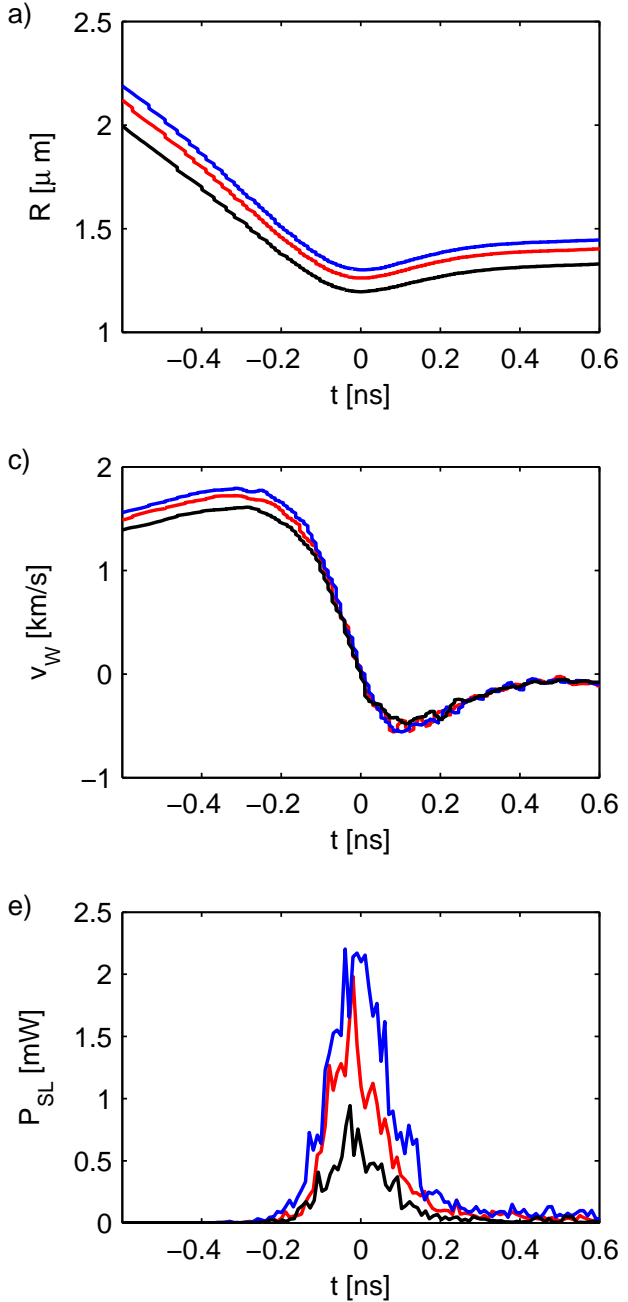
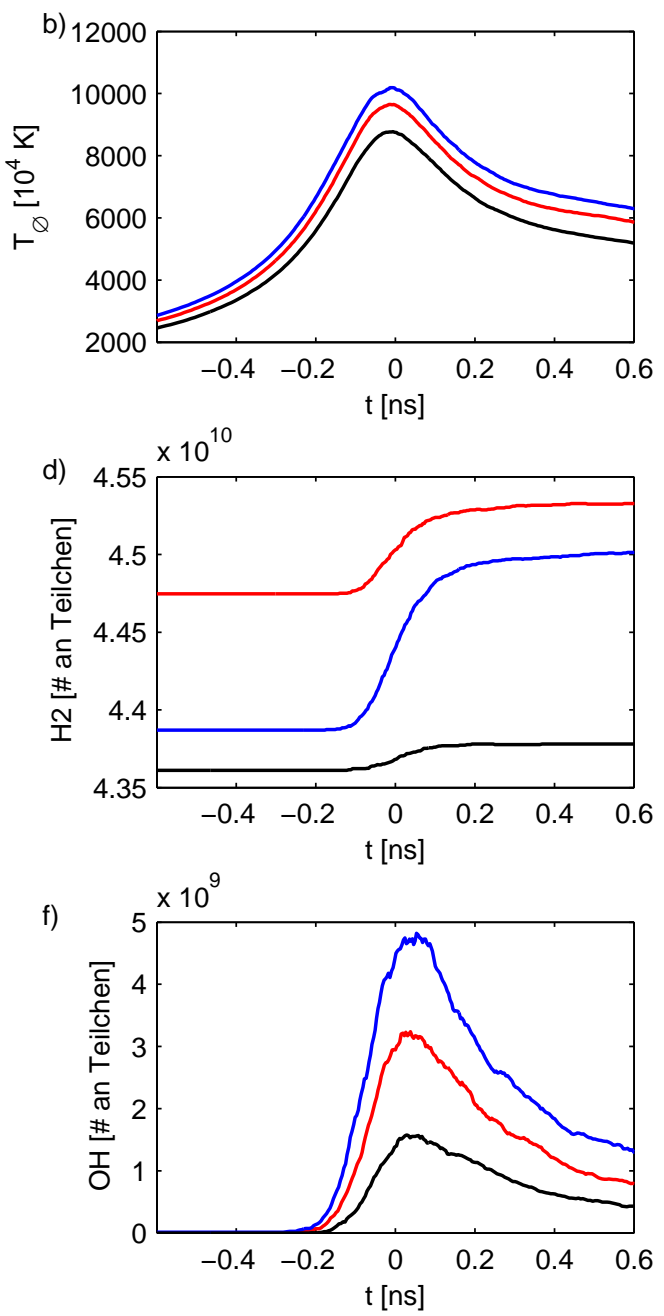

Abbildung 7.15: Verlauf von Radius (a), Durchschnittstemperatur (b), Blasenwandgeschwindigkeit (c). der Anzahl an $\mathrm{H}_{2}$ - Teilchen (d), der emittierten Lichtleistung (e) sowie der Anzahl an $\mathrm{OH}$ - Teilchen (f) im Hauptkollaps der laser-induzierten Blasen mit akustische Anregung (Fall 3, 4 und 5 aus Tab. 7.3. Parameter siehe Tab. 7.2.

Legende: $\varphi_{S}=184^{\circ}(-) ; \varphi_{S}=192^{\circ}(-) ; \varphi_{S}=331^{\circ}(-)$. 


\section{Kapitel 8}

\section{Zusammenfassung und Ausblick}

In der vorliegenden Arbeit wurde das Innere kollabierender Blasen unterschiedlicher Herkunft durch ein Molekulardynamikmodell simuliert. Die Bewegung des Blasenrandes wurde dabei mittels der erweiterten Rayleigh-Plesset-Gleichung ermittelt, welche über die Druckwerte am Blasenrand an die Hartkugelsimulation des Inneren gekoppelt wird. Die Verdampfung und Kondensation von Wasserdampf wird berücksichtigt, ebenso chemische Reaktionen der Bestandteile des Wassers in der heißen Kollapsphase. Die Emission von Kavitationslumineszenz wird durch ein Bremsstrahlungsmodell abgeschätzt. Bei der Anwendung des Modells auf kollabierende Blasen unterschiedlicher Ausprägung wurden folgende Ergebnisse erzielt:

Zunächst wurden Blasen simuliert, die unter typischen SBSL-Bedingungen auftreten. Für diese wurden intensive Parameterstudien durchgeführt, bei denen im Einzelnen folgende Zusammenhänge gefunden wurden:

- Die Art und der Umfang der Wärmeübertragung von den Teilchen an das Blasenmedium beeinflußt stark die innere Dynamik der Blase. In Kap. 4.1 wird dargelegt, dass sich bei einem starken Wärmeübergang im Laufe des Kollaps eine dichte, kalte Schicht in der Nähe der Blasenwand ausbildet, da die Teilchen nicht mehr genug thermische Energie (Geschwindigkeit) aufweisen um sich von der schnellen Wand zu entfernen. Kurz vor Ende des Kollaps löst sich diese Schicht von der langsamer werdenden Blasenwand ab, konvergiert im Blasenzentrum und führt dort zu sehr hohen Temperaturen. Ein starkes Temperaturgefälle zum Blasenrand ist vorhanden.

Findet hingegen ein geringer oder kein Wärmeübergang statt sammeln die Teilchen durch wiederholte Stöße mit der Wand immer mehr Energie. Die 
Teilchen sind aufgrund der hohen Geschwindigkeiten in der gesamten Blase verteilt. Im Kollaps erwärmt sich die Blase gleichmäßiger - es werden höhere Durchschnittstemperaturen, aber niedrigere Maximaltemperaturen im Blasenzentrum erreicht. Anhand des Vergleichs mit experimentell ermittelten Pulslängen der Emission sowie der Geschwindigkeit der Blasenwand wurde für den thermischen Akkomodationskoeffizienten $\alpha_{t}$, welcher das Ausmaß der Energieübertragung der Teilchen an die Blasenwand steuert, ein Wert von $\alpha_{t}=0,3$ festgesetzt.

- Kap. 4.2 beschäftigt sich mit dem Einfluß des Wasserdampfgehalts der Blase auf die Blasendynamik sowie die erreichten Temperaturen. Dazu wird im Speziellen eine Studie des Akkomodationskoeffizienten $\alpha_{v}$, der die Wahrscheinlichkeit der Kondensation eines mit der Blasenwand kollidierenden Teilchens beschreibt, durchgeführt. Es wird gezeigt, dass im Kollaps die Kondensation von Wasserdampf nicht im Gleichgewicht geschieht, eine bedeutende Menge an $\mathrm{H}_{2} \mathrm{O}$-Teilchen verbleibt in der Blase ('vapor trapping'). Die Menge des gefangenen Wasserdampfes ist direkt von $\alpha_{v}$ abhängig. Die zusätzliche Masse der Wasserdampfteilchen führt aufgrund des in der Endphase des Kollaps adiabatischen Charakters des Blasensystems zu einer starken Reduktion der Temperaturen.

Zusätzlich wird Energie von Rotationsfreiheitsgraden der Wasserdampfmoleküle aufgenommen. Die Blasentemperaturen nehmen also mit steigendem $\alpha_{v}$ zu. Ein Vergleich der erreichten Temperaturen mit experimentellen Daten führt zu einer Wahl von $\alpha_{v}=0.4$ - ein Wert, der in Übereinstimmung mit anderen theoretischen Arbeiten von YASUI sowie STOREY und SzERI steht [105] 38]. Ergänzend wird ein temperaturabhängiger Akkomodationskoeffizient eingeführt, der die Verdampfung in der letzten Kollapsphase besser darzustellen vermag.

Es wird gezeigt, dass die Rotationsfreiheitsgrade des Wassers bedeutend Energie aufnehmen und für eine Reduktion der über die Blase gemittelten Durchschnittstemperaturen von ca. $15.000 \mathrm{~K}$ auf ca. 10.000 K sorgen. Die durch die Abnahme der Teilchentemperatur bewirkte Reduktion des Gasdruckes an der Blasenwand führt zu einem deutlich kleineren Minimalradius.

- Der in der Blase gefangene Wasserdampf wird in der Endphase des Kollaps aufgrund der hohen Temperaturen dissoziiert. Es entstehen chemisch 
reaktive Teilchen ( $\mathrm{OH}$ und $\mathrm{H}$ ), die schnell zu anderen Spezies weiter reagieren. Das zur Simulation dieser Effekte eingesetzte chemische Modell wird in Kap. 4.3 vorgestellt und die Auswirkungen der chemischen Reaktionen auf das Blaseninnere erläutert.

Die Dissoziation des Wassers ist eine endotherme Reaktion, die aufgrund ihrer Häufigkeit die erreichten Temperaturen deutlich absenkt - die Durchschnittstemperaturen sinken von ca. $15.000 \mathrm{~K}$ auf ca. $11.500 \mathrm{~K}$. Dabei ist zu beachten, dass hier keine Rotation der Teilchen beachtet wurde, da die Kollisionsbedingungen chemisch reagierender Teilchen (Massenänderung und Energiegewinn/verlust) mit Rotation nicht ermittelt werden konnten.

Aus den Dissoziationsprodukten des Wassers bilden sich schnell chemisch stabilere Spezies, vor allem $\mathrm{H}_{2}$ und $\mathrm{O}_{2}$, in geringerem $\mathrm{Maße} \mathrm{HO}_{2}$ und $\mathrm{H}_{2} \mathrm{O}_{2}$. Die meisten Reaktionen, die zur Bildung dieser Spezies führen sind exotherm, wodurch deren Temperaturen (vor allem von $\mathrm{H}_{2}$ ) teilweise deutlich über dem Blasendurchschnitt liegen. Aus diesem Grund sind die entstehenden Spezies für einen Großteil der emittierten Lichtenergie verantwortlich. Das Edelgas und der Wasserdampf spielen für die Lichtemission trotz ihrer großen Anzahl eine geringe Rolle.

Geht man davon aus, dass die entstandenen Spezies nicht wasserlöslich sind sammeln sie sich über viele Perioden der Anregung in der Blase an. Nimmt man hingegen realistischer eine geringe Wahrscheinlichkeit für einen Übergang in die Flüssigkeit bei Wandkollisionen an, so verschwinden leichte (schnelle) Spezies während des Verlaufs einer Periode vollständig aus der Blase. Schwere (langsame) Spezies bleiben in kleineren Mengen vorhanden, eine signifikante Akkumulation findet aber nicht statt. Von den gebildeten Radikalen (OH, O, H) gelangt auf dem Weg der Kondensation nur ein kleiner Teil in das umgebende Blasenmedium. Für die meisten Radikale reicht die kurze Lebenszeit nicht aus, um vom heißen Blasenzentrum zum Rand zu gelangen. Eine größere Ausbeute an Radikalen außerhalb der Blase ist mittels der Ausspülung durch einen Jet oder einer andere Blasendeformation denkbar.

- Bei gegebener Systemgröße bestimmt die Anzahl der simulierten Teilchen, für wieviele reale Teilchen ein Simulationsteilchen steht. Diesen Wert bezeichnet der Skalierungsfaktor S. Die Abhängigkeit der Blasendynamik und der Ergebnisgenauigkeit von der Teilchenanzahl wird in Kap. 4.4 beleuchtet. Es wird deutlich, dass für die Simulation einer typischen SBSL-Blase $\left(10^{10}\right.$ 
bis $10^{11}$ reale Teilchen) eine Teilchenzahl von mindestens $10^{5}$ verwendet werden sollte, um aussagekräftige Ergebnisse zu erhalten. Geringere Teilchenzahlen lassen sich zumindest eingeschränkt zur Ermittlung von Trends benutzten.

Diffusionseffekte werden um den Faktor $S^{1 / 3}$ überschätzt, wodurch bei geringerer Teilchenzahl skaliert weniger Wasserdampfteilchen in der Blase verbleiben. MetTen fand einen Temperaturanstieg bei Erhöhung der Teilchenzahl, da die kleinere mittlere freie Weglänge eine Verstärkung der Verdichtungswellen in der Blase bewirkt [77]. Bei Berücksichtigung von Wasserdampf und chemischen Reaktionen sieht man jedoch, dass der Effekt der vergrößerten Stoßdynamik durch ein vermehrtes vapor trapping kompensiert wird. In der Endphase des Kollaps steigen in diesem erweiterten Modell daher die erreichten Temperaturen bei Verringerung der Teilchenzahl.

- Die experimentelle Beobachtung, dass die Lumineszenz von SL-Blasen bei Absenkung der Wassertemperatur $T_{\text {water }}$ stark zunimmt wird in Kap. 5.1 untersucht. Die Simulation von Blasen mit Wassertemperaturen zwischen $277 \mathrm{~K}$ und $310 \mathrm{~K}$ zeigte, dass hauptsächlich der mit $T_{\text {water }}$ sinkende Wasserdampfgehalt für steigende Blasentemperaturen und damit erhöhte Lumineszenz verantwortlich ist. Der Sättigungsdampfdruck von Wasser - dessen Wert die Anzahl an $\mathrm{H}_{2} \mathrm{O}$-Teilchen zu Beginn der Simulation bestimmt - ist exponentiell abhängig von der Wassertemperatur. Bei Erhöhung von $T_{\text {water }}$ werden immer mehr Wasserdampfteilchen in der Blase gefangen und setzen zunehmend die erreichten Temperaturen herunter. Das Maximum der Lichtemission wird bei $T_{\text {water }}=285 \mathrm{~K}$ gefunden, da hier die Emissionsregion größer ist als bei noch kleineren Wassertemperaturen.

Die chemische Aktivität nimmt bei Erhöhung von $T_{\text {water }}$ hingegen konstant zu: Der größere Vorrat an dissoziationsfähigen Wasserdampfpartikeln führt trotz verringerter Maximaltemperaturen zu deutlich steigender Produktion von chemischen Spezies.

- Kap. 5.2 beschäftigt sich mit der Entwicklung der Blasenparameter bei steigendem Anregungsdruck $P_{a}$ und unveränderter Anregungsfrequenz $\nu_{a}$. Es wird deutlich, dass es auf diese Weise nicht möglich ist, extremere Temperaturen in der Blase zu erreichen. Bei einer Erhöhung von $P_{a}$ wächst der Gleichgewichtsradius $R_{0}$ - die Blasen werden also größer, der Kollaps wird durch die erhöhte Teilchenzahl abgemildert und die erreichte Kompression sinkt. Entsprechend sinken auch die Temperaturen in der Blase. 
Die emittierte Lichtenergie $P_{S L}$ und die chemische Aktivität zeigen eine andere Abhängigkeit: die steigende Größe des minimalen Blasenradius führt zu einem Wachstum der heißen Region im Kollaps, wodurch bei hohem $P_{a}$ ein größeres Volumen zur Lichterzeugung und zur Auslösung chemischer Reaktionen zur Verfügung steht. Beim Übergang von $P_{a}=1,33$ bar auf 1,45 bar verfünffachen sich $P_{S L}$ und die Produktion chemischer Spezies, obwohl die Maximaltemperaturen von ca. $26.700 \mathrm{~K}$ auf ca. $22.000 \mathrm{~K}$ sinken.

- Kap. 5.3 beleuchtet den Einfluß des in der Flüssigkeit gelösten, und damit in der Blase vorkommenden, Edelgases auf die Dynamik der Blase. Die Edelgase Helium, Argon und Xenon werden betrachtet. Je schwerer das verwendete Edelgas ist, desto heißer werden die Blasen und entsprechend mehr Lichtenergie wird abgestrahlt. Schwere Teilchen können die von der Blasenwand übertragene Energie besser in kinetische Energie umsetzen, welche gegen Ende des Kollaps thermalisiert.

Weisen die Teilchen in der Blase stark unterschiedliche Massen auf (z.B. Xenon und Wasserdampf) kommt es im Laufe des Kollaps zu einer Segregation der Blasenbestandteile. Leichte Teilchen werden in das Blasenzentrum getrieben, schwere Teilchen verbleiben eher an der Blasenwand. Dieser Effekt wird auch bei Mischungen zweier Edelgase sichtbar.

Als Hauptursache der Segregation der Blasenbestandteile wird die DruckDiffusion identifiziert, die Thermo-Diffusion spielt eine untergeordnete Rolle.

Experimentelle Untersuchungen gaben Hinweise auf sehr hohe Temperaturen im Inneren von Blasen, die mit hohen Frequenzen und Drücken angeregt werden [135][137]. Diese Blasen sind aufgrund ihrer geringen Größe gute Objekte zur Simulation mittels Molekulardynamik, da der Skalierungsfaktor $S$ hier minimiert werden kann - z.T. sind Werte von $S=1$ möglich.

In Kap. 6 wurde die Dynamik von Vertretern von MBSL-Blasen mit $\nu_{a}=1 \mathrm{MHz}$ und $P_{a}=4$ bis 5 bar sowie solche mit $\nu_{a}=11 \mathrm{MHz}$ und $P_{a}=100$ bar untersucht. Es wurden jeweils mehrere Blasen mit unterschiedlichem Ruheradius $R_{0}$ berücksichtigt.

- Im Fall der mit $1 \mathrm{MHz}$ angeregten Blase zeigt sich, dass die Verhältnisse in solchen Blasen generell deutlich extremer sind als in Blasen, die im $\mathrm{kHz}$-Bereich angeregt werden. Die kleinen Maximalradien führen zu einem 
geringen Wasserdampfanteil, der hohe Anregungsdruck zu einem starken Kollaps. Mit sinkendem Ruheradius nehmen die Temperaturen weiter zu. Für Blasen mit Xenon als Edelgas werden, wie schon bei SBSL-Blasen, höhere Temperaturen und gesteigerte Lichtemission beobachtet. Eine Erhöhung der Anregung von 4 auf 5 bar erhöht die beobachteten Temperaturen nochmals (mit Xenon als Edelgas werden im Zentrum ca. 129.000 K erreicht). In allen Fällen wird nahezu der gesamte gefangene Wasserdampf dissoziiert.

- Die mit $11 \mathrm{MHz}$ angeregte Blase zeigt eine starke Abhängigkeit von $R_{0}$. Je größer die Blasen sind ( $R_{0}$ wird mit maximal $750 \mathrm{~nm}$ angenommen), desto nichtlinearer ist ihre Schwingung. Es treten zunehmend subharmonische Schwingungen der Anregungsfrequenz auf. Die Kompression sinkt, da Kollapse in Überdruckphasen meist von kleinen Maximalradien ausgehen. Kleine Blasen ( $R_{0}$ wird mit minimal $130 \mathrm{~nm}$ angenommen) zeigen deutlich regelmäßigere Oszillationen, der Kollaps geht von einem großen Maximalradius aus. Da zusätzlich die Teilchenanzahl klein ist werden sehr hohe Temperaturen erzielt. Bei $R_{0}=130 \mathrm{~nm}$ beträgt die maximale Temperatur in der Blase ca. 459.000 K. Mit Erhöhung des Ruheradius sinkt diese bis auf ca. $31.700 \mathrm{~K}$ bei $R_{0}=750 \mathrm{~nm}$. Das Maximum der Lichtemission liegt bei $R_{0}=400 \mathrm{~nm}$, da hier der beste Kompromiß aus Blasentemperaturen und Größe des Emissionsgebiets erzielt wird.

Kap. 7 befaßt sich mit der Simulation laser-indizierter Blasen, die teilweise von einem Schallfeld zusätzlich angetrieben werden. Es werden Vergleiche mit Experimenten von KuRZ durchgeführt [144].

- In der Anfangsphase heizt sich die Blase durch endotherme Reaktionen der durch die Laserenergie dissoziierten $\mathrm{H}_{2} \mathrm{O}$-Teilchen sehr schnell gleichmäßig bis auf ca. $16.000 \mathrm{~K}$ auf. Es werden relevant viele stabile Spezies wie $\mathrm{H}_{2}$ oder $\mathrm{O}_{2}$ gebildet, die je nach Grad der Kondensation an der Blasenwand, in der Blase verbleiben. Die Blase kühlt sich schnell wieder ab, nach 10 ns ist die durchschnittliche Blasentemperatur bereits unter $1.000 \mathrm{~K}$ abgefallen.

- Nichtgetriebene laser-induzierte Blasen führen nach dem anfänglichen Aufschwingen nur einen schwachen Kollaps durch, in welchem maximal ca. $650 \mathrm{~K}$ Zentrumstemperatur erreicht werden. Keinerlei chemische Aktivität oder Lumineszenz ist zu beobachten. 
- Wird die Blase hingegen durch ein Schallfeld (hier $P_{a}=1,59$ bar, $\nu_{a}=$ $44 \mathrm{kHz}$ ) angetrieben, so wird diese auf deutlich größere Radien aufgezogen und kollabiert heftiger. In Abhängigkeit der Phase der Entstehung $\varphi_{S}$ relativ zum Schallfeld werden verschiedene Radius-Zeit-Verläufe beobachtet, die die experimentell beobachteten qualitativ recht gut wiedergeben. Die erreichten Maximalradien werden allerdings überschätzt. Die auftretenden Temperaturen und die Lumineszenz variieren mit $\varphi_{S}$, da unterschiedliche Maximalradien erzielt werden und der Zeitpunkt des Kollaps zum Teil aus der Überdruckphase heraus verschoben wird. Die in [144] gefundenen Trend werden qualitativ bestätigt.

\section{Ausblick}

Das verwendete Modell kann an vielen Stellen erweitert werden, um die Gegebenheiten besser zu beschreiben oder neue Einsatzmöglichkeiten zu schaffen. Einige dieser Punkte werden hier dargestellt.

- Es könnte eine direkte Ionisation der Teilchen implementiert werden, um das Plasma in der Blase besser zu beschreiben. Überlegungen dazu finden sich in [77].

- Das Lichterzeugungsmodell könnte verfeinert werden, so dass z.B. anstelle der momentanen Mittelung der Teilchenparameter in einer Messzelle die bei einzelnen Kollisionen emittierte Strahlung direkt berechnet wird.

- Eine Modellierung des Wassers, z.B. mittels Smoothed Particle Hydrodynamics [148] würde es ermöglichen, erweiterte Aussagen über die Bedingungen außerhalb der Blase zu tätigen.

- Die Formulierung einer allgemeineren Form der Randbedingung würde die Simulation unsymmetrischer, recht beliebig geformter Blasen ermöglichen. Die Modellierung laser-induzierter Blasen könnte realistischer erfolgen, Störungen der Blasenoberfläche wie Einstülpungen oder gar Jets könnten erfaßt werden.

- Eine Erweiterung des chemischen Modells, zum Beispiel um die Stickstoffchemie, wäre wünschenswert, um umfassendere sonochemische Untersuchungen zu ermöglichen. 
- Die Berücksichtigung der Rotationsfreiheitsgrade chemisch reagierender Teilchen würde die Binnendynamik der Blasen realistischer darstellen.

- Erweiterte Untersuchungen zu den Anfangsbedingungen in laser-induzierten Blasen (Dissoziationsgrad, Anfangstemperatur) würden die Übereinstimmung der Simulation mit experimentellen Ergebnissen weiter verbessern. 


\section{Anhang A}

\section{Symbolverzeichnis}

Dieses Verzeichnis gibt die in der vorliegenden Arbeit gebräuchlichen Schreibweisen an sowie die eingesetzen SI-Einheiten.

Die Sortierung der Symbole erfolgt alphabetisch. 


\begin{tabular}{|c|c|c|}
\hline Symbol & Einheit & Bedeutung \\
\hline$\#_{M Z}$ & - & $\begin{array}{l}\text { Anzahl der Messzellen zur räumlichen Auflösung } \\
\text { der Hydrodynamik }\end{array}$ \\
\hline$A$ & $\mathrm{u}$ & Atommasse \\
\hline$A_{l}$ & $\mathrm{~K} \cdot \mathrm{m}$ & Faktor der spektralen Bremsleistungsdichte \\
\hline$A^{*}$ & - & $\begin{array}{l}\text { Der angeregte Komplex bei katalytischen Reaktionen } \\
\text { (Zwischenschritt des Lindemann-Mechanismus) }\end{array}$ \\
\hline$A_{v}$ & - & $\begin{array}{l}\text { Faktor des temperaturabhängigen } \mathrm{H}_{2} \mathrm{O} \text { - Akkomodations- } \\
\text { koeffizienten }\end{array}$ \\
\hline$\alpha_{t}$ & - & thermischer Akkomodationskoeffizient \\
\hline$\alpha_{v}$ & - & fester $\mathrm{H}_{2} \mathrm{O}$ - Akkomodationskoeffizient \\
\hline$\alpha_{v, v a r}$ & - & temperaturabhängiger $\mathrm{H}_{2} \mathrm{O}$ - Akkomodationskoeffizient \\
\hline$\alpha_{X}$ & - & Akkomodationskoeffizient der Spezies $X$ \\
\hline$\beta_{0}$ & - & Restitutionskoeffizient der Rotation bei Teilchenkollisionen \\
\hline$\chi_{1}$ & $\mathrm{eV}$ & 1. Ionisierungsenergie eines Atoms \\
\hline$D_{E k i n}$ & - & $\begin{array}{l}\text { Quotient der maximalen und initialen kinetischen Energie } \\
\text { der Edelgasteilchen }\end{array}$ \\
\hline$d_{S L}$ & $\mathrm{~m}$ & Teilchendurchmesser \\
\hline$d_{S L}$ & $\mathrm{~m}$ & Durchmesser der Licht emittierenden Region \\
\hline$\Delta t_{S L}$ & s & Halbwertsbreite der Lichtemission \\
\hline$\Delta p_{K}$ & $\mathrm{~kg} \cdot \mathrm{m} / \mathrm{s}$ & Der bei einer Kollision übertragene Impuls \\
\hline$\Delta K_{W}$ & - & $\begin{array}{l}\text { Anzahl der Wandkollisionen zwischen der Neuberechnung } \\
\text { der Wandparameter }\end{array}$ \\
\hline$\Delta t_{\Delta K W}$ & $\mathrm{~s}$ & $\begin{array}{l}\text { Zeitintervall zwischen der Neuberechnung der } \\
\text { Wandparameter. Variiert mit } \Delta K_{W}\end{array}$ \\
\hline$e$ & - & Restitutionskoeffizient bei Teilchenkollisionen \\
\hline$E_{a}$ & $\mathrm{~kJ} / \mathrm{mol}$ & Aktivierungsenergie einer chemischen Reaktion \\
\hline$E_{D}$ & $\mathrm{~kJ} / \mathrm{mol}$ & Reaktionsenthalpie einer chemischen Reaktion \\
\hline$E_{S L}$ & $\mathrm{~J}$ & emittierte Lichtenergie \\
\hline$\eta$ & - & Konstante des Ratenkoeffizienten einer chemischen Reaktion \\
\hline$\vec{g}_{12}$ & $\mathrm{~m} / \mathrm{s}$ & $\begin{array}{l}\text { Geschwindigkeitsdifferenz zweier kollidierender Teilchen } \\
1 \text { und } 2 \text { am Kontaktpunkt }\end{array}$ \\
\hline$\Gamma_{C}$ & - & Korrekturfaktor der Rate der Kondensation \\
\hline$I_{i}$ & $\mathrm{~kg} \cdot \mathrm{m}^{2}$ & Trägheitsmoment des Teilchen $i$ \\
\hline$K$ & - & Kompressionsfaktor der Blasenbewegung $\left(K=R_{\max } / R_{\min }\right)$ \\
\hline$k(T)$ & - & Ratenkoeffizient einer chemischen Reaktion \\
\hline$l$ & $\mathrm{~m}$ & mittlere freie Weglänge der Teilchen \\
\hline
\end{tabular}




\begin{tabular}{|c|c|c|}
\hline Symbol & Einheit & Bedeutung \\
\hline$L_{v}$ & $\mathrm{~J}$ & Latente Wärme beim Phasenübergang von Wassers \\
\hline$\Lambda$ & - & $\begin{array}{l}\text { Konstante des Ratenkoeffizienten einer } \\
\text { chemischen Reaktion }\end{array}$ \\
\hline$m$ & $\mathrm{~kg}$ & Teilchenmasse \\
\hline$M_{12}$ & $\mathrm{~kg}$ & reduzierte Masse zweier Teilchen 1 und 2 \\
\hline$M F_{X}$ & - & $\begin{array}{l}\text { Teilchenzahlverhältnis ('molar fraction') von Stoff X } \\
\text { zur gesamten Teilchenzahl }\end{array}$ \\
\hline$N_{N G}$ & - & Anzahl der simulierten Edelgasatome in der Blase \\
\hline$N_{X}$ & - & Anzahl der simulierten Teilchen der Spezies X \\
\hline$N_{\text {ges }}$ & - & Gesamtzahl der simulierten Teilchen in der Blase \\
\hline$N_{g e s, i}$ & - & initiale Gesamtzahl der simulierten Teilchen in der Blase \\
\hline$N_{r}$ & - & Gesamtzahl der realen Teilchen in der Blase \\
\hline$N_{X}^{r}$ & - & Reale Anzahl an Teilchen der Spezies X in der Blase \\
\hline$\nu$ & $\mathrm{m}^{2} / \mathrm{s}$ & Viskosität von Wasser \\
\hline$\nu_{a}$ & $\mathrm{~Hz}$ & Frequenz der Anregung \\
\hline$\omega_{a}$ & $1 / \mathrm{s}$ & Kreisfrequenz der Anregung $\left(\omega_{a}=2 \pi \nu_{a}\right.$ \\
\hline$\vec{\omega}_{i}$ & $\mathrm{~m} / \mathrm{s}$ & Winkelgeschwindigkeit des Teilchens $i$ \\
\hline$P_{0}$ & bar & Umgebungsdruck \\
\hline$P_{a}$ & bar & Anregungsdruck \\
\hline$P_{G}$ & bar & Gasdruck des in der Flüssigkeit gelösten Gases \\
\hline$P_{L}$ & bar & Druck im Wasser direkt an der Blasenwand \\
\hline$P_{S L}$ & W & Bremsstrahlungsleistung \\
\hline$P_{W}$ & bar & Innendruck an der Blasenwand \\
\hline$P_{\infty}$ & bar & Druck in großer Entfernung zur Blase \\
\hline$p_{R}$ & - & Reaktionswahrscheinlichkeit zweier kollidierender Teilchen \\
\hline$\varphi_{S}$ & $\circ$ & $\begin{array}{l}\text { Phase der Entstehung einer angeregten laser-induzierten } \\
\text { Blase relativ zum Schallfeld }\end{array}$ \\
\hline$Q$ & $\mathrm{~J}$ & Wärmemenge in der thermischen Grenzschicht \\
\hline$\dot{Q}$ & $\mathrm{~W}$ & $\begin{array}{l}\text { Wärmeleistung der thermischen Grenzschicht } \\
\text { pro Intervall }\end{array}$ \\
\hline$\dot{Q}_{G}$ & W & vom Gas an die Blasenwand abgegebene Wärmeleistung \\
\hline$\dot{Q}_{L}$ & W & Phasenübergangswärmeleistung \\
\hline$R$ & $\mathrm{~m}$ & der aktuelle Blasenradius \\
\hline$\vec{r}_{i}$ & $\mathrm{~m}$ & Ort des Teilchens $i$ \\
\hline$\vec{r}_{12}$ & $\mathrm{~m}$ & Ortsdifferenzvektor zweier Teilchen 1 und 2 \\
\hline$R_{0}$ & $\mathrm{~m}$ & Ruheradius (Gleichgewichtsradius) \\
\hline
\end{tabular}




\begin{tabular}{rll} 
Symbol & Einheit & Bedeutung \\
\hline$R_{\text {max }}$ & $\mathrm{m}$ & Maximalradius \\
$R_{\text {min }}$ & $\mathrm{m}$ & Minimalradius \\
$R_{v d W}$ & $\mathrm{pm}$ & van-der-Waals-Radius eines Atoms \\
$S$ & - & Skalierungsfaktor der Simulation; entspricht der \\
& & Anzahl an Atomen, für die ein Simulationsteilchen steht \\
$\sigma_{T}$ & $\mathrm{~m}^{2}$ & totaler Stoßquerschnitt \\
$\sigma_{R}$ & $\mathrm{~m}^{2}$ & totaler Reaktionsquerschnitt \\
$T_{\varnothing}$ & $\mathrm{K}$ & Maximum der räumlich gemittelten Durchschnittstemperatur \\
$t_{A^{*}}$ & $\mathrm{~s}$ & Lebenszeit des angeregten Zustands \\
& & im Lindemann - Mechanismus \\
$T_{i}$ & $\mathrm{~K}$ & Temperatur der auf die Blasenwand einfallenden Teilchen \\
$T_{\text {init }}$ & $\mathrm{K}$ & Anfangstemperatur der Teilchen. Zumeist $T_{\text {init }}=T_{w a t e r}$ \\
$t_{K o l l}$ & $\mathrm{~s}$ & Zeitpunkt des Hauptkollaps bei laser-induzierten Blasen \\
$T_{r}$ & $\mathrm{~K}$ & Temperatur der von der Blasenwand reflektierten Teilchen \\
$t_{R \text { min }}$ & $\mathrm{s}$ & Zeitpunkt des minimalen Blasenradius \\
$T_{W}$ & $\mathrm{~K}$ & Temperatur der Blasenwand \\
$T_{\text {water }}$ & $\mathrm{K}$ & Wassertemperatur \\
$\vec{v}_{i}$ & $\mathrm{~m} / \mathrm{s}$ & Geschwindigkeit des Teilchens $i$ \\
$V_{M Z}$ & $\mathrm{~m}{ }^{3}$ & Volumen einer Messzelle \\
$v_{N G, i n i t}$ & $\mathrm{~m} / \mathrm{s}$ & Geschwindigkeit der Edelgasteilchen bei Start der Simulation \\
$v_{N G, \text { max }}$ & $\mathrm{m} / \mathrm{s}$ & maximale Geschwindigkeit der Edelgasteilchen \\
$v_{W}$ & $\mathrm{~m} / \mathrm{s}$ & Geschwindigkeit der Blasenwand \\
$v_{W, \text { init }}$ & $\mathrm{m} / \mathrm{s}$ & Geschwindigkeit der Blasenwand bei Start \\
& & der MD-Simulation
\end{tabular}




\section{A.1 Naturkonstanten und Abkürzungen}

\begin{tabular}{rrll} 
Symbol & Wert & Einheit & Benennung \\
\hline$R_{\mathrm{g}}$ & $N_{\mathrm{A}} \cdot k_{\mathrm{B}}$ & $\mathrm{J} / \mathrm{K} / \mathrm{mol}$ & universelle Gaskonstante \\
$N_{\mathrm{A}}$ & $6,022 \cdot 10^{23}$ & $1 / \mathrm{mol}$ & Avogadro-Konstante \\
$k_{\mathrm{B}}$ & $1,381 \cdot 10^{-23}$ & $\mathrm{~J} / \mathrm{K}$ & Boltzmann-Konstante \\
$g_{\mathrm{z}}$ & 9.81 & $\mathrm{~m} / \mathrm{s}^{2}$ & Gravitationsbeschleunigung der Erde \\
$\varrho_{W}$ & 996,6 & $\mathrm{~kg} / \mathrm{m}^{3}$ & Dichte von Wasser \\
$\nu$ & $8,569 \cdot 10^{-7}$ & $\mathrm{~m}^{2} / \mathrm{s}$ & kinematische Viskosität von Wasser \\
$\sigma$ & 72,75 & $\mathrm{mN} / \mathrm{m}$ & Oberflächenspannung von Wasser \\
$c_{W}$ & 1481 & $\mathrm{~m} / \mathrm{s}$ & Schallgeschwindigkeit in Wasser \\
$q_{0}$ & $1,602 \cdot 10^{-19}$ & As & Elektronenladung \\
$\lambda_{W}$ & 0.609 & $\mathrm{~J} /(\mathrm{msK})$ & Wärmeleitzahl von Wasser
\end{tabular}

\section{Abkürzungen}

Abkürzungen Bedeutung

MBSL Mehrblasenlumineszenz

('Multi Bubble SonoLuminescence')

SBSL Einzelblasenlumineszenz

('Single Bubble SonoLuminescence')

MDS Molekulardynamiksimulation

N-S Navier-Stokes 


\section{Literaturverzeichnis}

[1] C. Pfleiderer: Strömungsmaschinen. Springer, Berlin, 1957. 5

[2] S. LuTHER: Theoretische Beschreibung und experimentelle Untersuchung raum-zeitlicher Strukturbildung in akustischen Kavitationsblasenfeldern. Doktorarbeit, Drittes Physikalisches Institut, Universität Göttingen, 2000. 5

[3] B. REWALD: Impact of climate change-induced drought on tree root hydraulic properties and competition belowground. Doktorarbeit, Universität Göttingen, 2008. 6

[4] R. GEISLER: Untersuchungen zur laserinduzierten Kavitation mit Nanosekunden- und Femtosekundenlasern. Doktorarbeit, Drittes Physikalisches Institut, Universität Göttingen, 2003. 6, 11, 12, 17, 19

[5] D. KRÖNInger: Particle Tracking Velocimetry - Messungen an kollabierenden Kavitationsblasen. Doktorarbeit, Drittes Physikalisches Institut, Universität Göttingen, 2008. 6

[6] R. Mettin: Bubble and particle dynamics in acoustic fields: Modern trends and applications, Kapitel Bubble structures in acoustic cavitation, Seiten 1-36. Research Signpost, Kerala (India), 2005. 7

[7] U. Parlitz, R. Mettin, S. Luther, I. Akhatov, M. Voss und W. LAUTERBorn: Spatio-temporal dynamics of acoustic cavitation bubble clouds. Phil. Trans. R. Soc. Lond. A 357, 313-334, 1999. 7

[8] W. Lauterborn, T. Kurz, R. Mettin und C. D. Ohl: Experimental and theoretical bubble dynamics. In: I. Prigogine und S. A. Rice (Herausgeber): Advanves in Chemical Physics, Band 110, Kapitel V, Seiten 295-380. John Wiley \& Sons, 1999. 7, 18 
[9] P. KосH: Partikelmodellierung der Strukturbildung akustischer Kavitationsblasen in Wechselwirkung mit dem Schalldruckfeld. Doktorarbeit, Drittes Physikalisches Institut, Universität Göttingen, 2006. 7, 142

[10] O. Lindau: Dynamik und Lumineszenz lasererzeugter Kavitationsblasen. Diplomarbeit, Drittes Physikalisches Institut, Universität Göttingen, 1998. 7

[11] E. N. HARVEY: A history of luminescence from the earliest times until 1900. Am. Phil. Soc. Press, Philadelphia, 1957. 10

[12] E. Domnitch und D. Gelfand: Camera lucida: A three-dimensional sonochemical observatory. Leonardo 37(5), 391-396, 2004. 10

[13] D. F. Gaitan: An experimental investigation of acoustic cavitation in gaseous liquids. Doktorarbeit, University of Mississippi, 1990. 10

[14] D. Gaitan, L. Crum, C. Church und R.A.Roy: Sonoluminescence and bubble dynamics for a single, stable cavitation bubble. J. Acoust. Soc Am. 91, 3166-3183, 1992. 10

[15] B. P. BArber und S. J. PUtTerman: Light scattering measurements of the repetitive supersonic implosion of a sonoluminescing bubble. Phys. Rev. Lett. 69(26), 3839-3842, 1992. 11

[16] Y. Tian, D. Riegel, J. Ketterling und R. E. Apfel: Direct observation of microbubble oscillations. The Journal of the Acoustical Society of America 98(5), 2922-2922, 1995. 11

[17] C. Wu und P. H. RoBeRTs: Shock-wave propagation in a sonoluminescing gas bubble. Phys. Rev. Lett. 70, 3424-3427, 1993. 12, 14, 22, 48

[18] W. C. Moss, D. B. Clarke, J. W. White und D. A. Young: Hydrodynamic simulations of bubble collapse and picosecond sonoluminescence. Phys. Fluids 6, 2979-2985, 1994. 12

[19] H. P. Greenspan und A. NADim: On sonoluminescence of an oscillating gas bubble. Physics of Fluids A: Fluid Dynamics 5(4), 1065-1067, 1993. 12

[20] V. Q. Vuong, A. J. Szeri und D. A. Young: Shock formation within sonoluminescence bubbles. Physics of Fluids 11(1), 10-17, 1999. 12, 59 
[21] B. P. Barber und S. J. Putterman: Observation of synchronous picosecond sonoluminescence. Nature 352, 318-320, 1991. 12

[22] W. C. Moss, D. B. Clarke, J. W. White und D. A. Young: Sonoluminescence and the prospects for table-top micro-thermonuclear fusion. Phys. Lett. A 211, 69-74, 1996. 12, 119

[23] R. Hiller, S. J. Putterman und B. P. Barber: Spectrum of synchronous picosecond sonoluminescence. Phys. Rev. Lett. 69(8), 1182-1184, 1992. 12, 111

[24] B. Gompf, R. Günther, G. Nick, R. Pecha und W. Eisenmenger: Resolving sonoluminescence pulse width with time-correlated single photon counting. Phys. Rev. Lett. 79, 1405-1408, 1997. 13, 61, 124

[25] R. Pecha, B. Gompf, G. Nick, Z. Wang und W. Eisenmenger: Resolving the sonoluminescence pulse shape with a streak camera. Phys. Rev. Lett. 81, 717-720, 1998. 13, 61, 124

[26] R. A. Hiller, S. J. Putterman und K. R. Weninger: Time-resolved spectra of sonoluminescence. Phys. Rev. Lett. 80(5), 1090-1093, 1998. 13, 61

[27] V. Q. Vuong und A. J. Szeri: Sonoluminescence and diffusive transport. Physics of Fluids 8(9), 2354-2364, 1996. 13, 22, 59

[28] K. Yasui: Alternative model of single-bubble sonoluminescence. Phys. Rev. E 56, 6750-6760, 1997. 13, 14, 21, 63, 67, 73, 82, 87, 102

[29] B. P. Barber, C. C. Wu, R. Löfstedt, P. H. Roberts und S. J. Putterman: Sensitivity of sonoluminescence to experimental parameters. Phys. Rev. Lett. 72(9), 1380-1383, 1994. 13, 111

[30] K. R. Weninger, R. Hiller, B. Barber, D. Lacoste und S. J. PutTERMAN: sonoluminescence from single bubbles in nonaqueous liquids: New parameter space for sonochemistry. J. Phys. Chem. 99(39), 14195-14197, 1995. 13, 111

[31] R. Hiller, K. Weninger, S. J. Putterman und B. P. Barber: Effect of noble gas doping in single-bubble sonoluminescence. Science 266(5183), 248-250, 1994. 13, 125 
[32] R. Löfstedt, K. Weninger, S. Putterman und B. P. Barber: Sonoluminescing bubbles and mass diffusion. Phys. Rev. E 51(5), 4400-4410, 1995. 13

[33] D. Lohse, M. Brenner, T. Dupont, S. Hilgenfeldt und B. JohnSTON: Sonoluminescing air bubbles rectify argon. Phys. Rev. Lett. 78, 13591362, 1996. 13, 119, 125

[34] J. A. Ketterling und R. E. Apfel: Experimental validation of the dissociation hypothesis for single bubble sonoluminescence. Phys. Rev. Lett. 81(22), 4991-4994, 1998. 13, 125

[35] T. J. Matula und L. A. CRum: Evidence for gas exchange in singlebubble sonoluminescence. Phys. Rev. Lett. 80(4), 865-868, 1998. 13, 125

[36] B. D. Storey und A. J. Szeri: Argon rectification and the cause of light emission in single-bubble sonoluminescence. Phys. Rev. Lett. 88(7), 074301, 2002. 13

[37] K. YAsui: Chemical reactions in a sonoluminescing bubble. J. Phys. Soc. Jpn. 1997, 2911-2920, 1997. 14

[38] B. D. Storey und A. J. Szeri: Water vapour, sonoluminescence and sonochemistry. Proc. Roy. Soc. Lond. A 456, 1685, 2000. 14, 22, 65, 67, 70, 78, 79, 82, 102, 188

[39] M. P. Brenner, S. Hilgenfeldt und D. Lohse: Single-bubble sonoluminescence. Rev. Mod. Phys. 74, 425-484, 2002. 14

[40] T. Lepoint, D. D. Pauw, F. Lepoint-Mullie, M. Goldman und A. Goldman: Sonoluminescence: An alternative 'electrohydrodynamic' hypothesis. J. Acoust. Soc. Am. 101(4), 2012-2030, 1997. 14

[41] A. Prosperetti: A new mechanism for sonoluminescence. J. Acoust. Soc. Am. 101, 2003-2007, 1997. 14

[42] V. Griffing: The chemical effects of ultrasonics. J. Chem. Phys. 20, 939-942, 1952. 14

[43] K. YAsui: Temperature in multibubble sonoluminescence. J. Chem. Phys. 115(7), 2893-2896, 2001. 14, 111 
[44] L. Frommhold und A. A. Atchley: Is sonoluminescence due to collision-induced Emission? Phys. Rev. Lett. 73(21), 2883-2886, 1994. 14

[45] L. Frommhold: Electron-atom bremsstrahlung and the sonoluminescence of rare gas bubbles. Phys. Rev. E 58(2), 1899-1905, 1998. 14

[46] W. C. Moss, D. B. Clarke und D. A. Young: Calculated pulse widths and spectra of a single sonoluminescing bubble. Science 276(5317), 13981401, 1997. 14, 61

[47] D. Hammer und L. Frommhold: Spectra of sonoluminescent rare-gas bubbles. Phys. Rev. Lett. 85(6), 1326-1329, 2000. 14

[48] C. Wu und P. Roberts: A model of sonoluminescence. Proc. R. Soc. Lond. A 455, 323-349, 1994. 14, 48

[49] T. J. Matula, R. A. Roy, P. D. Mourad, W. B. McNamara III und K. S. Suslick: Comparison of multibubble and single-bubble sonoluminescence spectra. Phys. Rev. Lett. 75(13), 2602-2605, 1995. 15

[50] K. YAsui: Single-Bubble and multibubble sonoluminescence. Phys. Rev. Lett. 83(21), 4297-4300, 1999. 15, 21

[51] J. B. Young, J. A. Nelson und W. Kang: Line emission in singlebubble sonoluminescence. Phys. Rev. Lett. 86(12), 2673-2676, 2001. 15

[52] D. J. Flannigan und K. S. Suslick: Plasma formation and temperature measurement during single-bubble cavitation. Nature 434, 52, 2005. 16

[53] W. Lauterborn: Kavitation durch Laserlicht. Acustica 31, 51, 1974. 17

[54] Y. Tomita und A. Shima: High-speed photographic observations of laserinduced cavitation bubbles in water. Acustica 51, 161, 1990. 17

[55] B. Ward und D. C. Emmony: Direct observation of the pressure developed in a liquid during cavitation-bubble collapse. Applied Physics Letters 59(18), 2228-2230, 1991. 17

[56] J. BILlE: Medizinische Physik Band 3: Medizinische Laserphysik. Springer, Berlin, 2005. 18 
[57] C.-D. Ohl, O. Lindau und W. Lauterborn: Luminescence from spherically and aspherically collapsing laser induced bubbles. Phys. Rev. Lett. 80(2), 393-396, 1998. 18

[58] C.-D. OHL: Probing luminescence from nonspherical bubble collapse. Physics of Fluids 14(8), 2700-2708, 2002. 18

[59] O. LORD RAYLEIGH: On the pressure development in a liquid during the collapse of a spherical cavity. Phil. Mag. 34, 94-98, 1917. 21, 44

[60] M. Plesset: The dynamics of cavitation bubbles. J. Appl. Mech. 16, 277282, 1949. 21, 44

[61] F. Gilmore: The growth or collapse of a spherical bubble in a viscous compressible liquid. California Institute of Technology, Pasadena, 1952. 21, 45

[62] J. B. Keller und M. Miksis: Bubble oscillations of large amplitude. J. Acoust. Soc. Am. 68(2), 628-633, 1980. 21, 45

[63] R. Löfstedt, B. P. Barber und S. J. Putterman: Toward a hydrodynamic theory of sonoluminescence. Physics of Fluids A: Fluid Dynamics 5(11), 2911-2928, 1993. 21

[64] K. YASUI: Effect of liquid temperature on sonoluminescence. Phys. Rev. E 64(1), 016310, 2001. 21, 111

[65] B. D. Storey und A. J. Szeri: A reduced model of cavitation physics for use in sonochemistry. Proc. Roy. Soc. A 457, 1685, 2001. 21

[66] L. Landau und E. Lifshitz: Fluid mechanics. Pergamon, Oxford, 1987. 21,22

[67] L. Kondic, J. I. Gersten und C. Yuan: Theoretical studies of sonoluminescence radiation: Radiative transfer and parametric dependence. Phys. Rev. E 52(5), 4976-4990, 1995. 22

[68] W. Moss, A. Young, J. Harte, J. Levatin, B. Rozsnyai, G. ZimMERMAn und I. Zimmerman: Computed optical emissions from a sonoluminescing bubble. Phys. Rev. E 59, 2986-2992, 1999. 22

[69] G. Hauke, D. Fuster und C. Dopazo: Dynamics of a single cavitating and reacting bubble. Phys. Rev. E 75, 066310, 2006. 22 
[70] E. G. Flekkoy und P. V. Coveney: From Molecular Dynamics to Dissipative Particle Dynamics. Phys. Rev. Lett. 83, 1775, 1999. 22

[71] P. Espanol und M. Revenga: Smoothed dissipative particle dynamics. Phys. Rev. E 67, 026705, 2003. 22

[72] T. Vladimiroff, Y. P. Carignan und A. K. MacPherson: The $d y$ namics of hard spheres in a collapsing spherical container. Molec. Phys. 71, 441, 1990. 22

[73] M. Matsumoto, K. Miyamato, K. Ohguchi und T. Kinjo: Molecular dynamics simulation of a collapsing bubble. Prog. Theor. Phys. Suppl. 138, 728, 2000. 22

[74] S. J. Ruuth, S. Putterman und B. Merriman: Molecular dynamics simulations of the response of a gas to a spherical piston: Implications for sonoluminescence. Phys. Rev. E 66, 036310, 2002. 23

[75] H. Okumura und N. Ito: Nonequilibrium molecular dynamics simulations of a bubble. Phys. Rev. E 67(4), 045301, 2003. 23

[76] P. Gaspard und J. Lutsko: Imploding shock wave in a fluid of hard-core particles. Phys. Rev. E. 70(2), 026306, 2004. 23

[77] B. Metten: Molekulardynamik-Simulationen zur Sonolumineszenz. Der Andere Verlag, Osnabrück, 2001. 24, 34, 52, 84, 105, 174, 190, 193

[78] B. Metten und W. Lauterborn: Molecular dynamics approach to single-bubble sonoluminescence. In: W. LAUTERBORN und T. KuRz (Herausgeber): Nonlinear Acoustics at The Turn of the Millenium: Proc. 15th Int. Symp. on Nonlinear Acoustics, Seite 429, Melville, New York, 2000. 24

[79] D. C. RAPAPORT: The art of molecular dynamics simulation. Cambridge University Press, Cambridge, 2nd. Auflage, 2004. 24, 26

[80] D. SchanZ: Molekulardynamiksimulation kollabierender Sonolumineszenzblasen, Diplom-Arbeit. Drittes Physikalisches Instiut, Universität Göttingen, 2003. 25, 27

[81] S. Miller und S. Luding: Event-driven molecular dynamics in parallel. J. Comp. Phys. 193, 306, 2004. 27 
[82] W. H. Press, S. A. Teukolsky, W. T. Vetterling und B. P. FlanNERY: Numerical recipes in $C$ : The art of scientific computing. Cambridge University Press, Cambridge, 1992. 28, 42, 45

[83] S. F. Foerster, M. Y. Louge, H. Chang und K. Allia: Measurements of the collision properties of small spheres. Physics of Fluids 6(3), 11081115, 1994. 30

[84] L. Labous, A. D. Rosato und R. N. Dave: Measurements of collisional properties of spheres using high-speed video analysis. Phys. Rev. E 56(5), 5717-5725, 1997. 31

[85] J. M. HAILE: Molecular dynamics simulation - elementary methods. Wiley, New York, 1992. 37

[86] F. REIF: Statistische Physik und Theorie der Wärme. Walter de Gruyter Verlag, 3rd. Auflage, 1987. 41

[87] J. Hirschfelder, C. Curtiss und R. Bird: Molecular theory of gases and liquids. Wiley \& Sons, 1954. 41

[88] E. LAx: Taschenbuch für Chemiker und Physiker. Springer-Verlag, 1967. 41

[89] M. Minnaert: On musical air-bubbles and the sounds of running water. Phil. Mag. 16(7), 235-248, 1933. 44

[90] H. Poritsky: The collapse or growth of a spherical bubble or cavity in a viscous fluid. In: 1st US National Congress on Applied Mechanics, Seiten 813-821, New York, 1952. 44

[91] A. Prosperetti und A. Lezzi: Bubble dynamics in a compressible liquid. Part 1. First-order theory. J. Fluid Mech. 168, 457-478, 1986. 45

[92] A. Prosperetti: Sonochemistry and sonoluminescence, Kapitel Oldfashioned bubble dynamics. Kluwer Academic Publishers, Dordrecht / Boston / London, 1999. 45

[93] H. Lin, B. D. Storey und A. J. Szeri: Inertially driven inhomogeneities in violently collapsing bubbles: The validity of the Rayleigh Plesset equation. Journal of Fluid Mechanics 452, 145-162, 2002. 45 
[94] S. Gasstone und R. LovberG: Controlled thermonuclear reactions. van Nostrand, New York, 1960. 48

[95] F. F. Chen: Introduction to plasma physics. Plenum, New York, 1974. 48

[96] H.-Y. KWAK und H. YANG: An aspect of sonoluminescence from hydrodynamic theory. Journal of the Physical Society of Japan 64, 1980-1992, 1995. 51

[97] H.-Y. KWAK und J. NA: Physical processes for single bubble sonoluminescence. Journal of the Physical Society of Japan 66(10), 3074-3083, 1997. 51

[98] M. J. Moran und D. SweIder: Measurements of sonoluminescence temporal pulse shape. Phys. Rev. Lett. 80(22), 4987-4990, 1998. 61

[99] K. R. Weninger, B. P. Barber und S. J. Putterman: Pulsed mie scattering measurements of the collapse of a sonoluminescing bubble. Phys. Rev. Lett. 78(9), 1997. 62

[100] S. Fujikawa und T. Akamatsu: Effects of the non-equilibrium condensation of vapour on the pressure wave produced by the collapse of a bubble in a liquid. J. Fluid Mech. 97, 481-512, 1980. 63

[101] K. YASUI: Effect of non-equilibrium evaporation and condensation on bubble dynamics near the sonoluminescence threshold. Ultrasonics 36, 575-580, 1998. 64

[102] M. Mozurkewich: Aerosol growth and the condensation coefficient of water: A review. Aerosol Sci. Technol 5:2, 223-236, 1986. 67

[103] I. W. EAmes, N. J. MARR und H. SABIR: The evaporation coefficient of water: A review. Int. J. Heat Mass Transf. 40, 2963-2973, 1995. 67

[104] M. Zientara, D. Jakubczyk, G. Derkachov, K. Kolwas und M. Kolwas: Simultaneous determination of mass and thermal accomodation coefficients from temporal evolution of an evaporating water microdroplet. J. Phys. D: Appl. Phys. 38, 1978-1983, 2005. 67

[105] K. YASUI: Variations of liquid temperature at bubble wall near the sonoluminescence yhreshold. J. Phys. Soc. Jap. 65, 2830-2840, 1995. 67, 70, 188 
[106] Y. Q. Li, P. Davidovits, Q. Shi, T. Jayne, C. Kolb und D. WorsNOP: Mass and thermal accomodation coefficient of $\mathrm{H}_{2} \mathrm{O}(\mathrm{g})$ on liquid water as a function of temperature. J. Phys. Chem. 105(47), 10627-10634, 2001. 73

[107] R. Marek und J. Straub: Analysis of the evaporation coefficient and the condensation coefficient of water. Int. J. Heat Mass Transf. 44, 39-53, 2001. 73

[108] M. SpIEGEL: Theory and problems of calculus of finite differences and difference equations. McGraw-Hill, New York, 1971. 73

[109] B. P. Barber, R. A. Hiller, R. Löfstedt, S. J. Putterman und K. Weninger: Defining the unknowns of sonoluminescence. Phys. Rep. 281, 65-143, 1997. 76

[110] S. Putterman und K. Weninger: Sonoluminescence: How bubbles turn sound into light. Annu. Rev. Fluid. Mech. 32, 445-476, 2000. 76

[111] S. Hilgenfeldt, S. Grossmann und D. Lohse: Sonoluminescence light emission. Phys. Fluids 11, 1318-1330, 1999. 76, 111, 112

[112] K. S. SusLICK: Sonochemistry. Science 247(4949), 1439-1445, 1990. 82

[113] K. S. Suslick: The chemical effects of ultrasound. Sci. Am. 260(2), 80-86, 1989. 82

[114] I. ElPiner: Ultrasound: Physical, chemical and biological Effects. Consultants Bureau, New York, 1964. 82

[115] T. J. MASON: Sonochemistry: Current uses and future prospects in the chemical and processing industries. Phil. Trans. R. Soc. Lond. 357(1751), 355-369, 1999. 82

[116] A. Colussi, L. Weavers und M. Hoffmann: Chemical bubble dynamics and quantitative sonochemistry. Journal of Physical Chemistry A 102(35), 6927-6934, 1998. 82

[117] T.Lepoint, F. Lepoint-Mullie und A. Henglein: Sonochemistry and sonoluminescence, Kapitel Single bubble sonochemistry. Kluwer Academic Publishers, Dordrecht / Boston / London, 1999. 82 
[118] G. A. BIRD: Molecular gas dynamics. NASA STI/Recon Technical Report A 76, 40225, 1976. 84

[119] S. L. Cole und J. W. Wilder: Gas phase decomposition by the lindemann mechanism. SIAM J. Appl. Math. 51(6), 1489-1497, 1991. 85

[120] G. P. Smith, D. M. Golden, M. Frenklach, N. W. Moriarty, B. Eiteneer, M. Goldenberg, C. T. Bowman, R. K. Hanson, S. Song, William, C. Gardiner, Jr., V. V. Lissianski und Z. Qin: Gri-Mech: Optimized detailed chemical reaction mechanism of natural gas flames and ignition. http://www.me.berkeley.edu/gri_mech/. 87

[121] V. Kamath und A. Prosperetti: A theoretical study of sonoluminescence. J. Acoust. Soc. Am. 94(1), 248-260, 1993. 87

[122] A. Takami, S. Kato, A. Shimono und S. Koda: Uptake coefficient of OH radical on aqueous surface. Chem. Phys. 231(2-3), 215-227, 1998. 97

[123] K. Yasui, T. Tuziuti, M. Sivakumar und Y. IIda: Theoretical study of single-bubble sonochemistry. J. Chem. Phys. 122(22), 224706, 2005. 97

[124] C. Gong und D. P. HarT: Ultrasound induced cavitation and sonochemical yields. J. Acoust. Soc. Am. 104(5), 2675-2682, 1997. 102

[125] P. JARMAn: Measurements of sonoluminescence from pure liquids and some aqueous solutions. Proceedings of the Physical Society 73(4), 628-640, 1959. 111

[126] P. K. Chendke und H. S. Fogler: Variation of sonoluminescence intensity of water with the liquid temperature. J. Phys. Chem. 89(9), 1673-1677, 1985. 111

[127] V. Q. Vuong, M. M. Fyrillas und A. J. Szeri: The influence of liquid temperature on the sonoluminescence hot spot. J. Acoust. Soc. Am. 104(4), 2073-2076, 1998. 111

[128] G. E. Vazquez und S. J. Putterman: Temperature and pressure dependence of sonoluminescence. Phys. Rev. Lett. 85(14), 3037-3040, 2000. 111

[129] B. P. Barber, K. Weninger, R. Löfstedt und S. Putterman: Observation of a new phase of sonoluminescence at low partial pressures. Phys. Rev. Lett. 74(26), 5276-5279, 1995. 119 
[130] M. Fyrillas und A. Szeri: Dissolution or growth of soluble spherical oscillating bubbles. J. Fluid Mech. 277, 381-407, 1994. 119

[131] G. Mark, A. Tauber, R. Laupert, H. Schuchmann, D. Schulz, A. Mues und C. von Sonntag: OH-radical formation by ultrasound in aqueous solutions - Part II. Ultrason. Sonochem. 5, 41-52, 1998. 124

[132] Y. T. Didenko und S. P. Pugach: Spectra of water sonoluminescence. Journal of Physical Chemistry 98(39), 9742-9749, 1994. 125

[133] B. D. Storey und A. J. Szeri: Mixture segregation within sonoluminescing bubbles. J. Fluid Mech. 396, 203, 1999. 141

[134] F. G. Blake: The onset of cavitation in liquids. I. Cavitation threshold sound pressures in water as a function of temperature and hydrostatic pressure. Technischer Bericht 12, Harvard University, Acoustic Research Laboratory, 1949. 142

[135] K. R. Weninger, C. G. Camara und S. J. Putterman: Observation of bubble dynamics within luminescent cavitation clouds: Sonoluminescence at the nano-scale. Phys. Rev. E 63(1), 016310, 2001. 142, 143, 153, 154, 191

[136] G. Vazquez, C. Camara, S. Putterman und K. Weninger: Sonoluminescence: Nature's smallest blackbody. Opt. Lett. 26, 575-577, 2000. 142

[137] C. Camara, S. Putterman und E. Kirilov: Sonoluminescence from a single bubble Driven at 1 megahertz. Phys. Rev. Lett. 92, 124301, 2004. 142, 143, 152, 191

[138] W. LAUterborn: Zu einer Theorie der Kavitationsschwellen. Acustica 22, 48-54, 1969/1970. 154

[139] W. Lauterborn: Eigenfrequenzen von Gasblasen in Flüssigkeiten. Acustica 20, 14-20, 1968. 154

[140] W. Lauterborn: Resonanzkurven von Gasblasen in Flüssigkeiten. Acustica 23, 73-81, 1970. 154

[141] W. LAUTERBORN: Numerical investigation of nonlinear oscillations of gas bubbles in liquids. J. Acoust, Soc. Am. 59, 283-293, 1974. 155 
[142] U. Parlitz, V. Englisch, Scheffczyk und W. Lauterborn: Bifurcation structure of bubble oscillators. J. Acoust. Soc. Am. 88, 1061-1077, 1990. 155

[143] C. Scheffczyk, U. Parlitz, T. Kurz, W. Knop und W. LauterBORN: Comparison of bifurcation structures of driven dissipative nonlinear oscillators. Phys. Rev. A 43, 6495-6502, 1990. 155

[144] T. Kurz, D. Kröninger, R. Geisler und W. Lauterborn: Optic cavitation in an ultrasonic field. Phys. Rev. E 74, 066307(7), 2006. 169, 177, 178, 179, 180, 181, 182, 184, 192, 193

[145] C.-D. OHL: Luminescence from acoustic-driven laser-induced cavitation bubbles. Phys. Rev. E 61(2), 1497-1500, 2000. 169

[146] I. Akhatov, O. Lindau, A. Topolnikov, R. Mettin, N. Vakhitova und W. LAUTERBorn: Collapse and rebound of a laser-induced cavitation bubble. Physics of Fluids 13(10), 2805-2819, 2001. 169

[147] D. KRÖNINGER: Laserinduzierte Kavitation im Schallfeld. Diplomarbeit, Drittes Physikalisches Institut, Universität Göttingen, 2004. 174, 179

[148] G. R. LiU und M. B. LiU: Smoothed particle hydrodynamics. A meshfree particle method. World Scientific, Singapore, 2003. 193 


\section{Danksagung}

An dieser Stelle möchte ich allen danken, die mich bei der Erstellung dieser Dissertation unterstützt haben. Dabei möchte ich folgende Personen hervorheben:

Herr Prof. Dr. Werner Lauterborn hat diese Arbeit ermöglicht. Er hat mich in seine Arbeitsgruppe aufgenommen und immer für die bestmöglichen Forschungsbedingungen gesorgt. Sein unermüdlicher Einsatz, die Tätigkeit am Dritten Physikalischen Institut so produktiv und dabei so angenehm wie möglich zu machen, hat die tägliche Arbeit sehr erleichtert. Die Ausstattung mit modernster Computer-Hardware, für die er immer gesorgt hat, ermöglichte die umfassenden Rechnungen, die Grundlage meiner Arbeit bilden. Sein immer vorhandenes Interesse an den erreichten Ergebnissen war eine große Motivation.

Herrn Prof. Dr. Martin Rein danke ich für die Übernahme des Korreferats.

Dr. Thomas Kurz danke ich für die Betreuung der Arbeit, die Beantwortung der vielen Fragen, die im Laufe der Zeit auftraten und seine generelle Unterstützung.

Bei Dr. Robert Mettin möchte ich mich für die Klärung vieler kleiner Fragen und die motivierenden Worte bei Problemen bedanken. Ebenso danke ich Herrn Prof. Dr. Ulrich Parlitz für kritische Ratschläge und Hinweise bei vielen Gelegenheiten im Laufe meiner Promotionsarbeit

Prof. Dr. Thierry Lepoint ermöglichte mir einen interessanten Forschungsaufenthalt am Institut Meurice in Brüssel. Dafür und für seine herzliche Art, mit der er mich dort aufgenommen hat danke ich ihm sehr.

Bei den für die Technik des Dritten Physikalischen Instituts verantwortlichen Personen Thomas Geiling und Gerhard König sowie Dr. Hans Werner Strube möchte ich mich für die hervorragende Rechner-Infrastruktur bedanken. Die sofortige Lösung auftretender Probleme sowie das gewissenhafte Backup aller Daten haben ein beruhigtes und immer effizientes Arbeiten ermöglicht. 
Gisa Kirschmann-Schröder half mir in allen Fragen der Fotographie und Bildbearbeitung. Dafür, dass sie immer aufmunternde Worte fand und eine positive Einstellung verbreitete danke ich ihr herzlich.

Den heutigen und früheren Angehörigen des Sekretariats - Elke Zech, Ulrike Schwartau und Anne-Katrin Kunze danke ich für die schnelle und zuverlässige Hilfe in formalen Dingen.

Den Mitarbeitern der elektronischen und mechanischen Werkstatt danke ich für die stets schnelle und hervorragende Unterstützung bei technischen Fragen und Problemen.

Ich danke allen weiteren Mitgliedern des Dritten Physikalischen Instituts für die stets angenehme Atmosphäre. Meine Mitstreiter beim Projekt Dissertation - Jörg Dittmar, David Engster, Dr. Philipp Koch, Karsten Köhler und Dennis Kröninger - möchte ich besonders hervorheben.

Die Arbeit wurde vom Graduiertenkolleg „Strömungsinstabilitäten und Turbulenz" finanziell unterstützt.

Meine Familie gab mir stets großen Rückhalt bei meinem Dissertationsvorhaben und hat mich in Schwierigkeiten immer gestärkt. Ohne ihre Unterstützung wäre mein Studium und diese Promotion nicht möglich gewesen. Dafür danke ich sehr Günther, Inge, Kurt und Ruth Schanz. Ich wünschte, wir könnten diesen Tag zu fünft feiern.

Meiner Freundin Kinga Varga danke ich von Herzen dafür, dass sie mir immer den Rücken freihielt und für ihr Verständnis, wenn die Arbeit manchmal viel Platz einnahm.

Patrick Neukäter und Dr. Volker Hesse haben immer mit Interesse meine Fortschritte verfolgt und in großen Teilen zu einer erfolgreichen Erholung in freien Zeiten beigetragen. 


\title{
Lebenslauf
}

\author{
Name: Daniel Alexander Schanz \\ Geboren: 22. September 1976, Mannheim \\ Eltern: Günther und Inge Schanz \\ Staatsangehörigkeit: deutsch
1982 - 1986 Besuch der Grundschule Elliehausen
1986 - 1988 Besuch der Orientierungsstufe Leinbergschule, Göttingen
1988 - 1995 Besuch des Felix-Klein-Gymnasium, Göttingen
1995 Abschluss mit der Allgemeinen Hochschulreife
1995 - 1996 Zivildienst in der Christophorusschule, Göttingen
1996 - 2003 Diplomstudiengang Physik an der Georg-August-Universität Göttingen \\ Februar 2003 Erwerb des Hochschulgrades Diplom-Physiker \\ März 2003 Beginn der Promotion Physik an der \\ Georg-August-Universität, Göttingen \\ Feb. 2003 - März 2005 Stipendiat des Graduiertenkollegs \\ „Strömungsinstabilitäten und Turbulenz“ \\ Apr. 2005 - Apr. 2008 Anstellung am Dritten Physikalischen Institut \\ der Georg-August-Universität Göttingen \\ als wissenschaftlicher Mitarbeiter
}

